'II|||||||||||

Amchitka Island, Alaska, Biological Monitoring Report 2011 Sampling Results

September 2013 
This page intentionally left blank 


\section{Amchitka Island, Alaska Biological Monitoring Report 2011 Sampling Results}

September 2013 
This page intentionally left blank 


\section{Contents}

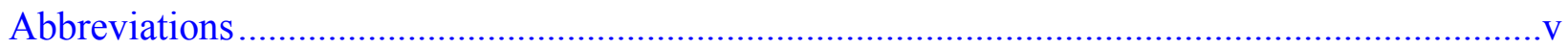

Executive Summary .................................................................................................... vii

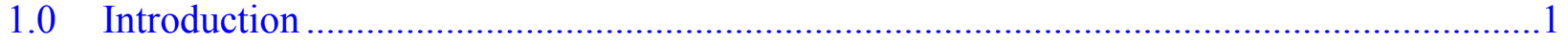

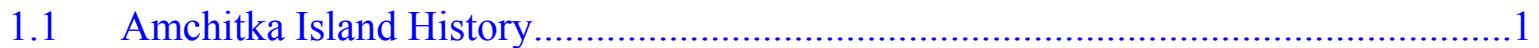

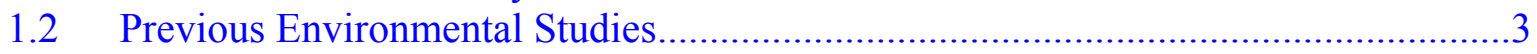

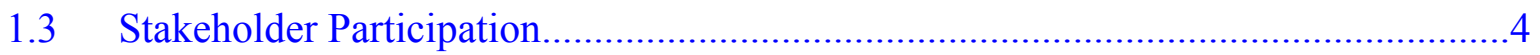

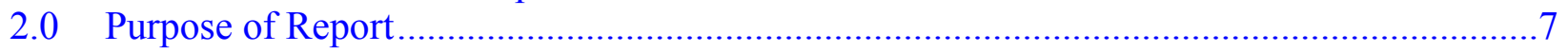

$2.1 \quad$ Data Quality Objectives ………………………….........................................

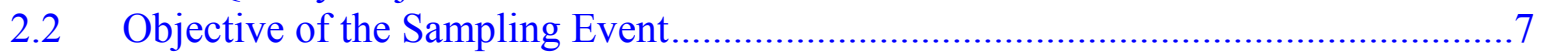

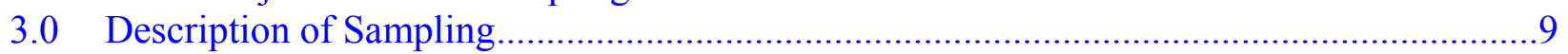

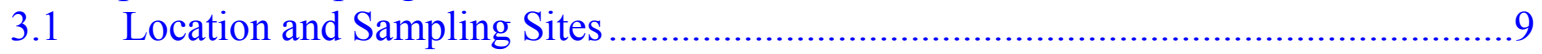

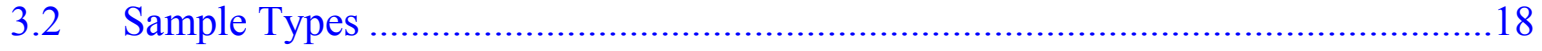

3.3 Sample Collection and Preparation .....................................................................19

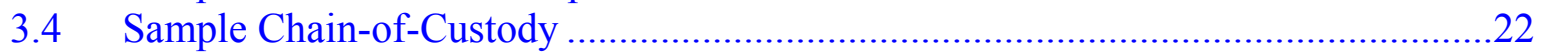

4.0 Laboratory Analysis, Quality Assurance, and Data Validation Procedures..........................25

4.1 Target Radionuclides and Selection Criteria for Methods of Analysis ......................25

4.2 Sample Laboratory Preparation Methods ..................................................................26

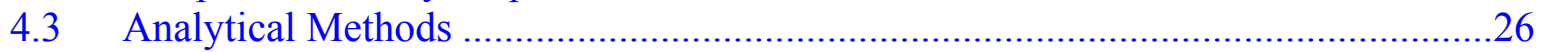

4.4 Quality Assurance and Data Validation ................................................................26

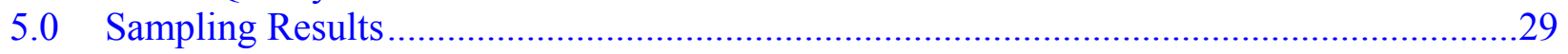

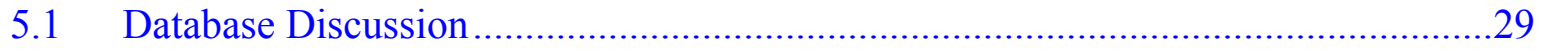

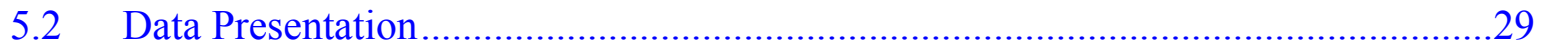

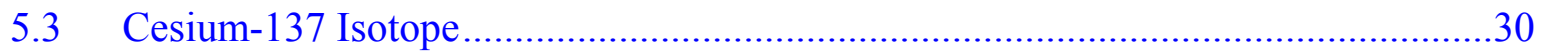

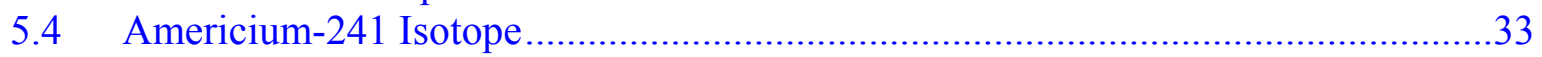

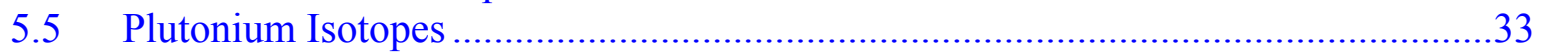

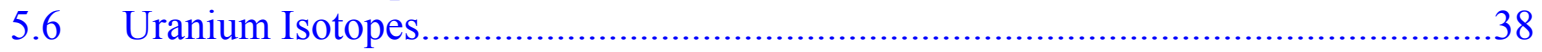

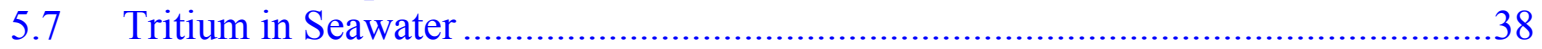

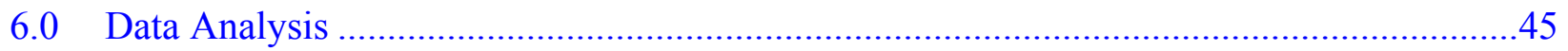

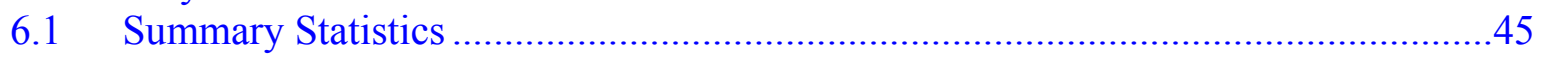

6.1.1 Cesium-137 Isotope …………………………...................................45

6.1.2 Americium-241 Isotope …………………….....................................45

6.1.3 Plutonium Isotopes............................................................................4

6.1.4 Uranium Isotopes ..............................................................................4

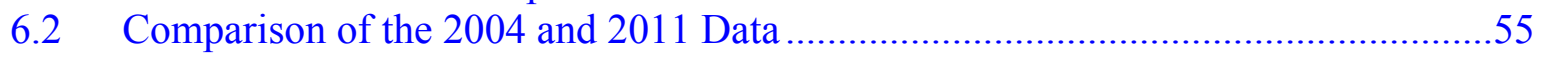

6.3 Comparison of Amchitka and Adak Data Collected in 2011 .....................................56

6.4 Comparison of 2011 Data with CRESP 2004 Data, Literature Sources, and

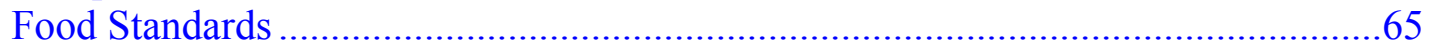

7.0 Human Health — Subsistence- and Commercial-Catch Seafood Consumption .....................67

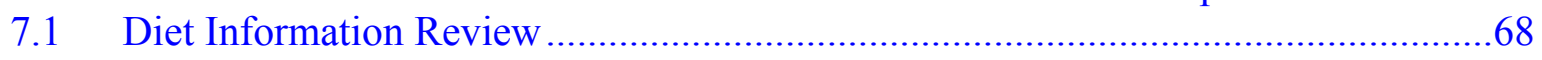

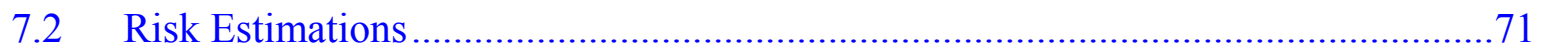

7.2.1 Slope Factors........................................................................................

7.2.2 Exposure Point Concentrations or Radionuclide Concentrations in

Marine Species.....................................................................................

7.2.2.1 EPCs for Sampled Species .........................................................76

7.2.2.2 EPCs for Other Fish and Clams ...............................................76

7.2.2.3 EPCs for Sea Lion and Seal ....................................................76 
7.2.2.4 EPCs for Salmon ................................................................77

7.2.2.5 EPCs for Crustaceans and Sea Birds........................................78

7.2.3 Risk Estimates...............................................................................

7.3 Summary of Potential Seafood Ingestion Risk Analysis.............................................90

7.4 Ecological Health Evaluation (Using the RESRAD-BIOTA Code) ..........................90

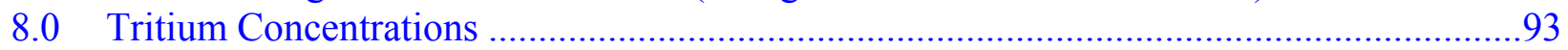

9.0 Cesium-137 Concentrations in Lichen, Soil Beneath Lichen, and Marine Sediment..........95

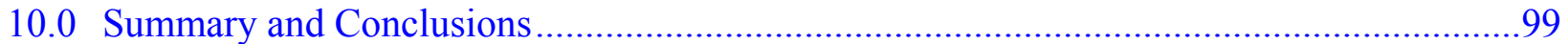

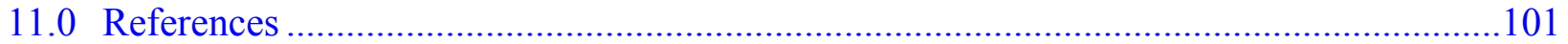

\section{Figures}

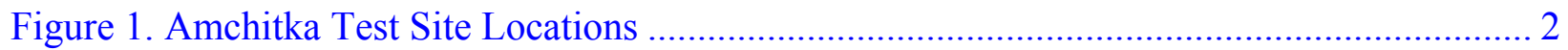

Figure 2. Amchitka Island Sample Locations ....................................................................... 10

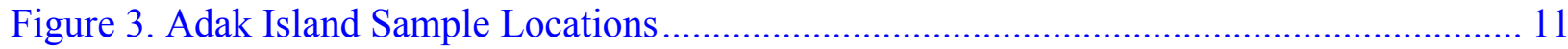

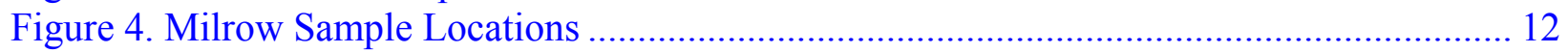

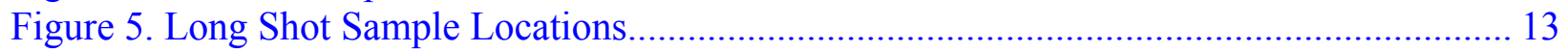

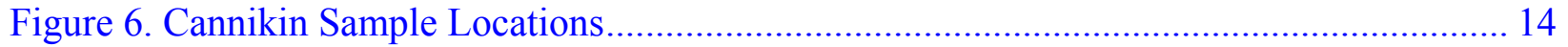

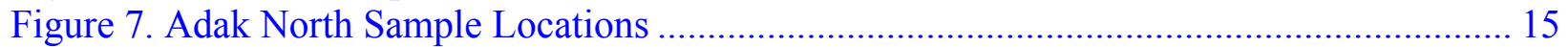

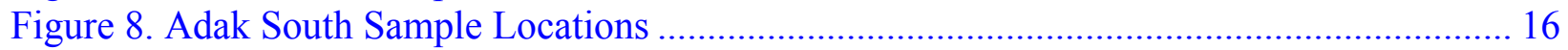

Figure 9. Cannikin Seawater Sample Locations ………....................................................... 17

Figure 10. ${ }^{137} \mathrm{Cs}$ in Selected Marine Vegetation, Fauna, and Gull Eggs ..................................... 31

Figure 11. ${ }^{137} \mathrm{Cs}$ in Dolly Varden, Lichen, Soil Beneath the Lichen, and Marine Sediment........ 32

Figure 12. ${ }^{241} \mathrm{Am}$ in Selected Marine Vegetation, Fauna, and Gull Eggs................................... 34

Figure 13. ${ }^{241} \mathrm{Am}$ in Dragon Kelp, Rockfish, Greenling, Irish Lord, Octopus, and Rockweed.... 35

Figure 14. ${ }^{239} \mathrm{Pu}$ in Selected Marine Vegetation, Fauna, and Gull Eggs ..................................... 36

Figure 15. ${ }^{240} \mathrm{Pu}$ in Selected Marine Vegetation, Fauna, and Gull Eggs ........................................ 37

Figure 16. ${ }^{234} \mathrm{U}$ in Selected Marine Vegetation, Fauna, and Gull Eggs........................................ 39

Figure 17. ${ }^{235} \mathrm{U}$ in Selected Marine Vegetation, Fauna, and Gull Eggs...................................... 40

Figure 18. ${ }^{238} \mathrm{U}$ in Selected Marine Vegetation, Fauna, and Gull Eggs...................................... 41

Figure 19. Zoom view of ${ }^{234} \mathrm{U}$ in Species and Media with Concentrations $<25 \mathrm{pCi} / \mathrm{kg}$................ 42

Figure 20. Zoom View of ${ }^{235} \mathrm{U}$ in Species and Media with Concentrations $<1 \mathrm{pCi} / \mathrm{kg}$.................. 43

Figure 21. Zoom View of ${ }^{238} \mathrm{U}$ in Species and Media with Concentrations $<1 \mathrm{pCi} / \mathrm{kg}$................. 44

Figure 22. 2011 Results That Exceed 2004 Results …………………..................................... 58

Figure 23. Comparison of Dry-Weight Concentrations (pCi/ $/ \mathrm{kg}$ ) at Amchitka and Adak ............. 60

Figure 24. Measure of Difference Between Amchitka and Adak Concentrations ........................ 61

Figure 25. Measurements of Isotopes and Species That Showed Statistically Significant Differences $(\mathrm{p}<0.05)$

Figure 26. Cumulative Probability Distribution of the Ratio of Differences Between Sites to

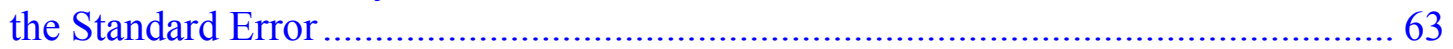

Figure 27. Measure of Amchitka and Adak Plutonium and Uranium Isotope Concentrations .... 64 


\section{Tables}

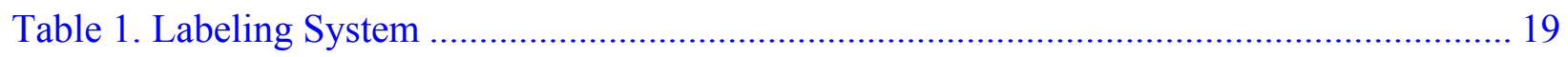

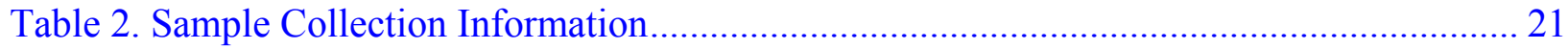

Table 3. Collection and Preparation of Samples.......................................................................... 23

Table 4. Techniques for Measurement of Target Radionuclide ............................................... 25

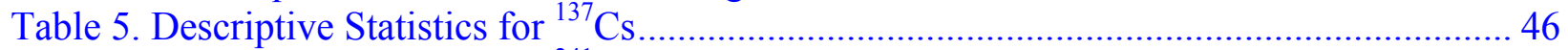

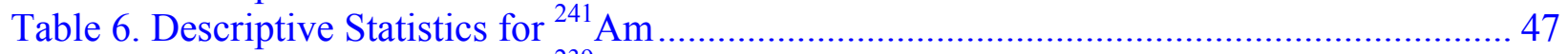

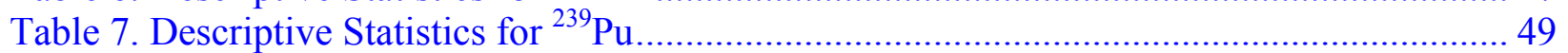

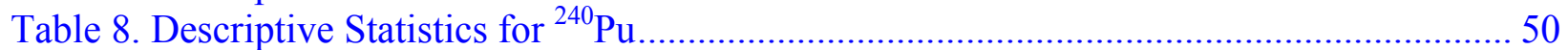

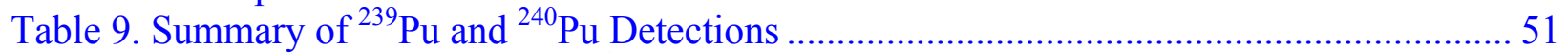

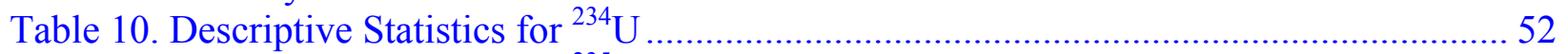

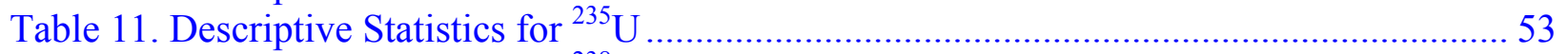

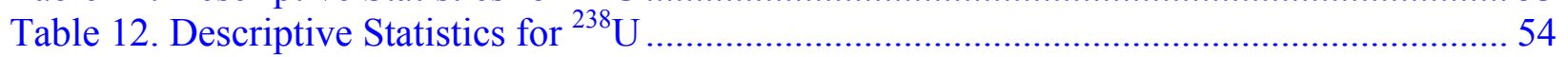

Table 13. Summary of Marine Species Sampled in Both 2004 (CRESP) and 2011 ..................... 55

Table 14. 2011 Results That Exceed 2004 (CRESP) Results …………………......................... 57

Table 15. Comparison of Amchitka Data to Worldwide Values and Food Safety Standards ...... 65

Table 16. Average Daily Intake Rates and Distributions of Seafood ............................................. 70

Table 17. Radionuclide Slope Factors Used for Estimating Risk for the Ingestion Pathway ...... 72

Table 18. Risk Calculation EPCs-Maximum Values of the Amchitka 2011 Sampling Data .... 72

Table 19. Risk Calculation EPCs-UCL ${ }_{95}$ Values of the Amchitka 2011 Sampling Data ........... 73

Table 20. Risk Calculation EPCs-Maximum Values of the Adak 2011 Sampling Data ............ 74

Table 21. Risk Calculation EPCs-UCL 95 Values of the Adak 2011 Sampling Data .................. 75

Table 22. BAFs Used for Estimating Radionuclide Concentrations in Marine Species................ 77

Table 23. Summary of Risk Estimates for the Seafood Ingestion Pathway ................................. 79

Table 24. Potential Risks to an Aleut Consuming Seafood Harvested from Amchitka and Adak Using the St. Paul Diet Data and Maximum Measured Concentrations............. 80

Table 25. Potential Risks to an Aleut Consuming Seafood Harvested from Amchitka and Adak Using the St. Paul Diet Data and UCL 95 Concentrations ................................... 81

Table 26. Potential Risks to an Aleut Consuming Seafood Harvested from Amchitka and Adak Using the Atka Diet Data and Maximum Measured Concentrations ................. 82

Table 27. Potential Risks to an Aleut Consuming Seafood Harvested from Amchitka and Adak Using the Atka Diet Data and UCL 95 Concentrations ....................................... 83

Table 28. Potential Risks to an Aleut Consuming Seafood Harvested from Amchitka and Adak Using the Nikolski Diet Data and Maximum Measured Concentrations ........... 84

Table 29. Potential Risks to an Aleut Consuming Seafood Harvested from Amchitka and Adak Using the Nikolski Diet Data and UCL 95 Concentrations ................................. 85

Table 30. Potential Risks to an Aleut Consuming Seafood Harvested from Amchitka and Adak Using Unalaska Diet Data and Maximum Measured Concentrations ................ 86

Table 31. Potential Risks to an Aleut Consuming Seafood Harvested from Amchitka and Adak Using the Unalaska Diet Data and UCL S5 Concentrations ................................ 87

Table 32. Potential Risks to an Aleut Consuming Seafood Harvested from Amchitka and Adak Using the Composite Diet Data and Maximum Measured Concentrations........ 88

Table 33. Potential Risks to an Aleut Consuming Seafood Harvested from Amchitka and Adak Using the Composite Diet Data and UCL ${ }_{95}$ Concentrations ............................. 89

Table 34. Tier 1 Analysis of Potential Ecological Risks to Marine Biota....................................... 92

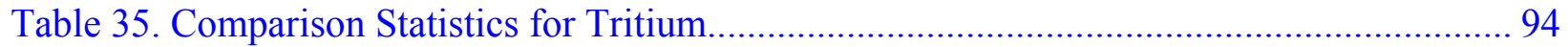


Table 36. ${ }^{134} \mathrm{Cs}$ and ${ }^{137} \mathrm{Cs}$ in Lichen, Soil Beneath the Lichen, and Marine Sediment, Amchitka and Adak Islands ............................................................................ 96

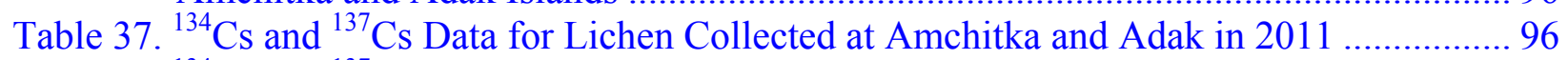

Table 38. ${ }^{134} \mathrm{Cs}$ and ${ }^{137} \mathrm{Cs}$ Data for Soil Collected at Amchitka and Adak in 2011 .................. 97

Table 39. ${ }^{134}$ Cs and ${ }^{137}$ Cs Data for Sediment Collected at Amchitka and Adak in 2011 ........... 97

Table 40. ${ }^{137} \mathrm{Cs}$ in Lichen at Amchitka and Adak Island 1970-1997 ....................................... 97

\section{Appendixes}

Appendix A Photographs of the Species Sampled

Appendix B Sample Identification

Appendix C Chain of Custody

Appendix D Laboratory Analytical Procedures

Appendix E Individual Sample Listing of Wet Weights, Dry Weights, and Ash Weights

Appendix F Laboratory Quality Assurance and Data Validation Procedures

Appendix G Activity Concentration of ${ }^{137} \mathrm{Cs}$

Appendix $\mathrm{H}$ Activity Concentration of ${ }^{241} \mathrm{Am}$

Appendix I Activity Concentration of Plutonium Isotopes

Appendix J Activity Concentration of Uranium Isotopes

Appendix K Tritium Results

Appendix L 2004 (CRESP) and 2011 Data Comparison Summary 


\section{Abbreviations}

$\begin{array}{ll}{ }^{241} \mathrm{Am} & \text { americium-241 } \\ { }^{134} \mathrm{Cs} & \text { cesium-134 } \\ { }^{137} \mathrm{Cs} & \text { cesium-137 } \\ { }^{3} \mathrm{H} & \text { tritium } \\ { }^{210} \mathrm{~Pb} & \text { lead-210 } \\ { }^{210} \mathrm{Po} & \text { polonium-210 } \\ { }^{239} \mathrm{Pu} & \text { plutonium-239 } \\ { }^{240} \mathrm{Pu} & \text { plutonium-240 } \\ { }^{234} \mathrm{U} & \text { uranium-234 } \\ { }^{235} \mathrm{U} & \text { uranium-235 } \\ { }^{236} \mathrm{U} & \text { uranium-236 } \\ { }^{238} \mathrm{U} & \text { uranium-238 }\end{array}$

ADEC Alaska Department of Environmental Conservation

AEC U.S. Atomic Energy Commission

ANL Argonne National Laboratory

APIA Aleutian Pribilof Islands Association

BAF bioaccumulation factor

BCG Biota Concentration Guide

COC chain-of-custody

CRESP Consortium for Risk Evaluation and Stakeholder Participation

DOD U.S. Department of Defense

DOE U.S. Department of Energy

DQO data quality objective

EPA U.S. Environmental Protection Agency

EPC exposure point concentration

g gram

g/day grams per day

HDPE high-density polyethylene

IAEA International Atomic Energy Agency

ICP-MS inductively coupled plasma mass spectrometry

kg kilogram

$\mathrm{kPa} \quad$ kilopascal 
L liter

LBV lower-box value

LLNL Lawrence Livermore National Laboratory

LM Office of Legacy Management

LTHMP Long-term Hydrologic Monitoring Program

LTS\&M long-term surveillance and maintenance

MDC minimum detectable concentration

mg milligram

$\mathrm{mL} \quad$ milliliter

oz ounce

$p \quad$ probability

$\mathrm{pCi} \quad$ picocuries

$\mathrm{pCi} / \mathrm{L} \quad$ picocuries per liter

$\mathrm{rad} \quad$ radiation absorbed dose

$\mathrm{R} / \mathrm{V} \quad$ research vessel

$\sigma \quad$ sigma

SD standard deviation

SF slope factor

TU Tritium Unit

UAF University of Alaska Fairbanks

UBV upper-box value

USFWS U.S. Fish and Wildlife Service 


\section{Executive Summary}

The Long-Term Surveillance and Maintenance (LTS\&M) Plan for the U.S. Department of Energy (DOE) Office of Legacy Management (LM) Amchitka Island sites describes how LM plans to conduct its mission to protect human health and the environment at the three nuclear test sites located on Amchitka Island, Alaska.

Amchitka Island, near the western end of the Aleutian Islands, is approximately 1,340 miles west-southwest of Anchorage, Alaska. Amchitka is part of the Aleutian Island Unit of the Alaska Maritime National Wildlife Refuge, which is administered by the U.S. Fish and Wildlife Service (USFWS). Since World War II, Amchitka has been used by multiple U.S. government agencies for various military and research activities. From 1943 to 1950, it was used as a forward air base for the U.S. Armed Forces. During the middle 1960s and early 1970s, the U.S. Department of Defense (DOD) and the U.S. Atomic Energy Commission (AEC) used a portion of the island as a site for underground nuclear tests. During the late 1980s and early 1990s, the U.S. Navy constructed and operated a radar station on the island.

Three underground nuclear tests were conducted on Amchitka Island. DOD, in conjunction with AEC, conducted the first nuclear test (named Long Shot) in 1965 to provide data that would improve the United States' capability of detecting underground nuclear explosions. The second nuclear test (Milrow) was a weapons-related test conducted by AEC in 1969 as a means to study the feasibility of detonating a much larger device. Cannikin, the third nuclear test on Amchitka, was a weapons-related test detonated on November 6, 1971. With the exception of small concentrations of tritium detected in surface water shortly after the Long Shot test, radioactive fission products from the tests remain in the subsurface at each test location.

As a continuation of the environmental monitoring that has taken place on Amchitka Island since before 1965, LM in the summer of 2011 collected biological and seawater samples from the marine and terrestrial environment of Amchitka Island adjacent to the three detonation sites and at a background or reference site, Adak Island, 180 miles to the east. Consistent with the goals of the Amchitka LTS\&M Plan, four data quality objectives (DQOs) were developed for the 2011 sampling event, as follows:

- The first objective was to collect selected marine flora and fauna, lichen, soil, and marine sediment and analyze the samples for test-related radionuclides to determine if subsistenceand commercial-catch seafood is safe to eat.

- The second objective provided the rationale for using biota data from the first objective for input in the RESRAD-BIOTA code to analyze the potential ecological risks from the radionuclides.

- The third objective supported the collection of tritium data in seawater.

- The fourth objective supported the collection of reindeer lichen and soil beneath the lichen and marine sediment and to analyze for cesium-137 $\left({ }^{137} \mathrm{Cs}\right)$.

Samples were analyzed for test-related radionuclides; americium-241 $\left({ }^{241} \mathrm{Am}\right),{ }^{137} \mathrm{Cs}$, plutonium-239 $\left({ }^{239} \mathrm{Pu}\right)$ and $-240\left({ }^{240} \mathrm{Pu}\right)$, and tritium as well as uranium-234 $\left({ }^{234} \mathrm{U}\right),-235\left({ }^{235} \mathrm{U}\right)$, and $-238\left({ }^{238} \mathrm{U}\right)$. In planning for the 2011 sampling event, a group of stakeholders representing the federal government, the State of Alaska, and the Aleutian Pribilof Island Association, 
collectively called the Amchitka Working Group, selected 14 biological species for analysis. A similar sampling event was conducted at Adak, which is used as a background or reference area unaffected by the underground nuclear tests at Amchitka. The previous investigations used the island of Kiska, Alaska, as the background or reference area, but the Amchitka Working Group decided that Adak would be a technically suitable reference or background location for the 2011 sampling event. The species sampled were identified based on diet information representing some of the subsistence- and commercial-catch seafood in the western Aleutian Islands.

Biological sampling began June 20, 2011, and continued through July 21, 2011. Two oceangoing research vessels provided the logistical support for the biological and seawater sampling operations. Biological sampling operations varied from personnel collecting samples from the island and along the shoreline to scientific divers performing two dives a day to collect the majority of the biological samples. Overall, a total of 350 biological samples and 166 seawater samples were collected.

The ranges of radionuclides measured in marine species and other media sampled during the 2011 monitoring program are summarized in Table ES-1.

A statistical comparison of the data collected from Amchitka and Adak (the reference location) indicates that the concentrations of nuclides are statistically consistent to each other. Overall, there is a pattern toward slightly higher concentration at the Amchitka site. The measures that seem to show the most deviation have limited data above the detection limits.

To address the first DQO, risk calculations were done using tissue data from the various seafood species collected in 2011 from Amchitka and Adak. A range of Aleut diets (based on published surveys of four villages) and a composite diet were used as dietary intake information for the risk estimates. The estimates for the five diets indicate that overall potential risks from the ingestion of seafood are similar and are at $2 \times 10^{-5}(2$ in 100,000) or lower (i.e., within the U.S. Environmental Protection Agency's acceptable risk range of $1 \times 10^{-6}$ to $1 \times 10^{-4}$ [1 in $1,000,000$ to 1 in 10,000$])$. The primary contributors to the risk estimates are the ${ }^{137} \mathrm{Cs}$ and ${ }^{234,235, \text { and }}{ }^{238} \mathrm{U}$ concentrations.

The risk estimates using Amchitka data are higher by $1 \times 10^{-6}(1$ in $1,000,000)$ than those using Adak data. This difference is lower than the $1 \times 10^{-5}(1$ in 100,000) risk level used by the State of Alaska as the benchmark for acceptable risk. Hence, based on the 2011 data collected for Amchitka and Adak, seafood harvested at Amchitka and Adak is considered safe for consumption at the intake levels for each of five diets evaluated. In addition, the increased cesium concentrations detected in the samples, due to the Fukushima Dai-ichi event, have not resulted in unacceptable risk levels from the ingestion of these seafoods.

For the second DQO, representative radionuclide concentrations in seawater, obtained from sampling data for nine marine species, were used as input to the RESRAD-BIOTA code for comparison with Biota Concentration Guidelines (BCGs) for water. The sum of ratios of radionuclide concentrations to BCGs was much lower than the threshold value of 1 and far below the potential radiation dose to marine species of $0.1 \mathrm{rad} /$ day; therefore, no further analysis was required. 
Table ES-1. Ranges of Radionuclides Measured in Marine Species and other Media: 2011 Monitoring

\begin{tabular}{|c|c|c|c|c|c|c|c|}
\hline Species & ${ }^{137} \mathrm{Cs}$ & ${ }^{241} \mathrm{Am}$ & ${ }^{239} \mathrm{Pu}$ & ${ }^{240} \mathrm{Pu}$ & ${ }^{234} \mathrm{U}$ & ${ }^{235} \mathrm{U}$ & ${ }^{238} \mathrm{U}$ \\
\hline $\begin{array}{l}\text { Dragon kelp } \\
\quad \text { Amchitka }(n=27) \\
\text { Adak }(n=18)\end{array}$ & $\begin{array}{c}0.24-3.2 \\
0.2-2.0\end{array}$ & $\begin{array}{l}0.003-0.033 \\
0.006-0.027\end{array}$ & $\begin{array}{l}0.003-0.16 \\
0.014-0.11\end{array}$ & $\begin{array}{c}0.007-0.13 \\
0.01-0.09\end{array}$ & $\begin{array}{c}12.4-44.6 \\
7.3-46.8\end{array}$ & $\begin{array}{l}0.41-1.5 \\
0.27-1.6\end{array}$ & $\begin{array}{l}9.6-35.4 \\
6.3-38.2\end{array}$ \\
\hline $\begin{array}{l}\text { Sea urchin } \\
\quad \text { Amchitka }(n=9) \\
\text { Adak }(n=6)\end{array}$ & $\begin{array}{c}1.8-9.9 \\
2.7-9.4 \\
\end{array}$ & $\begin{array}{c}0.08-0.2 \\
0.07-0.12 \\
\end{array}$ & $\begin{array}{l}0.033-0.09 \\
<0.04-0.07 \\
\end{array}$ & $\begin{array}{c}<0.02-0.065 \\
\text { ND }(<0.04) \\
\end{array}$ & $\begin{array}{l}16.7-26.4 \\
17.4-28.5 \\
\end{array}$ & \begin{tabular}{|l|}
$0.57-0.83$ \\
$0.20-0.83$ \\
\end{tabular} & $\begin{array}{c}13.5-19.5 \\
4.7-19.3 \\
\end{array}$ \\
\hline $\begin{array}{l}\text { Horse mussel } \\
\text { Amchitka }(n=9) \\
\text { Adak }(n=6)\end{array}$ & $\begin{array}{c}5.9-<44 \\
3.4-15 \\
\end{array}$ & $\begin{array}{c}0.11-<0.4 \\
0.09-0.15 \\
\end{array}$ & $\begin{array}{c}0.1-<2.0 \\
0.073-<0.2\end{array}$ & $\begin{array}{l}\text { ND }(<1.0) \\
\text { ND }(<0.1) \\
\end{array}$ & $\begin{array}{c}23.4-80.8 \\
9.7-49.8 \\
\end{array}$ & $\begin{array}{l}0.53-2.9 \\
0.29-1.8 \\
\end{array}$ & $\begin{array}{c}12.2-67.1 \\
6.8-41.1 \\
\end{array}$ \\
\hline $\begin{array}{l}\text { Chiton } \\
\text { Amchitka }(n=9) \\
\text { Adak }(n=6)\end{array}$ & $\begin{array}{l}1.6-4.1 \\
0.8-1.3 \\
\end{array}$ & $\begin{array}{c}0.07-0.14 \\
0.017-0.08\end{array}$ & $\begin{array}{c}0.03-<0.07 \\
<0.03-0.06\end{array}$ & $\begin{array}{l}\text { ND }(<0.04) \\
\text { ND }(<0.03)\end{array}$ & $\begin{array}{l}5.0-32.4 \\
5.1-32.5 \\
\end{array}$ & $\begin{array}{c}0.12-1.1 \\
0.15-0.99 \\
\end{array}$ & $\begin{array}{l}2.9-26.8 \\
3.6-23.2 \\
\end{array}$ \\
\hline $\begin{array}{l}\text { Rockfish } \\
\text { Amchitka }(n=26) \\
\text { Adak }(n=15) \\
\end{array}$ & $\begin{array}{l}1.1-6.4 \\
1.2-9.4 \\
\end{array}$ & $\begin{array}{l}0.009-0.07 \\
\text { ND }(<0.02)\end{array}$ & $\begin{array}{l}\text { ND }(<0.06) \\
\text { ND }(<0.06)\end{array}$ & $\begin{array}{l}\mathrm{ND}(<0.03) \\
\mathrm{ND}(<0.03) \\
\end{array}$ & $\begin{array}{l}2.3-19.5 \\
1.3-13.4 \\
\end{array}$ & \begin{tabular}{|l|}
$0.08-0.60$ \\
$0.04-0.48$ \\
\end{tabular} & $\begin{array}{l}1.9-14.2 \\
1.0-11.4 \\
\end{array}$ \\
\hline $\begin{array}{l}\text { Greenling } \\
\text { Amchitka }(n=27) \\
\text { Adak }(n=18)\end{array}$ & $\begin{array}{l}1.9-7.8 \\
0.9-7.4 \\
\end{array}$ & $\begin{array}{c}0.03-0.05 \\
0.009-0.033\end{array}$ & $\begin{array}{c}0.018-<0.08 \\
\text { ND }(<0.07)\end{array}$ & $\begin{array}{l}\text { ND }(<0.04) \\
\text { ND }(<0.04)\end{array}$ & $\begin{array}{l}2.5-11.4 \\
2.0-10.7 \\
\end{array}$ & \begin{tabular}{|l|}
$0.08-0.39$ \\
$0.05-0.31$ \\
\end{tabular} & $\begin{array}{l}1.8-9.1 \\
1.1-7.3 \\
\end{array}$ \\
\hline $\begin{array}{l}\text { Irish lord } \\
\quad \text { Amchitka }(n=27) \\
\text { Adak }(n=18)\end{array}$ & $\begin{array}{l}1.2-9.5 \\
1.8-13\end{array}$ & $\begin{array}{c}0.006-0.05 \\
<0.003-0.03\end{array}$ & $\begin{array}{l}\text { ND }(<0.08) \\
\text { ND }(<0.07)\end{array}$ & $\begin{array}{l}\text { ND }(<0.04) \\
\text { ND }(<0.04)\end{array}$ & $\begin{array}{c}3.0-19.3 \\
1.9-16\end{array}$ & $\begin{array}{l}0.09-0.68 \\
0.06-0.55\end{array}$ & $\begin{array}{c}2.0-16.1 \\
1.5-13\end{array}$ \\
\hline $\begin{array}{l}\text { Octopus } \\
\text { Amchitka }(n=3) \\
\text { Adak }(n=1)\end{array}$ & $\begin{array}{r}0.23-2.1 \\
0.7 \\
\end{array}$ & $\begin{array}{r}0.009-0.019 \\
0.015 \\
\end{array}$ & $\begin{array}{r}0.027-0.031 \\
0.02 \\
\end{array}$ & $\begin{array}{l}\text { ND }(<0.03) \\
\text { ND }(<0.01) \\
\end{array}$ & $\begin{array}{r}10.6-21.8 \\
13.6 \\
\end{array}$ & $\begin{array}{r}0.37-0.77 \\
0.45 \\
\end{array}$ & $\begin{array}{r}8.5-18.1 \\
10.7 \\
\end{array}$ \\
\hline $\begin{array}{l}\text { Pacific cod } \\
\text { Amchitka }(n=3) \\
\text { Adak }(n=2)\end{array}$ & $\begin{array}{l}2.1-3.8 \\
2.0-3.0 \\
\end{array}$ & $\begin{array}{l}\text { ND }(<0.003) \\
\text { ND }(<0.001) \\
\end{array}$ & $\begin{array}{l}\text { ND }(<0.01) \\
\text { ND }(<0.01)\end{array}$ & $\begin{array}{l}\mathrm{ND}(<0.003) \\
\mathrm{ND}(<0.003)\end{array}$ & $\begin{array}{r}2.5-3.0 \\
1.9 \\
\end{array}$ & \begin{tabular}{|l|}
$0.03-0.09$ \\
$0.05-0.06$ \\
\end{tabular} & $\begin{array}{l}0.8-2.1 \\
1.2-1.4 \\
\end{array}$ \\
\hline $\begin{array}{l}\text { Halibut } \\
\quad \text { Amchitka }(n=3) \\
\text { Adak }(n=1)\end{array}$ & $\begin{array}{r}0.82-1.5 \\
1.0 \\
\end{array}$ & $\begin{array}{l}\text { ND }(<0.001) \\
\text { ND }(<0.001)\end{array}$ & $\begin{array}{l}\text { ND }(<0.01) \\
\text { ND }(<0.01)\end{array}$ & $\begin{array}{l}\text { ND }(<0.003) \\
\text { ND }(<0.003)\end{array}$ & $\begin{array}{r}0.6-<1.6 \\
1.6 \\
\end{array}$ & $\begin{array}{r}0.01-0.03 \\
0.04 \\
\end{array}$ & $\begin{array}{c}0.2-0.65 \\
0.86\end{array}$ \\
\hline $\begin{array}{l}\text { Rockweed } \\
\text { Amchitka }(n=27) \\
\text { Adak }(n=18) \\
\end{array}$ & $\begin{array}{l}1.3-5.8 \\
1.0-7.3 \\
\end{array}$ & $\begin{array}{l}0.003-0.011 \\
0.006-0.015 \\
\end{array}$ & $\begin{array}{l}0.002-0.012 \\
0.002-0.019 \\
\end{array}$ & $\begin{array}{l}0.005-0.008 \\
0.006-0.011 \\
\end{array}$ & $\begin{array}{l}35.0-144 \\
45.5-144 \\
\end{array}$ & $\begin{array}{l}1.2-5.1 \\
1.6-5.1 \\
\end{array}$ & $\begin{array}{l}29-121 \\
37-120 \\
\end{array}$ \\
\hline $\begin{array}{l}\text { Reindeer lichen } \\
\text { Amchitka }(n=3) \\
\text { Adak }(n=3)\end{array}$ & $\begin{array}{c}404-1523 \\
2855-6236 \\
\end{array}$ & & & & & & \\
\hline $\begin{array}{l}\text { Gull eggs } \\
\text { Amchitka }(n=1)\end{array}$ & 4.2 & ND $(<0.008)$ & ND $(<0.03)$ & ND $(<0.02)$ & 0.6 & 0.0007 & 0.015 \\
\hline $\begin{array}{l}\text { Dolly Varden } \\
\text { Amchitka }(n=1) \\
\text { Adak }(n=1)\end{array}$ & $\begin{array}{r}98 \\
447 \\
\end{array}$ & $\begin{array}{l}\text { ND }(<0.001) \\
\text { ND }(<0.003) \\
\end{array}$ & $\begin{array}{l}\mathrm{ND}(<0.01) \\
\mathrm{ND}(<0.01)\end{array}$ & $\begin{array}{l}\text { ND }(<0.01) \\
\text { ND }(<0.01) \\
\end{array}$ & $\begin{array}{r}\mathrm{ND}(<1.3) \\
0.9 \\
\end{array}$ & $\begin{array}{l}0.01 \\
0.02 \\
\end{array}$ & $\begin{array}{l}0.23 \\
0.36 \\
\end{array}$ \\
\hline $\begin{array}{l}\text { Soil } \\
\quad \text { Amchitka }(n=3) \\
\quad \text { Adak }(n=3)\end{array}$ & $\begin{array}{r}312-6110 \\
1135-7264 \\
\end{array}$ & & & & & & \\
\hline $\begin{array}{l}\text { Sediment } \\
\text { Amchitka }(n=2)\end{array}$ & $11.9-36.3$ & & & & & & \\
\hline
\end{tabular}

All units in picocuries per kilogram (pCi/kg) wet weight except for soil and sediment, which are reported in dry weight. Maximum values listed are the maximum reported, even if below the minimum detectable concentration (MDC) (U-flagged). For example, the maximum ${ }^{137} \mathrm{Cs}$ for horse mussel on Amchitka listed above (44U pCi/kg) was below the MDC, vs. the maximum detected result of $14 \mathrm{pCi} / \mathrm{kg}$.

ND - Not Detected (all results <MDC). Reindeer lichen, soil, and sediment analyzed for ${ }^{137} \mathrm{Cs}$ only. 
For the third DQO, samples were collected in 2011 to develop a baseline of tritium concentrations for seawater near Amchitka and Adak. Previous studies and contaminant transport modeling predict that tritium will be the leading indicator of migration from the detonation points. Therefore, this study used low detection levels and targeted the most likely locations to obtain a baseline for tritium in seawater at Amchitka. The results showed that the activity of tritium in the marine environment surrounding Amchitka and Adak is similar to that resulting from global fallout from nuclear testing and natural tritium levels in the northern hemisphere. The data from the 2011 sampling event are in agreement with previous sampling results. Amchitka Island tritium levels from the 2011 sampling event ranged from 1.2 to 2.8 picocuries per liter.

For the fourth DQO, ${ }^{137} \mathrm{Cs}$ was measured in the reindeer lichen and soil on Amchitka and Adak. The data will provide perspective to long-term baseline data considered for Amchitka and Adak. The ${ }^{137} \mathrm{Cs}$ concentrations varied significantly among different samples, especially ${ }^{137} \mathrm{Cs}$ concentrations in soil beneath the lichen, for which the maximum values were 5-20 times higher than the minimum values. The lichen and soil beneath the lichen showed that ${ }^{137} \mathrm{Cs}$ levels in Adak were higher than the levels in Amchitka. The concentrations of ${ }^{134} \mathrm{Cs}$ in samples collected from Amchitka and Adak indicate that atmospheric transport of airborne contamination from the Fukushima Dai-ichi event may have contributed to the levels detected. 


\subsection{Introduction}

\subsection{Amchitka Island History}

Amchitka Island, near the western end of the Aleutian Islands, is approximately 1,340 miles west-southwest of Anchorage, Alaska (Figure 1). Amchitka is part of the Aleutian Island Unit of the Alaska Maritime National Wildlife Refuge, which is administered by the U.S. Fish and Wildlife Service (USFWS). Since World War II, Amchitka has been used by multiple U.S. government agencies for various military and research activities. From 1943 to 1950, it was used as a forward air base for the U.S. Armed Forces. During the middle 1960s and early 1970s, the U.S. Department of Defense (DOD) and the U.S. Atomic Energy Commission (AEC) used a portion of the island as a site for underground nuclear tests. During the late 1980s and early 1990s, the U.S. Navy constructed and operated a radar station on the island.

Three underground nuclear tests were conducted on Amchitka Island. DOD, in conjunction with AEC, conducted the first nuclear test (named Long Shot) in 1965 to provide data that would improve the United States' capability of detecting underground nuclear explosions. The second nuclear test (Milrow) was a weapons-related test conducted by AEC in 1969 as a means to study the feasibility of detonating a much larger device. Cannikin, the third nuclear test on Amchitka was a weapons-related test detonated on November 6, 1971. With the exception of tritium detected in surface water in several locations shortly after the Long Shot test, radioactive fission products from the tests remain in the subsurface at each test location.

The U.S. Department of Energy (DOE) Office of Legacy Management (LM) retains responsibility for those areas on Amchitka Island that were agreed to in the 2001 Letter of Agreement between USFWS and DOE (DOE 2001). As detailed in the USFWS Remedial Action/Removal Action Environmental Assessment Finding of No Significant Impact (USFWS 2000), LM has responsibility for portions of the island where drilling mud is encapsulated in engineered disposal cells. LM has long-term surveillance and maintenance (LTS\&M) responsibility for three underground test sites and seven surface-closed sites where drilling mud is stored.

The Long-Term Surveillance and Maintenance Plan for the U.S. Department of Energy Amchitka, Alaska, Site (DOE 2008) describes how LM intends to fulfill its mission to maintain protection of human health and the environment and fulfill its obligations for LTS\&M at Amchitka. The LTS\&M Plan is reviewed and updated, if necessary, every 5 years by LM. Surface remediation has been accomplished through capping the mud pits and closing the asphalt tanks in place. The Alaska Department of Environmental Conservation (ADEC) has accepted this work as being protective of human health and the environment (ADEC 2004).

Radioactive fission products remain in and around the subsurface cavities formed by the nuclear tests. Data collected since the detonations do not indicate that radionuclides have migrated into the accessible environment. Other than small quantities of radionuclides from Long Shot, there was no exposure to humans or the environment from the detonations (Merritt and Fuller 1977; DOE 1982; DOE 2000; Dasher et al. 2002; CRESP 2005; Burger et al. 2006; Burger et al. 2007a and 2007b). No feasible technology exists for removing the subsurface radioactivity associated with the nuclear test cavities; therefore, LM conducts monitoring near these sites to protect the public safety. 


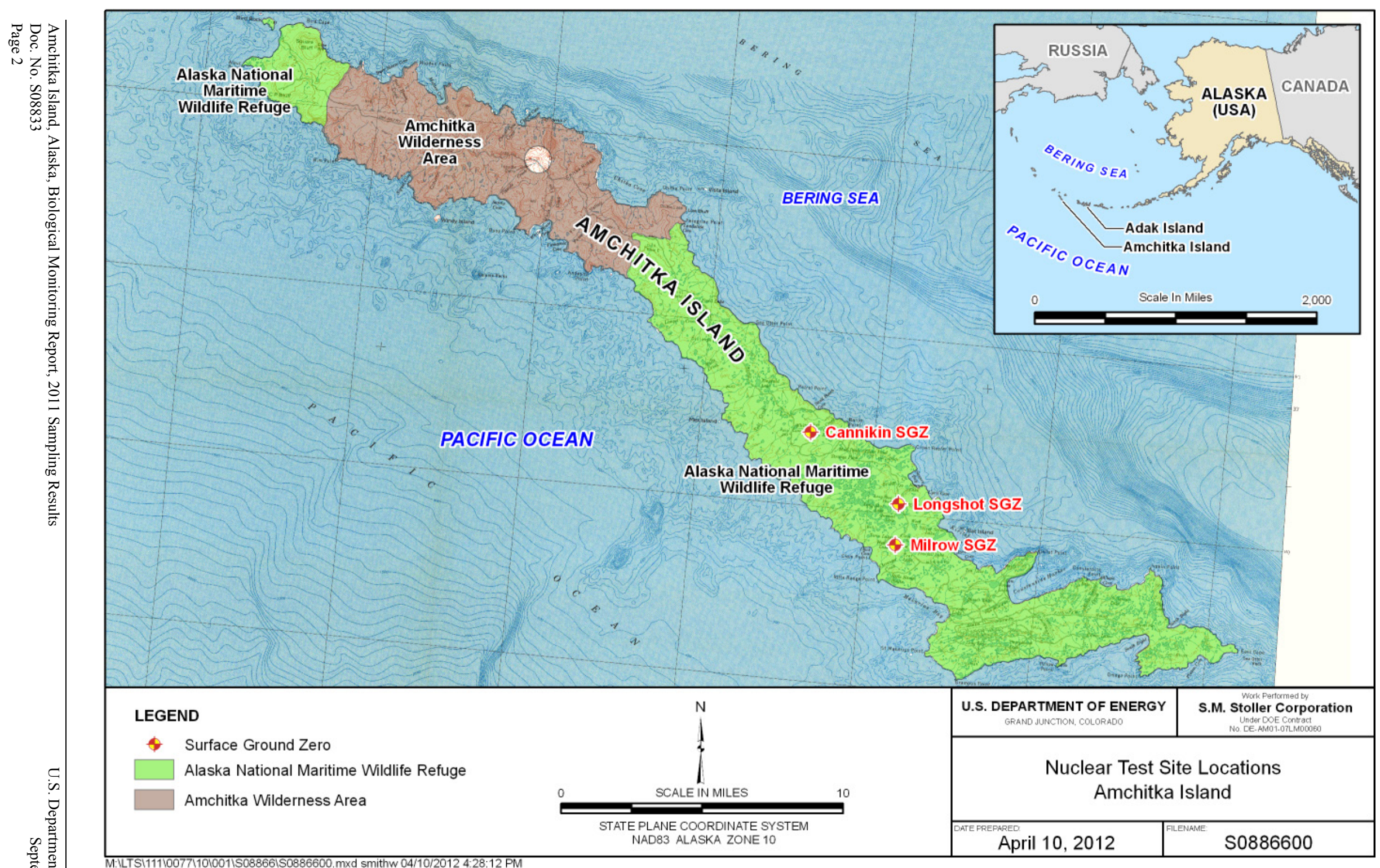

Figure 1. Amchitka Test Site Locations 
Computer modeling has indicated that over time, some of the subsurface residual radionuclides will migrate away from the blast cavities and may eventually reach the near-surface marine environment (DOE 2002b). Breakthrough of radionuclides to the marine environment is not expected for more than 2000 years (Hassan and Chapman 2006).

The potential for residual radionuclide release and the fact that Aleuts living in the Aleutian Islands harvest fish and other subsistence foods from this area and commercial fisheries operate in the area are the basis for the biological and terrestrial sampling conducted on Amchitka and Adak Islands during the late spring and early summer of 2011. LM wants to assess the possibility that selected residual radionuclides from the nuclear tests conducted on Amchitka Island might be entering the marine food chain, resulting in potential ecological and human health effects.

\subsection{Previous Environmental Studies}

This section summarizes previous scientific studies performed on and around Amchitka that were similar in purpose to the 2011 sampling event.

The Environment of Amchitka Island, Alaska (Merritt and Fuller 1977) is a multidisciplinary work that provides a concise review of the geology, ecology, and radionuclides in air, water, and biota with an emphasis on "the search for and identity of radionuclides of Amchitka origin in the samples and [contributing] to the general knowledge of the distribution of radionuclides in the environment." This evaluation found no evidence that any test-related radionuclides had escaped from the three underground nuclear test sites at Amchitka except for trace quantities of radionuclides, principally tritium, in water and soil gas samples taken in the immediate vicinity of the Long Shot test.

The Amchitka Radiobiological Program began in 1970 and continued through 1979. The program's principal objective was to collect biological and groundwater samples for radiobiological analyses and to determine the extent of radionuclide contamination from worldwide atmospheric fallout and from the detonation of the three underground nuclear tests on Amchitka. Migration of radionuclides from the underground test sites would be suspected if the concentration of radionuclides were significantly greater than could be attributed to worldwide fallout or if an unexpected assemblage of radionuclides were detected. In the Amchitka Radiobiological Program Final Report July 1970 to December 1979 (DOE 1982), it was determined that no radionuclides from the underground sites were detected, except for tritium from the Long Shot test, which produced increased tritium concentrations in surface water and freshwater plants near the site.

The Long Term Hydrologic Monitoring Program (LTHMP) monitored Amchitka radioactivity levels in groundwater as part of the Off-Site Environmental Monitoring Program for the Nevada Test Site and Other Test Areas Used for Underground Nuclear Detonations. Amchitka monitoring under this program began in 1977, and since then, sampling occurred from 1977 through 1989 and in 1991, 1993, 1997, and 2001. The U.S. Environmental Protection Agency (EPA) carried out this program and monitored Amchitka to measure levels and trends of radioactivity in the offsite environment surrounding the test areas to ensure that radioactivity levels are below existing radiation protection standards. 
In 1997, the LTHMP at Amchitka included radiobiological sampling and analysis. These samples were collected because of a survey of selected aquatic biota that Greenpeace conducted on the island (Greenpeace 1996). Greenpeace speculated that several long-lived manmade radionuclides were migrating into the surface environment from nuclear test cavities several thousand feet below the surface of the island (DOE 2000). The results of the 1997 LTHMP radiobiological sampling indicated there was no evidence of migration from the underground test cavities into the terrestrial or freshwater environments on Amchitka (DOE 2000; Dasher et al. 2002).

In 2004, the Consortium for Risk Evaluation and Stakeholder Participation II (CRESP), a group of independent universities, prepared the Amchitka Independent Science Assessment: Biological and Geophysical Aspects of Potential Radionuclide Exposures in the Amchitka Marine Environment (CRESP 2005). The CRESP study concluded that the foods consumed by humans are safe with respect to radionuclides, levels of radionuclides are well below published human health risk guidance levels, and data collected do not suggest that radionuclides in biota collected from Amchitka are attributable to the Amchitka test shots.

\subsection{Stakeholder Participation}

Amchitka Island is within the Aleutian Island Unit of the Alaska Maritime Refuge, created through an executive order by President William Howard Taft in 1913. LM has a Memorandum of Understanding with USFWS, the purpose of which is to define the roles and responsibilities of both agencies, specify the means of access to and egress from Amchitka, and explain how LM will exercise institutional controls. USFWS issued access permits to LM in conjunction with the 2011 monitoring and sampling events.

LM provides a financial assistance grant to ADEC and regularly meets with representatives from ADEC, which has partnered with the University of Alaska Fairbanks (UAF) to obtain technical support on the Amchitka project. LM collaborated with ADEC, UAF, and others to plan the 2011 sampling event. UAF provided technical assistance, and the UAF marine biology team supported the 2004 CRESP and 2011 sampling events by providing the scientific diving team that collected the biological samples from the marine environment.

LM provides a financial assistance grant to Aleutian Pribilof Islands Association Inc. (APIA). APIA is the federally recognized tribal organization of the Aleut people in Alaska and is an important component of the LM mission at Amchitka. APIA represents the interests of the Aleuts and assists LM with communications with the Aleut people and ADEC. APIA participates in developing work scope related to the LM mission on Amchitka, participates in regular planning meetings, and assisted with sample collection during the 2011 sampling event.

LM, ADEC, USFWS, and APIA, constitute the Amchitka Working Group. Additionally, LM retained Argonne National Laboratory (ANL) through a work order to help develop the biological Sampling Plan (DOE 2011) and provide technical support with data analysis, risk assessment (human health and ecological), and other technical issues, as required. ANL provided assistance with the sample collection during the 2011 sampling event and the data analysis and human health and ecological risk evaluation (Sections 6 through 9 of this report). 
LM also retained Lawrence Livermore National Laboratory (LLNL) through a work order to provide support in the radionuclide analysis of the biological samples collected in 2011 . The University of Miami Rosenstiel School of Marine and Atmospheric Science provided tritium data on all seawater samples collected. 
This page intentionally left blank 


\subsection{Purpose of Report}

\subsection{Data Quality Objectives}

LM, in conjunction with representatives from the Amchitka Working Group, ANL, and LLNL, met in late 2010 to plan for the 2011 sampling event. Their collaborative work resulted in the Sampling Plan for the Amchitka, Alaska, Site 2011 Sampling Event (DOE 2011), which outlined the following four sets of data quality objectives (DQOs) that provided the rationale for the 2011 sampling effort.

1. To ensure that subsistence- and commercial-catch seafood near Amchitka is safe to eat, the presence of selected (test-related) radionuclides will be determined from 14 marine plant and biological species.

2. The results from DQO \#1 will be entered into the RESRAD-BIOTA code to analyze the potential ecological risks from the radionuclides of interest.

3. Tritium data will be determined from seawater samples collected to establish a baseline tritium concentration in seawater.

4. Cesium-137 $\left({ }^{137} \mathrm{Cs}\right)$ data will be determined from reindeer lichen, soil beneath the lichen, and marine sediment to establish baseline ${ }^{137} \mathrm{Cs}$ concentrations in reindeer lichen, soil beneath the lichen, and marine sediments.

This report summarizes the work that was performed in the collection of the data to meet these DQOs, presents the results of the laboratory analyses from all the samples collected, and provides an evaluation of the ecological health of the Amchitka and Adak environments.

\subsection{Objective of the Sampling Event}

The objectives of this sampling program are to examine the concentrations of a selected group of radionuclides in selected microalgae, invertebrates, fish, gull eggs, and sediments in the terrestrial and marine ecosystem on and adjacent to Amchitka Island and to compare the results to the reference site of Adak Island. Interspecific (differences between species) and interisland changes in radionuclide concentrations have been evaluated, and the data have been entered into a database for the continued development of a baseline for past and future comparison and to assess whether the radionuclide concentrations found in the ecosystems of Amchitka and Adak Islands are similar to those found in other parts of the Northern Hemisphere.

To meet the objective of qualitatively comparing the 2011 data to the 2004 data, the sampling locations were in the same general areas as those included in the CRESP sampling event with one exception: Adak Island, instead of Kiska Island, was chosen to be the new reference location. The Amchitka Working Group selected Adak as the reference location for the 2011 sampling event because Adak is easier to access for sampling purposes. It was expected that, like Kiska, data from Adak would be representative of an area considered to be not influenced by the underground tests conducted at Amchitka and would therefore provide data useful for comparison with the Amchitka data.

For the samples collected during the 2011 sampling event, LLNL provided analytical methods with adequately low detection limits to measure the baseline activities of radionuclides in 
important biological media, ocean-bottom sediments, and seawater. The radionuclides analyzed were ${ }^{137} \mathrm{Cs}$, americium-241 $\left({ }^{241} \mathrm{Am}\right)$, plutonium-239 $\left({ }^{239} \mathrm{Pu}\right)$, plutonium-240 $\left({ }^{240} \mathrm{Pu}\right)$, uranium-234 $\left({ }^{234} \mathrm{U}\right)$, uranium-235 $\left({ }^{235} \mathrm{U}\right)$, and uranium-238 $\left({ }^{238} \mathrm{U}\right)$.

LM also collected baseline data on tritium $\left({ }^{3} \mathrm{H}\right)$ in seawater off the shoreline of the Cannikin site, and reindeer lichen and soil beneath the lichen were also collected and tested for ${ }^{137} \mathrm{Cs}$. Following the atmospheric releases from the Fukushima Dai-ichi nuclear power plant in March 2011, the Amchitka Working Group questioned whether these releases might affect the ability to interpret background activity concentrations of target radionuclides from the Amchitka 2011 sampling event. Therefore, reindeer lichen and soil beneath the lichen were also tested for cesium-134 $\left({ }^{134} \mathrm{Cs}\right)$. 


\subsection{Description of Sampling}

\subsection{Location and Sampling Sites}

The Amchitka and Adak sample locations are shown in Figure 2 and Figure 3, respectively. Adak Island, located approximately 200 miles east of Amchitka Island, was selected as the reference location (DOE 2011) for the 2011 sampling event.

Figure 4 through Figure 8 show the locations of the individual samples collected from the three sites on Amchitka and two areas on Adak. These figures also show the overnight anchorage location of the Research Vessel (R/V) Norseman during sampling activities. The R/V Norseman provided logistical support during the 2011 biological sampling event. As set forth in the biological Sampling Plan (DOE 2011), each sampling location consists of two separate areasthe shoreline transect and the 2-mile limit. For each sampling location, the shoreline transect extends from the shoreline to approximately 1,000 feet into the ocean and for approximately 3 miles along the shoreline. This 3-mile transect is broken into three separate transects, each approximately 1 mile in length. The shoreline transect is where the divers collected the biological species identified in the Sampling Plan as well as marine sediment. Sampling personnel working from the ship also employed hook-and-line fishing for fish in the shoreline transects. In addition, the terrestrial sampling team worked along the shoreline transects to collect littoral zone samples of algae.

In addition to the three transects at each of the five sites, a fourth transect extending 2 miles away from the shoreline at each of the five sites was employed. The 2-mile limit extended 2 miles into the ocean from the shoreline and was not divided into smaller transects. Sampling personnel at the 2-mile limit employed hook-and-line fishing to collect samples of rockfish, greenling, and/or Irish lords, halibut, and Pacific cod from the fantail of the R/V Norseman.

As depicted in Figure 4 through Figure 6, actual sample collection locations from the three areas on Amchitka conformed reasonably well to the sampling locations proposed in the Sampling Plan. However, the geographic features of Adak - a rugged coastline deeply indented by fiords, and coastline erosion that has created imposing cliffs as much as 2,500 feet above sea levelprevented access to some of the proposed sampling locations. As a result, each of the three transects along both the north and south sides of Adak were more spread out along the island's coastline, as shown in Figure 7 and Figure 8. Coordinates of sampling locations were obtained from the GPS units managed by the individual sampling teams. In some instances the coordinates mark the beginning and end of the transect, not each individual location where multiple samples of the same species were collected. Several individual samples may have been picked up at various locations along the same transect. For the final location to be depicted on the figure, either the beginning or the end transect location was chosen as the location placed on the figure for that composite sample.

Figure 9 shows the locations where seawater samples were collected off the coastline of the Cannikin site. The majority of the samples were collected from the deck of the R/V Ocean Pioneer during the vessel's support of the terrestrial inspection of the seven earthen caps on Amchitka. The seawater samples that were closer into the shoreline and not easily accessible by the larger R/V Ocean Pioneer were collected from the smaller R/V Norseman and from skiffs. 


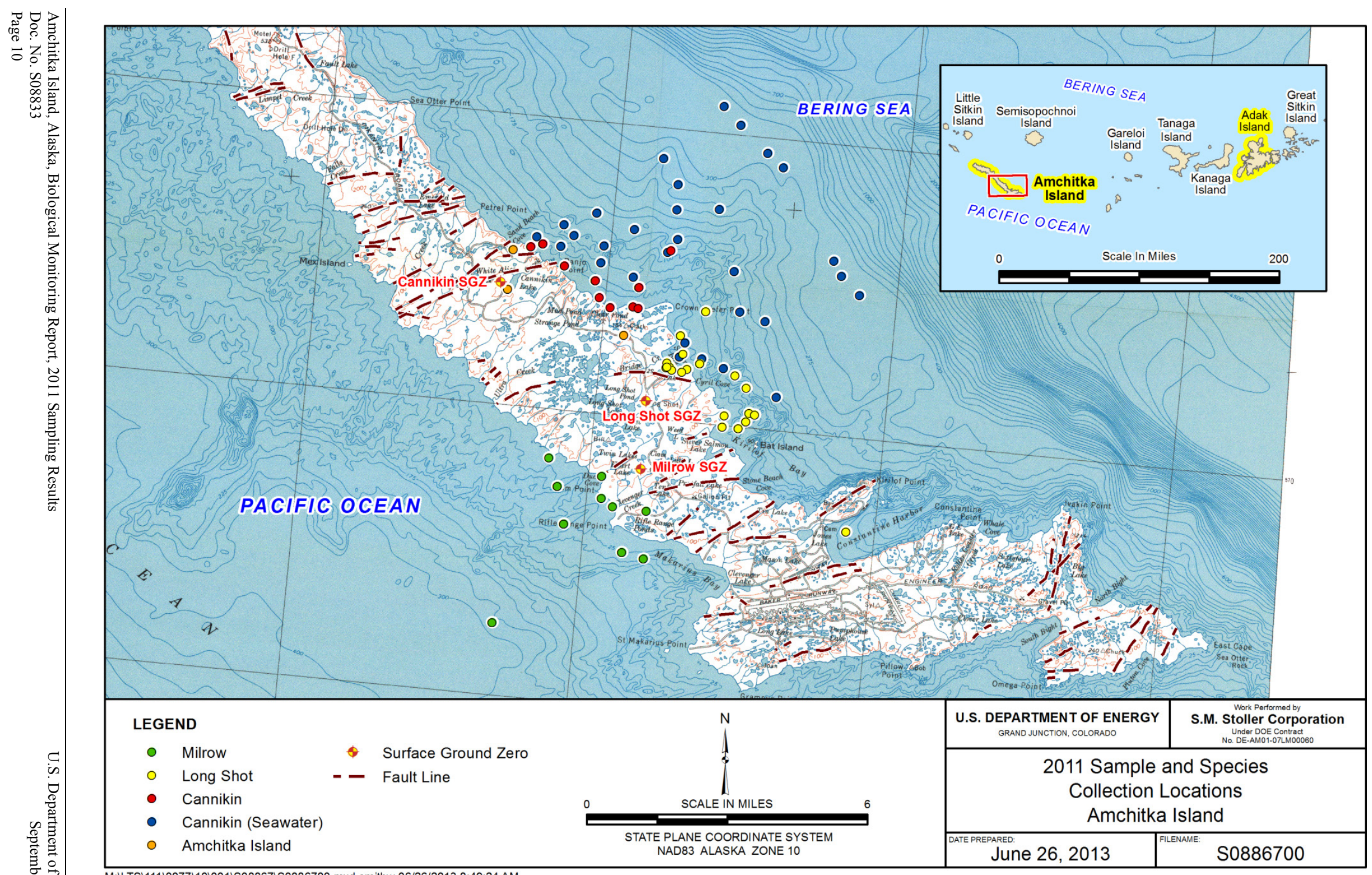

Figure 2. Amchitka Island Sample Locations 


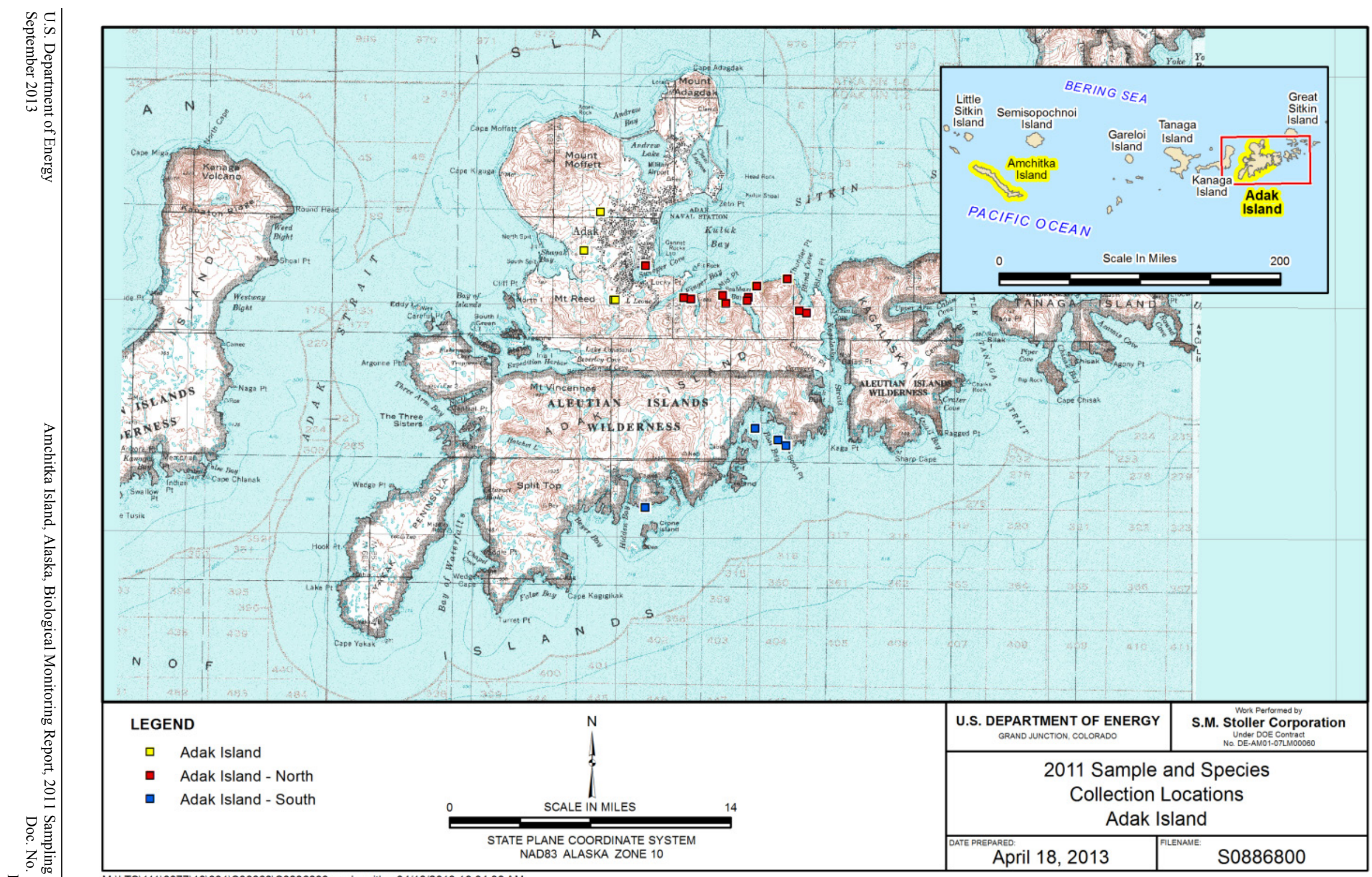

Figure 3. Adak Island Sample Locations 


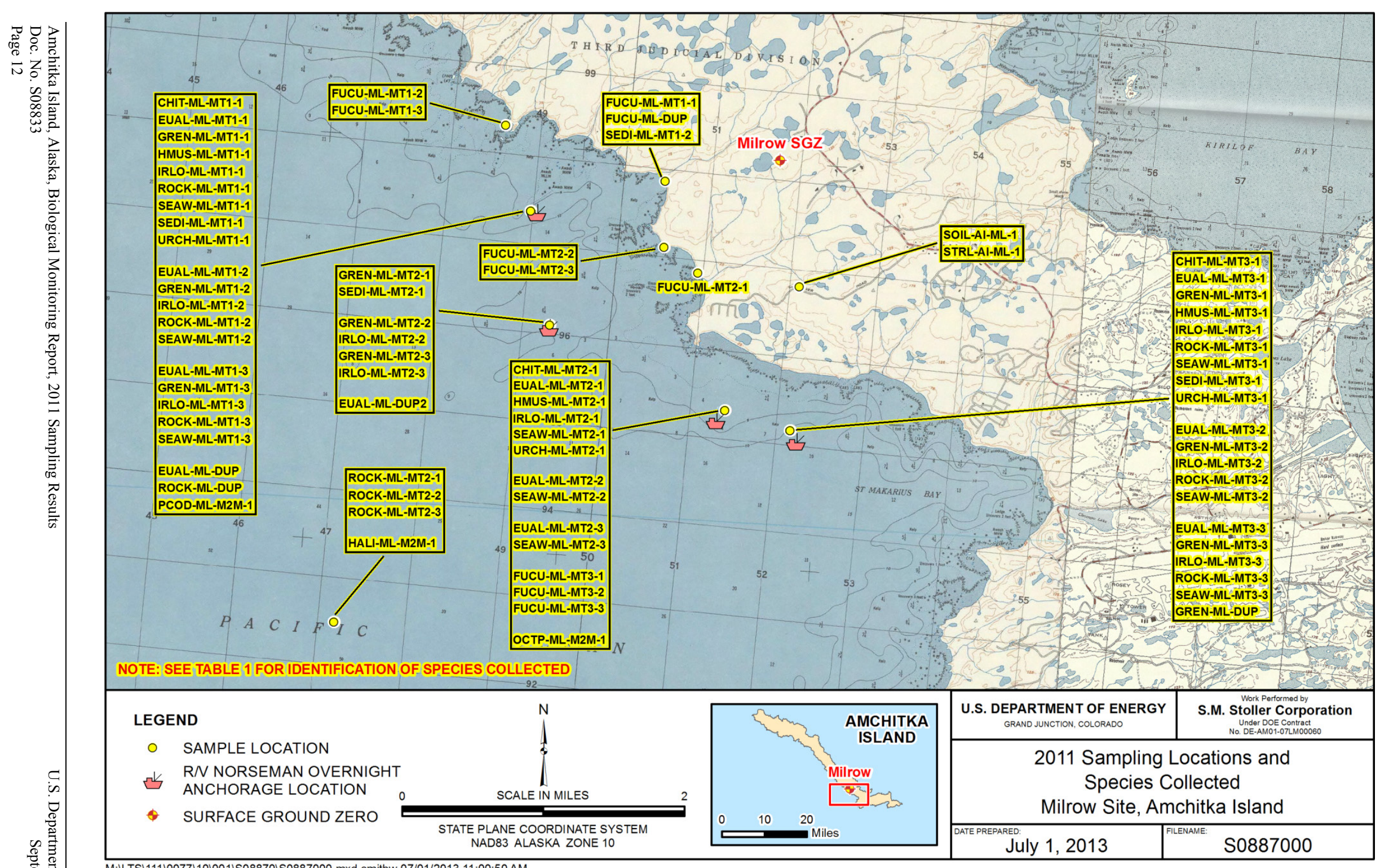

Figure 4. Milrow Sample Locations 


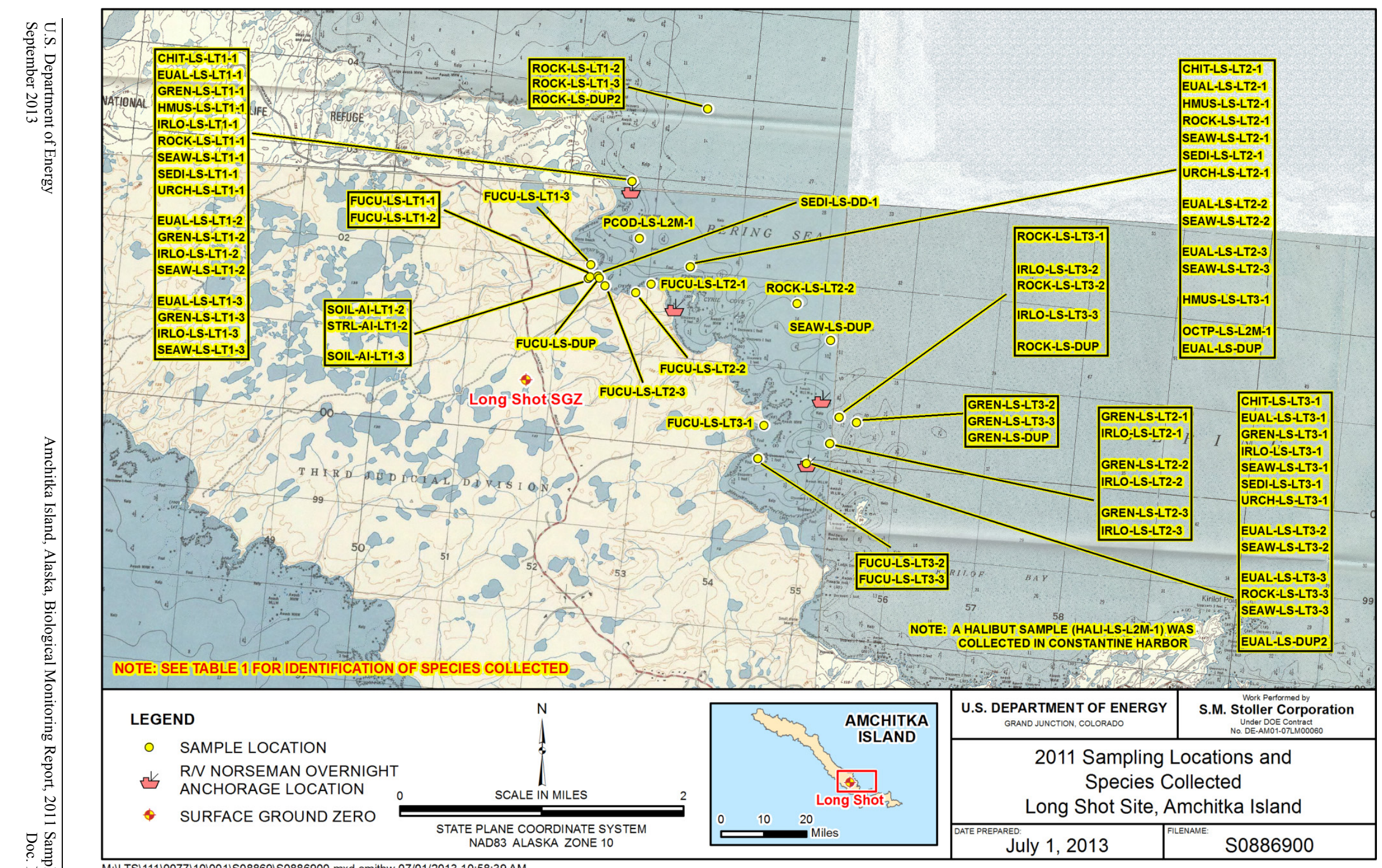

M:LTSI11110077/1010011S08869|S0886900.mxd smithw 07/01/2013 10:58:39 AM

Figure 5. Long Shot Sample Locations 


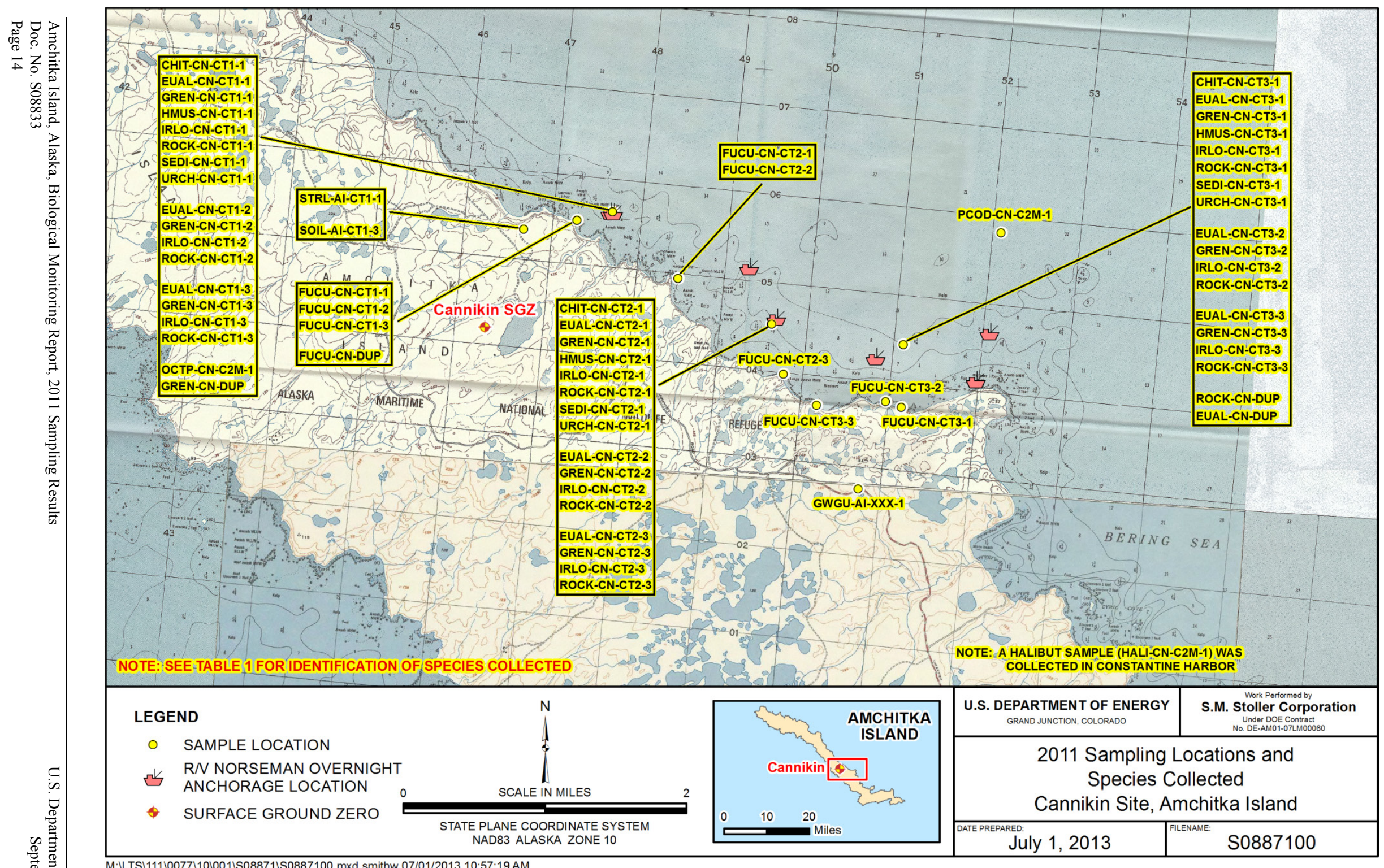

Figure 6. Cannikin Sample Locations 


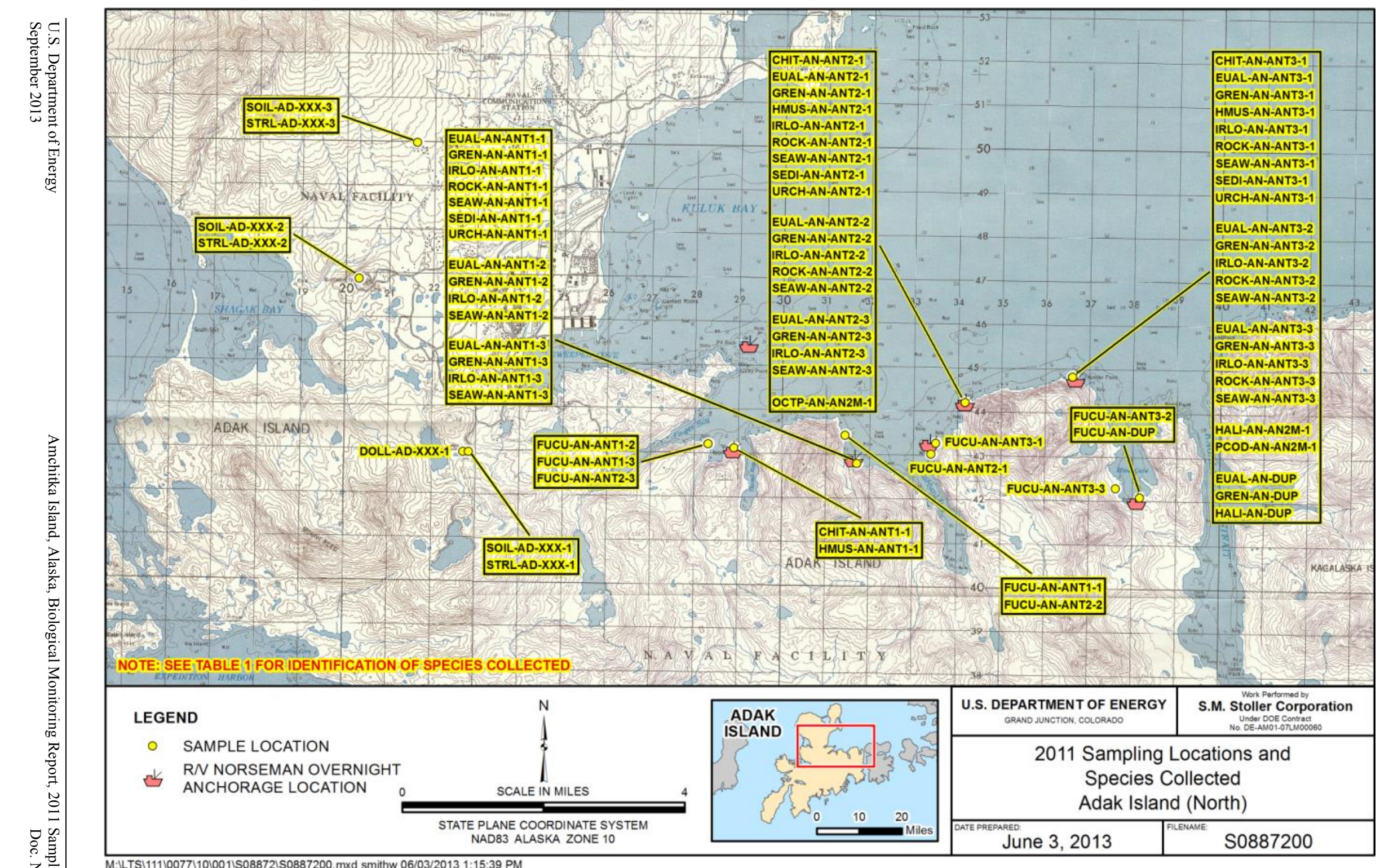

M:LTSI1111007711010011S088721S0887200.mxd smithw 06/03/2013 1:15:39 PM

Figure 7. Adak North Sample Locations 


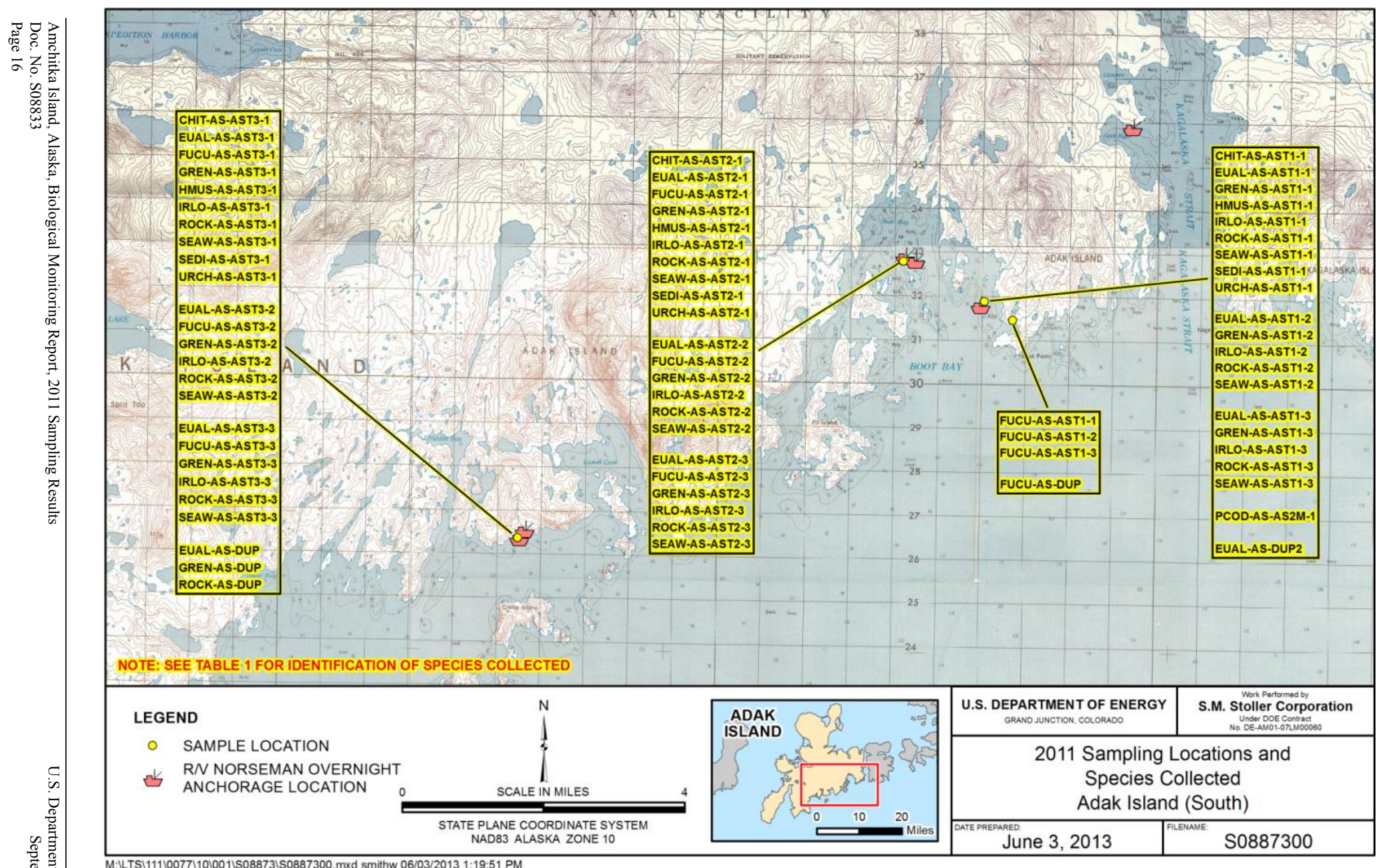

Figure 8. Adak South Sample Locations 


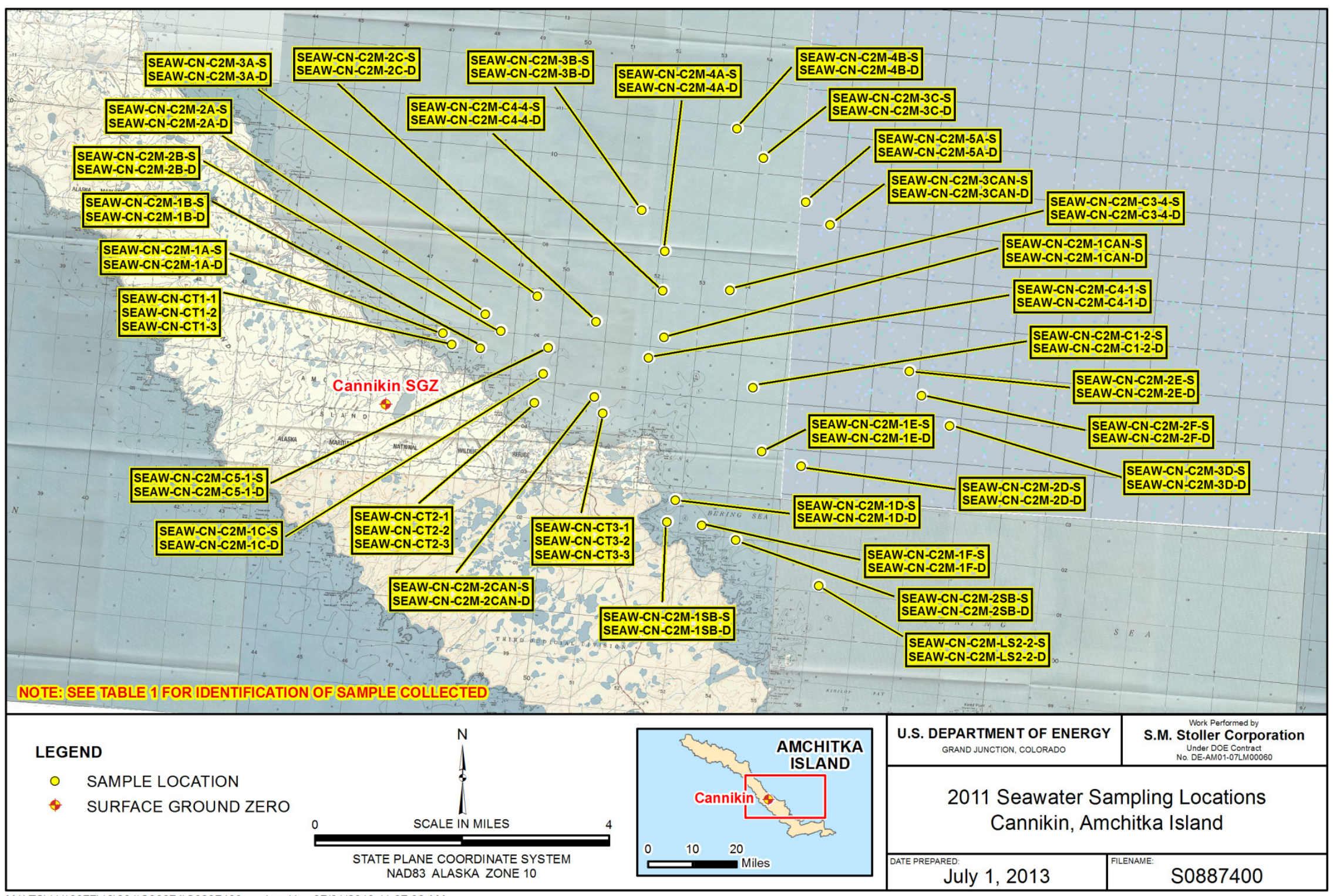

M:LTSI1111007711010011S08874|S0887400.mxd smithw 07/101/2013 11:07:03 AM

Figure 9. Cannikin Seawater Sample Locations 
Additional seawater samples were collected by divers within 1 meter of the seabed off the coastlines of Cannikin, Long Shot, and Milrow sites, as well as the northeast and southeast coastlines of Adak. Divers also collected marine sediment from the seabed from these same general areas.

Finally, sampling personnel collected gull eggs and reindeer lichen and soil beneath the lichen at random locations across both islands. No gull eggs were collected from Adak Island because either the eggs had already hatched or local island residents had collected them.

\subsection{Sample Types}

Results of biota sampling conducted by CRESP in 2004 indicate that concentrations of target radionuclides in biota (including subsistence- and commercial-catch species) are sufficiently low that the species are safe for human consumption (CRESP 2005), and the CRESP data do not suggest that radionuclides in biota collected from Amchitka are attributable to the Amchitka test shots. Subsistence- and commercial-catch species near Amchitka continue to be harvested for consumption; therefore, the species that are likely to be a food source were targeted for sampling in 2011 to verify that these species continue to be safe to eat. Reindeer lichen was sampled because it is a good indicator species for the uptake of ${ }^{137} \mathrm{Cs}$ in the local environment.

The following biological species were selected for sampling during 2011. Appendix A provides pictures of these species.

- Dragon kelp (Eualaria fistulosa),

- $\quad$ Rockweed (Fucus distichus),

- Reindeer lichen (Cladina reindeer lichen),

- Gumboot or Pacific chiton (Cryptochiton stelleri),

- $\quad$ Horse mussel (Modiolus modiolus),

- Sea urchin(Strongylocentrotus polyacanthus),

- $\quad$ Rockfish (Sebastes melanops and S. cilatus),

- $\quad$ Irish lord (Hemilepidotus jordani and H. hemilepidotus),

- Rock greenling (Hexagrammos lagocephalus),

- $\quad$ Pacific halibut (Hippoglossus stenolepis),

- $\quad$ Pacific cod (Gadus macrocephalus),

- $\quad$ North Pacific giant octopus (Enteroctopus dofleini),

- Dolly Varden (Salvelinus malma), and

- $\quad$ Glaucous-winged gull (Larus glaucescens) eggs.

The abbreviated sample names in Figure 4 through Figure 8 can be referenced to the following standard labeling system for all the samples collected:

[Species or sample material]-[Site Location]-[Transect Location]-[Sample Number] 
Table 1 shows the labeling system that was used for sample identification.

Table 1. Labeling System

\begin{tabular}{||l|c||}
\hline \multicolumn{1}{|c|}{ Species/Media to be Sampled } & Species/Media Identification \\
\hline Dragon kelp & EUAL \\
\hline Rockweed & FUCU \\
\hline Reindeer lichen & STRL \\
\hline Chiton & CHIT \\
\hline Horse mussel & HMUS \\
\hline Blue mussel & BMUS \\
\hline Sea urchin & URCH \\
\hline Rockfish & ROCK \\
\hline Red or yellow Irish lord & ILOR \\
\hline Rock greenling & GREN \\
\hline Halibut & HALI \\
\hline Pacific cod & PCOD \\
\hline Octopus & OCTP \\
\hline Dolly Varden & DOLL \\
\hline Glaucous-winged gull eggs & GWGU \\
\hline Seawater & SEAW \\
\hline Marine sediment & SEDI \\
\hline Soil & SOIL \\
\hline \multicolumn{1}{|c|}{ Site Location } & Location Identifier \\
\hline Amchitka Island & Al \\
\hline Adak Island & AD \\
\hline Cannikin & CN \\
\hline Long Shot & LS \\
\hline Milrow & ML \\
\hline Adak, North & AN \\
\hline Adak, South & AS \\
\hline & Transect Identifier \\
\hline Cannikin & CT1, CT2, CT3, and C2M \\
\hline Long Shot & LT1, LT2, LT3, and L2M \\
\hline Milrow & MT1, MT2, MT3, and M2M \\
\hline Adak, North & AST1, ANT2, ANT3, and AN2M , AST3, and AS2M \\
\hline Adak, South & $1-3$ \\
\hline Sample Number & Sample Number \\
\hline
\end{tabular}

\subsection{Sample Collection and Preparation}

Sampling began the week of June 19, 2011, with the arrival of the inspection and terrestrial sampling teams on Amchitka Island. The terrestrial sampling team consisted of Doug Dasher (ADEC), Karen Pletnikoff (APIA), Michael McCoy (APIA), Pauline Melovidov (APIA), Jason Nguyen (LM), Lauren Goodknight (S.M. Stoller Corporation [Stoller]), and Mary Picel (ANL). Doug and Karen were the co-leads directing the terrestrial sampling efforts. Rockweed, reindeer lichen, the soil beneath the lichen, gull eggs, and Dolly Varden were collected from the designated sampling areas along the shoreline where pedestrian access was possible (rockweed), Cannikin Lake (Dolly Varden), and opportunistically (gull eggs, reindeer lichen, and soil beneath the lichen) across Amchitka. The Amchitka terrestrial sampling was completed by noon on Friday, June 24, 2011. The majority of the seawater samples were collected from the deck of the R/V Ocean Pioneer on the afternoon and evening of Friday, June 24, 2011. The Ocean Pioneer 
arrived back in Adak on Sunday, June 26, and all samples were shipped out on Alaska Airlines to LLNL, the Tritium Laboratory at the University of Miami Rosenstiel School of Marine and Atmospheric Science in Miami, Florida, or the University of Alaska Anchorage that same day.

The terrestrial sampling on Adak Island began Sunday, June 26, and was completed on Wednesday, June 29. The samplers collected the lichen and soil beneath the lichen and collected Dolly Varden from lakes but were unable to collect samples of either rockweed or gull eggs on Adak. There are no roads to the island's coastline and thus no access to the proposed sampling locations. Also, apparently local residents collect gull eggs for personal consumption, and the sampling team was unable to locate any. All samples collected on Adak were shipped out on Alaska Airlines to LLNL on Thursday, June 30.

The R/V Norseman, the vessel chartered for the biological sampling, arrived at Sweepers Cove pier, Adak Island, on Wednesday, June 29. The dive team arrived via Alaska Airlines the following day. The scientific dive team from UAF included divemaster Stephen Jewett and divers Heloise Chenolet, Max Hoberg, Jared Weems, and Shona Snater. Also along as part of UAF's dive team were Piotr Kuklinski from the Institute of Oceanology Polish Academy of Sciences, David Scheel from the Alaska Pacific University, and a contract diver, Roger Clark. The scientific team aboard the R/V Norseman included Mark Kautsky, LM's site manager, Paul Darr, Stoller's project manager, Karen Pletnikoff and Michael McCoy from APIA, Lauren Goodknight, Stoller's sample coordinator, and Marty Dombrowski, Stoller's videographer.

Biological sampling activities began July 1 and continued through July 21 . Typically, dive operations occurred twice a day, once in the morning and once in the afternoon. There were three sites containing three transects each on Amchitka Island and two sites containing three transects on Adak Island. As the biological sampling project progressed, the dive teams were usually able to collect enough of all the specimens required from each transect during a period of two dives. The divers were limited to diving to a depth of 65 feet (20 meters); therefore, all samples were collected at depths less than 65 feet. The scientific team fished from the deck of the R/V Norseman to collect samples of the larger fish such as the Irish lords, greenlings, black rockfish, dusky rockfish, Pacific cod, and halibut.

With the exception of one rockfish duplicate from Milrow, all species proposed to be collected from Amchitka Island were collected. All species proposed to be collected from Adak Island were collected with the exception of one halibut and an octopus from south Adak Island and four rockfish from north Adak Island (one each from the three transects and a duplicate). As noted, no gull eggs were collected from Adak Island.

Where pedestrian access to rockweed was not possible on Amchitka and Adak, the biological sampling team of Karen Pletnikoff and Michael McCoy collected the rockweed samples from a skiff at all the remaining transect locations on both islands during times when the divers were not diving.

The remaining seawater samples off the coastline of the Cannikin site were collected from the deck of the R/V Norseman or from its skiff. 
Table 2 shows individual species collected, the number of samples of each species collected, and the average weight of the individual, or composited, prepared sample.

Table 2. Sample Collection Information

\begin{tabular}{|c|c|c|c|c|}
\hline Species & $\begin{array}{c}\text { Sample Wet } \\
\text { Weight Needed } \\
\text { (kg) }\end{array}$ & $\begin{array}{c}\text { Average } \\
\text { Weight } \\
\text { per Sample } \\
\text { (kg) }\end{array}$ & $\begin{array}{c}\text { Total } \\
\text { Number of } \\
\text { Individual } \\
\text { Samples } \\
\text { per Species }\end{array}$ & $\begin{array}{l}\text { Total Number } \\
\text { of Samples }^{a}\end{array}$ \\
\hline \multicolumn{5}{|c|}{ Ocean Sampling } \\
\hline Dragon kelp & 5 & 5.4 & 55 & $55^{b}$ \\
\hline Gumboot chiton & 0.5 & 0.59 & 40 & 15 \\
\hline Horse mussel & 0.1 & 0.48 & 164 & 15 \\
\hline Sea urchin & 0.5 & 0.62 & 92 & 15 \\
\hline Octopus & 5 & 3.56 & 8 & 4 \\
\hline Black or dusky rockfish & 0.5 & 1.04 & 47 & 46 \\
\hline Rock greenling & 0.5 & 0.62 & 51 & 50 \\
\hline Irish lord & 0.5 & 0.55 & 60 & 45 \\
\hline Halibut & 5 & 5.96 & 5 & 5 \\
\hline Pacific cod & 5 & 5.5 & 11 & 5 \\
\hline Seawater (diver-collected) ${ }^{\mathrm{c}}$ & NA & NA & NA & 50 \\
\hline Seawater (collected from vessel) ${ }^{\mathrm{c}}$ & NA & NA & NA & 66 \\
\hline Marine sediment & NA & NA & NA & 18 \\
\hline \multicolumn{5}{|c|}{ Terrestrial Sampling } \\
\hline Rockweed & 5 & 5.53 & 50 & 50 \\
\hline Reindeer lichen & 0.5 & 0.65 & 12 & $12^{\mathrm{d}}$ \\
\hline Soil beneath lichen & NA & 0.45 & NA & $12^{\mathrm{e}}$ \\
\hline Gull eggs & 2 & 1.2 & 12 & 1 \\
\hline Dolly Varden & 3 & 0.89 & 34 & 2 \\
\hline \multicolumn{4}{|l|}{ Total number of samples } & 466 \\
\hline
\end{tabular}

$\mathrm{kg}=$ kilogram

NA $=$ Not applicable

a Some samples were composited.

${ }^{\mathrm{b}} 5$ duplicate samples sent to UAF.

${ }^{\mathrm{c}}$ Samples sent to University of Miami.

${ }^{\mathrm{d}} 6$ duplicate samples sent to UAF.

${ }^{\mathrm{e}} 6$ duplicate samples sent to UAF.

When the dive team and samples were onboard the R/V Norseman after each dive, sample preparation began. With the help of members from the dive team, R/V Norseman crew, and certain members of the scientific team, samples were weighed, bagged, and labeled. Table 3 summarizes sampling and handling procedures for each species collected. Appendix B provides length and weight of samples collected. Once sample preparation was finished, all biological samples were frozen, and the seawater samples and sediment samples were stored in a cooler in the ship's hold.

Upon return of the R/V Norseman to the pier at Sweepers Cove on Thursday, July 21, all samples were placed into iced coolers with a completed chain-of-custody (COC) form, delivered to the airport, and shipped on Alaska Airlines to LLNL, the Tritium Laboratory at the University 
of Miami Rosenstiel School of Marine and Atmospheric Science in Miami, or the University of Alaska Anchorage that same day.

\subsection{Sample Chain-of-Custody}

The purpose of the COC was to provide documentation of the control and transfer of samples from the time of sample collection through shipping and receipt by the analytical laboratory. The $\mathrm{COC}$ form and sample labels were generated by the Stoller laboratory coordinator.

When the samples were physically transferred to the commercial carrier, the "Relinquisher" signed the COC forms and included the date and time of sample transfer. Employees of the commercial carrier were not required to sign the forms. The laboratory signed the "Received" blocks on the COC forms with the date and time the samples were received.

Each cooler was banded to ensure the contents were secure and the coolers would not open during transit. To ensure the integrity of the sample, custody seals were attached to the cooler openings. The original COC form was placed in a plastic zip bag and included inside one of the coolers being sent to the laboratory.

An example of a COC form is shown in Appendix C. The COC form contained the following information:

- $\quad$ Ticket number

- Data and time sample was collected

- $\quad$ Site

- Location where sample was collected

- Type and number of containers

- Preservative used on the sample

- Sample matrix

- $\quad$ Type of sample (composite or grab)

- Whether the sample was filtered or unfiltered

- Analyses requested 
Table 3. Collection and Preparation of Samples

\begin{tabular}{|c|c|c|c|c|}
\hline Species & Where & Collected By & Collection Procedure & Onboard Ship Handling/Storage \\
\hline \multicolumn{5}{|c|}{ Ocean Sampling } \\
\hline Dragon kelp & $\begin{array}{l}\text { Nearshore } \\
\text { subtidal }\end{array}$ & Dive team & $\begin{array}{l}\text { For each transect, three individual samples } \\
\text { weighing approximately } 5 \text { kilograms }(\mathrm{kg}) \text { each, or } \\
\text { three composited samples if necessary, were } \\
\text { collected. The holdfast was not collected. } \\
\text { A duplicate sample also was collected from one of } \\
\text { the three transects. The duplicate sample was split } \\
\text { with UAF. }\end{array}$ & $\begin{array}{l}\text { Sample bagging and labeling was performed onboard } \\
\text { the vessel. The sample was double bagged and } \\
\text { labeled twice with a unique location identification } \\
\text { number (once inside the bag and once on the outside) } \\
\text { and frozen. } \\
\text { The wet weight of each sample collected was recorded } \\
\text { in the database. }\end{array}$ \\
\hline Chiton & $\begin{array}{l}\text { Nearshore } \\
\text { subtidal }\end{array}$ & Dive team & $\begin{array}{l}\text { For each transect, whole chiton was collected and a } \\
\text { total combined weight of approximately } 0.5 \mathrm{~kg} \text { per } \\
\text { sample was obtained. }\end{array}$ & Labeling and packaging as above. \\
\hline Horse mussel & $\begin{array}{l}\text { Nearshore } \\
\text { subtidal }\end{array}$ & Dive team & $\begin{array}{l}\text { For each transect, the entire horse mussel was } \\
\text { collected and composited; a total combined weight } \\
\text { of approximately } 0.5 \mathrm{~kg} \text { per sample was obtained. } \\
\text { Approximately } 11 \text { horse mussels were collected } \\
\text { per sample. }\end{array}$ & Labeling and packaging as above. \\
\hline Sea urchin & $\begin{array}{l}\text { Nearshore } \\
\text { subtidal }\end{array}$ & Dive team & $\begin{array}{l}\text { For each transect, the entire sea urchin was } \\
\text { collected and composited, requiring approximately } \\
\text { six specimens per sample. }\end{array}$ & $\begin{array}{l}\text { For each composite sample, the wet weight of } \\
\text { individual urchins was measured, and the total weight } \\
\text { of the sample was calculated. Wide-mouth, 64-ounce } \\
\text { (oz) high-density polyethylene (HDPE) containers } \\
\text { were used to package the sea urchins. } \\
\text { Labels were attached to the containers, and then the } \\
\text { containers were placed in the freezer. }\end{array}$ \\
\hline Octopus & $\begin{array}{l}\text { Nearshore } \\
\text { subtidal }\end{array}$ & Dive team & $\begin{array}{l}\text { Divers collected specimens of adequate size } \\
\text { (if possible) within the } 2 \text {-mile zone. }\end{array}$ & $\begin{array}{l}\text { The total wet weight of the octopus was measured and } \\
\text { recorded in the database. Prior to the octopuses' arms } \\
\text { being severed and discarded, the octopus was } \\
\text { humanely euthanized by placing in a freezer. The } \\
\text { remaining portion was bagged, packaged, and labeled } \\
\text { the same as for dragon kelp. }\end{array}$ \\
\hline $\begin{array}{l}\text { Rockfish, rock } \\
\text { greenling, and } \\
\text { Irish lord }\end{array}$ & $\begin{array}{l}\text { Nearshore } \\
\text { subtidal } \\
\text { and } \\
\text { offshore }\end{array}$ & $\begin{array}{l}\text { Nearshore } \\
\text { collected by dive } \\
\text { team. Offshore } \\
\text { collected by } \\
\text { hook-and-line } \\
\text { crew. }\end{array}$ & $\begin{array}{l}\text { For each transect, one to two specimens of each } \\
\text { species and each transect were collected. }\end{array}$ & $\begin{array}{l}\text { Each fish had its taxonomic identification checked, sex } \\
\text { determined (if possible), length measured (total or fork } \\
\text { length, dependent on the fish species) in centimeters, } \\
\text { and weight recorded in grams. } \\
\text { Labeling and packaging same as for dragon kelp. }\end{array}$ \\
\hline Halibut & Offshore & $\begin{array}{l}\text { Hook-and-line } \\
\text { crew }\end{array}$ & $\begin{array}{l}\text { One fish per sample zone, not transect location, } \\
\text { was caught. }\end{array}$ & Same as for dragon kelp. \\
\hline
\end{tabular}


Table 3 (continued). Collection and Preparation of Samples

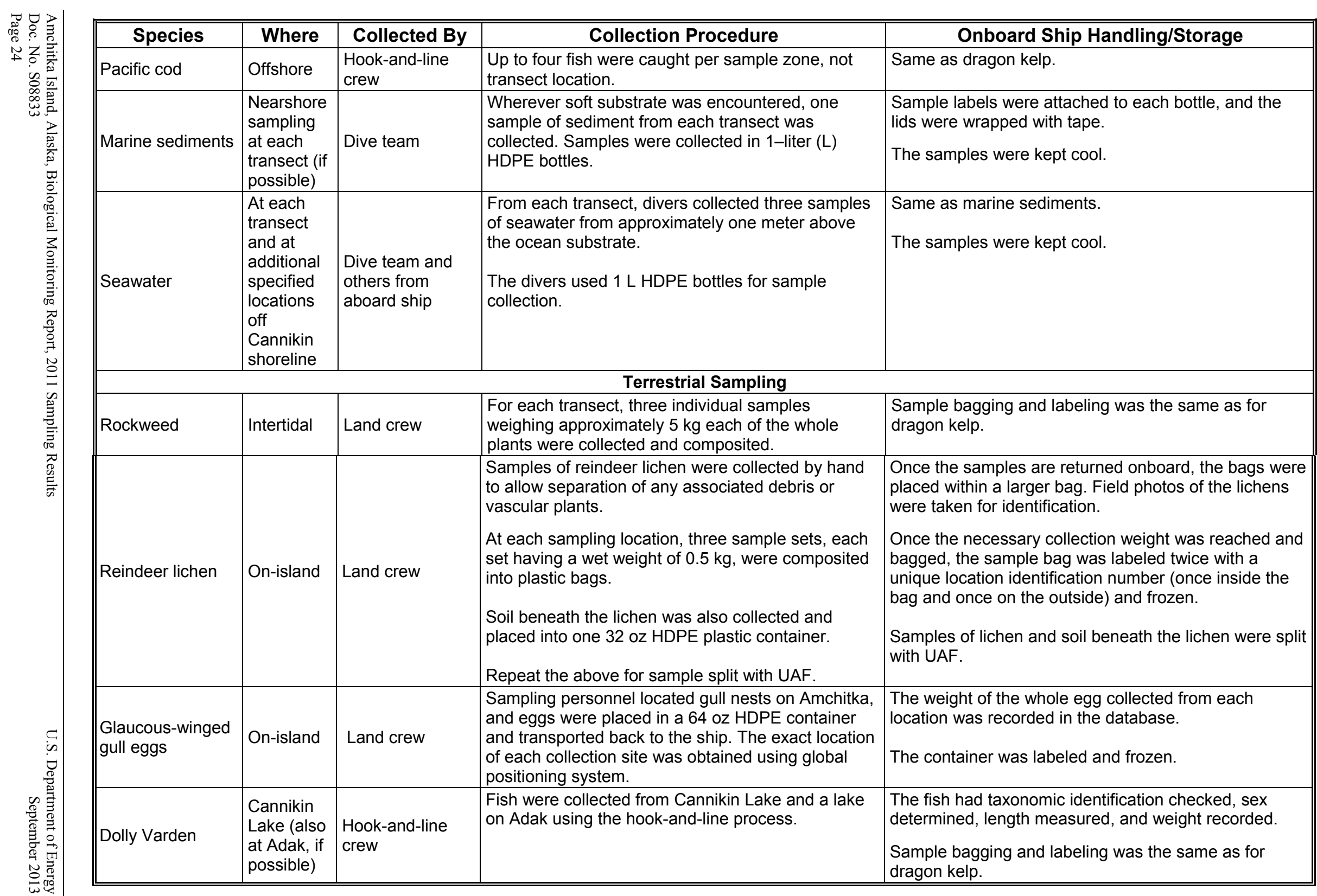




\subsection{Laboratory Analysis, Quality Assurance, and Data Validation Procedures}

\subsection{Target Radionuclides and Selection Criteria for Methods of Analysis}

Previous studies conducted by CRESP on biological aspects of potential radionuclide exposure in the Amchitka marine environment provided helpful insight into the likely range of observable radionuclide concentrations in a range of biological species. The aim of the 2011 sampling event was to optimize the design of the sampling and analysis program to help validate these measurements and provide a basis for analyzing future trends. A strategic planning process was laid out with DQOs that identified where and when to collect samples, how many samples needed to be collected (within practical limitations), and which measurement techniques (and at what cost) might be best suited to help reduce uncertainties in assessing food safety, fill any information gaps, and provide adequate detection sensitivity to support future statistical trending analyses. LLNL scientists also cited the advantages of developing isotope-specific data based on mass spectrometry to help characterize inputs from different potential source terms. During the post-planning phase of the 2011 sampling event, some consideration was also given to the possible complexities in interpreting background activity concentrations of target radionuclides due to atmospheric releases from the Fukushima Dai-ichi facility. A decision was therefore made to screen selected samples of soil and lichen for the presence of ${ }^{134} \mathrm{Cs}$ by gamma spectrometry.

Table 4 shows a list of recommended radiometric measurement techniques for analysis of target isotopes with nominal detection limits. The only deployable method outlined in the Sampling Plan for analysis of marine sediment, soil, and lichen was high-resolution gamma spectrometry. All other biological samples were to be analyzed for ${ }^{137} \mathrm{Cs},{ }^{234} \mathrm{U},{ }^{235} \mathrm{U},{ }^{238} \mathrm{U},{ }^{239} \mathrm{Pu},{ }^{240} \mathrm{Pu}$, and ${ }^{241} \mathrm{Am}$.

Table 4. Techniques for Measurement of Target Radionuclide

\begin{tabular}{||c|l|c||}
\hline Analyte & \multicolumn{1}{|c||}{ Analytical Technique } & $\begin{array}{c}\text { Minimal Detectable } \\
\text { Activity (pCi) }\end{array}$ \\
\hline${ }^{137} \mathrm{Cs}$ & Gas Proportional Beta Spectrometry ${ }^{\mathrm{b}}$ & 0.3 \\
\hline${ }^{234} \mathrm{U}$ & Quadrupole Inductively Coupled Plasma Mass Spectrometry (ICP-MS) & 0.003 \\
\hline${ }^{235} \mathrm{U}$ & Quadrupole Inductively Coupled Plasma Mass Spectrometry (ICP-MS) & $2 \times 10^{-6}$ \\
\hline${ }^{238} \mathrm{U}$ & Quadrupole Inductively Coupled Plasma Mass Spectrometry (ICP-MS) & $2 \times 10^{-7}$ \\
\hline${ }^{239} \mathrm{Pu}$ & Quadrupole Inductively Coupled Plasma Mass Spectrometry (ICP-MS) & 0.01 \\
\hline${ }^{240} \mathrm{Pu}$ & Quadrupole Inductively Coupled Plasma Mass Spectrometry (ICP-MS) & 0.02 \\
\hline${ }^{241} \mathrm{Am}$ & Alpha Spectrometry & $0.03-0.06$ \\
\hline
\end{tabular}

${ }_{\mathrm{pCi}}=$ picocuries

${ }^{\mathrm{b}}$ Gamma spectrometry to be employed for reindeer lichen, marine sediment, and soil 


\subsection{Sample Laboratory Preparation Methods}

Analytical results of previous sampling events showed that background activity concentrations of selected target radionuclides in the vicinity of Amchitka Island were at or below levels of detection. Planning for LM's 2011 sampling event included placing emphasis on collecting an adequate number and larger-sized samples to support future trending analysis. The mean sample dry weight of material for analysis of target radionuclides ranged from 40 grams ( $\mathrm{g}$ ) for horse mussel to well over 1,000 g for halibut, Pacific cod, dragon kelp, and rockweed. To reduce the sample mass to a more manageable size for wet chemistry, it was necessary to reduce the volume of material by combustion in a muffle furnace. Prior to combustion, the biological samples were dried by lyophilization in an industrial-size freezer. A summary of the laboratory preparation methods, including the initial sample processing and preparation procedures as well as sample combustion procedures, is included as Appendix D. Appendix E is the summary of individual samples' wet, dry, and ash weights.

\subsection{Analytical Methods}

The bulk of the ${ }^{137} \mathrm{Cs}$ analyses were performed at LLNL using gas proportional beta spectrometry. The remaining samples, including marine sediment, soil, and reindeer lichen, were analyzed by high-resolution gamma spectrometry. ${ }^{241} \mathrm{Am}$ measurements were performed at LLNL using alpha spectrometry. Plutonium isotope analyses were performed at LLNL on a Thermo Electron XSeries quadrupole inductively coupled plasma mass spectrometer (ICP-MS) fitted with a concentric nebulizer. Uranium isotope analyses were performed at LLNL on a Thermo Electron XSeries quadrupole ICP-MS fitted with a SeaSpray nebulizer. Tritium analysis was performed by the Tritium Laboratory at the University of Miami Rosenstiel School of Marine and Atmospheric Science using the enriched method and low-level gas proportional counters.

\subsection{Quality Assurance and Data Validation}

This section is a summary of the quality assurance and data validation process used for the radiometric analyses. Appendix F provides a detailed discussion of the process. The beta spectrometry system was calibrated using stock solutions of ${ }^{137} \mathrm{Cs}$ prepared by serial dilution of a Standard Reference Material 4233D supplied by the National Institute of Science and Technology. Data validation procedures included the analysis of archive water samples obtained under the DOE Mixed Analyte Performance Evaluation Program.

For ${ }^{241} \mathrm{Am}$, a conservative estimate of the minimum detectable concentration (MDC) was calculated based on a Stapleton approximation (adopted from EPA 2004) by using the mean background count rate for the alpha spectrometry system rather than a mean blank count rate. This approximation was made because a series of 10 replicate blank measurements all returned a zero count rate in the specific region of interest for ${ }^{241} \mathrm{Am}$. With this assumption, the largest number of ${ }^{241} \mathrm{Am}$ counts likely to be observed in a background count was 2, yielding an estimated MDC for measurement of ${ }^{241} \mathrm{Am}$ by alpha spectrometry of 0.01 picocurie (pCi).

The minimal detectable signal of the ICP-MS instrument for plutonium isotope measurements was estimated in a fashion similar to that described for uranium isotopes using procedures adopted from EPA (2004). Based on the standard deviation of 10 reported replicate blank 
measurements, the MDC, defined by units of activity for measurement of ${ }^{239} \mathrm{Pu}$ and ${ }^{240} \mathrm{Pu}$ by ICP-MS, is estimated to be around 0.03 and $0.02 \mathrm{pCi}$, respectively.

The minimal detectable signal of the ICP-MS instrument for uranium isotope measurements was defined by the standard deviation of 10 reported replicate blank measurements based on a noncentral $t$-distribution function, and assuming the variance of the signal between measurements was approximately constant (using procedures adopted from EPA 2004). 
This page intentionally left blank 


\subsection{Sampling Results}

\subsection{Database Discussion}

LM's Amchitka and Adak Islands' environmental data are stored in the LM environmental database known as SEEPRo (Site Environmental Evaluation for Projects). The Environmental Support Services Data Management group is responsible for ensuring that LM environmental data are captured, stored, organized, maintained, and preserved in reliable and accurate condition so that the data are available for use by other LM organizations.

\subsection{Data Presentation}

The box plots (also called box-and-whisker plots) in Figure 10 through Figure 21 show the distributions of results in samples collected from Amchitka and Adak islands, along with corresponding MDCs. These plots depict the median (central line bisecting the box), the lower and upper quartiles, the non-outlier range, and outliers. For each species, the corresponding number of samples collected from Amchitka and Adak islands is listed below the plots. With the exception of extreme outlier U-flagged results (undetected; values were below the MDC) and one horse mussel sample HMUS-LS-LT3-1 from Amchitka, box plots were generated using all data, including U-flagged results. To facilitate interpretation of the box plots, corresponding tables in Section 6 list detection frequencies and relevant summary statistics.

In the following figures, although all plot components - medians, lower and upper quartiles, non-outlier raw data, and outliers and extremes - are identified in the legends; the following introductory schematic provides more detail.

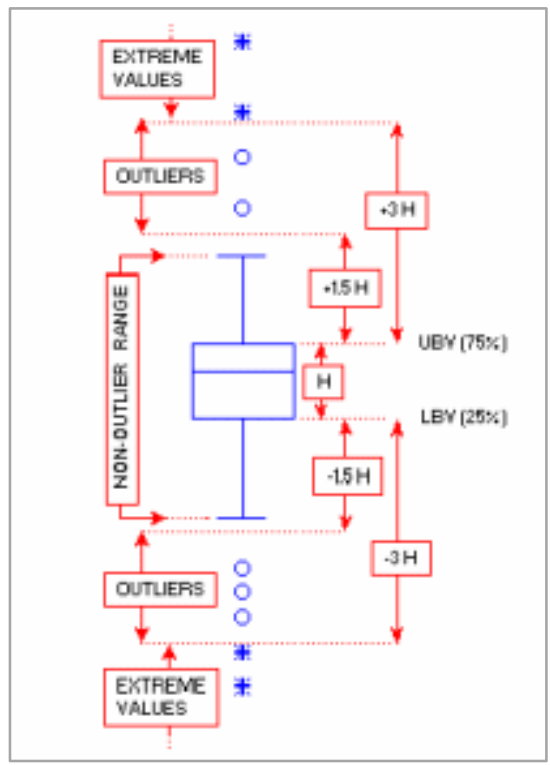

Source: Statistica 64, Version 10 Electronic Manual (StatSoft Inc.) 
For this analysis, a data point is considered an outlier if the following conditions hold:

Data point value $>\mathrm{UBV}+1.5(\mathrm{UBV}-\mathrm{LBV})$ or data point value $<\mathrm{LBV}-1.5(\mathrm{UBV}-\mathrm{LBV})$

where:

UBV is the upper value of the box in the box plot (75th percentile or upper quartile)

LBV is the lower value of the box in the box plot (25th percentile or lower quartile)

Similarly, a data point is deemed to be an extreme value if:

Data point value $>\mathrm{UBV}+3(\mathrm{UBV}-\mathrm{LBV})$ or data point value $<\mathrm{LBV}-3(\mathrm{UBV}-\mathrm{LBV})$.

\subsection{Cesium-137 Isotope}

A full listing of individual measurement data for ${ }^{137} \mathrm{Cs}$ in vegetation, marine fauna, marine sediment, and fauna collected during the Amchitka 2011 sampling event is given in Appendix G (Hamilton et al. 2012b). The data are reported in units of picocuries per kilogram (pCi/kg) wet weight for vegetation and fauna and in units of picocuries per kilogram dry weight for marine sediment and soil. All data are decay corrected to the date of sampling. For quick reference, data are sorted according to species type and location/grid. Gamma spectrometry measurements of ${ }^{137} \mathrm{Cs}$ and ${ }^{134} \mathrm{Cs}$ in marine sediment and soil are based on the $<2$ millimeter particle size fraction.

The ${ }^{137}$ Cs content of marine vegetation and fauna collected across the Adak and Amchitka sites is shown graphically in Figure 10 and Figure 11. Figure 10 shows ${ }^{137} \mathrm{Cs}$ in selected marine vegetation and fauna and gull eggs. Figure 11 shows ${ }^{137} \mathrm{Cs}$ in Dolly Varden, lichen, soil beneath the lichen, and marine sediment.

The ${ }^{137}$ Cs content of horse mussel from both sites shown in Figure 10 was highly variable, and excluding data developed for a single sample of gull eggs from Amchitka Island, contained the highest mean activity concentrations of ${ }^{137} \mathrm{Cs}$ at both islands. The mean activity concentrations of ${ }^{137} \mathrm{Cs}$ in horse mussel tissue from the Adak and Amchitka sites were 9.2 and $11.4 \mathrm{pCi} / \mathrm{kg}$ wet weight, respectively. This compares with reported levels up to several hundred $\mathrm{pCi} / \mathrm{kg}$ wet weight in Dolly Varden, and several thousand $\mathrm{pCi} / \mathrm{kg}$ wet weight in reindeer lichen. 


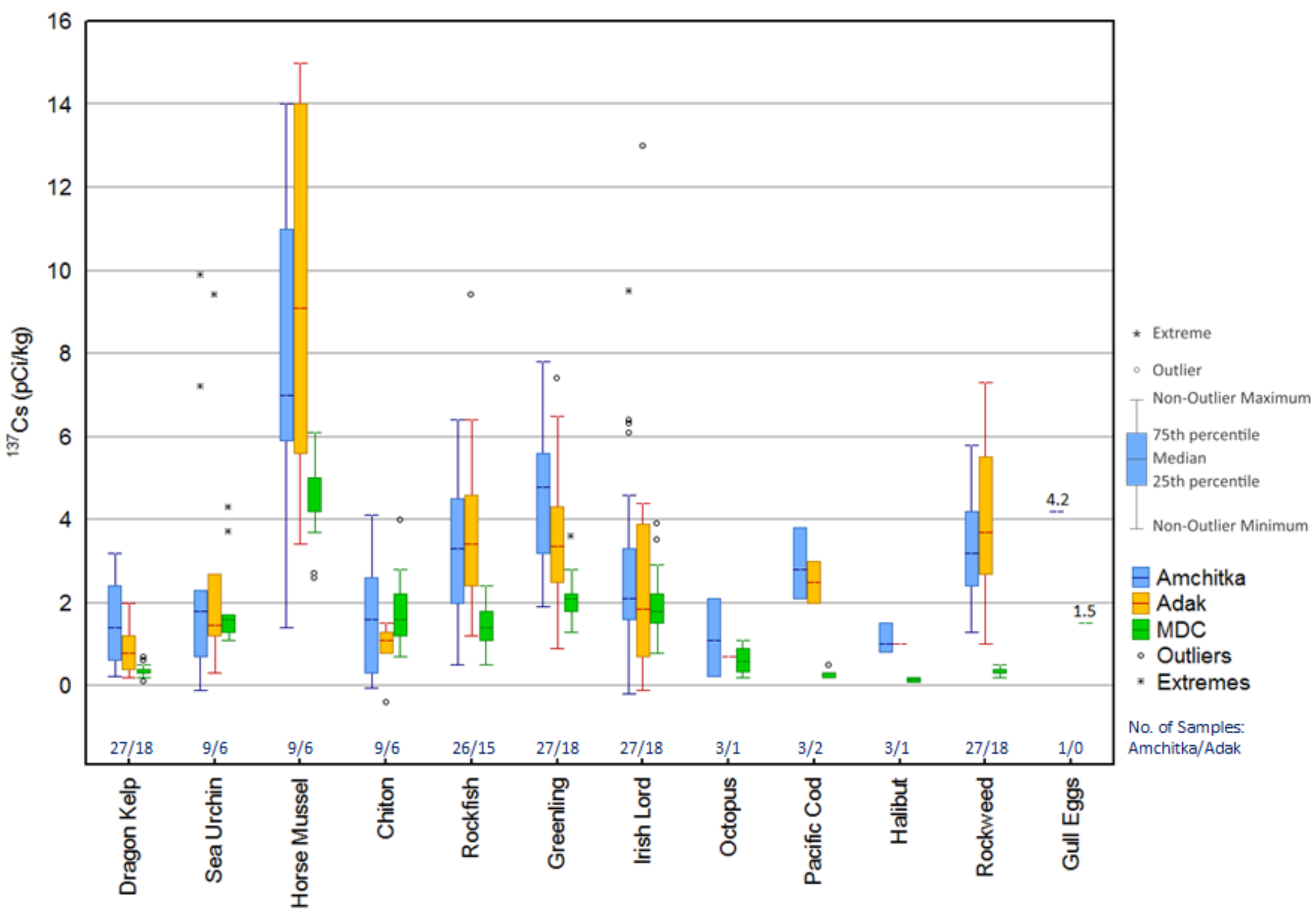

Figure $10 .{ }^{137} \mathrm{Cs}$ in Selected Marine Vegetation, Fauna, and Gull Eggs 


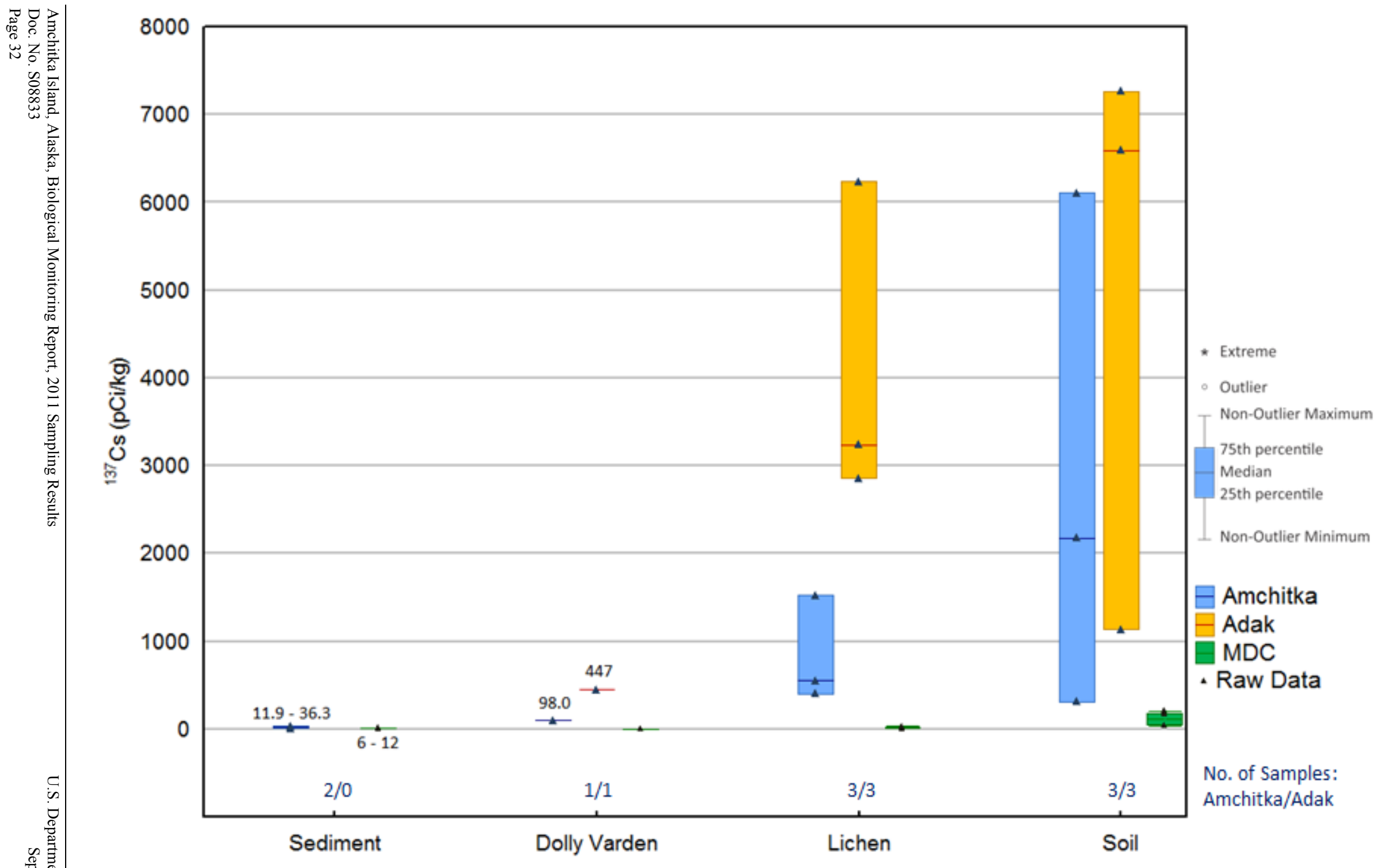

Figure 11. ${ }^{137}$ Cs in Dolly Varden, Lichen, Soil Beneath the Lichen, and Marine Sediment Raw data are also plotted, given the small number of samples collected for each of these species/media. 


\subsection{Americium-241 Isotope}

A full listing of individual measurement data for ${ }^{241} \mathrm{Am}$ in vegetation and marine fauna collected during the Amchitka 2011 sampling event is given in Appendix H (after Hamilton et al. 2012d). Data are reported in activity concentration units of picocuries per kilogram wet weight. For quick reference, data are sorted according to species type and location/grid. The results are reported as measured even if the reported value is less than the reported MDC. No weight corrections were made for the presence of sea salts in determining the ${ }^{241}$ Am content of rockweed and dragon kelp. The ${ }^{241}$ Am content of vegetation and fauna collected from the Adak and Amchitka sites is shown graphically in Figure 12 and Figure 13. Figure 12 depicts ${ }^{241}$ Am in selected marine vegetation, fauna, and gull eggs. Figure 13 depicts ${ }^{241} \mathrm{Am}$ in dragon kelp, rockfish, greenling, Irish lord, octopus, and rockweed.

${ }^{241}$ Am was measured by alpha spectrometry using ion-implanted-silicon charged-particle detectors. The estimated MDC for measurement of ${ }^{241} \mathrm{Am}$ by alpha spectrometry was $0.01 \mathrm{pCi}$. The mean reported sample measurement MDC for ${ }^{241} \mathrm{Am}$ expressed as activity concentration was $0.008 \mathrm{pCi} / \mathrm{kg}$ wet weight. The vast majority of samples analyzed under this sampling event contained ${ }^{241} \mathrm{Am}$ loadings that were at or below the reported MDC. Data also appear to be more highly variable across individual species of fauna. While there are no significant outliers, this may be indicative of the lower quantity of these data compared to the quantities obtained for other radionuclides.

The highest-quality data appear to have been obtained for sea urchin, octopus, and, to a lesser extent, dragon kelp. Few reliable laboratory intercomparison samples are available for performance testing of ${ }^{241} \mathrm{Am}$ at environmental concentrations. Quality assurance measures performed under this sampling event were limited to assessing the reproducibility of the alpha spectrometric measurement by conducting a series of cross counts on different detectors. In this case, the results satisfied internal laboratory data quality requirements within the quantifiable capabilities of the technique.

\subsection{Plutonium Isotopes}

Full individual measurement data for ${ }^{239} \mathrm{Pu}$ and ${ }^{240} \mathrm{Pu}$ in vegetation and fauna collected during the Amchitka 2011 sampling event are shown in Appendix I (after Hamilton et al. 2012c). The data are reported in units of picocuries per kilogram wet weight. For quick reference, data are sorted according to species type and location/grid. Data are reported as measured even if the reported value is less than the reported MDC. No weight corrections were made for the presence of sea salts in determining the plutonium content of rockweed and dragon kelp.

The ${ }^{239} \mathrm{Pu}$ and ${ }^{240} \mathrm{Pu}$ concentrations of vegetation and fauna collected from the Adak and Amchitka sites are shown graphically in Figure 14 and Figure 15 . Figure 14 depicts ${ }^{239} \mathrm{Pu}$ in selected marine vegetation and fauna, gull eggs, and lichen. Figure 15 depicts ${ }^{240} \mathrm{Pu}$ in selected marine vegetation and fauna, gull eggs, and lichen. 


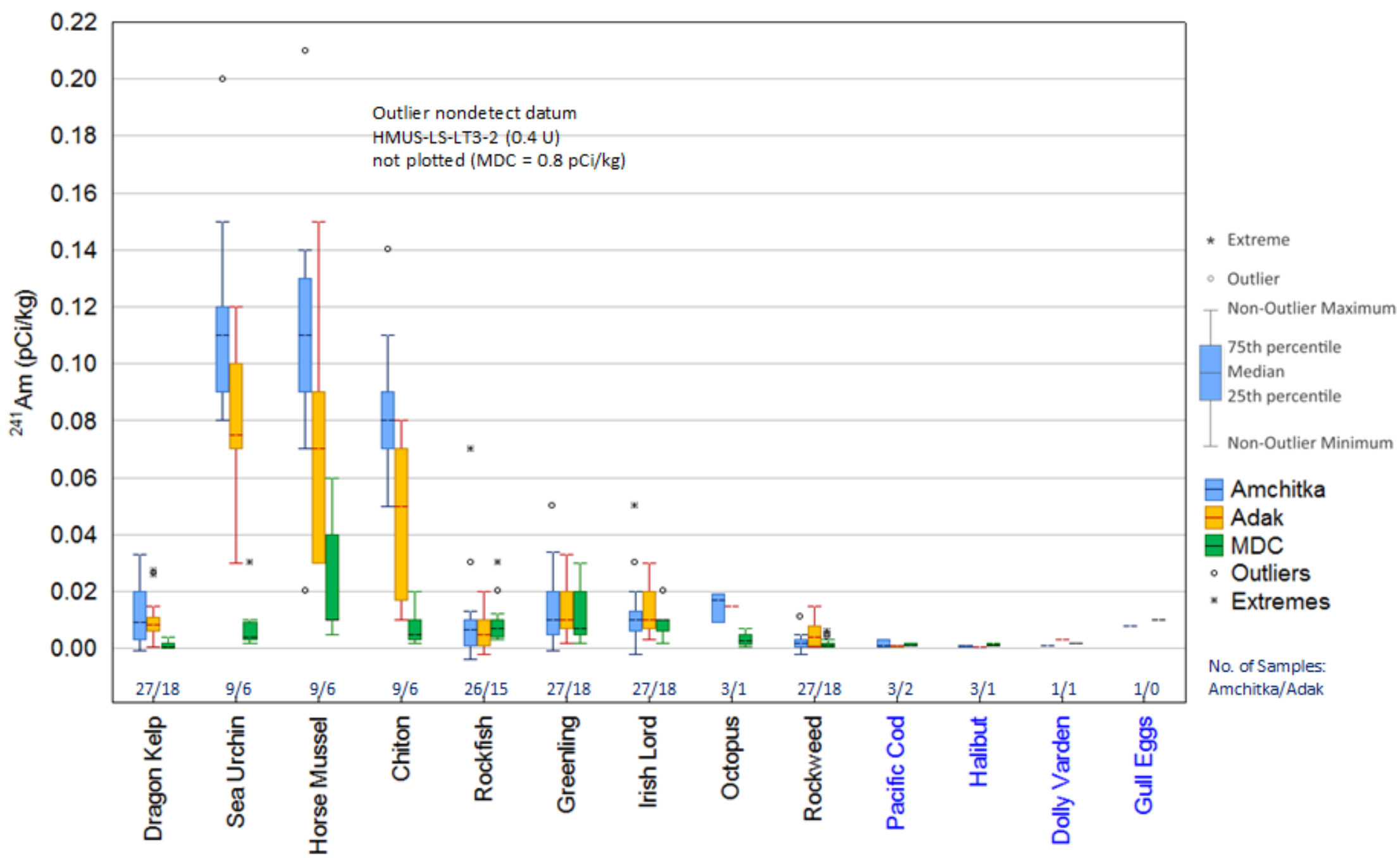

Figure 12. ${ }^{241} \mathrm{Am}$ in Selected Marine Vegetation, Fauna, and Gull Eggs

Species in the rightmost portion of graph (listed in blue font) are those for which all results were less than the MDC. A zoom-view of results for species with the lowest ${ }^{241} \mathrm{Am}$ concentrations is provided Figure 13. 


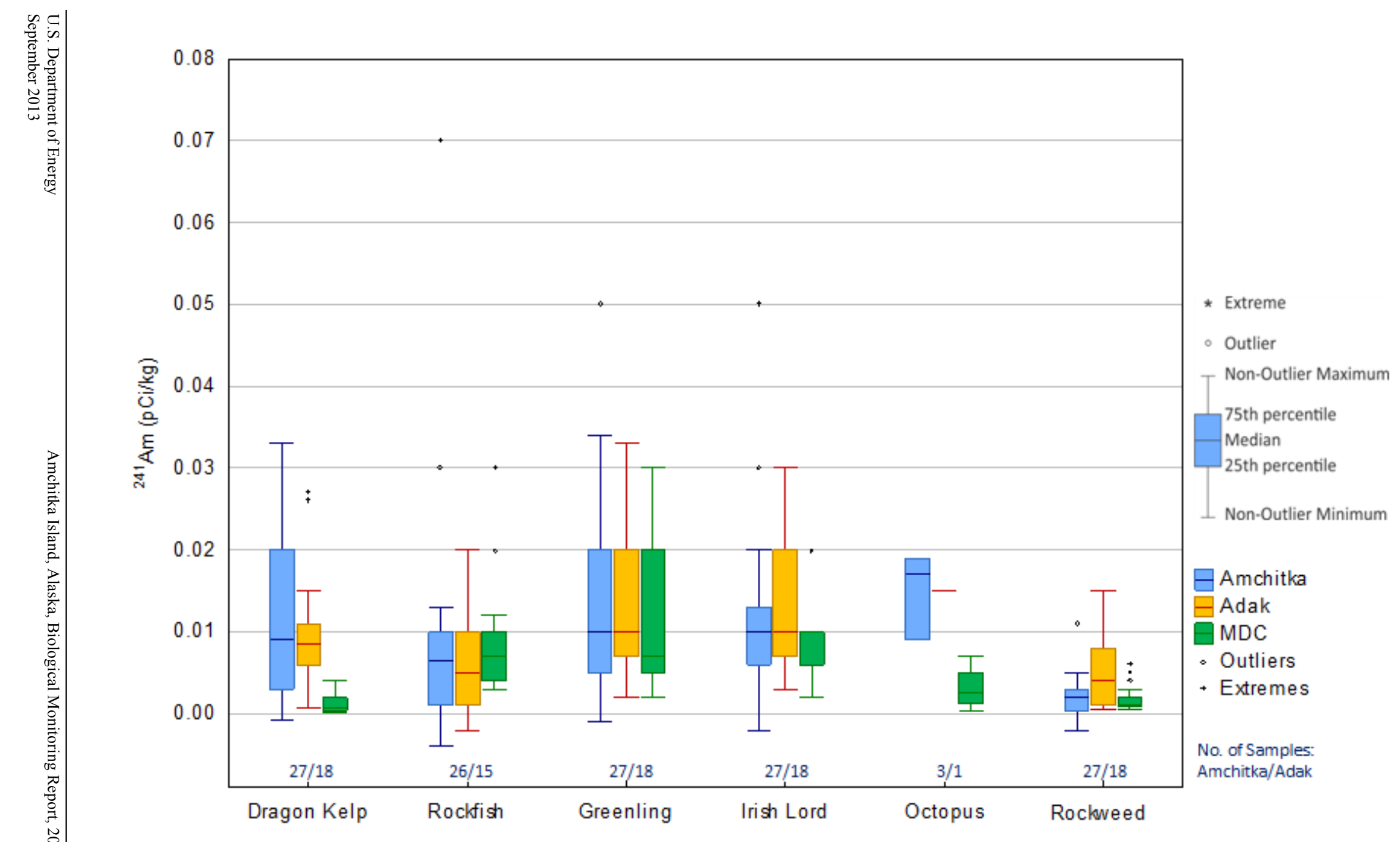

Figure 13. ${ }^{241}$ Am in Dragon Kelp, Rockfish, Greenling, Irish Lord, Octopus, and Rockweed Zoom-view of species with the lowest detectable ${ }^{241} \mathrm{Am}$ concentrations in Figure 12. Detection frequencies were low (<20 percent) for rockfish, greenling, and Irish lord. Interisland comparisons are significant only for rockweed. 


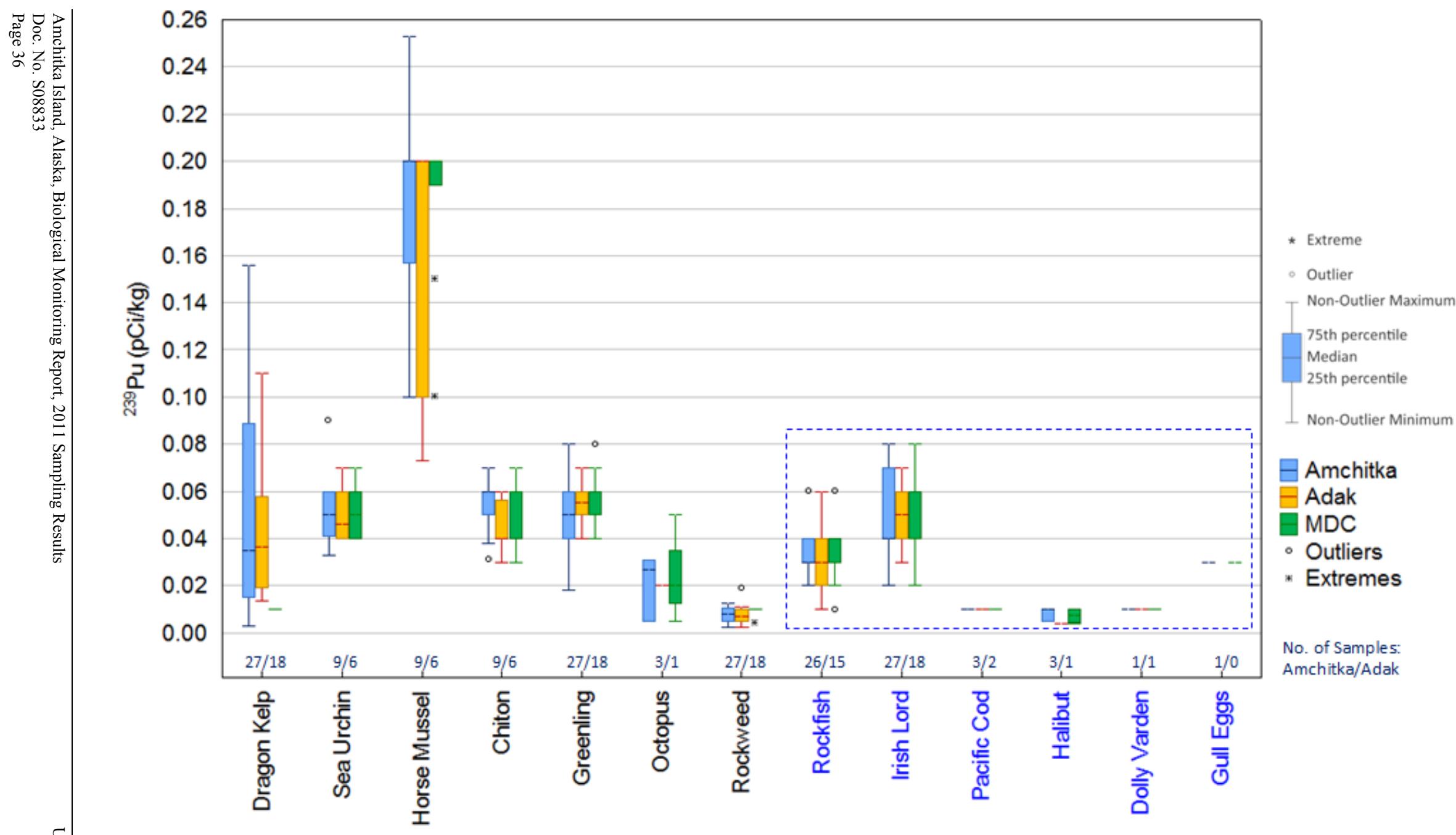

Figure 14. ${ }^{239} \mathrm{Pu}$ in Selected Marine Vegetation, Fauna, and Gull Eggs

Species in the rightmost portion of graph (blue font, outlined) are those for which all results were less than the MDC. This plot excludes outlier nondetect datum HMUS-LS-LT3-1 and corresponding MDC (2 pCi/kg). 


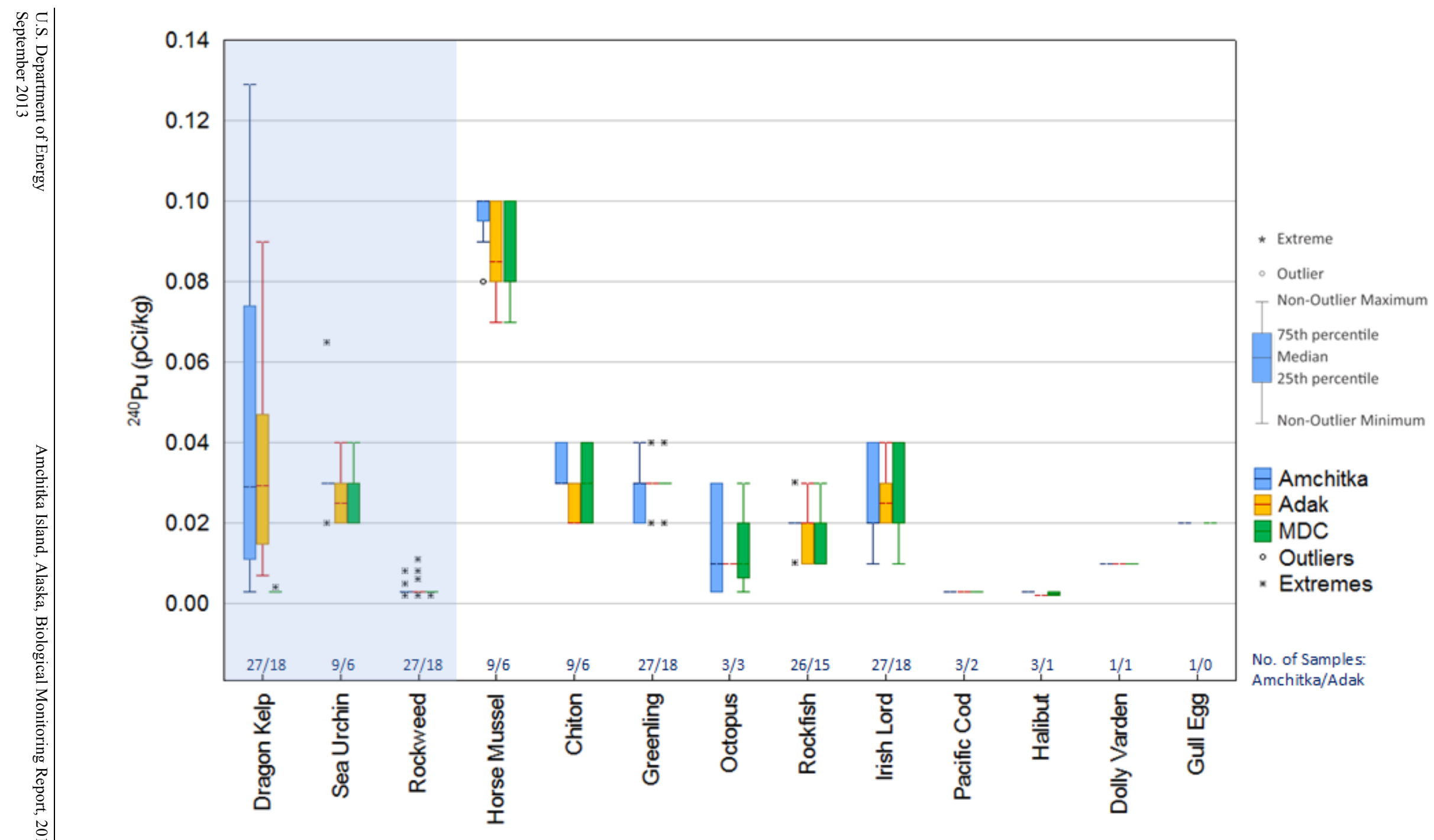

Figure 15. ${ }^{240} \mathrm{Pu}$ in Selected Marine Vegetation, Fauna, and Gull Eggs

Only three species/media_dragon kelp, sea urchin, and rockweed (shaded)—had detectable levels of ${ }^{240}$ Pu. Results for remaining species were all below corresponding MDCs. This plot excludes outlier nondetect datum HMUS-LS-LT3-1 and corresponding MDC (1 pCi/kg). 
Quantifiable concentrations of ${ }^{239} \mathrm{Pu}$ and/or ${ }^{240} \mathrm{Pu}$ were obtained in the vast majority of dragon kelp and rockweed samples along with selected samples of chiton, octopus, sea urchin, and horse mussel. Levels of ${ }^{239} \mathrm{Pu}$ and ${ }^{240} \mathrm{Pu}$ in fish species are consistently at or below the reported MDC.

Based on the standard deviation of 10 reported replicate blank measurements, the estimated MDC for ${ }^{239} \mathrm{Pu}$ and ${ }^{240} \mathrm{Pu}$ is 0.03 and $0.02 \mathrm{pCi}$, respectively. The mean reported sample measurement MDCs for ${ }^{239} \mathrm{Pu}$ and ${ }^{240} \mathrm{Pu}$ (excluding one outlier for a sample with a very low mass) expressed as activity concentrations were 0.04 and $0.02 \mathrm{pCi} / \mathrm{kg}$ wet weight, respectively. Analyses of mass ratio and concentration performed on certified reference materials satisfied laboratory data quality requirements for both precision and accuracy. Similarly, a series of duplicate measurement met applicable data quality requirements within the quantifiable capabilities of the technique. High-quality data appear to have been developed for plutonium isotopes in dragon kelp as evidenced by the internal consistency of ${ }^{240} \mathrm{Pu} /{ }^{239} \mathrm{Pu}$ atom ratios measured in this species. Moreover, it appears reasonable that dragon kelp and possibly rockweed could serve as good indicator species for future trending analysis of plutonium contamination in this region.

\subsection{Uranium Isotopes}

A full listing of individual measurement data for ${ }^{234} \mathrm{U},{ }^{235} \mathrm{U}$, and ${ }^{238} \mathrm{U}$ in vegetation and marine fauna collected during the Amchitka 2011 sampling event is given in Appendix J (after Hamilton et al. 2012e). Data are reported in units of picocuries per kilogram wet weight. For quick reference, data are sorted according to species type and location/grid. Quantifiable activity concentrations for ${ }^{234} \mathrm{U},{ }^{235} \mathrm{U}$, and ${ }^{238} \mathrm{U}$ with reasonable levels of precision are reported for all vegetation and fauna samples with the exception of ${ }^{234} \mathrm{U}$ in one horse mussel sample.

The ${ }^{234} \mathrm{U},{ }^{235} \mathrm{U}$, and ${ }^{238} \mathrm{U}$ content of vegetation and fauna collected from the Adak and Amchitka sites is shown graphically in Figure 16 through Figure 18, respectively. Additionally, with overall concentrations of ${ }^{234} \mathrm{U},{ }^{235} \mathrm{U}$, and ${ }^{238} \mathrm{U}$ being very low, Figure 19 through Figure 21 show box plots for selected species and media with the lowest $(<25 \mathrm{pCi} / \mathrm{kg}){ }^{234} \mathrm{U},{ }^{235} \mathrm{U}$, and ${ }^{238} \mathrm{U}$ concentrations, respectively.

No weight or analyte corrections were made for the presence of sea salts and inherent concentrations of uranium in ocean water for large-volume samples of rockweed and dragon kelp known to contain significant free and interstitial water.

\subsection{Tritium in Seawater}

A full listing of the tritium data for seawater collected during 2011 off the coastlines of Cannikin, Long Shot, and Milrow sites, as well as the north and south coastlines of Adak, is given in Appendix K. 


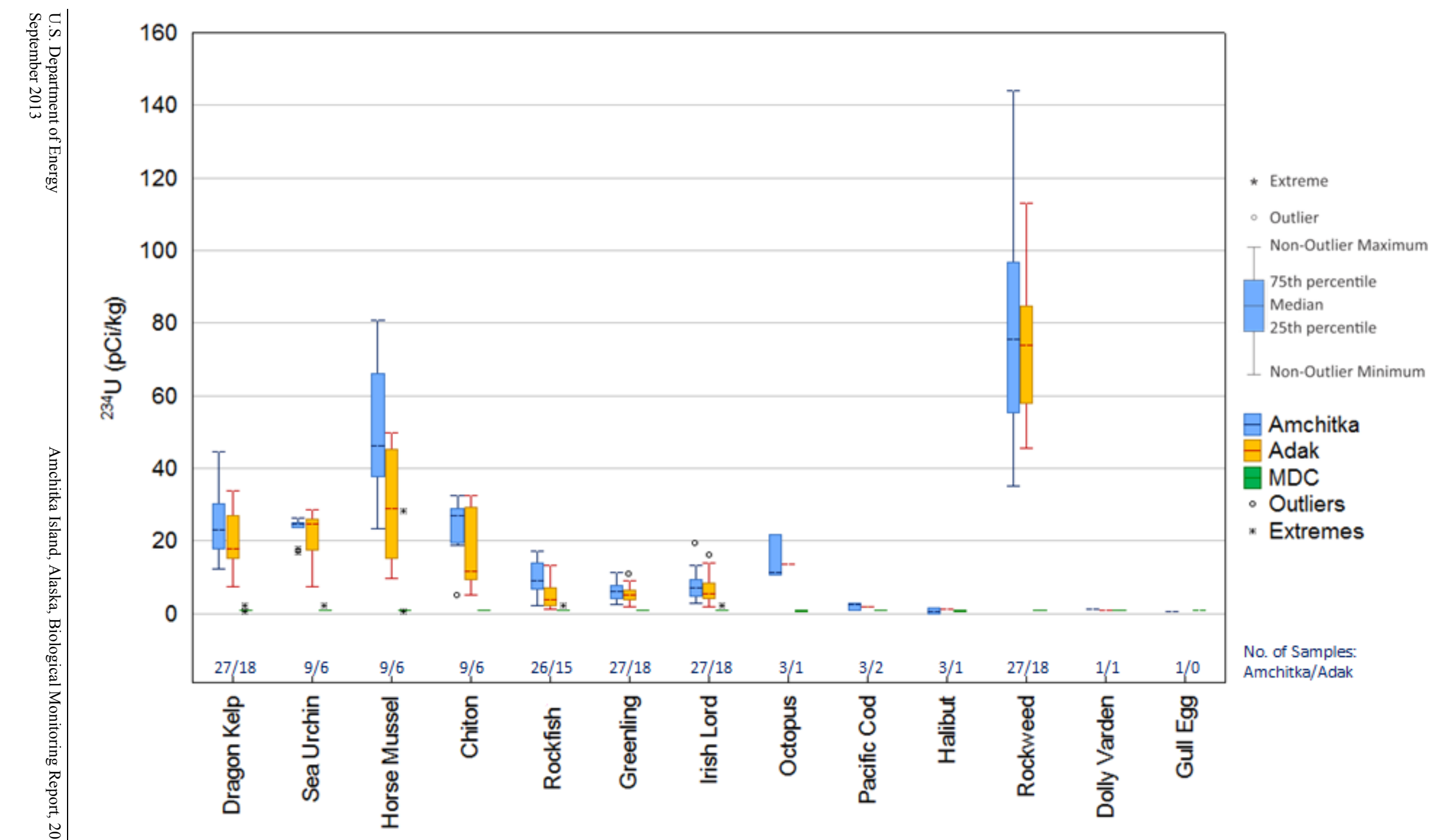

Figure 16. ${ }^{234} \mathrm{U}$ in Selected Marine Vegetation, Fauna, and Gull Eggs

As found for the other $U$ isotopes (Figures 17-18), ${ }^{234} U$ concentrations are highest in horse mussel and rockweed.

A zoom-view of results for species with lower ${ }^{234} \mathrm{U}$ concentrations $(<25 \mathrm{pCi} / \mathrm{kg})$ is provided in Figure 19. 


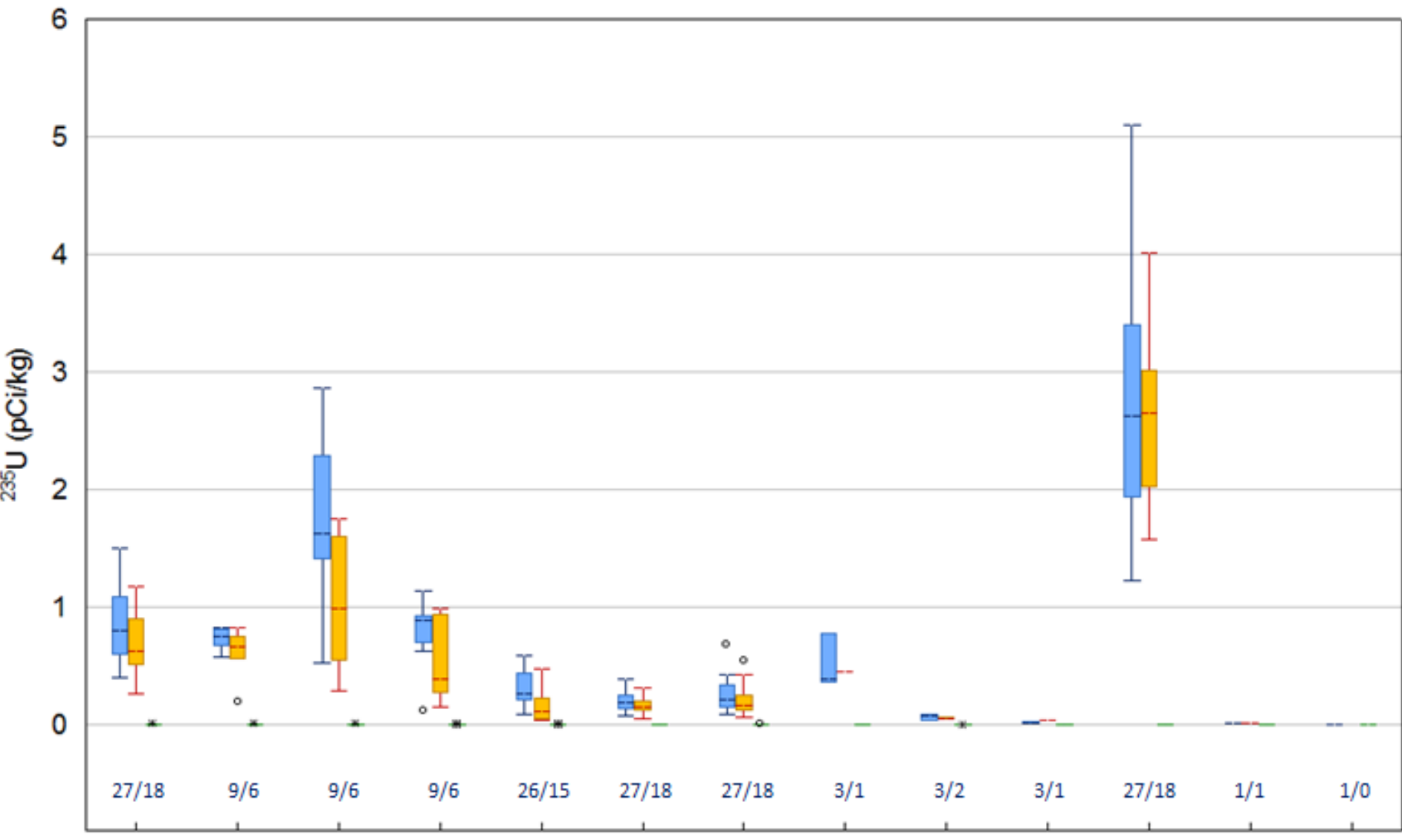

* Extreme

- Outlier

- Non-Outlier Maximum

75 th percentile

Median

25th percentile

Non-Outlier Minimum

Amchitka

Adak

MDC

- Outliers

* Extremes

No. of Samples: Amchitka/Adak

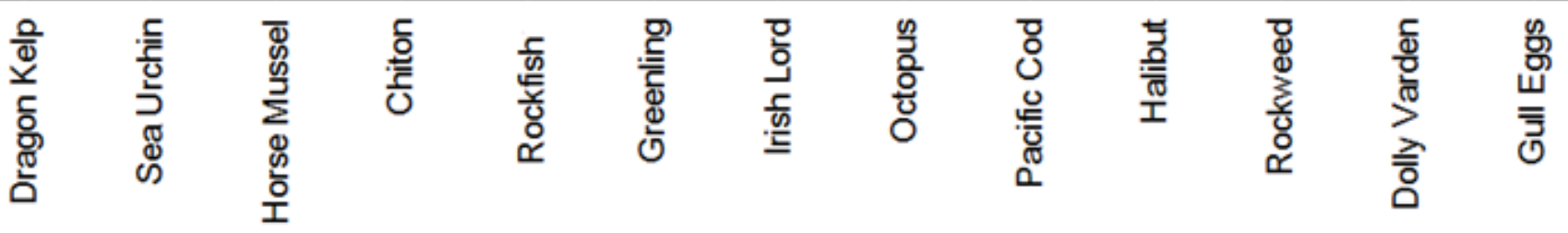

Figure 17. ${ }^{235} \mathrm{U}$ in Selected Marine Vegetation, Fauna, and Gull Eggs

As is the case for ${ }^{234} \mathrm{U}$ and ${ }^{238} \mathrm{U},{ }^{235} \mathrm{U}$ concentrations are highest in horse mussel and rockweed. A zoom-view of results for species with lower ${ }^{235} \mathrm{U}$ concentrations $(<1 \mathrm{pCi} / \mathrm{kg})$ is provided in Figure 20. 


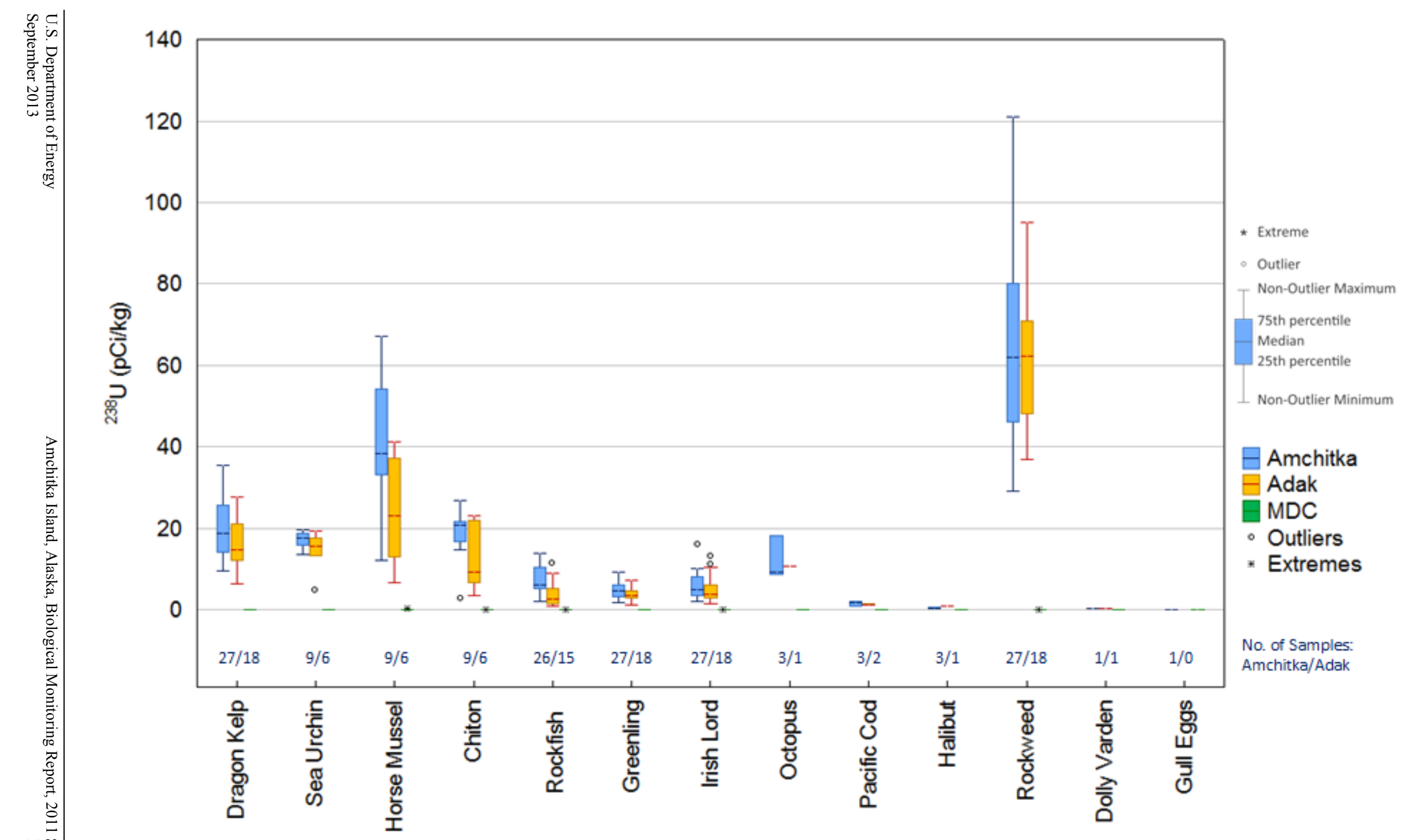

Figure 18. ${ }^{238} \mathrm{U}$ in Selected Marine Vegetation, Fauna, and Gull Eggs

${ }^{238} \mathrm{U}$ Magnitude and Distributions Are Very Similar to Those for ${ }^{234} \mathrm{U}$; Concentrations Are Highest in Horse Mussel and Rockweed.

A zoom-view of results for species with lower ${ }^{238} \mathrm{U}$ concentrations $(<20 \mathrm{pCi} / \mathrm{kg})$ is provided in Figure 21. 


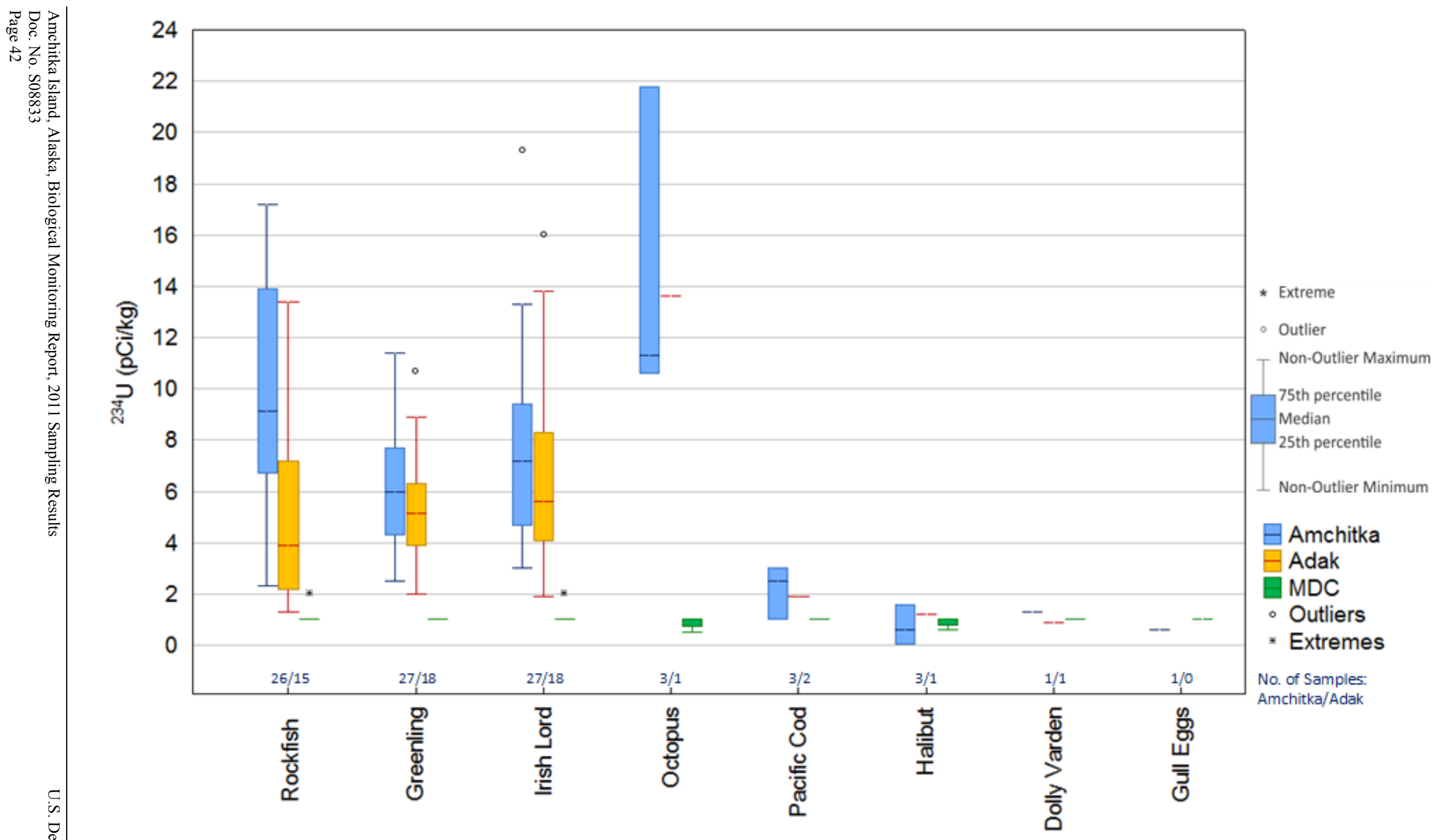

Figure 19. Zoom view of ${ }^{234} \mathrm{U}$ in Species and Media with Concentrations $<25 \mathrm{pCi} / \mathrm{kg}$ 


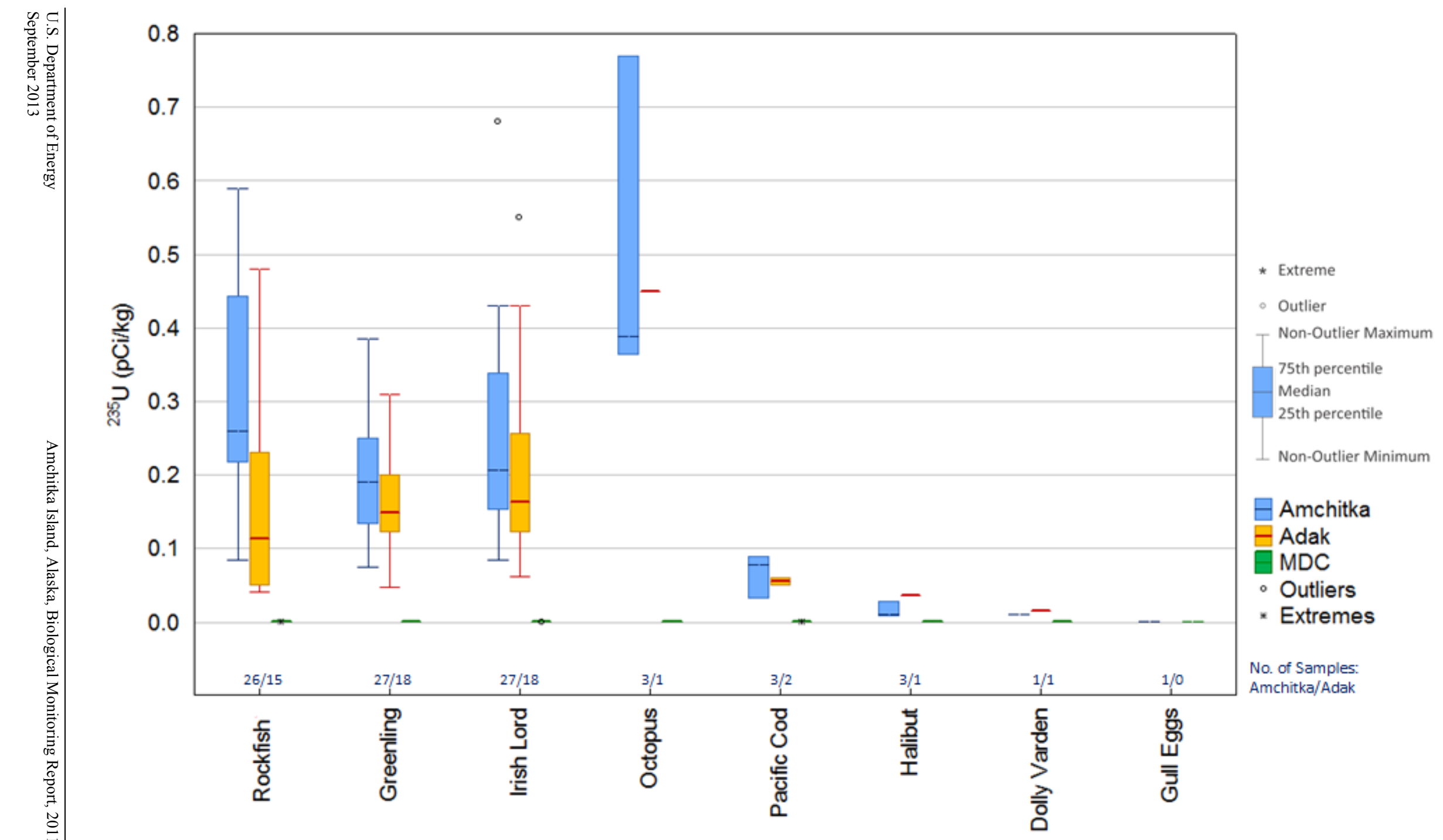

Figure 20. Zoom View of ${ }^{233} \mathrm{U}$ in Species and Media with Concentrations $<1 \mathrm{pCi} / \mathrm{kg}$ 


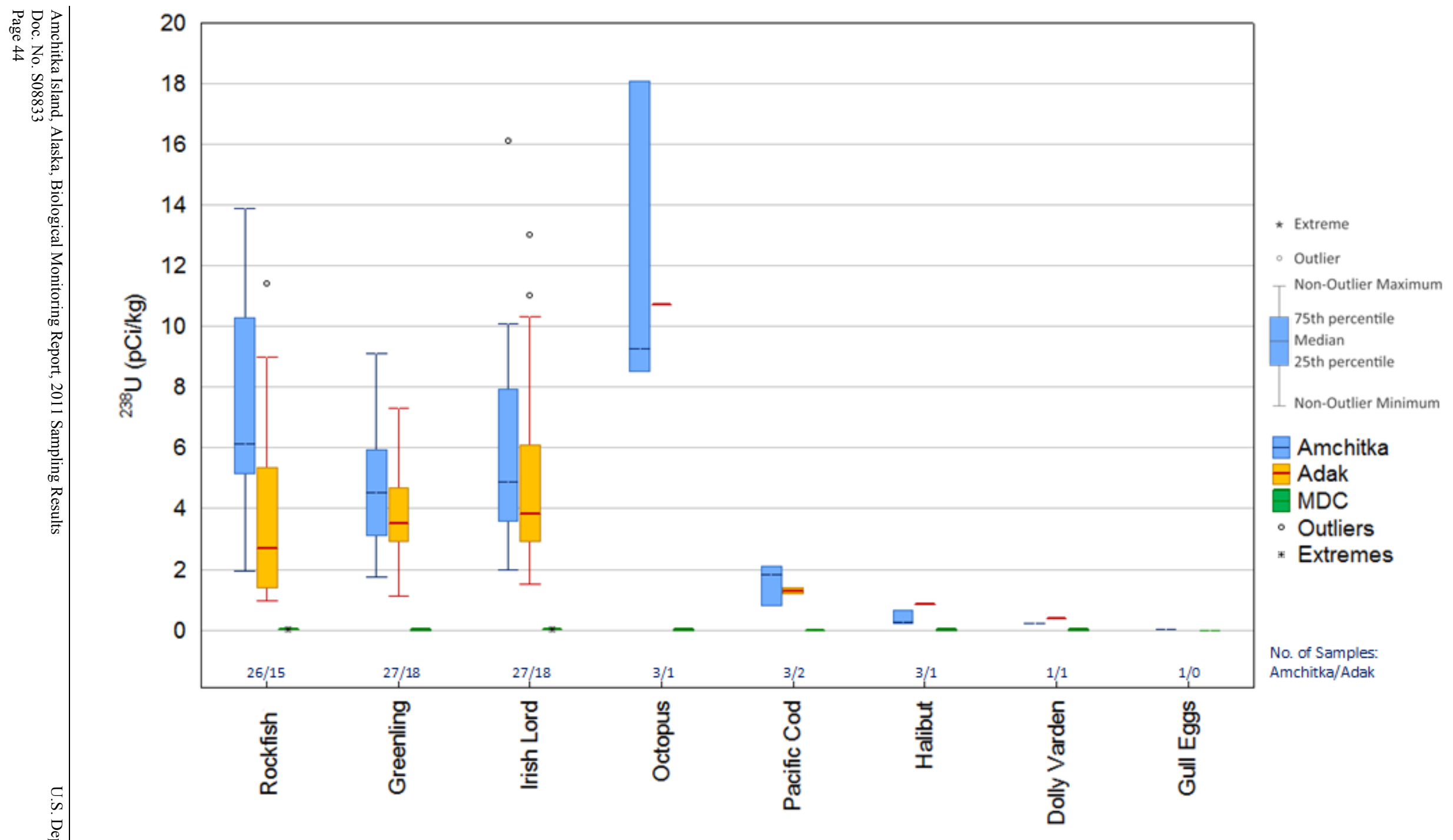

Figure 21. Zoom View of ${ }^{238} \mathrm{U}$ in Species and Media with Concentrations $<1 \mathrm{pCi} / \mathrm{kg}$ 


\subsection{Data Analysis}

\subsection{Summary Statistics}

Table 5 through Table 12 present the ranges of detected values (results at or greater than the MDC) and summary statistics for results from the 2011 sampling of Amchitka and Adak Islands. Nondetects are set equal to the MDC. Where duplicate sample results are available, the higher value of either the duplicate or the sample result is used. The species for which duplicate samples were collected are dragon kelp, rockweed, rockfish, greenling, and halibut. Refer to corresponding raw data summaries in Appendixes G-J and to LLNL (2012).

\subsubsection{Cesium-137 Isotope}

Table 5 shows the summary statistics for ${ }^{137} \mathrm{Cs}$. The established range for horse mussel excludes sample HMUS-LS-LT3 in which the MDC was $60 \mathrm{pCi} / \mathrm{kg}$ wet weight, and the subsequent result was $44 \mathrm{U} \pm 11 \mathrm{pCi} / \mathrm{kg}$ wet weight. U-flagged data are excluded from the statistical evaluation. The highest mean concentration of ${ }^{137} \mathrm{Cs}$ in marine fauna measured across both sites was observed in horse mussel. The mean concentration of ${ }^{137} \mathrm{Cs}$ in horse mussel was $11.5 \mathrm{pCi} / \mathrm{kg}$ wet weight for Amchitka and $9.4 \mathrm{pCi} / \mathrm{kg}$ wet weight for Adak. Mean and median concentrations in marine vegetation and fauna samples collected from Amchitka $(n$ [number of samples] $=27)$ were 1.1 and $1.0 \mathrm{pCi} / \mathrm{kg}$ wet weight, respectively, whereas those from Adak $(n=18)$ were 0.7 and $0.7 \mathrm{pCi} / \mathrm{kg}$. Maximum concentrations measured in marine vegetation and fauna samples from Amchitka and Adak were 14.0 and $15.0 \mathrm{pCi} / \mathrm{kg}$, respectively.

\subsubsection{Americium-241 Isotope}

Quantifiable concentrations of ${ }^{241}$ Am were detected (albeit at very low concentrations) for the vast majority of dragon kelp, sea urchin, chiton, and to some degree, horse mussels. Levels in all fish species appear to consistently fall at or below the MDC. Table 6 shows the summary statistics for ${ }^{241} \mathrm{Am}$.

The highest mean concentrations of ${ }^{241} \mathrm{Am}$ in samples of marine vegetation and fauna from both sites were typically observed in horse mussel, chiton, and urchin. For the Adak site, the mean concentration of ${ }^{241} \mathrm{Am}$ in horse mussel, $0.073 \mathrm{pCi} / \mathrm{kg}$ wet weight, and urchin, $0.078 \mathrm{pCi} / \mathrm{kg}$ wet weight, were indistinguishable from that in chitin, $0.046 / \mathrm{kg}$ wet weight, but were otherwise higher than for all other groups. Similarly, for the Amchitka site, the mean concentration of ${ }^{241} \mathrm{Am}$ in horse mussel, $0.143 \mathrm{pCi} / \mathrm{kg}$ wet weight, was indistinguishable from that in urchin, $0.116 \mathrm{pCi} / \mathrm{kg}$ wet weight, but was otherwise higher than for all other groups. The mean rockweed value reported for Adak, $0.005 \mathrm{pCi} / \mathrm{kg}$ wet weight, was higher than the mean reported for Amchitka, $0.002 \mathrm{pCi} / \mathrm{kg}$ wet weight, but the mean value measured for chiton was higher for samples collected from Amchitka, $0.084 \mathrm{pCi} / \mathrm{kg}$ wet weight, than for samples collected from Adak, $0.046 \mathrm{pCi} / \mathrm{kg}$ wet weight. 
Table 5. Descriptive Statistics for ${ }^{137} \mathrm{Cs}$

All units in $\mathrm{pCi} / \mathrm{kg}$ wet weight except for soil and sediment, which are reported in dry weight.

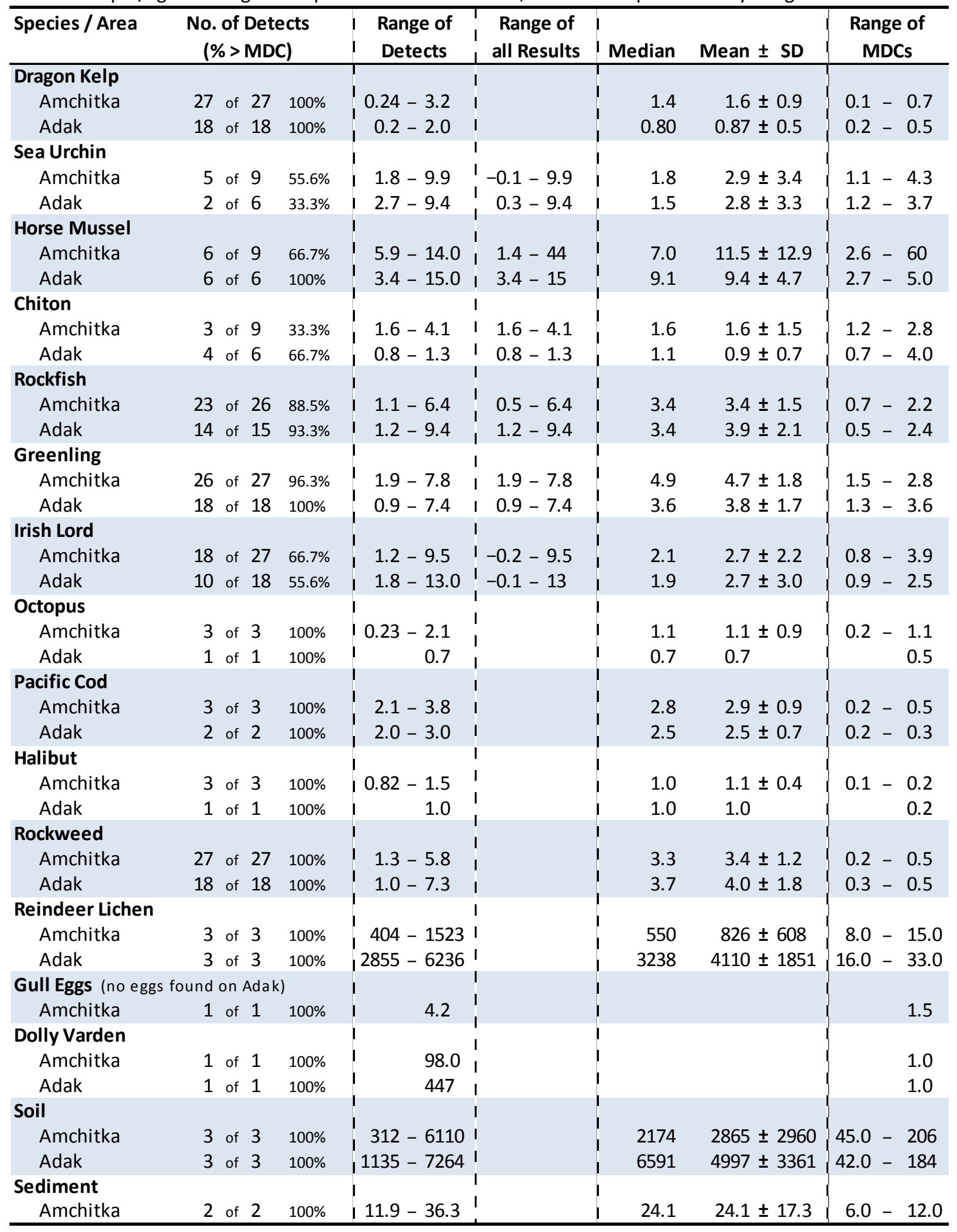

MDC - Minimum Detectable Concentration; SD - Standard Deviation. Statistics computed with values = MDC for nondetect The second range column-"Range of all Results"-includes U-flagged $(<M D C)$ results. This column is left blank for those species/media with $100 \%$ detection frequencies. Duplicate samples were collected for Dragon Kelp ( 3 Amchitka/2 Adak); Rockfish (4/1); greenling (3/2); halibut (0/1); and rockweed (3/2). The maximum result for each duplicate pair was used. 
Table 6. Descriptive Statistics for ${ }^{241} \mathrm{Am}$

All units in $\mathrm{pCi} / \mathrm{kg}$ wet weight.

\begin{tabular}{|c|c|c|c|c|c|c|c|}
\hline Species / Area & $\begin{array}{r}\text { No. of De } \\
(\%>M\end{array}$ & ects & $\begin{array}{c}\text { Range of } \\
\text { Detects }\end{array}$ & $\begin{array}{c}\text { Range of } \\
\text { all Results }\end{array}$ & Median & Mean \pm SD & Range of MDCs \\
\hline Dragon Kelp & & & & & & & \\
\hline Amchitka & 20 of 27 & $74.1 \%$ & $0.003-0.033$ & $-0.001-0.033$ & 0.009 & $0.012 \pm 0.010$ & $0.0003-0.004$ \\
\hline Adak & 15 of 18 & $83.3 \%$ & $0.006-0.027$ & $0.001-0.027$ & 0.009 & $0.010 \pm 0.007$ & $0.0002-0.002$ \\
\hline Sea Urchin & & & & & & & \\
\hline Amchitka & 8 of 9 & $88.9 \%$ & $0.08-0.20$ & $0.080-0.200$ & 0.110 & $0.116 \pm 0.038$ & $0.002-0.01$ \\
\hline Adak & 5 of 6 & $83.3 \%$ & $0.07-0.12$ & $0.030-0.120$ & 0.075 & $0.078 \pm 0.031$ & $0.003-0.03$ \\
\hline Horse Mussel & & & & 1 & & & 1 \\
\hline Amchitka & 6 of 9 & $66.7 \%$ & $0.11-0.21$ & $0.02-0.40$ & 0.11 & $0.14 \pm 0.11$ & $0.005-0.80$ \\
\hline Adak & 2 of 6 & $33.3 \%$ & $0.09-0.15$ & $0.03-0.15$ & 0.07 & $0.073 \pm 0.04$ & $0.009-0.05$ \\
\hline Chiton & & & 1 & & 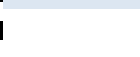 & & \\
\hline Amchitka & 8 of 9 & $88.9 \%$ & $0.070-0.14$ & $0.050-0.140$ & 0.08 & $0.084 \pm 0.027$ & $0.002-0.01$ \\
\hline Adak & 4 of 6 & $66.7 \%$ & $0.017-0.08$ & $0.010-0.080$ & 0.05 & $0.046 \pm 0.029$ & $0.002-0.02$ \\
\hline Rockfish & & & & 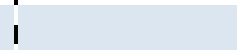 & & & 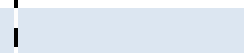 \\
\hline Amchitka & 3 of 26 & $11.5 \%$ & $0.009-0.07$ & $-0.004-0.07$ & 0.007 & $0.009 \pm 0.014$ & $0.003-0.03$ \\
\hline Adak & 0 of 15 & ND & & $1-0.002-0.02$ & & & $0.003-0.01$ \\
\hline Greenling & & & & & & & 1 \\
\hline Amchitka & 4 of 27 & $14.8 \%$ & $0.030-0.034$ & $-0.001-0.05$ & 0.01 & $0.014 \pm 0.012$ & $0.002-0.02$ \\
\hline Adak & 3 of 18 & $16.7 \%$ & $0.009 \quad 0.033$ & $0.002-0.033$ & 0.01 & $0.013 \pm 0.009$ & $0.002-0.03$ \\
\hline Irish Lord & & & 1 & & I & & \\
\hline Amchitka & 3 of 27 & $11.1 \%$ & $0.006 \quad 0.03$ & $-0.002-0.05$ & 0.010 & $0.011 \pm 0.010$ & $0.002-0.02$ \\
\hline Adak & 1 of 18 & $5.6 \%$ & 0.03 & $0.003-0.03$ & 0.010 & $0.013 \pm 0.008$ & $0.004-0.01$ \\
\hline Octopus & & & & 1 & & & 1 \\
\hline Amchitka & 2 of 3 & $66.7 \%$ & $0.017-0.019$ & $0.009-0.019$ & 0.017 & $0.015 \pm 0.005$ & $0.0004-0.007$ \\
\hline Adak & 1 of 1 & $100 \%$ & 0.015 & $1 \quad 0.015$ & 0.015 & 0.015 & 0.002 \\
\hline Pacific Cod & & & 1 & ! & 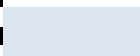 & & 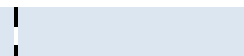 \\
\hline Amchitka & 0 of 3 & ND & 1 & $0.0004-0.003$ & & & $0.001-0.002$ \\
\hline Adak & 0 of 2 & ND & 1 & $0.001-0.001$ & & & 0.001 \\
\hline Halibut & & & & & & & 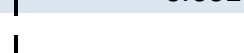 \\
\hline Amchitka & 0 of 3 & ND & & $0.0006-0.001$ & & & $0.001-0.002$ \\
\hline Adak & 0 of 1 & ND & & 0.001 & & & 0.002 \\
\hline Rockweed & & & & 1 & & & I \\
\hline Amchitka & 6 of 27 & $22.2 \%$ & $0.003-0.011$ & $-0.002-0.011$ & 0.002 & $0.002 \pm 0.003$ & $0.0005-0.006$ \\
\hline Adak & 7 of 18 & $38.9 \%$ & $0.006-0.015$ & $0.001-0.015$ & 0.004 & $0.005 \pm 0.005$ & $0.0005-0.005$ \\
\hline Gull Eggs (no & ound on $\mathrm{Ad}$ & & & & & & \\
\hline Amchitka & 0 of 1 & ND & t & 0.008 & & & 0.01 \\
\hline Dolly Varden & & & 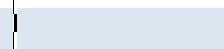 & & & & \\
\hline Amchitka & 0 of 1 & ND & 1 & 0.001 & & & 0.002 \\
\hline Adak & 0 of 1 & ND & & 0.003 & & & 0.002 \\
\hline
\end{tabular}

MDC - Minimum Detectable Concentration; ND - Not Detected (all results less than MDC); SD - Standard Deviation The second range column, "Range of all Results", includes U-flagged $(<M D C)$ results. Summary statistics were computed using values $=$ MDC for nondetects. Duplicate samples were collected for Dragon Kelp (3 Amchitka/2 Adak); Rockfish (4/1); greenling (3/2); halibut (0/1); and rockweed (3/2). The maximum result for each duplicate pair was used. Medians, means, and standard deviations were not calculated for species for which all results were $<$ MDC. 


\subsubsection{Plutonium Isotopes}

Statistical analyses show that where quantifiable data are obtained (e.g., for dragon kelp and rockweed), the activity concentrations of ${ }^{239} \mathrm{Pu}$ between the Adak and Amchitka sites are indistinguishable. Table 7 and Table 8 show the summary statistics for ${ }^{239} \mathrm{Pu}$ and ${ }^{240} \mathrm{Pu}$, respectively.

Plutonium isotopes were measured by quadrupole ICP-MS. The quantification capabilities of ICP-MS appear to be adequate for ${ }^{239} \mathrm{Pu}$ and ${ }^{240} \mathrm{Pu}$ measurements in dragon kelp samples and for ${ }^{239} \mathrm{Pu}$ measurements in rockweed samples. ${ }^{239} \mathrm{Pu}$ and ${ }^{240} \mathrm{Pu}$ loadings in all fish species and significant numbers of other fauna species were below the MDC. The mean dragon kelp value reported for Amchitka, $0.055 \mathrm{pCi} / \mathrm{kg}$ wet weight, was higher than the mean for samples collected from Adak, $0.048 \mathrm{pCi} / \mathrm{kg}$ wet weight, and rockweed mean values were equal for both Amchitka and Adak, $0.008 \mathrm{pCi} / \mathrm{kg}$ wet weight. Similarly, the mean ${ }^{240} \mathrm{Pu}$ value for dragon kelp is higher for Amchitka, $0.044 \mathrm{pCi} / \mathrm{kg}$ wet weight, than for Adak, $0.038 \mathrm{pCi} / \mathrm{kg}$ wet weight, but the rockweed mean value is just slightly higher for Adak, $0.004 \mathrm{pCi} / \mathrm{kg}$ wet weight, than for Amchitka, $0.003 \mathrm{pCi} / \mathrm{kg}$ wet weight.

The quantifiable concentrations of ${ }^{239} \mathrm{Pu}$ and/or ${ }^{240} \mathrm{Pu}$ in dragon kelp and rockweed shown in Table 9 fall in a relatively consistent range. For dragon kelp $(n=45)$, the mean value is $0.052 \mathrm{pCi} / \mathrm{kg}$ wet weight for ${ }^{239} \mathrm{Pu}$ and $0.042 \mathrm{pCi} / \mathrm{kg}$ wet weight for ${ }^{240} \mathrm{Pu}$. For rockweed $(n=45)$, the mean value is $0.0076 \mathrm{pCi} / \mathrm{kg}$ wet weight for ${ }^{239} \mathrm{Pu}$ and $0.0034 \mathrm{pCi} / \mathrm{kg}$ wet weight for ${ }^{240} \mathrm{Pu}$. These values suggest that reported levels for ${ }^{239} \mathrm{Pu}$ where data are available could be used without associated values for ${ }^{240} \mathrm{Pu}$ to estimate the total ${ }^{239+240} \mathrm{Pu}$ content of marine fauna for use in health and ecological assessments.

\subsubsection{Uranium Isotopes}

Table 10 through Table 12 show the summary statistics for ${ }^{234} \mathrm{U},{ }^{235} \mathrm{U}$, and ${ }^{238} \mathrm{U}$, respectively. The total uranium content of rockweed is statistically higher at both sites than that measured in all other species. Uranium in horse mussel is also statistically higher at both sites, but not as high as rockweed. The mean concentrations of ${ }^{234} \mathrm{U},{ }^{235} \mathrm{U}$, and ${ }^{238} \mathrm{U}$ in rockweed from samples collected at Amchitka were 81.6, 2.88, and $68.0 \mathrm{pCi} / \mathrm{kg}$ wet weight, respectively, all slightly higher than the mean concentrations of the same uranium isotopes in rockweed from samples collected at Adak, 72.7, 2.57, and $60.6 \mathrm{pCi} / \mathrm{kg}$ wet weight, respectively. The mean concentrations of ${ }^{234} \mathrm{U},{ }^{235} \mathrm{U}$, and ${ }^{238} \mathrm{U}$ in horse mussels collected at Amchitka were 49.5, 1.70, and $40.0 \mathrm{pCi} / \mathrm{kg}$ wet weight, respectively, and once again were higher than the mean concentrations of the same uranium isotopes in horse mussel samples collected at Adak, 29.6, 1.03, and $24.1 \mathrm{pCi} / \mathrm{kg}$ wet weight, respectively.

No quantifiable levels of anthropogenic uranium based on ${ }^{236} \mathrm{U}$ detection and measurement (results not shown) were observed in samples collected during this sampling event, although reportable MDCs were relatively high (LLNL 2012). 
Table 7. Descriptive Statistics for ${ }^{239} \mathrm{Pu}$

All units in $\mathrm{pCi} / \mathrm{kg}$ wet weight.

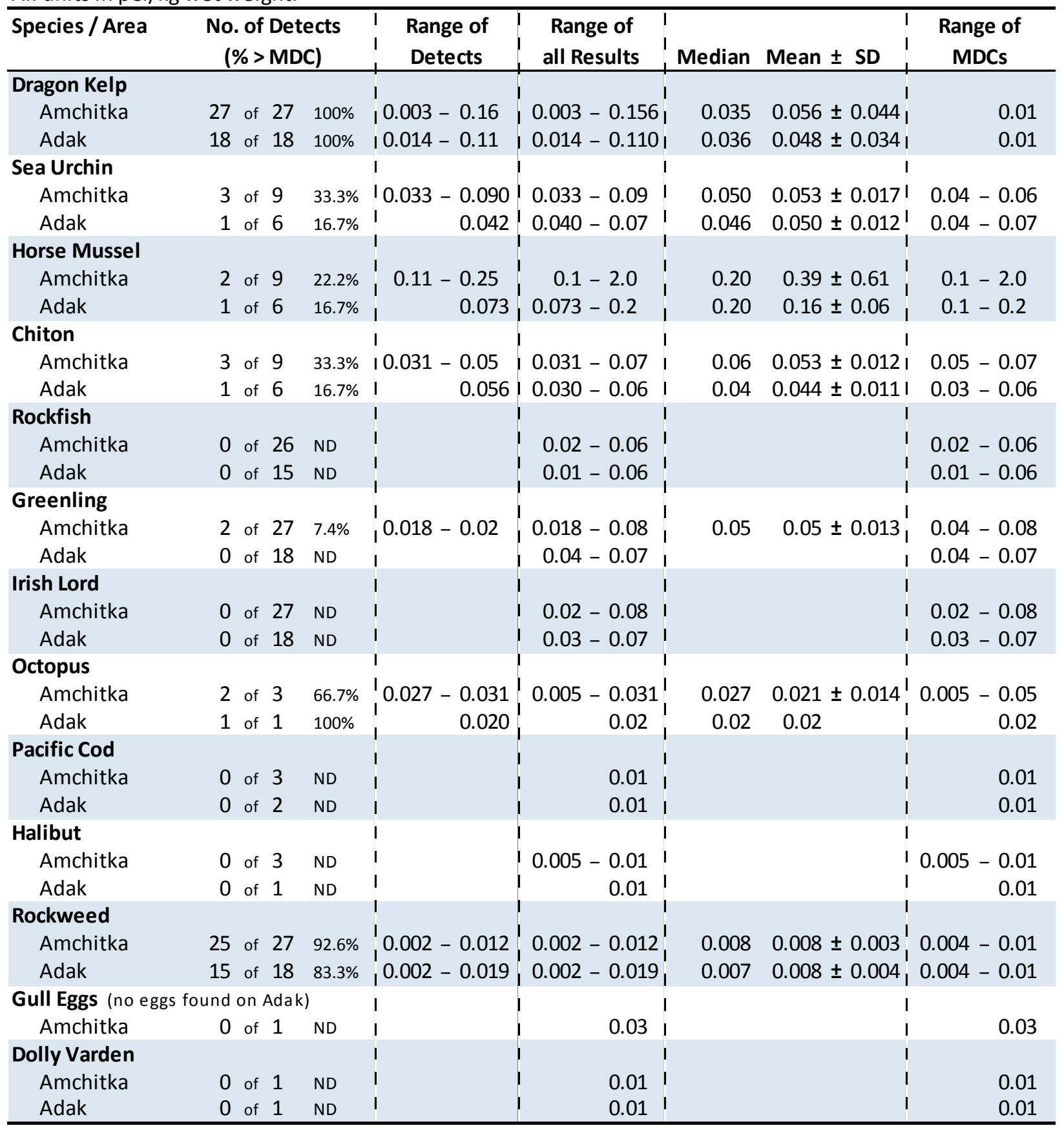

MDC - Minimum Detectable Concentration; ND - Not Detected (all results less than MDC); SD - Standard Deviation

The second range column, "Range of all Results", includes U-flagged ( $<M D C)$ results. Summary statistics were computed using values $=$ MDC for nondetects. Duplicate samples were collected for Dragon Kelp (3 Amchitka/2 Adak); Rockfish (4/1); greenling (3/2); halibut (0/1); and rockweed (3/2). The maximum result for each duplicate pair was used. Medians, means, and standard deviations were not calculated for species for which all results were $<$ MDC. 
Table 8. Descriptive Statistics for ${ }^{240} \mathrm{Pu}$

All units in $\mathrm{pCi} / \mathrm{kg}$ wet weight.

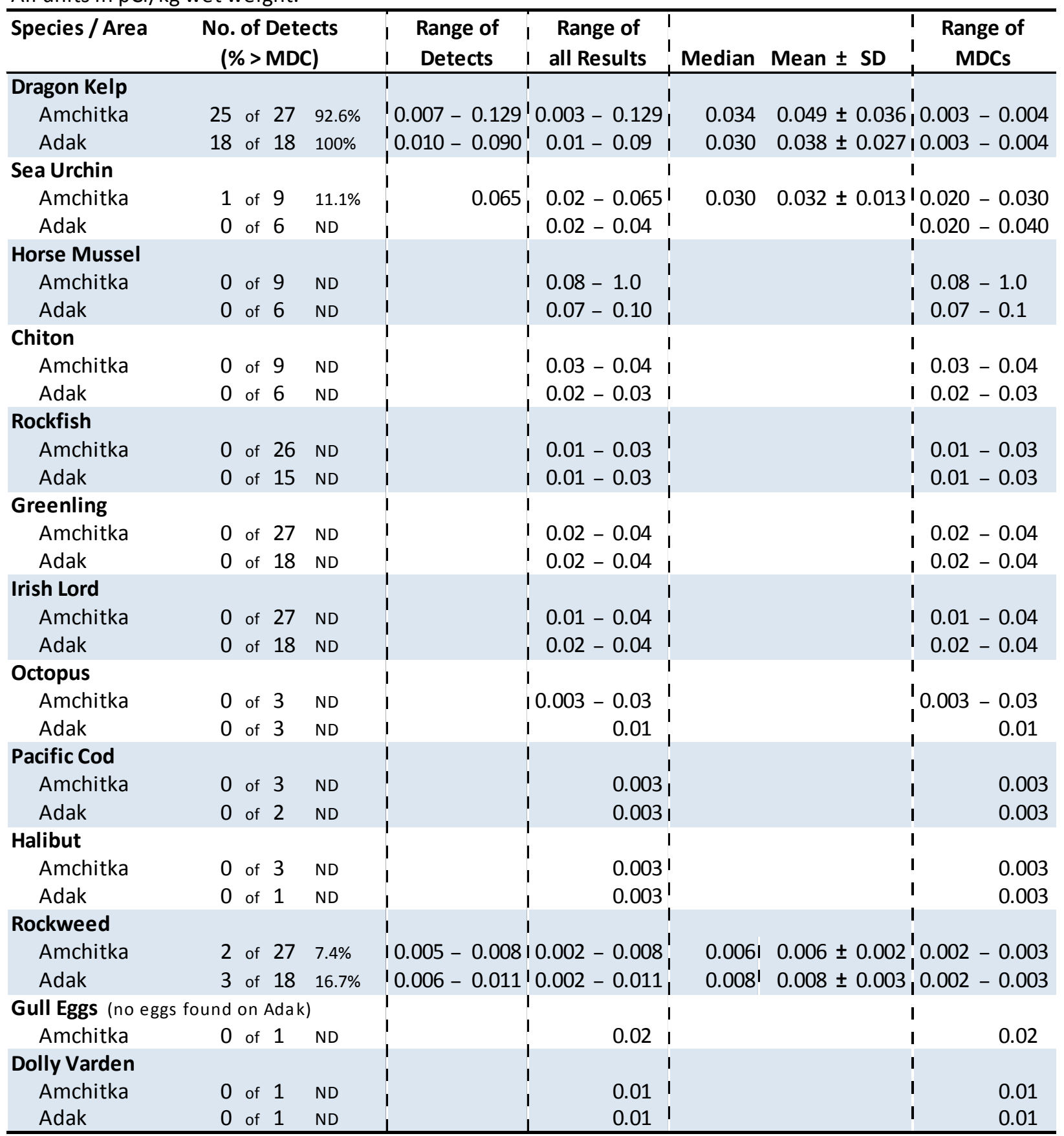

MDC - Minimum Detectable Concentration; ND - Not Detected (all results less than MDC); SD - Standard Deviation The second range column, "Range of all Results", includes U-flagged (<MDC) results. Summary statistics were computed using values $=$ MDC for nondetects. Duplicate samples were collected for Dragon Kelp $(3 \mathrm{Amchitka} / 2$ Adak); Rockfish (4/1); greenling (3/2); halibut (0/1); and rockweed (3/2). The maximum result for each duplicate pair was used. Medians, means, and standard deviations were not calculated for species for which all results were $<$ MDC. 
Table 9. Summary of ${ }^{239} \mathrm{Pu}$ and ${ }^{240} \mathrm{Pu}$ Detections

All units in $\mathrm{pCi} / \mathrm{kg}$ wet weight.

\begin{tabular}{|c|c|c|c|c|c|c|c|c|c|c|c|c|c|c|}
\hline \multirow[t]{2}{*}{ Species / Area } & \multirow{2}{*}{$\begin{array}{l}\text { Total No. } \\
\text { of Samples }\end{array}$} & \multicolumn{2}{|c|}{ No. of Detects } & \multicolumn{2}{|c|}{ Mean* } & \multicolumn{3}{|c|}{$\underline{\text { Range* }}^{*}$} & \multicolumn{2}{|c|}{ Max. Detected } & \multicolumn{2}{|c|}{ Max. MDC } & \multicolumn{2}{|c|}{ Pu-239, 240 (combined) ${ }^{* *}$} \\
\hline & & Pu-239 & Pu-240 & Pu-239 & Pu-240 & Pu-239 & $\mathrm{Pu}-2$ & & Pu-239 & $\mathrm{Pu}-240$ & Pu-239 & $\mathrm{Pu}-240$ & Mean & Range \\
\hline Dragon Kelp & 45 & 45 & 43 & 0.053 & 0.042 & $0.003-0.16$ & $0.003-$ & 0.13 & 0.16 & 0.13 & 0.01 & 0.004 & 0.095 & $0.0028-0.29$ \\
\hline Sea Urchin & 15 & 4 & 1 & 0.052 & 0.030 & $0.033-0.09$ & 0.020 & 0.065 & 0.09 & 0.065 & 0.07 & 0.04 & 0.056 & $0.033-0.16$ \\
\hline Rockweed & 45 & 40 & 5 & 0.008 & 0.003 & $0.002-0.019$ & $0.002-$ & 0.011 & 0.019 & 0.011 & 0.01 & 0.003 & 0.008 & $0.002-0.03$ \\
\hline Horse Mussel & 15 & 3 & 0 & 0.30 & 0.15 & $0.073-2.0$ & 0.070 & 1.0 & 0.25 & & 2.0 & 1.0 & 0.30 & $0.073-2.0$ \\
\hline Chiton & 15 & 4 & 0 & 0.05 & 0.03 & $0.03-0.07$ & I 0.02 - & 0.04 & 0.056 & & 0.07 & 0.04 & 0.05 & $0.03-0.07$ \\
\hline Greenling & 45 & 2 & 0 & 0.052 & 0.029 & $0.018-0.08$ & I 0.02 - & 0.04 & 0.020 & & 0.08 & 0.04 & 0.052 & $0.018-0.08$ \\
\hline Octopus & 4 & 3 & 0 & 0.021 & 0.013 & $0.005-0.031$ & $L^{\prime} 0.003-$ & 0.030 & 0.031 & & 0.05 & 0.03 & 0.021 & $0.005-0.031$ \\
\hline Rockfish & 41 & 0 & 0 & 0.035 & 0.018 & $0.01-0.06$ & 0.01 & 0.03 & & & 0.06 & 0.03 & 0.035 & $0.01-0.06$ \\
\hline Irish Lord & 45 & 0 & 0 & 0.05 & 0.027 & $0.02-0.08$ & 0.01 & 0.04 & & & 0.08 & 0.04 & 0.05 & $0.02-0.08$ \\
\hline Pacific Cod & 5 & 0 & 0 & 0.01 & 0.003 & 0.01 & & 0.003 & & & 0.01 & 0.003 & 0.01 & $0.01-0.01$ \\
\hline Halibut & 4 & 0 & 0 & 0.009 & 0.003 & $0.005-0.01$ & & 0.003 & & & 0.01 & 0.003 & 0.009 & $0.005-0.010$ \\
\hline Gull Eggs & 1 & 0 & 0 & 0.03 & 0.02 & 0.03 & & 0.02 & & & 0.03 & 0.02 & 0.03 & 0.03 \\
\hline Dolly Varden & 2 & 0 & 0 & 0.01 & 0.01 & 0.01 & & 0.01 & & & 0.01 & 0.01 & 0.01 & 0.01 \\
\hline
\end{tabular}

No. of Detects refers to results $\geq$ MDC (not U-flagged), where MDC = Minimum Detectable Concentration.

Dragon kelp, sea urchin, and rockweed (initial rows) were the only species with detectable levels of Pu-240. Shaded rows denote species for which only Pu-239 was detected.

* Means and ranges for individual Pu isotopes were computed using all results, including U-flagged data.

For dragon kelp, rockfish, greenling, halibut, and rockweed, the maximum result for each duplicate pair was used.

See preceding tables for radioisotope-specific statistics and Amchitka vs. Adak breakdowns.

** Approach for calculating summary statistics for Pu-239, 240 combined

If both Pu-239 and Pu-240 were detected ( $\geq \mathrm{MDC}$ ), results were summed. If Pu-239 detected, but not Pu-240, us ed only the Pu-239 result(s).

The opposite situation-Pu-240 detected but not Pu-239-does not apply to this data set.

If both Pu-239 and Pu-240 results were U-flagged $(<M D C)$, the maximum flagged value was used. E.g., if Pu- $239=4 \mathrm{U}$ and Pu- $240=2.0 \mathrm{U}$, used the 4.0 datum to calculate means. 
Table 10. Descriptive Statistics for ${ }^{234} U$

All units in $\mathrm{pCi} / \mathrm{kg}$ wet weight.

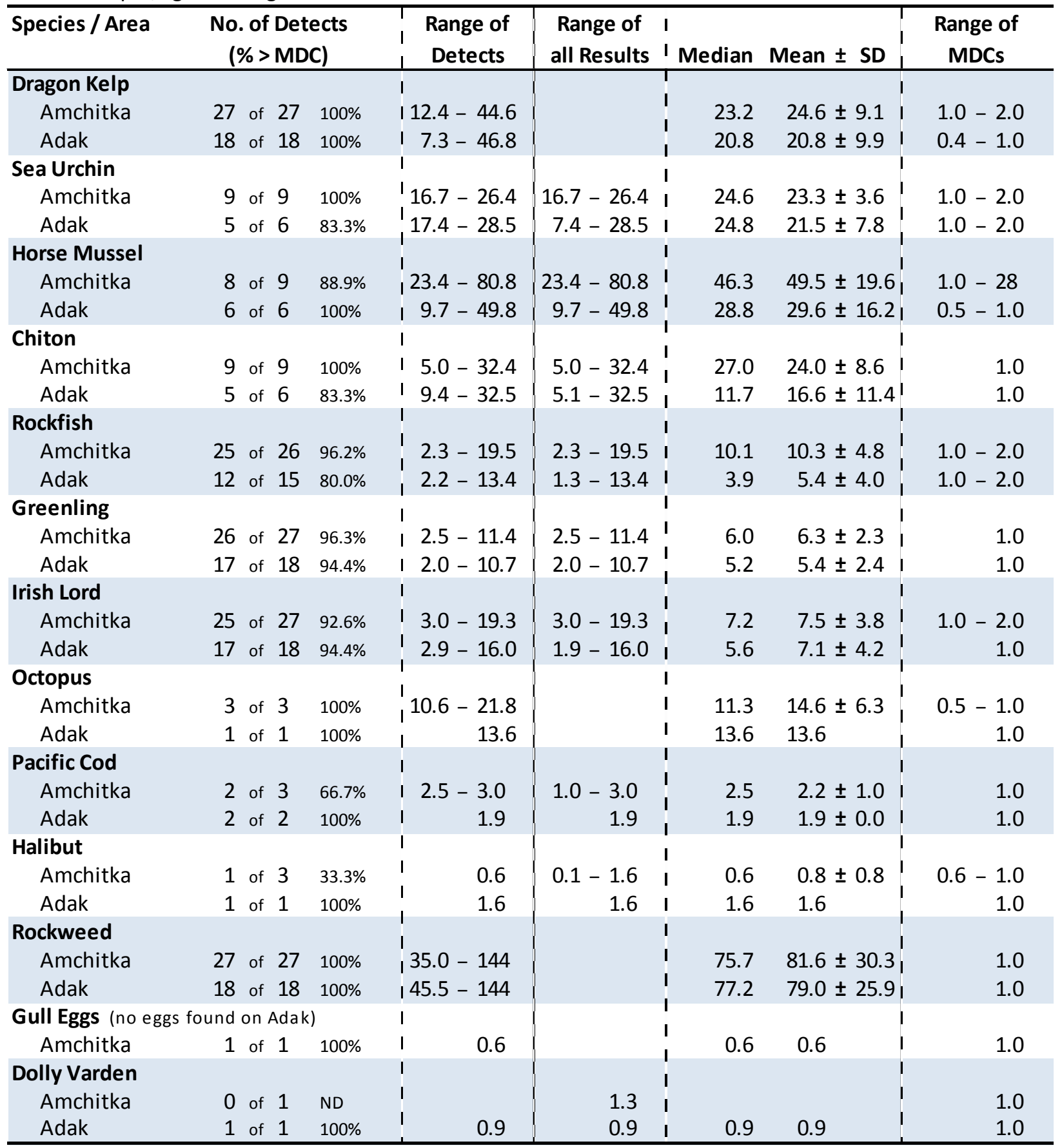

MDC - Minimum Detectable Concentration; SD - Standard Deviation. Statistics computed with values = MDC for nondetec1 The second range column-"Range of all Results"-includes U-flagged (<MDC) results. This column is left blank for those species/media with $100 \%$ detection frequencies. Duplicate samples were collected for Dragon Kelp ( 3 Amchitka/2 Adak); Rockfish (4/1); greenling (3/2); halibut (0/1); and rockweed (3/2). The maximum result for each duplicate pair was used. 
Table 11. Descriptive Statistics for ${ }^{235} \mathrm{U}$

All units in $\mathrm{pCi} / \mathrm{kg}$ wet weight.

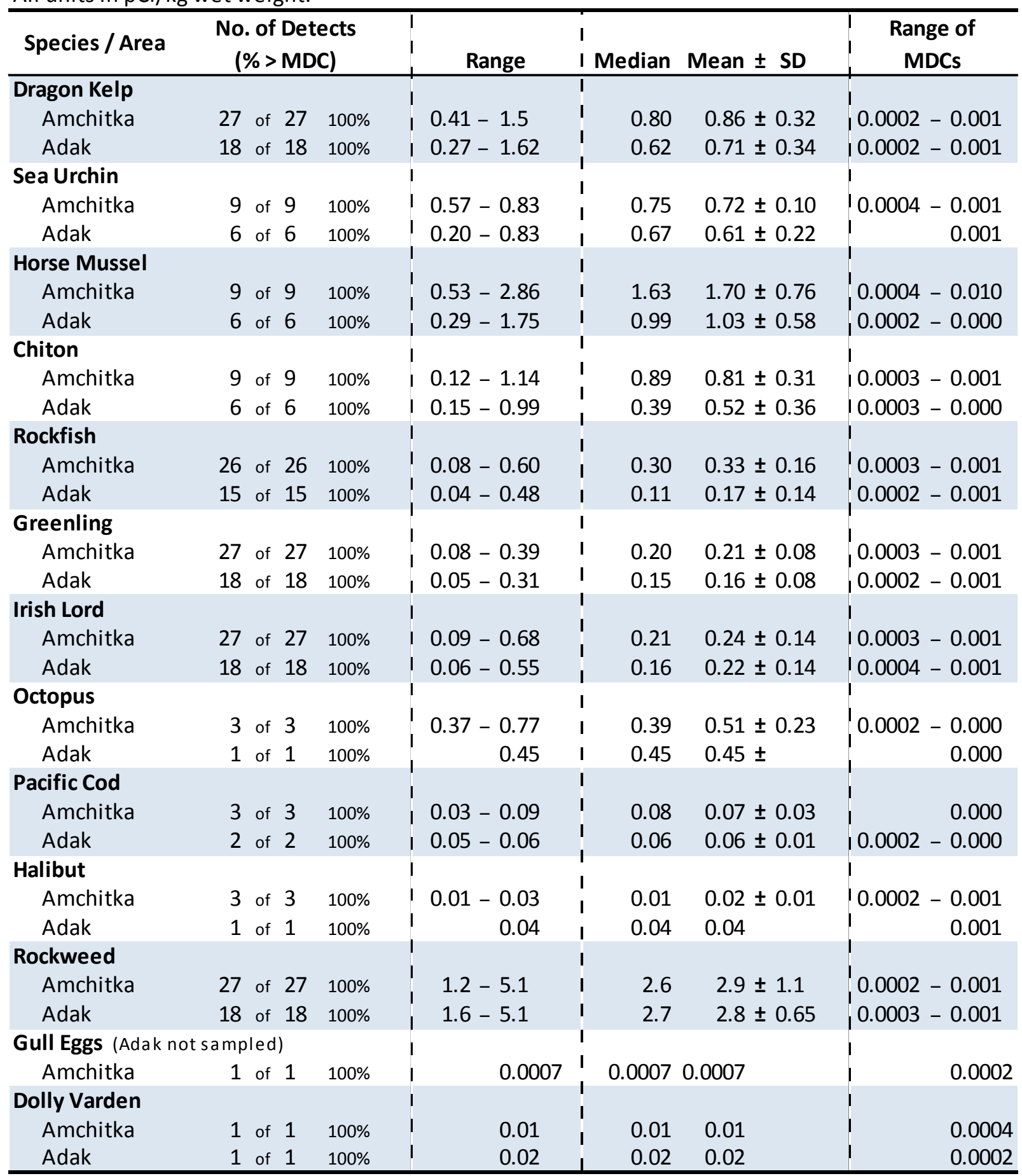

MDC - Minimum Detectable Concentration; SD - Standard Deviation.

Duplicate samples were collected for Dragon Kelp (3 Amchitka/2 Adak); Rockfish (4/1); greenling (3/2); halibut (0/1); and rockweed (3/2). The maximum result for each duplicate pair was used for this summary. 
Table 12. Descriptive Statistics for ${ }^{238} \mathrm{U}$

All units in $\mathrm{pCi} / \mathrm{kg}$ wet weight.

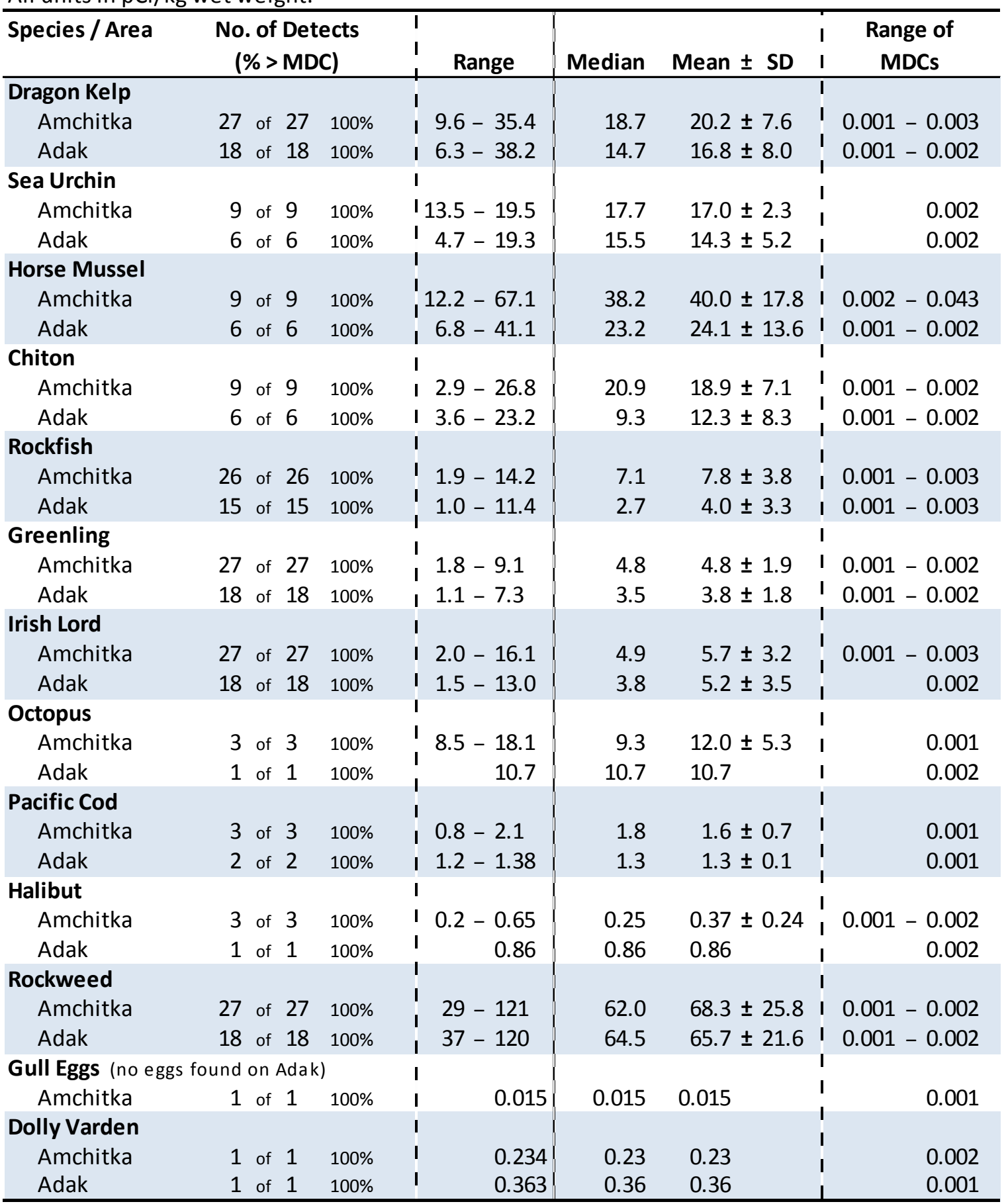

MDC - Minimum Detectable Concentration; SD - Standard Deviation.

Duplicate samples were collected for Dragon Kelp (3 Amchitka/2 Adak); Rockfish (4/1); greenling (3/2); halibut (0/1); and rockweed (3/2). The maximum result for each duplicate pair was used for this summary. 
Uranium isotopes were measured by direct aspiration quadrupole ICP-MS. Acceptable levels of precision were obtained for the vast majority of samples collected and analyzed for this sampling event. The mean reported measurement uncertainties $( \pm 1 \sigma)$ for measurement of ${ }^{234} \mathrm{U},{ }^{235} \mathrm{U}$, and ${ }^{238} \mathrm{U}$ were less than \pm 1 percent, \pm 1.2 percent, and \pm 5.5 percent, respectively. Data obtained for ${ }^{235} \mathrm{U}$ and ${ }^{238} \mathrm{U}$ appear to be of high quality, as evidenced by the internal consistency of ${ }^{235} \mathrm{U} /{ }^{238} \mathrm{U}$ mass ratios (not reported). The associated uranium isotope mean reported sample measurement MDCs using the total sample mass as supplied and expressed as activity concentrations were $1.2,0.0004$, and $0.002 \mathrm{pCi} / \mathrm{kg}$ wet weight, respectively. In practice, the actual quantity of sample used for uranium analysis was much less because high total dissolved solids content limits the instrument's ability to detect. Consequently, the actual mean working MDCs for detection and measurement of ${ }^{234} \mathrm{U},{ }^{235} \mathrm{U}$, and ${ }^{238} \mathrm{U}$ were around $0.007,2 \times 10^{-6}$, and $0.0001 \mathrm{pCi}$, respectively. Analyses performed on mass ratio and concentration standards satisfied all laboratory data quality requirements for both precision and accuracy. Similarly, a series of duplicate measurement met applicable laboratory data quality requirements within the quantifiable capabilities of the technique (LLNL 2012).

\subsection{Comparison of the 2004 and 2011 Data}

As stated earlier in this section, the 2011 data would be compared qualitatively to CRESP data (CRESP 2005) and will become the basis for future trending to begin in 2016. Appendix L provides a comparison of the CRESP and 2011 data. Table 13 summarizes the marine species sampled in both 2004 and 2011. This table is adapted from Appendix L, Table L-1. This summary is based on a comparison of the range of detected concentrations ( $\geq$ MDC), as documented in Tables L-2 through L-7. A large proportion of the 2004 results were below MDCs for ${ }^{137} \mathrm{Cs},{ }^{241} \mathrm{Am}$, and the plutonium isotopes. Central tendencies (e.g., mean or median concentrations) are not accounted for here. The highlighted cells denote species/isotopes for which 2011 results exceeded 2004 results.

Table 13. Summary of Marine Species Sampled in Both 2004 (CRESP) and 2011

\begin{tabular}{||l|c|c|c|c|c|c||}
\hline \multicolumn{1}{|c|}{ Species } & ${ }^{137} \mathbf{C s}$ & ${ }^{241} \mathbf{A m}$ & ${ }^{239,240} \mathbf{P u}$ & ${ }^{234} \mathbf{U}$ & ${ }^{235} \mathbf{U}$ & ${ }^{238} \mathbf{U}$ \\
\hline Dragon kelp & $\mathrm{X}$ & $\mathrm{X}$ & $\mathrm{X}$ & $\mathrm{X}$ & $\mathrm{X}$ & $\mathrm{X}$ \\
\hline Rockweed & $\mathrm{X}$ & $\mathrm{X}$ & $\mathrm{X}$ & $\mathrm{X}$ & $\mathrm{X}$ & $\mathrm{X}$ \\
\hline Gumboot or Pacific chiton & $\mathrm{X}$ & $\mathrm{X}$ & & & & \\
\hline Horse mussel & $\mathrm{X}$ & $\mathrm{X}$ & $\mathrm{X}$ & $\mathrm{X}$ & $\mathrm{X}$ & $\mathrm{X}$ \\
\hline Sea urchin & $\mathrm{X}$ & $\mathrm{X}$ & & & & \\
\hline Rockfish & $\mathrm{X}$ & $\mathrm{X}$ & $\mathrm{X}$ & $\mathrm{X}$ & $\mathrm{X}$ & $\mathrm{X}$ \\
\hline Red or yellow Irish lord & $\mathrm{X}$ & $\mathrm{X}$ & $\mathrm{X}$ & $\mathrm{X}$ & $\mathrm{X}$ & $\mathrm{X}$ \\
\hline Rock greenling & $\mathrm{X}$ & $\mathrm{X}$ & & & & \\
\hline Pacific halibut & $\mathrm{X}$ & $\mathrm{X}$ & $\mathrm{X}$ & $\mathrm{X}$ & $\mathrm{X}$ & $\mathrm{X}$ \\
\hline Pacific cod & $\mathrm{X}$ & $\mathrm{X}$ & $\mathrm{X}$ & $\mathrm{X}$ & $\mathrm{X}$ & $\mathrm{X}$ \\
\hline Octopus & $\mathrm{X}$ & $\mathrm{X}$ & & & & \\
\hline Dolly Varden & $\mathrm{X}$ & $\mathrm{X}$ & & & & \\
\hline Glaucous-winged gull eggs & $\mathrm{X}$ & $\mathrm{X}$ & & & & \\
\hline
\end{tabular}


Table 14 is an extract of Appendix L tables, specifically Tables L-2, L-5, and L-7, which summarize only those species/isotopes in which 2011 results exceeded corresponding 2004 results (yellow highlighting). Figure 22 is a graphical presentation of that same information. The bar chart provides a graphical comparison of the data listed in Table 13 and Table 14. For each grouping of data, the maximum is denoted by the darkest bar or column. The following summary can be made regarding comparison of 2004 to 2011 data:

- $\quad$ For ${ }^{137}$ Cs, 2011 maxima exceeding 2004 results correspond to samples collected from Adak.

- $\quad$ For ${ }^{234} \mathrm{U}$ and ${ }^{238} \mathrm{U}$, although maximum concentrations were detected in samples from Amchitka (2011), differences between 2004 results are nominal.

Furthermore, with respect to the uranium isotopes, uranium is a naturally occurring element found at low levels in virtually all rock, soil, and water. Uranium found naturally has three isotopes - ${ }^{238} \mathrm{U},{ }^{235} \mathrm{U}$, and ${ }^{234} \mathrm{U}$ - with the natural abundance of each of these isotopes, expressed as a percentage, of 99.27, 0.72, and 0.0055, respectively. Uranium in soil and rocks is distributed throughout the environment by wind, rain, and geologic processes. Rocks weather and break down to form soil, and soil can be washed by water and blown by wind, moving uranium into streams and lakes, and ultimately settling out and reforming as rock. The average crustal abundance of uranium is 2.8 parts per million and equates to an activity concentration of $1,781 \mathrm{pCi} / \mathrm{kg}$. This value is 6.5 and 8.4 times greater than the highest reported values of uranium isotopes detected in rockweed on Amchitka and Adak Islands, respectively.

\subsection{Comparison of Amchitka and Adak Data Collected in 2011}

The data were analyzed to compare the average dry weight concentrations from Amchitka with those from Adak. The Amchitka data were collected from the Long Shot, Cannikin, and Milrow sites. Measurements were below the MDCs in most ${ }^{241} \mathrm{Am}$ and plutonium data for the species rockfish, greenling, Irish lord, cod, halibut, Dolly Varden, gull eggs, and rockweed. To avoid introducing a bias for comparison, the raw data were used in all cases.

This section includes (1) concentration comparisons between sites for each combination of radionuclide and organism, (2) an aggregate comparison between the sites using all the combinations of radionuclide and organism, and (3) comparison of the isotopic concentrations of uranium and plutonium.

The analysis in this section focuses on the difference of the average concentrations, which is directly related to the health risks. In addition, further analysis would include comparing the average and also the standard deviation. For example, the sites might have similar averages but different variability. Tests with nonparametric statistics showed that most data are best handled with the average as the primary statistic. 
Table 14. 2011 Results That Exceed 2004 (CRESP) Results

Summary of ${ }^{137}$ Cs Results: 2004 (CRESP) vs. 2011 Sampling All units in $\mathrm{pCi} / \mathrm{kg}$ wet weight.

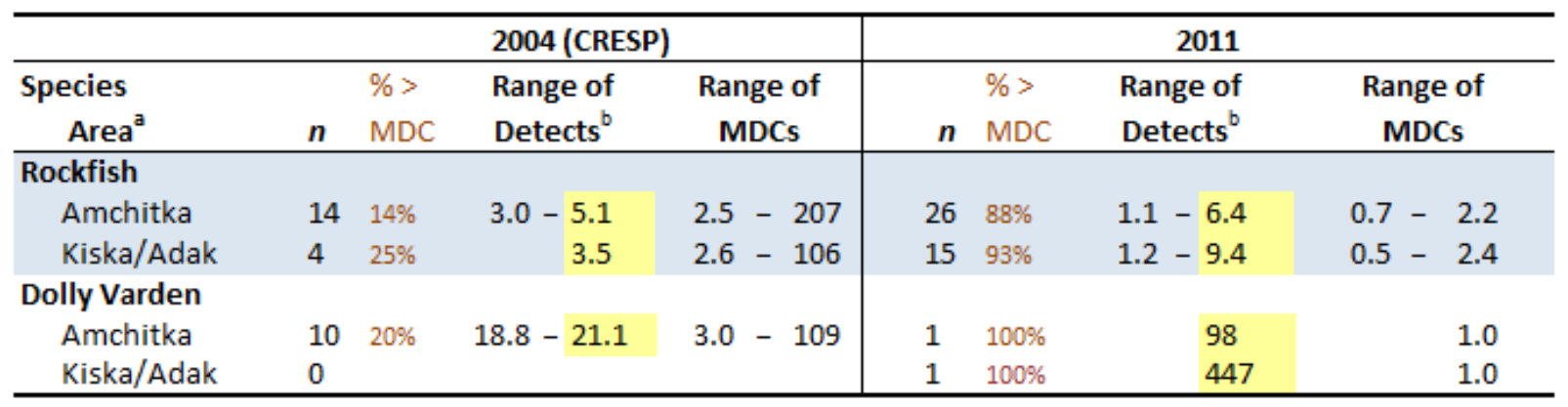

Summary of ${ }^{234} \mathrm{U}$ Results: 2004 (CRESP) vs. 2011 Sampling All units in $\mathrm{pCi} / \mathrm{kg}$ wet weight.

\begin{tabular}{|c|c|c|c|c|c|c|c|c|c|}
\hline \multirow{2}{*}{$\begin{array}{l}\begin{array}{l}\text { Species } \\
\text { Area }^{\mathrm{a}}\end{array} \\
\text { Horse Mussel }\end{array}$} & \multicolumn{4}{|c|}{2004 (CRESP) } & \multicolumn{5}{|c|}{2011} \\
\hline & $n$ & $\begin{array}{l}\%> \\
M D C\end{array}$ & $\begin{array}{l}\text { Range of } \\
\text { Detects }^{\circ}\end{array}$ & $\begin{array}{c}\text { Range of } \\
\text { MDCs }\end{array}$ & $n$ & $\begin{array}{l}\%> \\
M D C\end{array}$ & $\begin{array}{l}\text { Range of } \\
\text { Detects }^{\circ}\end{array}$ & \multicolumn{2}{|c|}{$\begin{array}{c}\text { Range of } \\
\text { MDCs }\end{array}$} \\
\hline Amchitka & 6 & $100 \%$ & $12-75.1$ & $0.8-1.4$ & 9 & $89 \%$ & $23.4-80.8$ & 1.0 & -28 \\
\hline Kiska/Adak & 2 & $100 \%$ & $8.4-12.6$ & $1.0-1.0$ & 6 & $100 \%$ & $9.7-49.8$ & 0.5 & -1.0 \\
\hline \multicolumn{10}{|l|}{ Irish Lord } \\
\hline Amchitka & 2 & $100 \%$ & $9.9-13.1$ & $0.73-1.0$ & 27 & $93 \%$ & $3.0-19.3$ & 1.0 & $-\quad 2.0$ \\
\hline Kiska/Adak & 1 & $100 \%$ & 15.3 & 1.0 & 18 & $94 \%$ & $2.9-16.0$ & & 1.0 \\
\hline \multicolumn{10}{|l|}{ Rockweed } \\
\hline Amchitka & 10 & $100 \%$ & $50-130$ & $0.8-1.5$ & 27 & $100 \%$ & $35.0-144$ & & 1.0 \\
\hline Kiska/Adak & 4 & $100 \%$ & $74-140$ & $0.4-1.3$ & 18 & $100 \%$ & $45.5-113$ & & 1.0 \\
\hline
\end{tabular}

Summary of ${ }^{238} \mathrm{U}$ Results: 2004 (CRESP) vs. 2011 Sampling All units in $\mathrm{pCi} / \mathrm{kg}$ wet weight.

\begin{tabular}{|c|c|c|c|c|c|c|c|c|}
\hline \multirow[b]{2}{*}{$\begin{array}{l}\text { Species } \\
\text { Area }^{\mathrm{a}}\end{array}$} & \multicolumn{4}{|c|}{2004 (CRESP) } & \multicolumn{4}{|c|}{2011} \\
\hline & $n$ & $\begin{array}{l}\%> \\
M D C\end{array}$ & $\begin{array}{l}\text { Range of } \\
\text { Detects }^{\circ}\end{array}$ & $\begin{array}{c}\text { Range of } \\
\text { MDCs }\end{array}$ & $n$ & $\begin{array}{l}\%> \\
M D C\end{array}$ & $\begin{array}{l}\text { Range of } \\
\text { Detects }^{b}\end{array}$ & $\begin{array}{c}\text { Range of } \\
\text { MDCs }\end{array}$ \\
\hline \multicolumn{9}{|l|}{ Horse Mussel } \\
\hline Amchitka & 6 & $100 \%$ & $11-61.6$ & $0.7-1.4$ & 9 & $100 \%$ & $12.2-67.1$ & $0.002-0.04$ \\
\hline Kiska/Adak & 2 & $100 \%$ & $7.5-12.6$ & 1.0 & 6 & $100 \%$ & $6.8-41.1$ & $0.001-0.002$ \\
\hline
\end{tabular}



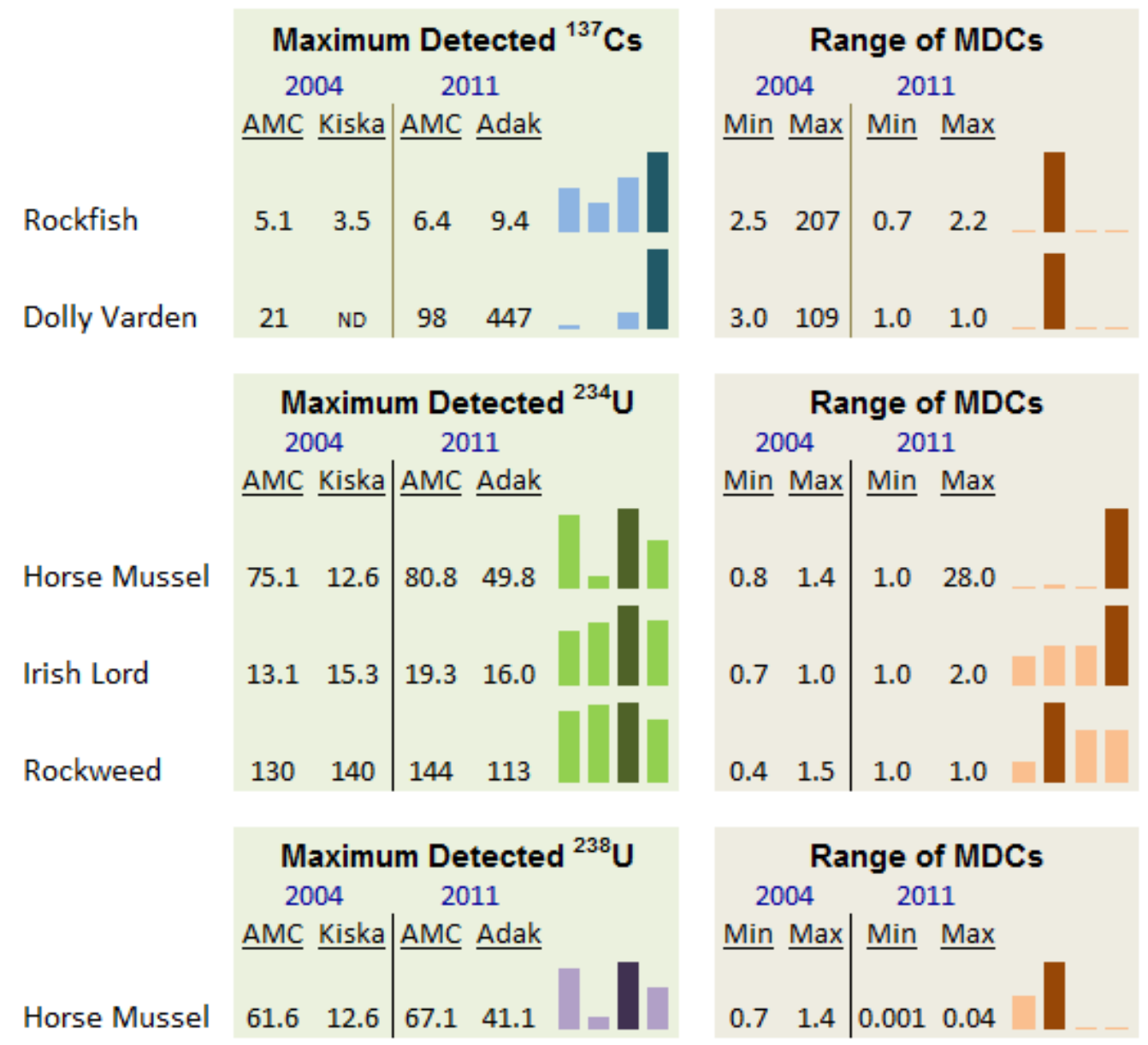

AMC - Amchitka; MDC - minimum detectable concentration All units in $\mathrm{pCi} / \mathrm{kg}$ wet weight.

\section{Legend:}
A B C D
A - Amchitka 2004 max result
A - CRESP 2004 min MDC
B - Kiska 2004 max result
C - Amchitka 2011 max result
D - Adak 2011 max result
B - CRESP 2004 max MDC
C - $2011 \min$ MDC
D - 2011 max MDC

Figure 22. 2011 Results That Exceed 2004 Results 
Figure 23 contains dry-weight concentrations at Amchitka and Adak for each radionuclide. Each plot contains the set of species along the horizontal axis. The average concentration from the two sites is compared for each species. Where possible, a standard error of the mean is also displayed. The data indicate a strong possibility of differences between the sites if the difference between the averages (height difference of the columns) is at least twice the combined standard error.

Figure 24 shows this measure (ratio of the difference between the sites to the measurement uncertainty, expressed as the combined standard error) for each species and radionuclide. If the bar is above the $\mathrm{t}$-Value $=2$ line shown in yellow, then there is a statistical significance between the results, with the higher concentration being at Amchitka (probability of random data having a value of 2 or above is about 2.5 percent). If the bar is below the $\mathrm{t}$-Value $=-2$ line, also shown in yellow, there is a similar chance that the Adak concentration is higher than the Amchitka concentration.

Figure 24 shows that there are more bars above than below the zero value. This indicates a slight bias towards larger concentration values at Amchitka. It is also expected, based on statistics $(p$-value $=0.05)$, that even if the two sites had similar concentrations, 5 percent of the values (i.e., 2 out of 40) are expected to fall either above or below the yellow lines. Actually, six are observed ( 3 above and 3 below) and are listed in Figure 25. There does not seem to be a pattern, in that each has a different species; each of the radionuclides (except the plutonium isotopes) has one observation higher and one observation lower at Adak compared to Amchitka.

A further test is to compare the distribution of $t$-Values to that expected from statistically sampling from similar concentrations. Figure 26 shows an expected distribution if the concentrations at the sites are the same (a cumulative normal distribution) compared with the actual distribution based on the data shown in Figure 24. The cumulative distribution is shifted to the right by 0.5 , which is the average $t$-Value, reflecting the bias of more bars on the top. The $\mathrm{S}$ portion of the curves match quite well, but the two values (shown as blue diamonds) on the bottom left show the greatest deviation from what is expected. These two values are the ${ }^{238} \mathrm{U}$ concentration in halibut and the ${ }^{241} \mathrm{Am}$ concentration in rockweed. With all the measurements combined in this way, another test can be done. The average $t$-Value of 0.5 can be compared to the standard error of this measure (with 44 measurements and a standard deviation of slightly larger than 1), resulting in an overall $t$-Value of approximately 2.4 . That is, the shift is over 2 times the shift expected (95 percent) from random sampling, indicating that overall the samples derive from different distributions. 


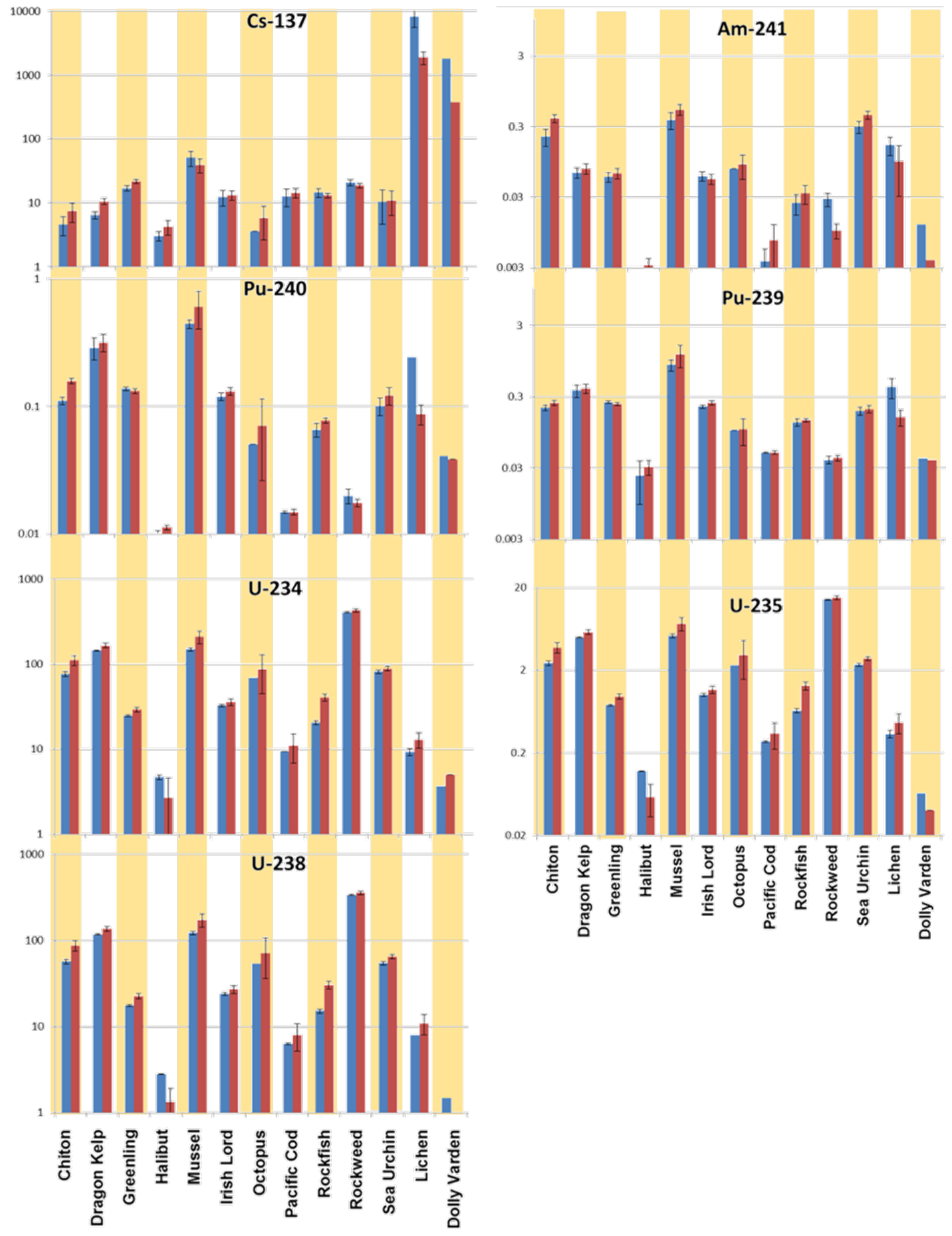

Note: Error bars show the standard error of the mean. Red is Amchitka and blue is Adak.

Figure 23. Comparison of Dry-Weight Concentrations (pCi/kg) at Amchitka and Adak 


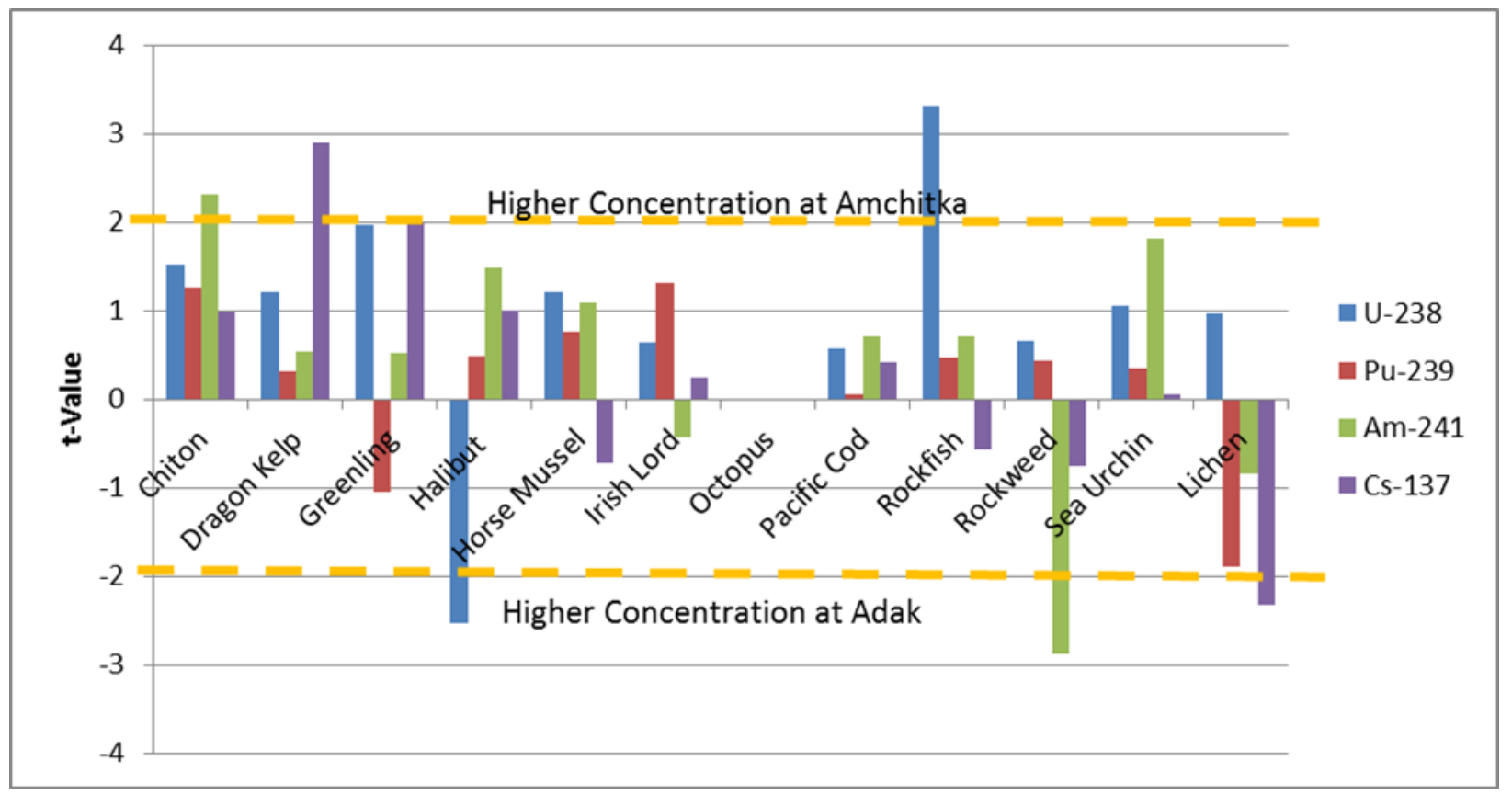

Figure 24. Measure of Difference Between Amchitka and Adak Concentrations

The ratios of the uranium and plutonium isotope concentrations were also analyzed and are shown in Figure 27. The average ratios of the activity concentrations of ${ }^{238} \mathrm{U},{ }^{235} \mathrm{U}$, and ${ }^{234} \mathrm{U}$ isotopes are 1: $0.042: 1.3$, which is within the variations of natural background (Zielinkaki et al. 1997 and 2008). The activity concentration of ${ }^{240} \mathrm{Pu}$ was about half that of ${ }^{239} \mathrm{Pu}$. Variations in this ratio were larger between organisms than between sites. This activity ratio corresponds to an atom ratio of 0.14 .

The analysis in this section used statistics based on the variation between samples and the mean of each species. Many factors contribute to uncertainty, including laboratory measurement techniques, sampling techniques, and the limited number of samples, which contributes to the variability observed in the data sets. Contributions of radionuclides in the environment may include naturally occurring radioactivity and anthropogenic influences such as fall-out from previous aboveground tests, nuclear power accidents such as at Fukushima Dai-Ichi, decommissioning submarines in the Arctic Ocean, and potential leaching from underground nuclear tests.

The data analyzed provide sufficient statistical evidence to infer that samples analyzed from both islands are equivalent for each nuclide and organism combination. Overall, there is a pattern toward slightly higher concentration (half a standard error of the mean, for example for uranium) at the Amchitka site. The two measurements that seem to show the most deviation have limited data above the MDCs. 


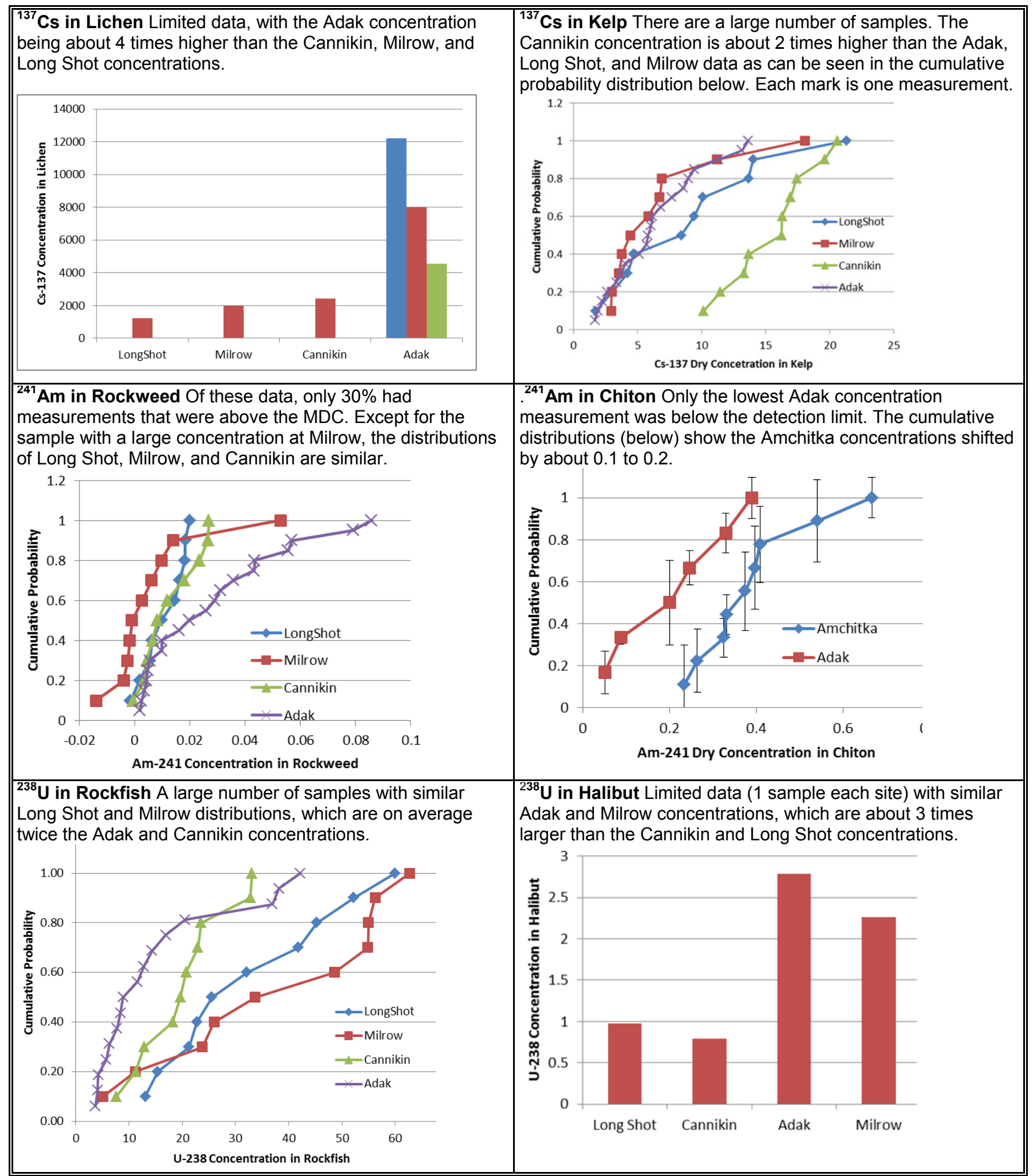

Note: The specific sample measurements are shown for each site. In four cases, the cumulative probability distribution is shown with each marker along a curve representing one of the measurements at that site.

Figure 25. Measurements of Isotopes and Species That Showed Statistically Significant Differences $(p<0.05)$ 


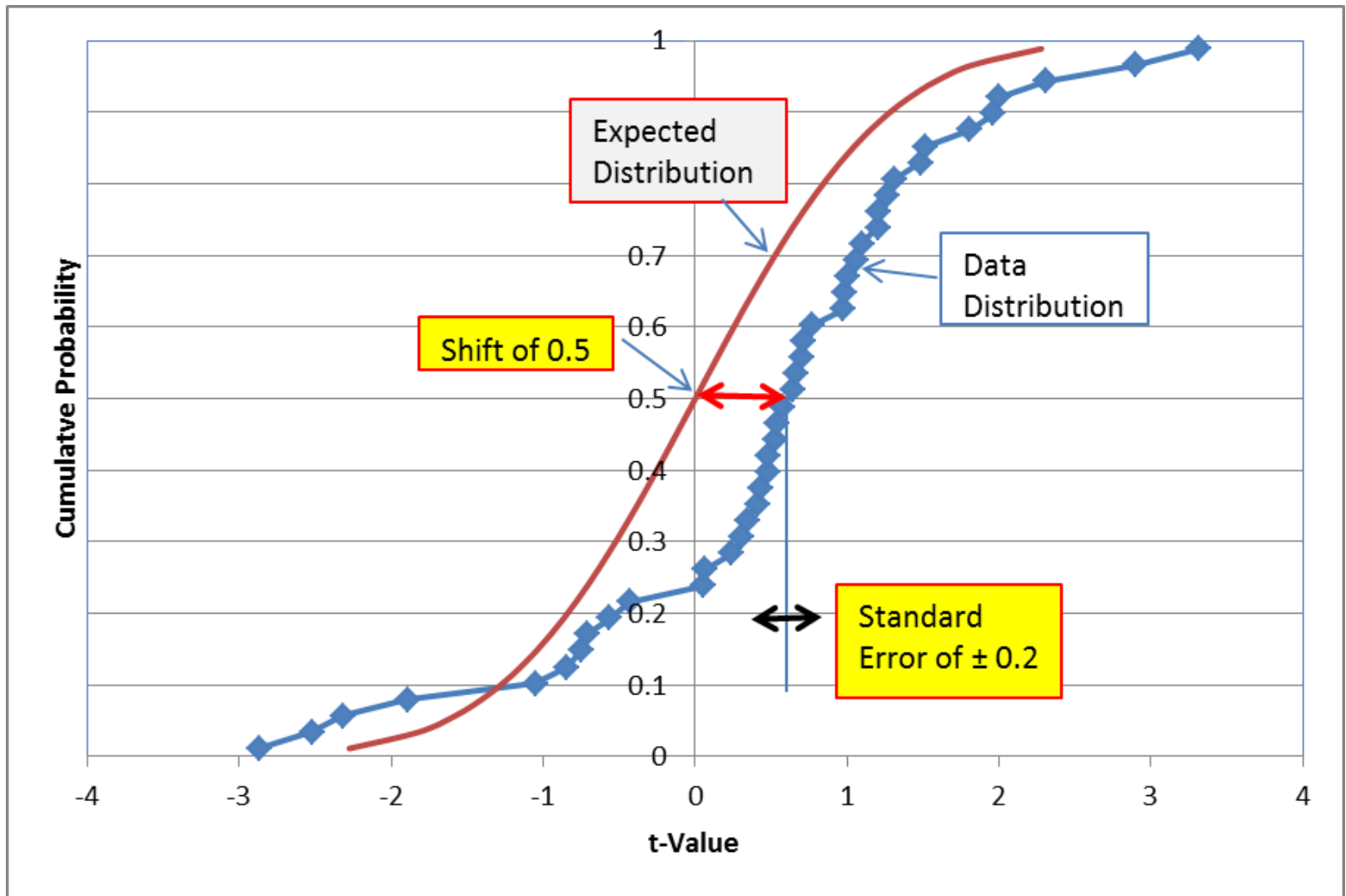

Note: This includes data from each nuclide and organism combination. The red curve is what is expected (i.e., a normal distribution around 0 with a standard deviation of 1.0). The blue curve is what is observed.

Figure 26. Cumulative Probability Distribution of the Ratio of Differences Between Sites to the Standard Error 


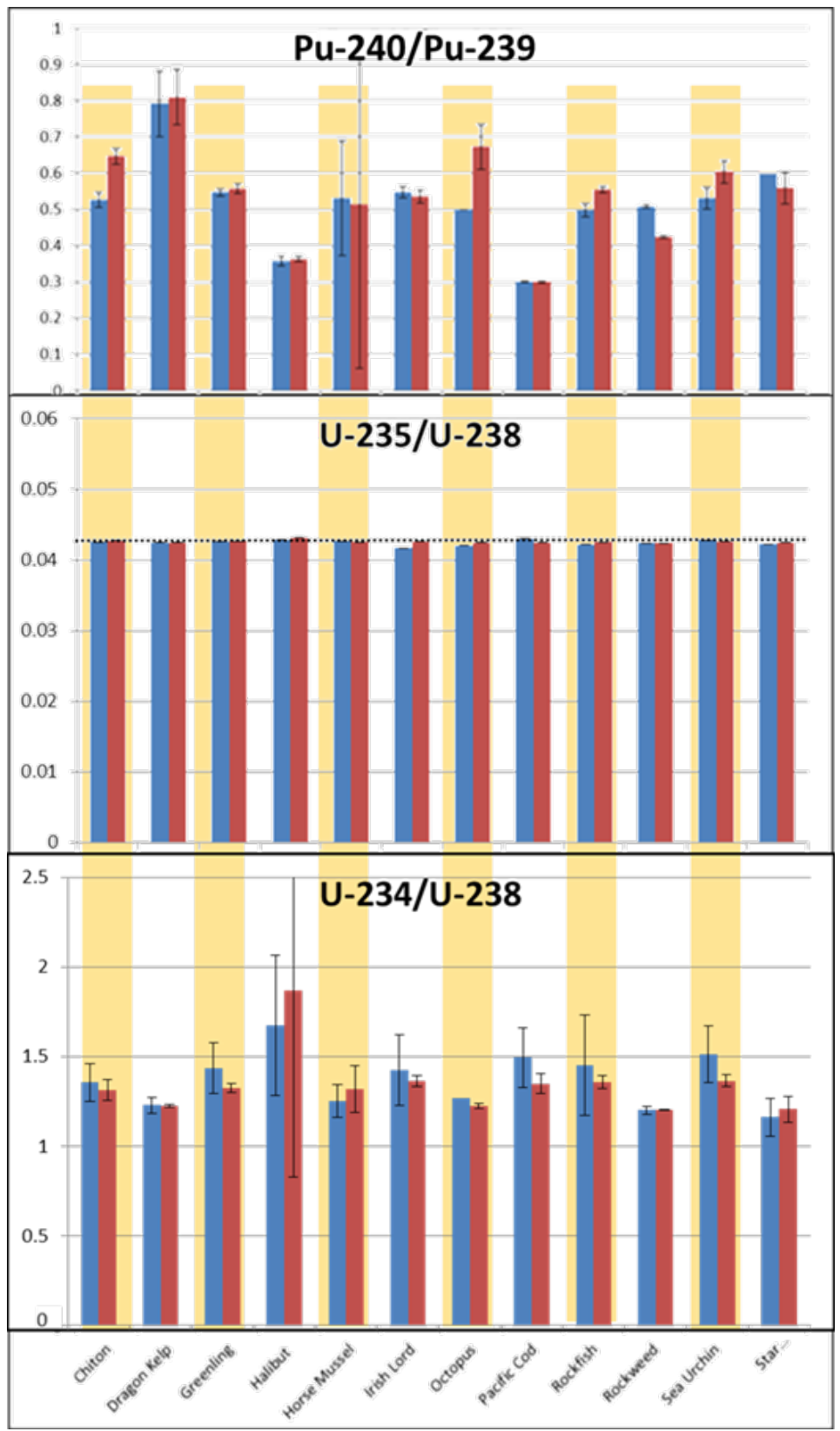

Note: Red is Amchitka and blue is Adak.

Figure 27. Measure of Amchitka and Adak Plutonium and Uranium Isotope Concentrations 


\subsection{Comparison of 2011 Data with CRESP 2004 Data, Literature Sources, and Food Standards}

A further evaluation of food safety was performed by comparing the radionuclide concentration data from this monitoring program to established food safety standards for each of the isotopes and to average values obtained in other studies. Table 15 compares Amchitka data to worldwide values, food safety standards, and CRESP's 2004 results. The second and third columns list the range of values for fish from the Northern Hemisphere sites generally and from the contaminated Irish Sea near Sellafield, England. The next two columns present the values for Amchitka in 2011 and CRESP's study in 2004. The next two columns are the Food and Drug Administration's (FDA's) minimum reporting level (MRL), which is the minimum concentration reported in the FDA food surveys (values below this are reported as zero), and the FDA Derived Intervention Level (DIL), which is a guidance level for food that is imported into the United States (FDA 2009). The DIL for radionuclides is based on short-term rather than lifetime exposure. The last column is the Codex Alimentarius Committee of the Food and Agriculture Organization (FAO) and World Health Organization's (WHO) food safety values for radionuclides and is intended to screen food sources as acceptable for consumption for a 1-year time frame (FAO/WHO 2004).

Table 15. Comparison of Amchitka Data to Worldwide Values and Food Safety Standards

\begin{tabular}{||c|c|c|c|c|c|c|c||}
\hline \hline Isotope & $\begin{array}{c}\text { Northern } \\
\text { Hemisphere } \\
\text { (range) }\end{array}$ & $\begin{array}{c}\text { Irish Sea } \\
\text { (range) }\end{array}$ & $\begin{array}{c}\text { Amchitka } \\
\mathbf{2 0 0 4} \\
\text { (range) }\end{array}$ & $\begin{array}{c}\text { Amchitka } \\
\mathbf{2 0 1 1} \\
\text { (range) }\end{array}$ & $\begin{array}{c}\text { FDA } \\
\text { MRL }\end{array}$ & $\begin{array}{c}\text { FDA } \\
\text { DIL }\end{array}$ & $\begin{array}{c}\text { Codex } \\
\text { Guide }\end{array}$ \\
\hline${ }^{137} \mathrm{Cs}$ & $8.9-10.8$ & $8.4-297.3$ & $\begin{array}{c}<\mathrm{MDC}-16.3 \\
3.8-16.7^{\mathrm{b}}\end{array}$ & $0.82-9.5$ & 135 & 32,432 & 27,027 \\
\hline${ }^{241} \mathrm{Am}$ & 0.03 & $0.0027-6.22$ & $<\mathrm{MDC}-7.3^{\mathrm{c}}$ & $0.006-0.07$ & 5405 & $54.05^{\mathrm{d}}$ & $27^{\mathrm{d}}$ \\
\hline${ }^{239 \% 240} \mathrm{Pu}$ & $0.0081-1.89$ & 0.00035 & $<\mathrm{MDC}-0.595$ & $0.018-0.020^{\mathrm{e}}$ & No MRL & $54.05^{\mathrm{d}}$ & $27^{\mathrm{d}}$ \\
\hline${ }^{234} \mathrm{U}$ & 0.486 & $0.0972-0.1405$ & $<\mathrm{MDC}-23.162$ & $0.6-19.3$ & No MRL & No DIL & No guideline \\
\hline${ }^{235} \mathrm{U}$ & No data & $\begin{array}{c}0.00081- \\
0.0081\end{array}$ & $<\mathrm{MDC}-14.32$ & $0.009-0.68$ & No MRL & No DIL & 2702 \\
\hline${ }^{238} \mathrm{U}$ & No data & $0.0946-0.1243$ & $<\mathrm{MDC}-21.05$ & $0.2-16.1$ & No MRL & No DIL & No guideline \\
\hline
\end{tabular}

All results in $\mathrm{pCi} / \mathrm{kg}$ wet weight.

${ }^{a}$ Range of means for Irish lord, rockfish, rock greenling, Pacific cod, and halibut

${ }^{b}$ Range of means for four species (Dolly Varden, walleye pollock, halibut, and Pacific cod)

${ }^{c}$ For all actinides, bone was analyzed as a conservative measure

${ }^{\mathrm{d}}$ Totals for all Am and $\mathrm{Pu}$ isotopes

${ }^{\mathrm{e}}$ Only two detects and only in rock greenling 
This page intentionally left blank 


\subsection{Human Health-Subsistence- and Commercial-Catch Seafood Consumption}

The first DQO for the 2011 sampling event was to collect data to determine if subsistence- and commercial-catch seafood that is part of the Aleut diet continues to be safe to eat. The 2011 sampling event provides the first set of data to address the objectives of the LTS\&M Plan (DOE 2008). As stated in the 2011 Sampling Plan (DOE 2011), results from the 2011 sampling event would be used to provide estimates of potential human health risk from the ingestion of seafood harvested or obtained through commercial catch on or near Amchitka. These data would be compared to data from Adak that were also collected during the 2011 sampling event. Adak is considered a reference location for the 2011 sampling effort. In addition, the 2011 data would also be compared qualitatively to CRESP (2005) data and will also become the basis for future trending to begin in 2016. Appendix L presents a comparison of the CRESP and 2011 data.

In addition to results of tissue analysis, other important input parameters for estimating human health risk from ingestion of seafood include (1) Aleut diet information, which was used to guide selection of the species collected for the sampling event in 2011 but also is used in this report to develop representative intake amounts for the various seafood species, (2) exposure frequencyhow often the seafood is ingested, (3) exposure duration-how many days/years ingested, and (4) ingestion toxicity information of the radionuclides of interest.

Potential adverse health effects associated with radionuclides under normal environmental exposure conditions (as opposed to catastrophic conditions) are primarily caused by the radiation the radionuclides emit while undergoing radioactive decay. The radiation can transfer sufficient localized energy to atoms and remove electrons from the electric field of their nucleus (ionization). With ingestion of food containing radionuclides, this transfer of energy can adversely affect the cells of biological tissues, and depending on the extent of potential damage, it could result in adverse health effects. The principal adverse health effects from low-level radiological exposures (which are typical for normal environmental exposures) are carcinogenicity, mutagenicity, and teratogenicity.

Carcinogenicity is the ability to cause cancer, which has been observed on both human and laboratory animals under relatively high doses or high dose rate of exposures. Studies of human populations exposed to low doses or low dose rates, such as those under normal environmental exposure conditions, have not adequately provided definitive risk information. Therefore, the potential cancer risks associated with low dose/dose rate exposures are estimated by extrapolating the data for high dose/dose rate exposures assuming a linear relationship between dose and risk and that there is no threshold. It is considered that radiation-induced cancers (both fatal and nonfatal) can occur at any point in a person's lifetime.

Mutagenicity is the ability to induce genetic mutation, which may be in the nucleus of either somatic (body) or germ (reproductive) cells. However, very few quantitative data are available on radiogenic mutations in humans, particularly from low-dose exposures, due to the mild mutagenic effects or similarities of the mutagenic effects to nonmutagenic effects. The frequency of serious genetic impairment caused by radiation is relatively small compared to that of the spontaneously arising genetic diseases (UNSCEAR 1982, 1988). The vast majority of data on radiation-induced mutation came from extensive animal studies in laboratories, and the results were extrapolated to humans. The risk of mutation per unit exposure is smaller than, or 
comparable to, the risk of cancer (EPA 1989). Moreover, radiation exposures to induce genetic mutation must occur during the 30 -year period of reproductive generation.

Teratogenicity is the ability to induce birth defects during the growth and development of an embryo. Although the risks per unit radiation exposure of serious teratogenic effects are greater than the risks of cancer, the radiation exposures to induce teratogenic effects must occur over a specific period during pregnancy, which is limited to 9 months.

On the basis of the length of induction periods and the severity of potential adverse effects, EPA determined that cancer risks appear to be the limiting risks and may be used as the sole basis for assessing human health effects from environmental radiation exposures (EPA 1989).

Besides radiation effects, some radioactive materials can cause adverse health effects by their chemical toxicities if their intake rates exceed specific threshold values. Based on the levels of exposure that are detailed in the following section, the intake rates would be much lower than the threshold values and, therefore, would not likely result in adverse health effects due to chemical toxicity.

Hence, the assessment of potential health risks resulting from seafood ingestion conducted for this report focused on the cancer risks associated with the radiation emitted by radionuclides of concern in the seafood.

\subsection{Diet Information Review}

Diet intake surveys previously conducted at four Alaska Native Villages-St. Paul, Atka, Nikolski, and Unalaska (Bartell et al. 1999; Hamrick et al. 2003; Hamrick and Smith 2003) were used for developing an Aleut diet for estimating the potential risk of ingesting seafood harvested (both from a subsistence and commercial basis) from Amchitka by an Aleut in Alaska. An average daily intake rate of seafood and the corresponding types or particular marine species (or distributions) that this intake comprised were determined by compositing the information from the survey data for the four villages. First, the total and species-specific intake rates were determined for each of the four villages assessed, these were then weighted by the number of villagers that participated in each of the surveys to obtain the total and species-specific intake rates for the composite diet. These average daily intake rates and distributions were used with radionuclide concentrations measured in marine species (or obtained from literature as in the case of salmon, or derived, as in the case of seal and sea lion) to estimate the potential risks associated with seafood ingestion. For ease of discussion, this Aleut diet will be referred to as the "composite diet" for the remainder of this section.

In addition to the composite diet, diet information for each of the four villages (as reported in the survey references mentioned above) was used to calculate the potential risk to an Aleut consuming seafood harvested from Amchitka. This was done to provide estimates reflecting a range of diets that include varying types of seafood and amounts of intake reflected in the survey data. It is noted that the villages (Atka, Nikolski, Unalaska, and St. Paul) that have survey data are a distance away from Amchitka and that the seafood being consumed at these villages would likely come from sources other than Amchitka. (No samples were collected from waters near these villages, as it is not within the scope and objective for LM's Amchitka project.) 
For the Atka diet data, diet information in terms of grams per day (g/day) (Bartell et al. 1999) were reported for three survey periods - June to September 1998, June to December 1998, and January to April 1999. Because the first period overlaps with the second period, the data for the second period were used, whenever available, with those for the third period to determine the average intake rates. The reported average daily intake rates for responders were used. Although the intake assessments do not cover an entire year, it is assumed that the diet pattern was applicable for the entire year.

The diet data for St. Paul (Hamrick et al. 2003), Nikolski, and Unalaska (Hamrick and Smith 2003) were reported as intake frequency expressed as the number of portions per year. Those results were multiplied by the corresponding amount in grams per portion, and then divided by 365 days per year to obtain the average daily intake rates in grams per day. For intake of seal and sea lion oil, each portion was assumed to be 1 tablespoon (equivalent to 0.5 fluid ounce with a density of 0.8 gram per cubic centimeter). Similar to the Atka diet data, the reported average frequencies for responders were used in the calculations.

For estimating potential health risks associated with seafood ingestion, the average daily intake rate of each seafood item is based on wet weight. The intake rates of dried seafood items, such as dried or smoked salmon, were converted to wet weight using the same wet weight to dry weight ratios determined from the 2011 sampling data (Hamilton et al. 2012a).

For a particular species of which multiple varieties are included in the diet, such as salmon for the St. Paul diet, the intake rates for silver, chum, red, king, and pink salmon, including dried, smoked, salted, cooked, or frozen, were all combined to give the total intake rate for salmon. This approach was necessary to provide a common basis for developing ingestion rates of different species with different sets of diet data because the level of detail regarding the varieties of species consumed is not similarly provided in the four diet survey data sets. The total intake rates derived for the 17 fish species are shown in Table 16. On the basis of these species-specific intake rates, the total seafood intake rate over all species was determined, and the distributions of the total intake rate among different species were calculated.

The intake rates for the composite diet and those for the four villages presented in Table 16 represent the seafood diet of an average adult because all the participants of the diet surveys were either males or females 15 years or older, except for a small number of younger participants in part of the Atka diet survey (Bartell et al. 1999). 
Table 16. Average Daily Intake Rates and Distributions of Seafood

\begin{tabular}{|c|c|c|c|c|c|c|c|c|c|c|}
\hline \multirow[b]{2}{*}{ Seafood item } & \multicolumn{2}{|c|}{ Atka Diet } & \multicolumn{2}{|c|}{ Nikolski Diet } & \multicolumn{2}{|c|}{ Unalaska Diet } & \multicolumn{2}{|c|}{ St. Paul Diet } & \multicolumn{2}{|c|}{ Composite Diet } \\
\hline & $\begin{array}{l}\text { Avg. } \\
\text { intake } \\
\text { rate } \\
\text { (g/day) }\end{array}$ & $\begin{array}{c}\% \text { of } \\
\text { total } \\
\text { intake } \\
\text { rate }\end{array}$ & $\begin{array}{l}\text { Avg. } \\
\text { intake } \\
\text { rate } \\
\text { (g/day) }\end{array}$ & $\begin{array}{c}\% \text { of } \\
\text { total } \\
\text { intake } \\
\text { rate }\end{array}$ & $\begin{array}{l}\text { Avg. } \\
\text { intake } \\
\text { rate } \\
\text { (g/day) }\end{array}$ & $\begin{array}{c}\% \text { of } \\
\text { total } \\
\text { intake } \\
\text { rate }\end{array}$ & $\begin{array}{l}\text { Avg. } \\
\text { intake } \\
\text { rate } \\
\text { (g/day) }\end{array}$ & $\begin{array}{c}\% \text { of } \\
\text { total } \\
\text { intake } \\
\text { rate }\end{array}$ & $\begin{array}{l}\text { Avg. } \\
\text { intake } \\
\text { rate } \\
\text { (g/day) }\end{array}$ & $\begin{array}{c}\% \text { of } \\
\text { total } \\
\text { intake } \\
\text { rate }\end{array}$ \\
\hline Fish, total & 100 & 63 & 520 & 89 & 140 & 77 & 10 & 43 & 100 & 76 \\
\hline Halibut & 19 & 12 & 160 & 28 & 30 & 17 & 5.2 & 22 & 27 & 20 \\
\hline Dolly Varden & 1.0 & 0.61 & 19 & 3.3 & 1.2 & 0.67 & $-{ }^{a}$ & - & 1.9 & 1.4 \\
\hline Cod & 10 & 6.3 & 11 & 1.9 & 4.6 & 2.6 & 0.080 & 0.34 & 3.9 & 2.8 \\
\hline Salmon & 55 & 33 & 330 & 56 & 98 & 55 & 4.9 & 21 & 67 & 49 \\
\hline Others & 18 & 11 & 1.4 & 0.23 & 2.0 & 1.1 & - & - & 3.3 & 2.4 \\
\hline $\begin{array}{l}\text { Mollusk and } \\
\text { crustacean, total }\end{array}$ & 7.0 & 4.3 & 14 & 2.4 & 12 & 6.7 & 1.5 & 6.6 & 6.8 & 5.0 \\
\hline Octopus & 3.9 & 2.4 & 1.9 & 0.32 & 1.1 & 0.63 & 0.19 & 0.81 & 1.1 & 0.84 \\
\hline Sea urchin & 1.7 & 1.0 & 0.93 & 0.16 & 1.1 & 0.62 & 0.32 & 1.4 & 0.82 & 0.60 \\
\hline Mussels & - & - & 5.4 & 0.93 & 0.090 & 0.051 & 0.012 & 0.051 & 0.43 & 0.31 \\
\hline Bidarkis & 1.5 & 0.91 & 5.4 & 0.93 & 0.21 & 0.12 & - & - & 0.67 & 0.49 \\
\hline Shrimp & - & - & 0.12 & 0.021 & 1.2 & 0.70 & - & - & 0.44 & 0.32 \\
\hline Crabs & - & - & 0.53 & 0.091 & 6.7 & 3.8 & 1.0 & 4.3 & 2.8 & 2.0 \\
\hline Clams & - & - & - & - & 1.4 & 0.79 & - & - & 0.48 & 0.35 \\
\hline Seabird eggs & 0.60 & 0.37 & 2.9 & 0.50 & 0.30 & 0.17 & 1.0 & 4.3 & 0.84 & 0.62 \\
\hline Seaweed/kelp & 0.10 & 0.061 & - & - & - & - & - & - & 0.014 & 0.010 \\
\hline Seals & 15 & 9.1 & 9.8 & 1.7 & 5.1 & 2.9 & 8.9 & 38 & 8.5 & 6.2 \\
\hline Sea lions & 32 & 20 & 5.9 & 1.0 & 2.8 & 1.6 & 1.6 & 7.0 & 6.6 & 4.9 \\
\hline Sea birds & 5.2 & 3.2 & 31 & 5.3 & 21 & 12 & 0.29 & 1.2 & 10 & 7.5 \\
\hline Total (g/day) & 160 & & 580 & & 180 & & 24 & & 140 & \\
\hline
\end{tabular}

a "-" = Not available 


\subsection{Risk Estimations}

Potential risk associated with ingestion of seafood was estimated with the following equation:

$$
\text { Risk }=\sum_{i} S F_{i} \times \sum_{j}\left(\text { Intake }_{j} \times C_{i, j}\right) \times 10^{-3} \times E F \times E D
$$

Where:

Risk = Excess lifetime cancer risk resulting from ingestion of seafood,

$S F_{i} \quad=$ Slope factor for radionuclide $i(1 / \mathrm{pCi})$,

$i \quad=$ Index for radionuclides,

$j \quad=$ Index for marine species,

Intake $_{j}=$ Total daily intake rate attributed to marine species $j$ (g/day),

$C_{i, j}=$ Concentration of radionuclide $i$ in marine species $j$, wet-weight based ( $\left.\mathrm{pCi} / \mathrm{kg}\right)$,

$10^{-3}=$ Unit conversion factor $(\mathrm{kg} / \mathrm{g})$,

$E F \quad=$ Exposure frequency (days/year) (365), and

$E D \quad=$ Exposure duration (years) $(30)$.

The assumed daily intake amount or rate for each marine species for the composite diet and for each of the four villages is based on the diet information presented in Table 16 and therefore varied with each village. Exposure frequency and exposure duration are conservatively assumed to be 365 days/year and 30 years, respectively. Slope factors used are those developed by EPA for use in estimating cancer risk. Radionuclide concentrations (or exposure point concentrations [EPCs]) for each species are based on the 2011 sampling data. Sections 7.2.1 and 7.2.2 provide more discussion on the slope factors and the radionuclide concentrations or EPCs used.

\subsubsection{Slope Factors}

To calculate potential cancer risks associated with seafood consumption, the EPA risk coefficients, known as slope factors (specific for the ingestion pathway), were used. EPA has developed pathway-specific slope factors for use in risk assessments. These slope factors are estimates of potential cancer risk per unit intake of radionuclides that were developed with considerations of age- and sex-specific factors for individual organs, along with organ-specific radiation absorbed doses. The slope factors are characterized as best-estimate values of the ageaveraged lifetime excess cancer risk per unit of intake for the radionuclides of concern. Detailed information on the derivation of slope factors and their application are available in several EPA documents (EPA 1991a,b; 1994, 1997; Eckerman et al. 1999). The method used in this human health risk assessment follows EPA risk assessment guidance (EPA 1991a; 1997).

The ingestion slope factor of a radionuclide provides an estimate of the potential lifetime excess cancer risk that could result from intake of $1 \mathrm{pCi}$ of that radionuclide. These slope factors were obtained from EPA Federal Guidance Report No. 13 (Eckerman et al. 1999) and are listed in Table 17. In the table, “+D" designations as applied to ${ }^{137} \mathrm{Cs},{ }^{235} \mathrm{U}$, and ${ }^{238} \mathrm{U}$, indicate that the potential risks contributed by the respective short-lived decay progenies (with a radioactive halflife less than 6 months) for each radionuclide were included in the values listed for these three radionuclides. It was assumed that concentrations of short-lived radionuclides in the various marine species were the same as the concentrations of their parent radionuclides, that is, the short-lived radionuclides are in secular equilibrium with the parent radionuclides. 
Table 17. Radionuclide Slope Factors Used for Estimating Risk for the Ingestion Pathway

\begin{tabular}{||c|c|c|c|c|c|c|c||}
\hline \multirow{3}{*}{$\begin{array}{c}\text { SF } \\
(\mathbf{1} / \mathbf{p C i} \mathbf{)})\end{array}$} & ${ }^{241} \mathbf{A m}$ & ${ }^{137} \mathbf{C s}+\mathbf{D}$ & ${ }^{239} \mathbf{P u}$ & ${ }^{240} \mathbf{P u}$ & ${ }^{234} \mathbf{U}$ & ${ }^{\mathbf{2 3 5}} \mathbf{U}+\mathbf{D}$ & ${ }^{\mathbf{2 3}} \mathbf{U}+\mathbf{D}$ \\
\cline { 2 - 8 } & $1.34 \times 10^{-10}$ & $3.74 \times 10^{-11}$ & $1.74 \times 10^{-10}$ & $1.74 \times 10^{-10}$ & $9.55 \times 10^{-11}$ & $9.76 \times 10^{-11}$ & $1.21 \times 10^{-10}$ \\
\hline
\end{tabular}

\subsubsection{Exposure Point Concentrations or Radionuclide Concentrations in Marine Species}

The EPCs of radionuclides used for the risk calculations were determined on the basis of Amchitka and Adak 2011 sampling data as presented in previous sections. The wet-weight concentrations from the laboratory analyses of the samples of the various species collected are considered to be the average value for the whole body of each sample for the various species. Table 18 and Table 19 present the maximum and the 95 percent upper confidence limit of the mean (UCL ${ }_{95}$ ) values, respectively, of the Amchitka data set. Table 20 and Table 21 present the maximum and UCL 95 values for Adak. Depending on the availability of sampling data, different methods were used to develop EPCs or radionuclide concentrations in the various species. The following sections provide more discussions on the different methods used.

Table 18. Risk Calculation EPCs_Maximum Values of the Amchitka 2011 Sampling Data

\begin{tabular}{|c|c|c|c|c|c|c|c|}
\hline \multirow{2}{*}{$\begin{array}{l}\text { Species Considered } \\
\text { for Intake }\end{array}$} & \multicolumn{7}{|c|}{ Radionuclide } \\
\hline & ${ }^{241} \mathrm{Am}$ & ${ }^{137} \mathrm{Cs}$ & ${ }^{239} \mathrm{Pu}$ & ${ }^{240} \mathrm{Pu}$ & ${ }^{234} U$ & ${ }^{235} U$ & ${ }^{238} U$ \\
\hline Halibut $^{\mathrm{a}}$ & 0.002 & 1.5 & 0.01 & 0.003 & 1.8 & 0.03 & 0.65 \\
\hline Dolly Varden ${ }^{a}$ & 0.002 & 98 & 0.01 & 0.01 & 1.3 & 0.01 & 0.23 \\
\hline $\operatorname{Cod}^{a}$ & 0.004 & 3.8 & 0.01 & 0.003 & 3.0 & 0.09 & 2.1 \\
\hline Salmon $^{\mathrm{b}}$ & 0.01 & 8.5 & 0.01 & 0.006 & 17 & 0.79 & 15 \\
\hline Other fish ${ }^{a}$, & 0.07 & 7.8 & 0.08 & 0.04 & 20 & 0.60 & 14 \\
\hline Octopus $^{a}$ & 0.019 & 2.1 & 0.03 & 0.03 & 22 & 0.77 & 18 \\
\hline${\text { Sea } \text { urchin }^{a}}^{a}$ & 0.20 & 9.9 & 0.09 & 0.07 & 26 & 0.83 & 20 \\
\hline Mussel $^{\mathrm{a}}$ & 0.80 & 44 & 2.0 & 1.0 & 81 & 2.9 & 67 \\
\hline Bidarkis (chiton) ${ }^{a}$ & 0.14 & 4.1 & 0.07 & 0.04 & 32 & 1.1 & 27 \\
\hline Shrimp $^{\mathrm{b}}$ & 0.03 & 12 & 0.07 & 0.04 & 15 & 0.53 & 12 \\
\hline Crabs $^{\mathrm{b}}$ & 0.03 & 12 & 0.07 & 0.04 & 15 & 0.53 & 12 \\
\hline Clams $^{a}$ & 0.80 & 44 & 2.0 & 1.0 & 81 & 2.9 & 67 \\
\hline Sea birds ${ }^{\mathrm{b}}$ & 0.02 & 110 & 0.32 & 0.16 & 62 & 2.1 & 50 \\
\hline Sea bird eggs $^{a}$ & 0.01 & 12 & 0.07 & 0.04 & 0.80 & 0.001 & 0.02 \\
\hline Seaweed $^{a}$ & 0.03 & 5.8 & 0.16 & 0.13 & 140 & 5.1 & 120 \\
\hline Seal $^{\mathrm{b}}$ & 0.02 & 50 & 0.77 & 0.39 & 6.2 & 0.21 & 5.0 \\
\hline Sea lion ${ }^{\mathrm{b}}$ & 0.02 & 50 & 0.77 & 0.39 & 6.2 & 0.21 & 5.0 \\
\hline
\end{tabular}

Units are $\mathrm{pCi} / \mathrm{kg}$

a EPCs were determined based on sampling data. For other fish, they are based on data for greenling, Pacific cod, and rockfish. For sea bird eggs, they are based on data for gull eggs and goose eggs. For seaweed, they are based on data for dragon kelp and rockweed. For clams, they are based on data for mussels.

${ }^{b}$ EPCs were derived using surface water concentrations and bioaccumulation factors (BAFs). For salmon, EPCs were derived based on water concentrations in the North Pacific Ocean reported in the literature (for ${ }^{241} \mathrm{Am}$, Lee et al. 2003, for ${ }^{137} \mathrm{Cs}$, Kamenik et al. 2013, for ${ }^{239} \mathrm{Pu}$ and ${ }^{240} \mathrm{Pu}$, Provinec et al. 2005, and for ${ }^{234} \mathrm{U}$, ${ }^{235} \mathrm{U}$, and ${ }^{238} \mathrm{U}$, Ku et al. 1977). EPCs for shrimp, crab, sea bird, seal, and sea lion are based on water concentrations derived from tissue concentrations of species collected in 2011 from Amchitka. 
Table 19. Risk Calculation EPCs_UCL ${ }_{95}$ Values of the Amchitka 2011 Sampling Data

\begin{tabular}{|c|c|c|c|c|c|c|c|}
\hline \multirow{2}{*}{$\begin{array}{l}\text { Species Considered } \\
\text { for Intake }\end{array}$} & \multicolumn{7}{|c|}{ "Radionuclide } \\
\hline & ${ }^{241} \mathrm{Am}$ & ${ }^{137} \mathrm{Cs}$ & ${ }^{239} \mathrm{Pu}$ & ${ }^{240} \mathrm{Pu}$ & ${ }^{234} U$ & ${ }^{235} \mathrm{U}$ & ${ }^{238} U$ \\
\hline Halibut $^{a}$ & 0.002 & 1.5 & 0.01 & 0.003 & 1.8 & 0.03 & 0.65 \\
\hline Dolly Varden ${ }^{a}$ & 0.002 & 98 & 0.01 & 0.01 & 1.3 & 0.01 & 0.23 \\
\hline $\mathrm{Cod}^{\mathrm{a}}$ & 0.004 & 3.8 & 0.01 & 0.003 & 3.0 & 0.09 & 2.1 \\
\hline Salmon $^{\mathrm{b}}$ & 0.01 & 8.5 & 0.01 & 0.006 & 17 & 0.79 & 15 \\
\hline Other fish ${ }^{a}$, & 0.04 & 7.7 & 0.07 & 0.04 & 13 & 0.44 & 10 \\
\hline Octopus $^{a}$ & 0.019 & 2.1 & 0.03 & 0.03 & 22 & 0.77 & 18 \\
\hline Sea urchin $^{\mathrm{a}}$ & 0.17 & 8.2 & 0.08 & 0.06 & 26 & 0.83 & 20 \\
\hline Mussel $^{a}$ & 0.51 & 29 & 1.2 & 0.61 & 76 & 2.7 & 64 \\
\hline Bidarkis (chiton) ${ }^{a}$ & 0.14 & 4.1 & 0.07 & 0.04 & 32 & 1.1 & 27 \\
\hline Shrimp $^{\mathrm{b}}$ & 0.03 & 12 & 0.07 & 0.04 & 15 & 0.53 & 12 \\
\hline Crabs $^{\mathrm{b}}$ & 0.03 & 12 & 0.07 & 0.04 & 15 & 0.53 & 12 \\
\hline Clams $^{a}$ & 0.51 & 29 & 1.2 & 0.61 & 76 & 2.7 & 64 \\
\hline Sea birds ${ }^{\mathrm{b}}$ & 0.02 & 110 & 0.32 & 0.16 & 62 & 2.1 & 50 \\
\hline Sea bird eggs ${ }^{a}$ & 0.01 & 12 & 0.07 & 0.04 & 0.80 & 0.001 & 0.02 \\
\hline Seaweed $^{\mathrm{a}}$ & 0.02 & 4.2 & 0.09 & 0.07 & 104 & 3.7 & 86 \\
\hline Seal $^{\mathrm{b}}$ & 0.02 & 50 & 0.77 & 0.39 & 6.2 & 0.21 & 5.0 \\
\hline Sea lion ${ }^{b}$ & 0.02 & 50 & 0.77 & 0.39 & 6.2 & 0.21 & 5.0 \\
\hline
\end{tabular}

Units are $\mathrm{pCi} / \mathrm{kg}$

${ }^{a}$ EPCs were determined based on sampling data. For other fish, they are based on data for greenling, Pacific cod, and rockfish. For sea bird eggs, they are based on data for gull eggs and goose eggs. For seaweed, they are based on data for dragon kelp and rockweed. For clams, they are based on data for mussels.

${ }^{b}$ EPCs were derived using surface water concentrations and bioaccumulation factors (BAFs). For salmon, EPCs were derived based on water concentrations in the North Pacific Ocean reported in the literature (for ${ }^{241} \mathrm{Am}$, Lee et al. 2003, for ${ }^{137} \mathrm{Cs}$, Kamenik et al. 2013, for ${ }^{239} \mathrm{Pu}$ and ${ }^{240} \mathrm{Pu}$, Provinec et al. 2005, and for ${ }^{234} \mathrm{U}$, ${ }^{235} \mathrm{U}$, and ${ }^{238} \mathrm{U}$, Ku et al. 1977). EPCs for shrimp, crab, sea bird, seal, and sea lion are based on water concentrations derived from tissue concentrations of species collected in 2011 from Amchitka. 
Table 20. Risk Calculation EPCs—Maximum Values of the Adak 2011 Sampling Data

\begin{tabular}{|c|c|c|c|c|c|c|c|}
\hline \multirow{2}{*}{$\begin{array}{l}\text { Species Considered } \\
\text { for Intake }\end{array}$} & \multicolumn{7}{|c|}{ Radionuclide } \\
\hline & ${ }^{241} \mathrm{Am}$ & ${ }^{137} \mathrm{Cs}$ & ${ }^{239} \mathrm{Pu}$ & ${ }^{240} \mathrm{Pu}$ & ${ }^{234} U$ & ${ }^{235} U$ & ${ }^{238} \mathrm{U}$ \\
\hline Halibut $^{a}$ & 0.002 & 1.0 & 0.01 & 0.003 & 1.60 & 0.04 & 0.86 \\
\hline Dolly Varden $^{a}$ & 0.003 & 450 & 0.01 & 0.01 & 0.90 & 0.02 & 0.36 \\
\hline $\mathrm{Cod}^{\mathrm{a}}$ & 0.002 & 3.0 & 0.01 & 0.003 & 1.90 & 0.06 & 1.4 \\
\hline Salmon $^{\mathrm{b}}$ & 0.01 & 8.5 & 0.01 & 0.006 & 17 & 0.79 & 15 \\
\hline Other fish ${ }^{\mathrm{a}}$, & 0.04 & 9.4 & 0.07 & 0.04 & 13 & 0.48 & 11 \\
\hline Octopus $^{a}$ & 0.015 & 0.70 & 0.02 & 0.01 & 14 & 0.45 & 11 \\
\hline Sea urchin $^{\mathrm{a}}$ & 0.12 & 9.4 & 0.07 & 0.04 & 29 & 0.83 & 19 \\
\hline Mussel $^{a}$ & 0.15 & 15 & 0.20 & 0.10 & 50 & 1.8 & 41 \\
\hline Bidarkis (chiton) $^{a}$ & 0.08 & 1.5 & 0.06 & 0.03 & 33 & 0.99 & 23 \\
\hline Shrimp $^{\mathrm{b}}$ & 0.03 & 9.9 & 0.03 & 0.02 & 9.4 & 0.33 & 7.9 \\
\hline Crabs $^{\mathrm{b}}$ & 0.03 & 9.9 & 0.03 & 0.02 & 9.4 & 0.33 & 7.9 \\
\hline Clams $^{a}$ & 0.15 & 15 & 0.20 & 0.10 & 50 & 1.8 & 41 \\
\hline Sea birds ${ }^{\mathrm{b}}$ & 0.02 & 90 & 0.13 & 0.07 & 38 & 1.3 & 31 \\
\hline Sea bird eggs ${ }^{c}$ & - & - & - & - & - & - & - \\
\hline Seaweed $^{\mathrm{a}}$ & 0.03 & 7.3 & 0.11 & 0.09 & 140 & 5.1 & 120 \\
\hline Seal $^{\mathrm{b}}$ & 0.02 & 41 & 0.32 & 0.17 & 3.8 & 0.13 & 3.1 \\
\hline Sea lion ${ }^{\mathrm{b}}$ & 0.02 & 41 & 0.32 & 0.17 & 3.8 & 0.13 & 3.1 \\
\hline
\end{tabular}

Units are $\mathrm{pCi} / \mathrm{kg}$

${ }^{a}$ EPCs were determined based on sampling data. For other fish, they are based on data for greenling, Pacific cod, and rockfish. For seaweed, they are based on data for dragon kelp and rockweed. For clams, they are based on data for mussels.

${ }^{b}$ EPCs were derived using surface water concentrations and bioaccumulation factors (BAFs). For salmon, EPCs were derived based on water concentrations in the North Pacific Ocean reported in the literature (for ${ }^{241} \mathrm{Am}$, Lee et al. 2003, for ${ }^{137} \mathrm{Cs}$, Kamenik et al. 2013, for ${ }^{239} \mathrm{Pu}$ and ${ }^{240} \mathrm{Pu}$, Provinec et al. 2005 , and for ${ }^{234} \mathrm{U}$, ${ }^{235} \mathrm{U}$, and ${ }^{238} \mathrm{U}$, Ku et al. 1977). EPCs for shrimp, crab, sea bird, seal, and sea lion are based on water concentrations derived from tissue concentrations of species collected in 2011 from Adak.

${ }^{\mathrm{c}}$ Sampling data for sea bird eggs are not available, as none were collected on Adak. 
Table 21. Risk Calculation EPCs_UCL $L_{95}$ Values of the Adak 2011 Sampling Data

\begin{tabular}{|c|c|c|c|c|c|c|c|}
\hline \multirow{2}{*}{$\begin{array}{l}\text { Species Considered } \\
\text { for Intake }\end{array}$} & \multicolumn{7}{|c|}{ Radionuclide } \\
\hline & ${ }^{241} \mathrm{Am}$ & ${ }^{137} \mathrm{Cs}$ & ${ }^{239} \mathrm{Pu}$ & ${ }^{240} \mathrm{Pu}$ & ${ }^{234} U$ & ${ }^{235} U$ & ${ }^{238} \mathrm{U}$ \\
\hline Halibut $^{a}$ & 0.002 & 1.0 & 0.01 & 0.003 & 1.60 & 0.04 & 0.86 \\
\hline Dolly Varden $^{a}$ & 0.003 & 450 & 0.01 & 0.01 & 0.90 & 0.02 & 0.36 \\
\hline $\mathrm{Cod}^{\mathrm{a}}$ & 0.002 & 3.0 & 0.01 & 0.003 & 1.90 & 0.06 & 1.4 \\
\hline Salmon $^{\mathrm{b}}$ & 0.01 & 8.5 & 0.01 & 0.006 & 17 & 0.79 & 15 \\
\hline Other fish ${ }^{\mathrm{a}}$, & 0.03 & 5.4 & 0.06 & 0.03 & 8.4 & 0.27 & 6.4 \\
\hline Octopus $^{a}$ & 0.02 & 0.70 & 0.02 & 0.01 & 14 & 0.45 & 11 \\
\hline Sea urchin $^{\mathrm{a}}$ & 0.12 & 8.2 & 0.07 & 0.04 & 29 & 0.83 & 19 \\
\hline Mussel $^{a}$ & 0.15 & 15 & 0.20 & 0.10 & 50 & 1.8 & 41 \\
\hline Bidarkis (chiton) $^{a}$ & 0.08 & 1.5 & 0.06 & 0.03 & 33 & 1.0 & 23 \\
\hline Shrimp $^{\mathrm{b}}$ & 0.03 & 9.9 & 0.03 & 0.02 & 9.4 & 0.33 & 7.9 \\
\hline Crabs $^{\mathrm{b}}$ & 0.03 & 9.9 & 0.03 & 0.02 & 9.4 & 0.33 & 7.9 \\
\hline Clams $^{a}$ & 0.15 & 15 & 0.20 & 0.10 & 50 & 1.8 & 41 \\
\hline Sea birds ${ }^{\mathrm{b}}$ & 0.02 & 90 & 0.13 & 0.07 & 38 & 1.3 & 31 \\
\hline Sea bird eggs ${ }^{c}$ & - & - & - & - & - & - & - \\
\hline Seaweed $^{\mathrm{a}}$ & 0.02 & 5.6 & 0.08 & 0.06 & 100 & 3.6 & 85 \\
\hline Seal $^{\mathrm{b}}$ & 0.02 & 41 & 0.32 & 0.17 & 3.8 & 0.13 & 3.1 \\
\hline Sea lion ${ }^{\mathrm{b}}$ & 0.02 & 41 & 0.32 & 0.17 & 3.8 & 0.13 & 3.1 \\
\hline
\end{tabular}

Units are $\mathrm{pCi} / \mathrm{kg}$

${ }^{a}$ EPCs were determined based on sampling data. For other fish, they are based on data for greenling, Pacific cod, and rockfish. For seaweed, they are based on data for dragon kelp and rockweed. For clams, they are based on data for mussels.

${ }^{b}$ EPCs were derived using surface water concentrations and bioaccumulation factors (BAFs). For salmon, EPCs were derived based on water concentrations in the North Pacific Ocean reported in the literature (for ${ }^{241} \mathrm{Am}$, Lee et al. 2003, for ${ }^{137} \mathrm{Cs}$, Kamenik et al. 2013, for ${ }^{239} \mathrm{Pu}$ and ${ }^{240} \mathrm{Pu}$, Provinec et al. 2005, and for ${ }^{234} \mathrm{U}, 235 \mathrm{U}$, and ${ }^{238} \mathrm{U}$, Ku et al. 1977). EPCs for shrimp, crab, sea bird, seal, and sea lion are based on water concentrations derived from tissue concentrations of species collected in 2011 from Adak.

${ }^{\mathrm{c}}$ Sampling data for sea bird eggs are not available, as none were collected on Adak. 


\subsubsection{EPCs for Sampled Species}

For the risk estimates discussed in this section, two calculations were performed, one using the maximum concentrations reported, and the other using the UCL 95 value. The UCL 95 value was calculated using the EPA recommended method (in particular the Chebyshev Inequality method) (EPA 2002). In addition, for risk estimates using UCL 95 values, when the number of samples was fewer than 3 , the maximum value was used. This approach is consistent with EPA recommendations for small numbers of samples because the calculated UCL 95 value would likely result in a value not within the range of measured values and likely greater than maximum. In the determination of maximum and $\mathrm{UCL}_{95}$ values, the sampling data reported as less than the MDCs were replaced with the MDCs. This would result in a higher $\mathrm{UCL}_{95}$ value and would be a conservative approach.

\section{$\mathrm{UCL}_{95}$}

For risk assessments, EPA recommends using the average concentration measured to represent "a reasonable estimate of the concentration likely to be contacted over time." However, because of the uncertainty associated with estimating the true average concentration at a given site, the 95 percent upper confidence limit $\left(\mathrm{UCL}_{95}\right)$ of the arithmetic mean should be used for this variable, which is also referred to as the exposure point concentration (EPC).

The marine species that were sampled (and listed in Table 16) included halibut, Dolly Varden, cod, octopus, sea urchin, mussel, bidarki (chiton), sea birds, seabird eggs, and seaweed.

Radionuclide concentrations were available for both gull eggs and goose eggs; the greater of the maximum or UCL 95 concentrations between these two types of eggs were selected as the EPC for seabird eggs in the calculations. Radionuclide concentrations were also available for two aquatic plants - dragon kelp and rockweed. Like the approach taken for seabird eggs, the greater of the maximum or UCL ${ }_{95}$ concentrations between these two types of aquatic plants were selected as the EPC for seaweed for risk calculations.

\subsubsection{EPCs for Other Fish and Clams}

EPCs used to calculate the potential risk for fish not sampled but reported in the diets are either the maximum or the UCL95 values selected from three fish species-greenling, Pacific cod, or rockfish. EPCs for clams are either the maximum or UCL 95 of the sampling data for mussels.

\subsubsection{EPCs for Sea Lion and Seal}

To develop EPCs for sea lions and seals, radionuclide concentrations in seawater around Amchitka and Adak were estimated using the measured concentrations for a marine species and the bioaccumulation factors (BAFs) reported for that species. The BAFs are ratios of concentrations in the species to concentrations in seawater. To obtain seawater concentrations, the average measured concentrations for nine marine species were used. The nine species included four benthic fish-Irish lord, greenling, halibut, and rockfish; three mollusks - octopus, horse mussel, and chiton; and two macroalgae - rockweed and dragon kelp. The average radionuclide concentrations for each of the nine species were divided by the representative BAFs for that species to obtain estimates of radionuclide concentrations in water. Nine sets of water concentrations were estimated (one from each species), and for each radionuclide, the maximum of the estimated values was selected as the representative concentration in seawater. The 
representative seawater concentrations were then multiplied by reported BAFs for marine mammals to obtain radionuclide concentrations in seals and sea lions.

The estimated seawater concentrations around Amchitka and Adak are compared with the concentrations for North Pacific Ocean reported in literature (Ku et al. 1977; Lee et al. 2003; Povinec et al. 2004, 2005; Hirose et al. 2006; Nakano and Povinec 2012; Kamenik et al. 2013). In general, the estimated concentrations for Amchitka and Adak are within the range of reported concentrations for North Pacific Ocean, except for the concentrations of ${ }^{239} \mathrm{Pu}$ and ${ }^{240} \mathrm{Pu}$. For these two radionuclides, the estimated concentrations are a few times greater. However, because the ${ }^{239} \mathrm{Pu}$ and ${ }^{240} \mathrm{Pu}$ concentrations are 2 to 4 orders of magnitude lower than the concentrations of ${ }^{137} \mathrm{Cs}$ and uranium isotopes; the seawater concentrations of ${ }^{239} \mathrm{Pu}$ and ${ }^{240} \mathrm{Pu}$ have little effect on the total cancer risks associated with seafood ingestion. Seawater concentrations are presented in Table 35 and further discussions on the comparison of seawater concentrations are provided in Section 7.4 .

Representative BAFs used for developing the EPCs of seals and sea lions were obtained primarily from the International Atomic Energy Agency (IAEA) wildlife transfer factors handbook (Howard et al. 2013), which is the most comprehensive database for BAFs available at present. The mean BAF values reported for each radionuclide and species were used. Besides the IAEA handbook (Howard et al. 2013), two others sources (ERICA 2013 and FASSET 2003) were also consulted to obtain BAFs; however, the values from these two sources were used only when BAFs were not available from the IAEA report (Howard et al. 2013). Table 22 lists the BAFs used for seals and sea lions (mammals) and other marine species.

Table 22. BAFs Used for Estimating Radionuclide Concentrations in Marine Species

\begin{tabular}{||l|c|c|c|c||}
\hline \multicolumn{1}{|c|}{ Marine Species $^{\text {a }}$} & Am & Cs & Pu & U \\
\hline Bottom fish & 320 & 71 & 2500 & 14 \\
\hline Predator fish & $58^{\mathrm{b}}$ & 79 & 190 & $14^{\mathrm{b}}$ \\
\hline Mollusk & 9900 & 50 & 1100 & 32 \\
\hline Mollusk-bivalve & $-^{\mathrm{c}}$ & 63 & 650 & 35 \\
\hline Crustacean & 500 & 53 & 120 & $10^{\mathrm{b}}$ \\
\hline Bird & $310^{\mathrm{d}}$ & 480 & $540^{\mathrm{d}}$ & $40^{4}$ \\
\hline Mammal & $280^{\mathrm{b}}$ & 220 & 1300 & $4^{\mathrm{e}}$ \\
\hline Macroalgae & 430 & 96 & 4100 & 83 \\
\hline
\end{tabular}

The primary source for BAFs is the wildlife transfer factors database compiled by IAEA (Howard et al. 2013) unless otherwise noted.

${ }^{b}$ Values are the defaults of ERICA (ERICA 2013).

c "-" denotes "not available."

d Values are from the FASSET database (FASSET 2003).

e Values is 10 times the default of ERICA (ERICA 2013).

\subsubsection{EPCs for Salmon}

Studies on the correlation between ${ }^{137} \mathrm{Cs}$ concentrations in Atlantic salmon and ${ }^{137} \mathrm{Cs}$ concentrations in seawater (Tucker et al. 1999 and Spares et al. 2007) showed that the concentrations in adult salmon reflected the entire range of values seen across the North Atlantic Ocean, which suggests that the marine life history of salmon is more panoceanic than previously thought, and they should be classified as a single trans-Atlantic straddling stock. Therefore, the 
EPCs for salmon were estimated using literature radionuclide concentrations for North Pacific Ocean (Ku et al. 1977, Lee et al. 2003, Povinec et al. 2005, and Kamenik et al. 2013) and the BAFs for piscivorous fish primarily from the IAEA wildlife transfer factors handbook (Howard et al. 2013). The literature radionuclide concentrations for uranium, ${ }^{241} \mathrm{Am}$, and

${ }^{239 / 240} \mathrm{Pu}$ in the North Pacific Ocean were for year 2000 or earlier; they were used directly for estimating the EPCs without correction for effective half-life in the ocean. These earlier concentrations are expected to be higher than the present levels. The ${ }^{137} \mathrm{Cs}$ concentration in seawater was based on 2012 measurement data (Kamenik et al. 2013), which were obtained after the Fukushima Dai-ichi accident in 2011. The radionuclide concentrations in the North Pacific Ocean used for estimating the salmon EPCs are listed in Table 23.

\subsubsection{EPCs for Crustaceans and Sea Birds}

After representative radionuclide concentrations in seawater around Amchitka and Adak were determined, they were multiplied by BAFs for marine crustaceans to obtain radionuclide concentrations for shrimp and crab. For sea birds, the radionuclides concentrations were obtained by multiplying the representative seawater concentrations with the BAFs for sea birds. The BAFs used in these calculations are also listed in Table 22.

\subsubsection{Risk Estimates}

Potential risks from ingestion of seafood were estimated for an individual consuming seafood harvested from Amchitka using a range of diets including those reported for Atka, Nikolski, Unalaska, and St. Paul, and the composite diet based on the diet information from these four villages as discussed previously. Similar calculation was done using Adak data to compare the Amchitka results with the designated reference location for this sampling event. Therefore, the intake rates attributed to different marine species as listed in Table 16 were used with concentrations

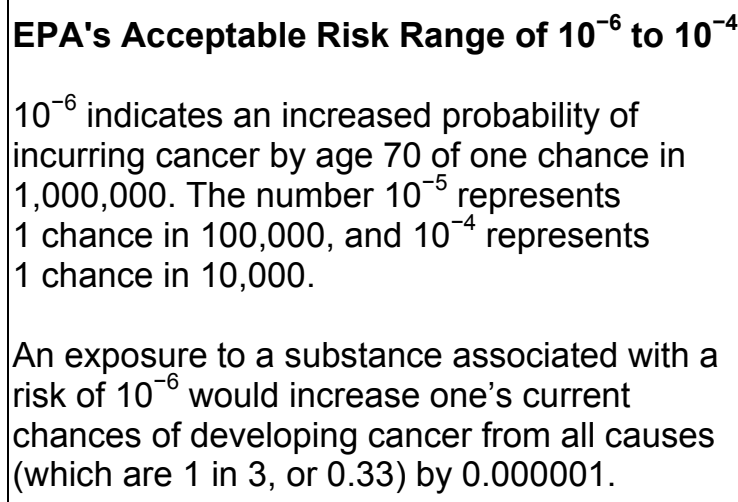

An exposure to a substance associated with a risk of $10^{-6}$ would increase one's current chances of developing cancer from all causes (which are 1 in 3 , or 0.33 ) by 0.000001 . or EPCs in different species from the Amchitka and Adak 2011 sampling data (Table 18 through Table 21). Table 23 summarizes the estimated risk. Detailed risk estimates are provided in Table 24 through Table 33. 
Table 23. Summary of Risk Estimates for the Seafood Ingestion Pathway

\begin{tabular}{|c|c|c|c|c|c|c|}
\hline \multirow{3}{*}{$\begin{array}{c}\text { Diet Data } \\
\text { Used }\end{array}$} & \multicolumn{4}{|c|}{ Radionuclide Concentrations or EPCs } & \multirow{2}{*}{\multicolumn{2}{|c|}{$\begin{array}{c}\text { Difference Between Risk } \\
\text { Estimates Based on } \\
\text { Amchitka Versus Adak Data }\end{array}$}} \\
\hline & \multicolumn{2}{|c|}{ Amchitka } & \multicolumn{2}{|c|}{ Adak } & & \\
\hline & Maximum & UCL95 & Maximum & UCL95 & Maximum & UCL95 \\
\hline St. Paul Diet & $7.4 \times 10^{-7}$ & $7.4 \times 10^{-7}$ & $5.9 \times 10^{-7}$ & $5.9 \times 10^{-7}$ & $1.5 \times 10^{-7}$ & $1.5 \times 10^{-7}$ \\
\hline Atka Diet & $6.4 \times 10^{-6}$ & $6.1 \times 10^{-6}$ & $5.4 \times 10^{-6}$ & $5.2 \times 10^{-6}$ & $1.0 \times 10^{-6}$ & $9.0 \times 10^{-7}$ \\
\hline Nikolski Diet & $2.3 \times 10^{-5}$ & $2.3 \times 10^{-5}$ & $2.3 \times 10^{-5}$ & $2.3 \times 10^{-5}$ & $-{ }^{a}$ & $-^{a}$ \\
\hline Unalaska Diet & $9.2 \times 10^{-6}$ & $9.2 \times 10^{-6}$ & $7.8 \times 10^{-6}$ & $7.8 \times 10^{-6}$ & $1.4 \times 10^{-6}$ & $1.4 \times 10^{-6}$ \\
\hline Composite & $6.1 \times 10^{-6}$ & $6.0 \times 10^{-6}$ & $5.4 \times 10^{-6}$ & $5.3 \times 10^{-6}$ & $7.0 \times 10^{-7}$ & $7.0 \times 10^{-7}$ \\
\hline
\end{tabular}

${ }^{a}$ There is no difference.

Note: Because the cancer risk estimates are very close, to show the differences, they are listed in two significant figures.

Based on Table 23, potential risks estimated with Amchitka and Adak sampling data are generally similar; however, risk estimates using Amchitka sampling data are slightly higher than those estimated using Adak sampling data. This result is consistent with the results of the statistical comparison of Amchitka and Adak data discussed in Section 6.3. The risk estimates for the five diets using data collected from Amchitka ranged from $7.4 \times 10^{-7}$ to $2.3 \times 10^{-5}$ using either maximums or UCL 95 values as EPCs. For Adak, the range is from $5.9 \times 10^{-7}$ to $2.3 \times 10^{-5}$, also using either maximums or UCL 95 values as EPCs. The estimates using Amchitka data are slightly higher than those for Adak but not more than $1.4 \times 10^{-6}$ (see Table 23 estimates for the Unalaska diet). The State of Alaska considers risk levels at or below $1 \times 10^{-5}$ above background (or in this case above estimates for the reference location) to be an acceptable risk benchmark. The results also indicate that the estimated potential risk is a direct function of the intake amounts, and given the same seafood intake rates, the cancer risk estimates based on maximum concentrations and UCL 95 concentrations for samples obtained in 2011 are almost the same.

Radionuclides that have significant contributions to the total risk can be identified by a comparison of the results shown in Table 24 through Table 33. Regardless of the choice of seafood intake data and radionuclide sampling locations, ${ }^{137} \mathrm{Cs},{ }^{234} \mathrm{U}$, and ${ }^{238} \mathrm{U}$ are the three radionuclides that contributed the most to the risk estimates.

Some uncertainty is inherent with any method for estimating risk. The potential uncertainties associated with the risk analysis presented in this report are typical for such an evaluation. The use of seawater data and BAFs published in the literature to derive EPCs in tissue for species not sampled but included in the diet (e.g., salmon) may have overestimated the potential risk. Reported seawater concentrations in the North Pacific Ocean were used to estimate concentrations in salmon. For ${ }^{241} \mathrm{Am},{ }^{239} \mathrm{Pu},{ }^{240} \mathrm{Pu}$, and uranium isotopes, the reported water concentrations corresponded to year 2000 or earlier. Because the reported values were used without correction for decay over time, the current concentrations are probably lower than the reported values. For ${ }^{137} \mathrm{Cs}$, the water concentration used was the maximum value for the North Pacific Ocean measured after the Fukushima Dai-ichi incident (an average value was not reported). The average ${ }^{137} \mathrm{Cs}$ concentration across the North Pacific Ocean could be less than this value. The use of maximum water concentrations for the North Pacific Ocean could lead to higher salmon tissue concentrations being estimated and used for risk calculation. Further, there are uncertainties in the BAFs used to calculate water concentrations around Amchitka and Adak 
Table 24. Potential Risks to an Aleut Consuming Seafood Harvested from Amchitka and Adak Using the St. Paul Diet Data and Maximum Measured Concentrations

\begin{tabular}{|c|c|c|c|c|c|c|c|c|c|c|c|c|c|c|c|c|}
\hline \multirow{2}{*}{$\begin{array}{l}\text { Seafood } \\
\text { species }\end{array}$} & \multicolumn{2}{|c|}{${ }^{241} \mathrm{Am}$} & \multicolumn{2}{|c|}{${ }^{137} \mathrm{Cs}+\mathrm{D}$} & \multicolumn{2}{|c|}{${ }^{239} \mathrm{Pu}$} & \multicolumn{2}{|c|}{${ }^{240} \mathrm{Pu}$} & \multicolumn{2}{|c|}{${ }^{234} U$} & \multicolumn{2}{|c|}{${ }^{235} U+D$} & \multicolumn{2}{|c|}{${ }^{238} U+D$} & \multicolumn{2}{|c|}{ Total } \\
\hline & Amchitka & Adak & Amchitka & Adak & Amchitka & Adak & Amchitka & Adak & Amchitka & Adak & nchitka & Adak & Imchitka & Adak & mchitka & Adak \\
\hline Halibut & $2 \times 10^{-11}$ & $2 \times 10^{-11}$ & $3 \times 10^{-9}$ & $2 \times 10^{-9}$ & $1 \times 10^{-10}$ & $1 \times 10^{-10}$ & $3 \times 10^{-11}$ & $3 \times 10^{-11}$ & $1 \times 10^{-8}$ & $9 \times 10^{-9}$ & $2 \times 10^{-10}$ & $2 \times 10^{-10}$ & $4 \times 10^{-9}$ & $6 \times 10^{-9}$ & $2 \times 10^{-8}$ & $2 \times 10^{-8}$ \\
\hline Dolly V & 0 & 0 & 0 & 0 & 0 & 0 & 0 & 0 & 0 & 0 & $v$ & $v$ & 0 & 0 & 0 & 0 \\
\hline Cod & $5 \times 10^{-13}$ & $2 \times 10^{-13}$ & $1 \times 10^{-10}$ & $1 \times 10^{-10}$ & $2 \times 10^{-12}$ & $2 \times 10^{-12}$ & $5 \times 10^{-13}$ & $5 \times 10^{-13}$ & $3 \times 10^{-10}$ & $2 \times 10^{-10}$ & $8 \times 10^{-12}$ & $5 \times 10^{-12}$ & $2 \times 10^{-10}$ & $1 \times 10^{-10}$ & $6 \times 10^{-10}$ & $4 \times 10^{-10}$ \\
\hline Salmon & $\times 10^{-11}$ & $7 \times 10^{-11}$ & $2 \times 10^{-8}$ & $2 \times 10^{-8}$ & $9 \times 10^{-11}$ & $9 \times 10^{-11}$ & $6 \times 10^{-11}$ & $6 \times 10^{-11}$ & $9 \times 10^{-8}$ & $9 \times 10^{-8}$ & $4 \times 10^{-9}$ & $4 \times 10^{-9}$ & $1 \times 10^{-7}$ & $1 \times 10^{-7}$ & $2 \times 10^{-7}$ & $2 \times 10^{-7}$ \\
\hline Other & 0 & 0 & 0 & 0 & 0 & 0 & 0 & 0 & 0 & 0 & 0 & 0 & 0 & 0 & 0 & 0 \\
\hline Octopus & $5 \times 10^{-12}$ & $4 \times 10^{-12}$ & $2 \times 10^{-10}$ & $5 \times 10^{-11}$ & $1 \times 10^{-11}$ & $7 \times 10^{-12}$ & $1 \times 10^{-11}$ & $4 \times 10^{-12}$ & $4 \times 10^{-9}$ & $3 \times 10^{-9}$ & $2 \times 10^{-10}$ & $9 \times 10^{-11}$ & $5 \times 10^{-9}$ & $3 \times 10^{-9}$ & $9 \times 10^{-9}$ & $6 \times 10^{-9}$ \\
\hline Sea urchin & $9 \times 10^{-11}$ & $6 \times 10^{-11}$ & $1 \times 10^{-9}$ & $1 \times 10^{-9}$ & $5 \times 10^{-11}$ & $4 \times 10^{-11}$ & $4 \times 10^{-11}$ & $2 \times 10^{-11}$ & $9 \times 10^{-9}$ & $1 \times 10^{-8}$ & $3 \times 10^{-10}$ & $3 \times 10^{-10}$ & $8 \times 10^{-9}$ & $8 \times 10^{-9}$ & $2 \times 10^{-8}$ & $2 \times 10^{-8}$ \\
\hline Mussel & $1 \times 10^{-11}$ & $3 \times 10^{-12}$ & $2 \times 10^{-10}$ & $7 \times 10^{-11}$ & $5 \times 10^{-11}$ & $5 \times 10^{-12}$ & $2 \times 10^{-11}$ & $2 \times 10^{-12}$ & $1 \times 10^{-9}$ & $6 \times 10^{-10}$ & $4 \times 10^{-11}$ & $2 \times 10^{-11}$ & $1 \times 10^{-9}$ & $7 \times 10^{-10}$ & $2 \times 10^{-9}$ & $1 \times 10^{-9}$ \\
\hline Bidarki (c & 0 & 0 & 0 & 0 & 0 & 0 & 0 & 0 & 0 & 0 & 0 & 0 & 0 & 0 & 0 & 0 \\
\hline Shrimp & 0 & 0 & 0 & 0 & 0 & 0 & 0 & 0 & 0 & 0 & 0 & 0 & 0 & 0 & 0 & 0 \\
\hline Crab & $\times 10^{-11}$ & $4 \times 10^{-11}$ & $5 \times 10^{-9}$ & $4 \times 10^{-9}$ & $1 \times 10^{-10}$ & $6 \times 10^{-11}$ & $7 \times 10^{-11}$ & $3 \times 10^{-11}$ & $2 \times 10^{-8}$ & $1 \times 10^{-8}$ & $6 \times 10^{-10}$ & $4 \times 10^{-10}$ & $2 \times 10^{-8}$ & $1 \times 10^{-8}$ & $4 \times 10^{-8}$ & $3 \times 10^{-8}$ \\
\hline Clam & 0 & 0 & 0 & 0 & 0 & 0 & 0 & 0 & 0 & 0 & 0 & 0 & 0 & 0 & 0 & 0 \\
\hline Sea bird & $8 \times 10^{-12}$ & $8 \times 10^{-12}$ & $1 \times 10^{-8}$ & $1 \times 10^{-8}$ & $2 \times 10^{-10}$ & $7 \times 10^{-11}$ & $9 \times 10^{-11}$ & $4 \times 10^{-11}$ & $2 \times 10^{-8}$ & $1 \times 10^{-8}$ & $7 \times 10^{-10}$ & $4 \times 10^{-10}$ & $2 \times 10^{-8}$ & $1 \times 10^{-8}$ & $5 \times 10^{-8}$ & $3 \times 10^{-8}$ \\
\hline Sea bird egg & $1 \times 10^{-11}$ & 0 & $5 \times 10^{-9}$ & 0 & $1 \times 10^{-10}$ & 0 & $8 \times 10^{-11}$ & 0 & $8 \times 10^{-10}$ & 0 & $9 \times 10^{-13}$ & 0 & $2 \times 10^{-11}$ & 0 & $6 \times 10^{-9}$ & 0 \\
\hline Seaweed & 0 & 0 & 0 & 0 & 0 & 0 & 0 & 0 & 0 & 0 & 0 & 0 & 0 & 0 & 0 & 0 \\
\hline Seal & $2 \times 10^{-10}$ & $2 \times 10^{-10}$ & $2 \times 10^{-7}$ & $2 \times 10^{-7}$ & $1 \times 10^{-8}$ & $6 \times 10^{-9}$ & $7 \times 10^{-9}$ & $3 \times 10^{-9}$ & $6 \times 10^{-8}$ & $4 \times 10^{-8}$ & $2 \times 10^{-9}$ & $1 \times 10^{-9}$ & $6 \times 10^{-8}$ & $4 \times 10^{-8}$ & $3 \times 10^{-7}$ & $2 \times 10^{-7}$ \\
\hline Sea lion & $4 \times 10^{-11}$ & $4 \times 10^{-11}$ & $3 \times 10^{-8}$ & $3 \times 10^{-8}$ & $2 \times 10^{-9}$ & $1 \times 10^{-9}$ & $1 \times 10^{-9}$ & $5 \times 10^{-10}$ & $1 \times 10^{-8}$ & $6 \times 10^{-9}$ & $4 \times 10^{-10}$ & $2 \times 10^{-10}$ & $1 \times 10^{-8}$ & $7 \times 10^{-9}$ & $6 \times 10^{-8}$ & $4 \times 10^{-8}$ \\
\hline Total & $5 \times 10^{-10}$ & $5 \times 10^{-10}$ & $3 \times 10^{-7}$ & $2 \times 10^{-7}$ & $2 \times 10^{-8}$ & $7 \times 10^{-9}$ & $8 \times 10^{-9}$ & $4 \times 10^{-9}$ & $2 \times 10^{-7}$ & $2 \times 10^{-7}$ & $8 \times 10^{-9}$ & $7 \times 10^{-9}$ & $2 \times 10^{-7}$ & $2 \times 10^{-7}$ & $7 \times 10^{-7}$ & $6 \times 10^{-7}$ \\
\hline
\end{tabular}


Table 25. Potential Risks to an Aleut Consuming Seafood Harvested from Amchitka and Adak Using the St. Paul Diet Data and UCL ${ }_{95}$ Concentrations

\begin{tabular}{|c|c|c|c|c|c|c|c|c|c|c|c|c|c|c|c|c|}
\hline \multirow{2}{*}{$\begin{array}{l}\text { Seafood } \\
\text { species }\end{array}$} & \multicolumn{2}{|c|}{${ }^{241} \mathrm{Am}$} & \multicolumn{2}{|c|}{${ }^{137} \mathrm{Cs}+\mathrm{D}$} & \multicolumn{2}{|c|}{${ }^{239} \mathrm{Pu}$} & \multicolumn{2}{|c|}{${ }^{240} \mathrm{Pu}$} & \multicolumn{2}{|c|}{${ }^{234} \mathrm{U}$} & \multicolumn{2}{|c|}{${ }^{235} \mathrm{U}+\mathrm{D}$} & \multicolumn{2}{|c|}{${ }^{238} U+D$} & \multicolumn{2}{|c|}{ Total } \\
\hline & Amchitka & Adak & Amchitka & Adak & Amchitka & Adak & Amchitka & Adak & Amchitka & Adak & Amchitka & Adak & Amchitka & Adak & Amchitka & Adak \\
\hline Halibut & $2 \times 10^{-11}$ & $2 \times 10^{-11}$ & $3 \times 10^{-9}$ & $2 \times 10^{-9}$ & $1 \times 10^{-10}$ & $1 \times 10^{-10}$ & $3 \times 10^{-11}$ & $3 \times 10^{-11}$ & $1 \times 10^{-8}$ & $9 \times 10^{-9}$ & $2 \times 10^{-10}$ & $2 \times 10^{-10}$ & $4 \times 10^{-9}$ & $6 \times 10^{-9}$ & $2 \times 10^{-8}$ & $2 \times 10^{-8}$ \\
\hline Dolly Var & 0 & 0 & 0 & 0 & 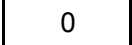 & 0 & 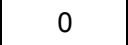 & 0 & 0 & 0 & 0 & 0 & 0 & 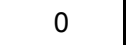 & 0 & 0 \\
\hline Cod & $5 \times 10^{-13}$ & $2 \times 10^{-13}$ & $1 \times 10^{-10}$ & $1 \times 10^{-10}$ & $2 \times 10^{-12}$ & $2 \times 10^{-12}$ & $5 \times 10^{-13}$ & $5 \times 10^{-13}$ & $3 \times 10^{-10}$ & $2 \times 10^{-10}$ & $8 \times 10^{-12}$ & $5 \times 10^{-12}$ & $2 \times 10^{-10}$ & $1 \times 10^{-10}$ & $6 \times 10^{-10}$ & $4 \times 10^{-10}$ \\
\hline Salmon & $7 \times 10^{-11}$ & $7 \times 10^{-11}$ & $2 \times 10^{-8}$ & $2 \times 10^{-8}$ & $9 \times 10^{-11}$ & $9 \times 10^{-11}$ & $6 \times 10^{-11}$ & $6 \times 10^{-11}$ & $9 \times 10^{-8}$ & $9 \times 10^{-8}$ & $4 \times 10^{-9}$ & $4 \times 10^{-9}$ & $1 \times 10^{-7}$ & $1 \times 10^{-7}$ & $2 \times 10^{-7}$ & $2 \times 10^{-7}$ \\
\hline Other fish & 0 & 0 & 0 & 0 & 0 & 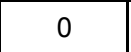 & 0 & 0 & 0 & 0 & 0 & 0 & 0 & 0 & 0 & 0 \\
\hline Octopus & $5 \times 10^{-12}$ & $4 \times 10^{-12}$ & $2 \times 10^{-10}$ & $5 \times 10^{-11}$ & $1 \times 10^{-11}$ & $7 \times 10^{-12}$ & $1 \times 10^{-11}$ & $4 \times 10^{-12}$ & $4 \times 10^{-9}$ & $3 \times 10^{-9}$ & $2 \times 10^{-10}$ & $9 \times 10^{-11}$ & $5 \times 10^{-9}$ & $3 \times 10^{-9}$ & $9 \times 10^{-9}$ & $6 \times 10^{-9}$ \\
\hline Sea urchin & $8 \times 10^{-11}$ & $6 \times 10^{-11}$ & $1 \times 10^{-9}$ & $1 \times 10^{-9}$ & $5 \times 10^{-11}$ & $4 \times 10^{-11}$ & $4 \times 10^{-11}$ & $2 \times 10^{-11}$ & $9 \times 10^{-9}$ & $1 \times 10^{-8}$ & $3 \times 10^{-10}$ & $3 \times 10^{-10}$ & $8 \times 10^{-9}$ & $8 \times 10^{-9}$ & $2 \times 10^{-8}$ & $2 \times 10^{-8}$ \\
\hline Mussel & $9 \times 10^{-12}$ & $3 \times 10^{-12}$ & $1 \times 10^{-10}$ & $7 \times 10^{-11}$ & $3 \times 10^{-11}$ & $5 \times 10^{-12}$ & $1 \times 10^{-11}$ & $2 \times 10^{-12}$ & $1 \times 10^{-9}$ & $6 \times 10^{-10}$ & $4 \times 10^{-11}$ & $2 \times 10^{-11}$ & $1 \times 10^{-9}$ & $7 \times 10^{-10}$ & $2 \times 10^{-9}$ & $1 \times 10^{-9}$ \\
\hline Bidarki (c & 0 & 0 & 0 & 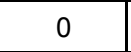 & 0 & 0 & 0 & 0 & 0 & 0 & 0 & 0 & 0 & 0 & 0 & 0 \\
\hline Shrimp & 0 & 0 & 0 & 0 & 0 & 0 & 0 & 0 & 0 & 0 & 0 & 0 & 0 & 0 & 0 & 0 \\
\hline Crab & $4 \times 10^{-11}$ & $4 \times 10^{-11}$ & $5 \times 10^{-9}$ & $4 \times 10^{-9}$ & $1 \times 10^{-10}$ & $6 \times 10^{-11}$ & $7 \times 10^{-11}$ & $3 \times 10^{-11}$ & $2 \times 10^{-8}$ & $1 \times 10^{-8}$ & $6 \times 10^{-10}$ & $4 \times 10^{-10}$ & $2 \times 10^{-8}$ & $1 \times 10^{-8}$ & $4 \times 10^{-8}$ & $3 \times 10^{-8}$ \\
\hline Clam & 0 & 0 & 0 & 0 & 0 & 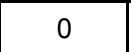 & 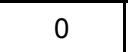 & 0 & 0 & 0 & 0 & 0 & 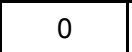 & 0 & 0 & 0 \\
\hline Sea bird & $8 \times 10^{-12}$ & $8 \times 10^{-12}$ & $1 \times 10^{-8}$ & $1 \times 10^{-8}$ & $2 \times 10^{-10}$ & $7 \times 10^{-11}$ & $9 \times 10^{-11}$ & $4 \times 10^{-11}$ & $2 \times 10^{-8}$ & $1 \times 10^{-8}$ & $7 \times 10^{-10}$ & $4 \times 10^{-10}$ & $2 \times 10^{-8}$ & $1 \times 10^{-8}$ & $5 \times 10^{-8}$ & $3 \times 10^{-8}$ \\
\hline Sea bird egg & $1 \times 10^{-11}$ & 0 & $5 \times 10^{-9}$ & 0 & $1 \times 10^{-10}$ & 0 & $8 \times 10^{-11}$ & 0 & $8 \times 10^{-10}$ & 0 & $9 \times 10^{-13}$ & 0 & $2 \times 10^{-11}$ & 0 & $6 \times 10^{-9}$ & 0 \\
\hline Seaweed & 0 & 0 & 0 & 0 & 0 & 0 & 0 & 0 & 0 & 0 & 0 & 0 & 0 & 0 & 0 & 0 \\
\hline Seal & $2 \times 10^{-10}$ & $2 \times 10^{-10}$ & $2 \times 10^{-7}$ & $2 \times 10^{-7}$ & $1 \times 10^{-8}$ & $6 \times 10^{-9}$ & $7 \times 10^{-9}$ & $3 \times 10^{-9}$ & $6 \times 10^{-8}$ & $4 \times 10^{-8}$ & $2 \times 10^{-9}$ & $1 \times 10^{-9}$ & $6 \times 10^{-8}$ & $4 \times 10^{-8}$ & $3 \times 10^{-7}$ & $2 \times 10^{-7}$ \\
\hline Sea lion & $4 \times 10^{-11}$ & $4 \times 10^{-11}$ & $3 \times 10^{-8}$ & $3 \times 10^{-8}$ & $2 \times 10^{-9}$ & $1 \times 10^{-9}$ & $1 \times 10^{-9}$ & $5 \times 10^{-10}$ & $1 \times 10^{-8}$ & $6 \times 10^{-9}$ & $4 \times 10^{-10}$ & $2 \times 10^{-10}$ & $1 \times 10^{-8}$ & $7 \times 10^{-9}$ & $6 \times 10^{-8}$ & $4 \times 10^{-8}$ \\
\hline Total & $5 \times 10^{-10}$ & $5 \times 10^{-10}$ & $3 \times 10^{-7}$ & $2 \times 10^{-7}$ & $2 \times 10^{-8}$ & $7 \times 10^{-9}$ & $8 \times 10^{-9}$ & $4 \times 10^{-9}$ & $2 \times 10^{-7}$ & $2 \times 10^{-7}$ & $8 \times 10^{-9}$ & $7 \times 10^{-9}$ & $2 \times 10^{-7}$ & $2 \times 10^{-7}$ & $7 \times 10^{-7}$ & $6 \times 10^{-7}$ \\
\hline
\end{tabular}


Table 26. Potential Risks to an Aleut Consuming Seafood Harvested from Amchitka and Adak Using the Atka Diet Data and Maximum Measured Concentrations

\begin{tabular}{|c|c|c|c|c|c|c|c|c|c|c|c|c|c|c|c|c|}
\hline \multirow{2}{*}{$\begin{array}{l}\text { Seafood } \\
\text { species }\end{array}$} & \multicolumn{2}{|c|}{${ }^{241} \mathrm{Am}$} & \multicolumn{2}{|c|}{${ }^{137} \mathrm{Cs}+\mathrm{D}$} & \multicolumn{2}{|c|}{${ }^{239} \mathrm{Pu}$} & \multicolumn{2}{|c|}{${ }^{240} \mathrm{Pu}$} & \multicolumn{2}{|c|}{${ }^{234} \mathrm{U}$} & \multicolumn{2}{|c|}{${ }^{235} \mathrm{U}+\mathrm{D}$} & \multicolumn{2}{|c|}{${ }^{238} \mathrm{U}+\mathrm{D}$} & \multicolumn{2}{|c|}{ Total } \\
\hline & Amchitka & Adak & Amchitka & Adak & Amchitka & Adak & Amchitka & Adak & Amchitka & Adak & Amchitka & Adak & Amchitka & Adak & Amchitka & Adak \\
\hline Halibut & $\times 10^{-11}$ & $6 \times 10^{-11}$ & $1 \times 10^{-8}$ & $8 \times 10^{-9}$ & $4 \times 10^{-10}$ & $4 \times 10^{-10}$ & $1 \times 10^{-10}$ & $1 \times 10^{-10}$ & $4 \times 10^{-8}$ & $3 \times 10^{-8}$ & $6 \times 10^{-8}$ & $8 \times 10^{-10}$ & $2 \times 10^{-8}$ & $2 \times 10^{-8}$ & $7 \times 10^{-8}$ & $6 \times 10^{-8}$ \\
\hline Dolly V & $\times 10^{-12}$ & $4 \times 10^{-12}$ & $4 \times 10^{-8}$ & $2 \times 10^{-7}$ & $2 \times 10^{-11}$ & $2 \times 10^{-11}$ & $2 \times 10^{-11}$ & $2 \times 10^{-11}$ & $1 \times 10^{-9}$ & $9 \times 10^{-11}$ & $1 \times 10^{-11}$ & $2 \times 10^{-11}$ & $3 \times 10^{-10}$ & $5 \times 10^{-10}$ & $4 \times 10^{-8}$ & $2 \times 10^{-7}$ \\
\hline Cod & $\times 10^{-11}$ & $3 \times 10^{-11}$ & $2 \times 10^{-8}$ & $1 \times 10^{-8}$ & $2 \times 10^{-10}$ & $2 \times 10^{-10}$ & $6 \times 10^{-11}$ & $6 \times 10^{-11}$ & $3 \times 10^{-8}$ & $2 \times 10^{-8}$ & $1 \times 10^{-9}$ & $7 \times 10^{-10}$ & $3 \times 10^{-8}$ & $2 \times 10^{-8}$ & $8 \times 10^{-8}$ & $5 \times 10^{-8}$ \\
\hline Salmon & $\times 10^{-10}$ & $8 \times 10^{-10}$ & $2 \times 10^{-7}$ & $2 \times 10^{-7}$ & $1 \times 10^{-9}$ & $1 \times 10^{-9}$ & $6 \times 10^{-10}$ & $6 \times 10^{-10}$ & $1 \times 10^{-6}$ & $1 \times 10^{-6}$ & $5 \times 10^{-8}$ & $5 \times 10^{-8}$ & $1 \times 10^{-6}$ & $1 \times 10^{-6}$ & $2 \times 10^{-6}$ & $\times 10^{-6}$ \\
\hline Other & $\times 10^{-9}$ & $1 \times 10^{-9}$ & $6 \times 10^{-8}$ & $7 \times 10^{-8}$ & $3 \times 10^{-9}$ & $2 \times 10^{-9}$ & $1 \times 10^{-9}$ & 年 & $4 \times 10^{-7}$ & $2 \times 10^{-7}$ & $1 \times 10^{-8}$ & $9 \times 10^{-9}$ & $3 \times 10^{-7}$ & $3 \times 10^{-7}$ & $8 \times 10^{-7}$ & $6 \times 10^{-7}$ \\
\hline Octopus & $\times 10^{-10}$ & $8 \times 10^{-11}$ & $3 \times 10^{-9}$ & $1 \times 10^{-9}$ & $2 \times 10^{-10}$ & $1 \times 10^{-10}$ & $2 \times 10^{-10}$ & $7 \times 10^{-11}$ & $9 \times 10^{-8}$ & $5 \times 10^{-8}$ & $3 \times 10^{-9}$ & $2 \times 10^{-9}$ & $9 \times 10^{-8}$ & $5 \times 10^{-8}$ & $2 \times 10^{-7}$ & $1 \times 10^{-7}$ \\
\hline Sea urchin & $\times 10^{-10}$ & $3 \times 10^{-10}$ & $7 \times 10^{-9}$ & $6 \times 10^{-9}$ & $3 \times 10^{-10}$ & $2 \times 10^{-10}$ & $2 \times 10^{-10}$ & $1 \times 10^{-10}$ & $5 \times 10^{-8}$ & $5 \times 10^{-8}$ & $1 \times 10^{-9}$ & $1 \times 10^{-9}$ & $4 \times 10^{-8}$ & $4 \times 10^{-8}$ & $1 \times 10^{-7}$ & $1 \times 10^{-7}$ \\
\hline Mussel & 0 & 0 & 0 & 0 & 0 & 0 & 0 & 0 & 0 & 0 & 0 & 0 & 0 & 0 & 0 & 0 \\
\hline Bidarki (chiton) & $\times 10^{-10}$ & $2 \times 10^{-10}$ & $2 \times 10^{-9}$ & $9 \times 10^{-10}$ & $2 \times 10^{-10}$ & $2 \times 10^{-10}$ & $1 \times 10^{-10}$ & $8 \times 10^{-11}$ & $5 \times 10^{-8}$ & $5 \times 10^{-8}$ & $2 \times 10^{-9}$ & $2 \times 10^{-9}$ & $5 \times 10^{-8}$ & $5 \times 10^{-8}$ & $1 \times 10^{-7}$ & $1 \times 10^{-7}$ \\
\hline Shrimp & 0 & 0 & 0 & 0 & 0 & 0 & 0 & 0 & 0 & 0 & 0 & 0 & 0 & 0 & 0 & 0 \\
\hline Crab & 0 & 0 & 0 & 0 & 0 & 0 & 0 & 0 & 0 & 0 & 0 & 0 & 0 & 0 & 0 & 0 \\
\hline Clam & 0 & 0 & 0 & 0 & 0 & 0 & $\sigma$ & 0 & 0 & 0 & 0 & 0 & 0 & 0 & 0 & 0 \\
\hline Sea bird & $1 \times 10^{-10}$ & $1 \times 10^{-10}$ & $2 \times 10^{-7}$ & $2 \times 10^{-7}$ & $3 \times 10^{-9}$ & $1 \times 10^{-9}$ & $2 \times 10^{-9}$ & $7 \times 10^{-10}$ & $3 \times 10^{-7}$ & $2 \times 10^{-7}$ & $1 \times 10^{-8}$ & $7 \times 10^{-9}$ & $3 \times 10^{-7}$ & $2 \times 10^{-7}$ & $9 \times 10^{-7}$ & $6 \times 10^{-7}$ \\
\hline Sea bird egg & $7 \times 10^{-12}$ & 0 & $3 \times 10^{-9}$ & 0 & $8 \times 10^{-11}$ & 0 & $5 \times 10^{-11}$ & 0 & $5 \times 10^{-10}$ & 0 & $5 \times 10^{-13}$ & 0 & $1 \times 10^{-11}$ & 0 & $4 \times 10^{-9}$ & 0 \\
\hline Seaweed & $5 \times 10^{-12}$ & $4 \times 10^{-12}$ & $2 \times 10^{-10}$ & $3 \times 10^{-10}$ & $3 \times 10^{-11}$ & $2 \times 10^{-11}$ & $2 \times 10^{-11}$ & $2 \times 10^{-11}$ & $2 \times 10^{-8}$ & $2 \times 10^{-8}$ & $5 \times 10^{-10}$ & $5 \times 10^{-10}$ & $2 \times 10^{-8}$ & $2 \times 10^{-8}$ & $3 \times 10^{-8}$ & $3 \times 10^{-8}$ \\
\hline Seal & $4 \times 10^{-10}$ & $4 \times 10^{-10}$ & $3 \times 10^{-7}$ & $3 \times 10^{-7}$ & $2 \times 10^{-8}$ & $9 \times 10^{-9}$ & $1 \times 10^{-8}$ & $5 \times 10^{-9}$ & $1 \times 10^{-7}$ & $6 \times 10^{-8}$ & $3 \times 10^{-9}$ & $2 \times 10^{-9}$ & $1 \times 10^{-7}$ & $6 \times 10^{-8}$ & $5 \times 10^{-7}$ & $4 \times 10^{-7}$ \\
\hline Sea lion & $8 \times 10^{-10}$ & $8 \times 10^{-10}$ & $7 \times 10^{-7}$ & $5 \times 10^{-7}$ & $5 \times 10^{-8}$ & $2 \times 10^{-8}$ & $2 \times 10^{-8}$ & $1 \times 10^{-8}$ & $2 \times 10^{-7}$ & $1 \times 10^{-7}$ & $7 \times 10^{-9}$ & $5 \times 10^{-9}$ & $2 \times 10^{-7}$ & $1 \times 10^{-7}$ & $1 \times 10^{-6}$ & $8 \times 10^{-7}$ \\
\hline Total & $5 \times 10^{-9}$ & $4 \times 10^{-9}$ & $2 \times 10^{-6}$ & $1 \times 10^{-6}$ & $8 \times 10^{-8}$ & $4 \times 10^{-8}$ & $4 \times 10^{-8}$ & $2 \times 10^{-8}$ & $2 \times 10^{-6}$ & $2 \times 10^{-6}$ & $9 \times 10^{-8}$ & $8 \times 10^{-8}$ & $2 \times 10^{-6}$ & $2 \times 10^{-6}$ & $6 \times 10^{-6}$ & $5 \times 10^{-6}$ \\
\hline
\end{tabular}


Table 27. Potential Risks to an Aleut Consuming Seafood Harvested from Amchitka and Adak Using the Atka Diet Data and UCL ${ }_{95}$ Concentrations

\begin{tabular}{|c|c|c|c|c|c|c|c|c|c|c|c|c|c|c|c|c|}
\hline \multirow{2}{*}{$\begin{array}{l}\text { Seafood } \\
\text { species }\end{array}$} & \multicolumn{2}{|c|}{${ }^{241} \mathrm{Am}$} & \multicolumn{2}{|c|}{${ }^{137} \mathrm{Cs}+\mathrm{D}$} & \multicolumn{2}{|c|}{${ }^{239} \mathrm{Pu}$} & \multicolumn{2}{|c|}{${ }^{240} \mathrm{Pu}$} & \multicolumn{2}{|c|}{${ }^{234} \mathrm{U}$} & \multicolumn{2}{|c|}{${ }^{235} U+D$} & \multicolumn{2}{|c|}{${ }^{238} U+D$} & \multicolumn{2}{|c|}{ Total } \\
\hline & Amchitka & Adak & Amchitka & Adak & Amchitka & Adak & Amchitka & Adak & Amchitka & Adak & Amchitka & Adak & Amchitka & Adak & Amchitka & Adak \\
\hline Halibut & $6 \times 10^{-11}$ & $6 \times 10^{-11}$ & $1 \times 10^{-8}$ & $8 \times 10^{-9}$ & $4 \times 10^{-10}$ & $4 \times 10^{-10}$ & $1 \times 10^{-10}$ & $1 \times 10^{-10}$ & $4 \times 10^{-8}$ & $3 \times 10^{-8}$ & $6 \times 10^{-8}$ & $8 \times 10^{-10}$ & $2 \times 10^{-8}$ & $2 \times 10^{-8}$ & $7 \times 10^{-8}$ & $6 \times 10^{-8}$ \\
\hline Dolly Varden & $3 \times 10^{-12}$ & $4 \times 10^{-12}$ & $4 \times 10^{-8}$ & $2 \times 10^{-7}$ & $2 \times 10^{-11}$ & $2 \times 10^{-11}$ & $2 \times 10^{-11}$ & $2 \times 10^{-11}$ & $1 \times 10^{-9}$ & $9 \times 10^{-11}$ & $1 \times 10^{-11}$ & $2 \times 10^{-11}$ & $3 \times 10^{-10}$ & $5 \times 10^{-10}$ & $4 \times 10^{-8}$ & $2 \times 10^{-7}$ \\
\hline Cod & $6 \times 10^{-11}$ & $3 \times 10^{-11}$ & $2 \times 10^{-8}$ & $1 \times 10^{-8}$ & $2 \times 10^{-10}$ & $2 \times 10^{-10}$ & $6 \times 10^{-11}$ & $6 \times 10^{-11}$ & $3 \times 10^{-8}$ & $2 \times 10^{-8}$ & $1 \times 10^{-9}$ & $7 \times 10^{-10}$ & $3 \times 10^{-8}$ & $2 \times 10^{-8}$ & $8 \times 10^{-8}$ & $5 \times 10^{-8}$ \\
\hline Salmon & $8 \times 10^{-10}$ & $8 \times 10^{-10}$ & $2 \times 10^{-7}$ & $2 \times 10^{-7}$ & $1 \times 10^{-9}$ & $1 \times 10^{-9}$ & $6 \times 10^{-10}$ & $6 \times 10^{-10}$ & $1 \times 10^{-6}$ & $1 \times 10^{-6}$ & $5 \times 10^{-8}$ & $5 \times 10^{-8}$ & $1 \times 10^{-6}$ & $1 \times 10^{-6}$ & $2 \times 10^{-6}$ & $2 \times 10^{-6}$ \\
\hline Other fish & $9 \times 10^{-10}$ & $7 \times 10^{-10}$ & $6 \times 10^{-8}$ & $4 \times 10^{-8}$ & $2 \times 10^{-9}$ & $2 \times 10^{-9}$ & $1 \times 10^{-9}$ & $1 \times 10^{-9}$ & $2 \times 10^{-7}$ & $2 \times 10^{-7}$ & $8 \times 10^{-9}$ & $5 \times 10^{-9}$ & $2 \times 10^{-7}$ & $2 \times 10^{-7}$ & $6 \times 10^{-7}$ & $4 \times 10^{-7}$ \\
\hline Octopus & $1 \times 10^{-10}$ & $8 \times 10^{-11}$ & $3 \times 10^{-9}$ & $1 \times 10^{-9}$ & $2 \times 10^{-10}$ & $1 \times 10^{-10}$ & $2 \times 10^{-10}$ & $7 \times 10^{-11}$ & $9 \times 10^{-8}$ & $5 \times 10^{-8}$ & $3 \times 10^{-9}$ & $2 \times 10^{-9}$ & $9 \times 10^{-8}$ & $5 \times 10^{-8}$ & $2 \times 10^{-7}$ & $1 \times 10^{-7}$ \\
\hline Sea urchin & $4 \times 10^{-10}$ & $3 \times 10^{-10}$ & $6 \times 10^{-9}$ & $6 \times 10^{-9}$ & $2 \times 10^{-10}$ & $2 \times 10^{-10}$ & $2 \times 10^{-10}$ & $1 \times 10^{-10}$ & $5 \times 10^{-8}$ & $5 \times 10^{-8}$ & $1 \times 10^{-9}$ & $1 \times 10^{-9}$ & $4 \times 10^{-8}$ & $4 \times 10^{-8}$ & $1 \times 10^{-7}$ & $1 \times 10^{-7}$ \\
\hline Mussel & 0 & 0 & 0 & 0 & 0 & 0 & 0 & 0 & 0 & 0 & 0 & 0 & 0 & 0 & 0 & 0 \\
\hline Bidarki (chiton) & $3 \times 10^{-10}$ & $2 \times 10^{-10}$ & $2 \times 10^{-9}$ & $9 \times 10^{-10}$ & $2 \times 10^{-10}$ & $2 \times 10^{-10}$ & $1 \times 10^{-10}$ & $8 \times 10^{-11}$ & $5 \times 10^{-8}$ & $5 \times 10^{-8}$ & $2 \times 10^{-9}$ & $2 \times 10^{-9}$ & $5 \times 10^{-8}$ & $5 \times 10^{-8}$ & $1 \times 10^{-7}$ & $1 \times 10^{-7}$ \\
\hline Shrimp & 0 & 0 & 0 & 0 & 0 & 0 & 0 & 0 & 0 & 0 & 0 & 0 & 0 & 0 & 0 & 0 \\
\hline Crab & 0 & 0 & 0 & 0 & 0 & 0 & 0 & 0 & 0 & 0 & 0 & 0 & 0 & 0 & 0 & 0 \\
\hline Clam & 0 & 0 & 0 & 0 & 0 & 0 & 0 & 0 & 0 & 0 & 0 & 0 & 0 & 0 & 0 & 0 \\
\hline Sea bird & $1 \times 10^{-10}$ & $1 \times 10^{-10}$ & $2 \times 10^{-7}$ & $2 \times 10^{-7}$ & $3 \times 10^{-9}$ & $1 \times 10^{-9}$ & $2 \times 10^{-9}$ & $7 \times 10^{-10}$ & $3 \times 10^{-7}$ & $2 \times 10^{-7}$ & $1 \times 10^{-8}$ & $7 \times 10^{-9}$ & $3 \times 10^{-7}$ & $2 \times 10^{-7}$ & $9 \times 10^{-7}$ & $6 \times 10^{-7}$ \\
\hline Sea bird egg & $7 \times 10^{-12}$ & 0 & $3 \times 10^{-9}$ & 0 & $8 \times 10^{-11}$ & 0 & $5 \times 10^{-11}$ & 0 & $5 \times 10^{-10}$ & 0 & $5 \times 10^{-13}$ & 0 & $1 \times 10^{-11}$ & 0 & $4 \times 10^{-9}$ & 0 \\
\hline Seaweed & $3 \times 10^{-12}$ & $2 \times 10^{-12}$ & $2 \times 10^{-10}$ & $2 \times 10^{-10}$ & $3 \times 10^{-11}$ & $1 \times 10^{-11}$ & $1 \times 10^{-11}$ & $1 \times 10^{-11}$ & $1 \times 10^{-8}$ & $1 \times 10^{-8}$ & $4 \times 10^{-10}$ & $4 \times 10^{-10}$ & $1 \times 10^{-8}$ & $1 \times 10^{-8}$ & $2 \times 10^{-8}$ & $2 \times 10^{-8}$ \\
\hline Seal & $4 \times 10^{-10}$ & $4 \times 10^{-10}$ & $3 \times 10^{-7}$ & $3 \times 10^{-7}$ & $2 \times 10^{-8}$ & $9 \times 10^{-9}$ & $1 \times 10^{-8}$ & $5 \times 10^{-9}$ & $1 \times 10^{-7}$ & $6 \times 10^{-8}$ & $3 \times 10^{-9}$ & $2 \times 10^{-9}$ & $1 \times 10^{-7}$ & $6 \times 10^{-8}$ & $5 \times 10^{-7}$ & $4 \times 10^{-7}$ \\
\hline Sea lion & $8 \times 10^{-10}$ & $8 \times 10^{-10}$ & $7 \times 10^{-7}$ & $5 \times 10^{-7}$ & $5 \times 10^{-8}$ & $2 \times 10^{-8}$ & $2 \times 10^{-8}$ & $1 \times 10^{-8}$ & $2 \times 10^{-7}$ & $1 \times 10^{-7}$ & $7 \times 10^{-9}$ & $5 \times 10^{-9}$ & $2 \times 10^{-7}$ & $1 \times 10^{-7}$ & $1 \times 10^{-6}$ & $8 \times 10^{-7}$ \\
\hline Total & $4 \times 10^{-9}$ & $3 \times 10^{-9}$ & $2 \times 10^{-6}$ & $1 \times 10^{-6}$ & $8 \times 10^{-8}$ & $3 \times 10^{-8}$ & $4 \times 10^{-8}$ & $2 \times 10^{-8}$ & $2 \times 10^{-6}$ & $2 \times 10^{-6}$ & $9 \times 10^{-8}$ & $7 \times 10^{-8}$ & $2 \times 10^{-6}$ & $2 \times 10^{-6}$ & $6 \times 10^{-6}$ & $5 \times 10^{-6}$ \\
\hline
\end{tabular}


Table 28. Potential Risks to an Aleut Consuming Seafood Harvested from Amchitka and Adak Using the Nikolski Diet Data and Maximum Measured Concentrations

\begin{tabular}{|c|c|c|c|c|c|c|c|c|c|c|c|c|c|c|c|c|}
\hline \multirow{2}{*}{$\begin{array}{l}\text { Seafood } \\
\text { species }\end{array}$} & \multicolumn{2}{|c|}{${ }^{241} \mathrm{Am}$} & \multicolumn{2}{|c|}{${ }^{137}$ Cs+D } & \multicolumn{2}{|c|}{${ }^{239} \mathrm{Pu}$} & \multicolumn{2}{|c|}{${ }^{240} \mathrm{Pu}$} & \multicolumn{2}{|c|}{${ }^{234} \mathrm{U}$} & \multicolumn{2}{|c|}{${ }^{235} \mathrm{U}+\mathrm{D}$} & \multicolumn{2}{|c|}{${ }^{238} U+D$} & \multicolumn{2}{|c|}{ Total } \\
\hline & Amchitka & Adak & Amchitka & Adak & Amchitka & Adak & Amchitka & Adak & Amchitka & Adak & Amchitka & Adak & Amchitka & Adak & Amchitka & Adak \\
\hline Halibut & $5 \times 10^{-10}$ & $5 \times 10^{-10}$ & $1 \times 10^{-7}$ & $7 \times 10^{-8}$ & $3 \times 10^{-9}$ & $3 \times 10^{-9}$ & $9 \times 10^{-10}$ & $9 \times 10^{-10}$ & $3 \times 10^{-7}$ & $3 \times 10^{-7}$ & $5 \times 10^{-9}$ & $6 \times 10^{-9}$ & $1 \times 10^{-7}$ & $2 \times 10^{-7}$ & $6 \times 10^{-7}$ & $5 \times 10^{-7}$ \\
\hline Dolly Varden & $6 \times 10^{-11}$ & $8 \times 10^{-11}$ & $8 \times 10^{-7}$ & $4 \times 10^{-6}$ & $4 \times 10^{-10}$ & $4 \times 10^{-10}$ & $4 \times 10^{-10}$ & $4 \times 10^{-10}$ & $3 \times 10^{-8}$ & $2 \times 10^{-8}$ & $2 \times 10^{-10}$ & $3 \times 10^{-10}$ & $6 \times 10^{-9}$ & $9 \times 10^{-9}$ & $8 \times 10^{-7}$ & $4 \times 10^{-6}$ \\
\hline Cod & $7 \times 10^{-11}$ & $3 \times 10^{-11}$ & $2 \times 10^{-8}$ & $1 \times 10^{-8}$ & $2 \times 10^{-10}$ & $2 \times 10^{-10}$ & $6 \times 10^{-11}$ & $6 \times 10^{-11}$ & $4 \times 10^{-8}$ & $2 \times 10^{-8}$ & $1 \times 10^{-9}$ & $7 \times 10^{-10}$ & $3 \times 10^{-8}$ & $2 \times 10^{-8}$ & $9 \times 10^{-8}$ & $6 \times 10^{-8}$ \\
\hline Salmon & $5 \times 10^{-9}$ & $5 \times 10^{-9}$ & $1 \times 10^{-6}$ & $1 \times 10^{-6}$ & $6 \times 10^{-9}$ & $6 \times 10^{-9}$ & $4 \times 10^{-9}$ & $4 \times 10^{-9}$ & $6 \times 10^{-6}$ & $6 \times 10^{-6}$ & $3 \times 10^{-7}$ & $3 \times 10^{-7}$ & $7 \times 10^{-6}$ & $7 \times 10^{-6}$ & $1 \times 10^{-5}$ & $1 \times 10^{-5}$ \\
\hline Other fish & $1 \times 10^{-10}$ & $8 \times 10^{-11}$ & $4 \times 10^{-9}$ & $5 \times 10^{-9}$ & $2 \times 10^{-10}$ & $2 \times 10^{-10}$ & $1 \times 10^{-10}$ & $1 \times 10^{-10}$ & $3 \times 10^{-8}$ & $2 \times 10^{-8}$ & $9 \times 10^{-10}$ & $7 \times 10^{-10}$ & $3 \times 10^{-8}$ & $2 \times 10^{-8}$ & $6 \times 10^{-8}$ & $5 \times 10^{-8}$ \\
\hline Octopus & $5 \times 10^{-11}$ & $4 \times 10^{-11}$ & $2 \times 10^{-9}$ & $5 \times 10^{-10}$ & $1 \times 10^{-10}$ & $7 \times 10^{-11}$ & $1 \times 10^{-10}$ & $4 \times 10^{-11}$ & $4 \times 10^{-8}$ & $3 \times 10^{-8}$ & $2 \times 10^{-9}$ & $9 \times 10^{-10}$ & $4 \times 10^{-8}$ & $3 \times 10^{-8}$ & $9 \times 10^{-8}$ & $5 \times 10^{-8}$ \\
\hline Sea urchin & $3 \times 10^{-10}$ & $2 \times 10^{-10}$ & $4 \times 10^{-9}$ & $4 \times 10^{-9}$ & $2 \times 10^{-10}$ & $1 \times 10^{-10}$ & $1 \times 10^{-10}$ & $7 \times 10^{-11}$ & $3 \times 10^{-8}$ & $3 \times 10^{-8}$ & $8 \times 10^{-10}$ & $8 \times 10^{-10}$ & $2 \times 10^{-8}$ & $2 \times 10^{-8}$ & $5 \times 10^{-8}$ & $6 \times 10^{-8}$ \\
\hline Mussel & $6 \times 10^{-9}$ & $1 \times 10^{-9}$ & $1 \times 10^{-7}$ & $3 \times 10^{-8}$ & $2 \times 10^{-8}$ & $2 \times 10^{-9}$ & $1 \times 10^{-8}$ & $1 \times 10^{-9}$ & $5 \times 10^{-7}$ & $3 \times 10^{-7}$ & $2 \times 10^{-8}$ & $1 \times 10^{-8}$ & $5 \times 10^{-7}$ & $3 \times 10^{-7}$ & $1 \times 10^{-6}$ & $6 \times 10^{-7}$ \\
\hline Bidarki (chiton) & $1 \times 10^{-9}$ & $6 \times 10^{-10}$ & $9 \times 10^{-9}$ & $3 \times 10^{-9}$ & $7 \times 10^{-10}$ & $6 \times 10^{-10}$ & $4 \times 10^{-10}$ & $3 \times 10^{-10}$ & $2 \times 10^{-7}$ & $2 \times 10^{-7}$ & $7 \times 10^{-9}$ & $6 \times 10^{-9}$ & $2 \times 10^{-7}$ & $2 \times 10^{-7}$ & $4 \times 10^{-7}$ & $4 \times 10^{-7}$ \\
\hline Shrimp & $5 \times 10^{-12}$ & $5 \times 10^{-12}$ & $6 \times 10^{-10}$ & $5 \times 10^{-10}$ & $2 \times 10^{-11}$ & $7 \times 10^{-12}$ & $8 \times 10^{-12}$ & $4 \times 10^{-12}$ & $2 \times 10^{-9}$ & $1 \times 10^{-9}$ & $7 \times 10^{-11}$ & $4 \times 10^{-11}$ & $2 \times 10^{-9}$ & $1 \times 10^{-9}$ & $5 \times 10^{-9}$ & $3 \times 10^{-9}$ \\
\hline Crab & $2 \times 10^{-11}$ & $2 \times 10^{-11}$ & $3 \times 10^{-9}$ & $2 \times 10^{-9}$ & $7 \times 10^{-11}$ & $3 \times 10^{-11}$ & $4 \times 10^{-11}$ & $2 \times 10^{-11}$ & $9 \times 10^{-9}$ & $5 \times 10^{-9}$ & $3 \times 10^{-10}$ & $2 \times 10^{-10}$ & $9 \times 10^{-9}$ & $6 \times 10^{-9}$ & $2 \times 10^{-8}$ & $1 \times 10^{-8}$ \\
\hline Clam & 0 & 0 & 0 & 0 & 0 & 0 & 0 & 0 & 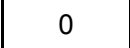 & 0 & 0 & 0 & 0 & 0 & 0 & 0 \\
\hline Sea bird & $8 \times 10^{-10}$ & $8 \times 10^{-10}$ & $1 \times 10^{-6}$ & $1 \times 10^{-6}$ & $2 \times 10^{-8}$ & $8 \times 10^{-9}$ & $1 \times 10^{-8}$ & $4 \times 10^{-9}$ & $2 \times 10^{-6}$ & $1 \times 10^{-6}$ & $7 \times 10^{-8}$ & $4 \times 10^{-8}$ & $2 \times 10^{-6}$ & $1 \times 10^{-6}$ & $5 \times 10^{-6}$ & $4 \times 10^{-6}$ \\
\hline Sea bird egg & $3 \times 10^{-11}$ & 0 & $1 \times 10^{-8}$ & 0 & $4 \times 10^{-10}$ & 0 & $2 \times 10^{-10}$ & 0 & $2 \times 10^{-9}$ & 0 & $3 \times 10^{-12}$ & 0 & $6 \times 10^{-11}$ & 0 & $2 \times 10^{-8}$ & 0 \\
\hline Seaweed & 0 & 0 & 0 & 0 & 0 & 0 & 0 & 0 & 0 & 0 & $0 \mathrm{E}+00$ & 0 & 0 & 0 & 0 & 0 \\
\hline Seal & $2 \times 10^{-10}$ & $2 \times 10^{-10}$ & $2 \times 10^{-7}$ & $2 \times 10^{-7}$ & $1 \times 10^{-8}$ & $6 \times 10^{-9}$ & $7 \times 10^{-9}$ & $3 \times 10^{-9}$ & $6 \times 10^{-8}$ & $4 \times 10^{-8}$ & $2 \times 10^{-9}$ & $1 \times 10^{-9}$ & $7 \times 10^{-8}$ & $4 \times 10^{-8}$ & $4 \times 10^{-7}$ & $3 \times 10^{-7}$ \\
\hline Sea lion & $1 \times 10^{-10}$ & $1 \times 10^{-10}$ & $1 \times 10^{-7}$ & $1 \times 10^{-7}$ & $9 \times 10^{-9}$ & $4 \times 10^{-9}$ & $4 \times 10^{-9}$ & $2 \times 10^{-9}$ & $4 \times 10^{-8}$ & $2 \times 10^{-8}$ & $1 \times 10^{-9}$ & $8 \times 10^{-10}$ & $4 \times 10^{-8}$ & $2 \times 10^{-8}$ & $2 \times 10^{-7}$ & $2 \times 10^{-7}$ \\
\hline Total & $1 \times 10^{-8}$ & $9 \times 10^{-9}$ & $4 \times 10^{-6}$ & $6 \times 10^{-6}$ & $7 \times 10^{-8}$ & $3 \times 10^{-8}$ & $4 \times 10^{-8}$ & $2 \times 10^{-8}$ & $9 \times 10^{-6}$ & $8 \times 10^{-6}$ & $4 \times 10^{-7}$ & $3 \times 10^{-7}$ & $1 \times 10^{-5}$ & $9 \times 10^{-6}$ & $2 \times 10^{-5}$ & $2 \times 10^{-5}$ \\
\hline
\end{tabular}


Table 29. Potential Risks to an Aleut Consuming Seafood Harvested from Amchitka and Adak Using the Nikolski Diet Data and UCL ${ }_{95}$ Concentrations

\begin{tabular}{|c|c|c|c|c|c|c|c|c|c|c|c|c|c|c|c|c|}
\hline \multirow{2}{*}{$\begin{array}{l}\text { Seafood } \\
\text { species }\end{array}$} & \multicolumn{2}{|c|}{${ }^{241} \mathrm{Am}$} & \multicolumn{2}{|c|}{${ }^{137} \mathrm{Cs}+\mathrm{D}$} & \multicolumn{2}{|c|}{${ }^{239} \mathrm{Pu}$} & \multicolumn{2}{|c|}{${ }^{240} \mathrm{Pu}$} & \multicolumn{2}{|c|}{${ }^{234} \mathrm{U}$} & \multicolumn{2}{|c|}{${ }^{235} U+D$} & \multicolumn{2}{|c|}{${ }^{238} U+D$} & \multicolumn{2}{|c|}{ Total } \\
\hline & Amchitka & Adak & Amchitka & Adak & Amchitka & Adak & Amchitka & Adak & Amchitka & Adak & Amchitka & Adak & Amchitka & Adak & Amchitka & Adak \\
\hline Halibut & $5 \times 10^{-10}$ & $5 \times 10^{-10}$ & $1 \times 10^{-7}$ & $7 \times 10^{-8}$ & $3 \times 10^{-9}$ & $3 \times 10^{-9}$ & $9 \times 10^{-10}$ & $9 \times 10^{-10}$ & $3 \times 10^{-7}$ & $3 \times 10^{-7}$ & $5 \times 10^{-9}$ & $6 \times 10^{-9}$ & $1 \times 10^{-7}$ & $2 \times 10^{-7}$ & $6 \times 10^{-7}$ & $5 \times 10^{-7}$ \\
\hline Dolly Varden & $6 \times 10^{-11}$ & $8 \times 10^{-11}$ & $8 \times 10^{-7}$ & $4 \times 10^{-6}$ & $4 \times 10^{-10}$ & $4 \times 10^{-10}$ & $4 \times 10^{-10}$ & $4 \times 10^{-10}$ & $3 \times 10^{-8}$ & $2 \times 10^{-8}$ & $2 \times 10^{-10}$ & $3 \times 10^{-10}$ & $6 \times 10^{-9}$ & $9 \times 10^{-9}$ & $8 \times 10^{-7}$ & $4 \times 10^{-6}$ \\
\hline Cod & $7 \times 10^{-11}$ & $3 \times 10^{-11}$ & $2 \times 10^{-8}$ & $1 \times 10^{-8}$ & $2 \times 10^{-10}$ & $2 \times 10^{-10}$ & $6 \times 10^{-11}$ & $6 \times 10^{-11}$ & $4 \times 10^{-8}$ & $2 \times 10^{-8}$ & $1 \times 10^{-9}$ & $7 \times 10^{-10}$ & $3 \times 10^{-8}$ & $2 \times 10^{-8}$ & $9 \times 10^{-8}$ & $6 \times 10^{-8}$ \\
\hline Salmon & $5 \times 10^{-9}$ & $5 \times 10^{-9}$ & $1 \times 10^{-6}$ & $1 \times 10^{-6}$ & $6 \times 10^{-9}$ & $6 \times 10^{-9}$ & $4 \times 10^{-9}$ & $4 \times 10^{-9}$ & $6 \times 10^{-6}$ & $6 \times 10^{-6}$ & $3 \times 10^{-7}$ & $3 \times 10^{-7}$ & $7 \times 10^{-6}$ & $7 \times 10^{-6}$ & $1 \times 10^{-5}$ & $1 \times 10^{-5}$ \\
\hline Other fish & $7 \times 10^{-11}$ & $5 \times 10^{-11}$ & $4 \times 10^{-9}$ & $3 \times 10^{-9}$ & $2 \times 10^{-10}$ & $2 \times 10^{-10}$ & $1 \times 10^{-10}$ & $9 \times 10^{-11}$ & $2 \times 10^{-8}$ & $1 \times 10^{-8}$ & $6 \times 10^{-10}$ & $4 \times 10^{-10}$ & $2 \times 10^{-8}$ & $1 \times 10^{-8}$ & $4 \times 10^{-8}$ & $3 \times 10^{-8}$ \\
\hline Octopus & $5 \times 10^{-11}$ & $4 \times 10^{-11}$ & $2 \times 10^{-9}$ & $5 \times 10^{-10}$ & $1 \times 10^{-10}$ & $7 \times 10^{-11}$ & $1 \times 10^{-10}$ & $4 \times 10^{-11}$ & $4 \times 10^{-8}$ & $3 \times 10^{-8}$ & $2 \times 10^{-9}$ & $9 \times 10^{-10}$ & $4 \times 10^{-8}$ & $\times 10^{-8}$ & $9 \times 10^{-8}$ & $5 \times 10^{-8}$ \\
\hline Sea urchin & $2 \times 10^{-10}$ & $2 \times 10^{-10}$ & $3 \times 10^{-9}$ & $3 \times 10^{-9}$ & $1 \times 10^{-10}$ & $1 \times 10^{-10}$ & $1 \times 10^{-10}$ & $7 \times 10^{-11}$ & $3 \times 10^{-8}$ & $3 \times 10^{-8}$ & $8 \times 10^{-10}$ & $8 \times 10^{-10}$ & $2 \times 10^{-8}$ & $2 \times 10^{-8}$ & $5 \times 10^{-8}$ & $6 \times 10^{-8}$ \\
\hline Mussel & $4 \times 10^{-9}$ & $1 \times 10^{-9}$ & $6 \times 10^{-8}$ & $3 \times 10^{-8}$ & $1 \times 10^{-8}$ & $2 \times 10^{-9}$ & $6 \times 10^{-9}$ & $1 \times 10^{-9}$ & $4 \times 10^{-7}$ & $3 \times 10^{-7}$ & $2 \times 10^{-8}$ & $1 \times 10^{-8}$ & $5 \times 10^{-7}$ & $3 \times 10^{-7}$ & $1 \times 10^{-6}$ & $6 \times 10^{-7}$ \\
\hline Bidarki (chiton) & $1 \times 10^{-9}$ & $6 \times 10^{-10}$ & $9 \times 10^{-9}$ & $3 \times 10^{-9}$ & $7 \times 10^{-10}$ & $6 \times 10^{-10}$ & $4 \times 10^{-10}$ & $3 \times 10^{-10}$ & $2 \times 10^{-7}$ & $2 \times 10^{-7}$ & $7 \times 10^{-9}$ & $6 \times 10^{-9}$ & $2 \times 10^{-7}$ & $2 \times 10^{-7}$ & $4 \times 10^{-7}$ & $4 \times 10^{-7}$ \\
\hline Shrimp & $5 \times 10^{-12}$ & $5 \times 10^{-12}$ & $6 \times 10^{-10}$ & $5 \times 10^{-10}$ & $2 \times 10^{-11}$ & $7 \times 10^{-12}$ & $8 \times 10^{-12}$ & $4 \times 10^{-12}$ & $2 \times 10^{-9}$ & $1 \times 10^{-9}$ & $7 \times 10^{-11}$ & $4 \times 10^{-11}$ & $2 \times 10^{-9}$ & $1 \times 10^{-9}$ & $5 \times 10^{-9}$ & $3 \times 10^{-9}$ \\
\hline Crab & $2 \times 10^{-11}$ & $2 \times 10^{-11}$ & $3 \times 10^{-9}$ & $2 \times 10^{-9}$ & $7 \times 10^{-11}$ & $3 \times 10^{-11}$ & $4 \times 10^{-11}$ & $2 \times 10^{-11}$ & $9 \times 10^{-9}$ & $5 \times 10^{-9}$ & $3 \times 10^{-10}$ & $2 \times 10^{-10}$ & $9 \times 10^{-9}$ & $6 \times 10^{-9}$ & $2 \times 10^{-8}$ & $1 \times 10^{-8}$ \\
\hline Clam & 0 & 0 & 0 & 0 & 0 & 0 & 0 & 0 & 0 & 0 & 0 & 0 & 0 & 0 & 0 & 0 \\
\hline Sea bird & $8 \times 10^{-10}$ & $8 \times 10^{-10}$ & $1 \times 10^{-6}$ & $1 \times 10^{-6}$ & $2 \times 10^{-8}$ & $8 \times 10^{-9}$ & $1 \times 10^{-8}$ & $4 \times 10^{-9}$ & $2 \times 10^{-6}$ & $1 \times 10^{-6}$ & $7 \times 10^{-8}$ & $4 \times 10^{-8}$ & $2 \times 10^{-6}$ & $1 \times 10^{-6}$ & $5 \times 10^{-6}$ & $4 \times 10^{-6}$ \\
\hline Sea birc & $3 \times 10^{-11}$ & 0 & $1 \times 10^{-8}$ & 0 & $4 \times 10^{-10}$ & 0 & $2 \times 10^{-10}$ & 0 & $2 \times 10^{-9}$ & 0 & $3 \times 10^{-12}$ & 0 & $6 \times 10^{-11}$ & 0 & $2 \times 10^{-8}$ & 0 \\
\hline Seaweed & 0 & 0 & 0 & 0 & 0 & 0 & 0 & 0 & 0 & 0 & 0 & 0 & 0 & 0 & 0 & 0 \\
\hline Seal & $2 \times 10^{-10}$ & $2 \times 10^{-10}$ & $2 \times 10^{-7}$ & $2 \times 10^{-7}$ & $1 \times 10^{-8}$ & $6 \times 10^{-9}$ & $7 \times 10^{-9}$ & $3 \times 10^{-9}$ & $6 \times 10^{-8}$ & $4 \times 10^{-8}$ & $2 \times 10^{-9}$ & $1 \times 10^{-9}$ & $7 \times 10^{-8}$ & $4 \times 10^{-8}$ & $4 \times 10^{-7}$ & $3 \times 10^{-7}$ \\
\hline Sea lion & $1 \times 10^{-10}$ & $1 \times 10^{-10}$ & $1 \times 10^{-7}$ & $1 \times 10^{-7}$ & $9 \times 10^{-9}$ & $4 \times 10^{-9}$ & $4 \times 10^{-9}$ & $2 \times 10^{-9}$ & $4 \times 10^{-8}$ & $2 \times 10^{-8}$ & $1 \times 10^{-9}$ & $8 \times 10^{-10}$ & $4 \times 10^{-8}$ & $2 \times 10^{-8}$ & $2 \times 10^{-7}$ & $2 \times 10^{-7}$ \\
\hline Total & $1 \times 10^{-8}$ & $9 \times 10^{-9}$ & $4 \times 10^{-6}$ & $6 \times 10^{-6}$ & $7 \times 10^{-8}$ & $3 \times 10^{-8}$ & $3 \times 10^{-8}$ & $2 \times 10^{-8}$ & $9 \times 10^{-6}$ & $8 \times 10^{-6}$ & $4 \times 10^{-7}$ & $3 \times 10^{-7}$ & $1 \times 10^{-5}$ & $9 \times 10^{-6}$ & $2 \times 10^{-5}$ & $2 \times 10^{-5}$ \\
\hline
\end{tabular}


Table 30. Potential Risks to an Aleut Consuming Seafood Harvested from Amchitka and Adak Using Unalaska Diet Data and Maximum Measured Concentrations

\begin{tabular}{|c|c|c|c|c|c|c|c|c|c|c|c|c|c|c|c|c|}
\hline \multirow{2}{*}{$\begin{array}{l}\text { Seafood } \\
\text { species }\end{array}$} & \multicolumn{2}{|c|}{${ }^{2441} \mathrm{Am}$} & \multicolumn{2}{|c|}{${ }^{137} \mathrm{Cs}+\mathrm{D}$} & \multicolumn{2}{|c|}{${ }^{239} \mathrm{Pu}$} & \multicolumn{2}{|c|}{${ }^{240} \mathrm{Pu}$} & \multicolumn{2}{|c|}{${ }^{234} U$} & \multicolumn{2}{|c|}{$\overline{{ }^{235} U+D}$} & \multicolumn{2}{|c|}{$\overline{238} U+D$} & \multicolumn{2}{|c|}{ Total } \\
\hline & Amchitka & Adak & Amchitka & Adak & Amchitka & Adak & Amchitka & Adak & chitka & Adak & chitka & Adak & nchitka & Adak & nchitka & Adak \\
\hline Halibut & 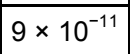 & 10 & $2 \times 1$ & 1 & 0 & 6 & $2 \times 1$ & -10 & 6 & -8 & 10 & 1 & -8 & $3 \times 10^{-8}$ & $1 \times 10^{-7}$ & $\times 10^{-7}$ \\
\hline olly & $10^{-12}$ & $\times 10^{-12}$ & $5 \times 10^{-8}$ & $2 \times 10^{-7}$ & $\times 10^{-11}$ & $2 \times 10^{-11}$ & $2 \times 10^{-11}$ & $2 \times 10^{-11}$ & $2 \times 10^{-9}$ & $1 \times 10^{-9}$ & $10^{-11}$ & $2 \times 10^{-11}$ & $4 \times 10^{-10}$ & $6 \times 10^{-10}$ & $5 \times 10^{-8}$ & $\times 10^{-7}$ \\
\hline Cod & $\times 10^{-11}$ & $1 \times 10^{-11}$ & $7 \times 10^{-9}$ & $6 \times 10^{-9}$ & $9 \times 10^{-11}$ & $9 \times 10^{-11}$ & $3 \times 10^{-11}$ & $3 \times 10^{-11}$ & $1 \times 10^{-8}$ & $9 \times 10^{-9}$ & $4 \times 10^{-10}$ & $3 \times 10^{-10}$ & $1 \times 10^{-8}$ & $8 \times 10^{-9}$ & $3 \times 10^{-8}$ & $2 \times 10^{-8}$ \\
\hline Salmon & $1 \times 10^{-9}$ & $1 \times 10^{-9}$ & $3 \times 10^{-7}$ & $3 \times 10^{-7}$ & $2 \times 10^{-9}$ & $2 \times 10^{-9}$ & $1 \times 10^{-9}$ & $1 \times 10^{-9}$ & $2 \times 10^{-6}$ & $2 \times 10^{-6}$ & $8 \times 10^{-8}$ & $8 \times 10^{-8}$ & $2 \times 10^{-6}$ & $2 \times 10^{-6}$ & $4 \times 10^{-6}$ & $4 \times 10^{-6}$ \\
\hline Other fish & $\times 10^{-10}$ & $1 \times 10^{-10}$ & $6 \times 10^{-9}$ & $8 \times 10^{-9}$ & $3 \times 10^{-10}$ & $3 \times 10^{-10}$ & $1 \times 10^{-10}$ & $2 \times 10^{-10}$ & $4 \times 10^{-8}$ & $3 \times 10^{-8}$ & $1 \times 10^{-9}$ & $1 \times 10^{-9}$ & $4 \times 10^{-8}$ & $3 \times 10^{-8}$ & $9 \times 10^{-8}$ & $7 \times 10^{-8}$ \\
\hline Octopus & $3 \times 10^{-11}$ & $2 \times 10^{-11}$ & $1 \times 10^{-9}$ & $3 \times 10^{-10}$ & $7 \times 10^{-11}$ & $4 \times 10^{-11}$ & $6 \times 10^{-11}$ & $2 \times 10^{-11}$ & $3 \times 10$ & $2 \times 10^{-8}$ & $9 \times 10^{-10}$ & $5 \times 10^{-10}$ & $3 \times 10^{-8}$ & $2 \times 10^{-8}$ & $5 \times 10^{-8}$ & $3 \times 10^{-8}$ \\
\hline Sea urchin & $\times 10^{-10}$ & $2 \times 10^{-10}$ & $4 \times 10^{-9}$ & $4 \times 10^{-9}$ & $2 \times 10^{-10}$ & $1 \times 10^{-10}$ & $1 \times 10^{-10}$ & $8 \times 10^{-11}$ & $3 \times 10^{-8}$ & $3 \times 10^{-8}$ & $1 \times 10^{-9}$ & $1 \times 10^{-9}$ & $3 \times 10^{-8}$ & $3 \times 10^{-8}$ & $6 \times 10^{-8}$ & $7 \times 10^{-8}$ \\
\hline Mussel & $\times 10^{-10}$ & $2 \times 10^{-11}$ & $2 \times 10^{-9}$ & $\times 10^{-10}$ & $3 \times 10^{-10}$ & $3 \times 10^{-11}$ & $2 \times 10^{-10}$ & $2 \times 10^{-11}$ & $8 \times 10^{-9}$ & $5 \times 10^{-9}$ & $3 \times 10^{-10}$ & $2 \times 10^{-10}$ & $8 \times 1$ & $5 \times 1$ & $10^{-8}$ & $\times 10^{-8}$ \\
\hline \begin{tabular}{|l|} 
Bidarki \\
(chiton)
\end{tabular} & $\times 10^{-11}$ & $2 \times 10^{-11}$ & $4 \times 10^{-10}$ & $1 \times 10^{-10}$ & $3 \times 10^{-11}$ & $2 \times 10^{-11}$ & $2 \times 10^{-11}$ & 11 & 7 & $7>$ & $10^{-10}$ & -10 & $7 \times 10^{-9}$ & -9 & $0^{-8}$ & $0^{-8}$ \\
\hline Shrimp & $\times 10^{-11}$ & $5 \times 10^{-11}$ & $6 \times 10^{-9}$ & $5 \times 10^{-9}$ & $2 \times 10^{-10}$ & $7 \times 10^{-11}$ & $9 \times 10^{-11}$ & $4 \times 10^{-11}$ & $2 \times 10^{-8}$ & $1 \times 10^{-8}$ & $7 \times 10^{-10}$ & $4 \times 10^{-10}$ & $2 \times 10^{-8}$ & $1 \times 10^{-8}$ & $5 \times 10^{-8}$ & $3 \times 10^{-8}$ \\
\hline Crab & $3 \times 10^{-10}$ & $3 \times 10^{-10}$ & $3 \times 10^{-8}$ & $3 \times 10^{-8}$ & $9 \times 10^{-10}$ & $4 \times 10^{-10}$ & $5 \times 10^{-10}$ & $2 \times 10^{-10}$ & $1 \times 10^{-7}$ & $7 \times 10^{-8}$ & $4 \times 10^{-9}$ & $2 \times 10^{-9}$ & $1 \times 10^{-7}$ & $7 \times 10^{-8}$ & $3 \times 10^{-7}$ & $2 \times 10^{-7}$ \\
\hline Clam & $2 \times 10^{-9}$ & $3 \times 10^{-10}$ & $3 \times 10^{-8}$ & $9 \times 10^{-9}$ & $5 \times 10^{-9}$ & $5 \times 10^{-10}$ & $3 \times 10^{-9}$ & $3 \times 10^{-10}$ & $1 \times 10^{-7}$ & $7 \times 10^{-8}$ & $4 \times 10^{-9}$ & $3 \times 10^{-9}$ & $1 \times 10^{-7}$ & $8 \times 10^{-8}$ & $3 \times 10^{-7}$ & $2 \times 10^{-7}$ \\
\hline Sea bird & $\times 10^{-10}$ & $6 \times 10^{-10}$ & $9 \times 10^{-7}$ & $8 \times 10^{-7}$ & $1 \times 10^{-8}$ & $5 \times 10^{-9}$ & $6 \times 10^{-9}$ & $3 \times 10^{-9}$ & $1 \times 10^{-6}$ & $8 \times 10^{-7}$ & $5 \times 10^{-8}$ & $3 \times 10^{-8}$ & $1 \times 10^{-6}$ & $9 \times 10^{-7}$ & $4 \times 10^{-6}$ & $2 \times 10^{-6}$ \\
\hline Sea bird egg & $4 \times 10^{-12}$ & 0 & $1 \times 10^{-9}$ & 0 & $4 \times 10^{-11}$ & 0 & $2 \times 10^{-11}$ & 0 & $3 \times 10^{-10}$ & 0 & $3 \times 10^{-13}$ & 0 & $6 \times 10^{-12}$ & 0 & $2 \times 10^{-9}$ & 0 \\
\hline Seaweed & 0 & 0 & 0 & 0 & 0 & 0 & 0 & 0 & 0 & 0 & 0 & 0 & 0 & 0 & 0 & 0 \\
\hline Seal & $1 \times 10^{-10}$ & $1 \times 10^{-10}$ & $1 \times 10^{-7}$ & $9 \times 10^{-8}$ & $8 \times 10^{-9}$ & $3 \times 10^{-9}$ & $4 \times 10^{-9}$ & $2 \times 10^{-9}$ & $3 \times 10^{-8}$ & $2 \times 10^{-8}$ & $1 \times 10^{-9}$ & $7 \times 10^{-10}$ & $3 \times 10^{-8}$ & $2 \times 10^{-8}$ & $2 \times 10^{-7}$ & $1 \times 10^{-7}$ \\
\hline Sea lion & $7 \times 10^{-11}$ & $7 \times 10^{-11}$ & $6 \times 10^{-8}$ & $5 \times 10^{-8}$ & $4 \times 10^{-9}$ & $2 \times 10^{-9}$ & $2 \times 10^{-9}$ & $9 \times 10^{-10}$ & $2 \times 10^{-8}$ & $1 \times 10^{-8}$ & $6 \times 10^{-10}$ & $4 \times 10^{-10}$ & $2 \times 10^{-8}$ & $1 \times 10^{-8}$ & $1 \times 10^{-7}$ & $7 \times 10^{-8}$ \\
\hline otal & $5 \times 10^{-9}$ & $3 \times 10^{-9}$ & $2 \times 10^{-6}$ & $2 \times 10^{-6}$ & $3 \times 10^{-8}$ & $1 \times 10^{-8}$ & $2 \times 10^{-8}$ & $8 \times 10^{-9}$ & $4 \times 10^{-6}$ & $3 \times 10^{-6}$ & $1 \times 10^{-7}$ & $1 \times 10^{-7}$ & $4 \times 10^{-6}$ & $3 \times 10^{-6}$ & $9 \times 10^{-6}$ & $8 \times 10^{-6}$ \\
\hline
\end{tabular}


Table 31. Potential Risks to an Aleut Consuming Seafood Harvested from Amchitka and Adak Using the Unalaska Diet Data and $U_{C L}{ }_{95}$ Concentrations

\begin{tabular}{|c|c|c|c|c|c|c|c|c|c|c|c|c|c|c|c|c|}
\hline \multirow{2}{*}{$\begin{array}{l}\text { Seafood } \\
\text { species }\end{array}$} & \multicolumn{2}{|c|}{${ }^{241} \mathrm{Am}$} & \multicolumn{2}{|c|}{${ }^{137} \mathrm{Cs}+\mathrm{D}$} & \multicolumn{2}{|c|}{${ }^{239} \mathrm{Pu}$} & \multicolumn{2}{|c|}{${ }^{240} \mathrm{Pu}$} & \multicolumn{2}{|c|}{${ }^{234} \mathrm{U}$} & \multicolumn{2}{|c|}{${ }^{235} \mathrm{U}+\mathrm{D}$} & \multicolumn{2}{|c|}{${ }^{238} U+D$} & \multicolumn{2}{|c|}{ Total } \\
\hline & Amchitka & Adak & Amchitka & Adak & Amchitka & Adak & Amchitka & Adak & Amchitka & Adak & Amchitka & Adak & $\begin{array}{c}\text { Amchitk } \\
\text { a }\end{array}$ & Adak & Imchitka & Adak \\
\hline Halibut & $\times 10^{-11}$ & $1 \times 10^{-10}$ & $2 \times 10^{-8}$ & ro & $\times 10^{-10}$ & $6 \times 10^{-10}$ & $2 \times 10^{-10}$ & $2 \times 10^{-10}$ & 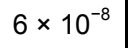 & $\times 10^{-8}$ & $9 \times 10^{-10}$ & $1 \times 10^{-9}$ & $3 \times 10^{-8}$ & $3 \times 10^{-8}$ & $0^{-7}$ & $\times 10^{-7}$ \\
\hline Dolly Varden & $3 \times 10^{-12}$ & $5 \times 10^{-12}$ & $5 \times 10^{-8}$ & $2 \times 10^{-7}$ & $2 \times 10^{-11}$ & $2 \times 10^{-11}$ & $2 \times 10^{-11}$ & $2 \times 10^{-11}$ & $2 \times 10^{-9}$ & $1 \times 10^{-9}$ & $1 \times 10^{-11}$ & $2 \times 10^{-11}$ & $\begin{array}{c}4 \times \\
10^{-10} \\
\end{array}$ & $6 \times$ & $5 \times 10^{-8}$ & $2 \times 10^{-7}$ \\
\hline Cod & $\times 10^{-11}$ & $1 \times 10^{-11}$ & $7 \times 10^{-9}$ & $6 \times 10^{-9}$ & $\times 10^{-11}$ & $9 \times 10^{-11}$ & $3 \times 10^{-11}$ & $3 \times 10^{-11}$ & $1 \times 10^{-8}$ & $9 \times 10^{-9}$ & $4 \times 10^{-10}$ & $3 \times 10^{-10}$ & $1 \times 10^{-8}$ & $8 \times 10^{-9}$ & $3 \times 10^{-8}$ & $2 \times 10^{-8}$ \\
\hline Salmon & $1 \times 10^{-9}$ & $1 \times 10^{-9}$ & $3 \times 10^{-7}$ & $3 \times 10^{-7}$ & $2 \times 10^{-9}$ & $2 \times 10^{-9}$ & $1 \times 10^{-9}$ & $1 \times 10^{-9}$ & $2 \times 10^{-6}$ & $2 \times 10^{-6}$ & $8 \times 10^{-8}$ & $8 \times 10^{-8}$ & $2 \times 10^{-6}$ & $2 \times 10^{-6}$ & $4 \times 10^{-6}$ & $4 \times 10^{-6}$ \\
\hline Other fish & $1 \times 10^{-10}$ & $8 \times 10^{-11}$ & $6 \times 10^{-9}$ & $4 \times 10^{-9}$ & $3 \times 10^{-10}$ & $2 \times 10^{-10}$ & $1 \times 10^{-10}$ & $1 \times 10^{-10}$ & $3 \times 10^{-8}$ & $2 \times 10^{-8}$ & $9 \times 10^{-10}$ & $6 \times 10^{-10}$ & $3 \times 10^{-8}$ & $2 \times 10^{-8}$ & $6 \times 10^{-8}$ & $4 \times 10^{-8}$ \\
\hline Octopus & $3 \times 10^{-11}$ & $2 \times 10^{-11}$ & $1 \times 10^{-9}$ & $3 \times 10^{-10}$ & $7 \times 10^{-11}$ & $4 \times 10^{-11}$ & $6 \times 10^{-11}$ & $2 \times 10^{-11}$ & $3 \times 10^{-8}$ & $2 \times 10^{-8}$ & $9 \times 10^{-10}$ & $5 \times 10^{-10}$ & $3 \times 10^{-8}$ & $2 \times 10^{-8}$ & $5 \times 10^{-8}$ & $3 \times 10^{-8}$ \\
\hline Sea urchin & $\times 10^{-10}$ & $2 \times 10^{-10}$ & $4 \times 10^{-9}$ & $4 \times 10^{-9}$ & $2 \times 10^{-10}$ & $1 \times 10^{-10}$ & $1 \times 10^{-10}$ & $8 \times 10^{-11}$ & $3 \times 10^{-8}$ & $3 \times 10^{-8}$ & $1 \times 10^{-9}$ & $1 \times 10^{-9}$ & $3 \times 10^{-8}$ & $3 \times 10^{-8}$ & $6 \times 10^{-8}$ & $7 \times 10^{-8}$ \\
\hline Mussel & $7 \times 10^{-11}$ & $2 \times 10^{-11}$ & $1 \times 10^{-9}$ & $6 \times 10^{-10}$ & $2 \times 10^{-10}$ & $3 \times 10^{-11}$ & $1 \times 10^{-10}$ & $2 \times 10^{-11}$ & $7 \times 10^{-9}$ & $5 \times 10^{-9}$ & $3 \times 10^{-10}$ & $2 \times 10^{-10}$ & $8 \times 10^{-9}$ & $5 \times 10^{-9}$ & $2 \times 10^{-8}$ & $1 \times 10^{-8}$ \\
\hline $\begin{array}{l}\text { Bidarki } \\
\text { (chiton) }\end{array}$ & $\times 10^{-11}$ & $2 \times 10^{-11}$ & $4 \times 10^{-10}$ & $1 \times 10^{-10}$ & $3 \times 10^{-11}$ & $2 \times 10^{-11}$ & $2 \times 10^{-11}$ & $1 \times 10^{-11}$ & $7 \times 10^{-9}$ & $7 \times 10^{-9}$ & $3 \times 10^{-10}$ & $2 \times 10^{-10}$ & $7 \times 10^{-9}$ & $6 \times 10^{-9}$ & $2 \times 10^{-8}$ & $1 x$ \\
\hline Shrimp & $5 \times 10^{-11}$ & $5 \times 10^{-11}$ & $6 \times 10^{-9}$ & $5 \times 10^{-9}$ & $2 \times 10^{-10}$ & $7 \times 10^{-11}$ & $9 \times 10^{-11}$ & $4 \times 10^{-11}$ & $2 \times 10^{-8}$ & $1 \times 10^{-8}$ & $7 \times 10^{-10}$ & $4 \times 10^{-10}$ & $2 \times 10^{-8}$ & $1 \times 10^{-8}$ & $5 \times 10^{-8}$ & $3 \times 10^{-8}$ \\
\hline Crab & $3 \times 10^{-10}$ & $3 \times 10^{-10}$ & $3 \times 10^{-8}$ & $3 \times 10^{-8}$ & $9 \times 10^{-10}$ & $4 \times 10^{-10}$ & $5 \times 10^{-10}$ & $2 \times 10^{-10}$ & $1 \times 10^{-7}$ & $7 \times 10^{-8}$ & $4 \times 10^{-9}$ & $2 \times 10^{-9}$ & $1 \times 10^{-7}$ & $7 \times 10^{-8}$ & $3 \times 10^{-7}$ & $2 \times 10^{-7}$ \\
\hline Clam & $1 \times 10^{-9}$ & $3 \times 10^{-10}$ & $2 \times 10^{-8}$ & $9 \times 10^{-9}$ & $3 \times 10^{-9}$ & $5 \times 10^{-10}$ & $2 \times 10^{-9}$ & $3 \times 10^{-10}$ & $1 \times 10^{-7}$ & $7 \times 10^{-8}$ & $4 \times 10^{-9}$ & $3 \times 10^{-9}$ & $1 \times 10^{-7}$ & $8 \times 10^{-8}$ & $3 \times 10^{-7}$ & $2 \times 10^{-7}$ \\
\hline Sea bird & $6 \times 10^{-10}$ & $6 \times 10^{-10}$ & $9 \times 10^{-7}$ & $8 \times 10^{-7}$ & $1 \times 10^{-8}$ & $5 \times 10^{-9}$ & $6 \times 10^{-9}$ & $3 \times 10^{-9}$ & $1 \times 10^{-6}$ & $8 \times 10^{-7}$ & $5 \times 10^{-8}$ & $3 \times 10^{-8}$ & $1 \times 10^{-6}$ & $9 \times 10^{-7}$ & $4 \times 10^{-6}$ & $2 \times 10^{-6}$ \\
\hline Sea bird egg & $4 \times 10^{-12}$ & 0 & $1 \times 10^{-9}$ & 0 & $4 \times 10^{-11}$ & 0 & $2 \times 10^{-11}$ & 0 & $3 \times 10^{-10}$ & 0 & $3 \times 10^{-13}$ & 0 & \begin{tabular}{|l|}
$6 \times$ \\
$10^{-12}$ \\
\end{tabular} & 0 & $2 \times 10^{-9}$ & 0 \\
\hline Seaweed & 0 & 0 & 0 & 2 & 0 & 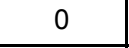 & 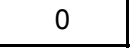 & 0 & 0 & 0 & 0 & 0 & 0 & 0 & 0 & 0 \\
\hline Seal & $1 \times 10^{-10}$ & $1 \times 10^{-10}$ & $1 \times 10^{-7}$ & $9 \times 10^{-8}$ & $8 \times 10^{-9}$ & $3 \times 10^{-9}$ & $4 \times 10^{-9}$ & $2 \times 10^{-9}$ & $3 \times 10^{-8}$ & $2 \times 10^{-8}$ & $1 \times 10^{-9}$ & $7 \times 10^{-10}$ & $3 \times 10^{-8}$ & $2 \times 10^{-8}$ & $2 \times 10^{-7}$ & $1 \times 10^{-7}$ \\
\hline Sea lion & $7 \times 10^{-11}$ & $7 \times 10^{-11}$ & $6 \times 10^{-8}$ & $5 \times 10^{-8}$ & $4 \times 10^{-9}$ & $2 \times 10^{-9}$ & $2 \times 10^{-9}$ & $9 \times 10^{-10}$ & $2 \times 10^{-8}$ & $1 \times 10^{-8}$ & $6 \times 10^{-10}$ & $4 \times 10^{-10}$ & $2 \times 10^{-8}$ & $1 \times 10^{-8}$ & $1 \times 10^{-7}$ & $7 \times 10^{-8}$ \\
\hline Total & $4 \times 10^{-9}$ & $3 \times 10^{-9}$ & $2 \times 10^{-6}$ & $2 \times 10^{-6}$ & $3 \times 10^{-8}$ & $1 \times 10^{-8}$ & $2 \times 10^{-8}$ & $8 \times 10^{-9}$ & $4 \times 10^{-6}$ & $3 \times 10^{-6}$ & $1 \times 10^{-7}$ & $1 \times 10^{-7}$ & $4 \times 10^{-6}$ & $3 \times 10^{-6}$ & $9 \times 10^{-6}$ & $8 \times 10^{-6}$ \\
\hline
\end{tabular}


Table 32. Potential Risks to an Aleut Consuming Seafood Harvested from Amchitka and Adak Using the Composite Diet Data and Maximum Measured Concentrations

\begin{tabular}{|c|c|c|c|c|c|c|c|c|c|c|c|c|c|c|c|c|}
\hline \multirow{2}{*}{$\begin{array}{l}\text { Seafood } \\
\text { species }\end{array}$} & \multicolumn{2}{|c|}{${ }^{2441} \mathrm{Am}$} & \multicolumn{2}{|c|}{${ }^{137} \mathrm{Cs}+\mathrm{D}$} & \multicolumn{2}{|c|}{${ }^{239} \mathrm{Pu}$} & \multicolumn{2}{|c|}{${ }^{240} \mathrm{Pu}$} & \multicolumn{2}{|c|}{${ }^{234} U$} & \multicolumn{2}{|c|}{$\overline{{ }^{235} U+D}$} & \multicolumn{2}{|c|}{$\overline{238} U+D$} & \multicolumn{2}{|c|}{ Total } \\
\hline & chitka & dak & chitka & Adak & hitka & Adak & itka & Adak & $a$ & dak & a & dak & a & dak & $a$ & dak \\
\hline alibut & 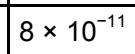 & s & $2 \times 10^{-8}$ & 1 & 0 & 5 & 2 & 2 & 8 & 8 & 10 & 1 & -8 & -8 & -8 & 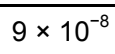 \\
\hline Dolly Varden & $0^{-12}$ & $0^{-12}$ & 8 & 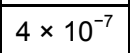 & $\times 10^{-11}$ & $10^{-11}$ & $10^{-11}$ & $10^{-11}$ & $10^{-9}$ & $10^{-9}$ & $0^{-11}$ & $\times 10^{-11}$ & $0^{-10}$ & $10^{-10}$ & -8 & $4 \times 10^{-7}$ \\
\hline Cod & $\times 10^{-11}$ & $1 \times 10$ & 6 & $5 \times 10^{-9}$ & $7 \times 1$ & $7 \times 10^{-11}$ & $2 \times 1$ & $2 \times 1$ & 1 . & $8 \times 10^{-9}$ & $4 \times 10^{-10}$ & $2 \times 10^{-10}$ & $1 \times 10^{-8}$ & $7 \times 10^{-9}$ & -8 & $10^{-8}$ \\
\hline almc & 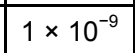 & 1 & $2 \times 10^{-7}$ & $2 \times 10^{-7}$ & 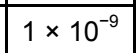 & 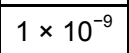 & 10 & 10 & $1 \times$ & 6 & 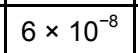 & -8 & $1 \times$ & -6 & 6 & $3 \times 10^{-6}$ \\
\hline Other & $\times 10^{-10}$ & $2 \times 10^{-10}$ & $1 \times 10^{-8}$ & $1 \times 10^{-8}$ & $5 \times 10^{-10}$ & $4 \times 10^{-10}$ & $2 \times 10^{-10}$ & $2 \times 10^{-10}$ & $7 \times 10^{-8}$ & $5 \times 10^{-8}$ & $2 \times 10^{-9}$ & $2 \times 10^{-9}$ & $6 \times 10^{-8}$ & $5 \times 10^{-8}$ & $1 \times 10^{-7}$ & $\times 10^{-7}$ \\
\hline Octopus & $10^{-11}$ & $x=10$ & $1 \times 10^{-9}$ & $\times 10^{-10}$ & $7 \times 10^{-11}$ & $4 \times 10^{-11}$ & $7 \times 10^{-11}$ & $2 \times 10^{-11}$ & $3 \times 10$ & $2 \times 10^{-8}$ & $9 \times 10^{-10}$ & $10^{-10}$ & $3 \times 10^{-8}$ & $10^{-8}$ & -8 & J \\
\hline ea L & $\times 10^{-10}$ & $1 \times 10^{-10}$ & $3 \times 10^{-9}$ & $3 \times 10^{-9}$ & $1 \times 10^{-10}$ & $1 \times 10^{-10}$ & $1 \times 10^{-10}$ & $6 \times 10^{-11}$ & $2 \times 10^{-8}$ & $2 \times 10^{-8}$ & $7 \times 10^{-10}$ & $7 \times 10^{-10}$ & $2 \times 10^{-8}$ & $2 \times 10^{-8}$ & $5 \times 10^{-8}$ & $\times 10^{-8}$ \\
\hline Mussel & $10^{-10}$ & $\times 10^{-11}$ & $8 \times 10$ & 10 & $2 \times 10^{0}$ & $2 \times 1$ & $8 \times 1$ & $8 \times 1$ & $4 \times 10^{0}$ & 10 & $1 \times 10^{-9}$ & 10 & $4 \times 10^{-8}$ & 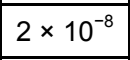 & -8 & 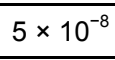 \\
\hline \begin{tabular}{|l|} 
Bidarki \\
(chiton)
\end{tabular} & $\times 10^{-10}$ & $8 \times 10^{-11}$ & $1 \times 10^{-9}$ & $0^{-10}$ & $9 \times 10^{-11}$ & 8 & $5 \times$ & & -8 & & 10 & & 2 & & $j^{-8}$ & $0^{\circ}$ \\
\hline Shrimp & $\times 10^{-11}$ & $\times 10^{-1}$ & $2 \times 10^{-9}$ & $2 \times 10^{-9}$ & $6 \times 10^{-11}$ & $2 \times 10^{-11}$ & $3 \times 10^{-11}$ & $1 \times 10^{-11}$ & $7 \times 10$ & $4 \times 10^{-9}$ & $2 \times 10^{-10}$ & $2 \times 10^{-10}$ & $7 \times 10^{-9}$ & $5 \times$ & $10^{-8}$ & $\times 10^{-8}$ \\
\hline $\mathrm{rab}$ & $\times 10^{-10}$ & $1 \times 10^{-10}$ & $1 \times 10^{-8}$ & $1 \times 10^{-8}$ & $4 \times 10^{-10}$ & $2 \times 10^{-10}$ & $2 \times 10^{-10}$ & $9 \times 10^{-11}$ & $5 \times 10^{-8}$ & $3 \times 10^{-8}$ & $2 \times 10^{-9}$ & $1 \times 10^{-9}$ & $5 \times 10^{-8}$ & $3 \times 10^{-8}$ & $1 \times 10^{-7}$ & $7 \times 10^{-8}$ \\
\hline Clam & $\times 10^{-10}$ & $1 \times 10^{-10}$ & $9 \times 10^{-9}$ & $3 \times 10^{-9}$ & $2 \times 10^{-9}$ & $2 \times 10^{-10}$ & $9 \times 10^{-10}$ & $9 \times 10^{-11}$ & $4 \times 10^{-8}$ & $3 \times 10^{-8}$ & $1 \times 10^{-9}$ & $9 \times 10^{-10}$ & $4 \times 10^{-8}$ & $3 \times 10^{-8}$ & $1 \times 10^{-7}$ & $6 \times 10^{-8}$ \\
\hline 0 & $\times 10^{-10}$ & $3 \times 10^{-10}$ & $5 \times 10^{-7}$ & $4 \times 10^{-7}$ & $6 \times 10^{-9}$ & $3 \times 10^{-9}$ & $3 \times 10^{-9}$ & $1 \times 10^{-9}$ & $7 \times 10^{-7}$ & $4 \times 10^{-7}$ & $2 \times 10^{-8}$ & $1 \times 10^{-8}$ & $7 \times 10^{-7}$ & $4 \times 10^{-7}$ & $2 \times 10^{-6}$ & $\times 10^{-6}$ \\
\hline Sea bird & $1 \times 10^{-11}$ & 0 & $4 \times 10^{-9}$ & 0 & $1 \times 10^{-10}$ & 0 & $6 \times 10^{-11}$ & 0 & $7 \times 10^{-10}$ & 0 & $7 \times 10^{-13}$ & 0 & $2 \times 10^{-11}$ & 0 & $5 \times 10^{-9}$ & 0 \\
\hline Seaweed & $7 \times 10^{-13}$ & $6 \times 10^{-13}$ & $3 \times 10^{-11}$ & $4 \times 10^{-11}$ & $4 \times 10^{-12}$ & $3 \times 10^{-12}$ & $3 \times 10^{-12}$ & $2 \times 10^{-12}$ & $2 \times 10^{-9}$ & $2 \times 10^{-9}$ & $8 \times 10^{-11}$ & $8 \times 10^{-11}$ & $2 \times 10^{-9}$ & $2 \times 10^{-9}$ & $4 \times 10^{-9}$ & $4 \times 10^{-9}$ \\
\hline Seal & $2 \times 10^{-10}$ & $2 \times 10^{-10}$ & $2 \times 10^{-7}$ & $1 \times 10^{-7}$ & $1 \times 10^{-8}$ & $5 \times 10^{-9}$ & $6 \times 10^{-9}$ & $3 \times 10^{-9}$ & $6 \times 10^{-8}$ & $3 \times 10^{-8}$ & $2 \times 10^{-9}$ & $1 \times 10^{-9}$ & $6 \times 10^{-8}$ & $4 \times 10^{-8}$ & $3 \times 10^{-7}$ & $2 \times 10^{-7}$ \\
\hline Sea lion & $2 \times 10^{-10}$ & $2 \times 10^{-10}$ & $1 \times 10^{-7}$ & $1 \times 10^{-7}$ & $1 \times 10^{-8}$ & $4 \times 10^{-9}$ & $5 \times 10^{-9}$ & $2 \times 10^{-9}$ & $4 \times 10^{-8}$ & $3 \times 10^{-8}$ & $2 \times 10^{-8}$ & $9 \times 10^{-10}$ & $4 \times 10^{-8}$ & $3 \times 10^{-8}$ & $2 \times 10^{-7}$ & $2 \times 10^{-7}$ \\
\hline otal & $\times 10^{-9}$ & $3 \times 10^{-9}$ & $1 \times 10^{-6}$ & $1 \times 10^{-6}$ & $4 \times 10^{-8}$ & $2 \times 10^{-8}$ & $2 \times 10^{-8}$ & $8 \times 10^{-9}$ & $2 \times 10^{-6}$ & $2 \times 10^{-6}$ & $9 \times 10^{-8}$ & $8 \times 10^{-8}$ & $2 \times 10^{-6}$ & $2 \times 10^{-6}$ & $6 \times 10^{-6}$ & $5 \times 10$ \\
\hline
\end{tabular}


Table 33. Potential Risks to an Aleut Consuming Seafood Harvested from Amchitka and Adak Using the Composite Diet Data and $U C L_{95}$ Concentrations

\begin{tabular}{|c|c|c|c|c|c|c|c|c|c|c|c|c|c|c|c|c|}
\hline & \multicolumn{2}{|c|}{${ }^{241} \mathrm{Am}$} & \multicolumn{2}{|c|}{${ }^{137} \mathrm{Cs}+\mathrm{D}$} & \multicolumn{2}{|c|}{${ }^{239} \mathrm{Pu}$} & \multicolumn{2}{|c|}{${ }^{240} \mathrm{Pu}$} & \multicolumn{2}{|c|}{${ }^{234} \mathrm{U}$} & \multicolumn{2}{|c|}{${ }^{235} U+D$} & \multicolumn{2}{|c|}{${ }^{238} U+D$} & \multicolumn{2}{|c|}{ Total } \\
\hline & nchitka & Adak & nchitka & Adak & nchitka & Adak & nchitka & Adak & mchitka & Adak & mchitka & Adak & hitka & Adak & tka & Adak \\
\hline Halibut & r & 正 & 年 & o & $10^{-10}$ & $10^{-10}$ & $2 \times 10^{-10}$ & $2 \times 10^{-10}$ & c & $\times 10^{-8}$ & $\times 10^{-10}$ & $1 \times 10^{-9}$ & $2 \times 10^{-8}$ & $10^{-8}$ & 8 & $x$ \\
\hline Dolly & -12 & -12 & 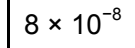 & 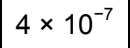 & -11 & $0^{-11}$ & $4 \times$ & $0^{-11}$ & & $0^{-9}$ & $2 \times 10^{-11}$ & $3 \times 10^{-11}$ & $6 \times 10^{-10}$ & $9 \times 10^{-10}$ & $\times 10^{-8}$ & $\times 10^{-7}$ \\
\hline Cod & -11 & , & 6 & 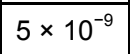 & $J^{-11}$ & $10^{-11}$ & 2 & 2 & 3 & 9 & $5^{-10}$ & $2 \times 1$ & $1 \times$ & -9 & 8 & $2 \times 10^{-8}$ \\
\hline $\mathrm{Imc}$ & $\times 10^{-9}$ & $\times 10^{-9}$ & $2 \times 10^{-7}$ & $2 \times$ & $\times 1$ & $1 \times 10^{-9}$ & $8 \times 10^{-10}$ & $8 \times 10^{-10}$ & $1 \times$ & $1 \times 10^{-6}$ & $6 \times 10^{-8}$ & $6 \times 10^{-8}$ & $1 \times 10^{-6}$ & $1 \times 10^{-6}$ & $3 \times 10^{-6}$ & $\times 10^{-6}$ \\
\hline Other & 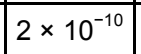 & 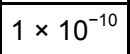 & 1 & $7 \times 10^{-9}$ & 0 & 0 & 2 & 2 & $5 \times 10^{-8}$ & $3 \times 10^{-8}$ & 2 & ${ }^{10}$ & 4 & 8 & 7 & $7 \times 10^{-8}$ \\
\hline Octop & $\times 10^{-11}$ & $3 \times 10^{-11}$ & $1 \times 10^{-9}$ & $3 \times 10^{-10}$ & $7 \times 10^{-11}$ & $4 \times 10^{-11}$ & $7 \times 10^{-11}$ & $2 \times 10^{-11}$ & 8 & $2 \times 10^{-8}$ & $9 \times 10^{-10}$ & $5 \times 10^{-10}$ & $3 \times 10^{-8}$ & $2 \times 10^{-8}$ & -8 & $\times 10^{-8}$ \\
\hline Sea ur & $\times 10^{-10}$ & $1 \times 10^{-10}$ & 10 & 10 & $\times 10^{-10}$ & $1 \times 10^{-10}$ & $1 \times 10^{-10}$ & $6 \times 10^{-11}$ & $\times 10$ & $2 \times 10^{-8}$ & $7 \times 10^{-10}$ & $7 \times 10^{-10}$ & 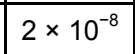 & 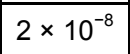 & 8 & 10 \\
\hline Muss & -10 & $9 \times 1$ & $5 \times 1$ & $x$ & $1 \times 1$ & $2 \times 10^{-10}$ & $5 \times 10^{-10}$ & $8 \times 10^{-11}$ & 5 & $2 \times 10^{-8}$ & $1 \times 10^{-9}$ & $8 \times 10^{-10}$ & $4 \times 10^{-8}$ & $2 \times 10^{-8}$ & -8 & $x$ \\
\hline \begin{tabular}{|l} 
Bidarki \\
(chiton)
\end{tabular} & $10^{-10}$ & $\times 10^{-11}$ & $1 \times 10^{-9}$ & $\times 10^{-10}$ & $0^{-11}$ & $8 \times 10^{-11}$ & 5 & 4 & 2 & ( & 10 & 10 & 2 & 2 & -8 & $4 \times 10^{-8}$ \\
\hline Shrimp & $\times 10^{-11}$ & $2 \times 10^{-11}$ & $2 \times 10^{-9}$ & $2 \times 10$ & $6 \times 10^{-11}$ & $2 \times 10^{-11}$ & $3 \times 10^{-11}$ & $1 \times 10^{-11}$ & $7 \times 10^{2}$ & $4 \times 10^{-9}$ & $2 \times 10^{-10}$ & $2 \times 10^{-10}$ & $7 \times 10^{-9}$ & $5 \times 10$ & $2 \times 10^{-8}$ & $1 \times 10$ \\
\hline Crab & $1 \times 10^{-10}$ & $1 \times 10^{-10}$ & $1 \times 10^{-8}$ & $1 \times 10^{-8}$ & $4 \times 10^{-10}$ & $2 \times 10^{-10}$ & $2 \times 10^{-10}$ & $9 \times 10^{-11}$ & $5 \times 10^{-8}$ & $3 \times 10^{-8}$ & $2 \times 10^{-9}$ & $1 \times 10^{-9}$ & $5 \times 10^{-8}$ & $3 \times 10^{-8}$ & $1 \times 10^{-7}$ & $7 \times 10^{-8}$ \\
\hline Clam & $\times 10^{-10}$ & $1 \times 10^{-10}$ & $6 \times 10^{-9}$ & $3 \times 10^{-9}$ & $1 \times 10^{-9}$ & $2 \times 10^{-10}$ & $6 \times 10^{-10}$ & $9 \times 10^{-11}$ & $4 \times 10^{-8}$ & $3 \times 10^{-8}$ & $1 \times 10^{-9}$ & $9 \times 10^{-10}$ & $4 \times 10^{-8}$ & $3 \times 10^{-8}$ & $9 \times 10^{-8}$ & $6 \times 10^{-8}$ \\
\hline Sea bird & $3 \times 10^{-10}$ & $3 \times 10^{-10}$ & $5 \times 10^{-7}$ & $4 \times 10^{-7}$ & $6 \times 10^{-9}$ & $3 \times 10^{-9}$ & $3 \times 10^{-9}$ & $1 \times 10^{-9}$ & $7 \times 10^{-7}$ & $4 \times 10^{-7}$ & $2 \times 10^{-8}$ & $1 \times 10^{-8}$ & $7 \times 10^{-7}$ & $4 \times 10^{-7}$ & $2 \times 10^{-6}$ & $1 \times 10^{-6}$ \\
\hline Sea b & $\times 10^{-11}$ & 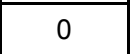 & $4 \times 10^{-9}$ & $v$ & $1 \times 10^{-10}$ & 0 & $6 \times 10^{-11}$ & 0 & $7 \times 10^{-10}$ & 0 & $7 \times 10^{-13}$ & 0 & $2 \times 10^{-11}$ & 0 & $5 \times 10^{-9}$ & 0 \\
\hline Seaweed & $4 \times 10^{-13}$ & $3 \times 10^{-13}$ & $2 \times 10^{-11}$ & $3 \times 10^{-11}$ & $2 \times 10^{-12}$ & $2 \times 10^{-12}$ & $2 \times 10^{-12}$ & $2 \times 10^{-12}$ & $2 \times 10^{-9}$ & $1 \times 10^{-9}$ & $6 \times 10^{-11}$ & $5 \times 10^{-11}$ & $2 \times 10^{-9}$ & $2 \times 10^{-9}$ & $3 \times 10^{-9}$ & $3 \times 10^{-9}$ \\
\hline Seal & $2 \times 10^{-10}$ & $2 \times 10^{-10}$ & $2 \times 10^{-7}$ & $1 \times 10^{-7}$ & $1 \times 10^{-8}$ & $5 \times 10^{-9}$ & $6 \times 10^{-9}$ & $3 \times 10^{-9}$ & $6 \times 10^{-8}$ & $3 \times 10^{-8}$ & $2 \times 10^{-9}$ & $1 \times 10^{-9}$ & $6 \times 10^{-8}$ & $4 \times 10^{-8}$ & $3 \times 10^{-7}$ & $2 \times 10^{-7}$ \\
\hline Se & $2 \times 10^{-10}$ & $2 \times 10^{-10}$ & $1 \times 10^{-7}$ & $1 \times 10^{-7}$ & $1 \times 10^{-8}$ & $4 \times 10^{-9}$ & $5 \times 10^{-9}$ & $2 \times 10^{-9}$ & $4 \times 10^{-8}$ & $3 \times 10^{-8}$ & $2 \times 10^{-8}$ & $9 \times 10^{-10}$ & $4 \times 10^{-8}$ & $3 \times 10^{-8}$ & $2 \times 10^{-7}$ & $2 \times 10^{-7}$ \\
\hline Total & $3 \times 10^{-9}$ & $2 \times 10^{-9}$ & $1 \times 10^{-6}$ & $1 \times 10^{-6}$ & $3 \times 10^{-8}$ & $2 \times 10^{-8}$ & $2 \times 10^{-8}$ & $8 \times 10^{-9}$ & $2 \times 10^{-6}$ & $2 \times 10^{-6}$ & $9 \times 10^{-8}$ & $8 \times 10^{-8}$ & $2 \times 10^{-6}$ & $2 \times 10^{-6}$ & $6 \times 10^{-6}$ & $5 \times 10$ \\
\hline
\end{tabular}


and to calculate the EPCs for several other species (e.g., sea lion and seal). The level of conservatism cannot be determined, but the values used were obtained from the latest and most comprehensive database compiled by IAEA (Howard et al. 2013) and should be appropriate for use in this assessment.

\subsection{Summary of Potential Seafood Ingestion Risk Analysis}

As discussed previously, the results of the risk calculations summarized in Table 23 and detailed in Table 24 through Table 33 for the five diets discussed in Section 7.1 indicate that overall potential risks from the ingestion of seafood based on the samples collected from Amchitka and Adak in 2011 are similar and are at $2 \times 10^{-5}$ or lower (i.e., within EPA's acceptable risk range of $1 \times 10^{-6}$ to $\left.1 \times 10^{-4}\right)$. The primary contributors to the risk estimates are the ${ }^{137} \mathrm{Cs}$ and ${ }^{234,235,238} \mathrm{U}$ concentrations.

The risk estimates using Amchitka data are slightly higher than those using Adak data by $1.4 \times 10^{-6}$. This is lower than the $1 \times 10^{-5}$ risk level (in excess of background) used by the State of Alaska as the benchmark for acceptable risk. For the 2011 sampling event, Adak served as the background location. Hence, based on the 2011 data collected for Amchitka and Adak, seafood harvested at Amchitka and Adak is considered safe for consumption at the intake levels for the five diets evaluated. In addition, the potential increase in cesium concentrations observed in the samples due to the Fukushima Dai-ichi event has not resulted in unacceptable risk estimates. These results are consistent with Decision Rule \#1 established for DQO\#1. Data from the 2011 sampling event will be documented for future comparisons.

\subsection{Ecological Health Evaluation (Using the RESRAD-BIOTA Code)}

The second DQO outlined in the 2011 Sampling Plan (DOE 2011) was to evaluate the ecological health of the biota species collected as part of the sampling. For this evaluation, the derived seawater concentrations as discussed in Section 7.2 are used for determining the ecological health of the species sampled by comparing the derived seawater concentrations with screening values incorporated into the RESRAD-BIOTA code (ISCORS 2004). If the derived seawater concentrations are greater than the screening values, then an analysis using the code to evaluate absorbed radiation dose to each species sampled would be conducted. The results of the code for each species is compared with the DOE guideline of $1 \mathrm{rad}$ per day set to protect aquatic organisms (DOE 2002a). 
The decision rule for this particular DQO discussed in the 2011 Sampling Plan stated that if radionuclide concentrations (in seawater around Amchitka and Adak) indicated that the ecological health index is below the guideline, no further data analysis will be performed. If the seawater concentrations indicated that the ecological health index could be greater than the guideline, additional ecological health analyses may be warranted.

RESRAD-BIOTA was developed by Argonne National Laboratory to implement DOE's guidance on evaluation of radiation doses to aquatic and terrestrial biota. According to DOE's guidance (DOE 2002a), a graded approach should be used for the evaluation. In Tier 1 analysis, representative environmental medium concentrations are compared with predetermined concentration limits called biota concentration guides (BCGs) to determine whether potential exists that radiation doses to biota species could exceed the target dose level. If the potential exists, then a Tier 2 or Tier 3 analysis, with the use of site- and species-specific information, can be conducted to obtain dose estimates to biota species for comparison with the target dose level. In this ecological health evaluation, the biota species of concern are aquatic organisms; therefore, the representative seawater concentrations around Amchitka and Adak Islands are compared with predetermined water BCGs in Tier 1 (i.e., screening) analysis. The water BCGs are the limiting water concentrations of individual radionuclides that were determined by DOE (DOE 2002a) through rigorous radiation dose analyses concerning a variety of aquatic organisms. If water concentration of a radionuclide is less than its BCG, potential radiation dose caused by that radionuclide to any aquatic organism living in the water is most likely to be less than the target dose level of $1 \mathrm{rad} /$ day.

In this evaluation, a Tier 1 analysis was conducted. Representative radionuclide concentrations in seawater near Amchitka and Adak islands, obtained using sampling data for nine marine species (see Section 7.2.2), were used as input to the RESRAD-BIOTA code for comparison with BCGs for water. Table 34 lists the representative water concentrations determined for Amchitka and Adak islands, respectively, and their ratios to the BCGs. Because a BCG for ${ }^{240} \mathrm{Pu}$ was not established, it was assumed to be the same as the BCG for ${ }^{239} \mathrm{Pu}$. Based on the listed values, the sum of ratios of radionuclide concentration to BCG for all radionuclides is much lower than the threshold value of 1 ; therefore, it is concluded that potential radiation doses to marine species of concern would be far below the target dose level ( $1 \mathrm{rad} /$ day), and no further analysis is required.

The representative seawater concentrations near Amchitka and Adak islands were compared with reported seawater concentrations for North Pacific Ocean obtained from literature, which were unit-converted and are also listed in Table 34. The comparison shows that representative seawater concentrations of ${ }^{241} \mathrm{Am}$ and uranium isotopes determined for Amchitka and Adak islands are within the range of reported concentrations for the North Pacific Ocean. For ${ }^{137} \mathrm{Cs}$, ${ }^{239} \mathrm{Pu}$, and ${ }^{240} \mathrm{Pu}$, the concentrations near Amchitka and Adak islands are somewhat higher than the reported concentrations for the North Pacific Ocean. (If the comparison included modeled concentrations for the North Pacific Ocean, the ${ }^{137}$ Cs concentrations near Amchitka and Adak islands would be within the range of concentrations for the North Pacific Ocean. This could be due to the conservatism associated with the selection of representative concentrations near Amchitka and Adak islands. The representative concentrations were determined by choosing the highest water concentrations back-calculated with sampling data of nine different species (see Section 7.2.2 for discussions). 
Table 34. Tier 1 Analysis of Potential Ecological Risks to Marine Biota

\begin{tabular}{|c|c|c|c|c|c|c|c|c|}
\hline \multirow[b]{2}{*}{$\begin{array}{c}\text { Concentration/ } \\
\text { Ratio }\end{array}$} & \multicolumn{8}{|c|}{ Radionuclide } \\
\hline & ${ }^{241} \mathrm{Am}$ & ${ }^{137} \mathrm{Cs}$ & ${ }^{239} \mathrm{Pu}$ & ${ }^{240} \mathrm{Pu}$ & ${ }^{234} U$ & ${ }^{235} U$ & ${ }^{238} \mathrm{U}$ & $\begin{array}{l}\text { Sum of } \\
\text { Ratios } \\
\text { to BCG }\end{array}$ \\
\hline BCG (pCi/L) & 438 & 42.6 & 187 & 187 & 202 & 217 & 223 & \\
\hline $\begin{array}{l}\text { Water conc. } \\
(\mathrm{pCi} / \mathrm{L})-A m c h i t k a\end{array}$ & 0.000059 & 0.23 & 0.00059 & 0.00030 & 1.6 & 0.053 & 1.3 & \\
\hline $\begin{array}{l}\text { Ratio to BCG- } \\
\text { Amchitka }\end{array}$ & $<0.0001$ & 0.0054 & $<0.0001$ & $<0.0001$ & 0.0077 & 0.0002 & 0.0056 & 0.0189 \\
\hline $\begin{array}{l}\text { Water conc. } \\
(\mathrm{pCi} / \mathrm{L}) \text {-Adak }\end{array}$ & 0.000059 & 0.19 & 0.00025 & 0.00013 & 0.94 & 0.033 & 0.79 & \\
\hline $\begin{array}{l}\text { Ratio to BCG- } \\
\text { Adak }\end{array}$ & $<0.0001$ & 0.0044 & $<0.0001$ & $<0.0001$ & 0.0047 & 0.0002 & 0.0035 & 0.0128 \\
\hline $\begin{array}{l}\text { Water conc. } \\
\text { (pCi/L)-North } \\
\text { Pacific Ocean }{ }^{a}\end{array}$ & $\begin{array}{c}0.000018 \\
\text { to } 0.00032\end{array}$ & $\begin{array}{c}0.043 \text { to } \\
0.11\end{array}$ & $\begin{array}{c}0.000042 \\
\text { to } 0.00015\end{array}$ & $\begin{array}{c}0.000035 \\
\text { to } 0.00012\end{array}$ & 1.24 & 0.057 & 1.09 & \\
\hline $\begin{array}{l}\text { Description of } \\
\text { water conc.- } \\
\text { North Pacific } \\
\text { Ocean }\end{array}$ & Low-high & Low-high & $\begin{array}{l}\text { Average- } \\
\text { Max. }\end{array}$ & $\begin{array}{l}\text { Average- } \\
\text { Max. }\end{array}$ & $\begin{array}{l}\text { Calculated } \\
\text { based on } \\
\text { total } \\
\text { uranium }\end{array}$ & $\begin{array}{c}\text { Calculated } \\
\text { based on } \\
\text { total } \\
\text { uranium }\end{array}$ & $\begin{array}{l}\text { Calculated } \\
\text { based on } \\
\text { total } \\
\text { uranium }\end{array}$ & \\
\hline $\begin{array}{l}\text { Ratio to BCG- } \\
\text { North Pacific } \\
\text { Ocean }\end{array}$ & $<0.0001$ & $\begin{array}{c}0.0010- \\
0.0025\end{array}$ & $<0.0001$ & $<0.0001$ & 0.0062 & 0.0003 & 0.0049 & $\begin{array}{l}0.0123- \\
0.0138\end{array}$ \\
\hline $\begin{array}{l}\mathrm{CG}=\text { Biota Concen } \\
\mathrm{Ci} / \mathrm{L}=\text { picocuries pe } \\
\text { Sources of literatur } \\
\text { average) and Hiros } \\
\text { data for individual } r \\
\text { the range } 0.17-0.28 \\
{ }^{235} \mathrm{U} \text {, and }{ }^{238} \mathrm{U} \text { ) with } \\
\text { multiplying the ratio }\end{array}$ & $\begin{array}{l}\text { ration Guide } \\
\text { liter } \\
\text { data: for }{ }^{241} \mathrm{~A} \\
\text { et al. } 2006 \text { ( } \\
\text { dionuclides ( } \\
\text { (Hirose et al. } \\
\text { an activity rat } \\
\text { of } 1.14 \text { betwe }\end{array}$ & $\begin{array}{l}\text { nax.); for } \\
\text { u and }{ }^{240} P \\
\text { 6). Litera } \\
1.14: 0.0 \\
{ }^{234} U \text { and }{ }^{2}\end{array}$ & $\begin{array}{l}03 \text {; for }{ }^{137} \\
U,{ }^{235} \mathrm{U} \text {, an } \\
\text { with an at } \\
\text { e data for } \\
: 1 \text {, where } \\
\mathrm{J} \text {, with the }\end{array}$ & $\begin{array}{l}\text { Kamenik } \\
{ }^{38} \mathrm{U}, \mathrm{Ku} \text { et } \\
\text { ratio of } 0 . \\
\text { uranium } \\
4 \text { and } 1 \mathrm{w} \\
\text { o of } 0.046\end{array}$ & $\begin{array}{l}\text { 2013; for } \\
\text { 77. Liter } \\
\text { petween } \\
\text { converte } \\
\text { om Ku et } \\
\text { jeen }{ }^{235} \mathrm{U}\end{array}$ & $\begin{array}{l}\text { and }{ }^{240} \mathrm{P} \\
\text { data for } \\
\text { and }{ }^{239} \mathrm{P} \\
\text { data for } \\
977 \text {, and } \\
{ }^{234} \mathrm{U} \text { in na }\end{array}$ & $\begin{array}{l}\text { rovinec e } \\
{ }^{0} \mathrm{Pu} \text { were } \\
\text { e ratio is } \\
\text { dual radi } \\
2 \text { was es } \\
\text { I uranium }\end{array}$ & $\begin{array}{l}2005 \text { (for } \\
\text { lerted to } \\
\text { verage of } \\
\text { ides }\left({ }^{234} \mathrm{U} \text {, }\right. \\
\text { ed by }\end{array}$ \\
\hline
\end{tabular}

The seawater concentrations of ${ }^{239} \mathrm{Pu}$ and ${ }^{240} \mathrm{Pu}$ are two to four orders of magnitude lower than the concentrations of ${ }^{137} \mathrm{Cs}$ and uranium isotopes; therefore, they contribute little influence to the overall impact caused by radionuclides to the marine ecosystem. The sum of ratios of the North Pacific seawater concentrations to the BCGs (0.0123) is also well below the threshold value of 1 , which indicates that the potential radiation doses to marine species in North Pacific Ocean are well below the target dose limit of $1 \mathrm{rad} /$ day set by DOE (2002a) to protect aquatic organisms.

Except for the concentrations of ${ }^{137} \mathrm{Cs}$, the reported concentrations for other radionuclides in North Pacific Ocean corresponded to year 2000 or earlier. Due to radioactive decay, present concentrations of these radionuclides are expected to be lower than the listed values. The reported ${ }^{137}$ Cs concentrations in Pacific Ocean corresponded to year 2012 after the Fukushima Dai-ichi incident and were measured in the Pacific Ocean north of the Hawaiian Islands (low value) and between Japan and Hawaii (high value) (Kamenik et al. 2013). According to the results of a mathematical modeling performed by Nakano and Povince (2012), the maximum ${ }^{137} \mathrm{Cs}$ concentration predicted for the Northwest Pacific Ocean after the Fukushima Dai-ichi incident was 21 becquerels per cubic meter in 2012, which converts to 0.567 picocuries per liter (pCi/L). The representative ${ }^{137} \mathrm{Cs}$ concentrations selected for Amchitka and Adak islands are about 30-40 percent of this modeled maximum, which provides indication of the level of conservatism associated with the seawater concentrations selected for Amchitka and Adak for this evaluation. 


\subsection{Tritium Concentrations}

The third DQO outlined in the 2011 Sampling Plan (DOE 2011) was to collect seawater samples off the coastline of the Cannikin site on the Bering Sea side of Amchitka, and from seawater samples collected from other Amchitka and Adak locations. Baseline tritium concentrations in seawater are being established starting with the tritium data collected in 2011 for seawater near Amchitka and Adak. The results from numerous historical sampling events have not adequately addressed whether measured values of tritium in seawater near Amchitka are from global fallout or whether they are related to the Amchitka nuclear tests. Tritium is one of the first radionuclides that would be seen in any leakage occurring from the underground test in the first 200 years.

Tritium (half-life $=12.43$ years) is the radioactive isotope of hydrogen that decays by beta to the noble gas helium-3. Tritium is produced naturally by interaction of cosmic rays with nitrogen and oxygen mainly in the upper atmosphere, and after oxidation to tritiated water, takes part in the hydrologic cycle. Shortly after the discovery of natural tritium in the environment, the potential of tritium as a tracer of water movement in natural water systems, including the ocean, was recognized (Lamont-Doherty Earth Observatory 2013).

Starting in the early 1950s, tritium from anthropogenic sources (mainly nuclear weapons tests) was added to the atmosphere in considerable amounts. By the mid-1960s, the natural background of tritium in precipitation was masked completely by so-called bomb tritium. The bomb tritium severely limited the use of natural tritium as a tracer because very few uncontaminated tritium data from the pre-bomb era are available. However, the anthropogenic tritium offered a new tool for studies of water movement in natural systems, that is, a dye introduced into the environment on a global scale at a relatively well-known rate. During the last 2 decades bomb tritium has developed into a routine parameter in studies of natural water systems.

Natural tritium concentrations in surface waters are of the order of $10^{-18}$ tritium atoms per hydrogen atom. For this reason, tritium concentrations are reported as Tritium Units (TU), which derive from a tritium-to-hydrogen ratio $([\mathrm{T}] /[\mathrm{H}])$ of $10^{-18}$ (1 TU is equivalent to an activity of $3.2 \mathrm{pCi} / \mathrm{L}$ in water). The production rate of natural tritium is about $0.5 \pm 0.3$ atoms tritium per square centimeter, leading to natural tritium values in mean continental precipitation of about $5 \mathrm{TU}(16 \mathrm{pCi} / \mathrm{L})$.

A major part of the bomb tritium was injected into the stratosphere, from where it mixed down into the troposphere and participates in the hydrologic cycle. Transfer to the surface waters of the oceans, which are the largest sink for tritium, occurs by water vapor exchange, precipitation, and continental runoff. Because most of the nuclear weapons tests were performed in the Northern Hemisphere, the tritium distribution is strongly asymmetric with relatively high concentrations in the Northern Hemisphere compared to those in the Southern Hemisphere.

Present day tritium concentrations (natural tritium and anthropogenic tritium) are of the order of $10 \mathrm{TU}(32 \mathrm{pCi} / \mathrm{L})$ in continental precipitation. In the ocean, the maximum tritium concentrations observed in the Northern Hemisphere surface water are of the order of $2 \mathrm{TU}(6.4 \mathrm{pCi} / \mathrm{L})$. Water bodies in regions with high runoff components, such as the Arctic Ocean, or a location surrounded by continents, such as the Mediterranean Sea, have elevated tritium concentrations. Present day tritium concentrations in Southern Ocean surface waters are of the order of $0.15 \mathrm{TU}$ $(0.48 \mathrm{pCi} / \mathrm{L})$. 
During a 2-year survey of the Aleutian Islands performed as part of ADEC's Alaska Monitoring and Assessment Program, seawater was sampled at random stations along the entire chain and analyzed for tritium (ADEC 2012). This sampling program covered the eastern part of the Aleutian Islands in 2006 and the western Aleutian Islands in 2007, during which a subset of seawater samples was collected off the coast of the Cannikin site.

Table 35 contains information on the mean, standard deviation, median, and range of each data set. The comparison, taking into account the MDC of measurement, shows that the activity of tritium in the marine environment surrounding Amchitka and Adak is similar to global fallout concentrations (i.e., previous sampling results). Data will be collected in subsequent sampling events to analyze trends.

Table 35. Comparison Statistics for Tritium

\begin{tabular}{||l|c|c|c|c|c|c|c||}
\hline \hline Tritium Sampling Effort & $\boldsymbol{n}$ & Mean & $\mathbf{\pm}$ & SD & Median & Range & Range of MDCs \\
\hline Eastern Aleutians $2006^{\mathrm{a}}$ & 62 & 4.3 & \pm & 2.5 & 4.2 & $-1.9-9.9$ & $6.6-11$ \\
\hline${\text { Western Aleutians } 2007^{\mathrm{a}}}^{\mathrm{a}}$ & 59 & 2.8 & \pm & 2.0 & 2.5 & $-0.7-9.1$ & $3.8-7.1$ \\
\hline Cannikin 2007 $^{\mathrm{a}}$ & 39 & 3.1 & \pm & 1.3 & 2.8 & $1.1-6.1$ & $2.6-5.3$ \\
\hline Cannikin 2011 & 69 & 1.6 & \pm & 0.2 & 1.6 & $1.2-2.2$ & 0.30 \\
\hline Long Shot 2011 & 9 & 1.7 & \pm & 0.2 & 1.7 & $1.5-2.0$ & 0.30 \\
\hline Milrow 2011 & 9 & 1.9 & \pm & 0.2 & 1.9 & $1.6-2.2$ & 0.30 \\
\hline Adak North 2011 & 9 & 1.7 & \pm & 0.2 & 1.7 & $1.5-2.2$ & 0.30 \\
\hline Adak South 2011 & 9 & 1.9 & \pm & 0.5 & 1.8 & $1.4-2.8$ & 0.30 \\
\hline Adak 2011 & 18 & 1.8 & \pm & 0.4 & 1.8 & $1.4-2.8$ & 0.30 \\
\hline
\end{tabular}

2006-2007 Alaska Monitoring and Assessment Program Data (ADEC 2012)

All units in $\mathrm{pCi} / \mathrm{L}$

$n=$ number of samples; $\mathrm{SD}=$ standard deviation; $\mathrm{MDC}=$ minimum detectable concentration

For all results reported here, no laboratory or data validation qualifiers were reported.

Duplicate samples were excluded from this summary. 


\subsection{Cesium-137 Concentrations in Lichen, Soil Beneath Lichen, and Marine Sediment}

The fourth DQO outlined in the 2011 Sampling Plan (DOE 2011) was to collect star reindeer lichen, soil beneath the lichen, and marine sediment and analyze those samples for ${ }^{137} \mathrm{Cs}$. Radioactive materials remain in the subsurface test cavities of the three nuclear test sites on Amchitka Island. Reindeer lichen, soil beneath the lichen, and marine sediment at Amchitka and the reference Adak site could provide information on whether the reindeer lichen or marine sediment have retained radionuclides and could provide additional data to support the interpretation of environmental monitoring results being collected for the Amchitka site.

The analytical data are used to establish baseline radionuclide $\left({ }^{134} \mathrm{Cs}\right.$ and $\left.{ }^{137} \mathrm{Cs}\right)$ concentrations in star reindeer lichen, the soil beneath the lichen, and marine sediment. The data from the 2011 sampling event are also compared with available data from literature sources or previous sampling events as available. Indications of uptake by star reindeer lichen, the soil beneath the lichen, and marine sediment will be considered in the planning for upcoming data collection.

On March 11, 2011, more than 3 months before LM's biological sampling event on Amchitka and Adak Islands, a 9.0 magnitude earthquake and subsequent massive tsunami struck the island of Japan, severely damaging several reactors at the Fukushima Dai-ichi Nuclear Power Plant and releasing radioactive materials into the environment. To determine what this direct release of radioactive materials into the atmosphere might have contributed to the background radiation on Amchitka and Adak Islands, semiquantitative gamma spectrometry measurements were made during the data validation process. The results imply that Dolly Varden, rockweed, and to a lesser extent, Irish lord appear to contain a significant cesium isotope signature from Fukushima Dai-ichi. The estimated ${ }^{134} \mathrm{Cs} /{ }^{137} \mathrm{Cs}$ activity ratios in pooled fauna samples at the time sampled ranged from $<30$ to about 60 percent. Observations of Fukushima-derived fallout impacting on this region are supported by findings of elevated levels of ${ }^{134} \mathrm{Cs}$ (and ${ }^{137} \mathrm{Cs}$ ) in lichen and soil collected from both the Adak and Amchitka regions. The activity concentrations of ${ }^{137} \mathrm{Cs}$ reported in Table 36 therefore represent maximal expected values measured without consideration of beta activity interference derived from ${ }^{134} \mathrm{Cs}$ (Hamilton et al. 2012b).

Table 36 through Table 39 summarize the sampling data for lichen, soil, and sediment collected at Amchitka and Adak in 2011. The concentrations vary significantly among different samples, especially ${ }^{137} \mathrm{Cs}$ concentrations in soil, for which the maximum values are 5-20 times higher than the minimum values. Sampling data of both lichen and soil show that ${ }^{134} \mathrm{Cs}$ and ${ }^{137} \mathrm{Cs}$ levels in Adak are higher than the levels in Amchitka.

More sediment samples were collected than reported here. Sediment samples were to be split with UAF, instead the entire sample was shipped to UAF and no split sample was sent to LLNL. The 2011 sediment samples currently in UAF's possession will be split at a later date and processed as part of the 2016 sampling event.

A large discrepancy was apparent in a comparison of the ratios calculated (based on average concentrations) from lichen and soil data shown in Tables 37 and 38. The ratio is about 27 with soil data but is about 1.2 with lichen data. For comparison, the ratio is about 2.2 with sediment data. The high ratio may be attributable to fallout due to the Fukushima Dai-ichi event. 
Table 36. ${ }^{134} \mathrm{Cs}$ and ${ }^{137} \mathrm{Cs}$ in Lichen, Soil Beneath the Lichen, and Marine Sediment, Amchitka and Adak Islands

\begin{tabular}{|c|c|c|c|c|c|c|c|c|c|}
\hline $\begin{array}{c}\text { Sample } \\
\text { ID }\end{array}$ & $\begin{array}{l}\text { Location } \\
\text { Code }\end{array}$ & $\begin{array}{c}\text { Sample } \\
\text { Types }\end{array}$ & $\begin{array}{l}\text { Dryl } \\
\text { wet } \\
\text { (\%) }\end{array}$ & $\begin{array}{c}\text { Ash/ } \\
\text { dry } \\
\text { (\%) }\end{array}$ & ${ }^{137} \mathrm{Cs}$ & Flag & ${ }^{134} \mathrm{Cs}$ & Flag & $\begin{array}{c}{ }^{134} \mathrm{Cs} /{ }^{137} \mathrm{Cs} \\
\text { Activity } \\
\text { Ratio }\end{array}$ \\
\hline \multicolumn{10}{|c|}{ Soil (pCi/kg dry wt) } \\
\hline JET-741 & SEDI-LS-LT1-1 & \begin{tabular}{|l} 
Marine \\
Sediment $^{\mathrm{a}}$
\end{tabular} & 81.9 & 99.0 & $36.3 \pm 5.6$ & - & 14 & U & - \\
\hline JET-743 & SEDI-ML-MT1-1 & \begin{tabular}{|l} 
Marine \\
Sediment
\end{tabular} & 64.4 & 95.9 & $11.9 \pm 3.6$ & - & 7 & U & - \\
\hline JDQ-904 & SOIL-AD-XXX-1 & Terrestrial Soil & 38.7 & 73.3 & $7264 \pm 114$ & - & 180 & $\mathrm{U}$ & - \\
\hline JDQ-905 & SOIL-AD-XXX-2 & Terrestrial Soil & 47.6 & 22.6 & $1135 \pm 26$ & - & $56 \pm 12$ & - & $0.05 \pm 0.01$ \\
\hline JDQ-906 & SOIL-AD-XXX-3 & Terrestrial Soil & 15.0 & 28.1 & $6591 \pm 133$ & - & $319 \pm 60$ & - & $0.05 \pm 0.01$ \\
\hline JDQ-901 & SOIL-AI-ML-1 & Terrestrial Soil & 68.1 & 79.1 & $312 \pm 22$ & - & 48 & $U$ & - \\
\hline JDQ-902 & SOIL-AI-LT1-2 & Terrestrial Soil & 12.5 & 73.5 & $6110 \pm 121$ & - & 221 & $\mathrm{U}$ & - \\
\hline JDQ-903 & SOIL-AI-CT1-3 & Terrestrial Soil & 33.8 & 69.0 & $2174 \pm 30$ & - & 50 & $U$ & - \\
\hline \multicolumn{10}{|c|}{ Lichen (pCi/kg wet wt) } \\
\hline JDQ-895 & STRL-AI-ML-1 & Lichen & 27.9 & 1.6 & $550 \pm 7$ & - & $460 \pm 4.2$ & - & $0.84 \pm 0.01$ \\
\hline JDQ-896 & STRL-AI-LT1-2 & Lichen & 33.0 & 1.5 & $404 \pm 7$ & - & $287 \pm 3.8$ & - & $0.71 \pm 0.02$ \\
\hline JDQ-897 & STRL-AD-XXX-1 & Lichen & 51.1 & 2.8 & $6236 \pm 42$ & - & $5352 \pm 25$ & - & $0.86 \pm 0.01$ \\
\hline JDQ-898 & STRL-AI-CT1-1 & Lichen & 63.1 & 12.1 & $1523 \pm 14$ & - & $\begin{array}{c}1282 \pm \\
8.4\end{array}$ & - & $0.84 \pm 0.01$ \\
\hline JDQ-899 & STRL-AD-XXX-2 & Lichen & 40.5 & 4.5 & $3238 \pm 119$ & - & $2666 \pm 11$ & - & $0.82 \pm 0.01$ \\
\hline JDQ-900 & STRL-AD-XXX-3 & Lichen & 62.9 & 4.1 & $2855 \pm 20$ & - & $2269 \pm 12$ & - & $0.79 \pm 0.01$ \\
\hline $\begin{array}{l}\text { JDQ-900- } \\
\text { DUP }\end{array}$ & STRL-AD-XXX-3 & Lichen & 63.7 & 4.1 & $2951 \pm 26$ & - & $2426 \pm 16$ & - & $0.82 \pm 0.01$ \\
\hline $\begin{array}{l}\text { JDQ-900- } \\
\text { DUP }\end{array}$ & STRL-AD-XXX-3 & Lichen & 63.7 & 4.1 & $2951 \pm 26$ & - & $2426 \pm 16$ & - & $0.82 \pm 0.01$ \\
\hline
\end{tabular}

${ }^{a}$ Marine sediment collected by the land-based team from the intertidal zone adjacent the Long Shot and Milrow areas.

Table 37. ${ }^{134}$ Cs and ${ }^{137}$ Cs Data for Lichen Collected at Amchitka and Adak in 2011

\begin{tabular}{||l|c|c|c|c||}
\hline \multirow{2}{*}{ Data Attribute } & \multicolumn{2}{|c|}{ Amchitka } & \multicolumn{2}{c||}{ Adak } \\
\cline { 2 - 5 } & ${ }^{134}$ Cs & ${ }^{137}$ Cs & ${ }^{134}$ Cs & ${ }^{137}$ Cs \\
\hline Number of samples & 3 & 3 & 3 & 3 \\
\hline \multirow{2}{*}{ Minimum concentration } & 287 (wet wt.) & 404 (wet wt.) & 2,269 (wet wt.) & 2,855 (wet wt.) \\
& 1,340 (dry wt.) & 1,890 (dry wt.) & 10,600 (dry wt.) & 13,300 (dry wt.) \\
\hline \multirow{2}{*}{ Maximum concentration } & 1,282 (wet wt.) & 1,523 (wet wt.) & 5,352 (wet wt.) & 6,236 (wet wt.) \\
& 5,990 (dry wt.) & 7,120 (dry wt.) & 25,000 (dry wt.) & 29,100 (dry wt.) \\
\hline \multirow{2}{*}{ Mean concentration } & 676 (wet wt.) & 826 (wet wt.) & 3,429 (wet wt.) & 4,110 (wet wt.) \\
& 3,160 (dry wt.) & 3,860 (dry wt.) & 16,000 (dry wt.) & 19,200 (dry wt.) \\
\hline \multirow{2}{*}{ Median concentration } & 460 (wet wt.) & 550 (wet wt.) & 2,666 (wet wt.) & 3,238 (wet wt.) \\
& 2,150 (dry wt.) & 2,570 (dry wt.) & 12,500 (dry wt.) & 15,100 (dry wt.) \\
\hline \multirow{2}{*}{ Standard deviation } & 434 (wet wt.) & 497 (wet wt.) & 1,369 (wet wt.) & 1,512 (wet wt.) \\
& 2,030 (dry wt.) & 2,320 (dry wt.) & 6,400 (dry wt.) & 7,070 (dry wt.) \\
\hline \hline
\end{tabular}

Units are $\mathrm{pCi} / \mathrm{kg}$.

Note: The 2011 sampling data are wet-weight based, which were converted to dry-weight-based concentrations using a dry-to-wet weight ratio of 0.214 for lichen (Hamilton et al. 2012a). 
Table 38. ${ }^{134}$ Cs and ${ }^{137}$ Cs Data for Soil Collected at Amchitka and Adak in 2011

\begin{tabular}{||l|c|c|c|c||}
\hline \multirow{2}{*}{\multicolumn{1}{|c|}{ Data Attribute }} & \multicolumn{2}{c|}{ Amchitka } & \multicolumn{2}{c||}{ Adak } \\
\cline { 2 - 5 } & ${ }^{\mathbf{1 3 4}} \mathbf{C s}$ & ${ }^{\mathbf{1 3 7}} \mathbf{C s}$ & ${ }^{\mathbf{1 3 4}} \mathbf{C s}$ & ${ }^{\mathbf{1 3 7}} \mathbf{C s}$ \\
\hline Number of samples & 3 & 3 & 3 & 3 \\
\hline Minimum concentration & 48 & 312 & 56 & 1135 \\
\hline Maximum concentration & 221 & 6110 & 319 & 7264 \\
\hline Mean concentration & 106 & 2865 & 185 & 4997 \\
\hline Median concentration & 50 & 2174 & 180 & 6591 \\
\hline Standard deviation & 81 & 2417 & 107 & 2744 \\
\hline
\end{tabular}

Units are $\mathrm{pCi} / \mathrm{kg}$.

Table 39. ${ }^{134}$ Cs and ${ }^{137}$ Cs Data for Sediment Collected at Amchitka and Adak in 2011

\begin{tabular}{||l|c|c|c|c||}
\hline \multirow{2}{*}{\multicolumn{1}{c|}{ Data Attribute }} & \multicolumn{2}{c|}{ Amchitka } & \multicolumn{2}{c||}{ Adak } \\
\cline { 2 - 5 } & ${ }^{134}$ Cs & ${ }^{137}$ Cs & ${ }^{134}$ Cs & ${ }^{137}$ Cs \\
\hline Number of samples ${ }^{a}$ & 2 & 2 & - & - \\
\hline Minimum concentration & 7 & 12 & - & - \\
\hline Maximum concentration & 14 & 36 & - & - \\
\hline Mean concentration & 11 & 24 & - & - \\
\hline
\end{tabular}

Units are $\mathrm{pCi} / \mathrm{kg}$.

Note: "-" data not available.

a Marine sediment collected by the land-based team from the intertidal zone adjacent the Long Shot and Milrow areas.

\section{Comparison of 2011 Lichen Data with Data from Literature Sources}

${ }^{137} \mathrm{Cs}$ concentrations in lichen have been used as indicators for studying the influence of global fallout in the Arctic region. Ranges of ${ }^{137} \mathrm{Cs}$ concentration reported in past sampling activities at Amchitka and Adak are summarized in Table 40. The reported concentrations are based on dry-weight material.

Table 40. ${ }^{137}$ Cs in Lichen at Amchitka and Adak Island 1970-1997

\begin{tabular}{|c|c|c|}
\hline Location & Number of Samples & Range of Data \\
\hline \multicolumn{3}{|c|}{${ }^{137} \mathrm{Cs}$ in Lichen Collected at Amchitka Island in $1970-71^{\mathrm{a}}$} \\
\hline Clam Lake & 7 & $8000 \pm 90-27,000 \pm 500$ \\
\hline \multicolumn{3}{|c|}{${ }^{137}$ Cs in Lichen Collected at Amchitka Island in 1971-79 } \\
\hline Clam Lake & 15 & $1500 \pm 3000-67,000 \pm 500$ \\
\hline Icebox Lake & 10 & $3000 \pm 80-16,000 \pm 300$ \\
\hline Cannikin Lake & 10 & $2500 \pm 120-21,000 \pm 200$ \\
\hline \multicolumn{3}{|c|}{${ }^{137} \mathrm{Cs}$ in Lichen Collected at Amchitka and Adak in $1997^{\mathrm{c}}$} \\
\hline Amchitka Island & 4 & $62 \pm 10-74 \pm 12$ \\
\hline Adak Island & 3 & $24 \pm 20-24 \pm 20$ \\
\hline
\end{tabular}

Unit is $\mathrm{pCi} / \mathrm{kg}$, dry wt., 2-sigma counting error.

${ }^{a}$ DOE 1982, Table 19

${ }^{\mathrm{b}}$ DOE 1982, Table 20

${ }^{\mathrm{c}}$ DOE 2000, Page 4.4 
The interisland comparison of the 2011 mean concentrations of ${ }^{137} \mathrm{Cs}$ in lichen from Amchitka and Adak (Table 37) shows that the mean concentration at Adak (19,200 pCi/kg dry wt.) is much higher than that at Amchitka $(3,860 \mathrm{pCi} / \mathrm{kg}$ dry wt.). Differences between radionuclide concentrations at the two islands are attributed to differing annual rainfall (56 inches at Adak, 33 inches at Amchitka) (Armstrong 1977). The $2011{ }^{137}$ Cs concentrations in lichen from Amchitka and Adak (Table 37) are higher than concentrations from the last sampling event (i.e., 1997), as shown in Table 40.

A complicating factor in evaluating ${ }^{137} \mathrm{Cs}$ results on Amchitka Island is the geographic situation of the island. Located at $51^{\circ}$ North, $179^{\circ}$ East, fallout would be expected to be at a maximum from detonations at Lop Nor, China (1964-1996), radioactive contamination from the Chernobyl and Fukushima Dai-ichi nuclear power plants (1986 and 2010, respectively), and worldwide fallout that is annually transferred to the troposphere through breaks in the tropopause located at $40^{\circ}$ North and $40^{\circ}$ South latitudes. Thus, the rates of fallout on Amchitka Island may have varied severalfold within a year from the 1970 s to 2010 due to such conditions. This may account for the substantial variability in radionuclide concentrations reported for samples of the same species collected at different times for the year; however, some variability in those values is probably due to ecological and biological factors that affect the metabolic rate of organisms (DOE 2000). Similar conclusions were reported by Seymour and Nelson (1977). 


\subsection{Summary and Conclusions}

DOD and AEC conducted underground nuclear tests during the middle 1960s and early 1970s on Amchitka Island, near the western end of the Aleutian Islands. Radioactive fission products remain in and around the subsurface cavities formed by the nuclear detonations. Data collected since the detonations do not indicate that the radionuclides have migrated from the test cavities to the accessible environment.

The primary focus of LM's long-term stewardship of the Amchitka Island sites is to ensure that conditions at Amchitka remain protective of human health and the environment. This was carried out by collecting data to address the four DQOs discussed in Section 2 of this report.

The first DQO addressed the Aleut consumption of subsistence- and commercial-catch seafood. LM achieved this DQO by collecting samples of marine species near Amchitka and analyzing the samples for selected radioisotopes indicative of the presence of test-related contamination. These radionuclides have other sources, including global fallout and natural occurrence in rocks, such that an understanding of background concentrations is important for monitoring. For the 2011 sampling event, a group of stakeholders that included LM, ADEC, and APIA identified 14 biological species to collect and analyze. A similar sampling event was conducted at Adak Island to assess conditions at a reference location. The species were chosen to represent the subsistence- and commercial-catch seafood present in the western Aleutian Islands.

The risk estimates for the five diets evaluated indicate that overall potential risks to an Aleut from the ingestion of seafood based on the samples collected from Amchitka and Adak in 2011 are similar and are at $2 \times 10^{-5}$ or lower (i.e., within EPA's acceptable risk range of $1 \times 10^{-6}$ to $\left.1 \times 10^{-4}\right)$. The primary contributors to the risk estimates are the ${ }^{137} \mathrm{Cs}$ and ${ }^{234,235,238} \mathrm{U}$ concentrations.

The risk estimates using Amchitka data are slightly higher than those using Adak data by $1.4 \times 10^{-6}$. This is lower than the $1 \times 10^{-5}$ risk level in excess of background (in this case, estimates from Adak are used as reference or background values) used by the State of Alaska as the benchmark for acceptable risk. Hence, based on the 2011 data collected for Amchitka and Adak, seafood harvested at Amchitka and Adak are considered safe for consumption at the intake levels for the five diets evaluated. These results confirmed earlier investigations, which showed that subsistence- and commercial-catch seafood is safe for human consumption. In addition, the potential increase in cesium concentrations observed in the samples due to the Fukushima Dai-ichi event has not resulted in unacceptable risk estimates.

The second DQO provided the rationale for using biota tissue data collected as input to the RESRAD-BIOTA code to estimate potential risks to these species from the radionuclides. This risk analysis was conducted by deriving representative radionuclide concentrations in seawater based on the tissue data reported for the biological species for comparison with the BCGs for water. The sum of ratios of radionuclide concentrations to BCGs was much lower than the threshold value of 1 ; therefore, the potential radiation doses to marine species are far below the target dose level of $0.1 \mathrm{rad} / \mathrm{day}$, and no further analysis was required. 
The third DQO supported the collection of tritium data in seawater. Tritium is the primary radionuclide at this time that would indicate leakage if concentrations exceed natural or global fallout values. Seawater samples were collected offshore of Amchitka in areas where groundwater may discharge to the sea. The analyses showed that the concentrations of tritium in the marine environment surrounding Amchitka and Adak are similar to global fallout concentrations measured previously by others.

Finally, the fourth DQO supported the collection of reindeer lichen and soil beneath the lichen and marine sediment. The ${ }^{137} \mathrm{Cs}$ concentrations varied significantly between different samples, especially ${ }^{137} \mathrm{Cs}$ concentrations in soil beneath the lichen, for which the maximum values were 5-20 times higher than the minimum values. The lichen and soil beneath the lichen showed that ${ }^{137} \mathrm{Cs}$ levels in Adak were higher than the levels in Amchitka. The concentrations of ${ }^{137} \mathrm{Cs}$ in samples collected from Amchitka and Adak indicate that atmospheric transport of airborne contamination from the Fukushima Dai-ichi event may have contributed to the levels detected. 


\subsection{References}

ADEC (Alaska Department of Environmental Conservation), 2004. Letter from John Halverson, Re: Amchitka, Alaska, Underground Nuclear Test Site-Amchitka Surface Closure Report, dated July 2003, September 27, 2004.

ADEC (Alaska Department of Environmental Conservation), 2012. Personal communication with Terri Lomax, Alaska Monitoring \& Assessment Program, Re: tritium data collected in the eastern Aleutian Islands in 2006 and in the western Aleutian islands in 2007 with additional tritium data collected off the coast of the Cannikin site, October 22.

Armstrong, R.H., 1977. "Weather and Climate," in The Environment of Amchitka Island, Alaska, TID-26712, M.L Merritt and R.G. Fuller (eds.), Technical Information Center, Energy Research and Development Administration, Washington, DC, September.

Bartell, S.M., E. Nobmann, and R.A. Ponce, 1999. What People Eat: Atka, Alaska, 1998-1999, Final Report, Project PF901329, submitted to University of Alaska and Aleutian/Pribilof Islands Association, Inc., IDM Consulting, Anchorage, Alaska, December.

Burger, J., M. Gochfeld, D.S. Kosson, C.W. Powers, S. Jewett, B. Friedlander, H. Chenelot, C.D. Volz, and C. Jeitner, 2006. "Radionuclides in marine macroalgae from Amchitka and Kiska islands in the Aleutians: establishing a baseline for future biomonitoring," Journal of Environmental Radiochemistry, 91: 27-40.

Burger, J., M. Gochfeld, and S.C. Jewett, 2007a. "Radionuclide Concentrations in Benthic Invertebrates from Amchitka and Kiska Islands in the Aleutian Chain, Alaska," Environmental Monitoring Assessment, 128 (2007): 329-341.

Burger, J., M. Gochfeld, D.S. Kosson, C.W. Powers, B. Friedlander, M. Stabin, D. Favret, S. Jewett, D. Snigaroff, T. Stamm, J. Weston, C. Jeitner, and C.D. Volz, 2007b. "Radionuclides in Marine Fishes and Birds from Amchitka and Kiska Islands in the Aleutians: Establishing a Baseline," Health Physics, 92 (2007): 265-278.

CRESP (Consortium for Risk Evaluation with Stakeholder Participation II), 2005. Amchitka Independent Science Assessment: Biological and Geophysical Aspects of Potential Radionuclide Exposure in the Amchitka Marine Environment, C.W. Powers, J. Burger, D. Kosson, M. Gochfeld, and D. Barnes, eds., Piscataway, New Jersey, August.

Dasher, D., W. Hanson, S. Read, S.H. Faller, D.E. Farmer, W. Efurd, J. Kelley, and R. Patrick, 2002. "An Assessment of the Reported Leakage of Anthropogenic Radionuclides from the Underground Nuclear Tests at Amchitka Island, Alaska, USA, to the Surface Environment," Journal of Environmental Radioactivity, 60(2002): 165-187.

DOE (U.S. Department of Energy), 1982. Amchitka Radiobiological Program Final Report July 1970 to December 1979, DOE/DP00269-39, November.

DOE (U.S. Department of Energy), 2000. Amchitka Island, Alaska, Special Sampling Project 1997, DOE/NV-624, Rev. 1, Las Vegas, Nevada, June. 
DOE (U.S. Department of Energy), 2001. Letter reply from K.A. Carlson, DOE Nevada Operations Office, to G. Siekaniec, U.S. Fish and Wildlife Service Manager, Alaska Maritime National Wildlife Refuge, Letter of Agreement for Contaminated Site Action List, Amchitka Island, Alaska, Las Vegas, Nevada, April 4.

DOE (U.S. Department of Energy), 2002a. Modeling Groundwater Flow and Transport of Radionuclides at Amchitka Island's Underground Nuclear Tests: Milrow, Long Shot, and Cannikin, DOE/NV/11508-51, Las Vegas, Nevada, October.

DOE (U.S. Department of Energy), 2002b. A Graded Approach for Evaluating Radiation Doses to Aquatic and Terrestrial Biota. Technical Standard DOE-STD-1153-2002. Module 1-3, Washington, DC.

DOE (U.S. Department of Energy), 2008. Long-Term Surveillance and Maintenance Plan for the U.S. Department of Energy Amchitka, Alaska, Site, LMS/AMC/S01980, Office of Legacy Management, Grand Junction, Colorado, September.

DOE (U.S. Department of Energy), 2011. Sampling Plan for the Amchitka, Alaska, Site 2011 Sampling Event, LMS/AMC/S05725, Office of Legacy Management, Grand Junction, Colorado, March.

Eckerman, K.F., R.W. Leggett, C.B. Nelson, J.S. Puskin, and A.C.B. Richardson, 1999. Cancer Risk Coefficients for Environmental Exposures to Radionuclides, Federal Guidance Report No. 13, EPA 402-R-99-001, prepared by Oak Ridge National Laboratory for the U.S. Environmental Protection Agency Office of Radiation and Indoor Air.

EPA (U.S. Environmental Protection Agency), 1989. Risk Assessment Guidance for Superfund, Volume I: Human Health Evaluation Manual, Interim Final, OSWER Directive 9285.7-01a, Office of Emergency and Remedial Response, September.

EPA (U.S. Environmental Protection Agency), 1991a. Risk Assessment Guidance for Superfund: Volume I-Human Health Evaluation Manual (Part B, Development of Risk-Based Preliminary Remediation Goals), EPA/540/R-92/003, Interim, Office of Emergency and Remedial Response, Washington, DC, December.

EPA (U.S. Environmental Protection Agency), 1991b. Risk Assessment Guidance for Superfund: Volume I-Human Health Evaluation Manual (Part C, Risk Evaluation of Remedial Alternatives), EPA/540/R-92/004, Interim, Office of Emergency Response, Washington, DC, December.

EPA (U.S. Environmental Protection Agency), 1994. Estimating Radiogenic Cancer Risks, EPA/402/R-93/076, Washington, DC.

EPA (U.S. Environmental Protection Agency), 1997. Health Effects Assessment Summary Tables FY-1997 Update, EPA/540/R-97/036, Washington, DC, July. 
EPA (U.S. Environmental Protection Agency), 2002. Calculating Upper Confidence Limits for Exposure Point Concentrations at Hazardous Waste Sites, OSWER 9385.6-10, Office of Emergency and Remedial Response, December.

EPA (U.S. Environmental Protection Agency), 2004. Multi-Agency Radiological Laboratory Analytical Protocols Manual (MARLAP), NUREG-1576, EPA-402-B-04-001C, NTIS PB2004-105421, July.

ERICA, 2013. ERICA Assessment Tool, available for download at http://www.erica-tool.com/, accessed 15 July 2013.

FASSET (Framework for Assessment of Environmental Impact), 2003. Deliverable 5: Appendix 1, "Transfer Factor and Dose Conversion Coefficient Look-up Tables," Handbook for Assessment of the Exposure of Biota to Ionizing Radiation from Radionuclides in the Environment, A Project within the EC 5th Framework Programme, J. Brown et al., eds., Norwegian Radiation Protection Agency (NRPA), October.

FDA (U.S. Food and Drug Administration), 2009. Compliance Policy Guide, 560.750 Radionuclides in Imported Foods-Level of Concern, December 9.

FAO/WHO (Food and Agriculture Organization of the United Nations and the World Health Organization), 2004. Joint FAO/WHO Food Standards Programme.

Greenpeace, 1996. Nuclear Flashback: The Return to Amchitka, Washington, DC.

Hamilton, T.F., S.K.G. Peters, R.E. Martinelli, S.R. Kehl, and M.W. Tamblin, 2012a. Initial Sample Processing (Wet, Dry and Ash Weights), Interim Report, AMCHITKA PROJECT, WFO Proposal D13386, Lawrence Livermore National Laboratory, LLNL-SR-535833.

Hamilton, T.F., S.K.G. Peters, R.E. Martinelli, S.R. Kehl, and M.W. Tamblin, $2012 b$. Preliminary Data Analysis on ${ }^{137}$ Cs, Data Report_Part 3, Amchitka Project, WFO Proposal D13386, Lawrence Livermore National Laboratory, LLNL-SR-568300.

Hamilton, T.F., S.K.G. Peters, R.E. Martinelli, S.R. Kehl, and M.W. Tamblin, 2012c. Analysis of ${ }^{239} \mathrm{Pu}$ and ${ }^{240} \mathrm{Pu}$, Data Report-Part 2, Amchitka Project, WFO Proposal D13386, Lawrence Livermore National Laboratory, LLNL-SR-562257.

Hamilton, T.F., S.K.G. Peters, R.E. Martinelli, S.R. Kehl, and M.W. Tamblin, 2012d. Analysis of ${ }^{241}$ Am, Data Report - Part 4, Amchitka Project, WFO Proposal D13386, Lawrence Livermore National Laboratory, LLNL-SR-557014.

Hamilton, T.F., S.K.G. Peters, R.E. Martinelli, S.R. Kehl, and M.W. Tamblin, 2012e. Analysis of ${ }^{238} U,{ }^{235} U$, and ${ }^{234} U$, Data Report-Part 1, Amchitka Project, WFO Proposal D13386, Lawrence Livermore National Laboratory, LLNL-SR-562021.

Hamrick, K. and J. Smith, 2003. Final Report, Subsistence Food Use in Unalaska and Nikolski, prepared for Aleutian/Pribilof Islands Association, Institute for Circumpolar Health Studies, University of Alaska Anchorage, August. 
Hamrick, K., J. Smith, S. Doucette, R. Nichols, A. Taylor, and S. Smith, 2003. Dietary Benefits and Risks in Alaska Native Villages, St. Paul Dietary Survey Results, prepared for Aleutian/Pribilof Islands Association, Institute for Circumpolar Health Studies, University of Alaska Anchorage, March.

Hassan, A., and J. Chapman, 2006. Verification and Uncertainty Reduction of Amchitka Underground Nuclear Testing Models, DOE/NV/13609-46, Publication No. 451216, Las Vegas, Nevada, Desert Research Institute, February.

Hirose, K., E. Igarashi, E. Ochiai, H. Seya, and S. Matsui, 2006. "Plutonium Isotopes in Seawater of the North Pacific: Effects of Close-in Fallout," Radioactivity in the Environment, 8: $67-82$.

Howard, B.J., N.A. Beresford, D. Copplestone, D. Telleria, G. Proehl, S. Fesenko, R. Jeffree, T. Yankovich, J. Brown, K. Higley, M. Johansen, H. Mulye, H. Vandenhove, S. Gashchak, M.D. Wood, H. Takata, P. Andersson, P. Dale, J. Ryan, A. Bollhöfer, C. Doering, C.L. Barnett, and C. Wells, 2013. "The IAEA Handbook on Radionuclide Transfer to Wildlife," Journal of Environmental Radioactivity, 121: 55-74, July.

ISCORS, 2004. User's Guide: RESRAD-BIOTA: A Tool for Implementing A Graded Approach to Biota Dose Evaluation, ISCORS Technical Report 2004-02, DOE/EH-0676.

Kamenik, J., H. Dulaiova1, K.O. Buesseler, S.M. Pike, and K. Štastná, 2013. "Cesium-134 and 137 activities in the central North Pacific Ocean after the Fukushima Dai-ichi nuclear power plant accident," Biogeosciences Discussion, 10: 5223-5244.

Ku, T.-L., K.G. Knauss, and G.S. Mathieu, 1977. "Uranium in open ocean: concentration and isotopic composition," Deep Sea Research, 24(11): 1005-1017.

Lee, S.-H., J. Gastaud, P. Povinec, G.-H. Hong, S.-H. Kim, C.-S. Chung, K.-W. Lee, H.B.L. Pettersson, 2003. "Distribution of plutonium and americium in the marginal seas of the northwest Pacific Ocean," Deep Sea Research Part II: Topical Studies in Oceanography, 50(17-21): 2727-2750.

Lamont-Doherty Earth Observatory, 2013. World Ocean Circulation Experiment, Columbia University. http://www.ldeo.columbia.edu/res/fac/etg.tmp/text/woce_proj.html, accessed 15 July 2013.

LLNL (Lawrence Livermore National Laboratory), 2012. Amchitka Project WFO Proposal D13386, Final Report, LLNL-SR-595292, October.

Merritt, M.L., and R.G. Fuller (eds), 1977. The Environment of Amchitka Island, Alaska, TID-26712, Technical Information Center, Energy Research and Development Administration, Washington, DC. 
Nakano, M. and P.P. Povinec, 2012. "Long-term simulations of the ${ }^{137}$ Cs dispersion from the Fukushima accident in the world ocean," Journal of Environmental Radioactivity, 111(0): 109-115.

Povinec, P.P., K. Hiroseb, T. Hondaa, T. Itoa, E.M. Scott, and O. Togawa, 2004. "Spatial distribution of ${ }^{3} \mathrm{H},{ }^{90} \mathrm{Sr},{ }^{137} \mathrm{Cs}$ and ${ }^{239,240} \mathrm{Pu}$ in surface waters of the Pacific and Indian OceansGLOMARD database," Journal of Environmental Radioactivity, 76(1-2): 113-137.

Povinec, P.P., A. Aarkrog, K.O. Buesseler, R. Delfanti, K. Hirose, G.H. Hong, T. Ito, H.D. Livingston, H. Nies, V.E. Noshkin, S. Shima, and O. Togawa, 2005. " ${ }^{90} \mathrm{Sr},{ }^{137} \mathrm{Cs}$ and ${ }^{239,240} \mathrm{Pu}$ concentration surface water time series in the Pacific and Indian Oceans-WOMARS results," Journal of Environmental Radioactivity 81(1): 63-87.

Seymour, A.H., and V.A. Nelson, 1977. "Radionuclides in Air, Water, and Biota" in The Environment of Amchitka Island Alaska, TID-26712, M.L Merritt and R.G. Fuller (eds.), Technical Information Center, Energy Research and Development Administration, Washington, DC.

Spares, A.D., M.J.W. Stokesbury, T. McDermott, T. Zikovsky, T.S. Avery, and M.J. Dadswell, 2007. "Inferring marine distribution of Canadian and Irish Atlantic salmon (Salmo salar L.) in the North Atlantic from tissue concentrations of bio-accumulated caesium 137," ICES Journal of Marine Science: Journal du Conseil, 64(2): 394-404.

Tucker, S.I., I. Pazzia, D. Rowan, and J.B. Rasmussen, 1999. "Detecting pan-Atlantic migration in salmon (Salmo salar) using ${ }^{137} \mathrm{Cs}$," Canadian Journal of Fisheries and Aquatic Sciences, 56(12): 2235-2239.

UNSCEAR (United Nation's Scientific Committee on the Effects of Atomic Radiation), 1982. Ionizing Radiation: Sources and Effects, United Nations, New York, NY.

UNSCEAR (United Nation's Scientific Committee on the Effects of Atomic Radiation), 1988. Sources, Effects, and Risks of Ionizing Radiation, United Nations, New York, NY.

USFWS (U.S. Fish and Wildlife Service), 2000. Remedial/Removal Action Environmental Assessment and Finding of No Significant Impact, Amchitka Island, Alaska, Homer, Alaska, December. (includes a letter from the governor of Alaska stating disagreement with this document).

Zielinski, R.A., D.T. Chafin, E. R. Banta, and B. J. Szabo, 1997. "Use of 234U and 238U isotopes to evaluate contamination of near-surface groundwater with uranium-mill effluent: a case study in south-central Colorado", U.S.A. Environmental Geology 32 (2) September 1997 Springer-Verlag.

Zielinski, R.A., J.K. Otton, R.R. Schumann, and L. Wirt, 2008. "Uranium in surface waters and sediments affected by historical mining in the Denver West 1:100,000 quadrangle, Colorado", U.S. Geological Survey Scientific Investigations Report 2007-5246, 54 p. 
This page intentionally left blank 


\section{Appendix A}

Photographs of the Species Sampled 
This page intentionally left blank 


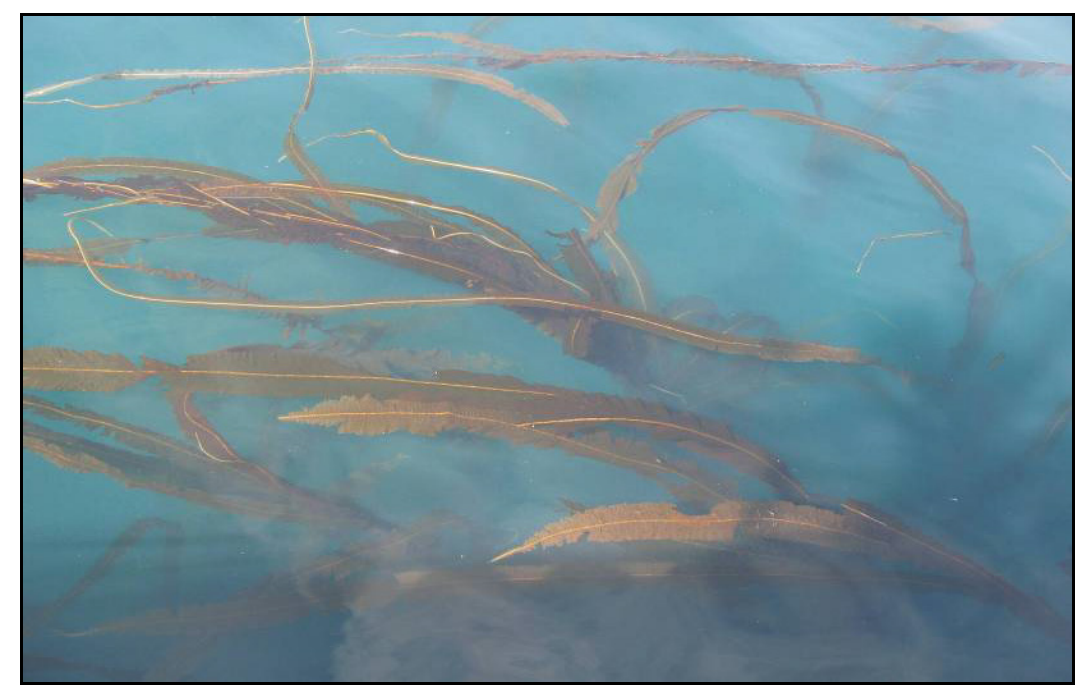

Dragon kelp (Eualaria fistulosa)

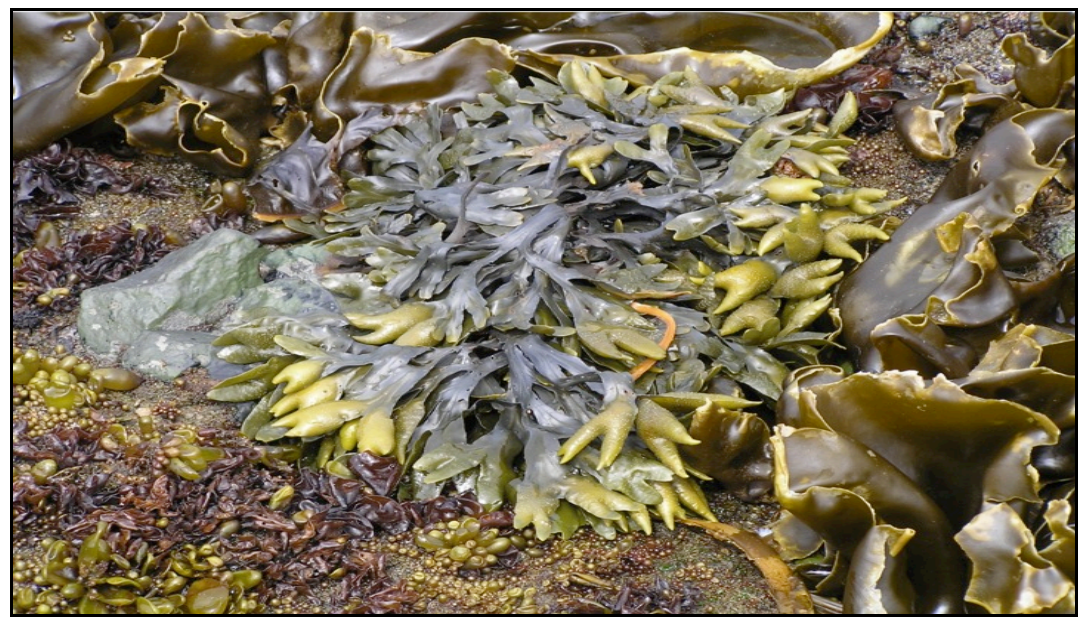

Rockweed (Fucus distichus)

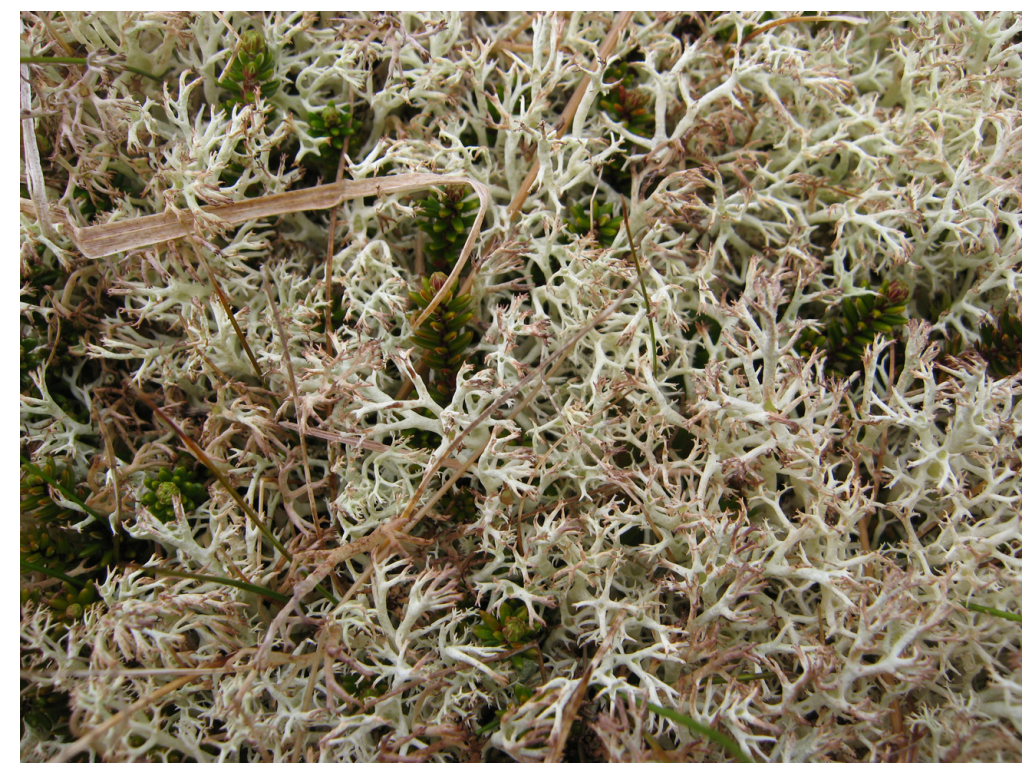

Star reindeer lichen (Cladina stellaris) 


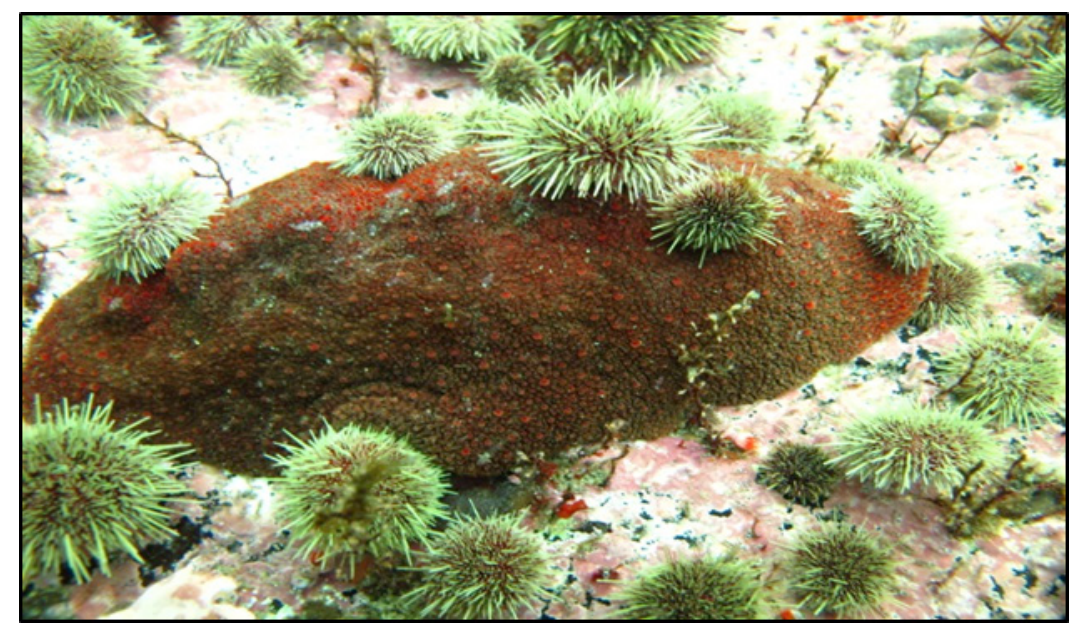

Gumboot or Pacific chiton (Cryptochiton stelleri)

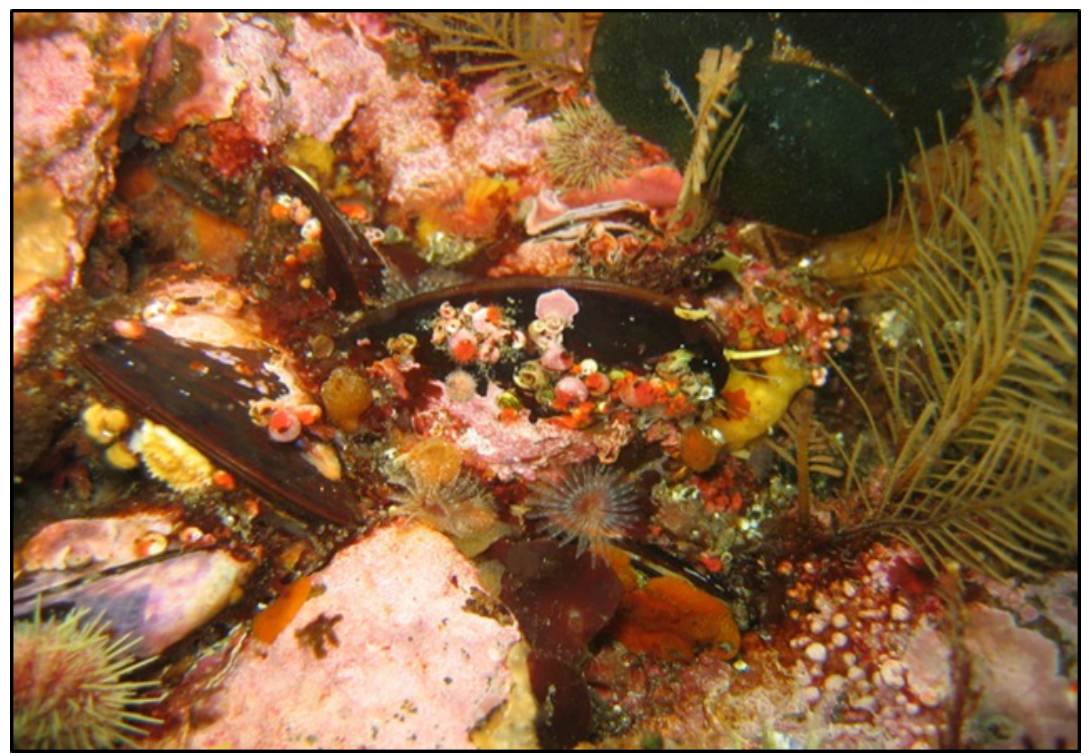

Horse mussel (Modiolus modiolus)

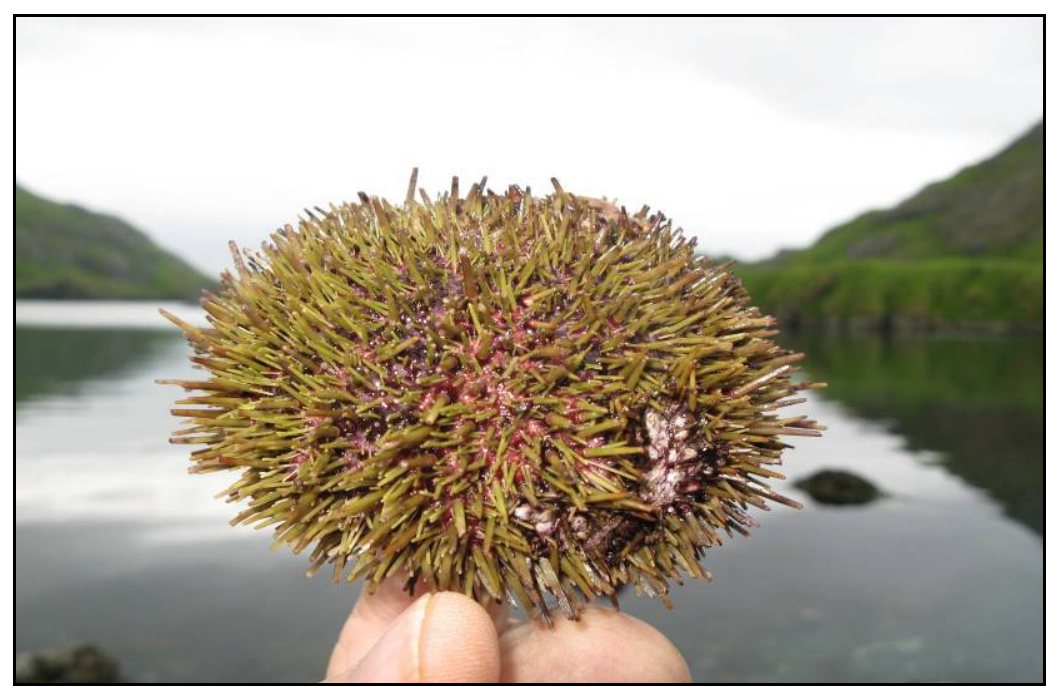

Sea urchin (Strongylocentrotus polyacanthus)

Amchitka Island, Alaska, Biological Monitoring Report, 2011 Sampling Results 


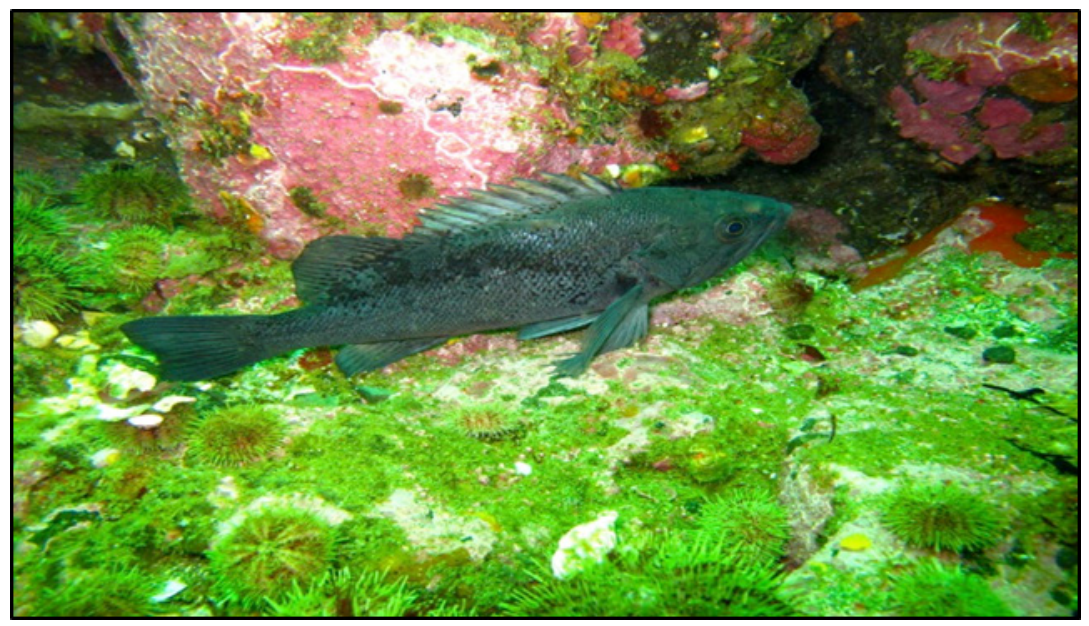

Rockfish (Sebastes melanops and S. cilatus)

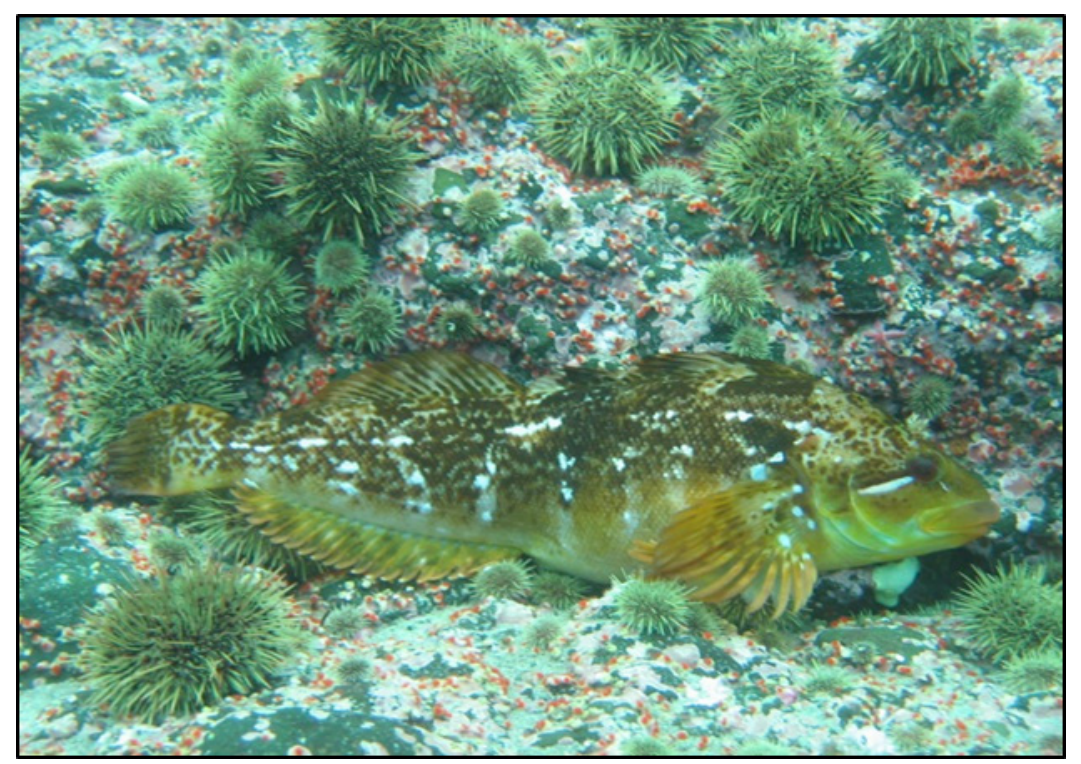

Rock greenling (Hexagrammos lagocephalus)

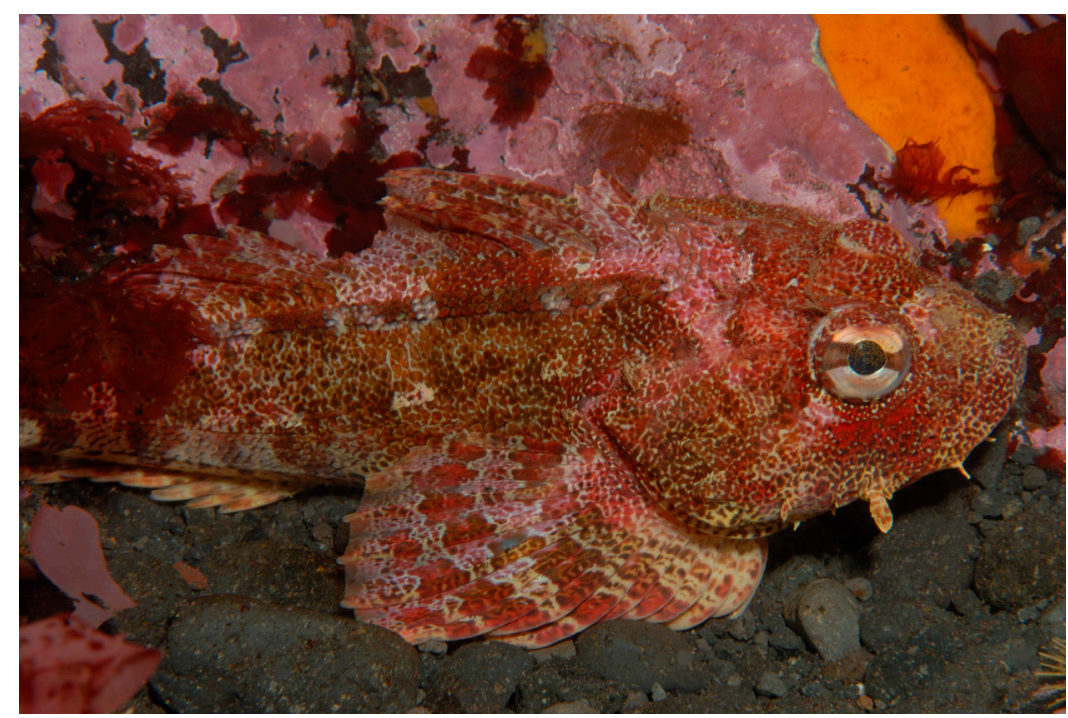

Irish lord (Hemilepidotus jordani and H. hemilepidotus) 


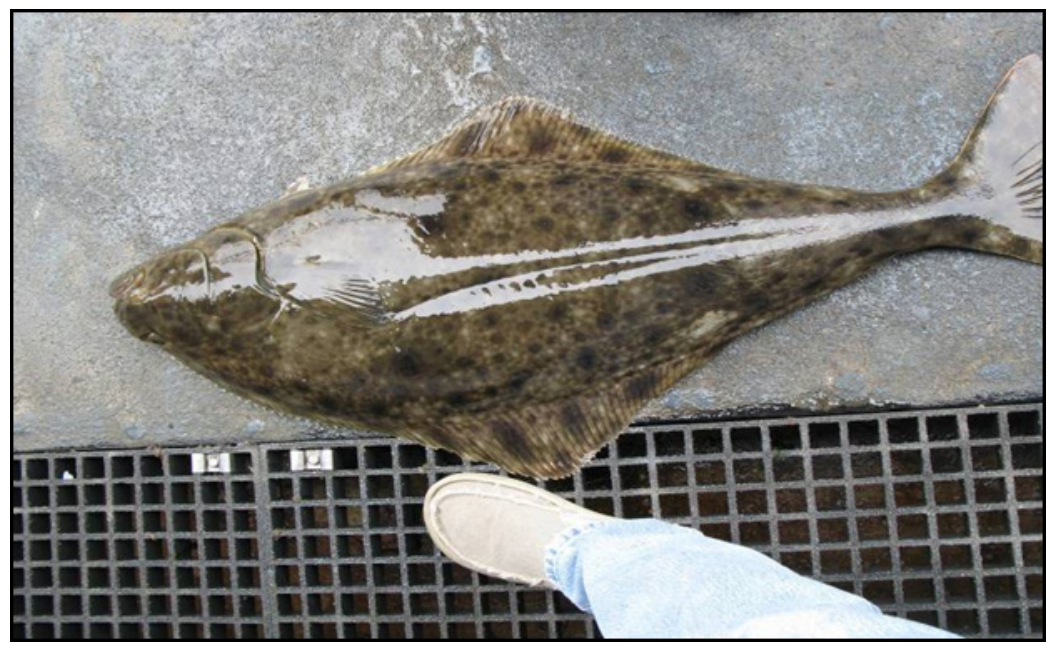

Pacific halibut (Hippoglossus stenolepis)

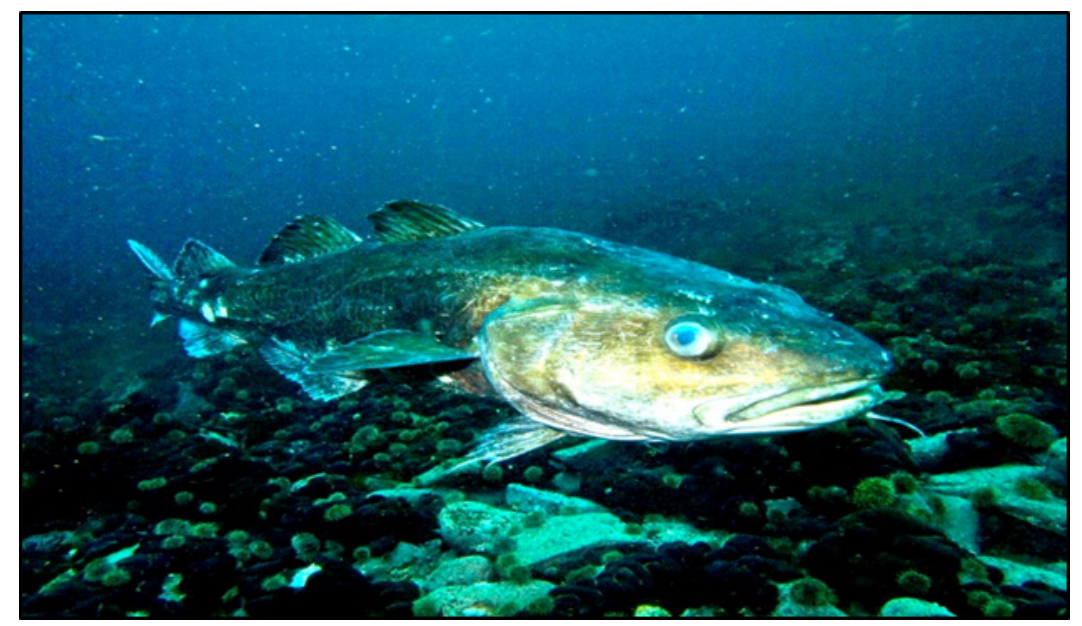

Pacific cod (Gadus macrocephalus)

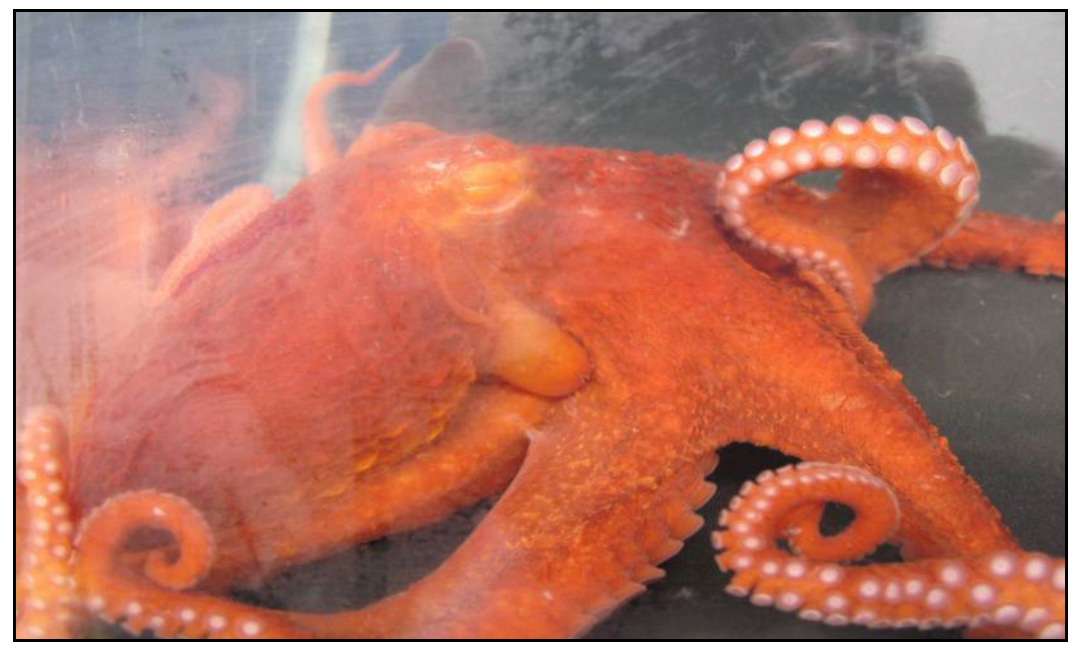

Octopus (Enteroctopus dofleini) 


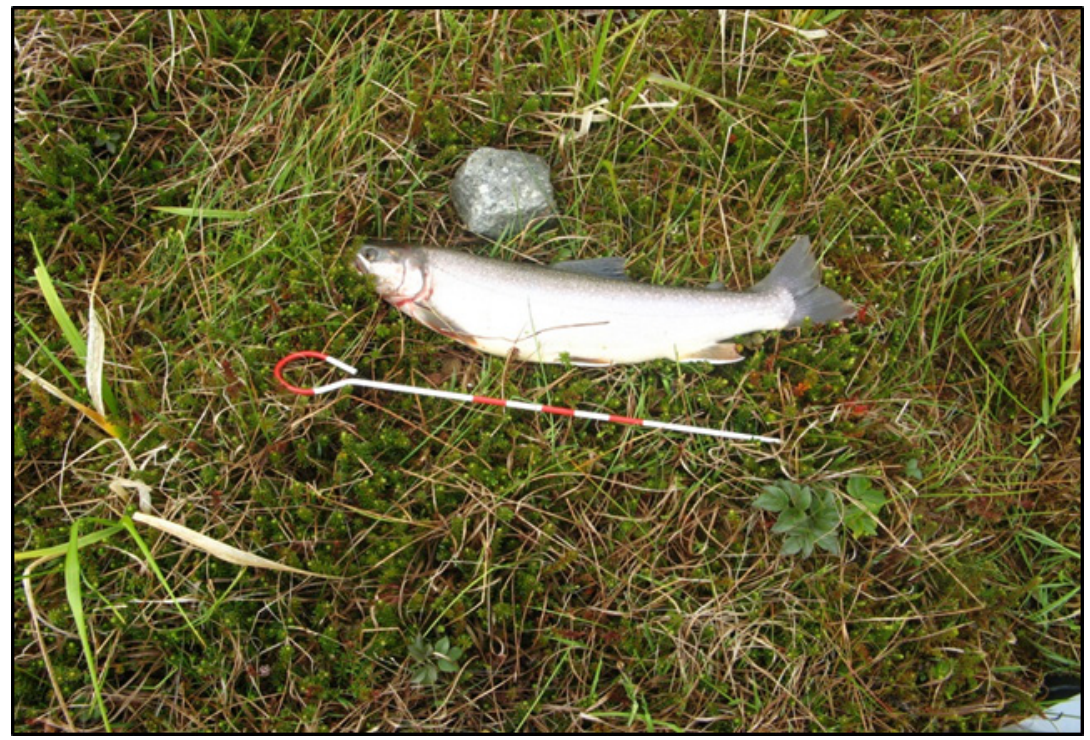

Dolly Varden (Salvelinus malma) 
This page intentionally left blank 
Appendix B

Sample Identification 
This page intentionally left blank 


\begin{tabular}{|c|c|c|c|c|c|c|c|c|}
\hline Ticket & Sample Date & Time & Location & $\begin{array}{l}\text { Number } \\
\text { Species } \\
\text { Sample }\end{array}$ & $\begin{array}{l}\text { Weight } \\
\text { (g) }\end{array}$ & $\begin{array}{l}\text { Length } \\
\text { (cm) }\end{array}$ & Sex & Notes \\
\hline JDQ 874 & $06 / 20 / 2011$ & $17: 15$ & FUCU-ML-DUP & 1 & 4990 & & & \\
\hline JDQ 865 & $06 / 20 / 2011$ & $17: 15$ & FUCU-ML-MT1-1 & 1 & 4990 & & & \\
\hline JDQ 868 & $06 / 20 / 2011$ & $17: 00$ & FUCU-ML-MT2-1 & 1 & 6000 & & & \\
\hline JET 743 & $06 / 20 / 2011$ & $16: 50$ & SEDI-ML-MT1-2 & 1 & --- & & & \\
\hline JGR 793 & $06 / 21 / 2011$ & $13: 54$ & FONT-AI-ML-1 & 1 & 4000 & & & UAF \\
\hline JDQ 866 & $06 / 21 / 2011$ & $12: 15$ & FUCU-ML-MT1-2 & 1 & 6000 & & & \\
\hline JDQ 869 & $06 / 21 / 2011$ & $11: 53$ & FUCU-ML-MT2-2 & 1 & 5000 & & & \\
\hline JDQ 870 & $06 / 21 / 2011$ & $11: 53$ & FUCU-ML-MT2-3 & 1 & 5000 & & & \\
\hline JDQ 901 & $06 / 21 / 2011$ & 9:50 & SOIL-AI-ML-1 & 1 & 1200 & & & 3 bags make up sample \\
\hline JES 503 & $06 / 21 / 2011$ & $9: 50$ & SOIL-AI-ML-1 & 1 & 1200 & & & UAF \\
\hline JDQ 895 & $06 / 21 / 2011$ & $9: 50$ & STRL-AI-ML-1 & 1 & 600 & & & \\
\hline JES 497 & $06 / 21 / 2011$ & 9:50 & STRL-AI-ML-1 & 1 & 600 & & & UAF \\
\hline JGR 794 & $06 / 22 / 2011$ & $10: 25$ & FONT-AI-LT1-1 & 1 & 6700 & & & UAF; Cloudberry Creek \\
\hline JGR 795 & $06 / 22 / 2011$ & $10: 25$ & FONT-AI-LT1-DUP & 1 & 6300 & & & UAF; Cloudberry Creek \\
\hline JDQ 864 & $06 / 22 / 2011$ & $16: 45$ & FUCU-LS-DUP & 1 & 5700 & & & \\
\hline JDQ 855 & $06 / 22 / 2011$ & $15: 25$ & FUCU-LS-LT1-1 & 1 & 5400 & & & \\
\hline JDQ 856 & $06 / 22 / 2011$ & $15: 50$ & FUCU-LS-LT1-2 & 1 & 5500 & & & \\
\hline JDQ 857 & $06 / 22 / 2011$ & $16: 45$ & FUCU-LS-LT1-3 & 1 & 6200 & & & \\
\hline JDQ 858 & $06 / 22 / 2011$ & $15: 15$ & FUCU-LS-LT2-1 & 1 & 7700 & & & \\
\hline JDQ 859 & $06 / 22 / 2011$ & $15: 41$ & FUCU-LS-LT2-2 & 1 & 7500 & & & \\
\hline JDQ 860 & $06 / 22 / 2011$ & $16: 15$ & FUCU-LS-LT2-3 & 1 & 7500 & & & \\
\hline JET 736 & $06 / 22 / 2011$ & $10: 45$ & LAKE-AI-LT1-1 & 1 & --- & & & Quonset Hut Lake \\
\hline JET 737 & $06 / 22 / 2011$ & $10: 45$ & LAKE-AI-LT1-DUP & 1 & --- & & & Quonset Hut Lake \\
\hline JGR 796 & $06 / 22 / 2011$ & $17: 20$ & SEDI-LS-DD-1 & 1 & --- & & & \\
\hline JET 741 & $06 / 22 / 2011$ & $17: 20$ & SEDI-LS-DD-1 & 1 & --- & & & \\
\hline JDQ 902 & $06 / 22 / 2011$ & $11: 15$ & SOIL-AI-LT1-2 & 1 & 500 & & & \\
\hline JES 504 & $06 / 22 / 2011$ & $11: 15$ & SOIL-AI-LT1-2 & 1 & 300 & & & UAF \\
\hline JES 505 & 06/22/2011 & $11: 15$ & SOIL-AI-LT1-3 & 1 & 300 & & & UAF \\
\hline JDQ 896 & $06 / 22 / 2011$ & $11: 15$ & STRL-AI-LT1-2 & 1 & 700 & & & \\
\hline JES 498 & $06 / 22 / 2011$ & $11: 15$ & STRL-AI-LT1-2 & 1 & 600 & & & UAF \\
\hline JDQ 845 & $06 / 23 / 2011$ & $12: 15$ & FUCU-CN-CT1-1 & 1 & 5200 & & & \\
\hline JDQ 846 & $06 / 23 / 2011$ & 11:05 & FUCU-CN-CT1-2 & 1 & 5100 & & & \\
\hline JDQ 847 & $06 / 23 / 2011$ & $11: 30$ & FUCU-CN-CT1-3 & 1 & 6000 & & & \\
\hline JDQ 854 & $06 / 23 / 2011$ & $12: 15$ & FUCU-CN-DUP & 1 & 5300 & & & \\
\hline JDQ 903 & $06 / 23 / 2011$ & $13: 45$ & SOIL-AI-CT1-3 & 1 & 400 & & & \\
\hline JDQ 898 & $06 / 23 / 2011$ & $13: 45$ & STRL-AI-CT1-1 & 1 & 500 & & & \\
\hline JES 499 & $06 / 23 / 2011$ & $13: 45$ & STRL-AI-CT1-1 & 1 & 800 & & & UAF; no soil collected \\
\hline \multirow{5}{*}{ JDQ 908} & 06/24/2011 & $12: 00$ & DOLL-AI-XXX-1 & 1 & 500 & 36.5 & M & \\
\hline & $06 / 24 / 2011$ & $12: 00$ & DOLL-AI-XXX-1 & 1 & 700 & 39 & M & \\
\hline & $06 / 24 / 2011$ & $12: 00$ & DOLL-AI-XXX-1 & 1 & 500 & 34 & $\mathrm{~F}$ & \\
\hline & $06 / 24 / 2011$ & $12: 00$ & DOLL-AI-XXX-1 & 1 & 400 & 32 & M & \\
\hline & 06/24/2011 & $12: 00$ & DOLL-AI-XXX-1 & 1 & 500 & 35 & $M$ & \\
\hline JGR 797 & $06 / 24 / 2011$ & $11: 25$ & FONT-AI-CN1-1 & 1 & 6600 & & & UAF \\
\hline JET 744 & $06 / 24 / 2011$ & $11: 00$ & GOOS-AI-XXX-1 & 5 & --- & & & \\
\hline
\end{tabular}




\begin{tabular}{|c|c|c|c|c|c|c|c|c|}
\hline Ticket & Sample Date & Time & Location & $\begin{array}{l}\text { Number } \\
\text { Species } \\
\text { Sample }\end{array}$ & $\begin{array}{l}\text { Weight } \\
\text { (g) }\end{array}$ & $\begin{array}{l}\text { Length } \\
\text { (cm) }\end{array}$ & Sex & Notes \\
\hline JDQ 907 & $06 / 24 / 2011$ & $12: 00$ & GWGU-AI-XXX-1 & 12 & 100 ea & & & \\
\hline JET 738 & $06 / 24 / 2011$ & $9: 54$ & LAKE-AI-CN-1 & 1 & --- & & & \\
\hline JET 739 & $06 / 24 / 2011$ & 9:54 & LAKE-AI-CN-DUP & 1 & --- & & & \\
\hline JDQ 772 & $06 / 24 / 2011$ & $21: 45$ & SEAW-CN-C2M-1CAN-D & 1 & --- & & & Salinity $=34$ \\
\hline JDQ 826 & $06 / 24 / 2011$ & $21: 50$ & $\begin{array}{l}\text { SEAW-CN-C2M-1CAN-D- } \\
\text { DUP }\end{array}$ & 1 & --- & & & Salinity $=34$ \\
\hline JDQ 771 & $06 / 24 / 2011$ & $21: 30$ & SEAW-CN-C2M-1CAN-S & 1 & --- & & & Salinity $=33$ \\
\hline JDQ 825 & $06 / 24 / 2011$ & $21: 35$ & $\begin{array}{l}\text { SEAW-CN-C2M-1CAN-S- } \\
\text { DUP }\end{array}$ & 1 & --- & & & Salinity = 34 \\
\hline JDQ 776 & $06 / 24 / 2011$ & $22: 37$ & SEAW-CN-C2M-1E-D & 1 & --- & & & Salinity $=35$ \\
\hline JDQ 775 & $06 / 24 / 2011$ & $22: 30$ & SEAW-CN-C2M-1E-S & 1 & --- & & & Salinity $=35$ \\
\hline JDQ 790 & $06 / 24 / 2011$ & $23: 07$ & SEAW-CN-C2M-2D-D & 1 & --- & & & Salinity $=35$ \\
\hline JDQ 789 & $06 / 24 / 2011$ & $22: 55$ & SEAW-CN-C2M-2D-S & 1 & --- & & & Salinity $=35$ \\
\hline JDQ 792 & $06 / 24 / 2011$ & $16: 18$ & SEAW-CN-C2M-2E-D & 1 & --- & & & Salinity $=35$ \\
\hline JDQ 791 & $06 / 24 / 2011$ & $16: 03$ & SEAW-CN-C2M-2E-S & 1 & -- & & & Salinity $=35$ \\
\hline JDQ 794 & $06 / 24 / 2011$ & $15: 50$ & SEAW-CN-C2M-2F-D & 1 & --- & & & Salinity $=35$ \\
\hline JDQ 793 & $06 / 24 / 2011$ & $15: 40$ & SEAW-CN-C2M-2F-S & 1 & --- & & & Salinity $=35$ \\
\hline JDQ 800 & $06 / 24 / 2011$ & $19: 55$ & SEAW-CN-C2M-3B-D & 1 & --- & & & Salinity $=33$ \\
\hline JDQ 799 & $06 / 24 / 2011$ & $19: 50$ & SEAW-CN-C2M-3B-S & 1 & --- & & & Salinity $=33$ \\
\hline JDQ 804 & $06 / 24 / 2011$ & $16: 54$ & SEAW-CN-C2M-3CAN-D & 1 & -- & & & Salinity $=34$ \\
\hline JDQ 803 & $06 / 24 / 2011$ & $16: 40$ & SEAW-CN-C2M-3CAN-S & 1 & --- & & & Salinity $=34$ \\
\hline JDQ 802 & $06 / 24 / 2011$ & $17: 45$ & SEAW-CN-C2M-3C-D & 1 & --- & & & Salinity $=34$ \\
\hline JDQ 801 & $06 / 24 / 2011$ & $17: 35$ & SEAW-CN-C2M-3C-S & 1 & -- & & & Salinity $=34$ \\
\hline JDQ 806 & $06 / 24 / 2011$ & $15: 20$ & SEAW-CN-C2M-3D-D & 1 & --- & & & Salinity $=35$ \\
\hline JDQ 805 & $06 / 24 / 2011$ & $15: 00$ & SEAW-CN-C2M-3D-S & 1 & --- & & & Salinity $=35$ \\
\hline JDQ 808 & $06 / 24 / 2011$ & $20: 20$ & SEAW-CN-C2M-4A-D & 1 & --- & & & Salinity $=34$ \\
\hline JDQ 807 & $06 / 24 / 2011$ & $20: 10$ & SEAW-CN-C2M-4A-S & 1 & --- & & & Salinity $=34$ \\
\hline JDQ 810 & $06 / 24 / 2011$ & 19:15 & SEAW-CN-C2M-4B-D & 1 & -- & & & Salinity $=35$ \\
\hline JDQ 809 & $06 / 24 / 2011$ & $18: 05$ & SEAW-CN-C2M-4B-S & 1 & --- & & & Salinity $=34$ \\
\hline JDQ 812 & $06 / 24 / 2011$ & $17: 20$ & SEAW-CN-C2M-5A-D & 1 & -- & & & Salinity $=34$ \\
\hline JDQ 811 & $06 / 24 / 2011$ & $17: 10$ & SEAW-CN-C2M-5A-S & 1 & --- & & & Salinity $=34$ \\
\hline JDQ 814 & $06 / 24 / 2011$ & $22: 12$ & SEAW-CN-C2M-C1-2-D & 1 & --- & & & Salinity $=35$ \\
\hline JDQ 813 & $06 / 24 / 2011$ & $22: 05$ & SEAW-CN-C2M-C1-2-S & 1 & --- & & & Salinity $=35$ \\
\hline JDQ 816 & $06 / 24 / 2011$ & $20: 45$ & SEAW-CN-C2M-C3-4-D & 1 & -- & & & Salinity $=34$ \\
\hline JDQ 815 & $06 / 24 / 2011$ & $20: 40$ & SEAW-CN-C2M-C3-4-S & 1 & --- & & & Salinity $=34$ \\
\hline JDQ 820 & $06 / 24 / 2011$ & $21: 15$ & SEAW-CN-C2M-C4-4-D & 1 & --- & & & Salinity $=33$ \\
\hline JDQ 819 & $06 / 24 / 2011$ & 21:05 & SEAW-CN-C2M-C4-4-S & 1 & --- & & & Salinity $=33$ \\
\hline JDQ 824 & $06 / 24 / 2011$ & $23: 45$ & SEAW-CN-C2M-LS2-2-D & 1 & --- & & & Salinity $=35$ \\
\hline \multirow{3}{*}{ JDQ 823} & $06 / 24 / 2011$ & 23:39 & SEAW-CN-C2M-LS2-2-S & 1 & -- & & & Salinity $=35$ \\
\hline & $06 / 26 / 2011$ & & BLCK-AN-ANT1-1 & 12 & --- & & & UAF; Doug Dasher \\
\hline & $06 / 26 / 2011$ & & BMUS-AN-ANT1 & 500 & --- & & & $\begin{array}{l}\text { UAF; Doug Dasher; } \\
\text { Sweeper Cove }\end{array}$ \\
\hline
\end{tabular}




\begin{tabular}{|c|c|c|c|c|c|c|c|c|}
\hline Ticket & Sample Date & Time & Location & $\begin{array}{l}\text { Number } \\
\text { Species } \\
\text { Sample }\end{array}$ & $\begin{array}{l}\text { Weight } \\
\text { (g) }\end{array}$ & $\begin{array}{l}\text { Length } \\
\text { (cm) }\end{array}$ & Sex & Notes \\
\hline \multirow{29}{*}{ JDQ 909} & $06 / 27 / 2011$ & $16: 50$ & DOLL-AD-XXX-1 & 1 & 300 & 31.5 & M & North Lake \\
\hline & 06/27/2011 & $16: 50$ & DOLL-AD-XXX-1 & 1 & 100 & 21.7 & $\mathrm{~F}$ & North Lake \\
\hline & $06 / 27 / 2011$ & $16: 50$ & DOLL-AD-XXX-1 & 1 & 100 & 22.9 & $\mathrm{~F}$ & North Lake \\
\hline & $06 / 27 / 2011$ & $16: 50$ & DOLL-AD-XXX-1 & 1 & 110 & 21.4 & $\mathrm{~F}$ & North Lake \\
\hline & $06 / 27 / 2011$ & $16: 50$ & DOLL-AD-XXX-1 & 1 & 110 & 24.2 & $\mathrm{~F}$ & North Lake \\
\hline & $06 / 27 / 2011$ & $19: 15$ & DOLL-AD-XXX-1 & 1 & 80 & 21.9 & $\mathrm{~F}$ & Pond above Mitt Lake \\
\hline & 06/27/2011 & $19: 15$ & DOLL-AD-XXX-1 & 1 & 90 & 21.8 & M & Pond above Mitt Lake \\
\hline & 06/27/2011 & $19: 15$ & DOLL-AD-XXX-1 & 1 & 100 & 23 & $\mathrm{~F}$ & Pond above Mitt Lake \\
\hline & $06 / 27 / 2011$ & 19:15 & DOLL-AD-XXX-1 & 1 & 100 & 22 & $\mathrm{~F}$ & Pond above Mitt Lake \\
\hline & $06 / 27 / 2011$ & $19: 15$ & DOLL-AD-XXX-1 & 1 & 100 & 21.1 & $\mathrm{~F}$ & Pond above Mitt Lake \\
\hline & $06 / 27 / 2011$ & 19:15 & DOLL-AD-XXX-1 & 1 & 100 & 23 & M & Pond above Mitt Lake \\
\hline & $06 / 27 / 2011$ & $19: 15$ & DOLL-AD-XXX-1 & 1 & 90 & 21.6 & $\mathrm{~F}$ & Pond above Mitt Lake \\
\hline & $06 / 27 / 2011$ & $19: 15$ & DOLL-AD-XXX-1 & 1 & 120 & 23.4 & $\mathrm{~F}$ & Pond above Mitt Lake \\
\hline & $06 / 28 / 2011$ & $16: 50$ & DOLL-AD-XXX-1 & 1 & 100 & 22.2 & $\mathrm{~F}$ & Tarn \\
\hline & $06 / 28 / 2011$ & $16: 50$ & DOLL-AD-XXX-1 & 1 & 130 & 25.5 & M & Tarn \\
\hline & $06 / 28 / 2011$ & $16: 50$ & DOLL-AD-XXX-1 & 1 & 150 & 25 & M & Tarn \\
\hline & $06 / 28 / 2011$ & $16: 50$ & DOLL-AD-XXX-1 & 1 & 100 & 22 & $\mathrm{~F}$ & Tarn \\
\hline & 06/28/2011 & $16: 50$ & DOLL-AD-XXX-1 & 1 & 120 & 23 & $\mathrm{~F}$ & Tarn \\
\hline & 06/28/2011 & $16: 50$ & DOLL-AD-XXX-1 & 1 & 100 & 24.4 & $\mathrm{~F}$ & Tarn \\
\hline & $06 / 28 / 2011$ & $16: 50$ & DOLL-AD-XXX-1 & 1 & 100 & 22.7 & $\mathrm{~F}$ & Tarn \\
\hline & $06 / 28 / 2011$ & $16: 50$ & DOLL-AD-XXX-1 & 1 & 100 & 22 & M & Tarn \\
\hline & 06/28/2011 & $16: 50$ & DOLL-AD-XXX-1 & 1 & 200 & 25.6 & $\mathrm{~F}$ & Tarn \\
\hline & $06 / 28 / 2011$ & $16: 50$ & DOLL-AD-XXX-1 & 1 & 110 & 22.5 & $\mathrm{~F}$ & Tarn \\
\hline & $06 / 28 / 2011$ & $16: 50$ & DOLL-AD-XXX-1 & 1 & 100 & 21.6 & $\mathrm{~F}$ & Tarn \\
\hline & $06 / 28 / 2011$ & $16: 50$ & DOLL-AD-XXX-1 & 1 & 300 & 26.4 & M & Tarn \\
\hline & $06 / 28 / 2011$ & $16: 50$ & DOLL-AD-XXX-1 & 1 & 100 & 20.6 & $\mathrm{~F}$ & Tarn \\
\hline & 06/28/2011 & $16: 50$ & DOLL-AD-XXX-1 & 1 & 80 & 19.5 & $\mathrm{~F}$ & Tarn \\
\hline & $06 / 28 / 2011$ & $16: 50$ & DOLL-AD-XXX-1 & 1 & 100 & 22.2 & $\mathrm{~F}$ & Tarn \\
\hline & $06 / 28 / 2011$ & $16: 50$ & DOLL-AD-XXX-1 & 1 & 110 & 22.3 & $\mathrm{~F}$ & Tarn \\
\hline JDQ 904 & $06 / 28 / 2011$ & $16: 50$ & SOIL-AD-XXX-1 & 1 & 350 & & & \\
\hline JES 506 & $06 / 28 / 2011$ & $16: 50$ & SOIL-AD-XXX-1 & 1 & 150 & & & \\
\hline JDQ 897 & $06 / 28 / 2011$ & $16: 50$ & STRL-AD-XXX-1 & 1 & 650 & & & Above Mitt Lake \\
\hline JES 500 & $06 / 28 / 2011$ & $16: 50$ & STRL-AD-XXX-1 & 1 & 650 & & & Above Mitt Lake \\
\hline JDQ 905 & $06 / 29 / 2011$ & $16: 15$ & SOIL-AD-XXX-2 & 1 & 30 & & & \\
\hline JES 507 & $06 / 29 / 2011$ & $12: 15$ & SOIL-AD-XXX-2 & 1 & 200 & & & UAF \\
\hline JDQ 906 & 06/29/2011 & $19: 25$ & SOIL-AD-XXX-3 & 1 & 200 & & & \\
\hline JES 508 & $06 / 29 / 2011$ & $19: 25$ & SOIL-AD-XXX-3 & 1 & 300 & & & UAF \\
\hline JDQ 899 & $06 / 29 / 2011$ & $12: 15$ & STRL-AD-XXX-2 & 1 & 700 & & & \\
\hline JES 501 & $06 / 29 / 2011$ & $12: 15$ & STRL-AD-XXX-2 & 1 & 700 & & & UAF \\
\hline JDQ 900 & 06/29/2011 & $19: 25$ & STRL-AD-XXX-3 & 1 & 600 & & & \\
\hline 150 & $06 / 29 / 2011$ & $19: 25$ & STRL-AD-XXX-3 & 1 & 650 & & & UAF \\
\hline JLU JUL & $06 / 30 / 2011$ & & BMUS-AN-ANT1 & 1000 & --- & & & UAF; Sweeper Cove \\
\hline JDR 008 & $07 / 02 / 2011$ & $18: 40$ & EUAL-LS-LT3-1 & 1 & 5800 & & & \\
\hline JDR 009 & $07 / 02 / 2011$ & $18: 10$ & EUAL-LS-LT3-2 & 1 & 5000 & & & \\
\hline
\end{tabular}




\begin{tabular}{|c|c|c|c|c|c|c|c|c|}
\hline Ticket & Sample Date & Time & Location & $\begin{array}{l}\text { Number } \\
\text { Species } \\
\text { Sample }\end{array}$ & $\begin{array}{l}\text { Weight } \\
\text { (g) }\end{array}$ & $\begin{array}{l}\text { Length } \\
(\mathrm{cm})\end{array}$ & Sex & Notes \\
\hline JDR 010 & 07/02/2011 & $18: 30$ & EUAL-LS-LT3-3 & 1 & 5100 & & & \\
\hline JDQ 861 & $07 / 02 / 2011$ & $18: 36$ & FUCU-LS-LT3-1 & 1 & 5400 & & & \\
\hline JDQ 862 & $07 / 02 / 2011$ & $19: 04$ & FUCU-LS-LT3-2 & 1 & 5400 & & & \\
\hline JDQ 863 & 07/02/2011 & $19: 04$ & FUCU-LS-LT3-3 & 1 & 5500 & & & \\
\hline \multirow{3}{*}{ JDR 028} & 07/02/2011 & $17: 30$ & IRLO-LS-LT3-1 & 1 & 500 & 33.1 & $\mathrm{~F}$ & $\begin{array}{l}\text { Composite; Originally } \\
\text { labeled as Rockfish }\end{array}$ \\
\hline & 07/02/2011 & $17: 30$ & IRLO-LS-LT3-1 & 1 & 375 & 28 & M & $\begin{array}{l}\text { Composite; Originally } \\
\text { labeled as Rockfish }\end{array}$ \\
\hline & $07 / 02 / 2011$ & $17: 30$ & IRLO-LS-LT3-1 & 1 & 480 & 31.1 & $\mathrm{~F}$ & $\begin{array}{l}\text { Composite; Originally } \\
\text { labeled as Rockfish }\end{array}$ \\
\hline JDR 020 & 07/02/2011 & $18: 10$ & URCH-LS-LT3-1 & 2 & 200 & & & \\
\hline JDR 013 & 07/03/2011 & $14: 50$ & CHIT-LS-LT2-1 & 2 & 590 & & & \\
\hline JDR 014 & 07/03/2011 & $10: 00$ & CHIT-LS-LT3-1 & 2 & 650 & & & \\
\hline JES 493 & 07/03/2011 & $15: 00$ & EUAL-LS-DUP & 1 & 5200 & & & UAF \\
\hline JDR 011 & 07/03/2011 & $15: 00$ & EUAL-LS-DUP2 & 1 & 5650 & & & \\
\hline JDR 005 & 07/03/2011 & $15: 00$ & EUAL-LS-LT2-1 & 1 & 5100 & & & \\
\hline JDR 006 & $07 / 03 / 2011$ & $15: 35$ & EUAL-LS-LT2-2 & 1 & 5250 & & & \\
\hline JDR 007 & 07/03/2011 & $15: 10$ & EUAL-LS-LT2-3 & 1 & 6000 & & & \\
\hline JET 752 & 07/03/2011 & $14: 20$ & GREN-LS-LT2-2 & 1 & 700 & 38 & $\mathrm{~F}$ & \\
\hline JET 753 & 07/03/2011 & $15: 10$ & GREN-LS-LT2-3 & 1 & 700 & 38.2 & $\mathrm{~F}$ & \\
\hline JDR 038 & 07/03/2011 & $11: 30$ & GREN-LS-LT3-1 & 1 & 800 & 40.3 & $\mathrm{~F}$ & \\
\hline JDR 017 & 07/03/2011 & $10: 00$ & HMUS-LS-LT3-1 & 1 & 40 & & & \\
\hline JDR 035 & $07 / 03 / 2011$ & $12: 20$ & IRLO-LS-LT2-1 & 1 & 600 & 33 & $\mathrm{~F}$ & \\
\hline JDR 036 & 07/03/2011 & $13: 45$ & IRLO-LS-LT2-2 & 1 & 540 & 31.4 & $\mathrm{~F}$ & \\
\hline JDR 037 & 07/03/2011 & $13: 50$ & IRLO-LS-LT2-3 & 1 & 500 & 31.5 & M & \\
\hline JDR 021 & 07/03/2011 & $15: 25$ & OCTP-LS-L2M-1 & 1 & 184000 & & & \\
\hline JDR 030 & $07 / 03 / 2011$ & $11: 45$ & ROCK-LS-LT3-3 & 1 & 1100 & 39.8 & M & \\
\hline JDQ 774 & 07/03/2011 & $17: 54$ & SEAW-CN-C2M-1D-D & 1 & --- & & & Salinity = 35 \\
\hline JDQ 773 & 07/03/2011 & $17: 53$ & SEAW-CN-C2M-1D-S & 1 & --- & & & Salinity $=35$ \\
\hline JDQ 778 & $07 / 03 / 2011$ & $18: 47$ & SEAW-CN-C2M-1F-D & 1 & --- & & & Salinity $=35$ \\
\hline JDQ 777 & 07/03/2011 & $18: 39$ & SEAW-CN-C2M-1F-S & 1 & --- & & & Salinity = 35 \\
\hline JDQ 780 & $07 / 03 / 2011$ & $18: 59$ & SEAW-CN-C2M-1SB-D & 1 & --- & & & Salinity = 35 \\
\hline JDQ 827 & 07/03/2011 & $19: 13$ & $\begin{array}{l}\text { SEAW-CN-C2M-1SB-D- } \\
\text { DUP }\end{array}$ & 1 & --- & & & Salinity = 35 \\
\hline JDQ 779 & 07/03/2011 & $19: 03$ & SEAW-CN-C2M-1SB-S & 1 & --- & & & Salinity = 35 \\
\hline JDQ 828 & $07 / 03 / 2011$ & 19:08 & $\begin{array}{l}\text { SEAW-CN-C2M-1SB-S- } \\
\text { DUP }\end{array}$ & 1 & --- & & & Salinity $=35$ \\
\hline JDQ 796 & $07 / 03 / 2011$ & 19:16 & SEAW-CN-C2M-2SB-D & 1 & --- & & & Salinity $=35$ \\
\hline JDQ 795 & 07/03/2011 & 19:11 & SEAW-CN-C2M-2SB-S & 1 & --- & & & Salinity = 35 \\
\hline JDQ 728 & $07 / 03 / 2011$ & $15: 30$ & SEAW-LS-LT2-1 & 1 & --- & & & Salinity $=33$ \\
\hline JDQ 729 & 07/03/2011 & $15: 20$ & SEAW-LS-LT2-2 & 1 & --- & & & Salinity = 34 \\
\hline JDQ 730 & $07 / 03 / 2011$ & $15: 40$ & SEAW-LS-LT2-3 & 1 & --- & & & Salinity = 35 \\
\hline JDQ 731 & 07/03/2011 & $10: 40$ & SEAW-LS-LT3-1 & 1 & --- & & & Salinity = 35 \\
\hline JDQ 732 & 07/03/2011 & 11:00 & SEAW-LS-LT3-2 & 1 & --- & & & Salinity $=35$ \\
\hline JDQ 733 & $07 / 03 / 2011$ & $11: 20$ & SEAW-LS-LT3-3 & 1 & --- & & & Salinity = 35 \\
\hline JIT 788 & 07/03/2011 & $15: 30$ & SEDI-LS-LT2-1 & 1 & --- & & & \\
\hline JIT 789 & 07/03/2011 & $10: 40$ & SEDI-LS-LT3-1 & 1 & --- & & & \\
\hline
\end{tabular}

Amchitka Island, Alaska, Biological Monitoring Report, 2011 Sampling Results 


\begin{tabular}{|c|c|c|c|c|c|c|c|c|}
\hline Ticket & Sample Date & Time & Location & $\begin{array}{l}\text { Number } \\
\text { Species } \\
\text { Sample }\end{array}$ & $\begin{array}{l}\text { Weight } \\
\text { (g) }\end{array}$ & $\begin{array}{l}\text { Length } \\
\text { (cm) }\end{array}$ & Sex & Notes \\
\hline JDR 019 & $07 / 03 / 2011$ & $16: 00$ & URCH-LS-LT2-1 & 6 & $100 \mathrm{EA}$ & & & \\
\hline JDR 012 & $07 / 04 / 2011$ & $15: 05$ & CHIT-LS-LT1-1 & 1 & 500 & & & \\
\hline JDR 002 & $07 / 04 / 2011$ & $14: 45$ & EUAL-LS-LT1-1 & 1 & 5250 & & & \\
\hline JDR 003 & 07/04/2011 & $14: 55$ & EUAL-LS-LT1-2 & 1 & 6250 & & & \\
\hline JDR 004 & $07 / 04 / 2011$ & $15: 10$ & EUAL-LS-LT1-3 & 1 & 5500 & & & \\
\hline JDR 032 & $07 / 04 / 2011$ & $15: 10$ & GREN-LS-LT1-1 & 1 & 400 & 32.5 & $\mathrm{~F}$ & \\
\hline JDR 033 & $07 / 04 / 2011$ & $15: 30$ & GREN-LS-LT1-2 & 1 & 600 & 35.5 & $\mathrm{~F}$ & \\
\hline JET 751 & $07 / 04 / 2011$ & $12: 15$ & GREN-LS-LT2-1 & 1 & 620 & 35.7 & $\mathrm{~F}$ & \\
\hline JDR 016 & $07 / 04 / 2011$ & 9:30 & HMUS-LS-LT2-1 & 15 & 610 & & & \\
\hline JDR 039 & $07 / 04 / 2011$ & $14: 45$ & IRLO-LS-LT1-1 & 1 & 500 & 32.6 & M & \\
\hline JDR 040 & $07 / 04 / 2011$ & $14: 55$ & IRLO-LS-LT1-2 & 1 & 500 & 37 & M & \\
\hline JDR 044 & $07 / 04 / 2011$ & 9:30 & PCOD-LS-L2M-1 & 1 & 7600 & 90 & M & \\
\hline JDR 022 & $07 / 04 / 2011$ & $15: 00$ & ROCK-LS-LT1-1 & 1 & 600 & 36.4 & $\mathrm{~F}$ & \\
\hline JDR 025 & $07 / 04 / 2011$ & 9:30 & ROCK-LS-LT2-1 & 1 & 1000 & 37.6 & M & \\
\hline JDQ 725 & $07 / 04 / 2011$ & $15: 00$ & SEAW-LS-LT1-1 & 1 & --- & & & Salinity $=36$ \\
\hline JDQ 726 & $07 / 04 / 2011$ & $14: 45$ & SEAW-LS-LT1-2 & 1 & --- & & & Salinity $=36$ \\
\hline JDQ 727 & $07 / 04 / 2011$ & 9:56 & SEAW-LS-LT1-3 & 1 & --- & & & Salinity $=36$ \\
\hline JIT 787 & 07/04/2011 & $15: 00$ & SEDI-LS-LT1-1 & 1 & --- & & & \\
\hline JDR 018 & $07 / 04 / 2011$ & $15: 02$ & URCH-LS-LT1-1 & 5 & 600 & & & \\
\hline JDQ 971 & $07 / 05 / 2011$ & $15: 40$ & CHIT-CN-CT3-1 & 2 & 500 & & & \\
\hline JDQ 965 & $07 / 05 / 2011$ & $15: 15$ & EUAL-CN-CT3-1 & 1 & 5900 & & & \\
\hline JDQ 966 & $07 / 05 / 2011$ & $15: 40$ & EUAL-CN-CT3-2 & 1 & 5200 & & & \\
\hline JDQ 967 & $07 / 05 / 2011$ & $15: 30$ & EUAL-CN-CT3-3 & 1 & 5800 & & & \\
\hline JDQ 995 & $07 / 05 / 2011$ & $15: 30$ & GREN-CN-CT3-1 & 1 & 650 & 37 & $\mathrm{~F}$ & \\
\hline JDQ 996 & $07 / 05 / 2011$ & $15: 45$ & GREN-CN-CT3-2 & 1 & 700 & 36.7 & M & \\
\hline JDQ 997 & $07 / 05 / 2011$ & $16: 00$ & GREN-CN-CT3-3 & 1 & 800 & 38.8 & $\mathrm{~F}$ & \\
\hline JDR 034 & $07 / 05 / 2011$ & $10: 12$ & GREN-LS-LT1-3 & 1 & 500 & 34.6 & $\mathrm{~F}$ & \\
\hline JDQ 974 & 07/05/2011 & $16: 10$ & HMUS-CN-CT3-1 & 16 & 500 & & & \\
\hline JDR 015 & $07 / 05 / 2011$ & $9: 57$ & HMUS-LS-LT1-1 & 12 & 500 & & & \\
\hline JET 747 & $07 / 05 / 2011$ & $14: 45$ & IRLO-CN-CT2-2 & 1 & 300 & 27.9 & $\mathrm{~F}$ & \\
\hline JET 747 & 07/05/2011 & $14: 45$ & IRLO-CN-CT2-2 & 1 & 340 & 27.6 & $\mathrm{~F}$ & \\
\hline JDQ 999 & $07 / 05 / 2011$ & $16: 10$ & IRLO-CN-CT3-1 & 1 & 550 & 32.7 & $\mathrm{~F}$ & \\
\hline JET 746 & $07 / 05 / 2011$ & $15: 30$ & IRLO-CN-CT3-2 & 1 & 840 & 42.6 & $\mathrm{~F}$ & \\
\hline JDR 041 & $07 / 05 / 2011$ & $10: 15$ & IRLO-LS-LT1-3 & 1 & 900 & 42.2 & U & \\
\hline JDQ 706 & $07 / 05 / 2011$ & $15: 30$ & SEAW-CN-CT3-1 & 1 & --- & & & Salinity = 35 \\
\hline JDQ 707 & $07 / 05 / 2011$ & $15: 20$ & SEAW-CN-CT3-2 & 1 & --- & & & Salinity $=35$ \\
\hline JIT 786 & $07 / 05 / 2011$ & $15: 30$ & SEDI-CN-CT3-1 & 1 & --- & & & \\
\hline JDQ 977 & $07 / 05 / 2011$ & $16: 00$ & URCH-CN-CT3-1 & 6 & 750 & & & \\
\hline JDQ 962 & 07/06/2011 & $16: 15$ & EUAL-CN-CT2-1 & 1 & 5550 & & & \\
\hline JDQ 963 & 07/06/2011 & $16: 25$ & EUAL-CN-CT2-2 & 1 & 5250 & & & \\
\hline JDQ 723 & 07/06/2011 & 9:35 & EUAL-CN-DUP & 1 & 5450 & & & UAF \\
\hline JDQ 968 & 07/06/2011 & 9:35 & EUAL-CN-DUP & 1 & 5250 & & & \\
\hline JDQ 851 & 07/06/2011 & $10: 49$ & FUCU-CN-CT3-1 & 1 & 5350 & & & \\
\hline JDQ 852 & $07 / 06 / 2011$ & $11: 19$ & FUCU-CN-CT3-2 & 1 & 5350 & & & \\
\hline
\end{tabular}




\begin{tabular}{|c|c|c|c|c|c|c|c|c|}
\hline Ticket & Sample Date & Time & Location & $\begin{array}{l}\text { Number } \\
\text { Species } \\
\text { Sample }\end{array}$ & $\begin{array}{l}\text { Weight } \\
\text { (g) }\end{array}$ & $\begin{array}{l}\text { Length } \\
\text { (cm) }\end{array}$ & Sex & Notes \\
\hline JDQ 853 & 07/06/2011 & $11: 43$ & FUCU-CN-CT3-3 & 1 & 5450 & & & \\
\hline \multirow{2}{*}{ JDR 001} & 07/06/2011 & $14: 55$ & PCOD-CN-C2M-1 & 1 & 3300 & 63.8 & $\mathrm{~F}$ & 2 bags for sample \\
\hline & 07/06/2011 & $14: 55$ & PCOD-CN-C2M-1 & 1 & 2000 & 57.4 & M & \\
\hline JDQ 982 & 07/06/2011 & $14: 30$ & ROCK-CN-CT2-1 & 1 & 1200 & 40.7 & $U$ & \\
\hline JDQ 983 & 07/06/2011 & $15: 00$ & ROCK-CN-CT2-2 & 1 & 975 & 36.5 & $M$ & \\
\hline JDQ 984 & 07/06/2011 & $15: 15$ & ROCK-CN-CT2-3 & 1 & 1050 & 39 & M & \\
\hline JDQ 985 & 07/06/2011 & $9: 50$ & ROCK-CN-CT3-1 & 1 & 1150 & 40.8 & $\mathrm{~F}$ & \\
\hline JDQ 986 & 07/06/2011 & $9: 55$ & ROCK-CN-CT3-2 & 1 & 1250 & 41.1 & $\mathrm{~F}$ & \\
\hline JDQ 987 & 07/06/2011 & $10: 10$ & ROCK-CN-CT3-3 & 1 & 900 & 35.5 & M & \\
\hline JDQ 988 & $07 / 06 / 2011$ & $10: 20$ & ROCK-CN-DUP & 1 & 900 & 35.5 & $M$ & \\
\hline JDQ 770 & 07/06/2011 & $15: 35$ & SEAW-CN-C2M-1C-D & 1 & --- & & & Salinity $=35$ \\
\hline JDQ 769 & 07/06/2011 & $15: 28$ & SEAW-CN-C2M-1C-S & 1 & -- & & & Salinity $=35$ \\
\hline JDQ 782 & $07 / 06 / 2011$ & $14: 35$ & SEAW-CN-C2M-2A-D & 1 & --- & & & Salinity $=35$ \\
\hline JDQ 781 & 07/06/2011 & $14: 25$ & SEAW-CN-C2M-2A-S & 1 & --- & & & Salinity $=35$ \\
\hline JDQ 784 & 07/06/2011 & $14: 57$ & SEAW-CN-C2M-2B-D & 1 & --- & & & Salinity = 34 \\
\hline JDQ 830 & 07/06/2011 & $15: 05$ & $\begin{array}{l}\text { SEAW-CN-C2M-2B-D- } \\
\text { DUP }\end{array}$ & 1 & --- & & & Salinity $=34$ \\
\hline JDQ 783 & 07/06/2011 & $14: 43$ & SEAW-CN-C2M-2B-S & 1 & --- & & & Salinity $=35$ \\
\hline JDQ 829 & 07/06/2011 & $14: 50$ & $\begin{array}{l}\text { SEAW-CN-C2M-2B-S- } \\
\text { DUP }\end{array}$ & 1 & --- & & & Salinity $=35$ \\
\hline JDQ 788 & 07/06/2011 & $13: 20$ & SEAW-CN-C2M-2CAN-D & 1 & --- & & & Salinity $=35$ \\
\hline JDQ 787 & 07/06/2011 & $13: 10$ & SEAW-CN-C2M-2CAN-S & 1 & --- & & & Salinity $=35$ \\
\hline JDQ 786 & $07 / 06 / 2011$ & $14: 00$ & SEAW-CN-C2M-2C-D & 1 & -- & & & Salinity $=35$ \\
\hline JDQ 785 & 07/06/2011 & $13: 51$ & SEAW-CN-C2M-2C-S & 1 & --- & & & Salinity $=35$ \\
\hline JDQ 798 & 07/06/2011 & $14: 18$ & SEAW-CN-C2M-3A-D & 1 & --- & & & Salinity $=35$ \\
\hline JDQ 797 & 07/06/2011 & $14: 10$ & SEAW-CN-C2M-3A-S & 1 & --- & & & Salinity $=35$ \\
\hline JDQ 818 & 07/06/2011 & $13: 40$ & SEAW-CN-C2M-C4-1-D & 1 & --- & & & Salinity $=35$ \\
\hline JDQ 817 & 07/06/2011 & $13: 30$ & SEAW-CN-C2M-C4-1-S & 1 & -- & & & Salinity $=35$ \\
\hline JDQ 822 & 07/06/2011 & $15: 20$ & SEAW-CN-C2M-C5-1-D & 1 & --- & & & Salinity $=34$ \\
\hline JDQ 821 & 07/06/2011 & $15: 14$ & SEAW-CN-C2M-C5-1-S & 1 & -- & & & Salinity $=35$ \\
\hline JDQ 703 & 07/06/2011 & $14: 30$ & SEAW-CN-CT2-1 & 1 & --- & & & Salinity $=35$ \\
\hline JDQ 708 & 07/06/2011 & 9:40 & SEAW-CN-CT3-3 & 1 & --- & & & Salinity $=35$ \\
\hline JDQ 724 & 07/06/2011 & $9: 45$ & SEAW-CN-DUP & 1 & --- & & & Salinity $=35$ \\
\hline JIT 785 & 07/06/2011 & $16: 30$ & SEDI-CN-CT2-1 & 1 & -- & & & \\
\hline JDQ 970 & 07/07/2011 & $15: 00$ & CHIT-CN-CT2-1 & 4 & 585 & & & \\
\hline JDQ 964 & 07/07/2011 & $10: 45$ & EUAL-CN-CT2-3 & 1 & 5450 & & & \\
\hline JDQ 992 & 07/07/2011 & $14: 40$ & GREN-CN-CT2-1 & 1 & 800 & 37.9 & $\mathrm{~F}$ & \\
\hline JDQ 993 & 07/07/2011 & $14: 50$ & GREN-CN-CT2-2 & 1 & 650 & 36.3 & $\mathrm{~F}$ & \\
\hline JDQ 994 & 07/07/2011 & $14: 55$ & GREN-CN-CT2-3 & 1 & 750 & 37.7 & $\mathrm{~F}$ & \\
\hline JDQ 973 & 07/07/2011 & $15: 00$ & HMUS-CN-CT2-1 & 15 & 500 & & & \\
\hline \multirow{2}{*}{ JET 748} & 07/07/2011 & $10: 40$ & IRLO-CN-CT2-1 & 1 & 450 & 30.9 & $\mathrm{~F}$ & Composite \\
\hline & 07/07/2011 & $10: 40$ & IRLO-CN-CT2-1 & 1 & 400 & 27.9 & M & Composite \\
\hline JET 750 & 07/07/2011 & $14: 55$ & IRLO-CN-CT2-3 & 1 & 850 & 40.6 & $\mathrm{~F}$ & \\
\hline \multirow{2}{*}{ JET 749} & 07/07/2011 & $15: 40$ & IRLO-CN-CT3-3 & 1 & 350 & 28.9 & $\mathrm{~F}$ & \\
\hline & 07/07/2011 & $15: 40$ & IRLO-CN-CT3-3 & 1 & 400 & 33.7 & U & \\
\hline
\end{tabular}




\begin{tabular}{|c|c|c|c|c|c|c|c|c|}
\hline Ticket & Sample Date & Time & Location & $\begin{array}{l}\text { Number } \\
\text { Species } \\
\text { Sample }\end{array}$ & $\begin{array}{l}\text { Weight } \\
\text { (g) }\end{array}$ & $\begin{array}{l}\text { Length } \\
\text { (cm) }\end{array}$ & Sex & Notes \\
\hline JDQ 704 & $07 / 07 / 2011$ & $10: 15$ & SEAW-CN-CT2-2 & 1 & 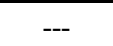 & & & Salinity $=35$ \\
\hline JDQ 705 & $07 / 07 / 2011$ & $10: 20$ & SEAW-CN-CT2-3 & 1 & --- & & & Salinity $=32$ \\
\hline JDQ 976 & $07 / 07 / 2011$ & $10: 27$ & URCH-CN-CT2-1 & 4 & 500 & & & \\
\hline JDQ 969 & $07 / 08 / 2011$ & $10: 50$ & CHIT-CN-CT1-1 & 3 & 500 & & & \\
\hline JDQ 718 & $07 / 08 / 2011$ & $10: 30$ & EUAL-CN-CT1-1 & 1 & 5500 & & & \\
\hline JDQ 960 & $07 / 08 / 2011$ & $10: 50$ & EUAL-CN-CT1-2 & 1 & 5700 & & & \\
\hline JDQ 961 & $07 / 08 / 2011$ & $10: 50$ & EUAL-CN-CT1-3 & 1 & 5600 & & & \\
\hline JDQ 848 & $07 / 08 / 2011$ & $17: 45$ & FUCU-CN-CT2-1 & 1 & 5450 & & & \\
\hline JDQ 849 & 07/08/2011 & $17: 55$ & FUCU-CN-CT2-2 & 1 & 5600 & & & \\
\hline JDQ 989 & $07 / 08 / 2011$ & $10: 50$ & GREN-CN-CT1-1 & 1 & 650 & 36.6 & $\mathrm{~F}$ & \\
\hline JDQ 990 & $07 / 08 / 2011$ & $10: 25$ & GREN-CN-CT1-2 & 1 & 550 & 35.4 & $\mathrm{~F}$ & \\
\hline JDQ 991 & $07 / 08 / 2011$ & $10: 30$ & GREN-CN-CT1-3 & 1 & 650 & 36.6 & $M$ & \\
\hline JDQ 998 & 07/08/2011 & $14: 25$ & GREN-CN-DUP & 1 & 600 & 35.5 & $\mathrm{~F}$ & \\
\hline JDQ 972 & $07 / 08 / 2011$ & $10: 37$ & HMUS-CN-CT1-1 & 12 & 505 & & & \\
\hline \multirow{2}{*}{ JIV 536} & $07 / 08 / 2011$ & $10: 37$ & IRLO-CN-CT1-1 & 1 & 250 & 24 & $\mathrm{~F}$ & Composite \\
\hline & $07 / 08 / 2011$ & $10: 37$ & IRLO-CN-CT1-1 & 1 & 300 & 26.5 & M & Composite \\
\hline \multirow{2}{*}{ JIV 537} & $07 / 08 / 2011$ & $15: 25$ & IRLO-CN-CT1-2 & 1 & 450 & 28.7 & $\mathrm{~F}$ & Composite \\
\hline & $07 / 08 / 2011$ & $15: 25$ & IRLO-CN-CT1-2 & 1 & 400 & 29.5 & $\mathrm{~F}$ & Composite \\
\hline JIV 538 & $07 / 08 / 2011$ & $15: 15$ & IRLO-CN-CT1-3 & 1 & 500 & 31.1 & $\mathrm{~F}$ & \\
\hline JDQ 978 & 07/08/2011 & $17: 00$ & OCTP-CN-C2M-1 & 1 & 210 & & & $\begin{array}{l}\text { DUP; Composite; } 2 \text { bags } \\
\text { for sample }\end{array}$ \\
\hline JDQ 978 & 07/08/2011 & $17: 00$ & OCTP-CN-C2M-1 & 1 & 850 & & & DUP; Composite \\
\hline JDQ 979 & $07 / 08 / 2011$ & $14: 30$ & ROCK-CN-CT1-1 & 1 & 850 & 36.3 & $\mathrm{~F}$ & \\
\hline JDQ 980 & $07 / 08 / 2011$ & $14: 45$ & ROCK-CN-CT1-2 & 1 & 1200 & 40.1 & $\mathrm{~F}$ & \\
\hline JDQ 981 & $07 / 08 / 2011$ & $15: 00$ & ROCK-CN-CT1-3 & 1 & 1000 & 37.7 & $M$ & \\
\hline JDQ 766 & 07/08/2011 & $16: 56$ & SEAW-CN-C2M-1A-D & 1 & --- & & & Salinity $=35$ \\
\hline JDQ 765 & $07 / 08 / 2011$ & $16: 47$ & SEAW-CN-C2M-1A-S & 1 & --- & & & Salinity $=35$ \\
\hline JDQ 768 & $07 / 08 / 2011$ & $17: 20$ & SEAW-CN-C2M-1B-D & 1 & --- & & & Salinity $=35$ \\
\hline JDQ 767 & $07 / 08 / 2011$ & $17: 10$ & SEAW-CN-C2M-1B-S & 1 & --- & & & Salinity $=35$ \\
\hline JDQ 700 & $07 / 08 / 2011$ & $10: 40$ & SEAW-CN-CT1-1 & 1 & --- & & & Salinity $=35$ \\
\hline JDQ 701 & $07 / 08 / 2011$ & $10: 28$ & SEAW-CN-CT1-2 & 1 & --- & & & Salinity $=35$ \\
\hline JDQ 702 & 07/08/2011 & $10: 37$ & SEAW-CN-CT1-3 & 1 & --- & & & Salinity $=35$ \\
\hline JIT 784 & $07 / 08 / 2011$ & $10: 45$ & SEDI-CN-CT1-1 & 1 & --- & & & \\
\hline JDQ 975 & $07 / 08 / 2011$ & $10: 40$ & URCH-CN-CT1-1 & 6 & 500 & & & \\
\hline JDR 055 & 07/09/2011 & $13: 15$ & CHIT-ML-MT1-1 & 4 & 500 & & & \\
\hline JDR 054 & $07 / 09 / 2011$ & $16: 45$ & EUAL-ML-DUP & 1 & 5500 & & & \\
\hline JDR 045 & 07/09/2011 & 13:05 & EUAL-ML-MT1-1 & 1 & 5500 & & & \\
\hline JDR 046 & $07 / 09 / 2011$ & $13: 15$ & EUAL-ML-MT1-2 & 1 & 5200 & & & \\
\hline JDR 047 & 07/09/2011 & $13: 10$ & EUAL-ML-MT1-3 & 1 & 5600 & & & \\
\hline JDQ 867 & 07/09/2011 & $14: 35$ & FUCU-ML-MT1-3 & 1 & 5300 & & & \\
\hline JDR 075 & $07 / 09 / 2011$ & $15: 10$ & GREN-ML-MT1-1 & 1 & 700 & 36.5 & $M$ & \\
\hline JDR 076 & $07 / 09 / 2011$ & $13: 05$ & GREN-ML-MT1-2 & 1 & 750 & 39.1 & & \\
\hline JDR 077 & 07/09/2011 & $15: 40$ & GREN-ML-MT1-3 & 1 & 800 & 38.4 & $\mathrm{~F}$ & \\
\hline JDR 058 & 07/09/2011 & $17: 40$ & HMUS-ML-MT1-1 & 10 & 500 & & & \\
\hline JDR 085 & $07 / 09 / 2011$ & $13: 10$ & IRLO-ML-MT1-1 & 1 & 400 & 29 & $\mathrm{~F}$ & Composite \\
\hline
\end{tabular}

U.S. Department of Energy September 2013
Amchitka Island, Alaska, Biological Monitoring Report, 2011 Sampling Results Doc. No. S08833 Page B-7 


\begin{tabular}{|c|c|c|c|c|c|c|c|c|}
\hline Ticket & Sample Date & Time & Location & $\begin{array}{l}\text { Number } \\
\text { Species } \\
\text { Sample }\end{array}$ & $\begin{array}{l}\text { Weight } \\
\text { (g) }\end{array}$ & $\begin{array}{l}\text { Length } \\
\text { (cm) }\end{array}$ & Sex & Notes \\
\hline & $07 / 09 / 2011$ & $13: 10$ & IRLO-ML-MT1-1 & 1 & 400 & 30.6 & $\mathrm{~F}$ & Composite \\
\hline \multirow{2}{*}{ JET 757} & 07/09/2011 & $13: 00$ & IRLO-ML-MT1-2 & 1 & 425 & 30 & $\mathrm{~F}$ & Composite \\
\hline & 07/09/2011 & $13: 00$ & IRLO-ML-MT1-2 & 1 & 400 & 28 & $\mathrm{~F}$ & Composite \\
\hline JET 758 & 07/09/2011 & $12: 30$ & IRLO-ML-MT1-3 & 1 & 500 & 31.4 & $\mathrm{~F}$ & \\
\hline JET 759 & 07/09/2011 & $16: 00$ & ROCK-ML-DUP & 1 & 950 & 37.8 & $\mathrm{~F}$ & \\
\hline JDR 065 & $07 / 09 / 2011$ & $12: 20$ & ROCK-ML-MT1-1 & 1 & 1050 & 40.8 & $\mathrm{~F}$ & \\
\hline JDR 066 & 07/09/2011 & $13: 15$ & ROCK-ML-MT1-2 & 1 & 800 & 36.3 & $M$ & \\
\hline JDR 067 & 07/09/2011 & $15: 00$ & ROCK-ML-MT1-3 & 1 & 900 & 37.6 & $M$ & \\
\hline JDQ 744 & 07/09/2011 & $13: 05$ & SEAW-ML-DUP & 1 & --- & & & Salinity $=36$ \\
\hline JDQ 735 & 07/09/2011 & $12: 55$ & SEAW-ML-MT1-1 & 1 & --- & & & Salinity $=36$ \\
\hline JDQ 736 & 07/09/2011 & $12: 47$ & SEAW-ML-MT1-2 & 1 & --- & & & Salinity $=34$ \\
\hline JDQ 737 & $07 / 09 / 2011$ & $13: 00$ & SEAW-ML-MT1-3 & 1 & --- & & & Salinity $=36$ \\
\hline JIT 790 & 07/09/2011 & $13: 10$ & SEDI-ML-MT1-1 & 1 & --- & & & \\
\hline JDR 061 & 07/09/2011 & $13: 10$ & URCH-ML-MT1-1 & 9 & 830 & & & \\
\hline JDR 056 & $07 / 10 / 2011$ & $17: 15$ & CHIT-ML-MT2-1 & 2 & 560 & & & \\
\hline JDR 057 & 07/10/2011 & $10: 50$ & CHIT-ML-MT3-1 & 2 & 500 & & & \\
\hline JDR 048 & $07 / 10 / 2011$ & $17: 00$ & EUAL-ML-MT2-1 & 1 & 5000 & & & \\
\hline JDR 049 & $07 / 10 / 2011$ & $17: 00$ & EUAL-ML-MT2-2 & 1 & 5600 & & & \\
\hline JDR 050 & 07/10/2011 & $17: 30$ & EUAL-ML-MT2-3 & 1 & 5600 & & & \\
\hline JDR 051 & $07 / 10 / 2011$ & $10: 40$ & EUAL-ML-MT3-1 & 1 & 5400 & & & \\
\hline JDR 052 & $07 / 10 / 2011$ & $10: 55$ & EUAL-ML-MT3-2 & 1 & 5200 & & & \\
\hline JDR 053 & 07/10/2011 & $10: 30$ & EUAL-ML-MT3-3 & 1 & 5300 & & & \\
\hline JDQ 871 & 07/10/2011 & $15: 00$ & FUCU-ML-MT3-1 & 1 & 5500 & & & \\
\hline JDQ 872 & 07/10/2011 & $15: 10$ & FUCU-ML-MT3-2 & 1 & 5500 & & & \\
\hline JDQ 873 & 07/10/2011 & $15: 20$ & FUCU-ML-MT3-3 & 1 & 5300 & & & \\
\hline JDR 084 & $07 / 10 / 2011$ & $12: 30$ & GREN-ML-DUP & 1 & 600 & 36 & $\mathrm{~F}$ & \\
\hline JDR 081 & 07/10/2011 & $12: 10$ & GREN-ML-MT3-1 & 1 & 675 & 36.9 & $\mathrm{~F}$ & \\
\hline JDR 082 & 07/10/2011 & $11: 55$ & GREN-ML-MT3-2 & 1 & 550 & 34.3 & $\mathrm{~F}$ & \\
\hline JDR 083 & 07/10/2011 & $12: 10$ & GREN-ML-MT3-3 & 1 & 600 & 35.7 & $\mathrm{~F}$ & \\
\hline JDR 060 & $07 / 10 / 2011$ & $11: 10$ & HMUS-ML-MT3-1 & 15 & 500 & & & \\
\hline \multirow{2}{*}{ JIV 541} & 07/10/2011 & $17: 15$ & IRLO-ML-MT2-1 & 1 & 350 & 27.4 & M & \\
\hline & $07 / 10 / 2011$ & $17: 15$ & IRLO-ML-MT2-1 & 1 & 400 & 29.2 & $\mathrm{~F}$ & \\
\hline JET 760 & $07 / 10 / 2011$ & $11: 00$ & IRLO-ML-MT3-1 & 1 & 860 & 42.5 & $\mathrm{~F}$ & \\
\hline JIV 539 & 07/10/2011 & $10: 30$ & IRLO-ML-MT3-2 & 1 & 800 & 40 & $\mathrm{~F}$ & \\
\hline JIV 540 & $07 / 10 / 2011$ & $11: 00$ & IRLO-ML-MT3-3 & 1 & 900 & 42.7 & $\mathrm{~F}$ & \\
\hline \multirow{2}{*}{ JDR 064} & 07/10/2011 & $17: 00$ & OCTP-ML-M2M-1 & 1 & 400 & & M & Composite \\
\hline & $07 / 10 / 2011$ & $17: 00$ & OCTP-ML-M2M-1 & 1 & 300 & & $M$ & Composite \\
\hline \multirow{4}{*}{ JDR 087} & $07 / 10 / 2011$ & $16: 30$ & PCOD-ML-M2M-1 & 1 & 1300 & 50 & $\mathrm{~F}$ & Composite \\
\hline & 07/10/2011 & $16: 30$ & PCOD-ML-M2M-1 & 1 & 1000 & 46.5 & $\mathrm{~F}$ & Composite \\
\hline & $07 / 10 / 2011$ & $16: 30$ & PCOD-ML-M2M-1 & 1 & 700 & 42.4 & $M$ & Composite \\
\hline & 07/10/2011 & $16: 30$ & PCOD-ML-M2M-1 & 1 & 2150 & & & Composite \\
\hline JDR 071 & $07 / 10 / 2011$ & $10: 15$ & ROCK-ML-MT3-1 & 1 & 800 & 37 & M & \\
\hline JDR 072 & $07 / 10 / 2011$ & $14: 30$ & ROCK-ML-MT3-2 & 1 & 1300 & 43.7 & $M$ & \\
\hline JDR 073 & $07 / 10 / 2011$ & $14: 25$ & ROCK-ML-MT3-3 & 1 & 2100 & 47.3 & $\mathrm{~F}$ & \\
\hline
\end{tabular}

Amchitka Island, Alaska, Biological Monitoring Report, 2011 Sampling Results 


\begin{tabular}{|c|c|c|c|c|c|c|c|c|}
\hline Ticket & Sample Date & Time & Location & $\begin{array}{l}\text { Number } \\
\text { Species } \\
\text { Sample }\end{array}$ & $\begin{array}{l}\text { Weight } \\
\text { (g) }\end{array}$ & $\begin{array}{l}\text { Length } \\
\text { (cm) }\end{array}$ & Sex & Notes \\
\hline JDQ 738 & 07/10/2011 & $17: 00$ & SEAW-ML-MT2-1 & 1 & --- & & & Salinity = 34 \\
\hline JDQ 739 & $07 / 10 / 2011$ & $17: 05$ & SEAW-ML-MT2-2 & 1 & --- & & & Salinity $=34$ \\
\hline JDQ 740 & 07/10/2011 & $17: 05$ & SEAW-ML-MT2-3 & 1 & --- & & & Salinity = 34 \\
\hline JDQ 741 & 07/10/2011 & $10: 50$ & SEAW-ML-MT3-1 & 1 & --- & & & Salinity $=35$ \\
\hline JDQ 742 & $07 / 10 / 2011$ & $11: 00$ & SEAW-ML-MT3-2 & 1 & --- & & & Salinity $=35$ \\
\hline JDQ 743 & 07/10/2011 & $11: 10$ & SEAW-ML-MT3-3 & 1 & --- & & & Salinity = 34 \\
\hline JIT 792 & 07/10/2011 & $11: 15$ & SEDI-ML-MT3-1 & 1 & --- & & & UAF \\
\hline JDR 062 & 07/10/2011 & $17: 20$ & URCH-ML-MT2-1 & 7 & 695 & & & \\
\hline JDR 063 & 07/10/2011 & $11: 20$ & URCH-ML-MT3-1 & 7 & 560 & & & \\
\hline JES 494 & $07 / 11 / 2011$ & $10: 50$ & EUAL-ML-DUP2 & 1 & 4200 & & & \\
\hline JDR 078 & $07 / 11 / 2011$ & $11: 10$ & GREN-ML-MT2-1 & 1 & 600 & 35.2 & $\mathrm{~F}$ & \\
\hline JDR 079 & 07/11/2011 & $10: 35$ & GREN-ML-MT2-2 & 1 & 600 & 35.1 & $\mathrm{~F}$ & \\
\hline JDR 080 & $07 / 11 / 2011$ & $10: 40$ & GREN-ML-MT2-3 & 1 & 625 & 36.4 & $\mathrm{~F}$ & \\
\hline JDR 086 & $07 / 11 / 2011$ & $15: 30$ & HALI-ML-M2M-1 & 1 & 5800 & & & \\
\hline JDR 059 & 07/11/2011 & $11: 30$ & HMUS-ML-MT2-1 & 17 & 535 & & & \\
\hline JDR 068 & $07 / 11 / 2011$ & $10: 00$ & IRLO-ML-MT2-2 & 1 & 500 & 31.3 & $\mathrm{~F}$ & \\
\hline \multirow{2}{*}{ JDR 069} & $07 / 11 / 2011$ & $10: 20$ & IRLO-ML-MT2-3 & 1 & 200 & 22.8 & $M$ & \\
\hline & 07/11/2011 & $10: 20$ & IRLO-ML-MT2-3 & 1 & 300 & 26.3 & $\mathrm{~F}$ & \\
\hline JIV 555 & $07 / 11 / 2011$ & $13: 30$ & ROCK-ML-MT2-1 & 1 & 900 & 37.5 & $\mathrm{~F}$ & \\
\hline JIV 556 & $07 / 11 / 2011$ & $13: 45$ & ROCK-ML-MT2-2 & 1 & 800 & 35 & M & \\
\hline JDR 070 & $07 / 11 / 2011$ & $14: 00$ & ROCK-ML-MT2-3 & 1 & 900 & 36 & M & \\
\hline JIT 791 & 07/11/2011 & $11: 20$ & SEDI-ML-MT2-1 & 1 & --- & & & \\
\hline JDQ 850 & $07 / 12 / 2011$ & $17: 30$ & FUCU-CN-CT2-3 & 1 & 5200 & & & \\
\hline JIW 344 & $07 / 12 / 2011$ & $14: 30$ & GREN-LS-DUP & 1 & 700 & 39 & $\mathrm{~F}$ & \\
\hline JIW 342 & 07/12/2011 & $14: 00$ & GREN-LS-LT3-2 & 1 & 650 & 37.2 & $\mathrm{~F}$ & \\
\hline JIW 343 & $07 / 12 / 2011$ & $14: 15$ & GREN-LS-LT3-3 & 1 & 600 & 35.4 & $\mathrm{~F}$ & \\
\hline JDR 042 & 07/12/2011 & $12: 10$ & IRLO-LS-LT3-1 & 1 & 850 & 41.5 & M & \\
\hline JET 755 & $07 / 12 / 2011$ & $12: 15$ & IRLO-LS-LT3-3 & 1 & 1100 & 44.2 & M & \\
\hline JDR 031 & 07/12/2011 & $13: 17$ & ROCK-LS-DUP & 1 & 1000 & 39 & $\mathrm{~F}$ & \\
\hline JDR 027 & 07/12/2011 & $16: 30$ & ROCK-LS-DUP2 & 1 & 1100 & 39.1 & $M$ & \\
\hline JDR 023 & $07 / 12 / 2011$ & $16: 05$ & ROCK-LS-LT1-2 & 1 & 900 & 37.1 & $\mathrm{~F}$ & \\
\hline JDR 024 & $07 / 12 / 2011$ & $16: 30$ & ROCK-LS-LT1-3 & 1 & 1000 & 38.4 & $\mathrm{~F}$ & \\
\hline JDR 026 & $07 / 12 / 2011$ & $15: 10$ & ROCK-LS-LT2-2 & 1 & 900 & 36.5 & M & \\
\hline JET 754 & $07 / 12 / 2011$ & $13: 10$ & ROCK-LS-LT3-1 & 1 & 1000 & 38.4 & $\mathrm{~F}$ & \\
\hline JDR 029 & $07 / 12 / 2011$ & $13: 12$ & ROCK-LS-LT3-2 & 1 & 1150 & 40.5 & $\mathrm{~F}$ & \\
\hline JDQ 734 & $07 / 12 / 2011$ & $15: 00$ & SEAW-LS-DUP & 1 & --- & & & $\begin{array}{l}\text { Diver collected with } \\
\text { NISKIN; Salinity = } 35\end{array}$ \\
\hline JDR 000 & 07/13/2011 & $8: 30$ & HALI-CN-C2M-1 & 1 & 9000 & 915 & $\mathrm{~F}$ & \\
\hline JDR 043 & $07 / 13 / 2011$ & 9:29 & HALI-LS-L2M-1 & 1 & 13750 & 1000 & $\mathrm{~F}$ & \\
\hline JDR 143 & $07 / 14 / 2011$ & $10: 20$ & CHIT-AS-AST3-1 & 2 & 550 & & & \\
\hline JDR 137 & 07/14/2011 & $14: 45$ & EUAL-AS-AST3-1 & 1 & 5650 & & & \\
\hline JDR 138 & 07/14/2011 & $14: 45$ & EUAL-AS-AST3-2 & 1 & 5100 & & & \\
\hline JDR 139 & 07/14/2011 & $14: 25$ & EUAL-AS-AST3-3 & 1 & 5400 & & & \\
\hline JDR 140 & $07 / 14 / 2011$ & $10: 15$ & EUAL-AS-DUP & 1 & 5550 & & & \\
\hline JDQ 891 & $07 / 14 / 2011$ & $16: 15$ & FUCU-AS-AST3-1 & 1 & 5250 & & & \\
\hline
\end{tabular}

U.S. Department of Energy September 2013
Amchitka Island, Alaska, Biological Monitoring Report, 2011 Sampling Results Doc. No. S08833 Page B-9 


\begin{tabular}{|c|c|c|c|c|c|c|c|c|}
\hline Ticket & Sample Date & Time & Location & $\begin{array}{l}\text { Number } \\
\text { Species } \\
\text { Sample }\end{array}$ & $\begin{array}{l}\text { Weight } \\
\text { (g) }\end{array}$ & $\begin{array}{l}\text { Length } \\
\text { (cm) }\end{array}$ & Sex & Notes \\
\hline JDQ 892 & $07 / 14 / 2011$ & $16: 20$ & FUCU-AS-AST3-2 & 1 & 5750 & & & \\
\hline JDQ 893 & $07 / 14 / 2011$ & $16: 25$ & FUCU-AS-AST3-3 & 1 & 6900 & & & \\
\hline \multirow{2}{*}{ JDR 167} & $07 / 14 / 2011$ & $10: 10$ & GREN-AS-AST3-1 & 1 & 300 & 28.2 & $\mathrm{~F}$ & \\
\hline & $07 / 14 / 2011$ & $10: 10$ & GREN-AS-AST3-1 & 1 & 350 & 31 & $\mathrm{~F}$ & \\
\hline JDR 168 & $07 / 14 / 2011$ & $10: 20$ & GREN-AS-AST3-2 & 1 & 500 & 35.5 & $\mathrm{~F}$ & \\
\hline JDR 169 & $07 / 14 / 2011$ & $14: 20$ & GREN-AS-AST3-3 & 1 & 500 & 32.9 & $\mathrm{~F}$ & \\
\hline JDR 170 & $07 / 14 / 2011$ & $14: 30$ & GREN-AS-DUP & 1 & 600 & 35.5 & $\mathrm{~F}$ & \\
\hline JDR 146 & $07 / 14 / 2011$ & $14: 40$ & HMUS-AS-AST3-1 & 9 & 500 & & & \\
\hline \multirow{2}{*}{ JDR 171} & $07 / 14 / 2011$ & $14: 45$ & IRLO-AS-AST3-1 & 1 & 350 & 25.1 & $\mathrm{~F}$ & Composite \\
\hline & $07 / 14 / 2011$ & $14: 45$ & IRLO-AS-AST3-1 & 1 & 650 & 37.6 & $\mathrm{~F}$ & Composite \\
\hline JET 766 & $07 / 14 / 2011$ & $14: 25$ & IRLO-AS-AST3-2 & 1 & 800 & 38 & $\mathrm{~F}$ & \\
\hline JET 767 & $07 / 14 / 2011$ & $14: 30$ & IRLO-AS-AST3-3 & 1 & 1000 & 42.9 & M & \\
\hline JDR 157 & $07 / 14 / 2011$ & $10: 10$ & ROCK-AS-AST3-1 & 1 & 800 & 36.9 & $\mathrm{~F}$ & \\
\hline JDR 158 & $07 / 14 / 2011$ & $10: 16$ & ROCK-AS-AST3-2 & 1 & 550 & 32.6 & $\mathrm{~F}$ & \\
\hline JDR 159 & $07 / 14 / 2011$ & $10: 29$ & ROCK-AS-AST3-3 & 1 & 1275 & 43.2 & M & \\
\hline JDR 160 & $07 / 14 / 2011$ & $14: 20$ & ROCK-AS-DUP & 1 & 1500 & 44.4 & $\mathrm{~F}$ & \\
\hline JDQ 761 & $07 / 14 / 2011$ & $14: 25$ & SEAW-AS-AST3-1 & 1 & --- & & & Salinity $=36$ \\
\hline JDQ 762 & $07 / 14 / 2011$ & $14: 45$ & SEAW-AS-AST3-2 & 1 & --- & & & Salinity = 35 \\
\hline JDQ 763 & $07 / 14 / 2011$ & $14: 30$ & SEAW-AS-AST3-3 & 1 & --- & & & Salinity = 37 \\
\hline JDQ 764 & $07 / 14 / 2011$ & $14: 40$ & SEAW-AS-DUP & 1 & --- & & & Salinity $=36$ \\
\hline JIT 798 & $07 / 14 / 2011$ & $14: 25$ & SEDI-AS-AST3-1 & 1 & --- & & & UAF \\
\hline JDR 149 & $07 / 14 / 2011$ & $14: 30$ & URCH-AS-AST3-1 & 5 & 500 & & & \\
\hline JDR 141 & $07 / 15 / 2011$ & $10: 25$ & CHIT-AS-AST1-1 & 4 & 560 & & & \\
\hline JDR 131 & $07 / 15 / 2011$ & $10: 52$ & EUAL-AS-AST1-1 & 1 & 5300 & & & \\
\hline JDR 132 & $07 / 15 / 2011$ & $10: 40$ & EUAL-AS-AST1-2 & 1 & 5000 & & & \\
\hline JDR 133 & $07 / 15 / 2011$ & $10: 30$ & EUAL-AS-AST1-3 & 1 & 5300 & & & \\
\hline JES 495 & $07 / 15 / 2011$ & $10: 30$ & EUAL-AS-DUP2 & 1 & 5200 & & & \\
\hline JDR 161 & $07 / 15 / 2011$ & $10: 25$ & GREN-AS-AST1-1 & 1 & 600 & 34.5 & $\mathrm{~F}$ & \\
\hline JDR 162 & $07 / 15 / 2011$ & $10: 40$ & GREN-AS-AST1-2 & 1 & 600 & 35.4 & $\mathrm{~F}$ & \\
\hline JDR 163 & $07 / 15 / 2011$ & $10: 47$ & GREN-AS-AST1-3 & 1 & 600 & 34.5 & $\mathrm{~F}$ & \\
\hline JDR 144 & $07 / 15 / 2011$ & $10: 37$ & HMUS-AS-AST1-1 & 17 & 500 & & & \\
\hline JET 768 & $07 / 15 / 2011$ & $10: 40$ & IRLO-AS-AST1-1 & 1 & 500 & 31.7 & $\mathrm{~F}$ & \\
\hline JET 769 & $07 / 15 / 2011$ & $10: 50$ & IRLO-AS-AST1-2 & 1 & 650 & 34.6 & $\mathrm{~F}$ & \\
\hline JET 770 & $07 / 15 / 2011$ & $11: 20$ & IRLO-AS-AST1-3 & 1 & 600 & 32.8 & $\mathrm{~F}$ & \\
\hline \multirow{2}{*}{ JDR 173} & $07 / 15 / 2011$ & $14: 50$ & PCOD-AS-AS2M-1 & 1 & 1775 & 56.2 & M & Composite \\
\hline & $07 / 15 / 2011$ & $14: 50$ & PCOD-AS-AS2M-1 & 1 & 5200 & 70.5 & $M$ & Composite \\
\hline JDR 151 & $07 / 15 / 2011$ & $10: 45$ & ROCK-AS-AST1-1 & 1 & 500 & 30.5 & M & \\
\hline JDR 152 & $07 / 15 / 2011$ & $11: 00$ & ROCK-AS-AST1-2 & 1 & 850 & 35.6 & M & \\
\hline JDR 153 & $07 / 15 / 2011$ & 11:05 & ROCK-AS-AST1-3 & 1 & 1500 & 43.5 & M & \\
\hline JDQ 755 & $07 / 15 / 2011$ & $10: 55$ & SEAW-AS-AST1-1 & 1 & --- & & & Salinity = 34 \\
\hline JDQ 756 & $07 / 15 / 2011$ & $10: 51$ & SEAW-AS-AST1-2 & 1 & --- & & & Salinity = 35 \\
\hline JDQ 757 & $07 / 15 / 2011$ & $10: 33$ & SEAW-AS-AST1-3 & 1 & --- & & & Salinity $=35$ \\
\hline JIT 796 & $07 / 15 / 2011$ & $10: 40$ & SEDI-AS-AST1-1 & 1 & --- & & & \\
\hline JDR 147 & $07 / 15 / 2011$ & $10: 47$ & URCH-AS-AST1-1 & 5 & 620 & & & \\
\hline
\end{tabular}

Amchitka Island, Alaska, Biological Monitoring Report, 2011 Sampling Results 


\begin{tabular}{|c|c|c|c|c|c|c|c|c|}
\hline Ticket & Sample Date & Time & Location & $\begin{array}{l}\text { Number } \\
\text { Species } \\
\text { Sample }\end{array}$ & $\begin{array}{l}\text { Weight } \\
\text { (g) }\end{array}$ & $\begin{array}{l}\text { Length } \\
\text { (cm) }\end{array}$ & Sex & Notes \\
\hline JDR 142 & $07 / 16 / 2011$ & $10: 20$ & CHIT-AS-AST2-1 & 3 & 940 & & & \\
\hline JDR 134 & $07 / 16 / 2011$ & $10: 20$ & EUAL-AS-AST2-1 & 1 & 5300 & & & \\
\hline JDR 135 & $07 / 16 / 2011$ & $10: 30$ & EUAL-AS-AST2-2 & 1 & 5500 & & & \\
\hline JDR 136 & 07/16/2011 & $10: 05$ & EUAL-AS-AST2-3 & 1 & 5200 & & & \\
\hline JDQ 885 & $07 / 16 / 2011$ & $11: 37$ & FUCU-AS-AST1-1 & 1 & 5300 & & & \\
\hline JDQ 886 & $07 / 16 / 2011$ & $11: 48$ & FUCU-AS-AST1-2 & 1 & 5400 & & & \\
\hline JDQ 887 & $07 / 16 / 2011$ & $12: 10$ & FUCU-AS-AST1-3 & 1 & 5100 & & & \\
\hline JDQ 888 & $07 / 16 / 2011$ & $15: 45$ & FUCU-AS-AST2-1 & 1 & 5200 & & & \\
\hline JDQ 889 & $07 / 16 / 2011$ & $16: 15$ & FUCU-AS-AST2-2 & 1 & 5300 & & & \\
\hline JDQ 890 & $07 / 16 / 2011$ & $16: 44$ & FUCU-AS-AST2-3 & 1 & 5200 & & & \\
\hline JDQ 894 & $07 / 16 / 2011$ & $11: 44$ & FUCU-AS-DUP & 1 & 5300 & & & \\
\hline JDR 164 & $07 / 16 / 2011$ & $10: 12$ & GREN-AS-AST2-1 & 1 & 900 & 40.1 & M & \\
\hline JDR 165 & $07 / 16 / 2011$ & $9: 55$ & GREN-AS-AST2-2 & 1 & 650 & 36.1 & $\mathrm{~F}$ & \\
\hline JDR 166 & $07 / 16 / 2011$ & $10: 12$ & GREN-AS-AST2-3 & 1 & 700 & 38.7 & $\mathrm{~F}$ & \\
\hline JDR 145 & $07 / 16 / 2011$ & $10: 18$ & HMUS-AS-AST2-1 & 6 & 550 & & & \\
\hline JIV 545 & $07 / 16 / 2011$ & $14: 40$ & IRLO-AS-AST2-1 & 1 & 600 & 32.9 & M & \\
\hline JIV 546 & $07 / 16 / 2011$ & $14: 45$ & IRLO-AS-AST2-2 & 1 & 500 & 31.9 & $\mathrm{~F}$ & \\
\hline JIV 547 & 07/16/2011 & $18: 10$ & IRLO-AS-AST2-3 & 1 & 650 & 34.4 & $\mathrm{~F}$ & \\
\hline JDR 154 & $07 / 16 / 2011$ & $13: 25$ & ROCK-AS-AST2-1 & 1 & 800 & 36.8 & $\mathrm{M}$ & \\
\hline JDR 155 & $07 / 16 / 2011$ & $13: 30$ & ROCK-AS-AST2-2 & 1 & 1225 & 40.8 & $\mathrm{~F}$ & \\
\hline JDR 156 & $07 / 16 / 2011$ & $13: 50$ & ROCK-AS-AST2-3 & 1 & 950 & 38.4 & M & \\
\hline JDQ 758 & $07 / 16 / 2011$ & $10: 00$ & SEAW-AS-AST2-1 & 1 & --- & & & Salinity $=35$ \\
\hline JDQ 759 & $07 / 16 / 2011$ & $10: 00$ & SEAW-AS-AST2-2 & 1 & --- & & & Salinity = 35 \\
\hline JDQ 760 & $07 / 16 / 2011$ & $10: 15$ & SEAW-AS-AST2-3 & 1 & --- & & & Salinity = 35 \\
\hline JIT 797 & $07 / 16 / 2011$ & $10: 00$ & SEDI-AS-AST2-1 & 1 & --- & & & \\
\hline JDR 148 & $07 / 16 / 2011$ & $10: 15$ & URCH-AS-AST2-1 & 7 & 530 & & & \\
\hline JDR 100 & $07 / 17 / 2011$ & $16: 30$ & CHIT-AN-ANT3-1 & 3 & 950 & & & \\
\hline JDR 094 & $07 / 17 / 2011$ & $16: 30$ & EUAL-AN-ANT3-1 & 1 & 5100 & & & \\
\hline JDR 095 & 07/17/2011 & $16: 25$ & EUAL-AN-ANT3-2 & 1 & 5600 & & & \\
\hline JDR 096 & $07 / 17 / 2011$ & $16: 15$ & EUAL-AN-ANT3-3 & 1 & 5100 & & & \\
\hline JDR 097 & $07 / 17 / 2011$ & $16: 25$ & EUAL-AN-DUP & 1 & 5700 & & & \\
\hline JES 496 & $07 / 17 / 2011$ & $16: 25$ & EUAL-AN-DUP & 1 & 5700 & & & UAF; DUP \\
\hline JDR 124 & $07 / 17 / 2011$ & $16: 15$ & GREN-AN-ANT3-1 & 1 & 550 & 35.9 & $\mathrm{~F}$ & \\
\hline JDR 125 & $07 / 17 / 2011$ & $16: 40$ & GREN-AN-ANT3-2 & 1 & 650 & 36.2 & $\mathrm{~F}$ & \\
\hline JDR 126 & $07 / 17 / 2011$ & $16: 10$ & GREN-AN-ANT3-3 & 1 & 550 & 36.4 & $\mathrm{~F}$ & \\
\hline JDR 127 & $07 / 17 / 2011$ & $16: 10$ & GREN-AN-DUP & 1 & 525 & 35.9 & $\mathrm{~F}$ & \\
\hline JDR 129 & $07 / 17 / 2011$ & $14: 30$ & HALI-AN-AN2M-1 & 1 & 27215 & 122 & $\mathrm{~F}$ & \\
\hline JET 761 & $07 / 17 / 2011$ & $14: 20$ & HALI-AN-DUP & 1 & 5000 & 105 & $\mathrm{~F}$ & \\
\hline JDR 128 & $07 / 17 / 2011$ & $16: 40$ & IRLO-AN-ANT3-1 & 1 & 750 & 36.4 & $\mathrm{~F}$ & \\
\hline JET 762 & $07 / 17 / 2011$ & $16: 10$ & IRLO-AN-ANT3-2 & 1 & 825 & 36.7 & $\mathrm{~F}$ & \\
\hline JET 763 & $07 / 17 / 2011$ & $16: 45$ & IRLO-AN-ANT3-3 & 1 & 400 & 28.6 & M & Composite \\
\hline JET 763 & $07 / 17 / 2011$ & $16: 45$ & IRLO-AN-ANT3-3 & 1 & 400 & 29 & $\mathrm{~F}$ & Composite \\
\hline \multirow{2}{*}{ JDR 130} & 07/17/2011 & $15: 30$ & PCOD-AN-AN2M-1 & 1 & 3100 & 66.6 & $U$ & Composite \\
\hline & $07 / 17 / 2011$ & $15: 30$ & PCOD-AN-AN2M-1 & 1 & 2100 & 58.7 & $\mathrm{~F}$ & Composite \\
\hline
\end{tabular}




\begin{tabular}{|c|c|c|c|c|c|c|c|c|}
\hline Ticket & Sample Date & Time & Location & $\begin{array}{l}\text { Number } \\
\text { Species } \\
\text { Sample }\end{array}$ & $\begin{array}{l}\text { Weight } \\
\text { (g) }\end{array}$ & $\begin{array}{l}\text { Length } \\
\text { (cm) }\end{array}$ & Sex & Notes \\
\hline JDR 114 & 07/17/2011 & $16: 25$ & ROCK-AN-ANT3-1 & 1 & 1050 & 38.7 & M & \\
\hline JDR 115 & $07 / 17 / 2011$ & $16: 10$ & ROCK-AN-ANT3-2 & 1 & 1200 & 40 & M & \\
\hline \multirow{2}{*}{ JDR 116} & $07 / 17 / 2011$ & $16: 20$ & ROCK-AN-ANT3-3 & 1 & 200 & 23.5 & $M$ & Composite \\
\hline & $07 / 17 / 2011$ & $16: 20$ & ROCK-AN-ANT3-3 & 1 & 400 & 27 & M & Composite \\
\hline JDQ 751 & $07 / 17 / 2011$ & $16: 35$ & SEAW-AN-ANT3-1 & 1 & --- & & & Salinity $=35$ \\
\hline JDQ 752 & $07 / 17 / 2011$ & $16: 20$ & SEAW-AN-ANT3-2 & 1 & --- & & & Salinity = 35 \\
\hline JDQ 753 & 07/17/2011 & $16: 15$ & SEAW-AN-ANT3-3 & 1 & --- & & & Salinity = 35 \\
\hline JDQ 754 & $07 / 17 / 2011$ & $16: 25$ & SEAW-AN-DUP & 1 & --- & & & Salinity = 35 \\
\hline JIT 795 & $07 / 17 / 2011$ & $16: 20$ & SEDI-AN-ANT3-1 & 1 & --- & & & \\
\hline JDR 106 & $07 / 17 / 2011$ & $16: 40$ & URCH-AN-ANT3-1 & 5 & 750 & & & \\
\hline JDR 099 & $07 / 18 / 2011$ & $15: 40$ & CHIT-AN-ANT2-1 & 3 & 910 & & & \\
\hline JDR 091 & 07/18/2011 & $16: 15$ & EUAL-AN-ANT2-1 & 1 & 5500 & & & \\
\hline JDR 092 & $07 / 18 / 2011$ & $16: 00$ & EUAL-AN-ANT2-2 & 1 & 5800 & & & \\
\hline JDR 093 & $07 / 18 / 2011$ & $15: 30$ & EUAL-AN-ANT2-3 & 1 & 5150 & & & \\
\hline JDQ 878 & 07/18/2011 & $14: 11$ & FUCU-AN-ANT2-1 & 1 & 5200 & & & \\
\hline JDQ 879 & $07 / 18 / 2011$ & $16: 57$ & FUCU-AN-ANT2-2 & 1 & 5400 & & & \\
\hline JDQ 880 & $07 / 18 / 2011$ & $17: 35$ & FUCU-AN-ANT2-3 & 1 & 5430 & & & \\
\hline JDQ 881 & 07/18/2011 & 9:02 & FUCU-AN-ANT3-1 & 1 & 5200 & & & \\
\hline JDQ 882 & $07 / 18 / 2011$ & 13:00 & FUCU-AN-ANT3-2 & 1 & 5100 & & & \\
\hline JDQ 883 & $07 / 18 / 2011$ & $13: 35$ & FUCU-AN-ANT3-3 & 1 & 5300 & & & \\
\hline JDQ 884 & $07 / 18 / 2011$ & $13: 00$ & FUCU-AN-DUP & 1 & 5200 & & & \\
\hline JDR 121 & $07 / 18 / 2011$ & $15: 40$ & GREN-AN-ANT2-1 & 1 & 600 & 36.8 & $\mathrm{~F}$ & \\
\hline JDR 122 & 07/18/2011 & $15: 40$ & GREN-AN-ANT2-2 & 1 & 650 & 38 & $\mathrm{~F}$ & \\
\hline JDR 123 & $07 / 18 / 2011$ & $15: 43$ & GREN-AN-ANT2-3 & 1 & 550 & 34.7 & $\mathrm{~F}$ & \\
\hline JDR 102 & $07 / 18 / 2011$ & $16: 00$ & HMUS-AN-ANT2-1 & 5 & 530 & & & \\
\hline JDR 103 & $07 / 18 / 2011$ & $10: 30$ & HMUS-AN-ANT3-1 & 7 & 500 & & & \\
\hline JDR 107 & $07 / 18 / 2011$ & $16: 00$ & OCTP-AN-AN2M-1 & 3 & 1200 & & & \\
\hline JDR 111 & $07 / 18 / 2011$ & $16: 00$ & ROCK-AN-ANT2-1 & 1 & 2500 & 51.5 & $\mathrm{~F}$ & \\
\hline JDR 112 & $07 / 18 / 2011$ & $16: 10$ & ROCK-AN-ANT2-2 & 1 & 1700 & 47.5 & M & \\
\hline JDQ 748 & $07 / 18 / 2011$ & $15: 25$ & SEAW-AN-ANT2-1 & 1 & --- & & & Salinity = 35 \\
\hline JDQ 749 & $07 / 18 / 2011$ & $15: 30$ & SEAW-AN-ANT2-2 & 1 & --- & & & Salinity $=35$ \\
\hline JDQ 750 & $07 / 18 / 2011$ & $15: 40$ & SEAW-AN-ANT2-3 & 1 & --- & & & Salinity $=35$ \\
\hline JIT 794 & $07 / 18 / 2011$ & $15: 20$ & SEDI-AN-ANT2-1 & 1 & --- & & & \\
\hline JDR 105 & $07 / 18 / 2011$ & $15: 50$ & URCH-AN-ANT2-1 & 9 & 820 & & & \\
\hline JDR 088 & 07/19/2011 & $14: 55$ & EUAL-AN-ANT1-1 & 1 & 5200 & & & \\
\hline JDR 089 & $07 / 19 / 2011$ & $14: 50$ & EUAL-AN-ANT1-2 & 1 & 5600 & & & \\
\hline JDR 090 & 07/19/2011 & $14: 40$ & EUAL-AN-ANT1-3 & 1 & 5200 & & & \\
\hline JDQ 875 & $07 / 19 / 2011$ & 13:09 & FUCU-AN-ANT1-1 & 1 & 5300 & & & \\
\hline JDR 118 & 07/19/2011 & $14: 38$ & GREN-AN-ANT1-1 & 1 & 500 & 33.9 & M & \\
\hline JDR 119 & 07/19/2011 & $14: 45$ & GREN-AN-ANT1-2 & 1 & 525 & 34.3 & M & \\
\hline JDR 120 & 07/19/2011 & $14: 55$ & GREN-AN-ANT1-3 & 1 & 675 & 36.6 & $\mathrm{~F}$ & \\
\hline
\end{tabular}




\begin{tabular}{|c|c|c|c|c|c|c|c|c|}
\hline Ticket & Sample Date & Time & Location & $\begin{array}{l}\text { Number } \\
\text { Species } \\
\text { Sample }\end{array}$ & $\begin{array}{l}\text { Weight } \\
\text { (g) }\end{array}$ & $\begin{array}{l}\text { Length } \\
\text { (cm) }\end{array}$ & Sex & Notes \\
\hline \multirow{2}{*}{ JIV 543} & 07/19/2011 & $15: 05$ & IRLO-AN-ANT1-1 & 1 & 300 & 28.5 & $\mathrm{~F}$ & Composite \\
\hline & 07/19/2011 & $15: 05$ & IRLO-AN-ANT1-1 & 1 & 350 & 28.4 & $\mathrm{~F}$ & Composite \\
\hline JIV 544 & 07/19/2011 & $14: 39$ & IRLO-AN-ANT1-2 & 1 & 550 & 32.6 & $\mathrm{~F}$ & \\
\hline JDR 113 & 07/19/2011 & $14: 47$ & IRLO-AN-ANT1-3 & 1 & 1025 & 37.7 & $\mathrm{~F}$ & \\
\hline JET 764 & 07/19/2011 & $9: 40$ & IRLO-AN-ANT2-1 & 1 & 680 & 37.3 & M & \\
\hline JET 765 & 07/19/2011 & $9: 50$ & IRLO-AN-ANT2-2 & 1 & 750 & 34.1 & $\mathrm{~F}$ & \\
\hline \multirow{2}{*}{ JIV 542} & 07/19/2011 & 9:35 & IRLO-AN-ANT2-3 & 1 & 450 & 31.3 & $\mathrm{M}$ & Composite \\
\hline & 07/19/2011 & 9:35 & IRLO-AN-ANT2-3 & 1 & 650 & 35.5 & $M$ & Composite \\
\hline JDR 108 & 07/19/2011 & $16: 00$ & ROCK-AN-ANT1-1 & 1 & 1350 & 40.9 & M & \\
\hline JDQ 745 & 07/19/2011 & $14: 50$ & SEAW-AN-ANT1-1 & 1 & --- & & & Salinity $=35$ \\
\hline JDQ 746 & 07/19/2011 & $14: 38$ & SEAW-AN-ANT1-2 & 1 & --- & & & Salinity $=35$ \\
\hline JDQ 747 & 07/19/2011 & $14: 40$ & SEAW-AN-ANT1-3 & 1 & -- & & & Salinity $=35$ \\
\hline JIT 793 & 07/19/2011 & $14: 55$ & SEDI-AN-ANT1-1 & 1 & --- & & & \\
\hline JDR 104 & 07/19/2011 & $14: 55$ & URCH-AN-ANT1-1 & 9 & 780 & & & \\
\hline JDR 098 & 07/20/2011 & $10: 00$ & CHIT-AN-ANT1-1 & 3 & 685 & & & \\
\hline JDQ 876 & $07 / 20 / 2011$ & $11: 06$ & FUCU-AN-ANT1-2 & 1 & 5200 & & & \\
\hline JDQ 877 & 07/20/2011 & $11: 20$ & FUCU-AN-ANT1-3 & 1 & 5250 & & & \\
\hline JDR 101 & $07 / 20 / 2011$ & $10: 10$ & HMUS-AN-ANT1-1 & 7 & 555 & & & \\
\hline
\end{tabular}


This page intentionally left blank 


\section{Appendix C}

Chain of Custody 
This page intentionally left blank 


\section{University of Miami Tritium Laboratory}

\section{Stoller \\ Legacy Management Team}

COC: 11023622.1 .1

Chain of Custody/Sample Submittal Form

RIN: 11023622

Sampler(s):

Laboratory: University of Miami Tritium Laboratory

Address: 4600 Rickenbacker Causeway

Phone: 305.421 .4000

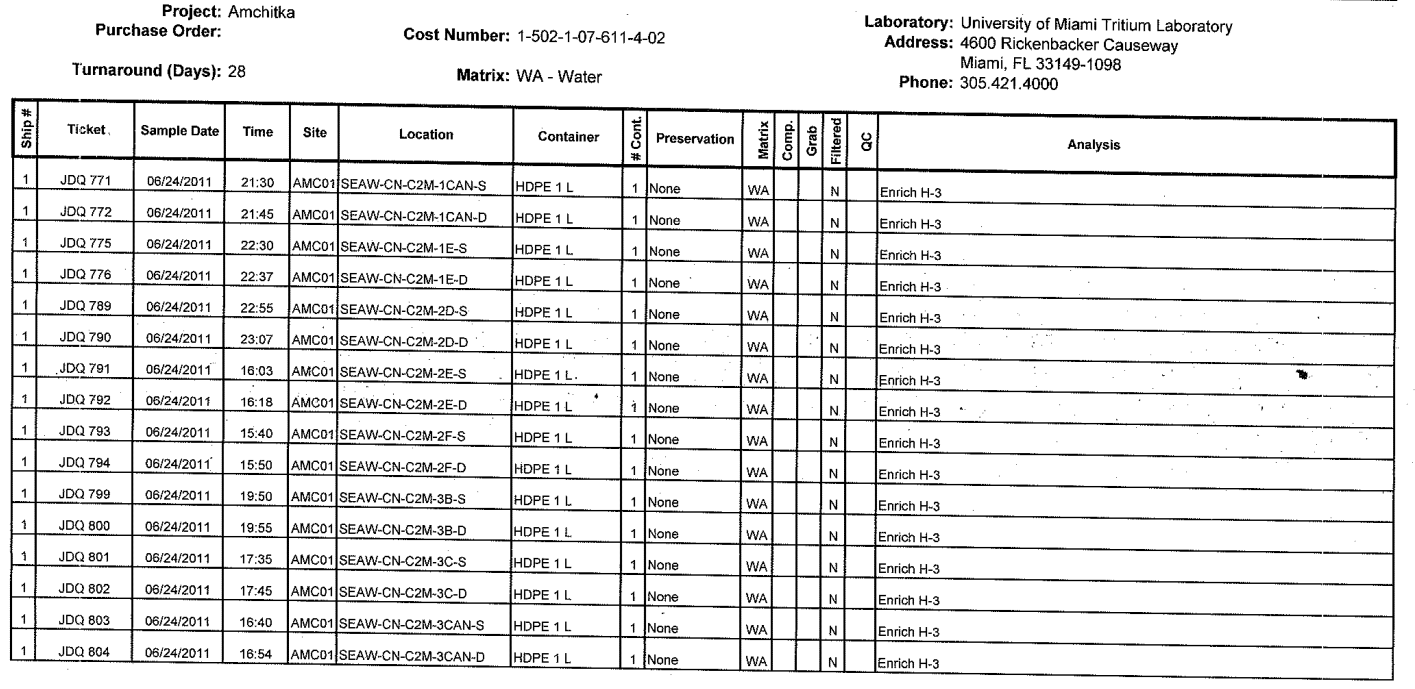

\begin{tabular}{|c|c|c|c|c|c|c|c|}
\hline 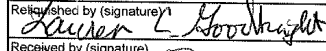 & $92 x / 11 /$ Timer & Reliquished by (signature) & Date & Time & Reliquished by (signature) & Date & Time \\
\hline Recoived by (signature) o Pure & 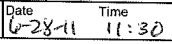 & Received by (signature) & Date & Time & Received by (signature) & Date & Time \\
\hline
\end{tabular}

Page 1 of 3

Stoller

Legacy Management Team
Chain of Custody/Sample Submittal Form

RIN: 11023622

Sampler(s):

Laboratory: University of Miami Tritium Laboratory

Address: 4600 Rickenbacker Causeway

Phone: 305.421 .4000

\begin{tabular}{|c|c|c|c|c|c|c|c|c|c|c|c|c|c|c|}
\hline & $\begin{array}{r}\text { Pur } \\
\text { Turnar }\end{array}$ & $\begin{array}{l}\text { Project: } \\
\text { hase Order: } \\
\text { und (Days): }\end{array}$ & & & $\begin{array}{r}\text { Cost Numbe } \\
\text { Matri }\end{array}$ & WA - Water & $1-4$ & & & & & $\begin{array}{r}\text { Laboratory: } \\
\text { Address: } \\
\text { Phone: }\end{array}$ & $\begin{array}{l}\text { Miami Tritium Laboratory } \\
\text { acker Causeway } \\
149-1098\end{array}$ & \\
\hline 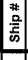 & Ticket & Sample Date & Time & Site & Location & Container & 8 & Preservation & \begin{tabular}{|l|}
$x$ \\
iv \\
\end{tabular} & $\frac{10}{50}$ & 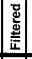 & $\mathscr{8}$ & Analysis & \\
\hline 1 & 300805 & 06/24/2011 & 15:00 & AMCO1 & SEAW-CN-C2M-3D-S & HDPE 1L & 1 & None & wa & & $\mathrm{N}$ & Enrich H-3 & & \\
\hline 1 & $J D Q 806$ & $06 / 24 / 2011$ & 15:20 & AMCO1 & SEAW-CN-C2M-3D-D & HDPE $1 L$ & 1 & None & wa & & $\mathrm{N}$ & Enrich $\mathrm{H}-3$ & & \\
\hline 1 & JDQ 807 & 06/24/2011 & 20:10 & AMC01 & SEAW-CN-C2M-AA-S & HDPE 1L & 1 & None & wa & & $\mathrm{N}$ & Enrich H-3 & & \\
\hline 1 & JDQ 808 & 06/24/2011 & $20: 20$ & AMCO1 & SEAW-CN-C2M-4A-D & HDPE $1 L$ & 1 & None & wa & & $\mathrm{N}$ & Enrich $\mathrm{H}-3$ & & \\
\hline 1 & Jo0 809 & \begin{tabular}{|l|}
$06 / 24 / 2011$ \\
\end{tabular} & 18:05 & AMCO1 & SEAW-CN-C2M-4B-S & HDPE 1L & 1 & None & wa & & $\mathrm{N}$ & Enrich H-3 & & \\
\hline 1 & JDQ 810 & $06 / 24 / 2011$ & 19:15 & AMCO1 & SEAW-CN-C2M-4B-D & HDPE $1 \mathrm{~L}$ & 1 & None & wa & & $\mathrm{N}$ & Enrich $\mathrm{H}-3$ & & \\
\hline 1 & J00811 & $06 / 24 / 2011$ & 17:10 & AMCO1 & SEAW-CN-C2M-5A-S & HDPE $1 \mathrm{~L}$ & 1 & None & $w_{A}$ & & $\mathrm{~N}$ & Enrich H-3 & & \\
\hline 1 & $3 D Q: 812$ & 06/24/2011 & $17: 20$ & AMCO1 & SEAW-CN-C2M-5A-D & HDPE 1L & 1 & None & wa & & $\mathrm{N}$ & Enrich $\mathrm{H}-3$ & & . \\
\hline 1 & JDQ 813 & 06/24/2011 & $22: 05$ & AMC01 & SEAW-CN-C2M-C1-2-S & HDPE 11 & 1 & None & wa & & $\mathrm{N}$ & Enrich H-3 & & \\
\hline 1 & JDQ 814 & \begin{tabular}{|l|}
$06 / 24 / 2011$ \\
\end{tabular} & 22:12 & AMCO1 & SEAW-CN-CZM-C1-2-D & HDPE $1 \mathrm{~L}$ & 1 & None & wa & & $\mathrm{N}$ & Enrich $\mathrm{H}-3$ & & \\
\hline 1 & $\mathrm{JDO} 815$ & 06/24/2011 & $20: 40$ & AMCO1 & SEAW-CN-C2M-C3-4-S & HDPE 1L & 1 & None & wa & & $\mathrm{N}$ & Enrich $\mathrm{H}-3$ & & \\
\hline 1 & JDQ 816 & \begin{tabular}{|l|}
$06 / 24 / 2011$ \\
\end{tabular} & $20: 45$ & AMCO1 & SEAW-CN-C2M-C3-4-D & HDPE 1L & 1 & None & wa & & $\mathrm{N}$ & Enrich $\mathrm{H}-3$ & & \\
\hline 1 & JDQ 819 & 06/24/2011 & 21:05 & AMCO1 & SEAW-CN-C2M-C4-4-S & HDPE 1L & 1 & None & wa & & $\mathrm{N}$ & Enrich $\mathrm{H}-3$ & & \\
\hline 1 & $J D Q 820$ & 06/24/2011 & $21: 15$ & AMCO1 & SEAW-CN-C2M-C4-4-D & HDPE $1 \mathrm{~L}$ & 1 & None & wa & & $\mathrm{N}$ & Enrich $\mathrm{H}-3$ & . & \\
\hline 1 & $J D Q 823$ & 06/24/2011 & 23:39 & AMC01 & SEAW-CN-C2M-LS2-2-S & HDPE 1 L & 1 & None & wa & & $\mathrm{N}$ & Enrich $\mathrm{H}-3$ & & \\
\hline 1 & JDO 824 & 06/24/2011 & $23: 45$ & AMC01 & SEAW-CN-C2M-LS2-2-D & HDPE 1L & 1 & None & wa & & ${ }_{N}$ & Enrich H-3 & & \\
\hline
\end{tabular}

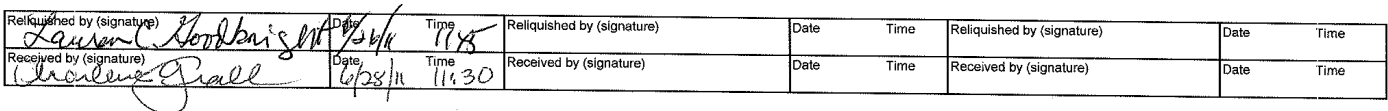

Page 2 of 3

U.S. Department of Energy September 2013
Amchitka Island, Alaska, Biological Monitoring Report, 2011 Sampling Results Doc. No. S08833

Page C-1 


\section{University of Miami Tritium Laboratory}

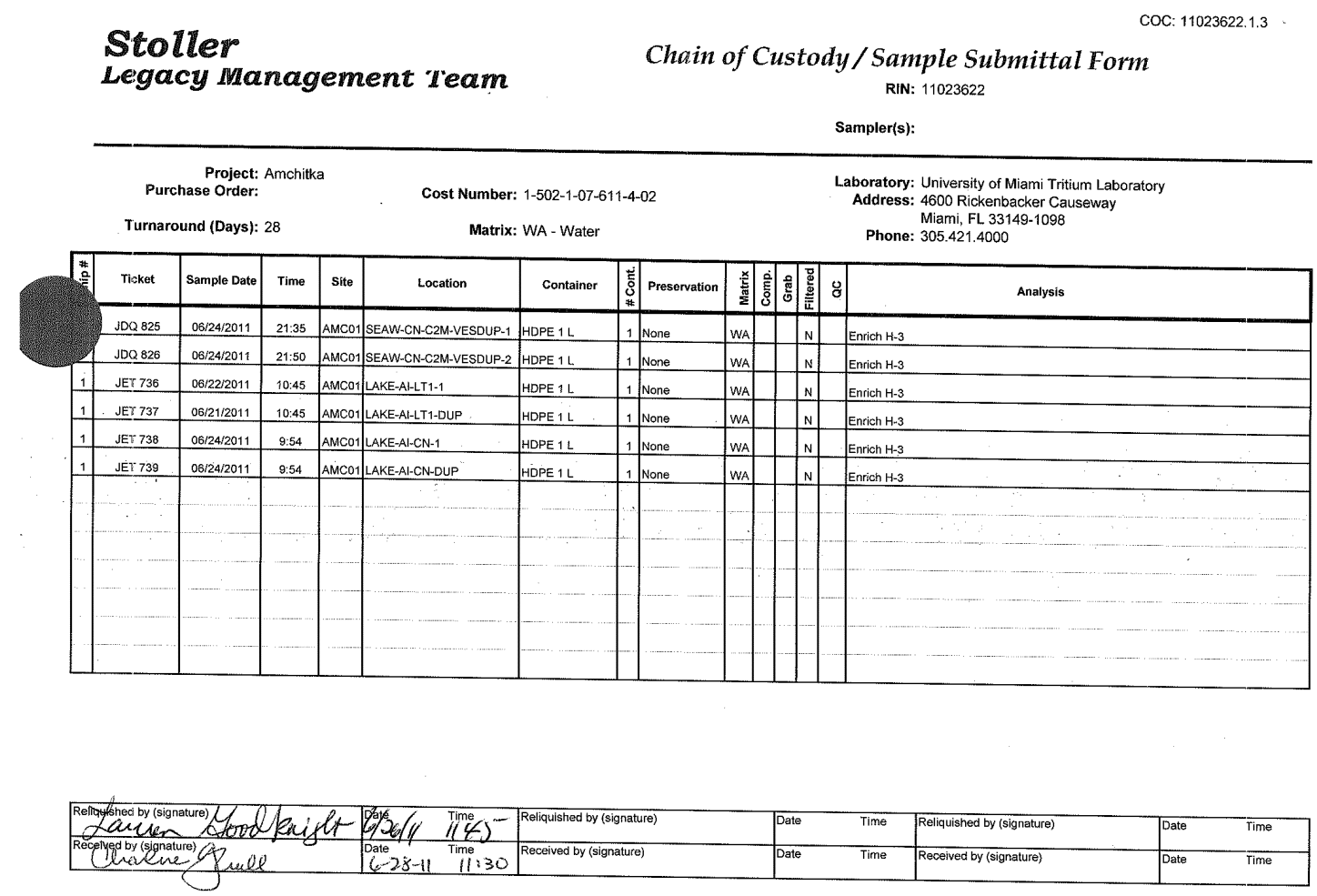

Page 3 of 3 


\section{University of Miami Tritium Laboratory}

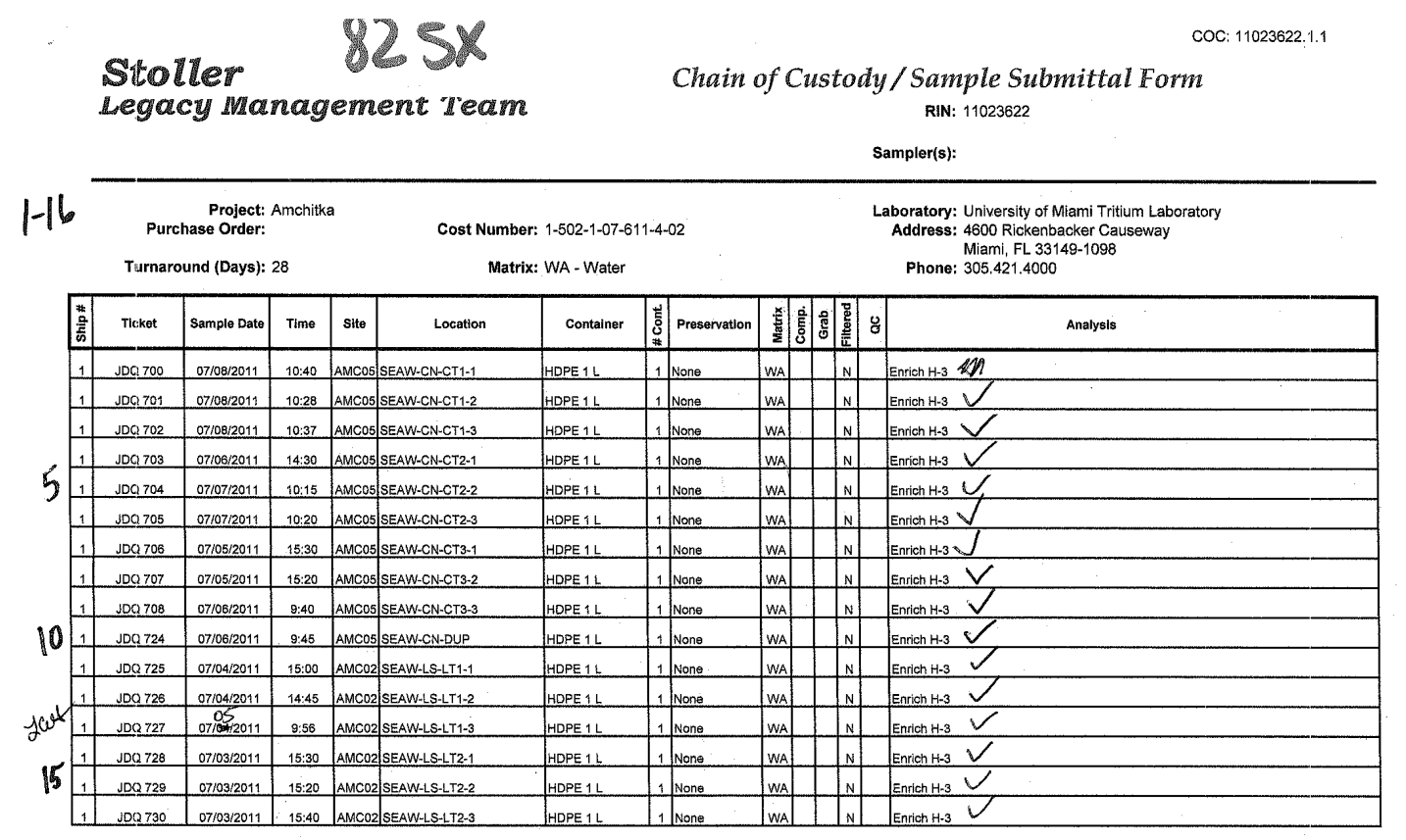

\begin{tabular}{|c|c|c|c|c|c|c|c|}
\hline Zaiguished by (signaturge) Hood knight & $79 \% 111450$ & Relifquished by (signgature) & Date & Time & Rellquished by (signature) & Date & Time \\
\hline Reved by signature & $77 / 25 / 11$ 10:55 & ignature) & Date & Time & Received by (signature) & Date & Time \\
\hline
\end{tabular}

Stoller Legacy Management Team
COC: 11023622.1 .2

Chain of Custody/Sample Submittal Form

RIN: 11023622

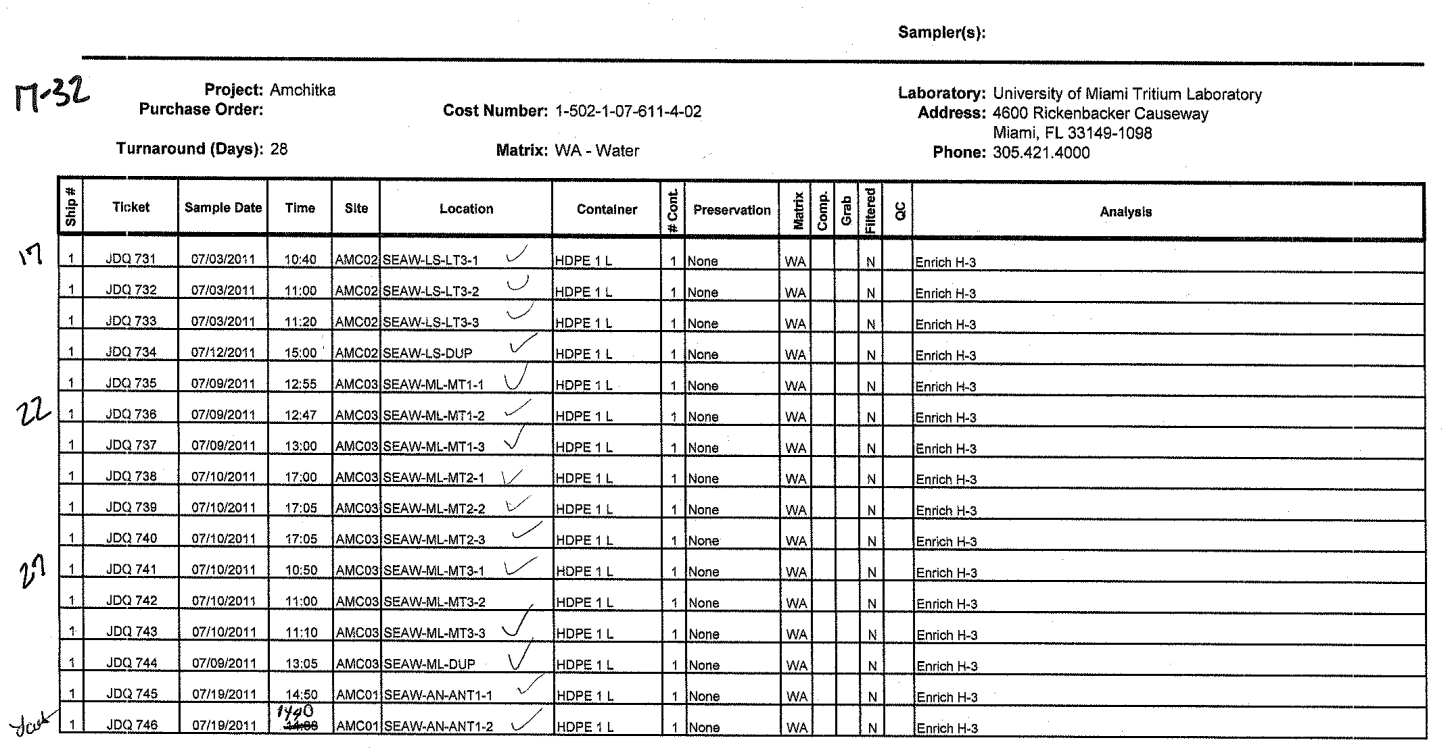

\begin{tabular}{|c|c|c|c|c|c|c|c|}
\hline Relliguished by (signature) Nool knisht & $294 / 1,1450$ & Reliquished by (signature) & Date & Time & Reliquished by (signature) & Date & Time \\
\hline Recoived by (signature) of ware & $\begin{array}{ll} & \text { Time } \\
7 / 25 / 11 & 10: 55\end{array}$ & Received by (signature) & Date & Time & Received by (signature) & Date & Time \\
\hline
\end{tabular}

Page 2 of 6 


\section{University of Miami Tritium Laboratory}

Stoller Legacy Management ream
Chain of Custody/Sample Submittal Form

RIN: 11023622

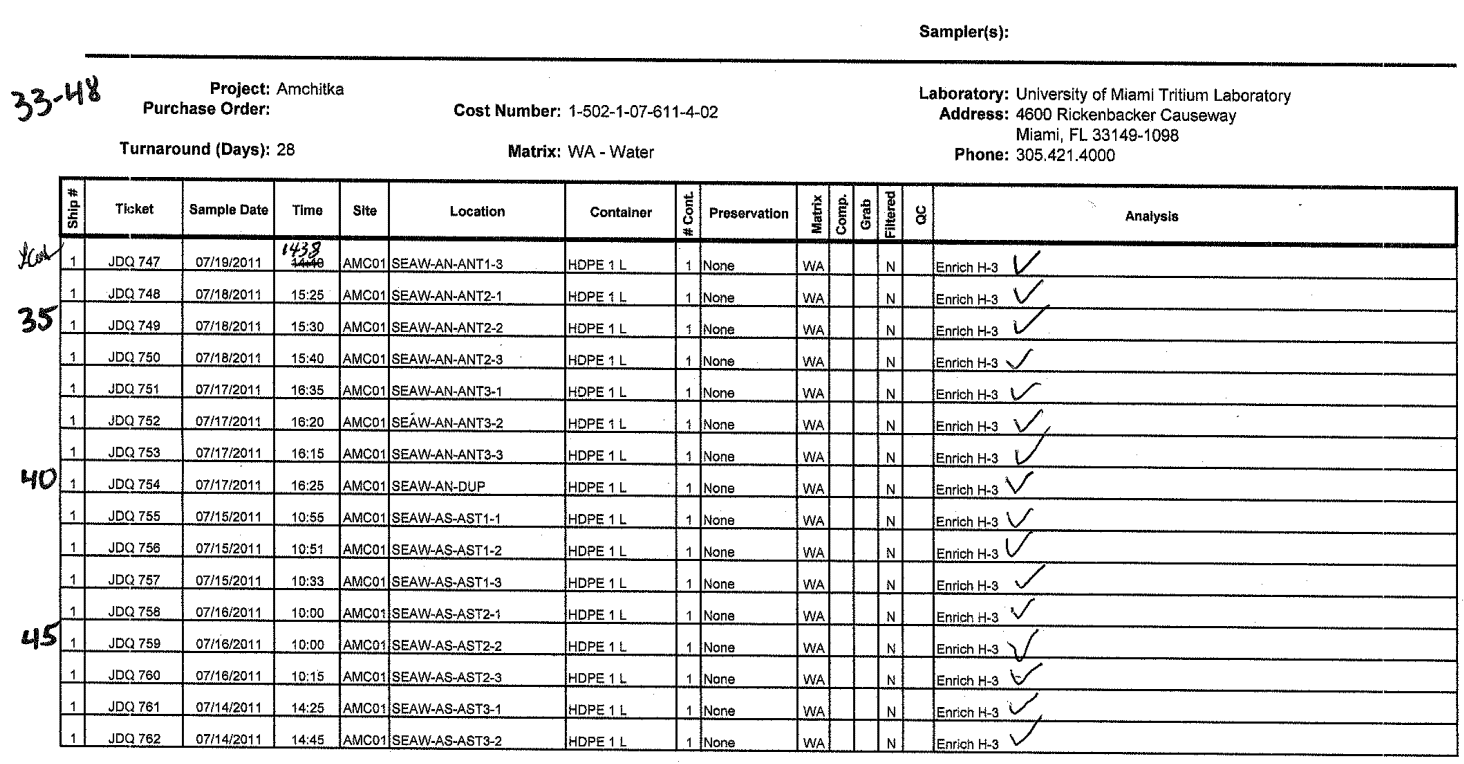

\begin{tabular}{|c|c|c|c|c|c|c|c|}
\hline Reyquishec by (signatue) on derisit & $7 \% 21111450$ & Rellquished by (signature) & Date & Time & Reliquished by (signature) & Date & Time \\
\hline Recaived by (signature) 9 inee & Dat $7 / 25 / 11$ Time $10: 55$ & Received by (signature) & Date. & Time & Received by (signature) & Date & Time \\
\hline
\end{tabular}

Page 3 of 6

Stoller Legacy Management Team
COC: 11023622.1 .4

Chain of Custody/Sample Submittal Form

$$
\text { RIN: } 11023622
$$

Sampler(s):

Laboratory: University of Miami Tritium Laboratory

Address: 4600 Rickenbacker Causeway

Phone: 305.421 .4000

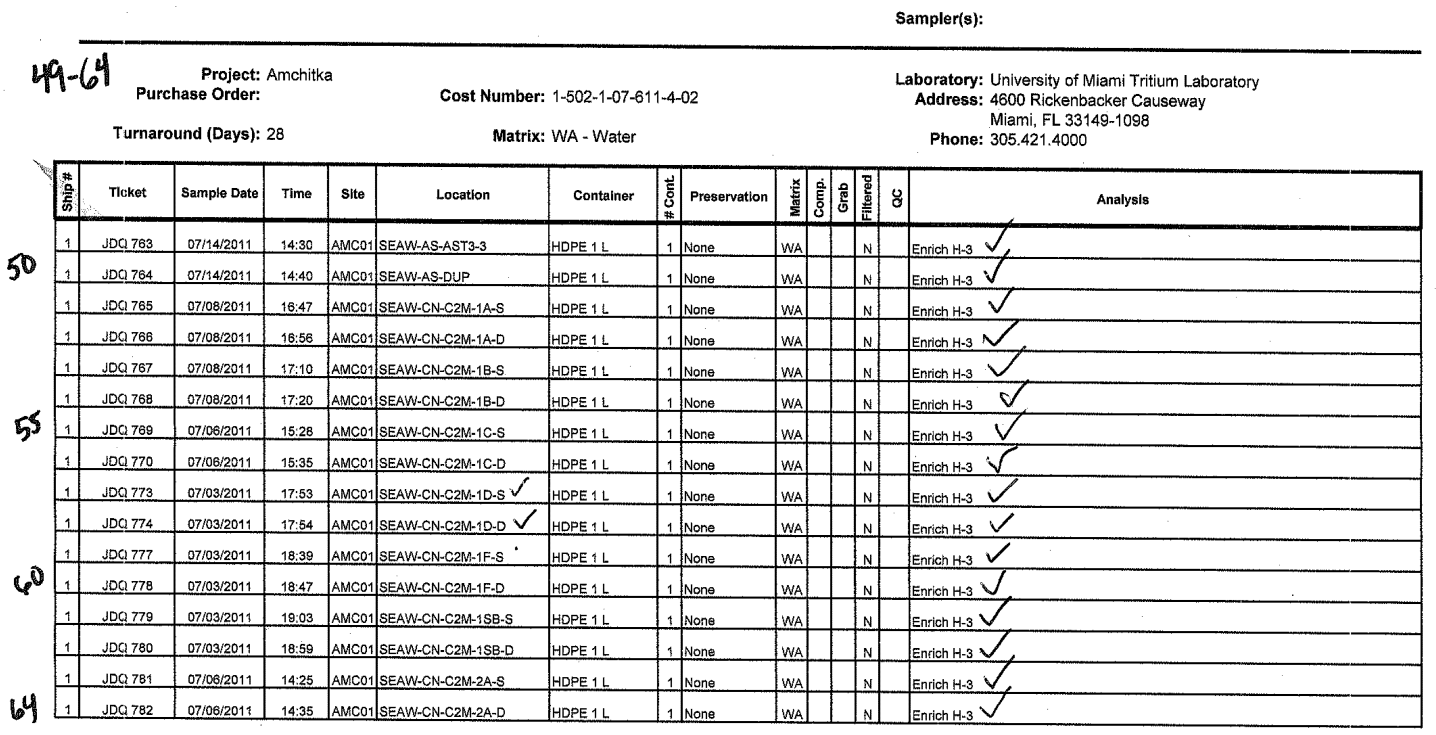

\begin{tabular}{|c|c|c|c|c|c|c|c|}
\hline Religuished by (signature Y yooleniset & $a_{20} / 11 / 450$ & Reliquished by (signature) & Date & Time & Reliquished by (signature) & Date & Time \\
\hline Red Hed by (signature) Taule & $\begin{array}{ll}0.10 \\
7 / 25 / 11 & 10.55 \\
\end{array}$ & Received by (signature) & Date & Time & Received by (signature) & Date & Time \\
\hline
\end{tabular}




\section{University of Miami Tritium Laboratory}

Stoller

Legacy Management Team
Chain of Custody/Sample Submittal Form

RIN: 11023622

Sampler(s):

Laboratory: University of Miami Tritium Laboratory

Cost Number: 1-502-1-07-611-4-02

Matrix: WA - Water

Address: 4600 Rickenbacker Causeway

Miami, FL 33149-1098

\begin{tabular}{|c|c|c|c|c|c|c|c|c|c|c|c|}
\hline Tlcket & Sample Date & Time & Site & Location & Contalner & \begin{tabular}{|l|} 
\\
\\
0 \\
0 \\
0
\end{tabular} & Preservation & 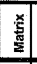 & 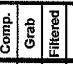 & 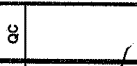 & Analysis \\
\hline $\mathrm{JDQ} 783$ & 07/06/2011 & 14:43 & AMCO 1 & SEAW-CN-C2M-2B-S & HDPE 1L & 1 & None & $w_{A}$ & $\mathrm{~N}$ & Enrich $\mathrm{H}-3 \mathrm{3}$ & \\
\hline$J D Q 784$ & 07/06/2011 & $14: 57$ & AMCO1 & SEAW-CN-C2M-2B-D & HDPE 1L & 1 & None & WA & $N$ & Enrich $\mathrm{H}-3 \mathrm{~V}$ & \\
\hline$J D Q 785$ & 07/06/2011 & 13:54 & AMCO1 & SEAW-CN-C2M-2C-S & HDPE 1L & 1 & None & wa & $\mathrm{N}$ & Enrich $\mathrm{H}-3 \mathrm{~V}$ & \\
\hline$J D Q 786$ & 07/06/2011 & 14:00 & AMCO1 & SEAW-CN-C2M-2C-D & HDPE $1 L$ & 1 & None & wa & $\mathrm{N}$ & Enrich $\mathrm{H}-3 \sqrt{ }$ & \\
\hline JDQ 787 & $07 / 06 / 2011$ & 13:10 & AMCO1 & SEAW-CN-C2M-2CAN-S & HOPE $1 \mathrm{~L}$ & 1 & None & WA & $\mathrm{N}$ & Enrich $\mathrm{H}-3$ & \\
\hline $\mathrm{JDQ} 788$ & \begin{tabular}{|l|}
$07 / 06 / 2011$ \\
\end{tabular} & 13:20 & AMCO1 & SEAW-CN-C2M-ZCAN-D & HDPE $1 L$ & 1 & None & wa & $\mathrm{N}$ & Enrich $\mathrm{H}-3 \mathrm{~V}$ & \\
\hline JDQ 795 & $07 / 03 / 2011$ & $19: 11$ & AMCO1 & SEAW-CN-C2M-2SB-S & HDPE $1 \mathrm{~L}$ & 1 & None & wa & $\mathrm{N}$ & Enrich $\mathrm{H}-3 \mathrm{~V}$ & \\
\hline$J D Q 796$ & 07/03/2011 & 19:16 & AMCO1 & SEAW-CN-C2M-2SB-D & HDPE $1 \mathrm{~L}$ & 1 & None & $w_{A}$ & N & Enrich $\mathrm{H}-3 \mathrm{~V}$ & \\
\hline$J D Q 797$ & 07/08/2011 & 14:10 & AMCO1 & SEAW-CN-C2M-3A-S & HDPE 1 L & 1 & None & wa & $\mathrm{N}$ & Enrich $\mathrm{H}-3 \mathrm{~J}$ & \\
\hline JDQ 798 & 07/06/2011 & 14:18 & AMCO1 & SEAW-CN-C2M-3A-D & HDPE $1 \mathrm{~L}$ & 1 & None & wa & $\mathrm{N}$ & Enrich $\mathrm{H}-3 \quad \sqrt{ }$ & \\
\hline$J D Q 817$ & 07/06/2011 & 13:30 & AMCO1 & SEAW-CN-C2M-C4-1-S & HDPE $1 \mathrm{~L}$ & 1 & None & $w_{A}$ & $\mathrm{~N}$ & Enrich $\mathrm{H}-3 \mathrm{~V}$ & \\
\hline JDQ 818 & 07/06:2011 & $13: 40$ & AMCO1 & SEAW-CN-C2M-C4-1-D & HOPE 1 L & 1 & None & wa & $N$ & Enrieh $\mathrm{H}-3 \mathrm{~V}$ & \\
\hline$J D Q 821$ & 07/06/2011 & $15: 14$ & AMCO 1 & SEAW-CN-C2M-C5-1-S & HDPE $1 L$ & 1 & None & wa & N & Enrich H-3 & \\
\hline $\mathrm{JDQ} 822$ & 07/06/2011 & $15: 20$ & AMCO1 & SEAW-CN-C2M-C5-1-D & HDPE 1 L & 1 & None & $w_{A}$ & $\mathrm{~N}$ & Enrich $\mathrm{H}-3 \quad$ & \\
\hline JDQ 827 & 07/03/2011 & 19:13 & AMCO1 & SEAW-CN-C2M-1SB-D-DUP & HDPE $1 L$ & 1 & None & wa & $\mathrm{N}$ & Envich $\mathrm{H}-3 \mathrm{~V}$ & \\
\hline$J D Q 828$ & 07/03/2011 & $19: 08$ & AMC01 & SEAW-CN-C2M-1SB-S-DUP & HDPE 1L & 1 & None & wa & N & Enrich $\mathrm{H}_{-3} \checkmark$ & \\
\hline
\end{tabular}

\begin{tabular}{|c|c|c|c|c|c|c|c|}
\hline $\begin{array}{l}\text { Religuished by (signature) } \\
\text { Lau forlen eniset }\end{array}$ & $77_{2 i 1 / 1} \quad 1450$ & Reliquished by (signature) & Date & Time & Reliquished by (signature) & Date & Time \\
\hline $\begin{array}{l}\text { Recived by (signature) } \\
\text { Qhacenef }\end{array}$ & $7 / 25 / 11 \quad 10: 55$ & Received by (signature) & Date & Time & Received by (signature) & Date & Time \\
\hline
\end{tabular}

Page 5 of 6

\section{Stoller Legacy Management Team}

COC: 11023622.1 .6

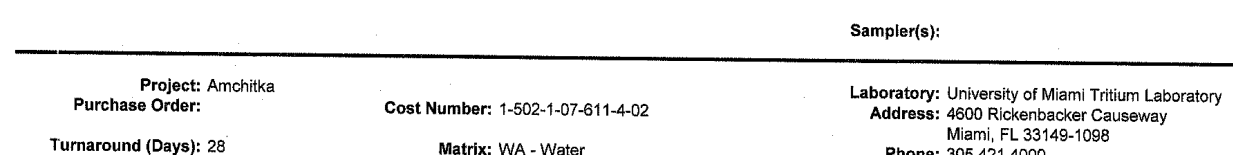

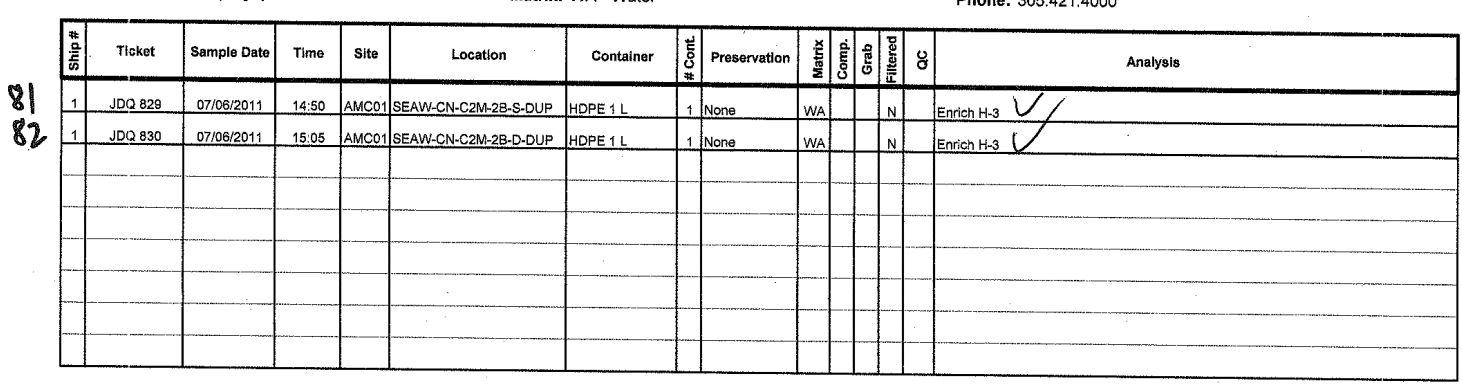

\begin{tabular}{|c|c|c|c|c|c|c|c|}
\hline Raviguishec by (signaturg) Soolkn' cht & $7 / 21119450$ & Reliquished by (signature) & Date & Time & Relliquished by (signature) & Date & Time \\
\hline $\begin{array}{l}\text { Recoived by (signature) } \\
\text { luacene }\end{array}$ & $7 / 25 / 11$ Timo:55 & Received by (signature) & Date & Time & Received by (signature) & Date & Time \\
\hline
\end{tabular}




\section{Lawrence Livermore National Laboratory}

Stoller

Legacy Maragement leam
Chain of Custody/Sample Submittal Form RIN: 11023623

Sampler(s):

\begin{tabular}{|c|c|c|c|c|c|c|c|c|c|c|c|}
\hline \multirow[b]{2}{*}{ 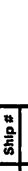 } & \multicolumn{4}{|c|}{$\begin{array}{l}\text { Project: Amchitka } \\
\text { Purchase Order: } \\
\text { Turnaround (Days): } 28\end{array}$} & \multicolumn{5}{|c|}{$\begin{array}{l}\text { Cost Number: 1-502-1-07-611-4-02 } \\
\text { Matrix: FA - Fauna SO-Soil VG - Vegetation }\end{array}$} & \multicolumn{2}{|r|}{$\begin{array}{l}\text { Laboratory: Lawrence Livermore National Laboratory } \\
\text { Address: Mr. Michael Tamblin } \\
7000 \text { East Avenue; Livermore, CA } 84550 \\
\text { Phone: } 925-424-2619\end{array}$} \\
\hline & Teket & Sample Date & Timo & Stto & Location & Contalner & \begin{tabular}{|l|l|}
\multirow{5}{\mathbf{g}}{} & Presenvation \\
\end{tabular} & & \begin{tabular}{l|l|l|}
0 \\
\end{tabular} & 量 & Anatysis \\
\hline 1 & JDQ BA5 & 08/23/2011 & 12:15 & AMCOS & FUCU-CN-CT1-1 & Plastic Bag & $\begin{array}{ll}1 & \text { None } \\
\end{array}$ & va & & & G Spec,Am,Pu, Uiso \\
\hline 1 & $\mathrm{JDQ} 846$ & 08/23/2011 & 11:05 & AMcos & FUCU-CN-CT1-2 & Plastic Bag & \begin{tabular}{|l|l|}
1 & None \\
\end{tabular} & vg & & & G Spec.Am,Pu,Uiso \\
\hline 1 & JDQ 847 & 08/23/2011 & 11:30 & AMCOS & FUCU-CN-CT1-3 & Plastic Bag & 1 None & vg & & & G Spec,Am, Pu.Uiso \\
\hline 1 & J0Q 854 & 08/23/2011 & i2:15 & AMCOS & FUCU-CN-DUP & Piastic Bag & 1. None & vg & & & G Spec.Am,Pu, Uiso \\
\hline 1 & J00 855 & 08/22/2011 & 15:25 & AMCOS & FUCULLS-LT1-1 & Plastic Bag & 1) None & va & & & G Spec,Am,Pu,Uiso \\
\hline 1 & JDQ 856 & 08/22/2011 & 15:50 & AMCO5 & FUCU-LS-LT1-2 & Plastic Bag & 1 None & vo & & & G Spec,Am,Pu,Uiso \\
\hline 1 & JDQ 857 & 08/2212011 & 16:45 & AMCOS & FUCU-LS-LT1-3 & Plastic Bag & 1 None & vg & & & G Spec,Am,Pu, Uiso \\
\hline 1 & JDQ 858 & $08 / 22 / 2011$ & 15:15 & AMCO5 & FUCU-LS-LT2-1 & Plastic Bag & 1 None & vG & & & G Spec,Am,Pu, Uiso \\
\hline 1 & J0Q 859 & 08/22/2011 & 15:41 & Amcos & FUCU-LS-LT2-2 & Plastic Bag & 1 Nane & vg & & & G Spec,Am.Pu,Uiso \\
\hline 1 & JDQ 860 & $08 / 22 / 2011$ & 16:15 & AMcos & FUCU-LS-LT2-3 & Plastic Bag & 1 None & va & & & 6 Spec,Am, Pu, Uiso \\
\hline 1 & $\mathrm{JDOQB64}$ & 06/22/2011 & 16:45 & AMCOS & FUCU-LS-DUP & Plastic Bag & 1 None & va & & & G Spec,Am,Pu, Uiso \\
\hline 1 & JDQ 865 & 08/20/2011 & $17: 15$ & AMCOS & FUCU-ML-MT1-1 & Plastic Bag & 1. None & vG & & & G Spec,Am,Pu, Uiso \\
\hline 1 & JDQ 868 & 08/21/2011 & 12:15 & AMCO5 & FUCU-ML-ATT1-2 & Plastic Bag & 1 None & va & & & G Spec,Am,Pu, Uiso \\
\hline 1 & J0Q 868 & 08/20/2011 & 17:00 & AMCO5 & FUCU-ML-MT2-1 & Plastic Bag & 1 Nane & va & & & G Spec,Am,Pu, Uiso \\
\hline 1 & JDQ 869 & 00/21/2011 & 11:53 & AMCOS & FUCU-ML-MT2-2 & Plastic Bas & 1 None & vg & & & G Spec,Am,Pu, Uiso \\
\hline 1 & JDQ 870 & 08/21/2011 & 11:53 & AMCOS & FUCU-ML-MT2-3 & Plastic Bag & 1 None & vol & & & G Spec,Am,Pu,Uiso \\
\hline
\end{tabular}

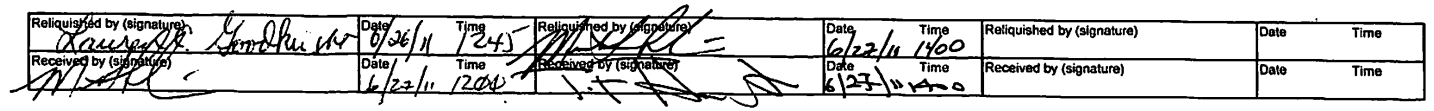

Page 1 of 2

\section{Stoller Legacy Management Team}

\section{Chain of Custody/Sample Submittal Form}

RIN: 11023623

Sampler(s)

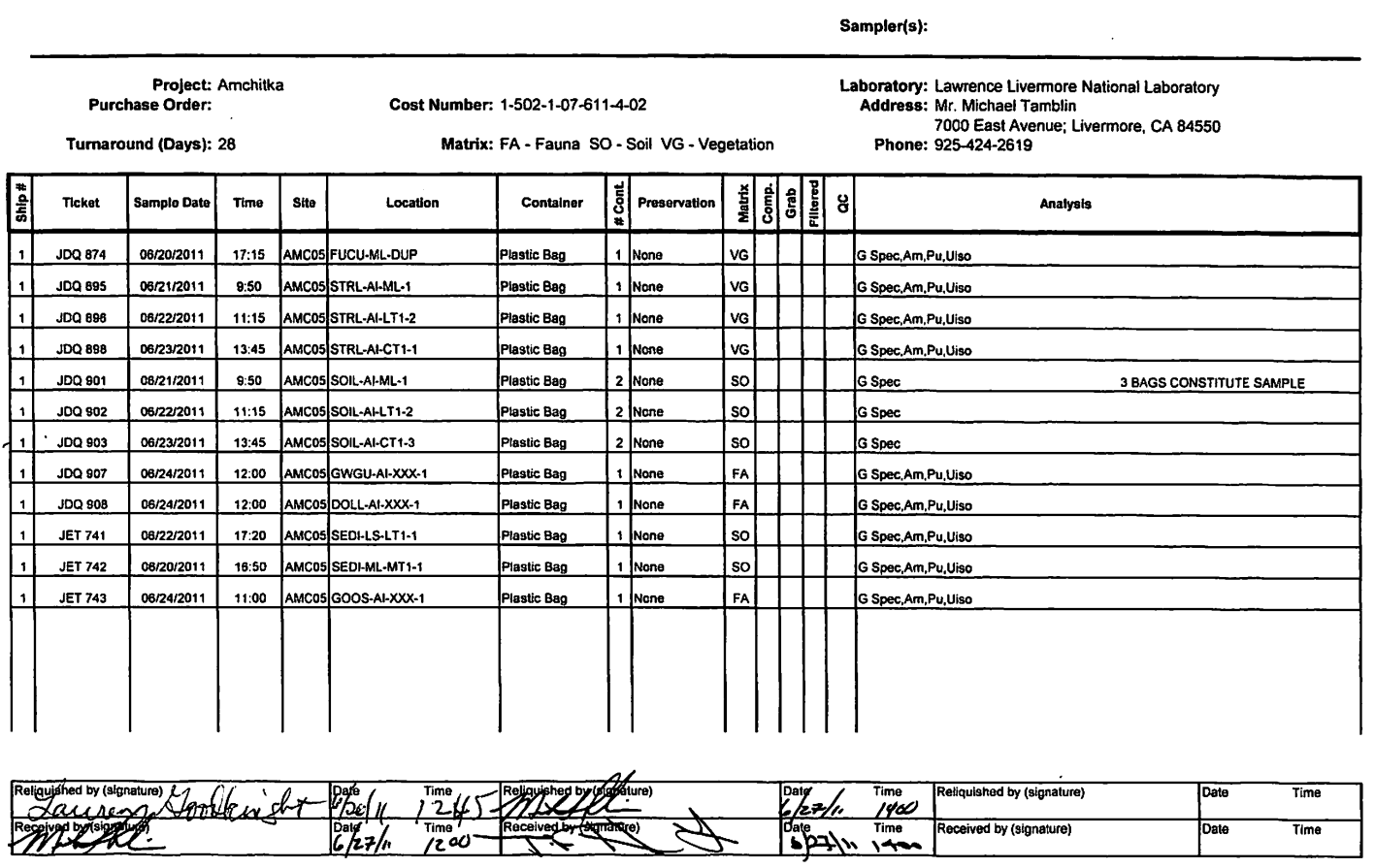




\section{Lawrence Livermore National Laboratory}

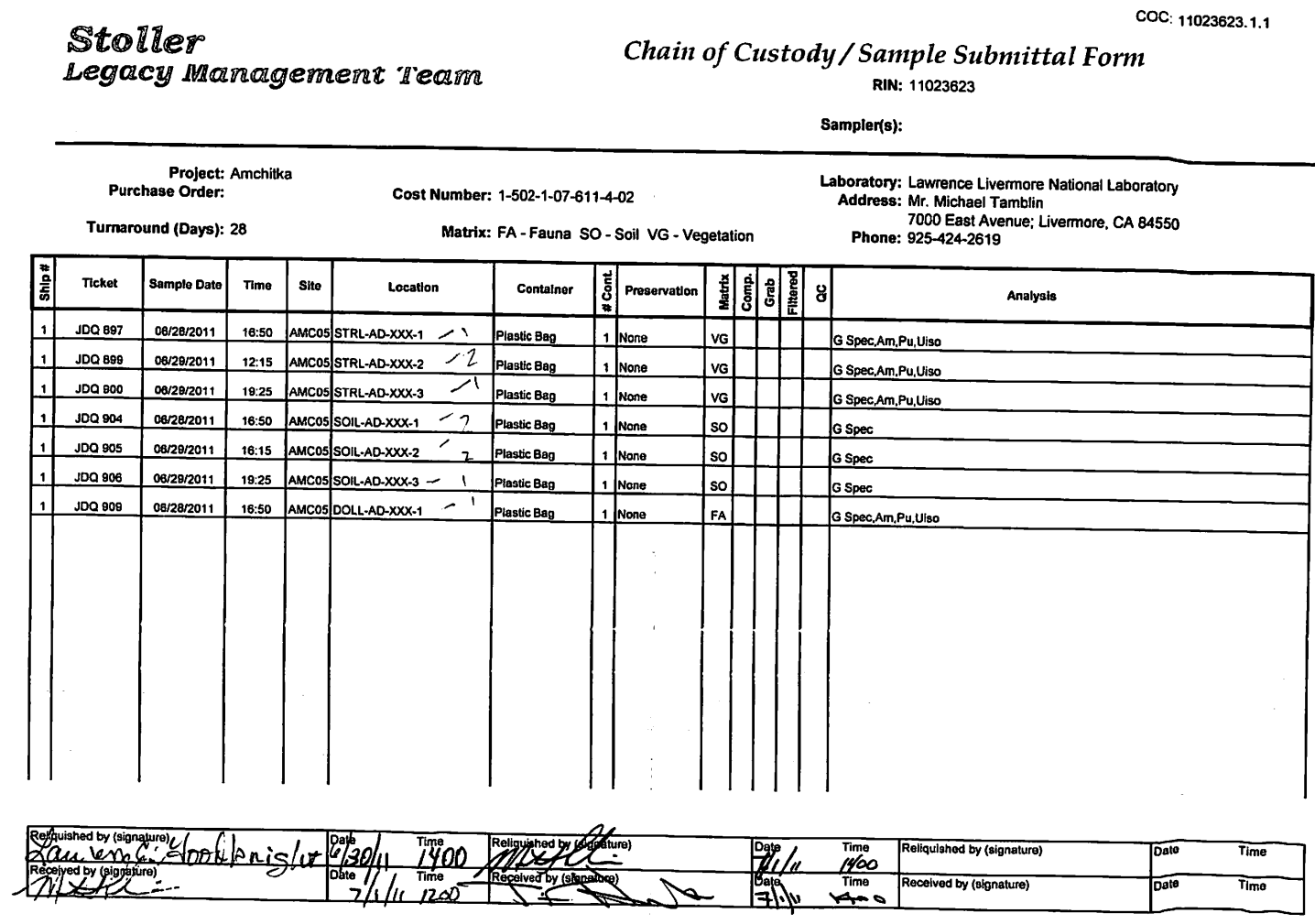

Page 1 of 1

COC: 11023623.1 .1

Sitoller
Legory Management Heam
Chain of Custody/Sample Submittal Forni

RIN: 11023623

Sampler(s):

Laboratory: Lawrence Livermore National Laboratory

Address: Mr. Micheel Tamblin

Phone: $925-424-2619$

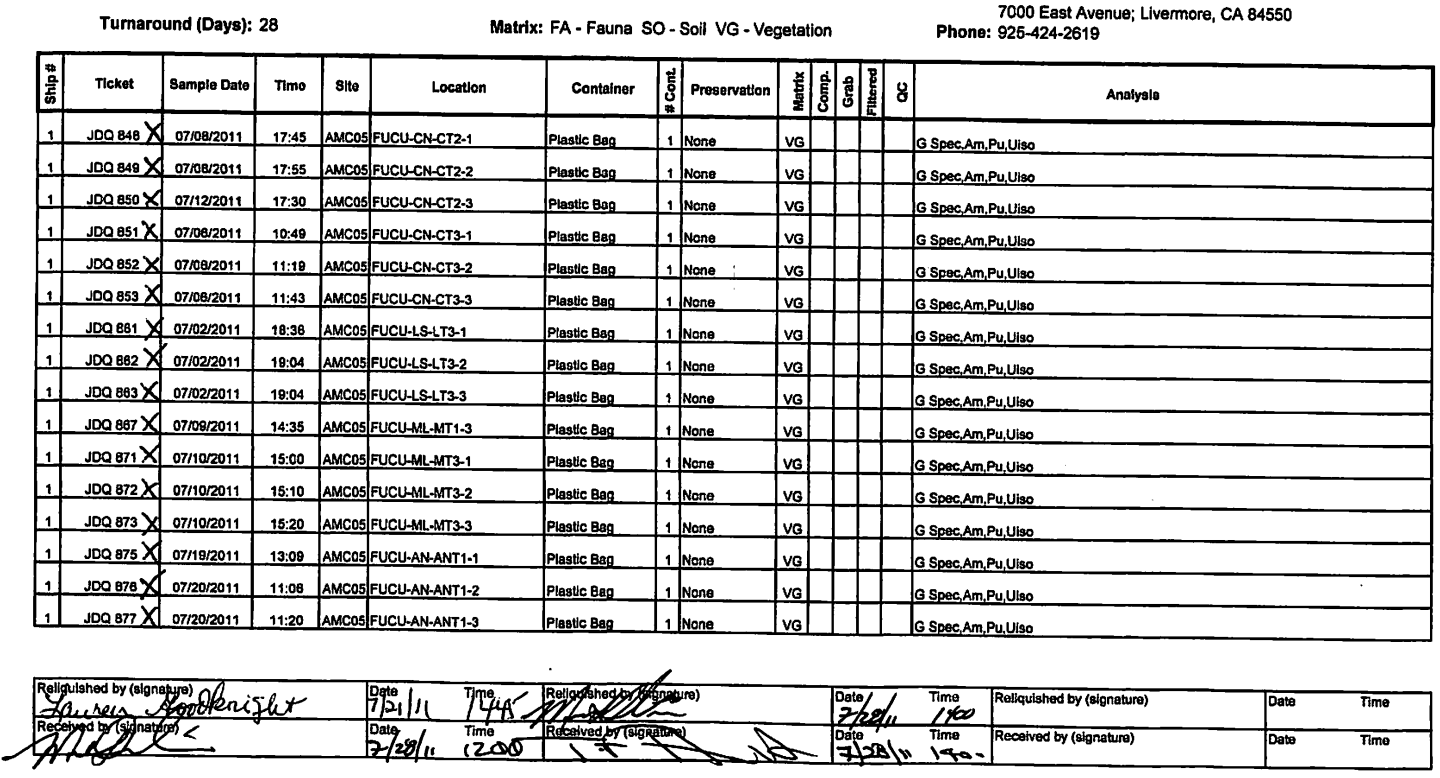




\section{Lawrence Livermore National Laboratory}

Sitolder

Legach Marnagement' 'Team
Chain of Custody/Sample Subnittal Form

RIN: 11023623

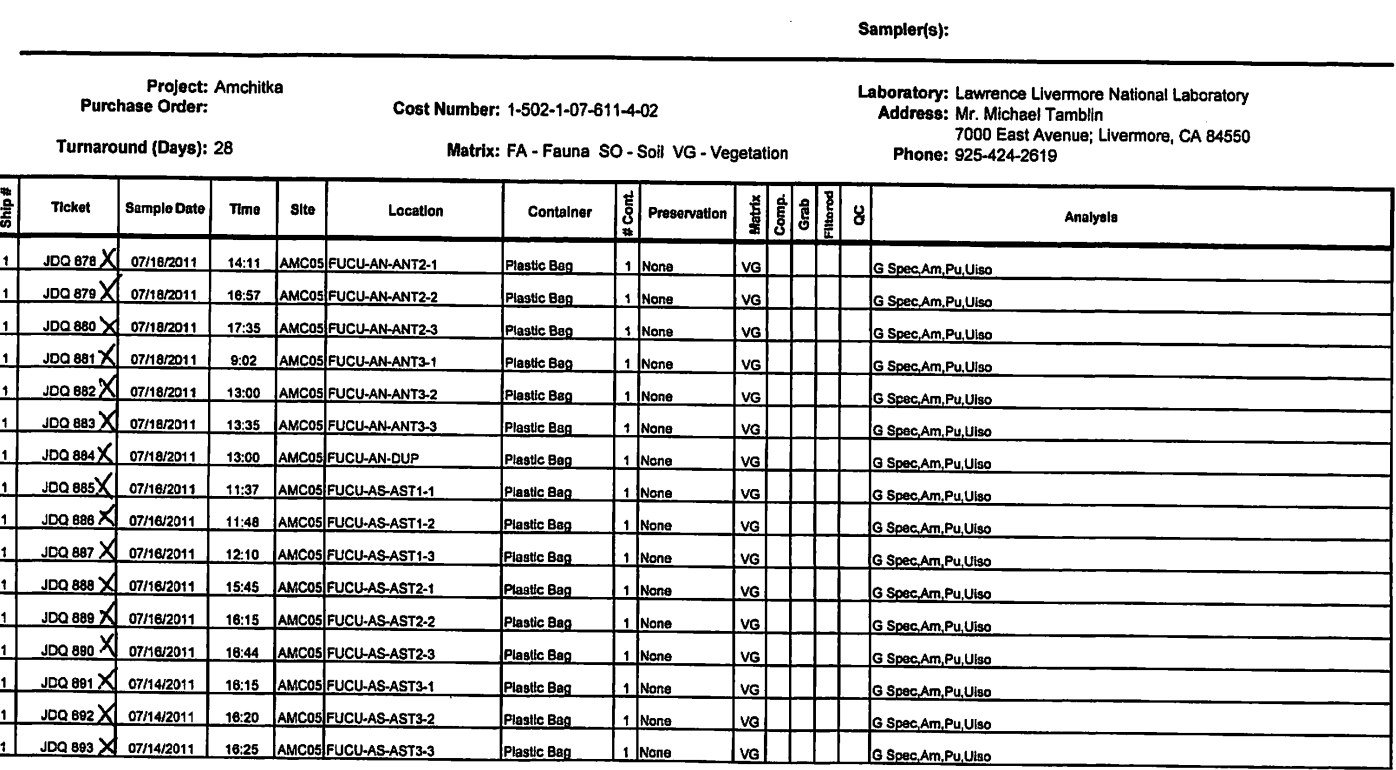

\begin{tabular}{|c|c|c|c|c|c|}
\hline 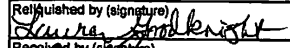 & 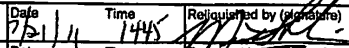 & $\begin{array}{ll}\text { Dato } \\
\text { pof }\end{array}$ & Relliquished by (signeture) & Date & Time \\
\hline & $\begin{array}{c}\text { Date } \\
7 / 20 / 11\end{array}$ & alos h, ine & Received by (signature) & Date & Time \\
\hline
\end{tabular}

Page 2 of 3

Stoller

Legacy management lieam
Chain of Custody/Sample Submittal Form

\author{
RIN: 11023623
}

ampler(s):

Laboratory: Lawrence Livermore National Laboratory

Address: Mr. Michael Tamblin

Phone: 9000 East Aven

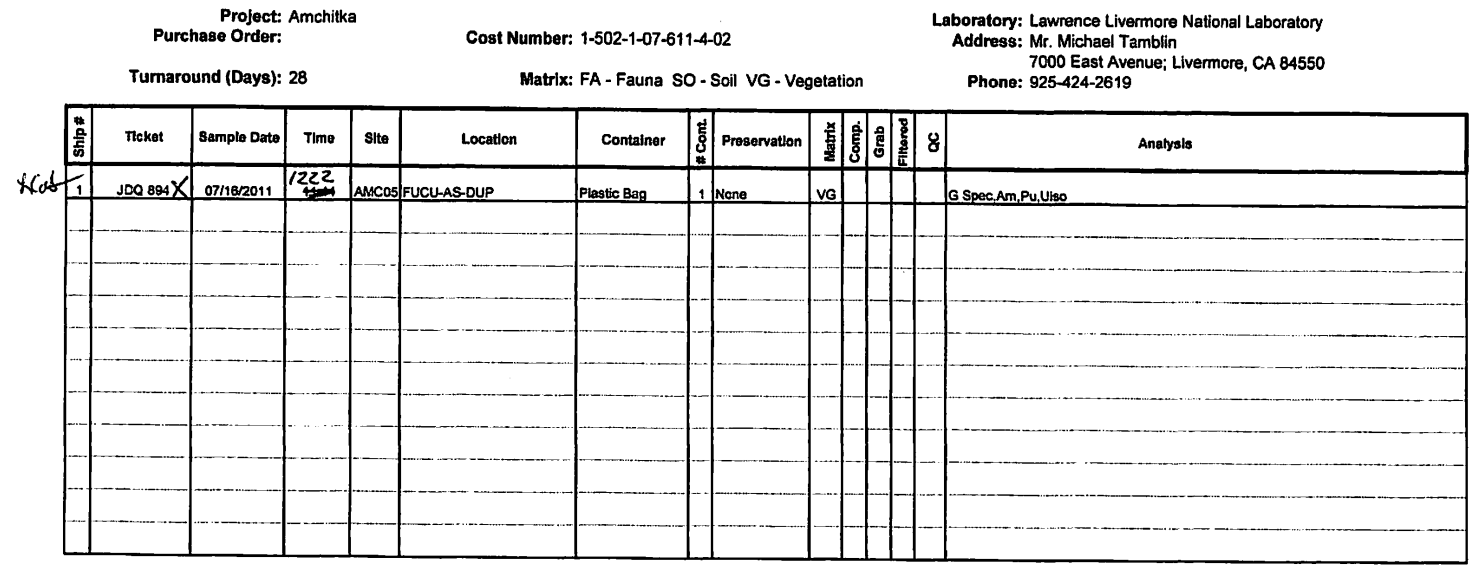

\begin{tabular}{|c|c|c|c|c|c|}
\hline Rellgulshod by (signatyon olleright & 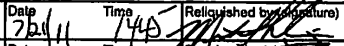 & $7 / 28 / 11$ Time 1100 & Rellquilshed by (signaturea) & Date & Time \\
\hline Recolyedyen & 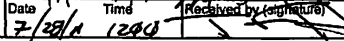 & Date & Recelved by (signature) & Dato & $\pi i m e$ \\
\hline
\end{tabular}

Page 3 of 3 


\section{Lawrence Livermore National Laboratory}

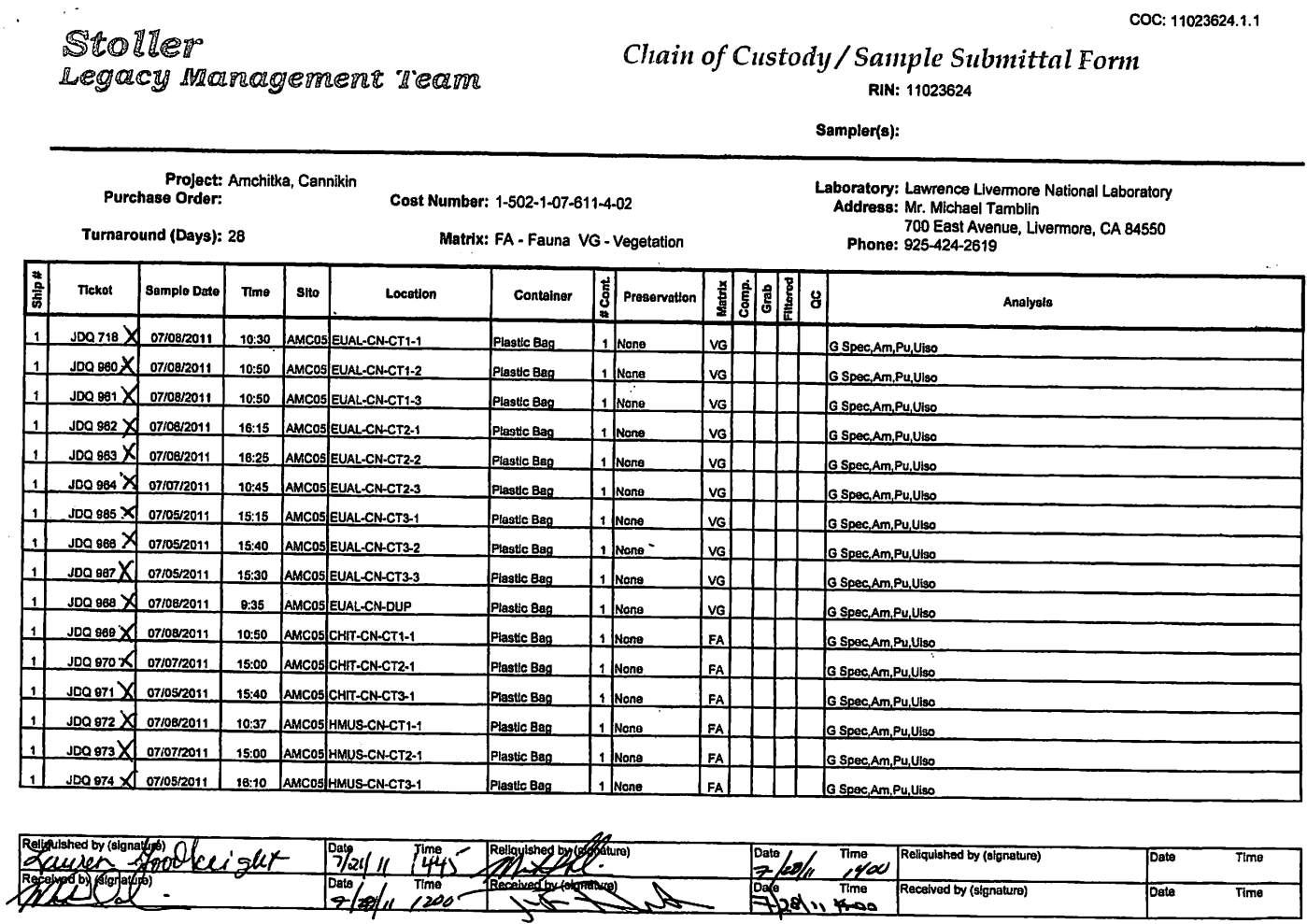

\section{Stoller Legacy Management I'eam}

Chain of Custody/Sample Submittal Form RIN: 11023624

Sampler(s):

Laboratory: Lawrence Livermore National Laboratory Address: Mr. Michael Tamblin 700 East Avenue, Livermore, CA 8455 Project: Amchilka, Cannikin Purchase Order: Cost Number: 1-502-1-07-611-4-02 Matrix: FA - Fauna VG - Vegetation

\begin{tabular}{|c|c|c|c|c|c|c|c|c|c|c|c|}
\hline 望 & Neket & Sample Data & Time & site & Location & Contalnor & 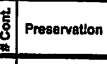 & 熄 & 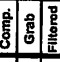 & 8 & Analyols \\
\hline 1 & JDa975X & 07100/2011 & 10:40 & Aacos & URCH-CN-CTH-1 & $88 \propto 2$ WM HOPE & 1 None & $\mathrm{FA}$ & & & ospec,Am,Pu, Uiso \\
\hline 1 & JDQ $976 X$ & 071072011 & 10:27 & Aacos & URCH-CN-CT2-1 & BS OZ WM HOPE & 1 None & $\mathrm{FA}$ & & & G Spec,Am,Pu, Uiso \\
\hline 1 & $\operatorname{Jpag} \theta 7$ & $07 / 105 / 2011$ & 18:00 & Ancos & URCH.CN-CT3-1 & 68 OZ WM HDPE & 1 None & FA & & & G Spec,Am,Pu, Uiso \\
\hline 1 & $J 0 Q 978 X$ & 071082011 & $17: 00$ & AMCOS & OCTP.CN-C2M-1 & Plaste Bag & 1 None & FA & & & G Spec,Am,PU,Uiso 2 PACKAGES FCR SAMPLE Cl+2 OF2) \\
\hline 1 & J00979X & 0710082011 & 14:30 & AMCOS & ROCK_CN.CT1-1 & Piastic Bag & 1 None & $F A$ & & & G Spec,Am,Pu, Uliso \\
\hline 1 & $\mathrm{~J} J 00980 \mathrm{X}$ & 07/08/2011 & 14:45 & ANCOS & ROCK-CN-Cr1.2 & Piastic Bag & 1 None & $\mathrm{FA}$ & & & o Spec,Am,Pu, Uliso \\
\hline 1 & $J D Q 881 X$ & $07708 / 2011$ & $15: 00$ & Amcos & ROCK-CNECT1-3 & Plastlc Bag & 1 None & $\mathrm{FA}$ & & & o Spec,Am,Pu, Uiso \\
\hline 1 & $100982 X$ & 077082011 & 14:30 & AMc05 & ROCK-CN-CT2-1 & Plastic Bag & 1 None & $\mathrm{FA}$ & & & 6 Spec,Am,Pu, Uliso \\
\hline 1 & $J D Q 983 X$ & $07108 / 2011$ & $15: 00$ & Amcos & ROCK-CN-CT2-2 & plastic Bag & 1 None & $\mathrm{FA}$ & & & G Spec,Am,Pu, Uliso \\
\hline 1 & JDQ 984X & $07 / 108 / 2011$ & 15:15 & Amicos & ROCK-CN-CT2-3 & Plastic Bas & 1 None & $\mathrm{FA}$ & & & o Spec,Am, Pu, Uliso \\
\hline 1 & $J 00985 X$ & $07100 / 2011$ & 9:50 & Ancos & ROCK-CN-CTY-1 & Plastic 8an & 1 None & FA & & & o Spec,Am,Pu, Uliso \\
\hline 1 & $100989 X$ & $07108 / 2011$ & $9: 55$ & AMcos & ROCK-CN-CT3-2 & Plastic Bag & 1 None & FA & & & G Spec,Am,Pu, Uitso \\
\hline 1 & $300887 x$ & $07 / 06 / 2011$ & 10:10 & AMCOS & $\mathrm{ROCK}-\mathrm{CN}-\mathrm{CT} 3-3$ & Plastic Bag & 1 None & FA & & & G Spec,Am,Pu, Uliso \\
\hline 1 & $100888 X$ & $07 / 108 / 2011$ & 10:20 & AMCOS & ROCK-CN-DUP & Plastic 8ag & 1 None & $\mathrm{FA}$ & & & o Spec,Am,Pu, Uliso \\
\hline 1 & $J D Q 989 X$ & 07108/2011 & 10:50 & AMCOS & GREN-CN-CT11-1 & Plastic Bag & 1 Nane & $\mathrm{FA}$ & & & oSpec,Am,Pu, Uliso \\
\hline 1 & $J 00990 X$ & 0710812011 & $10: 25$ & AMCOS & GREN-CN-CT11-2 & Plastic Bag & 1 Nene & FA & & & G Specam, Pu,Uliso \\
\hline
\end{tabular}

\begin{tabular}{|c|c|c|c|c|c|}
\hline 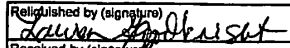 & 7 pely Time - Relloughed by & Date/ $/ 2$ Time & Rellqulahed by (signature) & Date & Time \\
\hline Recelvedpy gigefures & $\mid \begin{array}{ll}\text { Date } \\
7 / 20 / 11 & 1200\end{array}$ & Date & Rocelved by (signature) & Dato & Time \\
\hline
\end{tabular}

Page 2 of 4

U.S. Department of Energy September 2013
Amchitka Island, Alaska, Biological Monitoring Report, 2011 Sampling Results Doc. No. S08833 Page C-9 


\section{Lawrence Livermore National Laboratory}

Stoller

Legacy namagement Ieam
Chain of Custody/Sample Submittal Form

RIN: 11023624

Sampler(s):

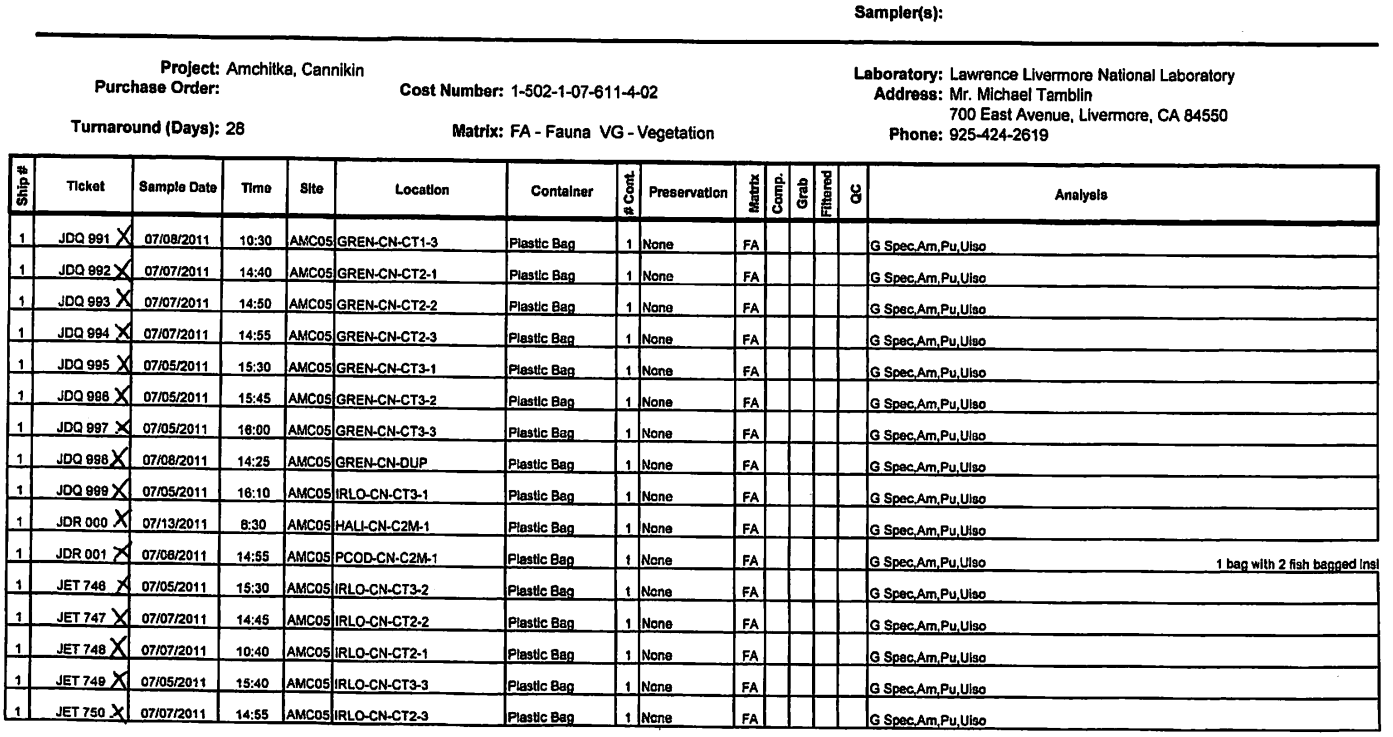

\begin{tabular}{|c|c|c|c|c|c|}
\hline 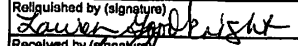 & 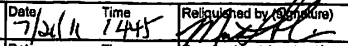 & 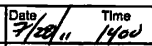 & 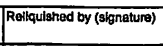 & Date & Time \\
\hline 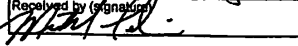 & 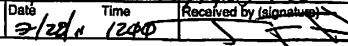 & Dato & Recelved by (signatura) & pale & $\pi m \theta$ \\
\hline
\end{tabular}

Page 3 of 4

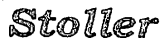

Legacy Marngement leam
Chain of Custody/Sample Subuittal Form RIN: 11023625

\begin{tabular}{|c|c|c|}
\hline & & Sampler(8): \\
\hline $\begin{array}{l}\text { Project: Amchitka, Long Shot } \\
\text { Purchase Order: } \\
\text { Turnaround (Days): } 28\end{array}$ & $\begin{array}{l}\text { Cost Number: 1-502-1-07-611-4-02 } \\
\text { Matrix: FA - Fauna VG - Vegetation }\end{array}$ & $\begin{array}{l}\text { Laboratory: Lawrence Livermore National Laboratory } \\
\text { Address: Mr. Michael Tamblin } \\
700 \text { East Avenue, Livermore, CA } 84550 \\
\text { Phone: } 925-424-2619\end{array}$ \\
\hline
\end{tabular}

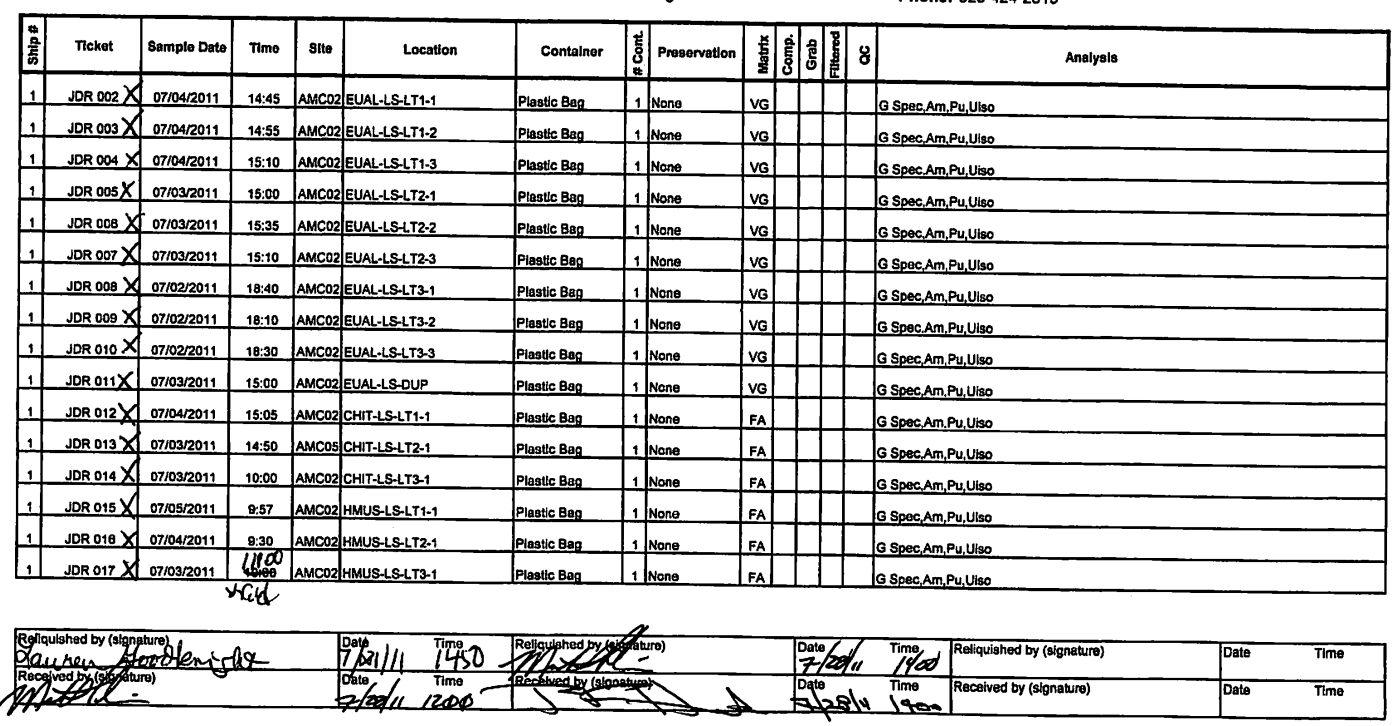




\section{Lawrence Livermore National Laboratory}

Stoller

Legacy Management Heam
COC: 11023625.1 .2

Chain of Custody/Sample Submittal Form

RIN: 11023625

Sampler(s):
Project: Amchitka, Long Shot

Purchase Order:

Turnaround (Days): 28
Cost Number: 1-502-1-07-611-4-02

Matrix: FA - Fauna VG - Vegetation
Laboratory: Lawrence Livermore National Laboratory 700 Encel Tamblin

700 East Avenue, Livermore, CA 84550

\begin{tabular}{|c|c|c|c|c|c|c|c|c|c|c|c|}
\hline \begin{tabular}{|l} 
* \\
के \\
\end{tabular} & ncket & Sample Dato & Nime & sito & Location & Container & \begin{tabular}{l|l} 
s. & Prosenvation \\
\end{tabular} & 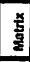 & \begin{tabular}{|l|l|}
$d$ & \\
& 0 \\
\end{tabular} & 8 & Analysis \\
\hline 1 & JDR $018 X$ & 0710412011 & 15:02 & $\mathrm{AMCO2}$ & URCHELS-LT1-1 & 68 oz HDPE WM & None & $\mathrm{FA}$ & & & G Spec,Am,Pu, Uliso \\
\hline 1 & JDR $019 X$ & 07/03/2011 & $18: 00$ & AMCO2 & URCH-LS.LT2-1 & 88 O2 HDPE WM & 1 None & FA & & & G Spoc,Am,Pu, Ulso \\
\hline 1 & JDR $020 X$ & 07/102/2011 & 18:10 & AMCO2 & URCH-LS-LT3-1 & 68 OZ HDPE WM & 1 None & FA & & & G Spec,Am,Pu,Uiso \\
\hline 1 & JDR $021 X$ & 07103/2011 & 15:25 & $\mathrm{AMCO2}_{2}$ & OCTP-LS-L2M-1 & Plastic Bag & 1 None & $\mathrm{FA}$ & & & G Spoc,Am,Pu,Uiso \\
\hline 1 & JOR $022 X$ & 078042011 & $15: 00$ & AMCO2 & ROCK-LS-LT1-I & Plastc Beg & 1 None & $\mathrm{FA}$ & & & G Spec,Am,Pu,Uiso \\
\hline 1 & JDR $023 X$ & 0711212011 & $18: 05$ & AMCO2 & ROCK-LS-LT1-2 & Plastc Bag & 1 None & FA & & & G Spec,Am,PU,Uiso \\
\hline 1 & JDR $024 X$ & 07/12/2011 & 16:30 & AMCO2 & ROCK-LS-LT1.3 & Plastic Bag & 1 None & $\mathrm{FA}$ & & & G Spec,Am,Pu, Uliso \\
\hline 1 & JDR $025 X$ & $07 / 04 / 2011$ & 8:30 & AMCO2 & ROCK-LS-LT2-1 & Plastic Bag & 1 None & FA & & & G Spec,Am,Pu,Uiso \\
\hline 1 & JOR 028 & $07 / 1222011$ & $15: 10$ & AMCO2 & ROCK-LS-LT2-2 & Plastic Bag & 1 Nene & FA & & & G Spec,Am,Pu, Uliso \\
\hline 1 & JDR $027 X$ & $07112 / 2011$ & 16:30 & AMCO2 & ROCK-LS-DUP2 & Plastic Bag & 1 None & FA & & & o Spoc,Am,Pu, Uiso \\
\hline 1 & JDR 020X & $071022 / 2011$ & 17:30 & AMCO2 & ROCK-LS-LT3-1 & Plastic Beg & 1 None & FA & & & G, Spec,Am,Pu,Ulso \\
\hline 1 & $J D R 028 X$ & 07/12/2011 & 13:12 & AMCO2 & ROCK-LS.LTT-2 & Plastic Bag & 1 None & FA & & & G Spec,Am,Pu, Uiso \\
\hline 1 & $J D R 030 X$ & 07/03/2011 & $11: 45$ & AMCO2 & ROCK-LS-LT3-3 & Plastic Bag & 1 None & FA & & & G Spec,Am,Pu,Uiso \\
\hline 1 & JDR 031 & 071122011 & 13:17 & AMCO2 & ROCK-LS-DUP & Plastic Bag & 1 Nene & FA & & & G Spec,Am,Pu, Uiso \\
\hline 1. & JOR 032X & $07104 / 2011$ & 15:10 & AMCO2 & GREN-LS-LT1-1 & Plastic Bag & 11 None & FA & & & G Spec,Am,Pu, Uiso \\
\hline 1 & JDR 033 X & 07/104/2011 & $15: 30$ & AMCO2 & GREN-LS-LTT1-2 & Plastic Beg & 1 None & FA & & & G Spec,Am,Pu, vilso \\
\hline
\end{tabular}

\begin{tabular}{|c|c|c|c|c|c|}
\hline 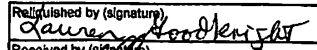 & 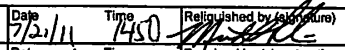 & 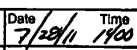 & Rellquished by (signature) & Date & Time \\
\hline 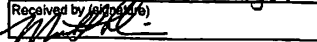 & 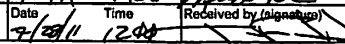 & $\sum_{i \rightarrow B}$ nime & Reoalved by (signature) & Deto & Time \\
\hline
\end{tabular}

Page 2 of 4

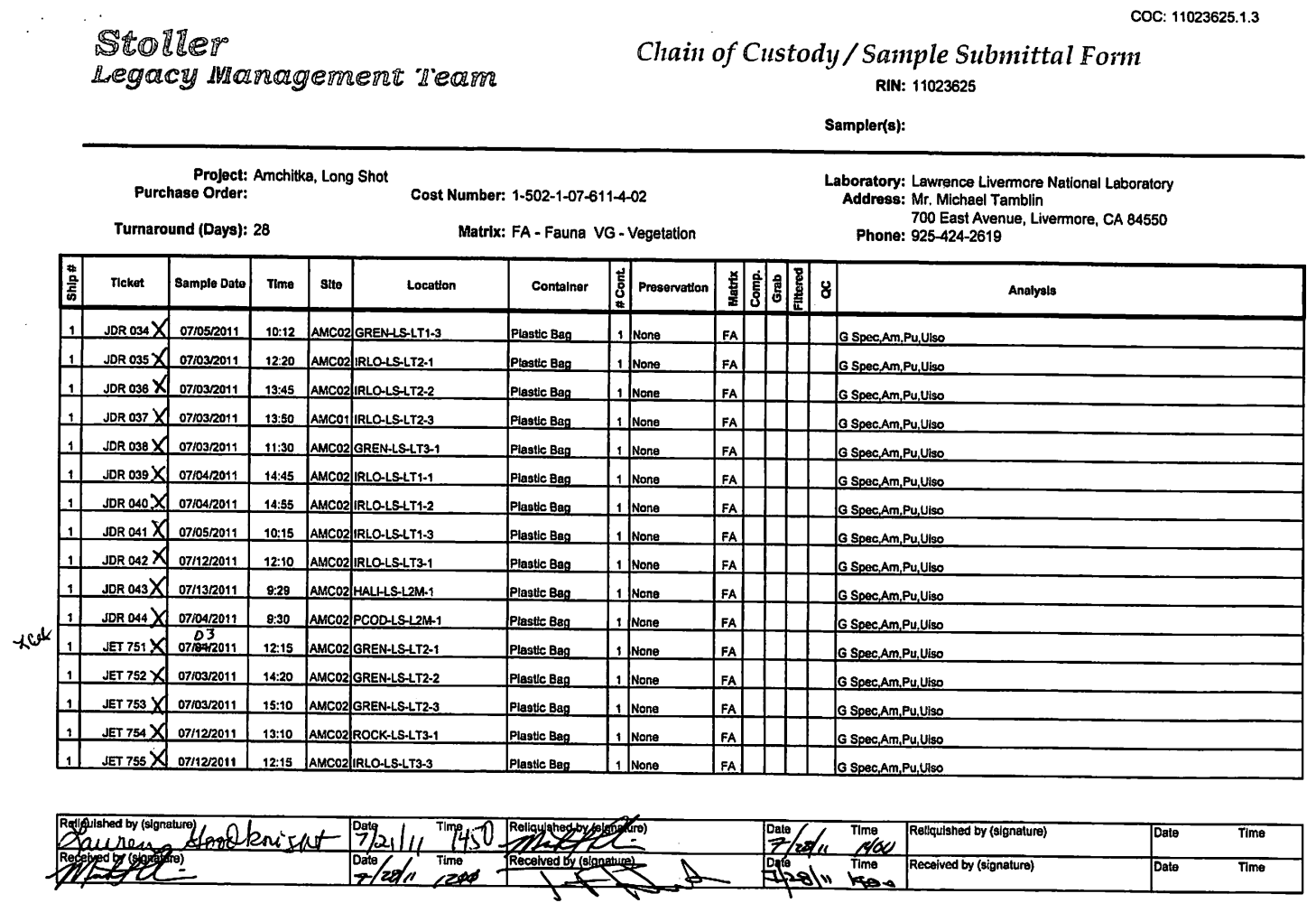

Page 3 of 4

U.S. Department of Energy September 2013
Amchitka Island, Alaska, Biological Monitoring Report, 2011 Sampling Results Doc. No. S08833

Page C-11 


\section{Lawrence Livermore National Laboratory}

COC: 11023626.1 .1

\section{Stoller}

Hegact Management Heam
Chain of Custody/Sample Submittal Form

RIN: 11023626

Sampler(s):

\begin{tabular}{|c|c|c|c|c|c|c|c|c|c|c|c|c|c|}
\hline & $\begin{array}{r}\text { Purct } \\
\text { Turnaro }\end{array}$ & $\begin{array}{l}\text { Project: } \\
\text { hase Order: } \\
\text { und (Days): }\end{array}$ & & & Cost Nu & $\begin{array}{l}: 1-502-1-07-6 \\
: F A-F a u n a\end{array}$ & G. & $\begin{array}{l}.02 \\
\text { Vegetation }\end{array}$ & & & & & $\begin{array}{l}\text { Iboratory: Lawrence Livermore National Laboratory } \\
\text { Address: Mr. Michael Tamblin } \\
700 \text { East Avenue, Livermore, CA } 84550 \\
\text { Phone: } 925-424-2619\end{array}$ \\
\hline & Tekot & Sample Date & $\pi / m e$ & site & Locattion & Contalner & 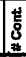 & Preservattor & 臬 & 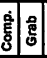 & 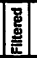 & \& & Analysis \\
\hline & JOR O45 X & 07700/2011 & 13:05 & AMCO3 & EUAL-ML-MT1-1 & Pleastic \&ogg & 1 & Vone & va & & $N$ & & G Spec,Am,Pu,Uliso \\
\hline & JDR 046 X & $0700 / 2011$ & 13:15 & AMCOS & EUAL-ML-MT1-2 & Plastc Bag & 1 & None & va & & $N$ & & G Spec,Am,Pu, Uiso \\
\hline & JDR 047 X & 07098/2011 & 13:10 & AMCO3 & EUAL-ML-MT1-3 & Plastc Bsg & 1 & None & vo & & N & & o Spec,Am,Pu, Ulso \\
\hline & JDR 040X & 07710/2011 & $17: 00$ & AMCO3 & EUAL-ML-NT2-1 & Plastc Bsg & 1 & None & vo & & $N$ & & G Spec,Am, Pu, Uliso \\
\hline & JOR 049X & 077102011 & 17:00 & AAACO3 & EUAL-ML-MT2-2 & Plagtc Bag & 1 & None & vo & & N & & o Spoc,Am,Pu, Ulso \\
\hline & JOR 050X & 07110/2011 & $17: 30$ & AMCO3 & EUAL-MLLMT2-3 & Plastic Beg & 1 & None & vo & & $N$ & & G Spec,Am,Pu, Uliso \\
\hline & JOR $051 X$ & 07/10/2011 & $10: 40$ & AMCO3 & EUAL-ML-MT3-1 & Plastic Beg & 1 & None & vo & & $N_{N}$ & & G Spec,Am,Pu, Uliso \\
\hline & JOR 052 X & 07710/2011 & 10:55 & AMCOS & EUAL-ML-MT3-2 & Plagtc Bag & 1 & None & vo & & $N$ & & G Spec,Am,Pu,Uliso \\
\hline & JOR 053X & 07710/2011 & 10:30 & AMCOS & EUAL-ML-MT3-3 & plastickag & 1 & None & $v_{G}$ & & $\mathrm{~N}$ & & G Spec,Am,Pu, Ulso \\
\hline & JDR 054X & 07rog/2011 & $16: 45$ & AMCO3 & EUAL-ML-DUP & Plestle Bag & 1 & None & vg & & $\mathrm{N}$ & & GSpec,Am,Pu, Uliso \\
\hline & JDR 055X & 07/08/2011 & 13:15 & AMCOS & CHIT-ML-MT1-1 & PlastcBag & 1 & None & FA & & $|N|$ & & G S Spec,Am,Pu, Uliso \\
\hline & JDR 05B X & 07/102011 & 17:15 & AMSOCO3 & CMIT-ALL-MT2-1 & Plastic Bsa & 1 & None & FA & & $N$ & & 6 Spec,Am,Pu, Uliso \\
\hline & JOR $057 X$ & 07710/2011 & 10:50 & AMCO3 & CHIT-ML-MT3-1 & Plastc B89 & 1 & Nene & FA & & $\mathrm{N}$ & & G Spec,Am,Pu, Uliso \\
\hline & JOR OSB X & 070092011 & 17:40 & AMCO3 & HMUS-ML-MT1-1 & Plastle Bag & 1 & None & FA & & $\mathrm{N}$ & & o Spec,Am,Pu, Uliso \\
\hline & JDR 059 X & 071112011 & 11:30 & AMCO3 & HMUS-MLATR2-1 & Plastc Bag & 1 & None & FA & & $|N|$ & & G Spec,Am,Pu, Uiso \\
\hline & $J D R 080 X$ & 07/10/2011 & $11: 10$ & AMCOS & HMUS-ML-MT3-1 & Plastc Bag & 1 & None & FA & & $|N|$ & & G Spec,Am,Pu, Iilso \\
\hline
\end{tabular}

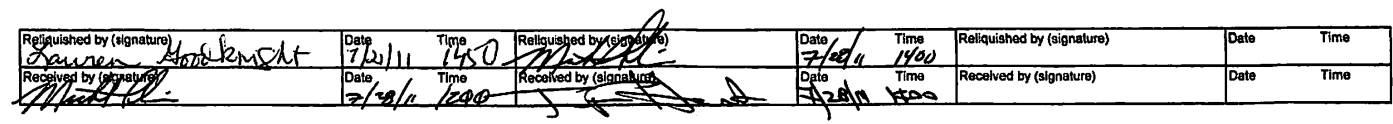

Page 1 of 4

COC: 11023626.1 .2

\author{
Stoller \\ Legacy Management Ieam
}

Chain of Custody/Sample Submittal Form

RIN: 11023626

Sampler(s):

Laboratory: Lawrence Livermore National Laboratory

Address: Mr. Michael Tamblin

Phone: 900 East Aven $424-2619$

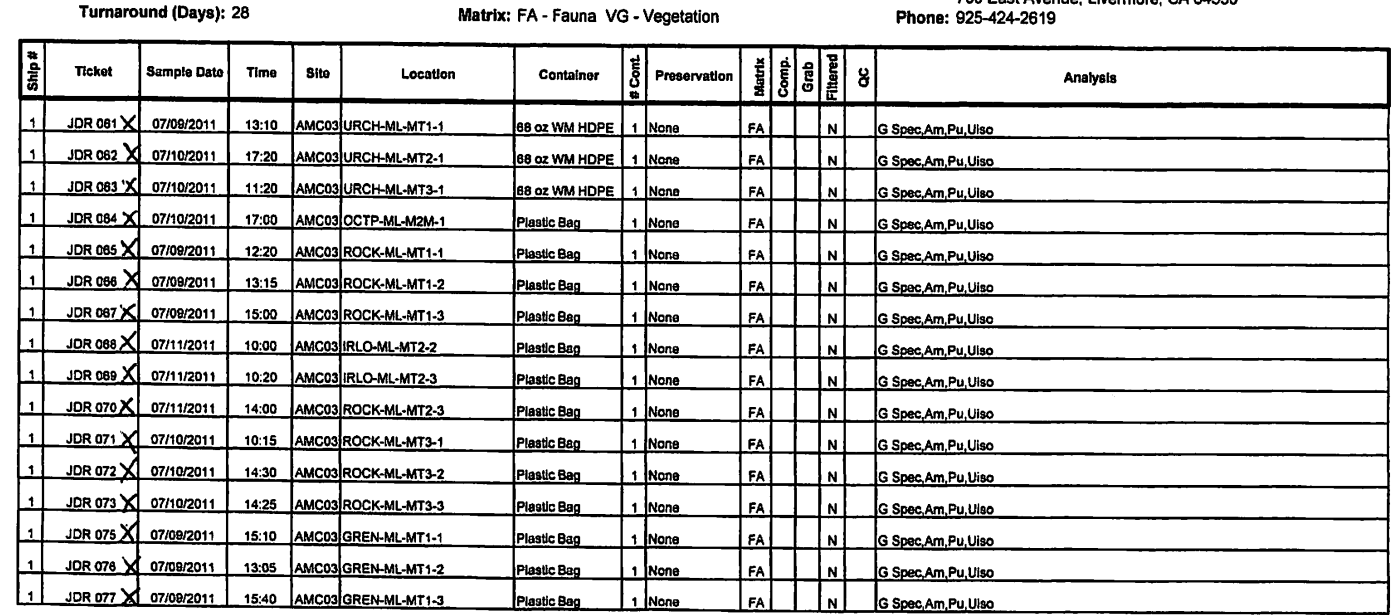




\section{Lawrence Livermore National Laboratory}

\section{Stolder \\ Legacy mangement "leam}

Chain of Custody/Sample Submittal Forn

RIN: 11023626

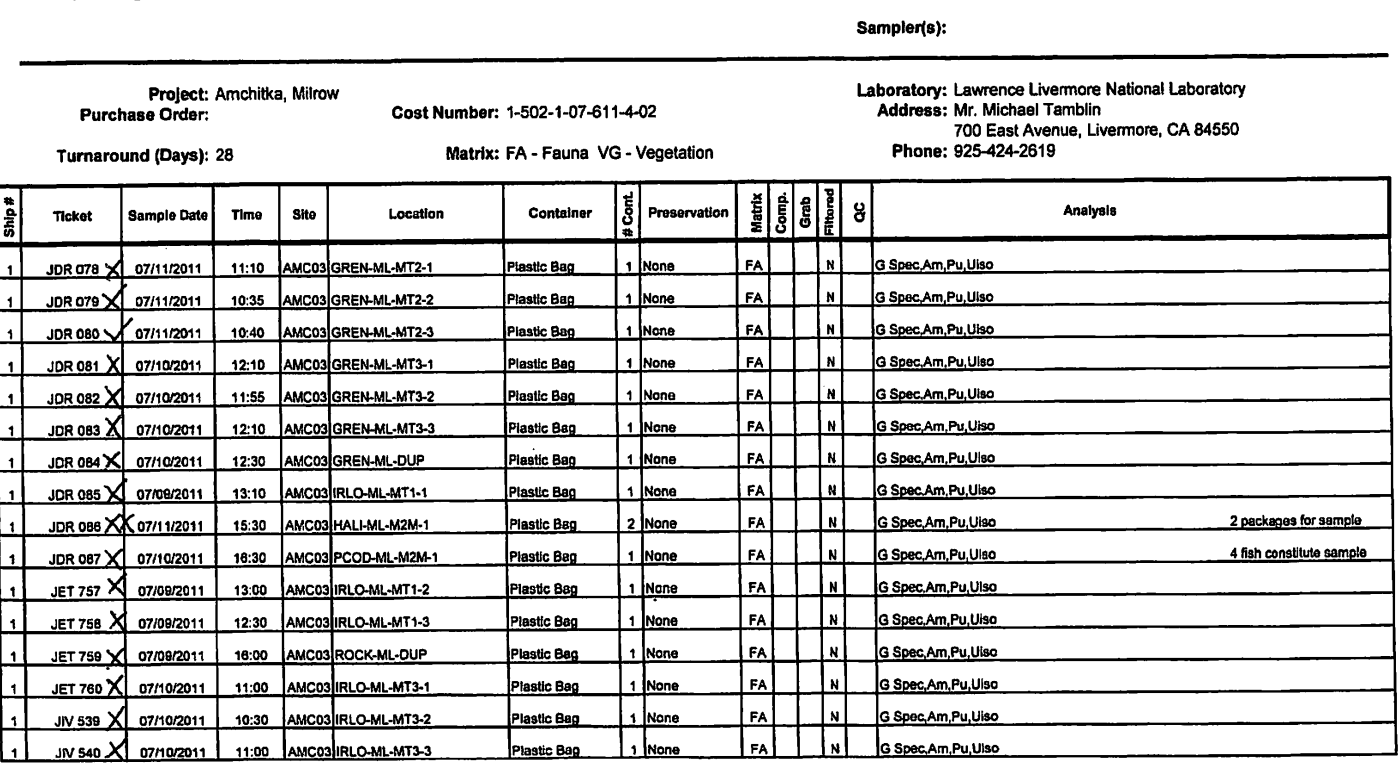

\begin{tabular}{|c|c|c|c|c|c|}
\hline Religuishod by (signaturty ofknisht & 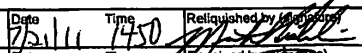 & 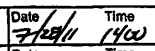 & Reliquished by (signature) & Date & Time \\
\hline 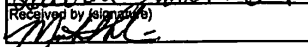 & 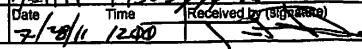 & Dated Time & Received by (stignatura) & Date & Time \\
\hline
\end{tabular}

Page 3 of 4

COC: 11023626.1 .4

\section{Stomer \\ Legacy Mnomement ream}

Chain of Custody/Sanple Subınittal Form

$$
\text { RIN: } 11023626
$$

Sampler(s):

Laboratory: Lawrence Livermore National Laboratory

Address: Mr. Michsel Tamblin 700 East Avenue, Livermore, CA 84550
Phone: $925-424-2619$

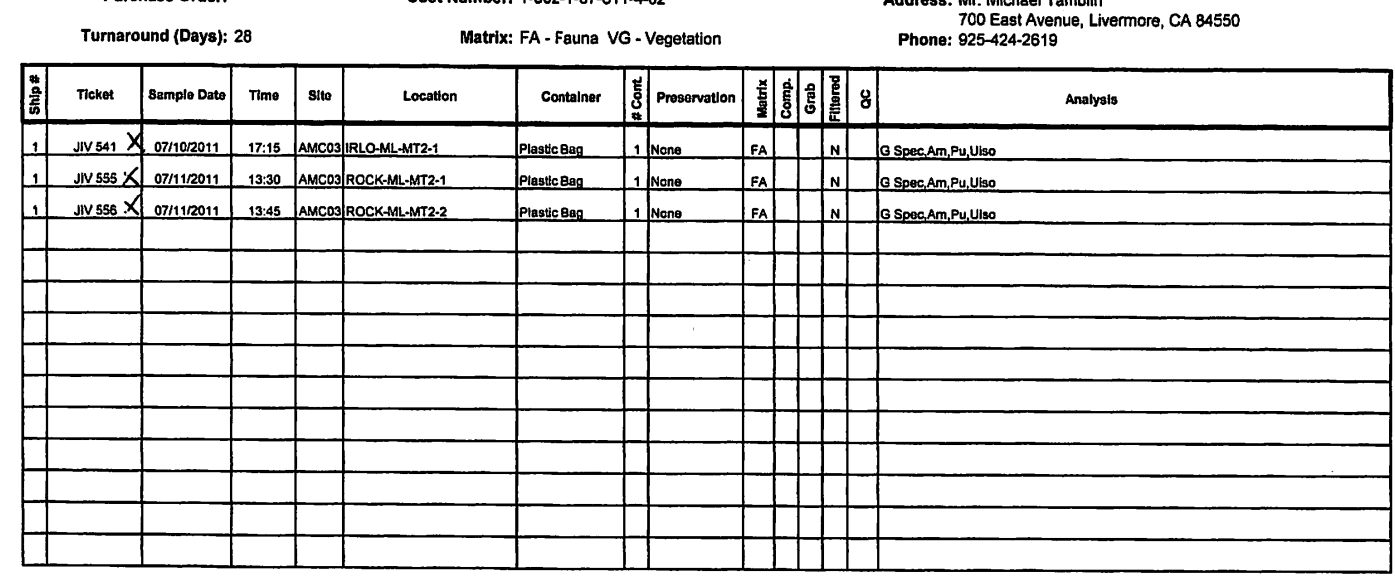

\begin{tabular}{|c|c|c|c|c|c|}
\hline 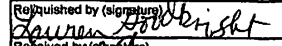 & 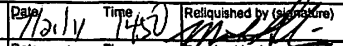 & 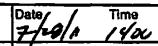 & Reliquished by (signatura) & Dato & Time \\
\hline 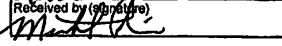 & 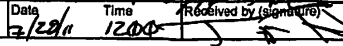 & tipol, ime & Recelved by (aignature) & Date & mit \\
\hline
\end{tabular}




\section{Lawrence Livermore National Laboratory}

\section{Stoller Legacy Management "eam}

Chain of Custody/Sample Subnittal Forn RIN: 11023627

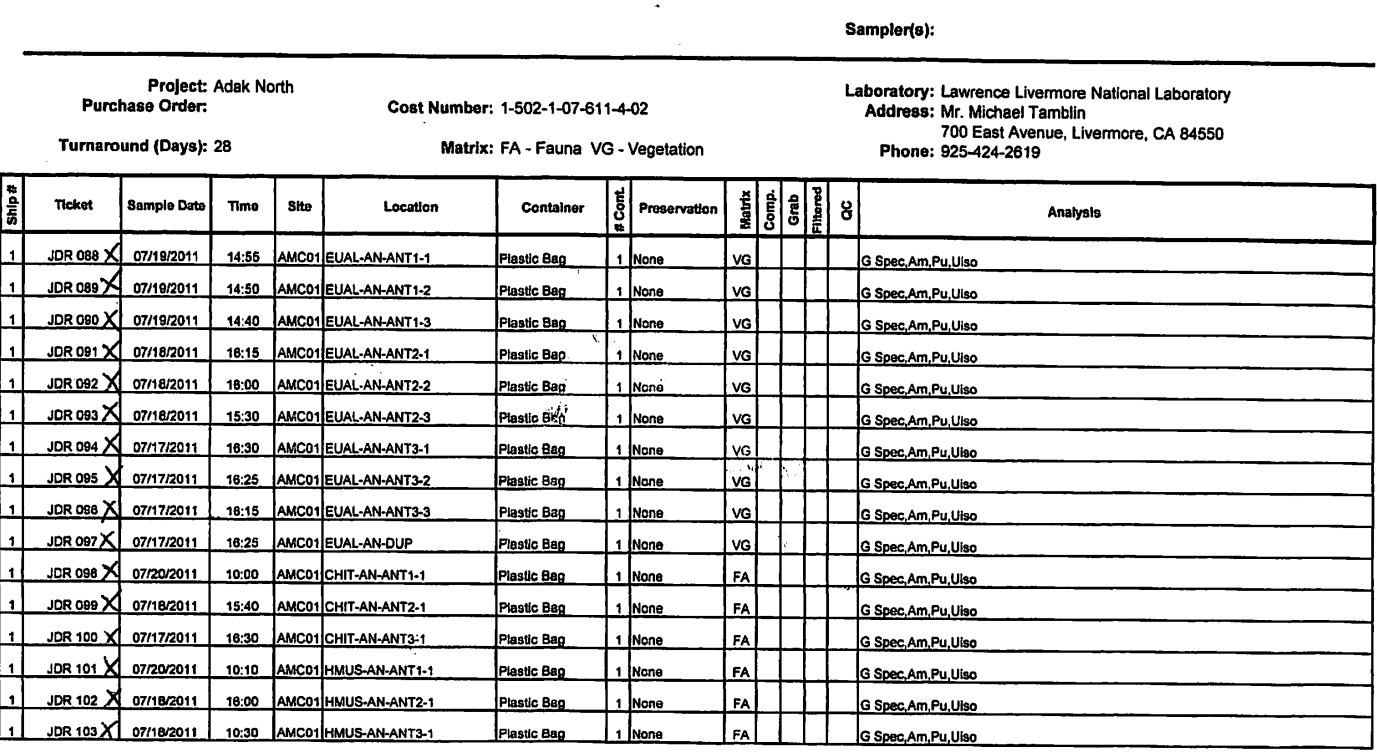

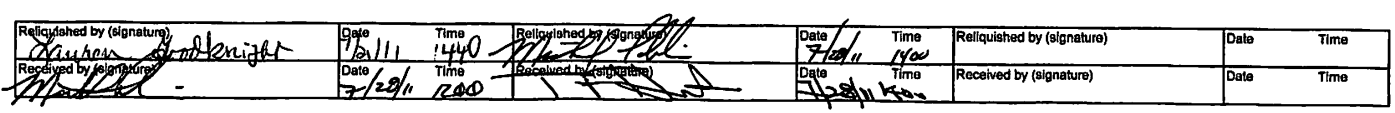

\section{Stoller \\ Legacy Management Iream}

Chain of Custody/Sample Submittal Form RIN: 11023627

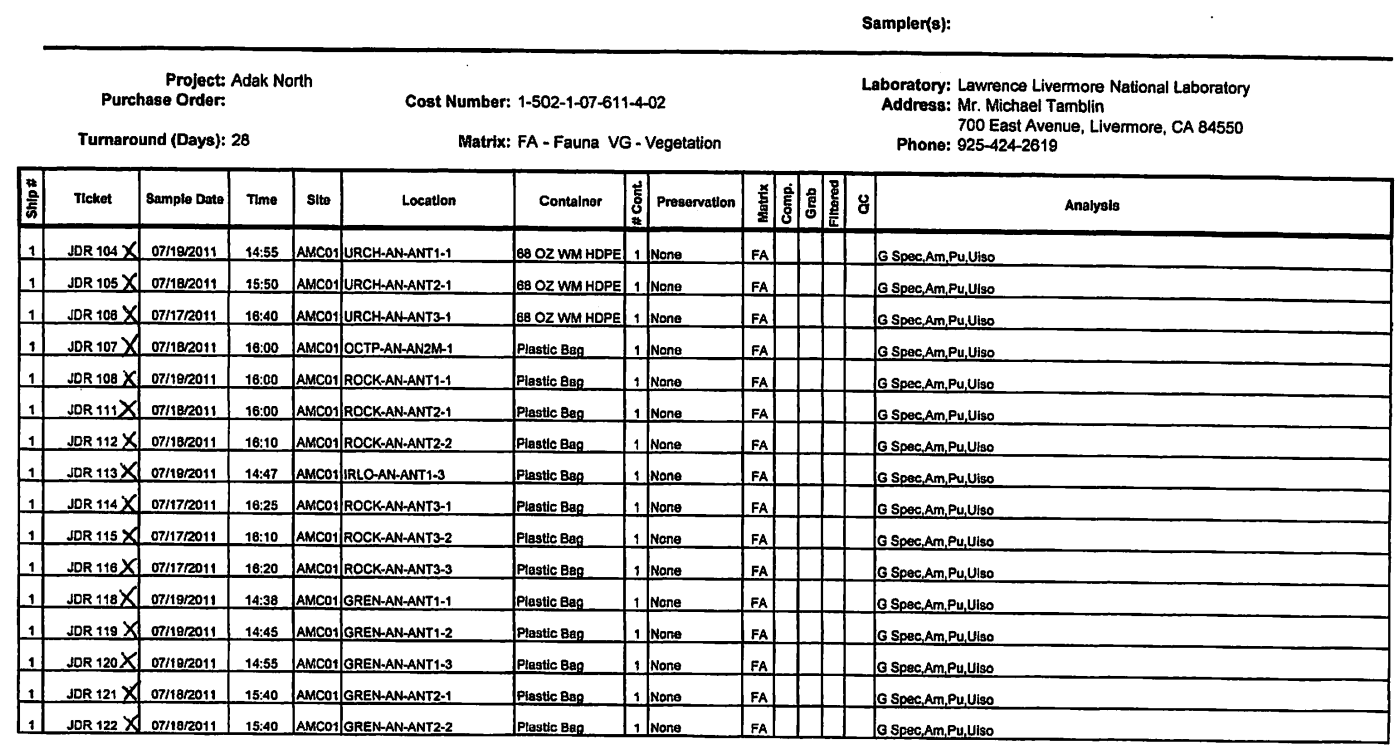

\begin{tabular}{|c|c|c|c|c|c|}
\hline 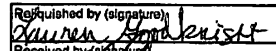 & $7 / 21 / 4 \quad$ Time 1440 Rellayshodpy & Date/relu Time 140 & Rellquished by (signature) & Tato & Time \\
\hline Receryed by & 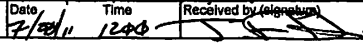 & 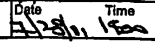 & Rocelved by (algnature) & Data & Time \\
\hline
\end{tabular}




\section{Lawrence Livermore National Laboratory}

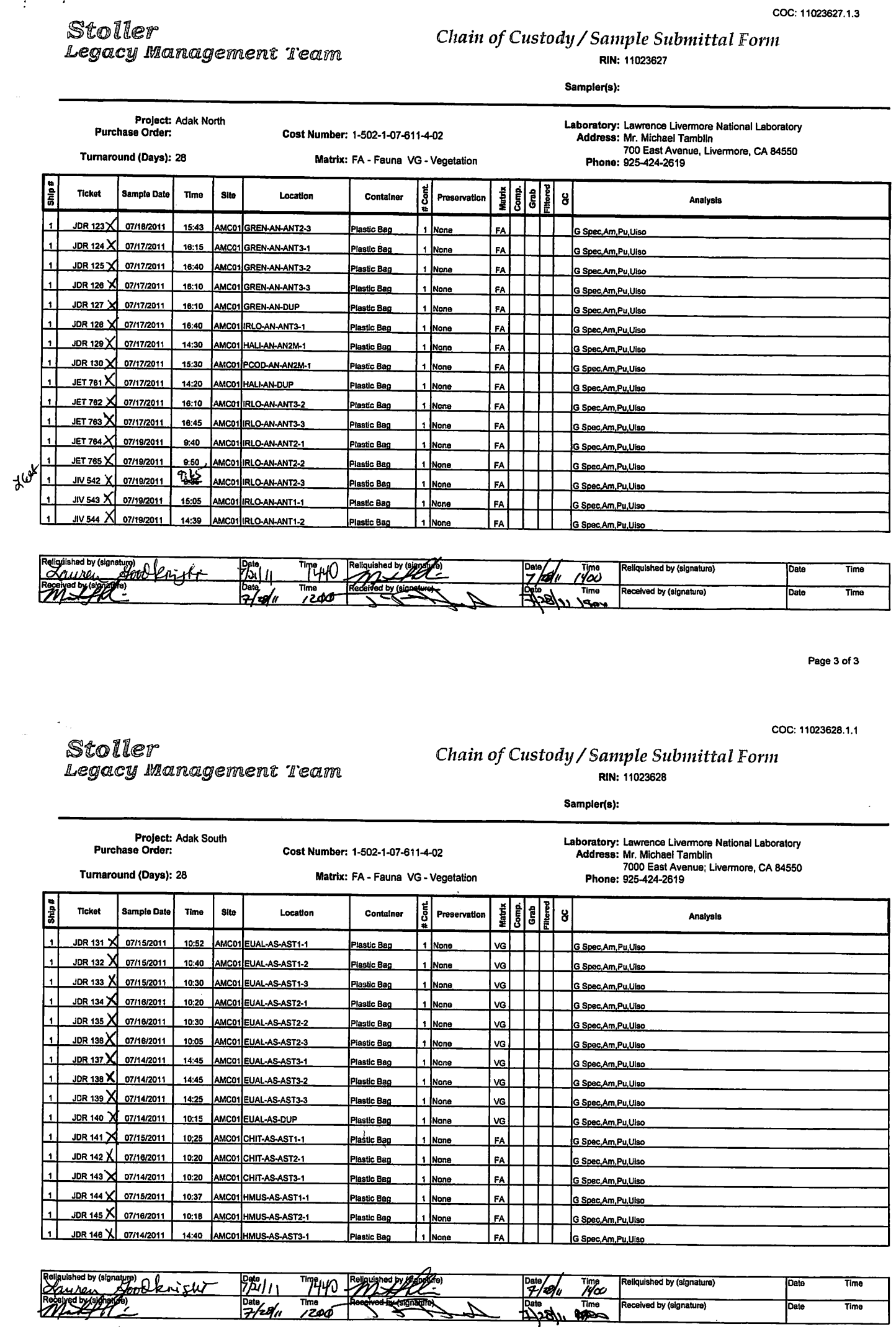

Page 1 of 4

U.S. Department of Energy September 2013
Amchitka Island, Alaska, Biological Monitoring Report, 2011 Sampling Results Doc. No. S08833

Page C-15 


\section{Lawrence Livermore National Laboratory}

\section{Stoller \\ Legary Mraragement Heam}

COC: 11023628.1 .2

Chain of Custody/Sample Submittal Form

RIN: 11023628

Sampler(s):

Laboratory: Lawrence Livermore National Laboratory

Address: Mr. Michael Tamblin

7000 East Avenue; Livermore, CA 84550

\begin{tabular}{|c|c|c|c|c|c|c|c|c|c|c|c|}
\hline 音 & Teket & Sampla Dato & $\pi / m e$ & 8ite & Locatton & Contalner & 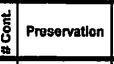 & है & 包 & 8 & Analyzls \\
\hline 1 & JOR $147 X$ & 07/15/2011 & $10: 47$ & AMCO1 & URCH-AS-AST1-1 & 6802 WM HOPE & Nene & $F A$ & & & G Spec,Am,Fu,Uliso \\
\hline 1 & ${ }_{\text {JOR }} 148 X$ & 07/18/2011 & 10:15 & AMCO1 & URCH-AS-AST2-1 & 68 OZ WM HDPE & 1 None & $\mathrm{FA}$ & & & G Spec,Am,Pu, Uiso \\
\hline 1 & JOR $148 X$ & 07/14/2011 & 14:30 & AMCO 1 & URCH-AS-AST3-1 & BB OZ WM HDPE & 1 None & $\mathrm{FA}$ & & & G Spec,Am,Pu,Ulso \\
\hline 1 & JDR $151 X$ & 07/15/2019 & 10:45 & AMCO1 & ROCK-AS-AST1-1 & Plastic Bag & 1 None & $\mathrm{FA}$ & & & GSpec,Am,Pu,Uliso \\
\hline 1 & JDR $152 X$ & 07/1582011 & 11:00 & AMCO1 & ROCK-AS-AST1-2 & Flagtic Bag & 1 None & $\mathrm{FA}$ & & & G Spec,Am,Pu, Uliso \\
\hline 1 & JDR 153X & $07115 / 2011$ & 11:08 & AMC01 & ROCK-AS-AST1-3 & Plastic Bag & 1 None & $\mathrm{FA}$ & & & G Spac,Am,Pu, Uiso \\
\hline 1 & JDR $154 X$ & 07/18/2011 & 13:25 & AMC01 & ROCK-AS-AST2-1 & Plastic Bag & 1 None & FA & & & G Spec,Am,Pu,Ulso \\
\hline 1 & JDR $155 X$ & 07/18/2011 & 19:30 & AMCO1 & ROCK-AS-AST2-2 & Plastc Bag & 1 None & $F A$ & & & G Spec,Am, Pu, Uliso \\
\hline 1 & JDR $156 X$ & 07/19/2011 & 13:50 & AMCO1 & ROCK-AS-AST2-3 & Plastic Bag & 1 None & $F A$ & & & G Spec,Am,Pu,Uliso \\
\hline 1 & $J D R 157 X$ & 07/14/2011 & $10: 10$ & AMCO1 & ROCK-AS-AST3-1 & Plastlc Bag & 1 None & $F A$ & & & G Spec,Am,Pu,Uliso \\
\hline 1 & JDR 15B X & $07 / 14 / 2011$ & 10:16 & AMCO1 & ROCK-AS-AST3-2 & Plastlc Bag & 1 None & $F A$ & & & G Spec,Am,Pu,Ulso \\
\hline 1 & $J D R 159 X$ & $07 / 1412011$ & 10:28 & AMCON & ROCK-AS-AST3.3 & Plastic Bag & 1 None & $F A$ & & & o Spec,Am,Pu,Uliso \\
\hline 1 & JOR $160 \mathrm{X}$ & 07/14/2011 & 14:20 & AMCO1 & ROCK-AS-DUP & Plastic Bag & 1 None & $F A$ & & & GSpec,Am,Pu,Ulso \\
\hline 1 & JDR $181 X$ & 07115/2011 & 10:25 & AMCO1 & GREN-AS-AST11-1 & Plastc Beg & 1 None & FA & & & GSpec,Am,Pu, Uliso \\
\hline 1 & JDR $182 X$ & 07/15/2011 & $10: 40$ & AMCO1 & GREN-AS-AST1-2 & Plastic 8ag & 1 Nene & $\mathrm{FA}$ & & & o Spec,Am,Pu, Uliso \\
\hline 1 & JDR $163 \mathrm{X}$ & $07115 / 2011$ & $10: 47$ & AMCO1 & GREN-AS-ASTT-3 & Plastic Bag. & $1 \int_{\text {None }}$ & $\mathrm{FAl}$ & & & Spec,Am,P \\
\hline
\end{tabular}

\begin{tabular}{|c|c|c|c|c|c|}
\hline 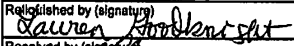 & 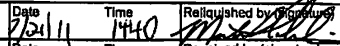 & $7 / 21 / a \quad$ Timed & Rellquighed by (slgnature) & Date & $T \operatorname{lm} \theta$ \\
\hline Recalved by fologer & 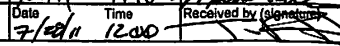 & 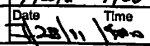 & Recelved by (stgnature) & Dato & Time \\
\hline
\end{tabular}

\section{Stoller \\ Legacy Management lieam}

Chain of Custody/Sample Submittal Form

$$
\text { RIN: } 11023628
$$

Sampler(3):
Laboratory: Lawrence Livermore National Laboratory
Address: Mr. Michael Tamblin 7000 East Avenue; Livermore, CA 8455 Purchagoject: Adak South

Turnaround (Days): 28
Cost Number: 1-502-1-07-611-4-02

Matrix: FA - Fauna VG - Vegetation Phone: 925-424-2619

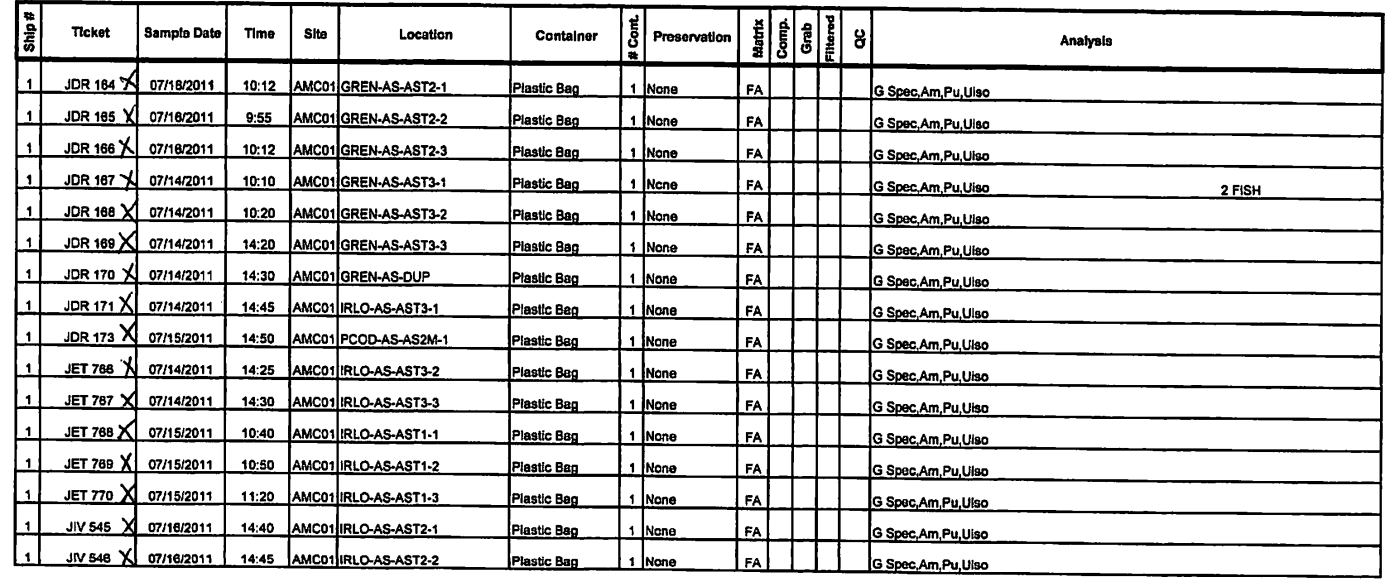

\begin{tabular}{|c|c|c|c|c|c|c|}
\hline 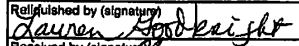 & $19921111, \quad$ Timo 1440 & 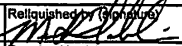 & $\begin{array}{ll}\text { Date } \\
7 / 20 / 4 & 1400\end{array}$ & Rellqulehed by (signature) & Date & Time \\
\hline 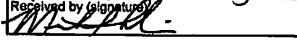 & $\begin{array}{l}\text { Dato } \\
7 / \mathrm{co} / \mathrm{d}\end{array}$ & pives rotign & 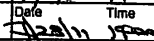 & Recelved by (signature) & Data & Time \\
\hline
\end{tabular}




\section{Lawrence Livermore National Laboratory}

Stoller

Legacy management leam
Chain of Custody/Sample Submittal Form

RIN: 11023628

Sampler(s):

Laboratory: Lawrence Livermore National Laboratory

Address: Mr. Michael Tamblin

Phone: 9000 East Aven

Matrix: FA - Fauna VG - Vegetation
COC: 11023628.1 .4

\begin{tabular}{|c|c|c|c|c|c|c|c|c|c|c|}
\hline 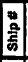 & Tickot & Sample Dato & Tlmo & stte & Locatton & Contalnor & \begin{tabular}{l|l} 
& Preservation \\
s.
\end{tabular} & 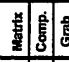 & \begin{tabular}{l|l|l} 
& 8 & 8 \\
& 0 & 8 \\
\end{tabular} & Analysis \\
\hline 1 & $\operatorname{JN} 547 X$ & 071182011 & 18:10 & AMCO1 & IRLO-AS-AST2-3 & Plastlc Bap & 1 None & $F A$ & & G Spec,Am,Pu, Ullso \\
\hline & & & & & & & & & & \\
\hline & & & & & & & & & & \\
\hline & & & & & & & & & & \\
\hline & & & & & & & & & & \\
\hline & & & & & & & & & & \\
\hline & & & & & & & & & & \\
\hline & & & & & & & & & & \\
\hline & & & & & & & & & & \\
\hline & & & & & & & & & & \\
\hline & & & & & & & & & & \\
\hline & & . & & & & & & & & \\
\hline & & & & & & & & & & \\
\hline & & & & & & & & & & \\
\hline
\end{tabular}

\begin{tabular}{|c|c|c|c|c|c|}
\hline 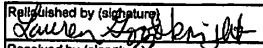 & 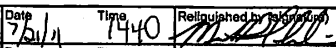 & 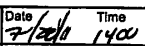 & Rellquished by (slgnature) & Date & Time \\
\hline paty & Date 7 /2olu & $\left(\frac{7}{7} 28\right) \times 1$ Time & Recelved by (slgnature) & Date & Time \\
\hline
\end{tabular}

Page 4 of 4 
This page intentionally left blank 
Appendix D

Laboratory Analytical Procedures 
This page intentionally left blank 


\section{D1 Sample Laboratory Preparation Methods}

LLNL was tasked with processing and analyzing over 300 environmental samples for ${ }^{137} \mathrm{Cs}$,

${ }^{241} \mathrm{Am}$, plutonium and uranium isotopes, and within the analyte detection and quantification capabilities of specified measurement techniques. Initial sample processing included sample dissection, freeze or oven drying, and sample volume reduction (sample combustion) prior to wet chemistry and radiometric analysis. The range of environmental samples for analysis included those discussed previously in Section 4.

\section{D1.1 Sample Shipping and Handling}

Vegetation, fauna, marine sediment, and soil collected in the field were typically sealed inside plastic bags or 1 L HDPE containers, labeled, and stored in a freezer or cool place without preservation. All fauna samples were collected and shipped as whole animals with exception of halibut and Pacific cod.

Samples were shipped overnight from Alaska to the San José airport in northern California, and then immediately transported by road (approximately 30 miles) to LLNL. The preferred shipping container type was a polystyrene cooler $(20 \mathrm{~L})$. Each sample shipment was accompanied by COC documentation showing a listing of samples identified by a shipment number, a ticket identification number, a sample site code, a sample location code, container type, level of preservation (if any), and a matrix identification code along with a listing of any analytical requirements. Upon arrival of the samples at LLNL, a validation check was performed on the basis of the COC sample documentation and any variances identified (Hamilton et al. 2012a). The samples were generally received in good condition.

Overall, LLNL analyzed 193 fauna samples, 96 vegetation samples, 6 soil samples, and 2 sediment samples along with 21 duplicates.

\section{D.1.2 Initial Sample Processing and Preparation Procedures}

Biological samples, including chiton, horse mussel, sea urchin, rockfish, halibut, Pacific cod, Irish lord, octopus, gull eggs, and Dolly Varden, were dried by lyophilization in an industrial-size freeze dryer (Northstar model 3666, $\sim 1.1$ cubic meter capacity) containing a modified condenser system $\left(-50^{\circ} \mathrm{C}\right)$ and liquid nitrogen $(\mathrm{LN})$ trap coupled to two large-capacity Sargent-Welch vacuum pumps running in series. The condenser and LN trap collect sublimed sample tissue water and require regular servicing to remove ice. Towards the end of the drying cycle, the freeze dryer chamber was opened up, and tissue samples were allowed to partially thaw at room temperature for several hours. This partial-thawing process allows residual tissue-bound water to migrate to the porous outer (dry) surface of the sample material and helps accelerate the drying process. The freeze dryer was otherwise operated continuously for a 16-week period with drying times for each load of samples varying between 3 to 6 weeks. Samples were considered dry after two or more consecutive weights showed little or no change based on self-imposed laboratory DQOs ( $<0.1 \%$ uncertainty) in reported normalization (dry/wet) weights. Biological samples were processed as received with the exceptions of rockweed and horse mussel. Rockweed samples were hand-picked after drying to remove foreign material. Horse mussels were sized, weighed, and then 'shucked' to remove soft tissue. Only the soft 
tissue of horse mussel was processed for analysis. The shells of gull eggs were also discarded prior to analysis.

The majority of the large-volume dragon kelp and rockweed samples (up to 5 kilograms [kg] each) were dried using a modified procedure to reduce processing times based on initial tests performed using lyophilization freeze drying. The remaining samples were weighed and then allowed to thaw (24-36 hours) sitting inside large plastic funnels propped up inside $50 \mathrm{~L}$ plastic containment bins. The free water (including sea salts) was allowed to drain directly into glass beakers sitting inside the containment bins. The amount of free liquid recovered from these samples averaged between 2 and $3 \mathrm{~kg}$. The beakers and residual biological material were then dried to constant weight in a fan-forced drying oven at $60^{\circ} \mathrm{C}$. Lichen samples were also oven dried to facilitate screening the samples for the presence of ${ }^{134} \mathrm{Cs}$ from Fukushima. In this instance, the dried lichen samples were homogenized in a laboratory blender, packed into standard geometry counting vials, and analyzed by high-resolution gamma spectrometry. The Marshall Islands Program gamma facility houses up to 24 high-resolution gamma detectors coupled to a DEC VAXStation operating under Canberra Nuclear Data Systems software. Details concerning detector calibration and quality assurance procedures are reported elsewhere (Brunk 1995; Hamilton et al. 2000).

Soil and sediments samples were also processed by oven drying to constant weight at $60{ }^{\circ} \mathrm{C}$ and then passed through a 2 millimeter $(\mathrm{mm})$ stainless steel standard sieve. Soil samples contained varying quantities of gravel ( $\geq 2 \mathrm{~mm}$ size fraction) and easily partitioned organic debris consisting mostly of plant roots and grass. Table D1shows the size fractionation of soils and sediments. SOIL-AD-XXX-3 contained a majority portion of organic debris. Measurement data are reported for the $<2 \mathrm{~mm}$ size fractions of soil and sediment only.

Table D1. Size Fractionation of Soils and Sediments

\begin{tabular}{|c|c|c|c|c|c|}
\hline \multirow[b]{2}{*}{ Ticket } & \multirow[b]{2}{*}{ Location } & \multirow[b]{2}{*}{$\begin{array}{l}\text { Total Sample } \\
\text { Dry Mass (g) }\end{array}$} & \multicolumn{3}{|c|}{ Size fraction (\%, dry weight) } \\
\hline & & & $<2 \mathrm{~mm}$ & $\geq 2 \mathrm{~mm}$ & $\begin{array}{c}\text { Easily Partitioned } \\
\text { Organic }\end{array}$ \\
\hline JET-741 & SEDI-LS-LT1-1 & 693.51 & 87 & 13.2 & 0.0 \\
\hline JET-742 & SEDI-ML-MT1-1 & 520.22 & 98 & 1.9 & 0.0 \\
\hline JDQ-901 & SOIL-AI-ML1-1 & 741.03 & 24 & 75.4 & 0.7 \\
\hline JDQ-902 & SOIL-AI-LT1-2 & 61.69 & 73 & 20.1 & 6.8 \\
\hline JDL-903 & SOIL-AI-CTI-3 & 124.57 & 95 & 3.0 & 1.8 \\
\hline JDQ-904 & SOIL-AD-XXX-1 & 88.69 & 59 & 27.2 & 14.2 \\
\hline JDQ-905 & SOIL-AD-XXX-2 & 143.63 & 67 & 21.2 & 12.2 \\
\hline JDQ-906 & SOIL-AD-XXX-3 & 26.49 & 34 & 20.6 & 45.0 \\
\hline
\end{tabular}

\section{D.1.3 Sample Combustion}

Analytical results of previous sampling events showed that background activity concentrations of selected target radionuclides in the vicinity of Amchitka Island were at or below levels of detection. Under Amchitka 2011, emphasis was placed on collecting an adequate number and larger-sized samples to support future trending analysis. The mean sample dry weight of material for analysis of target radionuclides ranged from 40 grams $(\mathrm{g})$ for horse mussel to well over $1,000 \mathrm{~g}$ for halibut, Pacific cod, dragon kelp, and rockweed. To reduce the sample mass to a 
more-manageable size for wet chemistry, it was necessary to reduce the volume of material by combustion in a muffle furnace.

Samples were typically loaded into aluminum pans or glass beakers and ashed at $450{ }^{\circ} \mathrm{C}$ for 3 to 4 days after slowly taking the muffle furnace up to temperature through a series of ramping steps. Rockweed was handpicked for foreign material prior to ashing. The foreign material included small rocks, snails, sea urchins and starfish, amphipods, and foreign plant species, and varied in mass between 1 and $44 \mathrm{~g}$.

It was not always possible to produce a total organic-free ash, but experience had shown that residual trace quantities of organic material (largely charcoal) can be effectively destroyed by addition of hydrogen peroxide during the acid dissolution steps to follow. The full listing of normalization dry/wet weights as well as ash/dry weights is provided in Appendix A.

Special modified handling and drying procedures were employed for large-volume dragon kelp and rockweed to reduce drying times (Hamilton et al. 2012a). Also, previous experience had shown that allowing the free liquid and polysaccharide material to drain from these materials prior to combustion in a muffle furnace significantly helps reduce the tendency of the material to caramelize into hard clumps. In this case, the free liquid was processed separately and then recombined with the dry ash prior to wet sample and dissolution. Additional details concerning sample processing and handling, and size fractionation of marine sediment and soils are given elsewhere (Hamilton et al. 2012a).

Marine sediment, soil, and lichen were initially oven dried to constant weight at $60{ }^{\circ} \mathrm{C}$. The dried lichen samples were homogenized in a laboratory blender, packed into standard geometry counting vials, and initially analyzed by high-resolution gamma spectrometry. The lichen material was recovered and used for subsequent analyses of uranium and plutonium isotopes and ${ }^{241} \mathrm{Am}$. Dried samples of vegetation and fauna were rendered down to a manageable volume using dry ashing techniques (Hamilton et al. 2012a) and, after addition of known quantities of stable $\mathrm{Cs},{ }^{242} \mathrm{Pu},{ }^{243} \mathrm{Am}$ yield tracers, digested in concentrated nitric acid $\left(\mathrm{HNO}_{3}\right)$ with periodic additions of hydrogen peroxide. After several days of treatment to produce a relatively clear, bright solution with minimal particulate residue, the sample digest was filtered and evaporated to near dryness. The dried sample residue was then rehydrated in dilute hydrochloric acid $(\mathrm{HCl})$. A small sub-aliquot of the original acid digest ( $<5$ percent) was consumed during the analysis of uranium isotopes by direct aspiration ICP-MS with all the remaining material saved for sequential analysis of ${ }^{137} \mathrm{Cs}$, plutonium isotopes, and ${ }^{241} \mathrm{Am}$.

Table D2 provides a summary of the data for each biological species and matrix type. In general, sample dry and ash weights proved to be relatively consistent within the same biological species. Dried dragon kelp and rockweed contained visible quantities of sea salt. Additionally, the high salt content of samples tended to add to the tedious nature of performing wet chemistry separations on what were already very large, bulky samples. Because seawater contains macro quantities of uranium isotopes at about 3.3 microgram $(\mu \mathrm{g})$ total uranium per liter, the presence of sea salt uranium should be evaluated as a possible contaminant in the final analysis. 
The normalization dry/wet weights as well as ash/dry weights (as an indication of organic content) of lichen samples were highly variable. However, it is not clear how these findings can be used to provide a quantitative basis for normalizing activity concentrations of target radionuclides (specifically cesium isotopes) found in lichen across different sample sites.

Table D2. Summary Data of Total Sample Wet, Dry and Ash Weights (and Normalization Dry/Wet and Ash/Dry Weight Ratios) by Species or Matrix Type

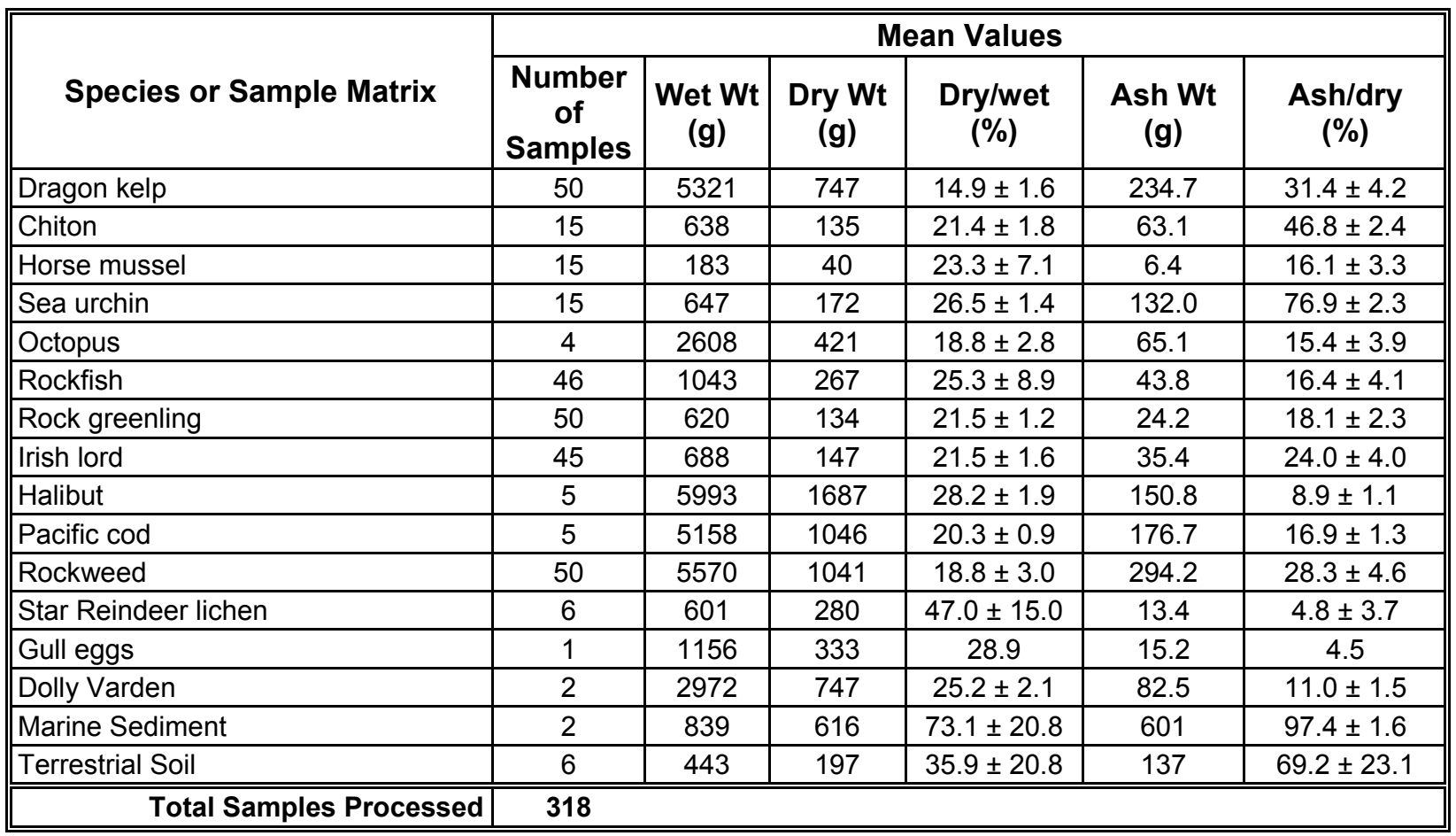

\section{D.2 Analytical Methods}

\section{D.2.1 Determination of ${ }^{137}$ Cesuim}

The bulk of the ${ }^{137} \mathrm{Cs}$ analyses were performed at LLNL using gas proportional betaspectrometry. The remaining samples, including marine sediment, soil, and star reindeer lichen, were analyzed by high-resolution gamma-spectrometry. Cesium isotopes, including a known quantity of stable cesium carrier (10 milligrams [mg]), were first separated from solution by extraction on microcrystalline ammonium molybdophosphate and then chemically isolated and purified using ion-exchange chromatography on BIO-REX-40 cation exchange resin (Hamilton et al. 2008). The column elution containing the purified cesium isotopes was then evaporated to dryness, rehydrated in dilute sodium hydroxide solution, and cesium isotopes were precipitated as $\mathrm{Cs}_{2} \mathrm{PtCl}_{6}$ by addition of chloroplatinic acid $\left(\mathrm{H}_{2} \mathrm{PtCl}_{6}\right)$. The purification of cesium is necessary to determine chemical recovery and remove naturally occurring potassium and rubidium from interfering with beta activity measurements of ${ }^{137} \mathrm{Cs}$ and ${ }^{134} \mathrm{Cs}$. The precipitate was then collected on a pre-weighed filter paper, dried and reweighed to a constant weight. The filter containing the $\mathrm{Cs}_{2} \mathrm{PtCl}_{6}$ precipitate was subsequently mounted on a ring and disc holder, and counted on a gas-proportional beta-spectrometry system (Gamma Products, Inc., G5000 with upgrades). 


\section{D.2.2 Determination of ${ }^{241}$ Americium}

${ }^{241}$ Am measurements were performed at LLNL using alpha spectrometry. Vegetation and fauna samples were first rendered down to manageable volumes using dry and wet ashing techniques as described previously. Americium and plutonium isotopes, including known quantities of ${ }^{243} \mathrm{Am}$ and ${ }^{242} \mathrm{Pu}$ added as isotopic dilution yield tracers, were first separated from the bulk sample digest solution by co-precipitation on aluminum phosphate. A secondary co-precipitation step was often employed for large, bulky samples. The analytes of interest, including plutonium isotopes and ${ }^{241} \mathrm{Am}$, were then chemically isolated and purified using ion-exchange chromatography. For analysis of ${ }^{241} \mathrm{Am}$, the effluent and washes from the TEVA plutonium column were taken to dryness, the residue rehydrated in a minimum volume of 2 moles (M) $\mathrm{HNO}_{3}$, and the solution passed through a TRU column preconditioned with 3 column volumes of $2 \mathrm{M} \mathrm{HNO}_{3}$. The TRU column was then washed with successive portions of $2 \mathrm{M} \mathrm{HNO}_{3}, 0.5 \mathrm{M}$ $\mathrm{HNO}_{3}$, and $9 \mathrm{M} \mathrm{HCl}$, and americium eluted using 10 column volumes of $4 \mathrm{M} \mathrm{HCl}$. The americium fraction in $4 \mathrm{M} \mathrm{HCl}$ was evaporated to dryness, the residue was rehydrated in 4 milliliter $(\mathrm{mL})$ of $9 \mathrm{M} \mathrm{HCl}$, the solution was passed through a two-layer ion-exchange column consisting of an upper layer of 2-3 mL Bio-Rad 50W-X8 cation-exchange resin (200-400 mesh) and a lower layer of 2-3 mL of Bio-Rad AG1-X8 anion-exchange resin (100-200 mesh), and the column was rinsed with five column volumes of $9 \mathrm{M} \mathrm{HCl}$. The two-layer ion-exchange column was employed to provide further separation from interfering alpha emitters, including trace quantities of polonium, plutonium, uranium, and thorium, as well as iron. The purified americium fraction from the column effluent was evaporated to dryness with addition of concentrated $\mathrm{HNO}_{3}$ and prepared for alpha-spectrometric detection and measurement by electrodeposition on stainless steel discs (modified after Wong et. al. 1994). Type I ultrapure water (resistivity $18.2 \mathrm{megohms}\left[\mathrm{M} \Omega\right.$ ] per $\cdot$ centimeter $(\mathrm{cm})$ at $25^{\circ} \mathrm{C}$; total organic carbon [TOC] $<10$ parts per billion [ppb]) was used for preparation of all solutions (produced from a Milli-Q synthesis A10 water purification system).

The americium source plates were counted on an EG\&G Ortec Octête PC alpha spectrometry system employing "ULTRA" ion-implanted-silicon charged-particle detectors and running on AlphaVision (version 5.3) and MAESTRO for Windows (model A65-B32; version 6.05). A counting time of approximately 72,000 seconds was originally established to yield a contract compliance minimum detectable activity (MDA) for ${ }^{241} \mathrm{Am}$ measurements of 0.03-0.06 picocurie ( $\mathrm{pCi}$ ). In practice, an effort was made to extend counting times to reduce minimum quantifiable values.

\section{D.2.3 Determination of Plutonium Isotopes}

Plutonium isotope analyses were performed at LLNL on a Thermo Electron XSeries quadrupole inductively coupled plasma mass spectrometer (ICP-MS) fitted with a concentric nebulizer. Americium and plutonium isotopes, including known quantities of ${ }^{243} \mathrm{Am}$ and ${ }^{242} \mathrm{Pu}$ added as isotopic dilution yield tracers, were first separated from the bulk sample digest solution by co-precipitation on aluminum phosphate. A secondary co-precipitation step was often employed for large, bulky samples. The analytes of interest, including plutonium isotopes and ${ }^{241} \mathrm{Am}$, were then chemically isolated and purified using ion-exchange chromatography. The ion-exchange purification step for plutonium involved rehydrating the aluminum-ferric precipitate in a minimal volume of $2.5 \mathrm{M} \mathrm{HNO}_{3}$ to $1 \mathrm{M}$ aluminum nitrate solution, and passing the sample through a 
preconditioned TEVA column. The TEVA column was then washed with $9 \mathrm{M} \mathrm{HCl}$ followed by successive rinses of $8 \mathrm{M} \mathrm{HCl}$, and plutonium isotopes eluted into a glass beaker using $20 \mathrm{~mL}$ $0.01 \mathrm{M} \mathrm{HCl}, 0.05 \mathrm{M}$ hydrofluoric acid, and $0.1 \mathrm{M}$ ammonium iodide solution. The column effluent and washings were reserved for analysis of americium isotopes. The purified plutonium fraction was then evaporated to dryness, treated with successive portions of $\mathrm{HNO}_{3}$, syringe filtered $(0.45$ micrometer $[\mu \mathrm{m}])$ into a $15 \mathrm{~mL}$ conical tube, and then made up to volume for ICP-MS analysis. Corrections for hydride $\left({ }^{238} \mathrm{UH}\right)$ interference for ICP-MS detection and measurement of ${ }^{239} \mathrm{Pu}$ were assessed as an integral part of the analysis. Type I ultrapure water (resistivity $18.2 \mathrm{M} \Omega / \mathrm{cm}$ at $25^{\circ} \mathrm{C}$; TOC $<10 \mathrm{ppb}$ ) was used for preparations of all solutions (produced from a Milli-Q synthesis A10 water purification system). Target plutonium isotopes of interest included ${ }^{239} \mathrm{Pu}$ and ${ }^{240} \mathrm{Pu}$.

\section{D.2.4 Determination of Uranium Isotopes}

Uranium isotope analyses were performed at LLNL on a Thermo Electron XSeries quadrupole ICP-MS fitted with a SeaSpray nebulizer. A direct dilute-and-shoot method was employed using 2 percent ultrapure $\mathrm{HNO}_{3}$ as the sample matrix after addition of a known quality of uranium-233 $\left({ }^{233} \mathrm{U}\right)$ isotope dilution tracer. A typical sample analysis involved taking a small sub-aliquot (1-2 $\mathrm{mL}$ ) of the original sample, digesting to dryness, and diluting the residue to volume (approximately $10 \mathrm{~mL}$ ) in an ICP-MS tube in the presence of ${ }^{233} \mathrm{U}$. All analyses were performed under established operational limitations set by the ICP-MS lab for total dissolved solids content $(<1-2$ percent) and total uranium content $(<0.2 \mu \mathrm{g} / \mathrm{mL})$. Type I ultrapure water (resistivity $18.2 \mathrm{M} \Omega / \mathrm{cm}$ at $25^{\circ} \mathrm{C}$; TOC $<10 \mathrm{ppb}$ ) was used for preparations of all solutions (produced from a Milli-Q synthesis A10 water purification system).

Target uranium isotopes of interest included ${ }^{238} \mathrm{U},\left({ }^{236} \mathrm{U}\right),{ }^{235} \mathrm{U}$, and ${ }^{234} \mathrm{U} .{ }^{236} \mathrm{U}$ was included in the analysis suite as a potential isotopic signature tracer of anthropogenic uranium, albeit at relatively high screening levels.

\section{D.2.5 Determination of Enriched Tritium}

Tritium concentrations are expressed in Tritium Units (TU), in which 1 TU indicates a Tritium/Hydrogen abundance ratio of $10^{-18}$. The values refer to the tritium scale recommended by the National Institute of Science and Technology (NIST, formerly National Bureau of Standards), and International Atomic Energy Agency (IAEA). The TU-numbers are based on the NIST tritium water standard 4926E. Age corrections and conversions are made using the recommended half-life of 12.32 years (i.e., a decay rate of lambda $=5.626$ percent per year [yr]). In this scale, $1 \mathrm{TU}$ is equivalent to 7.151 disintegrations per minute per kilogram $(\mathrm{dpm} / \mathrm{kg})$ water, or $3.222 \mathrm{pCi} / \mathrm{kg}$ water, or 0.1192 becquerel per kilogram $(\mathrm{Bq} / \mathrm{kg})$ water $(1 \mathrm{~Bq}=1$ disintegration per second or $60 \mathrm{dpm}$ ).

\section{D.2.5.1 Distillation}

The water sample is distilled with continuous reflux to dryness or near dryness. During the procedure, the still is vented to the ambient air through a drying agent to avoid contamination of the sample by atmospheric water vapor. 


\section{D.2.5.2 Electrolytic Enrichment}

The object of this procedure is to reduce the volume of the sample while preserving a large fraction of the tritium. A portion of the sample is transferred to the electrolytic enrichment cell. To that portion, $2 \mathrm{~mL}$ of concentrated sodium hydroxide solution (made from dead water [i.e., tritium-free water] and sodium peroxide or oxide) is added, and the remainder of the sample is transferred to a container on top of the cell.

The sample is electrolyzed in a cold water bath. Once a day the solution in the cell is topped up, and the procedure is continued. This procedure takes 10 to 14 days, and the remaining amount of water typically contains 80 percent of the original amount of tritium. The enriched water sample is then vacuum distilled from the sodium hydroxide, and the yield is weighed to $\pm 2 \mathrm{mg}$ ).

\section{D.2.5.3 Preparation of Sampling Gas}

About $3 \mathrm{~mL}$ of the enriched water sample is injected into a vacuum system. The water evaporates, and the vapor is reduced by hot magnesium metal to hydrogen gas, which is absorbed on activated charcoal at liquid nitrogen temperature in a stainless steel pressure cylinder. Approximately $4 \mathrm{~L}$ atmosphere of hydrogen is obtained this way.

\section{D.2.5.4 Low-Level Counting}

The low-level gas proportional counters have an active volume of $1 \mathrm{~L}$ and are shielded by $2.5 \mathrm{~cm}$ of selected lead, a ring of anti-coincidence Geiger counters, $10 \mathrm{~cm}$ of paraffin wax, boric acid and/or borated polyethylene, and at least $20 \mathrm{~cm}$ of iron, plus the walls and ceiling of the building. The counter is first filled with 10 pounds per square inch (psi) (67 kilopascals [kPa]) of propane. Thereafter, the sample hydrogen gas, under pressure in its cylinder, is added to the counter for a total pressure of $40 \mathrm{psi}(300 \mathrm{kPa})$. The counter is then sealed off, and the gas amplification is set to specifications by adjusting working voltage. After that, counting proceeds until criteria for accuracy or sensitivity have been met. The pulses are sorted into several channels, of which some are used for continuous control of the gas amplification. Counting times are 6 to 20 hours. A 1 TU original sample enriched from 275 to $6 \mathrm{~mL}$ typically shows 0.6 counts per minute (cpm) in the tritium channel above a background of $0.40 \mathrm{cpm}$, known to $\pm 0.02 \mathrm{cpm}$. The control of filling and counting procedures and calculation of results, as well as numerous checks on the performance of the machinery, are computerized.

When a sample is ready for activity measurement, the sample I.D. is merged with all the process information in the computer. Together, the data are entered into the particular sample file along with counter-fill data, sample pressure and temperature, a unique run number, and the time and date. Upon completion of the counting, temperature and pressure of the sample within the counter are again recorded for comparison and checked for computer input error. All records of the sample preparation information and counting results are stored in computer files. 
This page intentionally left blank 


\section{Appendix E}

Individual Sample Listing of Wet Weights, Dry Weights, and Ash Weights 
This page intentionally left blank 


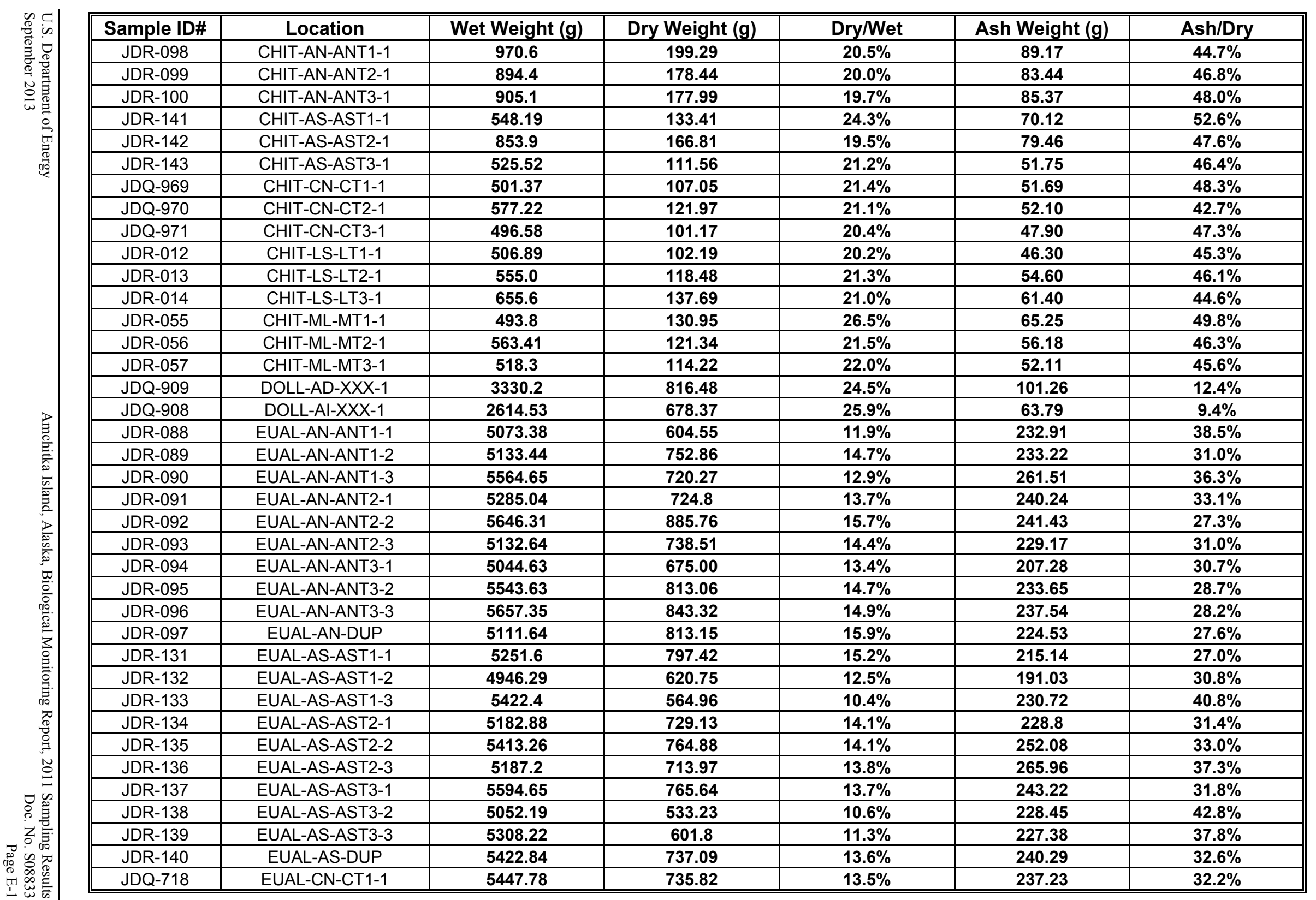




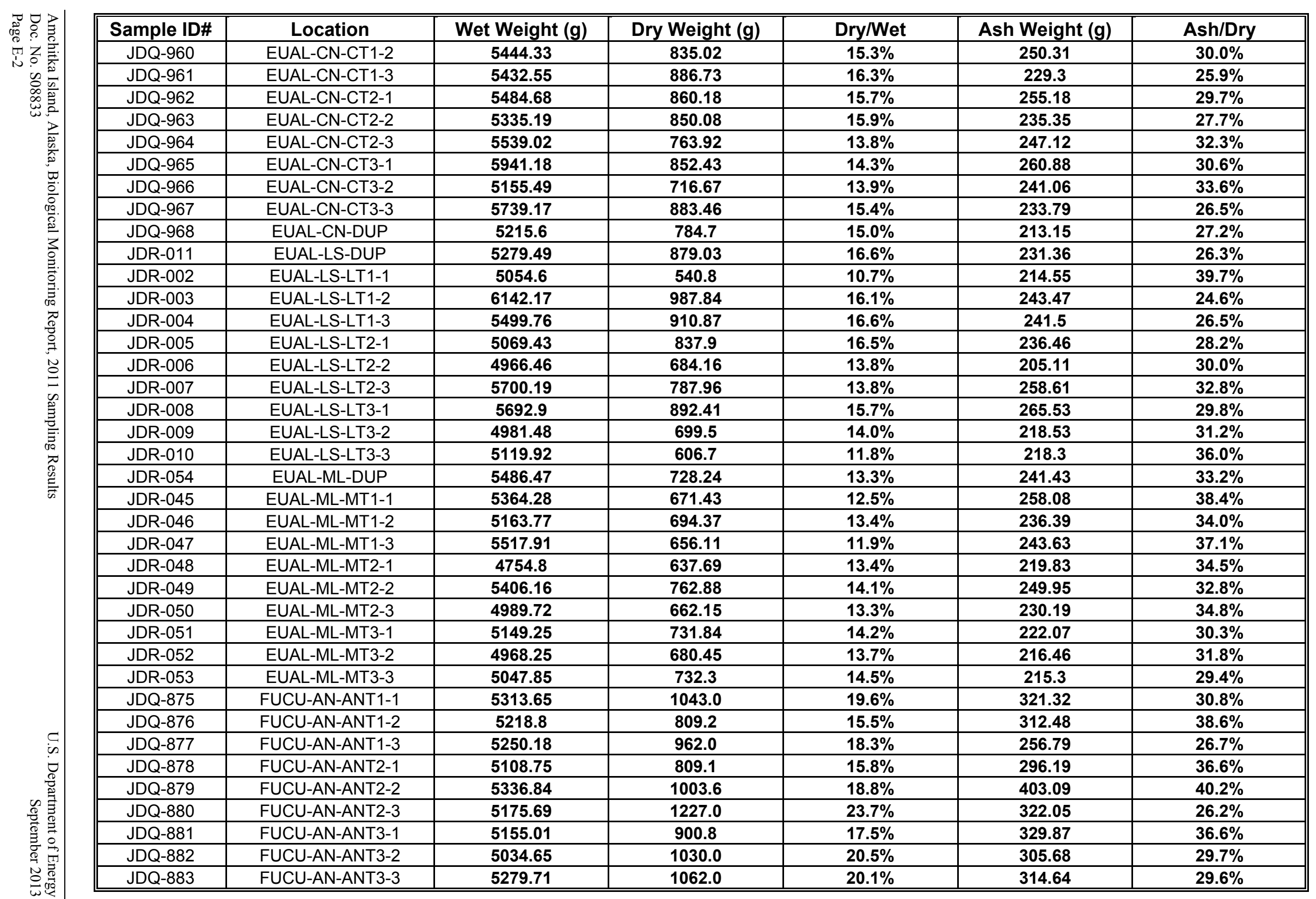




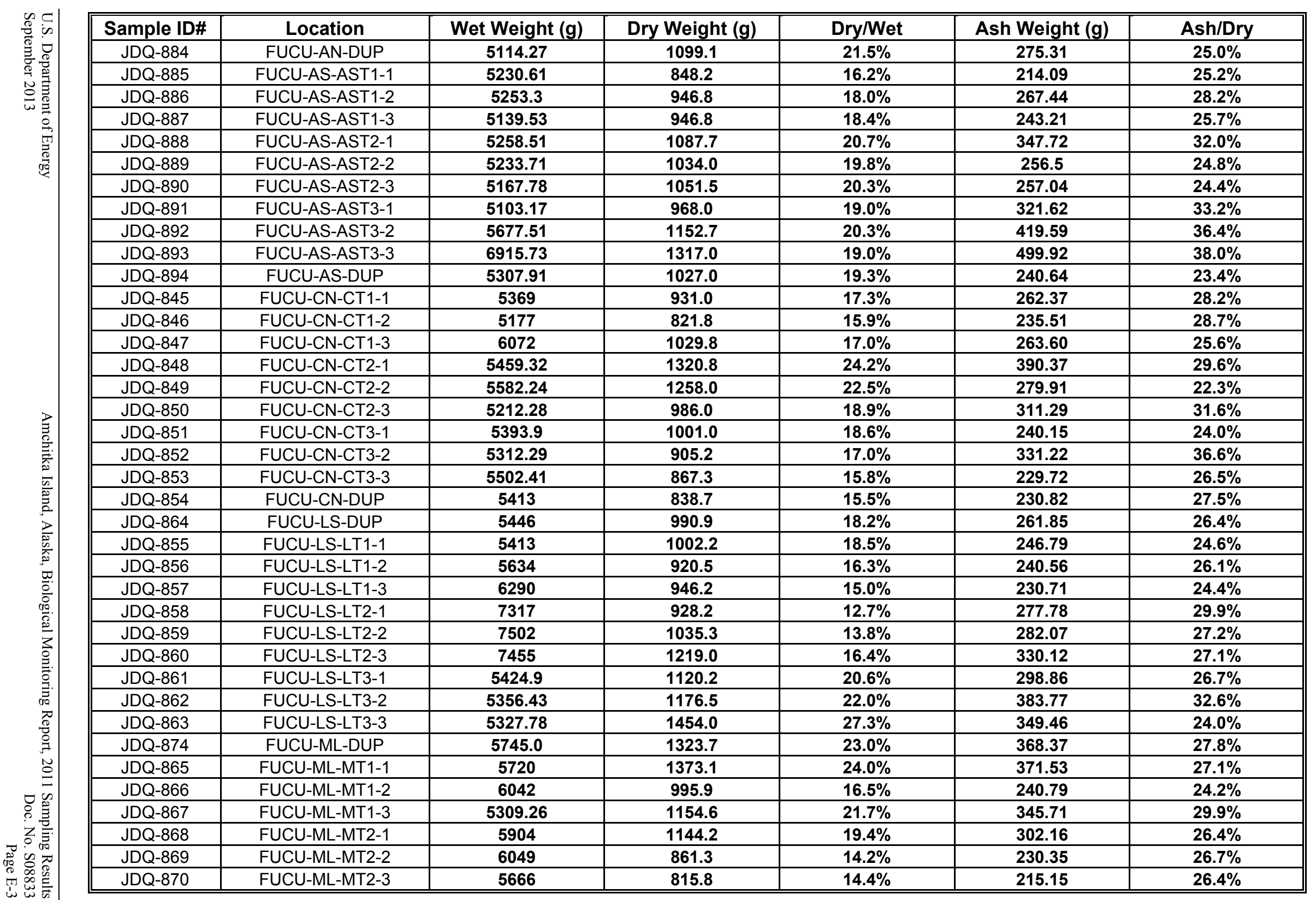




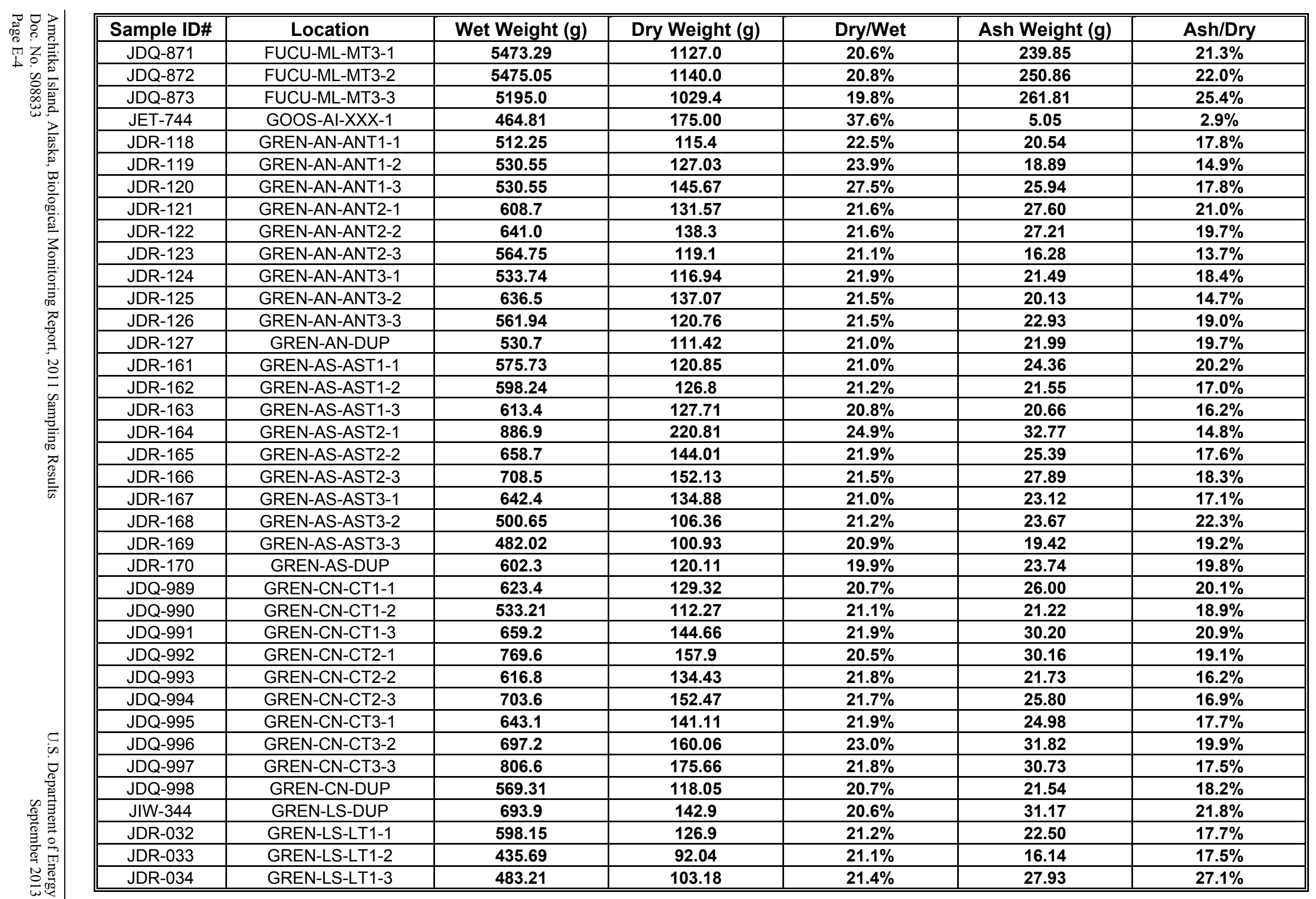




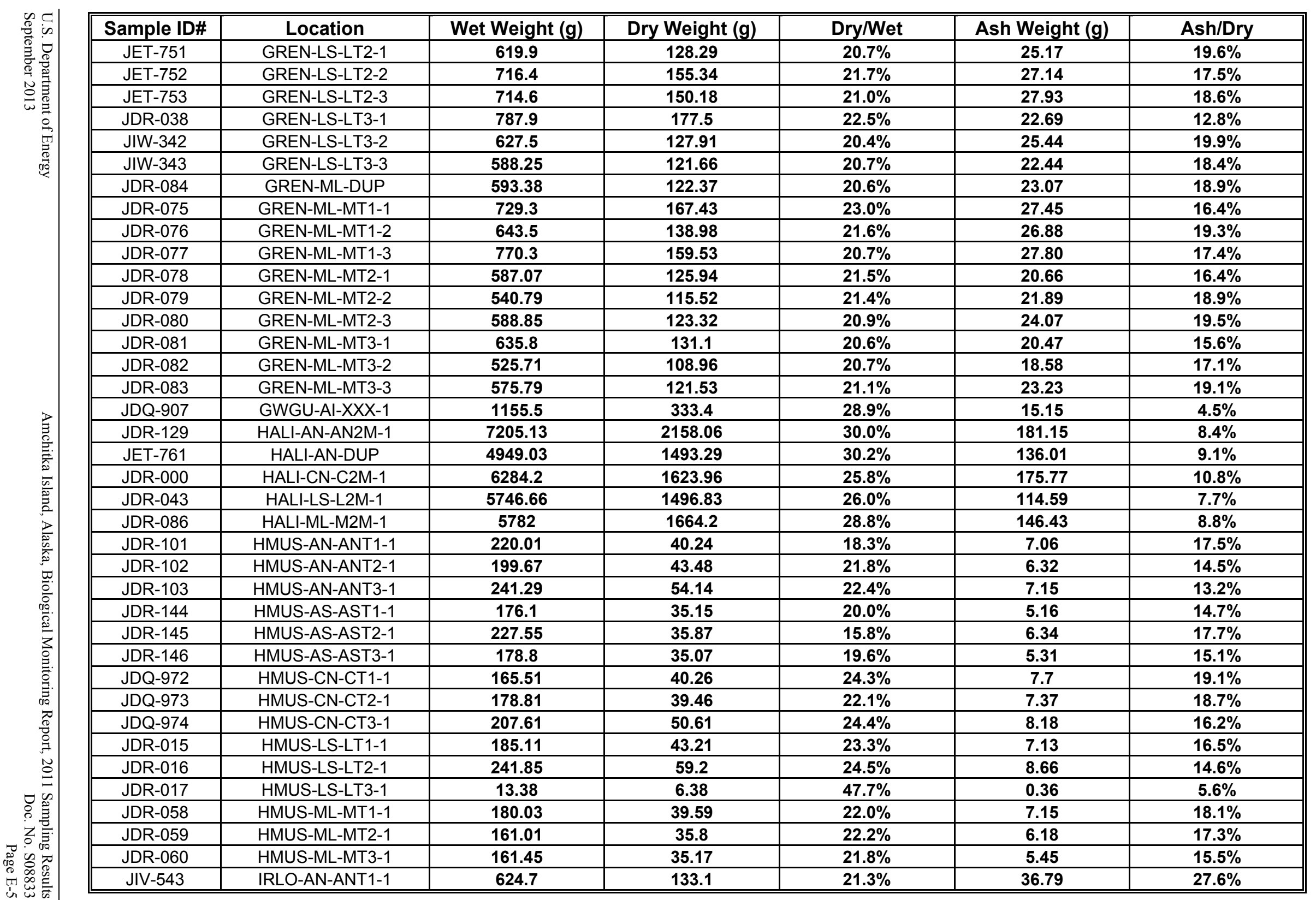




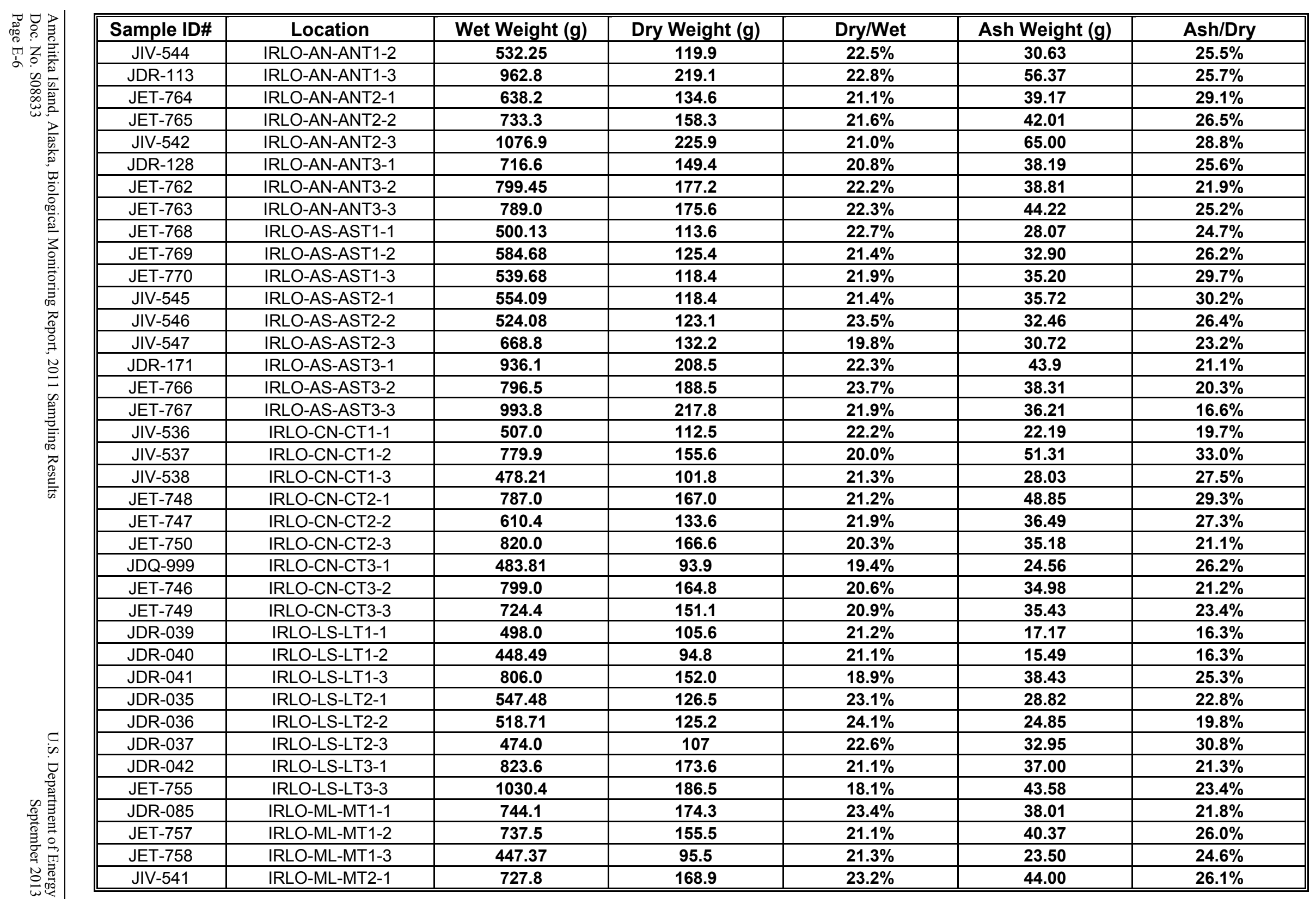




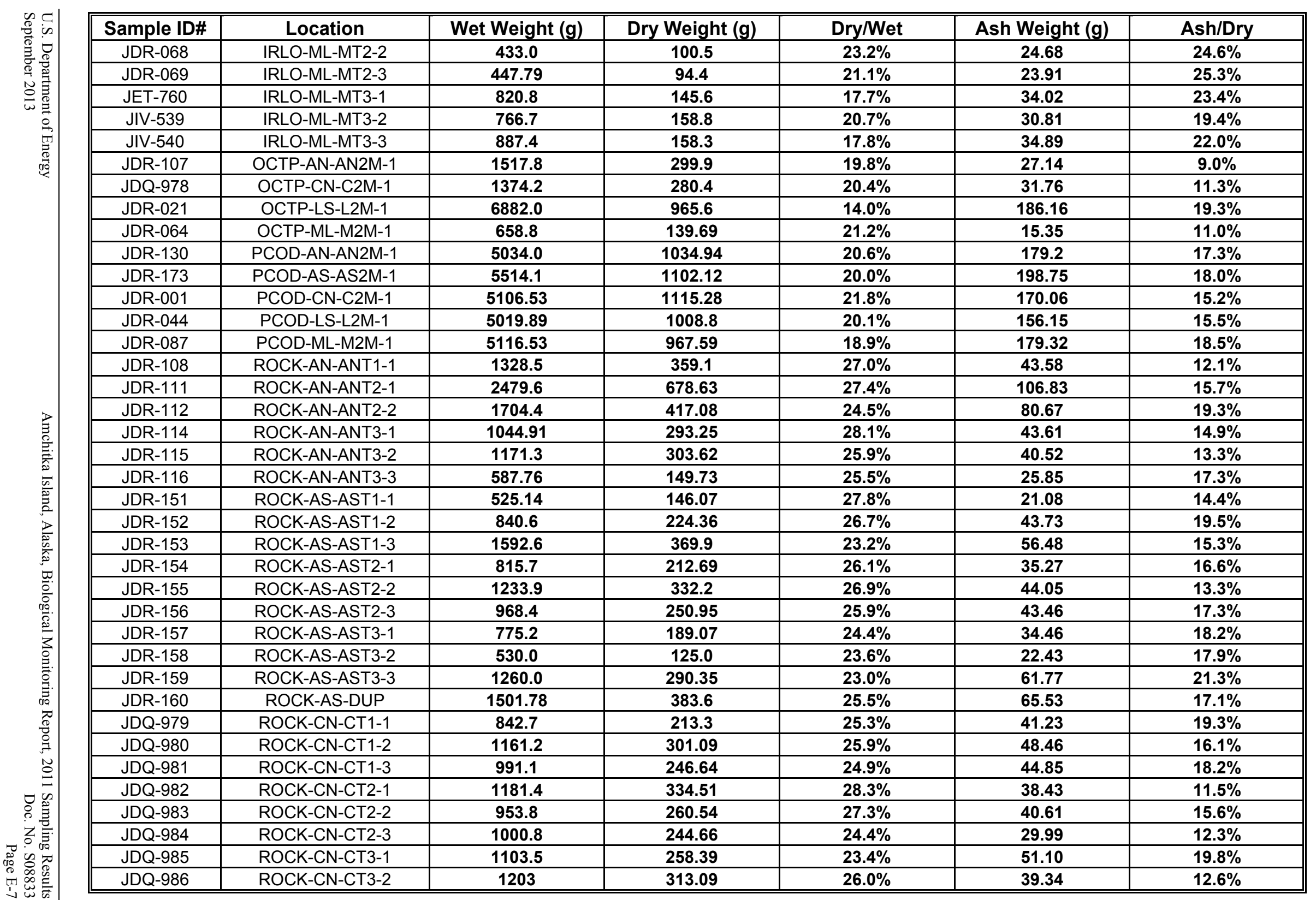




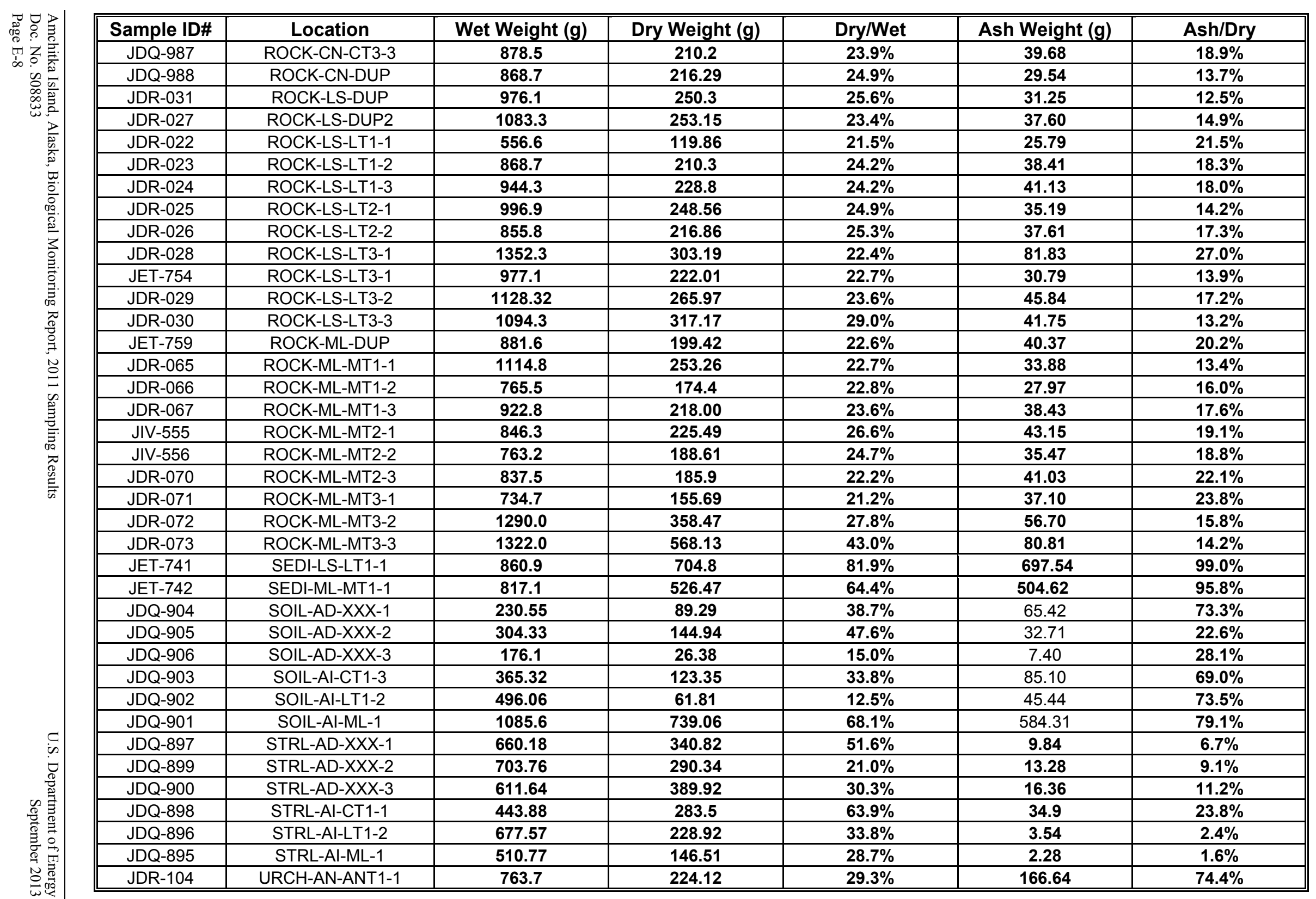




\begin{tabular}{|c|c|c|c|c|c|c|}
\hline Sample ID\# & Location & Wet Weight (g) & Dry Weight (g) & Dry/Wet & Ash Weight (g) & Ash/Dry \\
\hline JDR-105 & URCH-AN-ANT2-1 & 754.8 & 207.2 & $27.5 \%$ & 160.68 & $77.5 \%$ \\
\hline JDR-106 & URCH-AN-ANT3-1 & 720.1 & 189.43 & $26.3 \%$ & 142.38 & $75.2 \%$ \\
\hline JDR-147 & URCH-AS-AST1-1 & 577.99 & 152.17 & $26.3 \%$ & 121.39 & $79.8 \%$ \\
\hline JDR-148 & URCH-AS-AST2-1 & 479.6 & 118.8 & $24.8 \%$ & 86.18 & $72.5 \%$ \\
\hline JDR-149 & URCH-AS-AST3-1 & 537.38 & 143.91 & $26.8 \%$ & 111.06 & $77.2 \%$ \\
\hline JDQ-975 & URCH-CN-CT1-1 & 737.5 & 183.81 & $24.9 \%$ & 143.79 & $78.2 \%$ \\
\hline JDQ-976 & URCH-CN-CT2-1 & 534.9 & 156.1 & $29.2 \%$ & 112.91 & $72.3 \%$ \\
\hline JDQ-977 & URCH-CN-CT3-1 & 718.1 & 187.47 & $26.1 \%$ & 146.08 & $77.9 \%$ \\
\hline JDR-018 & URCH-LS-LT1-1 & 641.2 & 164.14 & $25.6 \%$ & 126.11 & $76.8 \%$ \\
\hline JDR-019 & URCH-LS-LT2-1 & 567.4 & 147.68 & $26.0 \%$ & 115.11 & $77.9 \%$ \\
\hline JDR-020 & URCH-LS-LT3-1 & 624.4 & 160.3 & $25.7 \%$ & 123.08 & $76.8 \%$ \\
\hline JDR-061 & URCH-ML-MT1-1 & 795.0 & 214.3 & $27.0 \%$ & 171.39 & $80.0 \%$ \\
\hline JDR-062 & URCH-ML-MT2-1 & 686.25 & 183.75 & $26.8 \%$ & 144.13 & $78.4 \%$ \\
\hline JDR-063 & URCH-ML-MT3-1 & 562.49 & 141.69 & $25.2 \%$ & 109.75 & $77.5 \%$ \\
\hline
\end{tabular}


This page intentionally left blank 


\section{Appendix F}

\section{Laboratory Quality Assurance and Data Validation Procedures}


This page intentionally left blank 


\section{F.1 Cesium-137 Isotope}

The beta-spectrometry system was calibrated using stock solutions of ${ }^{137} \mathrm{Cs}$ prepared by serial dilution of a Standard Reference Material 4233D supplied by NIST. Data validation procedures included the analysis of archive water samples obtained under the DOE Mixed Analyte Performance Evaluation Program (MAPEP) and are shown in Table F1. The average measurement bias across three test water samples each measured in triplicate was -3.4 percent. All performance test measurements easily satisfied the MAPEP acceptance range test criteria.

Table F1. Beta Spectrometric Measurements of Cesium Isotope Activity in MAPEP Performance Test Samples

\begin{tabular}{|c|c|c|c|c|c|c|c|c|}
\hline Sample ID & $\begin{array}{c}\text { Test Cs } \\
\text { Isotope } \\
\text { Activity } \\
\text { Level }^{\mathrm{a}} \\
\text { (pCi) }\end{array}$ & $\begin{array}{c}\text { Cs } \\
\text { Isotopes } \\
\text { Present }\end{array}$ & $\begin{array}{c}\text { Measured } \\
\text { Cs Isotope } \\
\text { Activity } \\
\text { Level } \\
\text { (pCi) }\end{array}$ & $\begin{array}{c}\text { Reference } \\
\text { Value }^{\mathrm{a}} \\
\left(\mathrm{pCi} \mathrm{L}^{-1}\right)\end{array}$ & $\begin{array}{c}\text { Measured } \\
\text { Value } \\
\left(\mathrm{pCi} \mathrm{L}^{-1}\right)\end{array}$ & $\begin{array}{c}\text { MAPEP } \\
\text { Acceptance } \\
\text { Range }^{\mathrm{a}} \\
\left(\mathrm{pCi} \mathrm{L}^{-1}\right)\end{array}$ & Test & Bias \\
\hline MAPEPW22_01 & 46.5 & ${ }^{13 /} \mathrm{Cs}$ & $44.1 \pm 1.6$ & 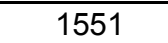 & 143 & 1095-2016 & PASS & $-7.7 \%$ \\
\hline MAPEPW22_02 & 46.5 & ${ }^{13 /} \mathrm{Cs}$ & $45.8 \pm 1.7$ & & 148 & & PASS & $-4.2 \%$ \\
\hline MAPEPW22_03 & 46.5 & ${ }^{13 /} \mathrm{Cs}$ & $48.5 \pm 1.8$ & 15 & $1572 \pm 58$ & 16 & PASS & $1.4 \%$ \\
\hline MAPEPW23_01 & 47.2 & ${ }^{3 /} \mathrm{Cs} /{ }^{134} \mathrm{Cs}$ & $46.4 \pm 1.8$ & 1573 & $1505 \pm 57$ & $1118-2059$ & PASS & $-4.4 \%$ \\
\hline MAPEPW23_02 & 47.2 & ${ }^{137} \mathrm{Cs} /{ }^{134} \mathrm{Cs}$ & $46.5 \pm 1.8$ & 1573 & $1508 \pm 57$ & 1118-2059 & PASS & $-4.2 \%$ \\
\hline MAPEPW23_03 & 47.2 & ${ }^{13 /} \mathrm{Cs} /{ }^{134} \mathrm{Cs}$ & $46.1 \pm 1.7$ & 1573 & $1495 \pm 57$ & 1118-2059 & PASS & $-5.0 \%$ \\
\hline MAPEPW24_02 & 33.3 & ${ }^{137} \mathrm{Cs} /{ }^{134} \mathrm{Cs}$ & $34.1 \pm 1.4$ & 1111 & $1107 \pm 44$ & $797-1464$ & PASS & $-0.4 \%$ \\
\hline MAPEPW24_02 & 33.3 & ${ }^{13 /} \mathrm{Cs} /{ }^{134} \mathrm{Cs}$ & $32.4 \pm 1.3$ & 1111 & $1051 \pm 42$ & $797-1464$ & PASS & $-5.4 \%$ \\
\hline MAPEPW24_03 & 33.3 & ${ }^{13 /} \mathrm{Cs} /{ }^{134} \mathrm{Cs}$ & $34.2 \pm 1.4$ & 1111 & $1108 \pm 44$ & $797-1464$ & PASS & $-0.3 \%$ \\
\hline
\end{tabular}

${ }^{\mathrm{a}}$ Decay corrected to count date.

The analyte detection and quantification capabilities of the ${ }^{137} \mathrm{Cs}$ measurements based on beta spectrometry were assessed using a graphical technique (Habaux and Vos 1970). A 6-point calibration curve was prepared in triplicate using sub-aliquots of MAPEPW22. Test levels ranged in activity between 0 and $47 \mathrm{pCi}$. The response curve yielded a straight-line calibration $\left(\mathrm{r}^{2}=0.9999\right)$ with a zero point intercept of $-0.07 \mathrm{pCi}$ and an estimated MDA of $0.65 \mathrm{pCi}$. For the purpose of this study, measurement MDAs for individual samples are expressed as activity concentrations (or the MDC [minimum detectable concentration]) in units of picocuries per kilogram wet weight, to account for variances in sample mass and analyte recovery.

During the data validation process, the purified beta spectrometry sources were pooled according to species type and counted by high-resolution gamma-spectrometry. These semiquantitative measurements were performed as a precautionary measure to check for the presence of ${ }^{134} \mathrm{Cs}$ (half-life $=2.05$ years) beta activity fallout originating from the Fukushima Dai-Ichi nuclear facility in Japan following the earthquake and tsunami on March 11, 2011. These measurements clearly identified the presence of ${ }^{134} \mathrm{Cs}$ in Dolly Varden and pooled samples of rockweed. Trace quantities of ${ }^{134} \mathrm{Cs}$ were also observed in pooled samples of Irish lord. In view of these findings, the associated measurement data for Dolly Varden, rockweed, and Irish lord were posted with a data qualifier indicating the presence of a known interference (Hamilton et al. 2012b). Because the Amchitka 2011 sampling event occurred soon after the Fukushima nuclear accident, all the reported ${ }^{137} \mathrm{Cs}$ beta spectrometry measurements may be variously affected by the same data qualifier and should therefore only be used as a conservative measure of ${ }^{137} \mathrm{Cs}$ activity concentrations for input into associated human health and ecological assessment models. By 
analogy, a conservative estimate of the activity concentration of ${ }^{134} \mathrm{Cs}$ in fauna for the 2011 sampling event could be made by assuming the cesium isotope activity observed at the time of the sampling was dominated by a Fukushima source-term, that is, ${ }^{134} \mathrm{Cs} /{ }^{137} \mathrm{Cs}$ activity ratio $\approx 1$, and multiplying the reported ${ }^{137} \mathrm{Cs}$ activity concentration by an isotope ratio-decay factor (assuming the time between sampling and the original analysis date was approximately 12 months) equal to the reported activity concentration of ${ }^{137} \mathrm{Cs}\left(0.59 \mathrm{pCi} \mathrm{kg}{ }^{-1}\right)$.

\section{F.2 Americium-241 Isotope}

A conservative estimate of the MDA was calculated based on a Stapleton approximation (adopted from MARLAP 2004) by using the mean background count rate for the alpha spectrometry system rather than a mean blank count rate. This approximation was made because a series of 10 replicate blank measurements all returned a zero count rate in the specific region of interest for ${ }^{241} \mathrm{Am}$. With this assumption, the largest number of ${ }^{241} \mathrm{Am}$ counts likely to be observed in a background count was 2, yielding an estimated MDA for measurement of ${ }^{241} \mathrm{Am}$ by alpha spectrometry of $0.01 \mathrm{pCi}$. For the purposes of this report, measurement MDAs for individual samples are expressed as activity concentrations (or the MDC) in units of picocuries per kilogram wet weight, to account for the variances in the sample mass and analyte recovery. The vast majority of samples analyzed for the 2011 sampling event contained ${ }^{241} \mathrm{Am}$ levels that were at or below the MDA. Where time permitted, the counting times for source plates were extended to improve on the minimum quantifiable values; nonetheless, in many cases this still proved to be inadequate in providing a reasonable level of measurement precision. The reproducibility of the per-kilogram counts on source plates was assessed using a small number of cross counts on different detectors. The cross counts satisfied internal laboratory quality requirements with a mean measurement bias $(\mathrm{N}=8)$ of 28 percent compared with an average individual sample measurement uncertainty $( \pm 1$ sigma $[\sigma])$ for the same source plates of 24 percent. By excluding duplicate counts on one outlier source plate (No. 6) containing ${ }^{241} \mathrm{Am}$ levels at or below the reported MDAs, the mean bias between cross counts is further improved to 16.1 percent, as shown in Table F2. 
Table F2. Cross-Count Data for Determination of ${ }^{241} \mathrm{Am}$ by Alpha Spectrometry

\begin{tabular}{|c|c|c|c|c|}
\hline No & Location & 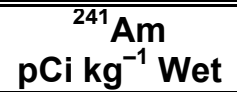 & $\% \mathrm{CV}$ & MDA \\
\hline \multirow{2}{*}{1} & \multirow{2}{*}{ CHIT-CN-CT2-1 } & $0.066 \pm 0.010$ & \multirow{2}{*}{3} & 0.003 \\
\hline & & $0.071 \pm 0.013$ & & 0.004 \\
\hline \multirow{2}{*}{2} & \multirow{2}{*}{ URCH-CN-CT2-1 } & $0.13 \pm 0.02$ & \multirow{2}{*}{12} & 0.004 \\
\hline & & $0.10 \pm 0.04$ & & 0.01 \\
\hline \multirow{2}{*}{3} & \multirow{2}{*}{ OCTP-CN-C2M-1 } & $0.015 \pm 0.005$ & \multirow{2}{*}{8} & 0.003 \\
\hline & & $0.017 \pm 0.005$ & & 0.003 \\
\hline \multirow{2}{*}{4} & \multirow{2}{*}{ ROCK-CN-CT2-3 } & $0.007 \pm 0.006$ & \multirow{2}{*}{26} & 0.007 \\
\hline & & $0.004 \pm 0.003$ & & 0.003 \\
\hline \multirow{2}{*}{5} & \multirow{2}{*}{ CHIT-AN-ANT1-1 } & $0.066 \pm 0.005$ & \multirow{2}{*}{9} & 0.002 \\
\hline & & $0.079 \pm 0.012$ & & 0.003 \\
\hline \multirow[b]{2}{*}{6} & \multirow{2}{*}{ ROCK-AS-AST1-1 } & $-0.001 \pm 0.001$ & \multirow{2}{*}{116} & 0.008 \\
\hline & & $0.013 \pm 0.007$ & & 0.007 \\
\hline \multirow{2}{*}{7} & \multirow{2}{*}{ PCOD-AS-AS2M-1 } & $0.002 \pm 0.001$ & \multirow{2}{*}{38} & 0.001 \\
\hline & & $0.001 \pm 0.001$ & & 0.001 \\
\hline \multirow{2}{*}{8} & \multirow{2}{*}{ IRLO-ML-MT1-3 } & $0.010 \pm 0.008$ & \multirow{2}{*}{13} & 0.009 \\
\hline & & $0.012 \pm 0.007$ & & 0.007 \\
\hline & & Average \%CV & $.4(16.1$ & \\
\hline
\end{tabular}

$\mathrm{CV}=$ coefficient of variation

\section{F3 Plutonium Isotopes}

The minimal detectable signal of the ICP-MS instrument for plutonium isotope measurements was estimated in a fashion similar to that described for uranium isotopes using procedures adopted from MARLAP (2004). Based on the standard deviation of 10 reported replicate blank measurements, the MDA defined by units of activity for measurement of ${ }^{239} \mathrm{Pu}$ and ${ }^{240} \mathrm{Pu}$ by ICP-MS is estimated to be around 0.03 and $0.02 \mathrm{pCi}$, respectively. For the purpose of this study, measurement MDAs for individual samples are expressed as activity concentrations (or the MDC) in units of picocuries per kilogram wet weight, to account for the variances in the sample mass.

Mass bias and concentration performance test measurements were performed using certified reference materials (CRMs) traceable to the NIST, namely CRM 128 containing a ${ }^{239} \mathrm{Pu} /{ }^{242} \mathrm{Pu}$ atom ratio of 0.9989 , and CRM 138 containing a ${ }^{240} \mathrm{Pu} /{ }^{239} \mathrm{Pu}$ atom ratio of 0.2409 . Each of these materials was originally certified as a primary isotopic ratio standard, but as the material was diluted to a known volume, they are subsequently also used in LLNL as laboratory concentration control standards. The performance tests samples as analyzed were prepared in the sub-nanogram concentration range to mimic expected activity concentrations in the Amchitka samples. Table F3 shows the average ${ }^{239} \mathrm{Pu} /{ }^{240} \mathrm{Pu}$ bias on six replicates measurements of test solutions of CRM 138 containing 0.004 nanogram per gram $\mathrm{ng} \mathrm{g}^{-1}\left(0.24 \mathrm{pCi} \mathrm{g}^{-1}\right){ }^{239} \mathrm{Pu}$ and $0.00034 \mathrm{ng} / \mathrm{g}\left(0.08 \mathrm{pCi} \mathrm{g}^{-1}\right){ }^{240} \mathrm{Pu}$ was -0.6 percent. Table $\mathrm{F} 4$ shows the replicate analyses $(\mathrm{N}=10)$ of test solutions of CRM 128 containing $0.25 \mathrm{ng}$ (or $16 \mathrm{pCi}$ ) of ${ }^{239} \mathrm{Pu}$ also met laboratory performance criteria for accuracy and precision. In this example, the mean measurement bias as reported for the ${ }^{239} \mathrm{Pu}$ concentration in the stock solution was 4.5 percent but satisfies laboratory performance acceptance criteria by falling within $3 \sigma$ of the mean individual measurement uncertainty or \pm 5.3 percent. 
In Table F5, a series of nonrandom Amchitka samples $(\mathrm{N}=48)$ were selected to run in duplicate on separate ICP-MS runs to assess the effects of run variability on the precision and accuracy of the plutonium isotopic measurements (Table F4). There were two considerations given to selection of the samples (1) a reasonable cross section of samples based on the different vegetation and fauna species, and (2) a reasonable selection of samples where the initial measurement provided quantifiable data for either ${ }^{239} \mathrm{Pu}$ or ${ }^{240} \mathrm{Pu}$, or both. The criteria applied for inclusion of the duplicate data in the statistical summary was also selective. In this case, only those duplicate measures in which the individual measurements exceeded the Critical Concentration (defined by half the reported MDC, or MDC/2) were included $(\mathrm{N}=25)$. Otherwise, the results of both measurements are shown as an MDA with no uncertainty.

Table F3. Mass Bias Performance Test Measurements of Plutonium Isotopes in Certified reference Material CRM 138 Based on ICP-MS Detection

\begin{tabular}{||c|c|c||}
\hline Sample & $\begin{array}{c}{ }^{\mathbf{2 4 0}} \mathbf{P u} /{ }^{\mathbf{2 3 9}} \mathbf{P u} \\
\text { Mass Ratio }\end{array}$ & Bias \\
\hline CRM138\#1 & $0.084 \pm 0.002$ & $-2.2 \%$ \\
\hline CRM138\#2 & $0.086 \pm 0.001$ & $0.1 \%$ \\
\hline CRM138\#3 & $0.086 \pm 0.002$ & $0.3 \%$ \\
\hline CRM138\#4 & $0.086 \pm 0.002$ & $0.5 \%$ \\
\hline CRM138\#5 & $0.087 \pm 0.002$ & $0.9 \%$ \\
\hline CRM138\#6 & $0.083 \pm 0.002$ & $-3.2 \%$ \\
\hline \hline Measured Mean Value & $\mathbf{0 . 0 8 5 4} \pm \mathbf{0 . 0 0 1 4}$ & $-\mathbf{0 . 6 \%}$ \\
\hline Reference Value & $\mathbf{0 . 0 8 5 9} \pm \mathbf{0 . 0 0 0 1}$ & \\
\hline
\end{tabular}

Table F4. Concentration Performance Test Measurement of ${ }^{239} \mathrm{Pu}$ Isotopes in Certified Reference Material CRM 128 Based on ICP-MS Detection

\begin{tabular}{|c|c|c|}
\hline Sample & 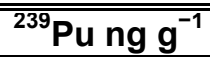 & Bias \\
\hline CRM128 \#1 & $27.5 \pm 0.4$ & $5.8 \%$ \\
\hline CRM128 \#2 & $27.5 \pm 0.3$ & $5.7 \%$ \\
\hline CRM128 \#3 & $27.3 \pm 0.4$ & $5.3 \%$ \\
\hline CRM128 \#4 & $27.5 \pm 0.7$ & $5.9 \%$ \\
\hline CRM128 \#5 & $26.8 \pm 0.5$ & $3.3 \%$ \\
\hline CRM128 \#6 & $27.1 \pm 0.4$ & $4.5 \%$ \\
\hline CRM128 \#7 & $27.1 \pm 0.3$ & $4.4 \%$ \\
\hline CRM128 \#8 & $26.8 \pm 0.3$ & $3.4 \%$ \\
\hline CRM128 \#9 & $26.8 \pm 0.6$ & $3.1 \%$ \\
\hline CRM128 \#10 & $27.0 \pm 0.8$ & $3.9 \%$ \\
\hline Measured Mean Value & $27.1 \pm 0.3$ & $4.5 \%$ \\
\hline Reference Value & 25.96 & \\
\hline
\end{tabular}


Table F5. Replicate Measurements of Plutonium Isotopes Based on ICP-MS

\begin{tabular}{|c|c|c|c|c|c|c|c|}
\hline \multirow{2}{*}{ No. } & \multirow{2}{*}{ Ticket Number } & \multicolumn{2}{|c|}{${ }^{239} \mathrm{Pu}, \mathrm{pCi} \mathrm{kg}^{-1} \mathrm{Wet}$} & \multicolumn{2}{|c|}{ 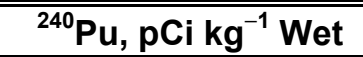 } & \multicolumn{2}{|c|}{ 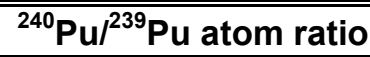 } \\
\hline & & Value & $\% \mathrm{CV}$ & Value & $\% \mathrm{CV}$ & Value & $\% \mathrm{CV}$ \\
\hline \multirow{2}{*}{1} & \multirow{2}{*}{ CHIT-AS-AST3-1 } & $0.056 \pm 0.004$ & \multirow{2}{*}{2.3} & 0.03 & \multirow{2}{*}{$\mathrm{L}$} & - & \multirow[b]{2}{*}{-} \\
\hline & & $0.054 \pm 0.004$ & & 0.03 & & - & \\
\hline \multirow{2}{*}{2} & \multirow{2}{*}{ CHIT-CN-CT1-1 } & 0.06 & \multirow[b]{2}{*}{-} & 0.04 & \multirow[b]{2}{*}{-} & - & \multirow[b]{2}{*}{ - } \\
\hline & & 0.06 & & 0.04 & & - & \\
\hline \multirow{2}{*}{3} & \multirow{2}{*}{ CHIT-CN-CT2-1 } & $0.052 \pm 0.006$ & \multirow{2}{*}{12} & 0.03 & \multirow{2}{*}{ - } & - & \multirow{2}{*}{ - } \\
\hline & & $0.066 \pm 0.005$ & & 0.03 & & - & \\
\hline & & 0.07 & - & 0.04 & 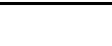 & - & \\
\hline 4 & CHIT-CN-CT3-1 & 0.07 & - & 0.04 & - & - & - \\
\hline 5 & CHIT_IS S-I T2-1 & $0.031 \pm 0.001$ & 21 & 0.03 & & - & \\
\hline 5 & |CHII-LS-LIL-1 & $0.021 \pm 0.004$ & 21 & 0.03 & - & - & - \\
\hline & & 0.01 & & 0.01 & & - & \\
\hline 6 & DOLL-AI-XXX-1 & 0.01 & - & 0.01 & - & - & - \\
\hline & & $0.051 \pm 0.001$ & & $0.040 \pm 0.001$ & & $0.21 \pm 0.01$ & \\
\hline 7 & EUAL-AN-ANI-1 & $0.048 \pm 0.004$ & 2.5 & $0.036 \pm 0.002$ & 5.3 & $0.20 \pm 0.03$ & 2.9 \\
\hline & & $0.100 \pm 0.001$ & & $0.081 \pm 0.002$ & & $0.22 \pm 0.01$ & \\
\hline 8 & | EUAL-AS-AS T-1 & $0.104 \pm 0.002$ & 2.0 & $0.085 \pm 0.003$ & 2.5 & $0.22 \pm 0.02$ & 0.5 \\
\hline$a$ & FUULACACT3 3 & $0.104 \pm 0.001$ & $23+2>0$ & $0.086 \pm 0.002$ & 05 & $0.23 \pm 0.01$ & \\
\hline 9 & | EUAL-AS-AST 3-3 & $0.108 \pm 0.001$ & 2.3 & $0.085 \pm 0.004$ & 0.5 & $0.22 \pm 0.02$ & - \\
\hline 10 & FEIALCN-CT1-1 & $0.074 \pm 0.001$ & 10 & $0.061 \pm 0.002$ & 33 & $0.23 \pm 0.01$ & \\
\hline 10 & EUAL-CN-CI1-1 & $0.075 \pm 0.004$ & 1.0 & $0.057 \pm 0.001$ & 3.3 & $0.21 \pm 0.01$ & 4.2 \\
\hline & & $\begin{array}{c}0.0113 \pm \\
0.0003\end{array}$ & & $0.007 \pm 0.001$ & & $0.18 \pm 0.05$ & \\
\hline 11 & EUAL-CN-CT1-2 & $\begin{array}{c}0.0133 \pm \\
0.0005\end{array}$ & 8.1 & $0.007 \pm 0.001$ & 0.0 & $0.15 \pm 0.05$ & 8.1 \\
\hline 12 & FUUAL_CN-CT2-3 & $0.061 \pm 0.001$ & 07 & $0.052 \pm 0.002$ & 08 & $0.23 \pm 0.02$ & 02 \\
\hline 12 & EUAL-CIN-CIL-O & $0.060 \pm 0.001$ & 0.1 & $0.051 \pm 0.002$ & 0.0 & $0.23 \pm 0.02$ & 0.2 \\
\hline 13 & 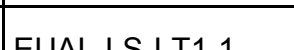 & $0.064 \pm 0.001$ & 07 & $0.051 \pm 0.002$ & 00 & $0.22 \pm 0.02$ & 07 \\
\hline 13 & EUAL-LS-L 1 1-1 & $0.065 \pm 0.004$ & 0.7 & $0.051 \pm 0.003$ & 0.0 & $0.22 \pm 0.02$ & 0.1 \\
\hline & & 0.005 & - & 0.003 & & - & - \\
\hline 14 & EUAL-LS-LT1-2 & 0.005 & - & 0.003 & - & - & - \\
\hline 15 & FUIAI _MI -MT1-3 & $0.094 \pm 0.001$ & 08 & $0.078 \pm 0.002$ & O & $0.23 \pm 0.01$ & 08 \\
\hline 15 & EUAL-ML-MI 1-3 & $0.096 \pm 0.004$ & 0.8 & $0.078 \pm 0.002$ & 0.0 & $0.23 \pm 0.01$ & 0.8 \\
\hline & & $0.116 \pm 0.001$ & & $0.096 \pm 0.003$ & & $0.23 \pm 0.01$ & \\
\hline 16 & EAUL-ML-MT2-1 & $0.117 \pm 0.001$ & 0.3 & $0.094 \pm 0.004$ & 1.0 & $0.22 \pm 0.02$ & 1.4 \\
\hline 17 & FAU -MI -MT2-2 & $0.100 \pm 0.001$ & 0 & $0.085 \pm 0.002$ & 28 & $0.23 \pm 0.01$ & 28 \\
\hline 17 & EAUL-ML-MI 2-2 & $0.100 \pm 0.001$ & 0.0 & $0.081 \pm 0.003$ & 2.8 & $0.22 \pm 0.02$ & 2.8 \\
\hline 18 & FUUI -MI -MT3-2 & $\begin{array}{c}0.0351 \pm \\
0.0004\end{array}$ & 16 & $0.026 \pm 0.001$ & 34 & $0.20 \pm 0.02$ & 19 \\
\hline & & $0.036 \pm 0.001$ & & $0.028 \pm 0.002$ & & $0.21 \pm 0.03$ & \\
\hline 19 & FUCU-AN-ANT1-1 & $\begin{array}{c}0.0190 \pm \\
0.0003\end{array}$ & 22 & $0.011 \pm 0.001$ & 0.0 & $0.15 \pm 0.03$ & 22 \\
\hline & & $0.012 \pm 0.001$ & & $0.011 \pm 0.001$ & & $0.24 \pm 0.06$ & \\
\hline & & 0.006 & & 0.003 & & - & \\
\hline 20 & FUCU-AN-ANT2-3 & 0.006 & - & 0.003 & - & - & - \\
\hline 21 & FIUCLAN_ANT3-1 & 0.006 & & 0.003 & & - & 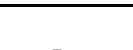 \\
\hline 21 & FUCU-AIN-ANIS-1 & 0.006 & - & 0.003 & - & - & - \\
\hline & & 0.006 & & 0.003 & & - & \\
\hline 22 & |FUCU-AS-AST3-1 & 0.006 & - & 0.003 & - & - & - \\
\hline 23 & FUCHLCN-CT2 2 & 0.006 & & 0.003 & & - & 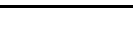 \\
\hline 23 & FUCU-CN-CT2-2 & 0.006 & - & 0.003 & - & - & - \\
\hline 24 & FUCHIS S-I T1-1 & 0.006 & -1 & 0.003 & - & - & - \\
\hline 24 & |FUCU-LS-LI 1-1 & 0.006 & - & 0.003 & - & - & - \\
\hline
\end{tabular}


Table F5 (continued). Replicate Measurements of Plutonium Isotopes Based on ICP-MS

\begin{tabular}{|c|c|c|c|c|c|c|c|}
\hline \multirow{2}{*}{ No. } & \multirow{2}{*}{ Ticket Number } & \multicolumn{2}{|c|}{${ }^{239} \mathrm{Pu}, \mathrm{pCi} \mathrm{kg}^{-1}$ Wet } & \multicolumn{2}{|c|}{${ }^{240} \mathrm{Pu}, \mathrm{pCi} \mathrm{kg}^{-1}$ Wet } & \multicolumn{2}{|c|}{${ }^{2{ }^{240}} \mathrm{Pu} /{ }^{239} \mathrm{Pu}$ atom ratio } \\
\hline & & Value & $\% \mathrm{CV}$ & Value & $\% \mathrm{CV}$ & Value & $\% \mathrm{CV}$ \\
\hline \multirow{2}{*}{25} & \multirow{2}{*}{ FUCU-LS-LT2-1 } & 0.05 & \multirow[b]{2}{*}{-} & 0.03 & \multirow[b]{2}{*}{ - } & - & \multirow[b]{2}{*}{-} \\
\hline & & 0.05 & & 0.03 & & - & \\
\hline \multirow{2}{*}{26} & \multirow{2}{*}{ GREN-CN-CT3-1 } & 0.05 & \multirow[b]{2}{*}{-} & 0.03 & \multirow{2}{*}{-} & - & \multirow{2}{*}{-} \\
\hline & & 0.05 & & 0.03 & & - & \\
\hline \multirow{2}{*}{27} & \multirow{2}{*}{ GREN-CN-CT3-2 } & 0.05 & \multirow[b]{2}{*}{ - } & 0.02 & \multirow[b]{2}{*}{-} & - & \multirow{2}{*}{-} \\
\hline & & 0.05 & & 0.02 & & - & \\
\hline 28 & CPEN I $C 1 \mathrm{~T}_{2} 1$ & 0.04 & & 0.02 & & - & \\
\hline 28 & GREIN-LS-LIJ-1 & 0.04 & - & 0.02 & - & - & - \\
\hline 29 & GWGIU-AI-XXX-1 & 0.04 & 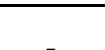 & 0.02 & 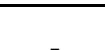 & - & 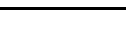 \\
\hline & & 0.04 & - & 0.02 & - & - & - \\
\hline & & 0.005 & & 0.003 & & - & \\
\hline 30 & HALI-CN-C2M-1 & 0.005 & - & 0.003 & - & - & - \\
\hline & & 0.006 & & 0.003 & 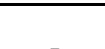 & - & \\
\hline 31 & HALI-LS-L2M-1 & 0.006 & - & 0.003 & - & - & - \\
\hline 22 & & $0.073 \pm 0.005$ & & 0.08 & & - & \\
\hline 32 & HIVIUS-AN-AINIT-1 & $0.052 \pm 0.008$ & 11 & 0.08 & - & - & - \\
\hline 33 & HMUIS-MI_MT1-1 & $0.114 \pm 0.0006$ & 17 & 0.10 & 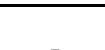 & - & \\
\hline 33 & | HIVIUS-IVIL-IVIIT-1 & $0.110 \pm 0.0006$ & 1.1 & 0.10 & - & - & - \\
\hline 34 & IRIO-AS-AST2_3 & 0.05 & - & 0.03 & 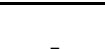 & - & - \\
\hline 34 & IRLU-AS-ASTL-S & 0.05 & - & 0.03 & - & - & - \\
\hline 35 & $1 I_{1} \Omega_{1} \mathrm{~S}_{1} \mathrm{~T}_{2} 3$ & 0.07 & 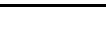 & 0.04 & 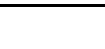 & - & 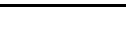 \\
\hline 35 & IRLO-LS-LI 2-3 & 0.07 & - & 0.04 & - & - & - \\
\hline 36 & OCTP_AN_AN2M-1 & 0.02 & - & 0.01 & - & - & - \\
\hline 30 & OCTP-AN-AN2M-1 & 0.02 & - & 0.01 & - & - & - \\
\hline 37 & OCTP_CN-C2M-1 & $0.031 \pm 0.002$ & 55 & 0.01 & 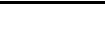 & - & 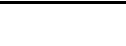 \\
\hline $3 r$ & OCTP-CN-C2M-1 & $0.027 \pm 0.003$ & 5.5 & 0.01 & - & - & - \\
\hline 38 & ОСТP-ML-M2M-1 & $0.027 \pm 0.004$ & $80-8$ & 0.03 & 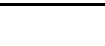 & - & 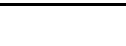 \\
\hline 30 & I & $0.023 \pm 0.003$ & 0.0 & 0.03 & - & - & - \\
\hline & & 0.006 & 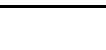 & 0.003 & 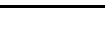 & - & 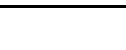 \\
\hline 39 & PCOD-AN-AN2M-1 & 0.006 & - & 0.003 & - & - & - \\
\hline 40 & STRL-AD-XXX-3 & $0.237 \pm 0.004$ & 36 & $0.13 \pm 0.01$ & 67 & $0.16 \pm 0.03$ & 3.0 \\
\hline 40 & SIRL-AD-XXX-3 & $0.255 \pm 0.005$ & 3.6 & $0.15 \pm 0.02$ & 6.1 & $0.17 \pm 0.04$ & 3.0 \\
\hline & & 0.04 & & 0.02 & - & - & - \\
\hline 41 & URCH-AN-ANI 2-1 & 0.04 & - & 0.02 & - & - & - \\
\hline 12 & $\mathrm{HRCH}-\mathrm{CN}-\mathrm{CT} 1-1$ & $0.090 \pm 0.002$ & 122 & $0.065 \pm 0.006$ & 105 & $0.20 \pm 0.04$ & 17 \\
\hline 42 & ORCA-CANCIT-I & $0.070 \pm 0.003$ & 12.2 & $0.053 \pm 0.006$ & 10.5 & $0.20 \pm 0.05$ & 1.1 \\
\hline & & 0.006 & & 0.003 & - & - & - \\
\hline 43 & URCH-CN-CI2-1 & 0.006 & - & 0.003 & - & - & - \\
\hline 44 & $\mathrm{HRCH}-\mathrm{CN}-\mathrm{CT} 3-1$ & $0.033 \pm 0.002$ & 28 & 0.02 & 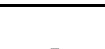 & - & 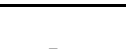 \\
\hline 44 & URCH-CN-CT3-1 & $0.019 \pm 0.005$ & 28 & 0.02 & - & - & - \\
\hline 45 & IRCH_IS-I T1-1 & $0.130 \pm 0.005$ & & 0.03 & & - & - \\
\hline 40 & URCH-LS-LT1-1 & $0.084 \pm 0.003$ & 21.7 & 0.03 & - & - & - \\
\hline 46 & IIRCH-I S-I T3-1 & 0.05 & - & 0.03 & 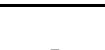 & - & \\
\hline 46 & & 0.05 & - & 0.03 & - & - & - \\
\hline & & $0.102 \pm 0.002$ & & 0.02 & & - & \\
\hline 47 & URCH-ML-MT1-1 & $0.071 \pm 0.003$ & 18 & 0.02 & - & - & - \\
\hline & & $0.149 \pm 0.005$ & & 0.03 & 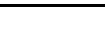 & - & - \\
\hline 48 & URCH-ML-MI 2-1 & $0.126 \pm 0.002$ & 8.2 & 0.03 & - & - & - \\
\hline & & Average CV & $8.0 \%$ & & $2.6 \%$ & & $3.8 \%$ \\
\hline
\end{tabular}


The mean CVs obtained on duplicate measurements of ${ }^{239} \mathrm{Pu},{ }^{240} \mathrm{Pu}$, and ${ }^{240} \mathrm{Pu} /{ }^{239} \mathrm{Pu}$ atom ratios meeting this criterion were 8.0, 2.6, and 3.8 percent, respectively. The mean individual measurement uncertainty for each measure at the $1 \sigma$ level was 3.2, 5.2, and 12 percent, respectively. The duplicate measurements were therefore within acceptable data quality performance criteria based on a 3 sigma rule. Under the reporting criterion given above, the plutonium isotope measurements performed for this sampling event based on ICP-MS appear to satisfy internal laboratory performance requirements for precision, accuracy, and quantification capability. In addition, individual data are provided (Appendix E, taken from Hamilton et al. 2012c) in which quantifiable data were obtained with a reasonable level of precision even if these values fall below the reported MDA. In this example, the duplicate measurements provide some evidence that quantifiable plutonium isotope data falling at or below the measurement MDA could be misleading, as a number of the initial measures showing quantifiable concentrations of the analyte could not be replicated in the subsequent analysis, supporting the use of the reported MDA value in any decision-making rule or assessment. This was especially true of measurements of ${ }^{239} \mathrm{Pu}$ in rockweed, where many reported results fall below the MDA of $0.006 \mathrm{pCi} \mathrm{kg}^{-1}$ wet weight.

\section{F4 Uranium Isotopes}

The minimal detectable signal of the ICP-MS instrument for uranium isotope measurements was defined by the standard deviation $(\sigma 0)$ of 10 reported replicate blank measurements based on a noncentral $t$ distribution function, and assuming the variance of the signal between measurements was approximately constant (using procedures adopted from MARLAP 2004). The MDA defined by units of activity for measurement of ${ }^{234} \mathrm{U},{ }^{235} \mathrm{U},\left({ }^{236} \mathrm{U}\right)$, and ${ }^{238} \mathrm{U}$ by ICP-MS is estimated to be around $0.007,2 \times 10^{-6}, 3 \times 10^{-5}$, and $1 \times 10^{-5} \mathrm{pCi}$, respectively. As a small, sub-aliquot of the sample was used for these measurements, the effective measurement MDA for each of the uranium isotopes is significantly high when accounting for the full mass of sample material as supplied. In this case, the mean effective MDA for measurement of ${ }^{234} \mathrm{U}$, ${ }^{235} \mathrm{U},\left({ }^{236} \mathrm{U}\right)$, and ${ }^{238} \mathrm{U}$ in the suite of Amchitka samples was around 2.5, 0.0009, 0.02, and $0.004 \mathrm{pCi}$, respectively.

The achievable quantifiable limits of uranium detection and measurement using this direct aspiration method was therefore less than what was expected but still appear to have satisfied measurement quality objectives to provide uranium isotopic data with reasonable precision and accuracy. For example, the mean, standard deviation, minimum and maximum ${ }^{235} \mathrm{U} /{ }^{238} \mathrm{U}$ mass ratios observed across the whole sample analysis suite $(\mathrm{N}=310)$ excluding one outlier was $0.0072,0.0001,0.0064$, and 0.0075 , respectively. The expected ${ }^{235} \mathrm{U} /{ }^{238} \mathrm{U}$ mass ratio of naturally occurring uranium in the environment is 0.0072 .

For the purpose of this study, measurement MDAs for individual samples are expressed as activity concentrations (or the MDC) in units of picocuries per kilogram wet weight to account for variances in the sample mass. In Table F6 and Table F7, mass bias and concentration performance test measurements were performed using a CRM traceable to the NIST, NBL CRM-129A, originally purchased from the Isotope Products Laboratories (now Eckert and Ziegler AG). The performance tests were performed at the nanogram total uranium level to mimic expected concentrations in samples. The mass fraction bias for ${ }^{234} \mathrm{U},{ }^{235} \mathrm{U}$, and ${ }^{238} \mathrm{U}$ was $2.1,0.7$, and $-0.01 \%$, respectively, or well within the expected tolerance limits for environmental measurements. 
Table F7 shows replicate analyses of the reference material and also shows that the accuracy and precision of the ICP-MS measurements satisfied laboratory performance measures with the mean measurement bias for ${ }^{234} \mathrm{U},{ }^{235} \mathrm{U}$, and ${ }^{238} \mathrm{U}$ falling within $3 \sigma$ of the individual measurement uncertainty.

Table F6. Mass Bias Performance Test Measurement of Uranium Isotopes in Certified Reference Material CRM129A Based on ICP-MS Detection

\begin{tabular}{||c|c|c|c||}
\hline $\begin{array}{c}\text { Mass Fraction } \\
(\mathbf{x} 100)\end{array}$ & Reference Value & Measured Value & Bias \\
\hline${ }^{234} U$ & $0.005208 \pm 0.000004$ & $0.00532 \pm 0.00005$ & $2.1 \%$ \\
\hline${ }^{235} U$ & $0.71183 \pm 0.0004$ & $0.717 \pm 0.0055$ & $0.7 \%$ \\
\hline${ }^{236} U$ & $0.0000096 \pm 0.0000012$ & $<1 \times 10^{-4}$ & - \\
\hline${ }^{238} U$ & $99.2830 \pm 0.0004$ & $99.3 \pm 0.92$ & $-0.01 \%$ \\
\hline
\end{tabular}

Table F7. Concentration Performance Test Measurements of Uranium Isotopes in Certified Reference Material CRM 129A Based on ICP-MS Detection

\begin{tabular}{||c|c|c|c|c|c|c||}
\hline Sample & ${ }^{\mathbf{2 3 4}} \mathbf{U}, \mathbf{n g}$ & $\mathbf{B i a s}$ & ${ }^{\mathbf{2 3 5} \mathbf{U}, \mathbf{n g}}$ & $\mathbf{B i a s}$ & ${ }^{\mathbf{2 3 8} \mathbf{U}, \mathbf{n g}}$ & Bias \\
\hline CRM129A \#1 & $0.059 \pm 0.002$ & $3.5 \%$ & $7.99 \pm 0.06$ & $2.6 \%$ & $1107 \pm 5$ & $1.9 \%$ \\
\hline CRM129A \#2 & $0.0595 \pm 0.0016$ & $4.4 \%$ & $7.99 \pm 0.06$ & $2.6 \%$ & $1115 \pm 7$ & $2.6 \%$ \\
\hline CRM129A \#3 & $0.057 \pm 0.002$ & $3.5 \%$ & $7.96 \pm 0.08$ & $2.2 \%$ & $1109 \pm 9$ & $2.1 \%$ \\
\hline CRM129A \#4 & $0.06 \pm 0.002$ & $5.3 \%$ & $7.96 \pm 0.07$ & $2.2 \%$ & $1102 \pm 8$ & $1.4 \%$ \\
\hline CRM129A \#5 & $0.0585 \pm 0.0012$ & $2.7 \%$ & $7.98 \pm 0.08$ & $2.4 \%$ & $1108 \pm 5$ & $2.0 \%$ \\
\hline CRM129A \#6 & $0.059 \pm 0.002$ & $3.5 \%$ & $7.95 \pm 0.05$ & $2.1 \%$ & $1098 \pm 6$ & $1.1 \%$ \\
\hline CRM129A \#7 & $0.058 \pm 0.002$ & $1.8 \%$ & $7.98 \pm 0.05$ & $2.4 \%$ & $1101 \pm 5$ & $1.3 \%$ \\
\hline CRM129A \#8 & $0.0595 \pm 0.0018$ & $4.4 \%$ & $7.97 \pm 0.06$ & $2.3 \%$ & $1099 \pm 5$ & $1.2 \%$ \\
\hline CRM129A \#9 & $0.0609 \pm 0.0008$ & $6.9 \%$ & $7.95 \pm 0.07$ & $2.1 \%$ & $1095 \pm 8$ & $0.8 \%$ \\
\hline CRM129A \#10 & $0.0589 \pm 0.0014$ & $3.4 \%$ & $7.88 \pm 0.05$ & $2.3 \%$ & $1091 \pm 3$ & $0.4 \%$ \\
\hline \hline Measured Mean Value & $\mathbf{0 . 0 5 9 0 \pm \mathbf { 0 . 0 0 1 1 }}$ & $\mathbf{3 . 6 \%}$ & $\mathbf{7 . 9 6} \pm \mathbf{0 . 0 3 2}$ & $\mathbf{2 . 2 \%}$ & $\mathbf{1 1 0 3 \pm \mathbf { 7 }}$ & $\mathbf{1 . 5 \%}$ \\
\hline Reference Value & $\mathbf{0 . 0 5 7 0}$ & & $\mathbf{7 . 7 8 9}$ & & $\mathbf{1 0 8 6 . 4}$ & \\
\hline
\end{tabular}

A series of Amchitka samples were analyzed in duplicate $(\mathrm{N}=26)$ on separate ICP-MS runs to assess the effects of run variability on the precision and accuracy of the uranium isotopic measurements. F8shows the results. The relative difference in the two duplicate measurements can be expressed as a coefficient of variation (CV). The mean CVs obtained for duplicate measurements of ${ }^{234} \mathrm{U},{ }^{235} \mathrm{U}$, and ${ }^{238} \mathrm{U}$ were 4.1 percent, 2.0 percent, and 1.6 percent, respectively. The CV values closely mirror typical individual sample measurement precision for the each of the uranium isotopes, and are therefore within acceptable laboratory quality performance criteria based on a $3 \sigma$ rule. The highest level of variability was observed for ${ }^{234} \mathrm{U}$ measurements (mean 4.1 percent, range $0-10.5$ percent). Moreover, there is some evidence for a positive bias for ${ }^{234} \mathrm{U}$ measurements because ${ }^{234} \mathrm{U} /{ }^{238} \mathrm{U}$ activity ratios (not reported) on samples of marine origin consistently fall above the characteristic ratio of 1.14 observed in seawater. 
Table F8. Replicate Measurements of Uranium Isotopes Based on ICP-MS

\begin{tabular}{|c|c|c|c|c|c|c|c|}
\hline \multirow{2}{*}{ No. } & \multirow{2}{*}{ Ticket Number } & \multicolumn{2}{|c|}{${ }^{238} \mathrm{U}, \mathrm{pCi} \mathrm{kg}{ }^{-1}$ Wet } & \multicolumn{2}{|c|}{${ }^{235} \mathrm{U}, \mathrm{pCi} \mathrm{kg}{ }^{-1}$ Wet } & \multicolumn{2}{|c|}{${ }^{234} \mathrm{U}, \mathrm{pCi} \mathrm{kg}^{-1}$ Wet } \\
\hline & & Value & $\% \mathrm{CV}$ & Value & $\% \mathrm{CV}$ & Value & $\% \mathrm{CV}$ \\
\hline \multirow{2}{*}{1} & \multirow{2}{*}{ FUCU-ML-MT1-1 } & $79.9 \pm 0.2$ & \multirow{2}{*}{2.4} & $3.38 \pm 0.01$ & \multirow{2}{*}{3.7} & $96.7 \pm 1.8$ & \multirow{2}{*}{2.4} \\
\hline & & $76.1 \pm 0.5$ & & $3.14 \pm 0.04$ & & $92.2 \pm 2.7$ & \\
\hline \multirow{2}{*}{2} & \multirow{2}{*}{ FUCU-AS-DUP } & $95.2 \pm 0.2$ & \multirow{2}{*}{0.3} & $4.01 \pm 0.02$ & \multirow{2}{*}{0.0} & $115 \pm 2$ & \multirow{2}{*}{0.7} \\
\hline & & $95.7 \pm 0.5$ & & $4.0 \pm 0.1$ & & $116 \pm 3$ & \\
\hline \multirow{2}{*}{3} & \multirow{2}{*}{ HMUS-CN-CT1-1 } & $20.2 \pm 0.1$ & \multirow{2}{*}{0.1} & $0.850 \pm 0.0007$ & 03 & $23.4 \pm 1.2$ & 10 \\
\hline & & $20.2 \pm 0.1$ & & $0.856 \pm 0.006$ & 0.3 & $24.3 \pm 1.2$ & 1.9 \\
\hline 1 & HMUSCNOT21 1 & $41.9 \pm 0.3$ & 00 & $1.78 \pm 0.02$ & 08 & $52.9 \pm 2.1$ & 26 \\
\hline 4 & HIVUS-CN-CT3-1 & $41.2 \pm 0.2$ & 0.9 & $1.75 \pm 0.02$ & 0.8 & $50.2 \pm 1.1$ & 2.6 \\
\hline 5 & HPCHCNCT1 1 & $16.3 \pm 0.1$ & 05 & $0.70 \pm 0.01$ & 00 & $24.6 \pm 3.5$ & 21 \\
\hline 5 & ORCA-CLN-CIT-T & $16.4 \pm 0.2$ & 0.5 & $0.70 \pm 0.04$ & 0.0 & $23.4 \pm 2.3$ & 2.4 \\
\hline 6 & & $3.3 \pm 0.1$ & & $0.143 \pm 0.003$ & & $4.8 \pm 0.6$ & \\
\hline 6 & ROCK-CN-CT1-2 & $3.31 \pm 0.02$ & 0.3 & $0.139 \pm 0.004$ & 1.6 & $4.6 \pm 0.6$ & 2.7 \\
\hline & & $5.7 \pm 0.1$ & & $0.247 \pm 0.004$ & & $7.2 \pm 0.8$ & \\
\hline$r$ & GREN-CN-C T1-2 & $5.1 \pm 0.1$ & 5.4 & $0.216 \pm 0.005$ & 6.1 & $7.2 \pm 0.8$ & 0.1 \\
\hline 8 & $|R| O-I S-I T 1-3$ & $4.20 \pm 0.01$ & 01 & $0.179 \pm 0.003$ & 01 & $5.3 \pm 0.8$ & 16 \\
\hline 8 & IRLU-LS-LT I-3 & $4.21 \pm 0.03$ & 0.1 & $0.178 \pm 0.002$ & 0.1 & $5.8 \pm 0.9$ & 4.6 \\
\hline$a$ & FILAL $\mathrm{AL}$ MTT2 3 & $18.09 \pm 0.04$ & $12+2+3$ & $0.770 \pm 0.01$ & 01 & $21.9 \pm 0.6$ & 00 \\
\hline 9 & EUAL-IVIL-IVIIL-3 & $18.5 \pm 0.2$ & 1.2 & $0.78 \pm 0.04$ & 0.4 & $21.8 \pm 1.1$ & 0.0 \\
\hline 10 & HMUIS-MI_MT2-1 & $34.5 \pm 0.1$ & 0 & $1.46 \pm 0.01$ & 05 & $40.1 \pm 0.8$ & 36 \\
\hline 10 & HIVIUS-IVIL-IVIIL-1 & $34.5 \pm 0.4$ & 0.0 & $1.44 \pm 0.02$ & 0.5 & $43.1 \pm 3.3$ & 3.6 \\
\hline 11 & ROCK ML AMT1 3 & $7.96 \pm 0.04$ & 07 & $0.340 \pm 0.003$ & 07 & $10.2 \pm 1.0$ & 2 \\
\hline II & RUCK-IVIL-IVIII-3 & $8.1 \pm 0.1$ & 0.7 & $0.345 \pm 0.006$ & 0.7 & $9.4 \pm 1.4$ & 4.2 \\
\hline & & $8.4 \pm 0.1$ & 31 & $0.364 \pm 0.006$ & 45 & $11.4 \pm 1.8$ & \\
\hline 12 & GRENN-MLL-MII J-1 & $7.9 \pm 0.1$ & 3.1 & $0.333 \pm 0.007$ & 4.5 & $10.3 \pm 0.8$ & 5.1 \\
\hline 13 & $P C O \cap-\triangle N_{-}-\triangle N 2 M-1$ & $1.18 \pm 0.01$ & 03 & $0.050 \pm 0.001$ & 01 & $1.9 \pm 0.5$ & 107 \\
\hline 13 & PCUD-AIN-AINZIVI-I & $1.19 \pm 0.02$ & 0.3 & $0.051 \pm 0.001$ & 0.1 & $1.5 \pm 0.6$ & 10.1 \\
\hline 14 & FIAI $1 \mathrm{C}^{-1} \mathrm{CT} 1 \mathrm{2}$ & $15.1 \pm 0.1$ & & $0.638 \pm 0.005$ & 01 & $17.8 \pm 1.2$ & 12 \\
\hline 14 & EUAL-AS-AST1-2 & $15.1 \pm 0.1$ & 0.0 & $0.643 \pm 0.007$ & 0.4 & $19.4 \pm 0.7$ & 4.2 \\
\hline 15 & FUAL_AS_AST2-1 & $13.1 \pm 0.2$ & 00 & $0.56 \pm 0.01$ & 04 & $15.3 \pm 1.0$ & 32 \\
\hline 15 & EUAL-AS-ASIL-1 & $13.1 \pm 0.1$ & 0.0 & $0.557 \pm 0.008$ & 0.4 & $16.3 \pm 0.7$ & 3.2 \\
\hline 16 & FUAI-AS-AST3-1 & $20.89 \pm 0.03$ & 02 & $0.882 \pm 0.006$ & 0.5 & $27.0 \pm 1.2$ & 24 \\
\hline 10 & EUAL-AS-AST3-1 & $21.0 \pm 0.1$ & 0.2 & $0.89 \pm 0.01$ & 0.5 & $25.7 \pm 1.1$ & 2.4 \\
\hline 17 & CHIT_AS_AST2-1 & $3.60 \pm 0.02$ & 07 & $0.153 \pm 0.002$ & 13 & $5.1 \pm 1.9$ & 40 \\
\hline 11 & CHII-AS-ASI 2-1 & $3.7 \pm 0.1$ & 0.1 & $0.156 \pm 0.005$ & 1.3 & $4.7 \pm 1.4$ & 4.0 \\
\hline 18 & URCH-AS-AST2-1 & $19.3 \pm 0.2$ & 08 & $0.83 \pm 0.01$ & 02 & $26.0 \pm 2.0$ & 35 \\
\hline 18 & URCA-AS-AS IL-1 & $19.6 \pm 0.2$ & 0.8 & $0.83 \pm 0.01$ & 0.2 & $24.2 \pm 2.3$ & 3.5 \\
\hline & & $2.91 \pm 0.02$ & & $0.123 \pm 0.002$ & & $3.9 \pm 0.6$ & \\
\hline 19 & GREN-AS-AST1-2 & $2.92 \pm 0.01$ & 0.2 & $0.125 \pm 0.001$ & 0.7 & $3.1 \pm 0.7$ & 10.5 \\
\hline 20 & $\mathrm{GRFN}_{-} \mathrm{S}_{-} \Delta \mathrm{ST}_{3}{ }_{1}$ & $2.15 \pm 0.04$ & 05 & $0.093 \pm 0.005$ & 11 & $2.9 \pm 0.7$ & 18 \\
\hline 20 & GREN-AS-ASI3-1 & $2.13 \pm 0.04$ & 0.5 & $0.090 \pm 0.002$ & 1.4 & $2.6 \pm 0.5$ & 4.8 \\
\hline 21 & & $8.8 \pm 0.1$ & 76 & $0.377 \pm 0.002$ & $8325-35$ & $12.3 \pm 1.9$ & 101 \\
\hline 21 & IRLO-CN-CT2-2 & $7.6 \pm 0.1$ & 7.6 & $0.319 \pm 0.009$ & 8.3 & $10.0 \pm 1.5$ & 10.1 \\
\hline & ROCK-I S-I T3-1 & $13.64 \pm 0.03$ & 01 & $0.574 \pm 0.002$ & 07 & $17.2 \pm 1.2$ & \\
\hline 22 & ROCK-LS-LI3-1 & $13.66 \pm 0.03$ & 0.1 & $0.582 \pm 0.005$ & 0.1 & $17.8 \pm 0.6$ & 1.1 \\
\hline 23 & ROCK-MI-DUP & $14.2 \pm 0.1$ & 129 & $0.598 \pm 0.007$ & 133 & $19.5 \pm 1.0$ & 66 \\
\hline 23 & RUCK-IVIL-EUP & $18.4 \pm 0.1$ & 12.9 & $0.781 \pm 0.007$ & 13.3 & $22.2 \pm 1.7$ & 6.6 \\
\hline & & $8.16 \pm 0.03$ & & $0.348 \pm 0.003$ & & $10.2 \pm 0.6$ & \\
\hline 24 & IRLO-ML-MT3-1 & $8.13 \pm 0.03$ & 0.2 & $0.347 \pm 0.005$ & 0.2 & $10.8 \pm 1.2$ & 3.1 \\
\hline 25 & $I R I O-M I-M T 3-3$ & $6.46 \pm 0.04$ & 3 & $0.281 \pm 0.003$ & 1 & $8.2 \pm 0.8$ & 34 \\
\hline 25 & IRLU-IVL-IVII $3-3$ & $6.05 \pm 0.03$ & 3.3 & $0.257 \pm 0.004$ & 4.4 & $7.7 \pm 0.9$ & 3.4 \\
\hline 26 & IRI O-AN-ANT2-3 & $10.31 \pm 0.04$ & 0 & $0.434 \pm 0.005$ & 0 & $12.3 \pm 1.2$ & 71 \\
\hline 26 & $|R L U-A N N-A| N \mid Z-3$ & $10.3 \pm 0.1$ & 0.0 & $0.435 \pm 0.004$ & 0.0 & $14.2 \pm 1.1$ & 1.1 \\
\hline & & Average \% CV & $1.6 \%$ & & $2.0 \%$ & & $4.1 \%$ \\
\hline
\end{tabular}




\section{F5 Tritium in Seawater}

At least once weekly each counter counts dead hydrogen gas (from petroleum). In addition, water from the deep Floridan aquifer (more than 10,000 year old water) is reduced to occasionally check on the tank hydrogen gas. This procedure sets the background count of the counting equipment. A further check on process blanks is that at least once a week a sample of dead water (from the Floridan aquifer) goes through all the same procedures, including enrichment, as the unknown samples. In order to check on the efficiency of the enrichment procedure, at least once a week a sample of known activity is processed through the entire system of enrichment, reduction, and counting. The efficiency of each counter is determined by counting hydrogen gas made by reduction of standard water in the regular preparation system. This standard water is prepared from NIST Standard Reference Material 4926 by dilution by weighing. The dependence of factors such as background and efficiency on parameters such as pressure, gas composition, and gas amplification is known, and the appropriate corrections are applied by the software of the computing system.

Periodically, usually about every 6 weeks, all measurements in all counters for the preceding time period are recomputed, applying statistical tests, and scrutinized for flaws in quality. Recomputation includes all measurements of unknowns, backgrounds, blanks, enrichment factors, and efficiencies. Only after this step is the result considered final. The results are then reported in Data Releases, one for each project or job.

Water samples for tritium analysis are received and inventoried using the chain of custody form supplied by the client. A computer worksheet listing sample name, volume, sample collection date, and date of arrival into lab, as well as client information, is generated. At this time, each order is given a unique job number, and each sample decimal numbered within that job. For example, the job-sample number (JB number) 123.05 indicates the fifth sample in the listing for job 123. The computer input is proofread, and the worksheet and labels are printed. An abbreviated copy of the worksheet listing is given to administrative personnel to be filed with the client's records. The preparation technician uses the worksheet to keep track of the progress of the samples. Preliminary results are recorded on this sheet as they become available through the computer. From the time the worksheet is printed, the sample is referred to by its JB number. Triple copy labels are attached to each sample bottle.

When processing begins, one of the labels bearing the JB number is attached to the processing vessel; the label then "follows" the sample through the preparation steps; i.e., it is physically transferred to the next process container. The same label is used from the beginning distillation through electrolytic enrichment, vacuum distillation, and reduction to hydrogen gas and is eventually attached to the face of the pressure gauge of the counter in which the sample is counted. During each step of sample preparation, a yellow preparation card reports the distillation date and unit number; electrolysis starting date; volume and cell number; and freezeout date and unit number. Recorded also are the order number through the reduction system and the cylinder number used to store the gas sample prior to counting. 


\section{Appendix G}

\section{Activity Concentration of ${ }^{137} \mathrm{Cs}$}


This page intentionally left blank 


\begin{tabular}{|c|c|c|c|c|c|c|c|c|c|c|c|}
\hline \multirow{5}{*}{ 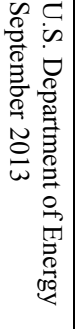 } & Matrix Subtype & Location Code & Matrix & $\begin{array}{c}\text { Date } \\
\text { Sampled }\end{array}$ & $\begin{array}{c}\text { Wet Result } \pm \\
\text { Uncertainty }\end{array}$ & $\begin{array}{c}\text { Wet } \\
\text { Detection } \\
\text { Limit }\end{array}$ & $\begin{array}{l}\text { Dry Result } \pm \\
\text { Uncertainty }\end{array}$ & $\begin{array}{c}\text { Dry } \\
\text { Detection } \\
\text { Limit }\end{array}$ & Qualifiers & Latitude & Longitude \\
\hline & \multicolumn{11}{|c|}{${ }^{137} \mathrm{Cs}(\mathrm{pCi} / \mathrm{kg})$} \\
\hline & \multicolumn{11}{|c|}{ Amchitka Site (AMC01) } \\
\hline & $\begin{array}{l}\text { Dolly Varden - } \\
\text { whole }\end{array}$ & DOLL-AI-XXX-1 & FAUNA & 6/24/2011 & $98 \pm 8$ & 1 & $377.705 \pm 30.833$ & 3.854 & $\mathrm{~J}$ & 51.468283 & 179.108052 \\
\hline & $\begin{array}{c}\text { Goose Egg - } \\
\text { no shell }\end{array}$ & GoOS-AI-XXX-1 & FAUNA & 6/24/2011 & $12 \pm 2$ & 2 & $31.873 \pm 5.312$ & 5.312 & & 51.491305 & 179.0595 \\
\hline & $\begin{array}{c}\text { Glaucous-winged } \\
\text { Gull Egg- } \\
\text { no shell }\end{array}$ & GWGU-AI-XXX-1 & FAUNA & 6/24/2011 & $4.2 \pm 1$ & 1.5 & $14.556 \pm 3.466$ & 5.199 & $\mathrm{~J}$ & 51.456388 & 179.167 \\
\hline & \multicolumn{11}{|c|}{ Amchitka Site (Long Shot) (AMC02) } \\
\hline & \multirow{3}{*}{ Chiton } & CHIT-LS-LT1-1 & FAUNA & $7 / 4 / 2011$ & $-0.04 \pm 0.4$ & 1.6 & $-0.198 \pm 1.984$ & 7.936 & $\mathrm{U}$ & 51.457555 & 179.195138 \\
\hline & & CHIT-LS-LT2-1 & FAUNA & $7 / 3 / 2011$ & $1.6 \pm 0.4$ & 1.2 & $7.495 \pm 1.874$ & 5.621 & $\mathrm{~J}$ & 51.44925 & 179.205583 \\
\hline & & CHIT-LS-LT3-1 & FAUNA & $7 / 3 / 2011$ & $0.002 \pm 0.6$ & 1.7 & $0.01 \pm 2.857$ & 8.094 & $U$ & 51.43 & 179.22667 \\
\hline & \multirow{10}{*}{ Dragon Kelp } & EUAL-LS-DUP & VEG & $7 / 3 / 2011$ & $1.4 \pm 0.2$ & 0.3 & $8.408 \pm 1.201$ & 1.802 & & 51.44925 & 179.205583 \\
\hline & & EUAL-LS-LT1-1 & VEG & $7 / 4 / 2011$ & $0.5 \pm 0.2$ & 0.4 & $4.672 \pm 1.869$ & 3.738 & $\mathrm{~J}$ & 51.457555 & 179.195138 \\
\hline & & EUAL-LS-LT1-2 & VEG & $7 / 4 / 2011$ & $2.2 \pm 0.4$ & 0.5 & $13.677 \pm 2.487$ & 3.108 & & 51.457555 & 179.195138 \\
\hline 气. & & EUAL-LS-LT1-3 & VEG & $7 / 4 / 2011$ & $0.7 \pm 0.2$ & 0.4 & $4.226 \pm 1.207$ & 2.415 & $\mathrm{~J}$ & 51.457555 & 179.195138 \\
\hline $\overrightarrow{\vec{t}}$ & & EUAL-LS-LT2-1 & VEG & $7 / 3 / 2011$ & $0.5 \pm 0.1$ & 0.2 & $3.024 \pm 0.605$ & 1.21 & $\mathrm{~J}$ & 51.44925 & 179.205583 \\
\hline$\frac{\bar{n}}{2}$ & & EUAL-LS-LT2-2 & VEG & $7 / 3 / 2011$ & $1.3 \pm 0.2$ & 0.4 & $9.438 \pm 1.452$ & 2.904 & & 51.44925 & 179.205583 \\
\hline 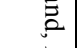 & & EUAL-LS-LT2-3 & VEG & $7 / 3 / 2011$ & $0.24 \pm 0.06$ & 0.1 & $1.736 \pm 0.434$ & 0.723 & $\mathrm{~J}$ & 51.44925 & 179.205583 \\
\hline$\vec{D}$ & & EUAL-LS-LT3-1 & VEG & $7 / 2 / 2011$ & $2.2 \pm 0.4$ & 0.4 & $14.041 \pm 2.553$ & 2.553 & & 51.43 & 179.22667 \\
\hline 茛 & & EUAL-LS-LT3-2 & VEG & $7 / 2 / 2011$ & $3 \pm 0.6$ & 0.7 & $21.347 \pm 4.269$ & 4.981 & & 51.43 & 179.22667 \\
\hline . & & EUAL-LS-LT3-3 & VEG & $7 / 2 / 2011$ & $1.2 \pm 0.2$ & 0.3 & $10.122 \pm 1.687$ & 2.53 & & 51.43 & 179.22667 \\
\hline 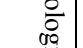 & \multirow{10}{*}{ Rockweed } & FUCU-LS-DUP & VEG & $6 / 22 / 2011$ & $3.6 \pm 0.4$ & 0.3 & $19.784 \pm 2.198$ & 1.649 & $\mathrm{~J}$ & 51.447472 & 179.190888 \\
\hline . & & FUCU-LS-LT1-1 & VEG & $6 / 22 / 2011$ & $2.2 \pm 0.4$ & 0.4 & $11.885 \pm 2.161$ & 2.161 & $\mathrm{~J}$ & 51.447527 & 179.189361 \\
\hline 3 & & FUCU-LS-LT1-2 & VEG & $6 / 22 / 2011$ & $3.2 \pm 0.4$ & 0.3 & $19.575 \pm 2.447$ & 1.835 & $\mathrm{~J}$ & 51.447527 & 179.189361 \\
\hline . & & FUCU-LS-LT1-3 & VEG & $6 / 22 / 2011$ & $2.9 \pm 0.4$ & 0.3 & $19.282 \pm 2.66$ & 1.995 & $\mathrm{~J}$ & 51.448777 & 179.189305 \\
\hline 惫. & & FUCU-LS-LT2-1 & VEG & $6 / 22 / 2011$ & $5.2 \pm 0.6$ & 0.3 & $41 \pm 4.731$ & 2.365 & $\mathrm{~J}$ & 51.447222 & 179.199361 \\
\hline$\stackrel{0}{\pi}$ & & FUCU-LS-LT2-2 & VEG & $6 / 22 / 2011$ & $3.4 \pm 0.4$ & 0.2 & $24.644 \pm 2.899$ & 1.45 & $\mathrm{~J}$ & 51.44625 & 179.196972 \\
\hline 要 & & FUCU-LS-LT2-3 & VEG & $6 / 22 / 2011$ & $4.2 \pm 0.4$ & 0.3 & $25.686 \pm 2.446$ & 1.835 & $\mathrm{~J}$ & 51.446722 & 179.191888 \\
\hline $\begin{array}{l}7 \\
n \\
n\end{array}$ & & FUCU-LS-LT3-1 & VEG & $7 / 2 / 2011$ & $3.7 \pm 0.4$ & 0.3 & $17.922 \pm 1.938$ & 1.453 & $\mathrm{~J}$ & 51.433611 & 179.219305 \\
\hline 吕 & & FUCU-LS-LT3-2 & VEG & $7 / 2 / 2011$ & $5.8 \pm 0.6$ & 0.2 & $26.393 \pm 2.73$ & 0.91 & $\mathrm{~J}$ & 51.430166 & 179.218694 \\
\hline$\forall \underset{:}{\mathscr{O}}$ & & FUCU-LS-LT3-3 & VEG & $7 / 2 / 2011$ & $4.2 \pm 0.6$ & 0.4 & $15.39 \pm 2.199$ & 1.466 & $\mathrm{~J}$ & 51.430166 & 179.218694 \\
\hline
\end{tabular}




\begin{tabular}{|c|c|c|c|c|c|c|c|c|c|c|}
\hline Matrix Subtype & Location Code & Matrix & $\begin{array}{c}\text { Date } \\
\text { Sampled }\end{array}$ & $\begin{array}{l}\text { Wet Result } \pm \\
\text { Uncertainty }\end{array}$ & $\begin{array}{c}\text { Wet } \\
\text { Detection } \\
\text { Limit }\end{array}$ & $\begin{array}{l}\text { Dry Result } \pm \\
\text { Uncertainty }\end{array}$ & $\begin{array}{c}\text { Dry } \\
\text { Detection } \\
\text { Limit }\end{array}$ & Qualifiers & Latitude & Longitude \\
\hline \multirow{10}{*}{ Greenling - whole } & GREN-LS-DUP & FAUNA & $7 / 12 / 2011$ & $3.7 \pm 1$ & 2.2 & $17.967 \pm 4.856$ & 10.683 & $\mathrm{~J}$ & 51.434527 & 179.234416 \\
\hline & GREN-LS-LT1-1 & FAUNA & $7 / 4 / 2011$ & $2.5 \pm 0.8$ & 2.1 & $11.784 \pm 3.771$ & 9.898 & $\mathrm{~J}$ & 51.457555 & 179.195138 \\
\hline & GREN-LS-LT1-2 & FAUNA & $7 / 4 / 2011$ & $3.3 \pm 1$ & 2.8 & $15.621 \pm 4.734$ & 13.254 & $\mathrm{~J}$ & 51.457555 & 179.195138 \\
\hline & GREN-LS-LT1-3 & FAUNA & $7 / 5 / 2011$ & $1.9 \pm 0.6$ & 1.7 & $8.898 \pm 2.81$ & 7.961 & $\mathrm{~J}$ & 51.457555 & 179.195138 \\
\hline & GREN-LS-LT2-1 & FAUNA & $7 / 3 / 2011$ & $2 \pm 0.8$ & 2.1 & $9.664 \pm 3.866$ & 10.147 & $U$ & 51.432194 & 179.230277 \\
\hline & GREN-LS-LT2-2 & FAUNA & $7 / 3 / 2011$ & $4.4 \pm 1$ & 2.2 & $20.292 \pm 4.612$ & 10.146 & $\mathrm{~J}$ & 51.432194 & 179.230277 \\
\hline & GREN-LS-LT2-3 & FAUNA & $7 / 3 / 2011$ & $2.4 \pm 0.8$ & 1.8 & $11.42 \pm 3.807$ & 8.565 & $\mathrm{~J}$ & 51.432194 & 179.230277 \\
\hline & GREN-LS-LT3-1 & FAUNA & $7 / 3 / 2011$ & $4.5 \pm 0.8$ & 1.5 & $19.975 \pm 3.551$ & 6.658 & & 51.43 & 179.22667 \\
\hline & GREN-LS-LT3-2 & \begin{tabular}{|l|} 
FAUNA \\
\end{tabular} & 7/12/2011 & $5.6 \pm 1$ & 2.2 & $27.472 \pm 4.906$ & 10.793 & $\mathrm{~J}$ & 51.434527 & 179.234416 \\
\hline & GREN-LS-LT3-3 & FAUNA & 7/12/2011 & $2.3 \pm 0.8$ & 1.9 & $11.121 \pm 3.868$ & 9.187 & $\mathrm{~J}$ & 51.434527 & 179.234416 \\
\hline Halibut - whole & HALI-LS-L2M-1 & FAUNA & $7 / 13 / 2011$ & $1 \pm 0.2$ & 0.1 & $3.839 \pm 0.768$ & 0.384 & & 51.40025 & 179.283361 \\
\hline \multirow{3}{*}{$\begin{array}{l}\text { Horse Mussel - } \\
\text { soft tissue only }\end{array}$} & HMUS-LS-LT1-1 & FAUNA & $7 / 5 / 2011$ & $2.2 \pm 1.2$ & 3.7 & $9.425 \pm 5.141$ & 15.851 & $U$ & 51.457555 & 179.195138 \\
\hline & HMUS-LS-LT2-1 & FAUNA & $7 / 4 / 2011$ & $1.4 \pm 0.8$ & 2.6 & $5.719 \pm 3.268$ & 10.622 & $U$ & 51.44925 & 179.205583 \\
\hline & HMUS-LS-LT3-1 & FAUNA & $7 / 3 / 2011$ & $44 \pm 22$ & 60 & $92.276 \pm 46.138$ & 125.831 & $U$ & 51.44925 & 179.205583 \\
\hline \multirow{9}{*}{ Irish Lord - whole } & IRLO-LS-LT1-1 & FAUNA & 7/4/2011 & $2.7 \pm 0.8$ & 1.8 & $12.733 \pm 3.773$ & 8.489 & $\bar{J}$ & 51.457555 & 179.195138 \\
\hline & IRLO-LS-LT1-2 & FAUNA & $7 / 4 / 2011$ & $2 \pm 0.8$ & 2.2 & $9.462 \pm 3.785$ & 10.408 & $U$ & 51.457555 & 179.195138 \\
\hline & IRLO-LS-LT1-3 & \begin{tabular}{|l|} 
FAUNA \\
\end{tabular} & $7 / 5 / 2011$ & $2.5 \pm 0.8$ & 2.3 & $13.257 \pm 4.242$ & 12.196 & $\mathrm{~J}$ & 51.457555 & 179.195138 \\
\hline & IRLO-LS-LT2-1 & FAUNA & $7 / 3 / 2011$ & $6.3 \pm 1.2$ & 2.5 & $27.266 \pm 5.193$ & 10.82 & $\bar{J}$ & 51.432194 & 179.230277 \\
\hline & IRLO-LS-LT2-2 & FAUNA & $7 / 3 / 2011$ & $1.7 \pm 0.6$ & 1.4 & $7.043 \pm 2.486$ & 5.8 & $\mathrm{~J}$ & 51.432194 & 179.230277 \\
\hline & IRLO-LS-LT2-3 & FAUNA & $7 / 3 / 2011$ & $3.3 \pm 1.2$ & 3.5 & $14.619 \pm 5.316$ & 15.505 & $\mathrm{~J}$ & 51.432194 & 179.230277 \\
\hline & IRLO-LS-LT3-1 & FAUNA & 7/2/2011 & $0.7 \pm 0.2$ & 0.8 & $3.122 \pm 0.892$ & 3.568 & $U$ & 51.43 & 179.22667 \\
\hline & IRLO-LS-LT3-2 & \begin{tabular}{|l|} 
FAUNA \\
\end{tabular} & $7 / 12 / 2011$ & $1.7 \pm 0.8$ & 1.9 & $8.065 \pm 3.795$ & 9.014 & $U$ & 51.434944 & 179.2315 \\
\hline & IRLO-LS-LT3-3 & FAUNA & $7 / 12 / 2011$ & $2.3 \pm 0.8$ & 1.8 & $12.707 \pm 4.42$ & 9.945 & $\bar{J}$ & 51.434944 & 179.2315 \\
\hline Octopus & OCTP-LS-L2M-1 & FAUNA & $7 / 3 / 2011$ & $0.23 \pm 0.06$ & 0.2 & $1.639 \pm 0.428$ & 1.425 & $\mathrm{~J}$ & 51.44925 & 179.205583 \\
\hline $\begin{array}{l}\text { Pacific Cod - } \\
\text { whole }\end{array}$ & PCOD-LS-L2M-1 & FAUNA & $7 / 4 / 2011$ & $2.1 \pm 0.2$ & 0.2 & .995 & 0.995 & & 51.451805 & 179.196972 \\
\hline \multirow{10}{*}{ Rockfish - whole } & ROCK-LS-DUP & \begin{tabular}{|l|} 
FAUNA \\
\end{tabular} & $7 / 12 / 2011$ & $2.4 \pm 0.8$ & 1.8 & $9.359 \pm 3.12$ & 7.019 & $\mathrm{~J}$ & 51.434944 & 179.2315 \\
\hline & ROCK-LS-DUP2 & FAUNA & 7/12/2011 & $1.3 \pm 0.4$ & 0.7 & $5.563 \pm 1.712$ & 2.995 & $\mathrm{~J}$ & 51.465472 & 179.206722 \\
\hline & ROCK-LS-LT1-1 & \begin{tabular}{|l|} 
FAUNA \\
\end{tabular} & $7 / 4 / 2011$ & $2 \pm 0.8$ & 2.2 & $9.288 \pm 3.715$ & 10.216 & $U$ & 51.457555 & 179.195138 \\
\hline & ROCK-LS-LT1-2 & FAUNA & $7 / 12 / 2011$ & $3.4 \pm 0.6$ & 1.3 & $14.045 \pm 2.478$ & 5.37 & $\mathrm{~J}$ & 51.465472 & 179.206722 \\
\hline & ROCK-LS-LT1-3 & FAUNA & $7 / 12 / 2011$ & $4.1 \pm 0.8$ & 1.4 & $16.921 \pm 3.302$ & 5.778 & $\mathrm{~J}$ & 51.465472 & 179.206722 \\
\hline & ROCK-LS-LT2-1 & FAUNA & $7 / 4 / 2011$ & $4.8 \pm 0.8$ & 1.5 & $19.251 \pm 3.209$ & 6.016 & & 51.44925 & 179.205583 \\
\hline & ROCK-LS-LT2-2 & FAUNA & $7 / 12 / 2011$ & $5.3 \pm 1$ & 2 & $20.916 \pm 3.946$ & 7.893 & $\mathrm{~J}$ & 51.44625 & 179.223416 \\
\hline & ROCK-LS-LT3-1 & \begin{tabular}{|l|} 
FAUNA \\
\end{tabular} & 7/12/2011 & $2.3 \pm 0.8$ & 1.9 & $10.123 \pm 3.521$ & 8.362 & $\mathrm{~J}$ & 51.434944 & 179.2315 \\
\hline & ROCK-LS-LT3-2 & FAUNA & $7 / 12 / 2011$ & $2.8 \pm 0.6$ & 1.4 & $11.878 \pm 2.545$ & 5.939 & $\mathrm{~J}$ & 51.434944 & 179.2315 \\
\hline & ROCK-LS-LT3-3 & FAUNA & $7 / 3 / 2011$ & $1.2 \pm 0.2$ & 0.7 & $4.14 \pm 0.69$ & 2.415 & $\mathrm{~J}$ & 51.43 & 179.22667 \\
\hline
\end{tabular}




\begin{tabular}{|c|c|c|c|c|c|c|c|c|c|c|}
\hline Matrix Subtype & Location Code & Matrix & $\begin{array}{c}\text { Date } \\
\text { Sampled }\end{array}$ & $\begin{array}{l}\text { Wet Result } \pm \\
\text { Uncertainty }\end{array}$ & $\begin{array}{c}\text { Wet } \\
\text { Detection } \\
\text { Limit }\end{array}$ & $\begin{array}{l}\text { Dry Result } \pm \\
\text { Uncertainty }\end{array}$ & \begin{tabular}{|c|} 
Dry \\
Detection \\
Limit
\end{tabular} & Qualifiers & Latitude & Longitude \\
\hline Reindeer Lichen & STRL-AI-LT1-2 & VEG & $6 / 22 / 2011$ & $404 \pm 14$ & 8 & $\begin{array}{c}1224.835 \pm \\
42.445\end{array}$ & 24.254 & & 51.447361 & 179.189111 \\
\hline \multirow{3}{*}{$\begin{array}{l}\text { Sea Urchin - } \\
\text { whole }\end{array}$} & URCH-LS-LT1-1 & FAUNA & $7 / 4 / 2011$ & $1.1 \pm 0.6$ & 1.7 & $4.297 \pm 2.344$ & 6.641 & $\mathrm{U}$ & 51.457555 & 179.195138 \\
\hline & URCH-LS-LT2-1 & FAUNA & $7 / 3 / 2011$ & $1.8 \pm 0.6$ & 1.6 & $6.916 \pm 2.305$ & 6.147 & $\mathrm{~J}$ & 51.44925 & 179.205583 \\
\hline & URCH-LS-LT3-1 & FAUNA & $7 / 2 / 2011$ & $9.9 \pm 2.7$ & 4.3 & $38.562 \pm 10.517$ & 16.749 & $\mathrm{~J}$ & 51.43 & 179.22667 \\
\hline \multicolumn{11}{|c|}{ Amchitka Site (Milrow) (AMC03) } \\
\hline \multirow{3}{*}{ Chiton } & CHIT-ML-MT1-1 & \begin{tabular}{|l|} 
FAUNA \\
\end{tabular} & $7 / 9 / 2011$ & $0.6 \pm 0.4$ & 1.2 & $2.263 \pm 1.508$ & 4.525 & $\mathrm{U}$ & 51.408472 & 179.139166 \\
\hline & CHIT-ML-MT2-1 & FAUNA & $7 / 10 / 2011$ & $2.1 \pm 0.8$ & 2.2 & $9.751 \pm 3.715$ & 10.215 & $U$ & 51.389416 & 179.173138 \\
\hline & CHIT-ML-MT3-1 & FAUNA & $7 / 10 / 2011$ & $0.3 \pm 0.4$ & 1.4 & $1.361 \pm 1.815$ & 6.353 & $\mathrm{U}$ & 51.387805 & 179.184 \\
\hline \multirow{10}{*}{ Dragon Kelp } & EUAL-ML-DUP & VEG & $7 / 9 / 2011$ & $0.5 \pm 0.2$ & 0.3 & $3.768 \pm 1.507$ & 2.261 & $\mathrm{~J}$ & 51.408472 & 179.139166 \\
\hline & EUAL-ML-MT1-1 & VEG & $7 / 9 / 2011$ & $1.4 \pm 0.2$ & 0.3 & $11.192 \pm 1.599$ & 2.398 & & 51.408472 & 179.139166 \\
\hline & EUAL-ML-MT1-2 & VEG & $7 / 9 / 2011$ & $0.4 \pm 0.2$ & 0.3 & $2.976 \pm 1.488$ & 2.232 & $\mathrm{~J}$ & 51.408472 & 179.139166 \\
\hline & EUAL-ML-MT1-3 & VEG & $7 / 9 / 2011$ & $0.35 \pm 0.1$ & 0.2 & $2.944 \pm 0.841$ & 1.682 & $\mathrm{~J}$ & 51.408472 & 179.139166 \\
\hline & EUAL-ML-MT2-1 & VEG & $7 / 10 / 2011$ & $0.9 \pm 0.2$ & 0.4 & $6.708 \pm 1.491$ & 2.981 & $\mathrm{~J}$ & 51.389416 & 179.173138 \\
\hline & EUAL-ML-MT2-2 & VEG & $7 / 10 / 2011$ & $0.5 \pm 0.2$ & 0.3 & $3.543 \pm 1.417$ & 2.126 & $\mathrm{~J}$ & 51.389416 & 179.173138 \\
\hline & EUAL-ML-MT2-3 & VEG & $7 / 10 / 2011$ & $2.4 \pm 0.4$ & 0.6 & $18.091 \pm 3.015$ & 4.523 & & 51.389416 & 179.173138 \\
\hline & EUAL-ML-MT3-1 & VEG & $7 / 10 / 2011$ & $0.63 \pm 0.1$ & 0.2 & $4.432 \pm 0.703$ & 1.407 & & 51.387805 & 179.184 \\
\hline & EUAL-ML-MT3-2 & VEG & $7 / 10 / 2011$ & $0.8 \pm 0.2$ & 0.4 & $5.845 \pm 1.461$ & 2.922 & $\mathrm{~J}$ & 51.387805 & 179.184 \\
\hline & EUAL-ML-MT3-3 & VEG & $7 / 10 / 2011$ & $1 \pm 0.2$ & 0.3 & $6.896 \pm 1.379$ & 2.069 & & 51.387805 & 179.184 \\
\hline \multirow{10}{*}{ Rockweed } & FUCU-ML-DUP & VEG & $6 / 20 / 2011$ & $1.8 \pm 0.2$ & 0.3 & $7.81 \pm 0.868$ & 1.302 & $\mathrm{~J}$ & 51.412449 & 179.160843 \\
\hline & FUCU-ML-MT1-1 & VEG & $6 / 20 / 2011$ & $2.1 \pm 0.2$ & 0.2 & $8.749 \pm 0.833$ & 0.833 & $\mathrm{~J}$ & 51.412449 & 179.160843 \\
\hline & FUCU-ML-MT1-2 & VEG & $6 / 21 / 2011$ & $2.3 \pm 0.2$ & 0.3 & $13.952 \pm 1.213$ & 1.82 & $\mathrm{~J}$ & 51.417083 & 179.134083 \\
\hline & FUCU-ML-MT1-3 & VEG & $7 / 9 / 2011$ & $1.7 \pm 0.2$ & 0.4 & $7.814 \pm 0.919$ & 1.839 & $\mathrm{~J}$ & 51.417083 & 179.134083 \\
\hline & FUCU-ML-MT2-1 & VEG & $6 / 20 / 2011$ & $2.9 \pm 0.4$ & 0.3 & $14.966 \pm 2.064$ & 1.548 & $\mathrm{~J}$ & 51.403305 & 179.167166 \\
\hline & FUCU-ML-MT2-2 & VEG & $6 / 21 / 2011$ & $3.4 \pm 0.4$ & 0.2 & $23.887 \pm 2.81$ & 1.405 & $\mathrm{~J}$ & 51.405694 & 179.161361 \\
\hline & FUCU-ML-MT2-3 & VEG & $6 / 21 / 2011$ & $2.9 \pm 0.4$ & 0.3 & $20.137 \pm 2.777$ & 2.083 & $\mathrm{~J}$ & 51.405694 & 179.161361 \\
\hline & FUCU-ML-MT3-1 & VEG & $7 / 10 / 2011$ & $3.1 \pm 0.4$ & 0.3 & $15.054 \pm 1.943$ & 1.457 & $\mathrm{~J}$ & 51.389416 & 179.173138 \\
\hline & FUCU-ML-MT3-2 & VEG & $7 / 10 / 2011$ & $2.4 \pm 0.2$ & 0.3 & $11.526 \pm 0.961$ & 1.441 & $\mathrm{~J}$ & 51.389416 & 179.173138 \\
\hline & FUCU-ML-MT3-3 & VEG & $7 / 10 / 2011$ & $1.9 \pm 0.4$ & 0.5 & $9.592 \pm 2.019$ & 2.524 & $\mathrm{~J}$ & 51.389416 & 179.173138 \\
\hline
\end{tabular}




\begin{tabular}{|c|c|c|c|c|c|c|c|c|c|c|}
\hline Matrix Subtype & Location Code & Matrix & $\begin{array}{c}\text { Date } \\
\text { Sampled }\end{array}$ & $\begin{array}{l}\text { Wet Result } \pm \\
\text { Uncertainty }\end{array}$ & $\begin{array}{c}\text { Wet } \\
\text { Detection } \\
\text { Limit }\end{array}$ & $\begin{array}{l}\text { Dry Result } \pm \\
\text { Uncertainty }\end{array}$ & $\begin{array}{c}\text { Dry } \\
\text { Detection } \\
\text { Limit }\end{array}$ & Qualifiers & Latitude & Longitude \\
\hline \multirow{10}{*}{ Greenling - whole } & GREN-ML-DUP & FAUNA & 7/10/2011 & $7.7 \pm 2$ & 2.4 & $37.338 \pm 9.698$ & 11.638 & & 51.387805 & 179.184 \\
\hline & GREN-ML-MT1-1 & \begin{tabular}{|l|} 
FAUNA \\
\end{tabular} & 7/9/2011 & $4.9 \pm 1$ & 2.2 & $21.344 \pm 4.356$ & 9.583 & $\mathrm{~J}$ & 51.408472 & 179.139166 \\
\hline & GREN-ML-MT1-2 & FAUNA & $7 / 9 / 2011$ & $3.2 \pm 0.8$ & 1.9 & $14.817 \pm 3.704$ & 8.797 & $\mathrm{~J}$ & 51.408472 & 179.139166 \\
\hline & GREN-ML-MT1-3 & FAUNA & 7/9/2011 & $5.7 \pm 1$ & 2 & $27.523 \pm 4.829$ & 9.657 & $\mathrm{~J}$ & 51.408472 & 179.139166 \\
\hline & GREN-ML-MT2-1 & FAUNA & $7 / 11 / 2011$ & $6.7 \pm 1$ & 1.7 & $31.232 \pm 4.662$ & 7.925 & & 51.396944 & 179.1435 \\
\hline & GREN-ML-MT2-2 & FAUNA & $7 / 11 / 2011$ & $2.5 \pm 0.8$ & 2.2 & $11.703 \pm 3.745$ & 10.299 & $\mathrm{~J}$ & 51.396944 & 179.1435 \\
\hline & GREN-ML-MT2-3 & FAUNA & 7/11/2011 & $3.4 \pm 0.8$ & 2.1 & $16.235 \pm 3.82$ & 10.027 & $\mathrm{~J}$ & 51.396944 & 179.1435 \\
\hline & GREN-ML-MT3-1 & FAUNA & $7 / 10 / 2011$ & $5.5 \pm 0.8$ & 1.5 & $26.674 \pm 3.88$ & 7.275 & & 51.387805 & 179.184 \\
\hline & GREN-ML-MT3-2 & \begin{tabular}{|l|} 
FAUNA \\
\end{tabular} & 7/10/2011 & $7.8 \pm 1.4$ & 2.7 & $37.633 \pm 6.755$ & 13.027 & $\mathrm{~J}$ & 51.387805 & 179.184 \\
\hline & GREN-ML-MT3-3 & FAUNA & 7/10/2011 & $3.9 \pm 1$ & 2.2 & $18.478 \pm 4.738$ & 10.423 & $\mathrm{~J}$ & 51.387805 & 179.184 \\
\hline Halibut - whole & HALI-ML-M2M-1 & FAUNA & $7 / 11 / 2011$ & $0.82 \pm 0.1$ & 0.1 & $2.849 \pm 0.347$ & 0.347 & & 51.365055 & 179.111472 \\
\hline \multirow{3}{*}{$\begin{array}{l}\text { Horse Mussel - } \\
\text { soft tissue only }\end{array}$} & HMUS-ML-MT1-1 & FAUNA & $7 / 9 / 2011$ & $7 \pm 1.8$ & 4.6 & $31.832 \pm 8.185$ & 20.918 & $\mathrm{~J}$ & 51.408472 & 179.139166 \\
\hline & HMUS-ML-MT2-1 & FAUNA & $7 / 11 / 2011$ & $5.9 \pm 2$ & 5.4 & $26.535 \pm 8.995$ & 24.286 & $\bar{J}$ & 51.389416 & 179.173138 \\
\hline & HMUS-ML-MT3-1 & FAUNA & 7/10/2011 & $6.7 \pm 2.4$ & 6.1 & $30.757 \pm 11.017$ & 28.002 & $\mathrm{~J}$ & 51.387805 & 179.184 \\
\hline \multirow{9}{*}{ Irish Lord - whole } & IRLO-ML-MT1-1 & FAUNA & 7/9/2011 & $1.2 \pm 0.4$ & 1.1 & $5.123 \pm 1.708$ & 4.696 & $\bar{J}$ & 51.408472 & 179.139166 \\
\hline & IRLO-ML-MT1-2 & FAUNA & $7 / 9 / 2011$ & $2.8 \pm 0.8$ & 1.8 & $13.28 \pm 3.794$ & 8.537 & $\bar{J}$ & 51.408472 & 179.139166 \\
\hline & IRLO-ML-MT1-3 & \begin{tabular}{|l|} 
FAUNA \\
\end{tabular} & $7 / 9 / 2011$ & $6.4 \pm 1.2$ & 2.7 & $29.981 \pm 5.621$ & 12.648 & $\mathrm{~J}$ & 51.408472 & 179.139166 \\
\hline & IRLO-ML-MT2-1 & FAUNA & $7 / 10 / 2011$ & $4.6 \pm 0.8$ & 1.8 & $19.822 \pm 3.447$ & 7.756 & $\bar{J}$ & 51.389416 & 179.173138 \\
\hline & IRLO-ML-MT2-2 & FAUNA & 7/11/2011 & $6.1 \pm 1.4$ & 2.9 & $26.282 \pm 6.032$ & 12.495 & $\mathrm{~J}$ & 51.396944 & 179.1435 \\
\hline & IRLO-ML-MT2-3 & FAUNA & $7 / 11 / 2011$ & $0.2 \pm 0.6$ & 1.7 & $0.949 \pm 2.846$ & 8.064 & $\mathrm{U}$ & 51.396944 & 179.1435 \\
\hline & IRLO-ML-MT3-1 & FAUNA & 7/10/2011 & $9.5 \pm 1.6$ & 1.2 & $53.555 \pm 9.02$ & 6.765 & $\mathrm{~J}$ & 51.387805 & 179.184 \\
\hline & IRLO-ML-MT3-2 & \begin{tabular}{|l|} 
FAUNA \\
\end{tabular} & 7/10/2011 & $1.8 \pm 0.4$ & 1.2 & $8.691 \pm 1.931$ & 5.794 & $\mathrm{~J}$ & 51.387805 & 179.184 \\
\hline & IRLO-ML-MT3-3 & FAUNA & $7 / 10 / 2011$ & $2.1 \pm 0.6$ & 1.5 & $11.772 \pm 3.363$ & 8.409 & $\mathrm{~J}$ & 51.387805 & 179.184 \\
\hline Octopus & OCTP-ML-M2M-1 & \begin{tabular}{|l|} 
FAUNA \\
\end{tabular} & 7/10/2011 & $1.1 \pm 0.4$ & 1.1 & $5.188 \pm 1.886$ & 5.188 & $\mathrm{~J}$ & 51.389416 & 179.173138 \\
\hline $\begin{array}{l}\text { Pacific Cod - } \\
\text { whole }\end{array}$ & PCOD-ML-M2M-1 & FAUNA & $7 / 10 / 2011$ & $2.8 \pm 0.2$ & 0.3 & $14.806 \pm 1.058$ & 1.586 & & 51.408472 & 179.139166 \\
\hline \multirow{10}{*}{ Rockfish - whole } & ROCK-ML-DUP & \begin{tabular}{|l|} 
FAUNA \\
\end{tabular} & $7 / 9 / 2011$ & $3.6 \pm 0.8$ & 1.7 & $15.915 \pm 3.537$ & 7.515 & $\mathrm{~J}$ & 51.408472 & 179.139166 \\
\hline & ROCK-ML-MT1-1 & FAUNA & 7/9/2011 & $1.1 \pm 0.4$ & 0.9 & $4.842 \pm 1.761$ & 3.962 & & 51.408472 & 179.139166 \\
\hline & ROCK-ML-MT1-2 & \begin{tabular}{|l|} 
FAUNA \\
\end{tabular} & $7 / 9 / 2011$ & $5 \pm 0.8$ & 1.4 & $21.947 \pm 3.511$ & 6.145 & & 51.408472 & 179.139166 \\
\hline & ROCK-ML-MT1-3 & FAUNA & 7/9/2011 & $1.9 \pm 0.4$ & 0.8 & $8.043 \pm 1.693$ & 3.386 & $\mathrm{~J}$ & 51.408472 & 179.139166 \\
\hline & ROCK-ML-MT2-1 & FAUNA & $7 / 11 / 2011$ & $0.5 \pm 0.4$ & 1.1 & $1.877 \pm 1.501$ & 4.128 & $U$ & 51.365055 & 179.111472 \\
\hline & ROCK-ML-MT2-2 & FAUNA & $7 / 11 / 2011$ & $4 \pm 0.8$ & 1.9 & $16.186 \pm 3.237$ & 7.688 & $\mathrm{~J}$ & 51.365055 & 179.111472 \\
\hline & ROCK-ML-MT2-3 & FAUNA & $7 / 11 / 2011$ & $5.2 \pm 1$ & 2.1 & $23.427 \pm 4.505$ & 9.461 & $\mathrm{~J}$ & 51.365055 & 179.111472 \\
\hline & ROCK-ML-MT3-1 & \begin{tabular}{|l|} 
FAUNA \\
\end{tabular} & 7/10/2011 & $0.6 \pm 0.4$ & 1.2 & $2.831 \pm 1.888$ & 5.663 & $U$ & 51.387805 & 179.184 \\
\hline & ROCK-ML-MT3-2 & FAUNA & $7 / 10 / 2011$ & $2.8 \pm 0.6$ & 1.1 & $10.076 \pm 2.159$ & 3.958 & $\mathrm{~J}$ & 51.387805 & 179.184 \\
\hline & ROCK-ML-MT3-3 & FAUNA & $7 / 10 / 2011$ & $3.9 \pm 0.8$ & 1.4 & $9.075 \pm 1.862$ & 3.258 & $\mathrm{~J}$ & 51.387805 & 179.184 \\
\hline
\end{tabular}




\begin{tabular}{|c|c|c|c|c|c|c|c|c|c|c|}
\hline Matrix Subtype & Location Code & Matrix & $\begin{array}{c}\text { Date } \\
\text { Sampled }\end{array}$ & $\begin{array}{l}\text { Wet Result } \pm \\
\text { Uncertainty }\end{array}$ & $\begin{array}{c}\text { Wet } \\
\text { Detection } \\
\text { Limit }\end{array}$ & $\begin{array}{l}\text { Dry Result } \pm \\
\text { Uncertainty }\end{array}$ & \begin{tabular}{|c|} 
Dry \\
Detection \\
Limit
\end{tabular} & Qualifiers & Latitude & Longitude \\
\hline Reindeer Lichen & STRL-AI-ML-1 & VEG & 6/21/2011 & $550 \pm 14$ & 8 & $\begin{array}{c}1968.354 \pm \\
50.104 \\
\end{array}$ & 28.631 & & 51.402583 & 179.183972 \\
\hline \multirow{3}{*}{$\begin{array}{l}\text { Sea Urchin - } \\
\text { whole }\end{array}$} & URCH-ML-MT1-1 & FAUNA & $7 / 9 / 2011$ & $2.3 \pm 0.6$ & 1.6 & $8.532 \pm 2.226$ & 5.936 & $\mathrm{~J}$ & 51.408472 & 179.139166 \\
\hline & URCH-ML-MT2-1 & FAUNA & $7 / 10 / 2011$ & $0.7 \pm 0.6$ & 1.6 & $2.614 \pm 2.241$ & 5.976 & $\mathrm{U}$ & 51.389416 & 179.173138 \\
\hline & URCH-ML-MT3-1 & FAUNA & $7 / 10 / 2011$ & $-0.1 \pm 0.4$ & 1.1 & $-0.397 \pm 1.588$ & 4.367 & $\mathrm{U}$ & 51.387805 & 179.184 \\
\hline \multicolumn{11}{|c|}{ Amchitka Site (Cannikin) (AMC05) } \\
\hline \multirow{3}{*}{ Chiton } & CHIT-CN-CT1-1 & FAUNA & $7 / 8 / 2011$ & $2.8 \pm 1$ & 2.8 & $13.114 \pm 4.684$ & 13.114 & $\mathrm{U}$ & 51.482972 & 179.123833 \\
\hline & CHIT-CN-CT2-1 & FAUNA & $7 / 7 / 2011$ & $4.1 \pm 0.8$ & 1.8 & $19.403 \pm 3.786$ & 8.518 & $\mathrm{~J}$ & 51.472611 & 179.151111 \\
\hline & CHIT-CN-CT3-1 & FAUNA & $7 / 5 / 2011$ & $2.6 \pm 1$ & 2.5 & $12.762 \pm 4.908$ & 12.271 & $\mathrm{~J}$ & 51.471416 & 179.172861 \\
\hline \multirow{10}{*}{ Dragon Kelp } & EUAL-CN-CT1-1 & VEG & $7 / 8 / 2011$ & $1.8 \pm 0.2$ & 0.3 & $13.324 \pm 1.48$ & 2.221 & & 51.482972 & 179.123833 \\
\hline & EUAL-CN-CT1-2 & VEG & $7 / 8 / 2011$ & $2.5 \pm 0.4$ & 0.5 & $16.299 \pm 2.608$ & 3.26 & & 51.482972 & 179.123833 \\
\hline & EUAL-CN-CT1-3 & VEG & $7 / 8 / 2011$ & $3.2 \pm 0.6$ & 0.7 & $19.6 \pm 3.675$ & 4.288 & $\mathrm{~J}$ & 51.482972 & 179.123833 \\
\hline & EUAL-CN-CT2-1 & VEG & $7 / 6 / 2011$ & $1.8 \pm 0.2$ & 0.4 & $11.48 \pm 1.276$ & 2.551 & & 51.472611 & 179.151111 \\
\hline & EUAL-CN-CT2-2 & VEG & 7/6/2011 & $2.7 \pm 0.4$ & 0.3 & $16.946 \pm 2.511$ & 1.883 & & 51.472611 & 179.151111 \\
\hline & EUAL-CN-CT2-3 & VEG & $7 / 7 / 2011$ & $1.4 \pm 0.2$ & 0.4 & $10.15 \pm 1.45$ & 2.9 & & 51.472611 & 179.151111 \\
\hline & EUAL-CN-CT3-1 & VEG & $7 / 5 / 2011$ & $2.5 \pm 0.4$ & 0.6 & $17.433 \pm 2.789$ & 4.184 & $\mathrm{~J}$ & 51.471416 & 179.172861 \\
\hline & EUAL-CN-CT3-2 & VEG & $7 / 5 / 2011$ & $1.9 \pm 0.4$ & 0.5 & $13.66 \pm 2.876$ & 3.595 & & 51.471416 & 179.172861 \\
\hline & EUAL-CN-CT3-3 & VEG & $7 / 5 / 2011$ & $2.5 \pm 0.4$ & 0.5 & $16.249 \pm 2.6$ & 3.25 & & 51.471416 & 179.172861 \\
\hline & EUAL-CN-DUP & VEG & $7 / 6 / 2011$ & $3.1 \pm 0.4$ & 0.4 & $20.598 \pm 2.658$ & 2.658 & & 51.471416 & 179.172861 \\
\hline \multirow{10}{*}{ Rockweed } & FUCU-CN-CT1-1 & VEG & $6 / 23 / 2011$ & $3 \pm 0.4$ & 0.2 & $17.301 \pm 2.307$ & 1.153 & $\mathrm{~J}$ & 51.481888 & 179.118138 \\
\hline & FUCU-CN-CT1-2 & VEG & $6 / 23 / 2011$ & $3.2 \pm 0.4$ & 0.3 & $20.154 \pm 2.519$ & 1.889 & $\mathrm{~J}$ & 51.481888 & 179.118138 \\
\hline & FUCU-CN-CT1-3 & VEG & $6 / 23 / 2011$ & $5.4 \pm 0.6$ & 0.3 & $31.834 \pm 3.537$ & 1.769 & $\mathrm{~J}$ & 51.481888 & 179.118138 \\
\hline & FUCU-CN-CT2-1 & VEG & $7 / 8 / 2011$ & $3.3 \pm 0.4$ & 0.4 & $13.637 \pm 1.653$ & 1.653 & $\mathrm{~J}$ & 51.476638 & 179.135277 \\
\hline & FUCU-CN-CT2-2 & VEG & $7 / 8 / 2011$ & $4.2 \pm 0.4$ & 0.3 & $18.636 \pm 1.775$ & 1.331 & $\mathrm{~J}$ & 51.476638 & 179.135277 \\
\hline & FUCU-CN-CT2-3 & VEG & $7 / 12 / 2011$ & $1.3 \pm 0.2$ & 0.3 & $6.872 \pm 1.057$ & 1.586 & $\mathrm{~J}$ & 51.467583 & 179.153583 \\
\hline & FUCU-CN-CT3-1 & VEG & $7 / 6 / 2011$ & $3.3 \pm 0.4$ & 0.3 & $17.782 \pm 2.155$ & 1.617 & $\mathrm{~J}$ & 51.465027 & 179.173166 \\
\hline & FUCU-CN-CT3-2 & VEG & 7/6/2011 & $5.4 \pm 0.6$ & 0.3 & $31.696 \pm 3.522$ & 1.761 & $\mathrm{~J}$ & 51.465472 & 179.170583 \\
\hline & FUCU-CN-CT3-3 & VEG & 7/6/2011 & $3 \pm 0.4$ & 0.5 & $19.038 \pm 2.538$ & 3.173 & $\mathrm{~J}$ & 51.464638 & 179.159305 \\
\hline & FUCU-CN-DUP & VEG & $6 / 23 / 2011$ & $3.3 \pm 0.2$ & 0.2 & $21.291 \pm 1.29$ & 1.29 & $\mathrm{~J}$ & 51.481888 & 179.118138 \\
\hline
\end{tabular}




\begin{tabular}{|c|c|c|c|c|c|c|c|c|c|c|}
\hline Matrix Subtype & Location Code & Matrix & $\begin{array}{c}\text { Date } \\
\text { Sampled }\end{array}$ & $\begin{array}{l}\text { Wet Result } \pm \\
\text { Uncertainty }\end{array}$ & $\begin{array}{c}\text { Wet } \\
\text { Detection } \\
\text { Limit }\end{array}$ & $\begin{array}{l}\text { Dry Result } \pm \\
\text { Uncertainty }\end{array}$ & \begin{tabular}{|c|} 
Dry \\
Detection \\
Limit
\end{tabular} & Qualifiers & Latitude & Longitude \\
\hline \multirow{10}{*}{ Greenling - whole } & GREN-CN-CT1-1 & FAUNA & $7 / 8 / 2011$ & $6.6 \pm 1.2$ & 2.2 & $31.816 \pm 5.785$ & 10.605 & & 51.482972 & 179.123833 \\
\hline & GREN-CN-CT1-2 & FAUNA & $7 / 8 / 2011$ & $5.5 \pm 1$ & 2.1 & $26.121 \pm 4.749$ & 9.974 & $\mathrm{~J}$ & 51.482972 & 179.123833 \\
\hline & GREN-CN-CT1-3 & FAUNA & $7 / 8 / 2011$ & $6.4 \pm 1$ & 1.9 & $29.164 \pm 4.557$ & 8.658 & & 51.482972 & 179.123833 \\
\hline & GREN-CN-CT2-1 & FAUNA & 7/7/2011 & $5.1 \pm 1$ & 2.2 & $24.857 \pm 4.874$ & 10.723 & $\mathrm{~J}$ & 51.472611 & 179.151111 \\
\hline & GREN-CN-CT2-2 & FAUNA & $7 / 7 / 2011$ & $5.4 \pm 1$ & 1.8 & $24.777 \pm 4.588$ & 8.259 & & 51.472611 & 179.151111 \\
\hline & GREN-CN-CT2-3 & FAUNA & $7 / 7 / 2011$ & $3.9 \pm 0.8$ & 2 & $17.997 \pm 3.692$ & 9.229 & $\mathrm{~J}$ & 51.472611 & 179.151111 \\
\hline & GREN-CN-CT3-1 & FAUNA & $7 / 5 / 2011$ & $7.7 \pm 1$ & 1.8 & $35.092 \pm 4.557$ & 8.203 & & 51.471416 & 179.172861 \\
\hline & GREN-CN-CT3-2 & FAUNA & $7 / 5 / 2011$ & $4.9 \pm 1$ & 2 & $21.344 \pm 4.356$ & 8.712 & $\mathrm{~J}$ & 51.471416 & 179.172861 \\
\hline & GREN-CN-CT3-3 & FAUNA & 7/5/2011 & $4.8 \pm 1$ & 2 & $22.041 \pm 4.592$ & 9.184 & $\mathrm{~J}$ & 51.471416 & 179.172861 \\
\hline & GREN-CN-DUP & FAUNA & $7 / 8 / 2011$ & $2.7 \pm 0.8$ & 2 & $13.021 \pm 3.858$ & 9.645 & $\mathrm{~J}$ & 51.482972 & 179.123833 \\
\hline Halibut - whole & HALI-CN-C2M-1 & FAUNA & $7 / 13 / 2011$ & $1.5 \pm 0.2$ & 0.2 & $5.805 \pm 0.774$ & 0.774 & & 51.40025 & 179.283361 \\
\hline \multirow{3}{*}{$\begin{array}{l}\text { Horse Mussel - } \\
\text { soft tissue only }\end{array}$} & HMUS-CN-CT1-1 & FAUNA & $7 / 8 / 2011$ & $14 \pm 4$ & 5 & $57.554 \pm 16.444$ & 20.555 & $\mathrm{~J}$ & 51.482972 & 179.123833 \\
\hline & HMUS-CN-CT2-1 & FAUNA & $7 / 7 / 2011$ & $11 \pm 2$ & 5 & $49.846 \pm 9.063$ & 22.657 & $\mathrm{~J}$ & 51.472611 & 179.151111 \\
\hline & HMUS-CN-CT3-1 & FAUNA & 7/5/2011 & $11 \pm 2$ & 5 & $45.124 \pm 8.204$ & 20.511 & $\mathrm{~J}$ & 51.471416 & 179.172861 \\
\hline \multirow{9}{*}{ Irish Lord - whole } & IRLO-CN-CT1-1 & FAUNA & $7 / 8 / 2011$ & $1.7 \pm 0.6$ & 1.8 & $7.661 \pm 2.704$ & 8.112 & $\bar{U}$ & 51.482972 & 179.123833 \\
\hline & IRLO-CN-CT1-2 & FAUNA & $7 / 8 / 2011$ & $1.6 \pm 0.6$ & 1.5 & $8.02 \pm 3.007$ & 7.518 & $\mathrm{~J}$ & 51.482972 & 179.123833 \\
\hline & IRLO-CN-CT1-3 & FAUNA & $7 / 8 / 2011$ & $3.4 \pm 1.4$ & 3.9 & $15.972 \pm 6.577$ & 18.32 & $U$ & 51.482972 & 179.123833 \\
\hline & IRLO-CN-CT2-1 & FAUNA & $7 / 7 / 2011$ & $1.4 \pm 0.6$ & 1.5 & $6.598 \pm 2.828$ & 7.069 & $\bar{U}$ & 51.472611 & 179.151111 \\
\hline & IRLO-CN-CT2-2 & FAUNA & 7/7/2011 & $-0.2 \pm 0.4$ & 1.7 & $-0.914 \pm 1.828$ & 7.767 & $\bar{U}$ & 51.472611 & 179.151111 \\
\hline & IRLO-CN-CT2-3 & FAUNA & $7 / 7 / 2011$ & $2.6 \pm 0.8$ & 2.2 & $12.797 \pm 3.938$ & 10.828 & $\mathrm{~J}$ & 51.472611 & 179.151111 \\
\hline & IRLO-CN-CT3-1 & FAUNA & $7 / 5 / 2011$ & $2.7 \pm 1$ & 2.5 & $13.911 \pm 5.152$ & 12.881 & $\mathrm{~J}$ & 51.471416 & 179.172861 \\
\hline & IRLO-CN-CT3-2 & FAUNA & $7 / 5 / 2011$ & $2 \pm 0.8$ & 1.9 & $9.697 \pm 3.879$ & 9.212 & $\mathrm{~J}$ & 51.471416 & 179.172861 \\
\hline & IRLO-CN-CT3-3 & FAUNA & $7 / 5 / 2011$ & $0.3 \pm 0.4$ & 1.3 & $1.438 \pm 1.918$ & 6.232 & $U$ & 51.471416 & 179.172861 \\
\hline Octopus & OCTP-CN-C2M-1 & FAUNA & $7 / 8 / 2011$ & $2.1 \pm 0.4$ & 0.7 & $10.292 \pm 1.96$ & 3.431 & & 51.482972 & 179.123833 \\
\hline $\begin{array}{l}\text { Pacific Cod - } \\
\text { whole }\end{array}$ & PCOD-CN-C2M-1 & FAUNA & $7 / 6 / 2011$ & $3.8 \pm 0.6$ & 0.5 & $17.399 \pm 2.747$ & 2.289 & & 51.483527 & 179.187583 \\
\hline \multirow{10}{*}{ Rockfish - whole } & ROCK-CN-CT1-1 & FAUNA & $7 / 8 / 2011$ & $3.3 \pm 0.8$ & 1.7 & $13.038 \pm 3.161$ & 6.716 & $\mathrm{~J}$ & 51.482972 & 179.123833 \\
\hline & ROCK-CN-CT1-2 & FAUNA & $7 / 8 / 2011$ & $3.3 \pm 0.6$ & 1.2 & $12.727 \pm 2.314$ & 4.628 & $\mathrm{~J}$ & 51.482972 & 179.123833 \\
\hline & ROCK-CN-CT1-3 & FAUNA & $7 / 8 / 2011$ & $4.5 \pm 0.6$ & 1.1 & $18.083 \pm 2.411$ & 4.42 & & 51.482972 & 179.123833 \\
\hline & ROCK-CN-CT2-1 & FAUNA & 7/6/2011 & $3.2 \pm 0.6$ & 1.3 & $11.302 \pm 2.119$ & 4.591 & $\mathrm{~J}$ & 51.472611 & 179.151111 \\
\hline & ROCK-CN-CT2-2 & FAUNA & $7 / 6 / 2011$ & $3.3 \pm 0.6$ & 1.2 & $12.081 \pm 2.197$ & 4.393 & $\mathrm{~J}$ & 51.472611 & 179.151111 \\
\hline & ROCK-CN-CT2-3 & FAUNA & $7 / 6 / 2011$ & $1.9 \pm 0.6$ & 1.6 & $7.772 \pm 2.454$ & 6.545 & $\mathrm{~J}$ & 51.472611 & 179.151111 \\
\hline & ROCK-CN-CT3-1 & FAUNA & $7 / 6 / 2011$ & $3.7 \pm 0.8$ & 1.5 & $15.802 \pm 3.417$ & 6.406 & $\mathrm{~J}$ & 51.471416 & 179.172861 \\
\hline & ROCK-CN-CT3-2 & FAUNA & $7 / 6 / 2011$ & $4.5 \pm 0.6$ & 1.2 & $17.291 \pm 2.305$ & 4.611 & & 51.471416 & 179.172861 \\
\hline & ROCK-CN-CT3-3 & FAUNA & $7 / 6 / 2011$ & $6.4 \pm 1$ & 2 & $26.748 \pm 4.179$ & 8.359 & & 51.471416 & 179.172861 \\
\hline & ROCK-CN-DUP & FAUNA & $7 / 6 / 2011$ & $4 \pm 1$ & 2.1 & $16.065 \pm 4.016$ & 8.434 & $\mathrm{~J}$ & 51.471416 & 179.172861 \\
\hline
\end{tabular}




\begin{tabular}{|c|c|c|c|c|c|c|c|c|c|c|}
\hline Matrix Subtype & Location Code & Matrix & $\begin{array}{c}\text { Date } \\
\text { Sampled }\end{array}$ & $\begin{array}{l}\text { Wet Result } \pm \\
\text { Uncertainty }\end{array}$ & $\begin{array}{c}\text { Wet } \\
\text { Detection } \\
\text { Limit }\end{array}$ & $\begin{array}{l}\text { Dry Result } \pm \\
\text { Uncertainty }\end{array}$ & $\begin{array}{c}\text { Dry } \\
\text { Detection } \\
\text { Limit }\end{array}$ & Qualifiers & Latitude & Longitude \\
\hline Reindeer Lichen & STRL-AI-CT1-1 & VEG & $6 / 23 / 2011$ & $1523 \pm 27$ & 15 & $2414.39 \pm 42.803$ & 23.779 & & 51.480583 & 179.109472 \\
\hline \multirow{3}{*}{$\begin{array}{l}\text { Sea Urchin - } \\
\text { whole }\end{array}$} & URCH-CN-CT1-1 & FAUNA & $7 / 8 / 2011$ & $0.7 \pm 0.4$ & 1.3 & $2.809 \pm 1.605$ & 5.216 & $\mathrm{U}$ & 51.482972 & 179.123833 \\
\hline & URCH-CN-CT2-1 & FAUNA & $7 / 7 / 2011$ & $7.2 \pm 1$ & 1.7 & $24.672 \pm 3.427$ & 5.825 & & 51.472611 & 179.151111 \\
\hline & URCH-CN-CT3-1 & FAUNA & $7 / 5 / 2011$ & $2.3 \pm 0.6$ & 1.2 & $8.81 \pm 2.298$ & 4.597 & $\mathrm{~J}$ & 51.471416 & 179.172861 \\
\hline \multicolumn{11}{|c|}{ Amchitka Site (Adak Island) (AMC13) } \\
\hline $\begin{array}{l}\text { Dolly Varden - } \\
\text { whole }\end{array}$ & DOLL-AD-XXX-1 & FAUNA & 6/28/2011 & $447 \pm 37$ & 1 & $\begin{array}{c}1823.192 \pm \\
150.913 \\
\end{array}$ & 4.079 & $\mathrm{~J}$ & 51.8375 & -176.672555 \\
\hline \multirow{3}{*}{ Reindeer Lichen } & STRL-AD-XXX-1 & VEG & $6 / 28 / 2011$ & $6236 \pm 82$ & 33 & $\begin{array}{c}12206.851 \pm \\
160.513\end{array}$ & 64.597 & & 51.837527 & -176.670694 \\
\hline & STRL-AD-XXX-2 & VEG & $6 / 29 / 2011$ & $3238 \pm 37$ & 16 & $8000.474 \pm 91.42$ & 39.533 & & 51.872833 & -176.707444 \\
\hline & STRL-AD-XXX-3 & VEG & 6/29/2011 & $2855 \pm 39$ & 19 & $\begin{array}{c}4535.668 \pm \\
61.958\end{array}$ & 30.185 & & 51.900805 & -176.688527 \\
\hline \multicolumn{11}{|c|}{ Amchitka Site (Adak Island North) (AMC14) } \\
\hline \multirow{3}{*}{ Chiton } & CHIT-AN-ANT1-1 & FAUNA & $7 / 20 / 2011$ & $0.9 \pm 0.2$ & 0.7 & $4.383 \pm 0.974$ & 3.409 & $\mathrm{~J}$ & 51.838722 & -176.582805 \\
\hline & CHIT-AN-ANT2-1 & FAUNA & $7 / 18 / 2011$ & $0.8 \pm 0.2$ & 0.7 & $4.01 \pm 1.002$ & 3.509 & $\mathrm{~J}$ & 51.848361 & -176.506277 \\
\hline & \begin{tabular}{|l|} 
CHIT-AN-ANT3-1 \\
\end{tabular} & FAUNA & 7/17/2011 & $1.3 \pm 0.6$ & 1.4 & $6.611 \pm 3.051$ & 7.119 & $\mathrm{~J}$ & 51.853722 & -176.470694 \\
\hline \multirow{10}{*}{ Dragon Kelp } & EUAL-AN-ANT1-1 & VEG & $7 / 19 / 2011$ & $0.4 \pm 0.2$ & 0.3 & $3.354 \pm 1.677$ & 2.516 & $\mathrm{~J}$ & 51.835694 & -176.542138 \\
\hline & EUAL-AN-ANT1-2 & VEG & $7 / 19 / 2011$ & $1 \pm 0.2$ & 0.3 & $6.817 \pm 1.363$ & 2.045 & & 51.835694 & -176.542138 \\
\hline & EUAL-AN-ANT1-3 & VEG & $7 / 19 / 2011$ & $0.8 \pm 0.2$ & 0.4 & $6.183 \pm 1.546$ & 3.092 & $\mathrm{~J}$ & 51.835694 & -176.542138 \\
\hline & EUAL-AN-ANT2-1 & VEG & $7 / 18 / 2011$ & $0.7 \pm 0.2$ & 0.3 & $5.103 \pm 1.458$ & 2.187 & $\mathrm{~J}$ & 51.848361 & \begin{tabular}{|l}
-176.506277 \\
\end{tabular} \\
\hline & EUAL-AN-ANT2-2 & VEG & $7 / 18 / 2011$ & $0.35 \pm 0.08$ & 0.2 & $2.23 \pm 0.51$ & 1.274 & $\mathrm{~J}$ & 51.848361 & -176.506277 \\
\hline & EUAL-AN-ANT2-3 & VEG & $7 / 18 / 2011$ & $0.24 \pm 0.08$ & 0.2 & $1.667 \pm 0.556$ & 1.389 & $\mathrm{~J}$ & 51.848361 & -176.506277 \\
\hline & EUAL-AN-ANT3-1 & VEG & $7 / 17 / 2011$ & $1.2 \pm 0.2$ & 0.4 & $8.969 \pm 1.495$ & 2.99 & & 51.853722 & \begin{tabular}{|l|}
-176.470694 \\
\end{tabular} \\
\hline & EUAL-AN-ANT3-2 & VEG & $7 / 17 / 2011$ & $2 \pm 0.4$ & 0.4 & $13.638 \pm 2.728$ & 2.728 & & 51.853722 & \begin{tabular}{|l}
-176.470694 \\
\end{tabular} \\
\hline & EUAL-AN-ANT3-3 & VEG & $7 / 17 / 2011$ & $0.9 \pm 0.2$ & 0.3 & $6.04 \pm 1.342$ & 2.013 & & 51.853722 & \begin{tabular}{|l}
-176.470694 \\
\end{tabular} \\
\hline & EUAL-AN-DUP & VEG & $7 / 17 / 2011$ & $1.5 \pm 0.4$ & 0.8 & $9.432 \pm 2.515$ & 5.03 & $\mathrm{~J}$ & 51.853722 & -176.470694 \\
\hline \multirow{10}{*}{ Rockweed } & FUCU-AN-ANT1-1 & VEG & $7 / 19 / 2011$ & $5.5 \pm 0.6$ & 0.3 & $28.022 \pm 3.057$ & 1.528 & $\mathrm{~J}$ & 51.841444 & -176.546083 \\
\hline & FUCU-AN-ANT1-2 & VEG & $7 / 20 / 2011$ & $4.1 \pm 0.4$ & 0.3 & $26.45 \pm 2.58$ & 1.935 & $\mathrm{~J}$ & 51.8395 & -176.591305 \\
\hline & FUCU-AN-ANT1-3 & VEG & $7 / 20 / 2011$ & $6.7 \pm 1$ & 0.4 & $36.564 \pm 5.457$ & 2.183 & $\mathrm{~J}$ & 51.8395 & -176.591305 \\
\hline & FUCU-AN-ANT2-1 & VEG & $7 / 18 / 2011$ & $3.3 \pm 0.4$ & 0.3 & $20.84 \pm 2.526$ & 1.895 & $\mathrm{~J}$ & 51.837666 & \begin{tabular}{|l}
-176.517527 \\
\end{tabular} \\
\hline & FUCU-AN-ANT2-2 & VEG & 7/18/2011 & $3.3 \pm 0.4$ & 0.3 & $17.542 \pm 2.126$ & 1.595 & $\mathrm{~J}$ & 51.841444 & -176.546083 \\
\hline & FUCU-AN-ANT2-3 & VEG & $7 / 18 / 2011$ & $5.6 \pm 0.6$ & 0.3 & $23.623 \pm 2.531$ & 1.266 & $\mathrm{~J}$ & 51.8395 & -176.591305 \\
\hline & FUCU-AN-ANT3-1 & VEG & $7 / 18 / 2011$ & $7.3 \pm 0.8$ & 0.3 & $41.766 \pm 4.577$ & 1.716 & $\mathrm{~J}$ & 51.839944 & -176.515916 \\
\hline & FUCU-AN-ANT3-2 & VEG & $7 / 18 / 2011$ & $6.6 \pm 1$ & 0.4 & $32.263 \pm 4.888$ & 1.955 & $\mathrm{~J}$ & 51.828972 & -176.448305 \\
\hline & FUCU-AN-ANT3-3 & VEG & $7 / 18 / 2011$ & $2.9 \pm 0.4$ & 0.3 & $14.418 \pm 1.989$ & 1.492 & $\mathrm{~J}$ & 51.830833 & -176.456361 \\
\hline & FUCU-AN-DUP & VEG & 7/18/2011 & $3.5 \pm 0.4$ & 0.4 & $16.287 \pm 1.861$ & 1.861 & $\mathrm{~J}$ & 51.828972 & -176.448305 \\
\hline
\end{tabular}




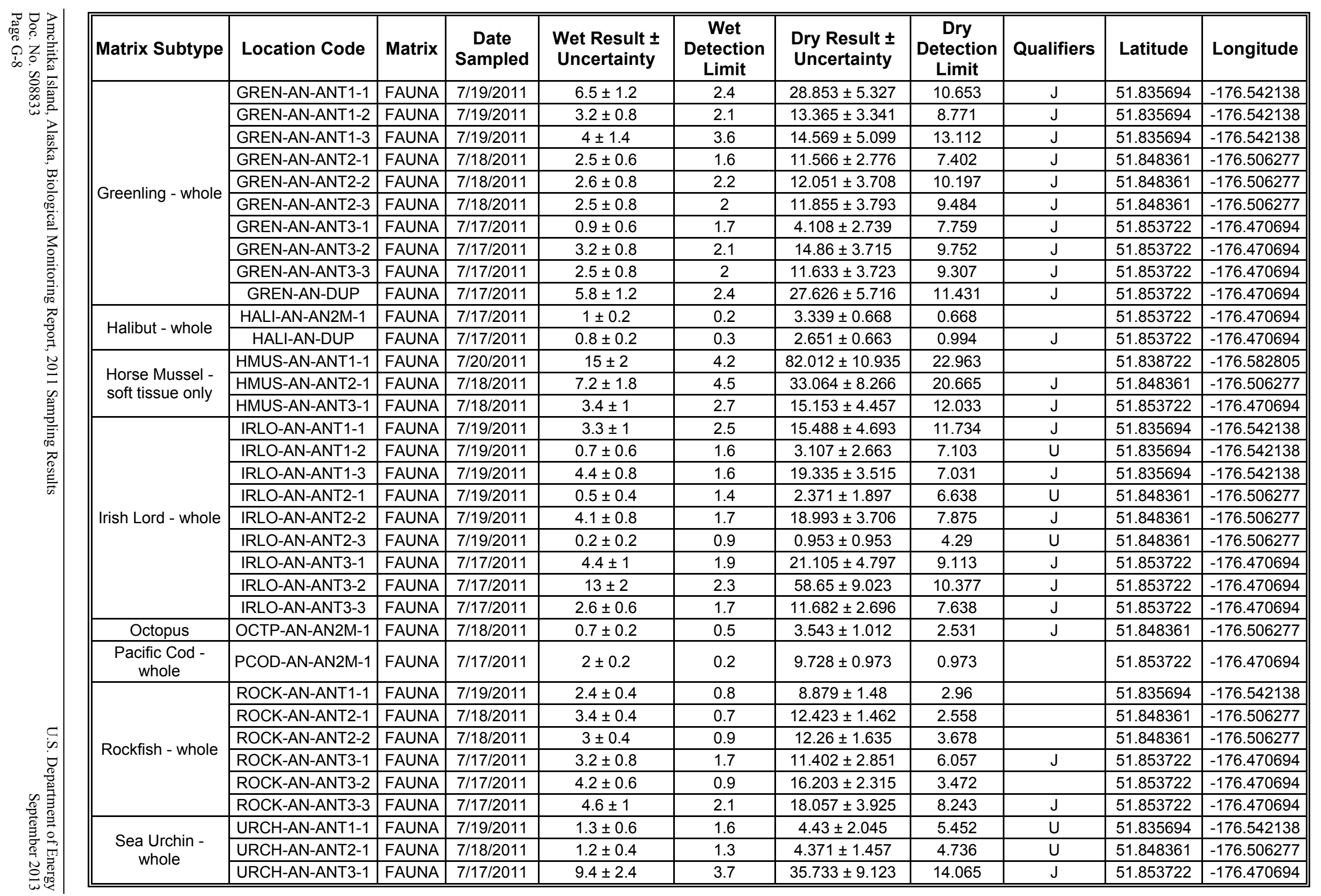




\begin{tabular}{|c|c|c|c|c|c|c|c|c|c|c|}
\hline Matrix Subtype & Location Code & Matrix & $\begin{array}{c}\text { Date } \\
\text { Sampled }\end{array}$ & $\begin{array}{l}\text { Wet Result } \pm \\
\text { Uncertainty }\end{array}$ & $\begin{array}{c}\text { Wet } \\
\text { Detection } \\
\text { Limit }\end{array}$ & $\begin{array}{l}\text { Dry Result } \pm \\
\text { Uncertainty }\end{array}$ & $\begin{array}{c}\text { Dry } \\
\text { Detection } \\
\text { Limit }\end{array}$ & Qualifiers & Latitude & Longitude \\
\hline \multicolumn{11}{|c|}{ Amchitka Site (Adak Island South) (AMC15) } \\
\hline \multirow{3}{*}{ Chiton } & CHIT-AS-AST1-1 & FAUNA & $7 / 15 / 2011$ & $-0.4 \pm 0.4$ & 4 & $-1.644 \pm 1.644$ & 16.436 & $U$ & 51.737472 & -176.48025 \\
\hline & CHIT-AS-AST2-1 & FAUNA & $7 / 16 / 2011$ & $1.3 \pm 0.4$ & 1.2 & $6.655 \pm 2.048$ & 6.143 & $\mathrm{~J}$ & 51.745666 & -176.507055 \\
\hline & CHIT-AS-AST3-1 & FAUNA & $7 / 14 / 2011$ & $1.5 \pm 0.6$ & 1.9 & $7.066 \pm 2.826$ & 8.95 & $\mathrm{U}$ & 51.688333 & -176.63375 \\
\hline \multirow{10}{*}{ Dragon Kelp } & EUAL-AS-AST1-1 & VEG & $7 / 15 / 2011$ & $1.3 \pm 0.2$ & 0.5 & $8.567 \pm 1.318$ & 3.295 & $\mathrm{~J}$ & 51.737472 & -176.48025 \\
\hline & EUAL-AS-AST1-2 & VEG & $7 / 15 / 2011$ & $0.33 \pm 0.08$ & 0.2 & $2.628 \pm 0.637$ & 1.593 & $\mathrm{~J}$ & 51.737472 & -176.48025 \\
\hline & EUAL-AS-AST1-3 & VEG & $7 / 15 / 2011$ & $0.8 \pm 0.2$ & 0.2 & $7.677 \pm 1.919$ & 1.919 & & 51.737472 & -176.48025 \\
\hline & EUAL-AS-AST2-1 & VEG & $7 / 16 / 2011$ & $0.8 \pm 0.2$ & 0.2 & $5.688 \pm 1.422$ & 1.422 & & 51.745666 & -176.507055 \\
\hline & EUAL-AS-AST2-2 & VEG & $7 / 16 / 2011$ & $1.6 \pm 0.2$ & 0.5 & $11.321 \pm 1.415$ & 3.538 & & 51.745666 & -176.507055 \\
\hline & EUAL-AS-AST2-3 & VEG & $7 / 16 / 2011$ & $0.8 \pm 0.2$ & 0.3 & $5.812 \pm 1.453$ & 2.179 & $\mathrm{~J}$ & 51.745666 & -176.507055 \\
\hline & EUAL-AS-AST3-1 & VEG & $7 / 14 / 2011$ & $1.8 \pm 0.4$ & 0.4 & $13.148 \pm 2.922$ & 2.922 & & 51.688333 & -176.63375 \\
\hline & EUAL-AS-AST3-2 & VEG & $7 / 14 / 2011$ & $0.2 \pm 0.2$ & 0.3 & $1.896 \pm 1.896$ & 2.844 & $\mathrm{~J}$ & 51.688333 & -176.63375 \\
\hline & EUAL-AS-AST3-3 & VEG & $7 / 14 / 2011$ & $0.46 \pm 0.08$ & 0.2 & $4.056 \pm 0.705$ & 1.763 & $\mathrm{~J}$ & 51.688333 & -176.63375 \\
\hline & EUAL-AS-DUP & VEG & $7 / 14 / 2011$ & $0.5 \pm 0.2$ & 0.2 & $3.679 \pm 1.472$ & 1.472 & $\mathrm{~J}$ & 51.688333 & -176.63375 \\
\hline \multirow{10}{*}{ Rockweed } & FUCU-AS-AST1-1 & VEG & $7 / 16 / 2011$ & $5.1 \pm 0.6$ & 0.3 & $31.46 \pm 3.701$ & 1.851 & $\mathrm{~J}$ & 51.733666 & -176.470805 \\
\hline & FUCU-AS-AST1-2 & VEG & $7 / 16 / 2011$ & $1.8 \pm 0.2$ & 0.4 & $9.985 \pm 1.109$ & 2.219 & $\mathrm{~J}$ & 51.733666 & -176.470805 \\
\hline & FUCU-AS-AST1-3 & VEG & $7 / 16 / 2011$ & $1.4 \pm 0.2$ & 0.4 & $7.599 \pm 1.086$ & 2.171 & $\mathrm{~J}$ & 51.733666 & -176.470805 \\
\hline & FUCU-AS-AST2-1 & VEG & $7 / 16 / 2011$ & $1 \pm 0.2$ & 0.3 & $4.834 \pm 0.967$ & 1.45 & $\mathrm{~J}$ & 51.745666 & -176.507055 \\
\hline & FUCU-AS-AST2-2 & VEG & $7 / 16 / 2011$ & $4.1 \pm 0.4$ & 0.4 & $20.754 \pm 2.025$ & 2.025 & $\mathrm{~J}$ & 51.745666 & -176.507055 \\
\hline & FUCU-AS-AST2-3 & VEG & $7 / 16 / 2011$ & $4.3 \pm 0.6$ & 0.4 & $21.124 \pm 2.948$ & 1.965 & $\mathrm{~J}$ & 51.745666 & -176.507055 \\
\hline & FUCU-AS-AST3-1 & VEG & $7 / 14 / 2011$ & $2.7 \pm 0.4$ & 0.5 & $14.234 \pm 2.109$ & 2.636 & $\mathrm{~J}$ & 51.688333 & -176.63375 \\
\hline & FUCU-AS-AST3-2 & VEG & $7 / 14 / 2011$ & $2.3 \pm 0.4$ & 0.4 & $11.326 \pm 1.97$ & 1.97 & $\mathrm{~J}$ & 51.688333 & -176.63375 \\
\hline & FUCU-AS-AST3-3 & VEG & $7 / 14 / 2011$ & $3.2 \pm 0.4$ & 0.3 & $16.804 \pm 2.101$ & 1.575 & $\mathrm{~J}$ & 51.688333 & -176.63375 \\
\hline & FUCU-AS-DUP & VEG & $7 / 16 / 2011$ & $2.6 \pm 0.2$ & 0.3 & $13.438 \pm 1.034$ & 1.551 & $\mathrm{~J}$ & 51.733666 & -176.470805 \\
\hline \multirow{10}{*}{ Greenling - whole } & GREN-AS-AST1-1 & FAUNA & $7 / 15 / 2011$ & $4.3 \pm 1$ & 2.1 & $20.485 \pm 4.764$ & 10.004 & $\mathrm{~J}$ & 51.737472 & -176.48025 \\
\hline & GREN-AS-AST1-2 & \begin{tabular}{|l|} 
FAUNA \\
\end{tabular} & $7 / 15 / 2011$ & $7.4 \pm 1.8$ & 2.1 & $34.913 \pm 8.492$ & 9.908 & & 51.737472 & -176.48025 \\
\hline & GREN-AS-AST1-3 & FAUNA & $7 / 15 / 2011$ & $2.2 \pm 0.6$ & 1.4 & $10.567 \pm 2.882$ & 6.724 & $\mathrm{~J}$ & 51.737472 & -176.48025 \\
\hline & GREN-AS-AST2-1 & FAUNA & $7 / 16 / 2011$ & $4.4 \pm 0.8$ & 1.5 & $17.673 \pm 3.213$ & 6.025 & $\mathrm{~J}$ & 51.745666 & -176.507055 \\
\hline & GREN-AS-AST2-2 & FAUNA & $7 / 16 / 2011$ & $1.8 \pm 0.6$ & 1.3 & $8.233 \pm 2.744$ & 5.946 & $\mathrm{~J}$ & 51.745666 & -176.507055 \\
\hline & GREN-AS-AST2-3 & \begin{tabular}{|l|} 
FAUNA \\
\end{tabular} & $7 / 16 / 2011$ & $4.1 \pm 1$ & 2.1 & $19.095 \pm 4.657$ & 9.78 & $\mathrm{~J}$ & 51.745666 & -176.507055 \\
\hline & GREN-AS-AST3-1 & FAUNA & $7 / 14 / 2011$ & $5.2 \pm 1$ & 2.2 & $24.766 \pm 4.763$ & 10.478 & $\mathrm{~J}$ & 51.688333 & -176.63375 \\
\hline & GREN-AS-AST3-2 & FAUNA & $7 / 14 / 2011$ & $3.5 \pm 1$ & 2.7 & $16.475 \pm 4.707$ & 12.709 & $\mathrm{~J}$ & 51.688333 & -176.63375 \\
\hline & GREN-AS-AST3-3 & \begin{tabular}{|l|} 
FAUNA \\
\end{tabular} & $7 / 14 / 2011$ & $3.6 \pm 1$ & 2.7 & $17.193 \pm 4.776$ & 12.895 & $\mathrm{~J}$ & 51.688333 & -176.63375 \\
\hline & GREN-AS-DUP & FAUNA & $7 / 14 / 2011$ & $3.3 \pm 0.8$ & 2 & $16.548 \pm 4.012$ & 10.029 & $\mathrm{~J}$ & 51.688333 & -176.63375 \\
\hline
\end{tabular}




\begin{tabular}{|c|c|c|c|c|c|c|c|c|c|c|}
\hline Matrix Subtype & Location Code & Matrix & $\begin{array}{c}\text { Date } \\
\text { Sampled }\end{array}$ & $\begin{array}{l}\text { Wet Result } \pm \\
\text { Uncertainty }\end{array}$ & $\begin{array}{c}\text { Wet } \\
\text { Detection } \\
\text { Limit }\end{array}$ & $\begin{array}{l}\text { Dry Result } \pm \\
\text { Uncertainty }\end{array}$ & \begin{tabular}{|c|} 
Dry \\
Detection \\
Limit
\end{tabular} & Qualifiers & Latitude & Longitude \\
\hline \multirow{3}{*}{$\begin{array}{l}\text { Horse Mussel - } \\
\text { soft tissue only }\end{array}$} & HMUS-AS-AST1-1 & FAUNA & $7 / 15 / 2011$ & $11 \pm 2$ & 5 & $55.11 \pm 10.02$ & 25.05 & $\mathrm{~J}$ & 51.737472 & -176.48025 \\
\hline & \begin{tabular}{|l|} 
HMUS-AS-AST2-1 \\
\end{tabular} & FAUNA & $7 / 16 / 2011$ & $14 \pm 4$ & 5 & $88.812 \pm 25.375$ & 31.719 & $\mathrm{~J}$ & 51.745666 & -176.507055 \\
\hline & \begin{tabular}{|l|} 
HMUS-AS-AST3-1 \\
\end{tabular} & FAUNA & $7 / 14 / 2011$ & $5.6 \pm 1.8$ & 4.8 & $28.551 \pm 9.177$ & 24.472 & $\mathrm{~J}$ & 51.688333 & -176.63375 \\
\hline \multirow{9}{*}{ Irish Lord - whole } & \begin{tabular}{|l|} 
IRLO-AS-AST1-1 \\
\end{tabular} & FAUNA & 7/15/2011 & $-0.1 \pm 0.4$ & 1.8 & $-0.44 \pm 1.761$ & 7.925 & $U$ & 51.737472 & -176.48025 \\
\hline & IRLO-AS-AST1-2 & FAUNA & 7/15/2011 & $3.9 \pm 0.8$ & 1.6 & $18.184 \pm 3.73$ & 7.46 & $\mathrm{~J}$ & 51.737472 & -176.48025 \\
\hline & IRLO-AS-AST1-3 & FAUNA & $7 / 15 / 2011$ & $1.4 \pm 0.6$ & 1.9 & $6.381 \pm 2.735$ & 8.66 & $U$ & 51.737472 & -176.48025 \\
\hline & IRLO-AS-AST2-1 & FAUNA & $7 / 16 / 2011$ & $1.4 \pm 0.8$ & 2.5 & $6.552 \pm 3.744$ & 11.7 & $\mathrm{U}$ & 51.745666 & -176.507055 \\
\hline & IRLO-AS-AST2-2 & FAUNA & $7 / 16 / 2011$ & $1.5 \pm 0.6$ & 1.9 & $6.386 \pm 2.554$ & 8.089 & $\mathrm{U}$ & 51.745666 & -176.507055 \\
\hline & IRLO-AS-AST2-3 & FAUNA & $7 / 16 / 2011$ & $0.3 \pm 0.6$ & 1.9 & $1.518 \pm 3.035$ & 9.612 & $U$ & 51.745666 & -176.507055 \\
\hline & IRLO-AS-AST3-1 & FAUNA & $7 / 14 / 2011$ & $2.6 \pm 0.6$ & 1.7 & $11.673 \pm 2.694$ & 7.632 & $\mathrm{~J}$ & 51.688333 & -176.63375 \\
\hline & IRLO-AS-AST3-2 & FAUNA & $7 / 14 / 2011$ & $1.9 \pm 0.6$ & 1.4 & $8.028 \pm 2.535$ & 5.916 & $\mathrm{~J}$ & 51.688333 & -176.63375 \\
\hline & IRLO-AS-AST3-3 & FAUNA & $7 / 14 / 2011$ & $1.8 \pm 0.6$ & 1.6 & $8.213 \pm 2.738$ & 7.301 & $\mathrm{~J}$ & 51.688333 & -176.63375 \\
\hline $\begin{array}{l}\text { Pacific Cod - } \\
\text { whole }\end{array}$ & PCOD-AS-AS2M-1 & FAUNA & 7/15/2011 & $3 \pm 0.4$ & 0.3 & $15.01 \pm 2.001$ & 1.501 & $J$ & 51.737472 & -176.48025 \\
\hline \multirow{10}{*}{ Rockfish - whole } & ROCK-AS-AST1-1 & FAUNA & $7 / 15 / 2011$ & $6.4 \pm 1.2$ & 2.2 & $23.009 \pm 4.314$ & 7.909 & $\mathrm{~J}$ & 51.737472 & -176.48025 \\
\hline & \begin{tabular}{|l|} 
ROCK-AS-AST1-2 \\
\end{tabular} & FAUNA & $7 / 15 / 2011$ & $5.7 \pm 1$ & 1.9 & $21.356 \pm 3.747$ & 7.119 & & 51.737472 & -176.48025 \\
\hline & \begin{tabular}{|l|} 
ROCK-AS-AST1-3 \\
\end{tabular} & FAUNA & $7 / 15 / 2011$ & $2.2 \pm 0.4$ & 0.6 & $9.472 \pm 1.722$ & 2.583 & & 51.737472 & -176.48025 \\
\hline & ROCK-AS-AST2-1 & FAUNA & $7 / 16 / 2011$ & $1.8 \pm 0.6$ & 1.8 & $6.903 \pm 2.301$ & 6.903 & $U$ & 51.745666 & -176.507055 \\
\hline & \begin{tabular}{|l|} 
ROCK-AS-AST2-2 \\
\end{tabular} & FAUNA & $7 / 16 / 2011$ & $4 \pm 0.6$ & 1.2 & $14.857 \pm 2.229$ & 4.457 & & 51.745666 & -176.507055 \\
\hline & \begin{tabular}{|l|} 
ROCK-AS-AST2-3 \\
\end{tabular} & FAUNA & $7 / 16 / 2011$ & $3.9 \pm 0.8$ & 1.6 & $15.05 \pm 3.087$ & 6.174 & $\mathrm{~J}$ & 51.745666 & -176.507055 \\
\hline & \begin{tabular}{|l|} 
ROCK-AS-AST3-1 \\
\end{tabular} & FAUNA & $7 / 14 / 2011$ & $1.2 \pm 0.4$ & 1 & $4.92 \pm 1.64$ & 4.1 & $\mathrm{~J}$ & 51.688333 & -176.63375 \\
\hline & ROCK-AS-AST3-2 & FAUNA & $7 / 14 / 2011$ & $9.4 \pm 2.2$ & 2.4 & $39.856 \pm 9.328$ & 10.176 & & 51.688333 & -176.63375 \\
\hline & ROCK-AS-AST3-3 & FAUNA & $7 / 14 / 2011$ & $2.4 \pm 0.4$ & 0.5 & $10.415 \pm 1.736$ & 2.17 & & 51.688333 & -176.63375 \\
\hline & ROCK-AS-DUP & FAUNA & $7 / 14 / 2011$ & $1 \pm 0.2$ & 0.5 & $3.915 \pm 0.783$ & 1.957 & $\mathrm{~J}$ & 51.688333 & -176.63375 \\
\hline \multirow{3}{*}{$\begin{array}{l}\text { Sea Urchin - } \\
\text { whole }\end{array}$} & \begin{tabular}{|l|} 
URCH-AS-AST1-1 \\
\end{tabular} & FAUNA & $7 / 15 / 2011$ & $1.6 \pm 0.6$ & 1.7 & $6.077 \pm 2.279$ & 6.457 & $U$ & 51.737472 & -176.48025 \\
\hline & \begin{tabular}{|l|} 
URCH-AS-AST2-1 \\
\end{tabular} & FAUNA & $7 / 16 / 2011$ & $0.3 \pm 0.4$ & 1.2 & $1.211 \pm 1.615$ & 4.844 & $U$ & 51.745666 & -176.507055 \\
\hline & \begin{tabular}{|l|} 
URCH-AS-AST3-1 \\
\end{tabular} & FAUNA & $7 / 14 / 2011$ & $2.7 \pm 0.6$ & 1.6 & $10.082 \pm 2.24$ & 5.975 & $\mathrm{~J}$ & 51.688333 & -176.63375 \\
\hline
\end{tabular}

Qualifier definitions:

$U$ - Analytical result below detection limit

$\mathrm{J}$ - Estimated 


\section{Appendix H}

\section{Activity Concentration of ${ }^{241} \mathrm{Am}$}


This page intentionally left blank 


\begin{tabular}{|c|c|c|c|c|c|c|c|c|c|c|c|}
\hline \multirow{2}{*}{\multicolumn{2}{|c|}{ 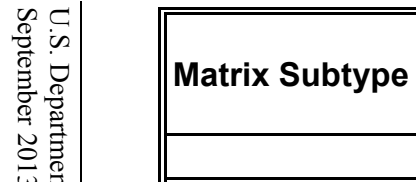 }} & Location Code & Matrix & $\begin{array}{c}\text { Date } \\
\text { Sampled }\end{array}$ & $\begin{array}{c}\text { Wet Result } \pm \\
\text { Uncertainty }\end{array}$ & $\begin{array}{c}\text { Wet } \\
\text { Detection } \\
\text { Limit }\end{array}$ & $\begin{array}{l}\text { Dry Result } \pm \\
\text { Uncertainty }\end{array}$ & $\begin{array}{c}\text { Dry } \\
\text { Detection } \\
\text { Limit }\end{array}$ & Qualifiers & Latitude & Longitude \\
\hline & & \multicolumn{10}{|c|}{${ }^{241} \mathrm{Am}(\mathrm{pCi} / \mathrm{kg})$} \\
\hline$\omega \underset{0}{ \pm}$ & \multicolumn{11}{|c|}{ Amchitka Site (AMC01) } \\
\hline $\overrightarrow{\overrightarrow{0}}$ & $\begin{array}{l}\text { Dolly Varden - } \\
\text { whole }\end{array}$ & DOLL-AI-XXX-1 & FAUNA & $6 / 24 / 2011$ & $0.001 \pm 0.002$ & 0.002 & $0.004 \pm 0.008$ & 0.008 & $U$ & 51.468283 & 179.108052 \\
\hline & $\begin{array}{c}\text { Goose Egg - no } \\
\text { shell }\end{array}$ & GOOS-AI-XXX-1 & FAUNA & $6 / 24 / 2011$ & $-0.004 \pm 0.008$ & 0.04 & $-0.011 \pm 0.021$ & 0.106 & $U$ & 51.491305 & 179.0595 \\
\hline & $\begin{array}{c}\text { Glaucous-winged } \\
\text { Gull Egg - no } \\
\text { shell }\end{array}$ & GWGU-AI-XXX-1 & FAUNA & $6 / 24 / 2011$ & $0.008 \pm 0.016$ & 0.01 & $0.028 \pm 0.055$ & 0.035 & $\mathrm{U}$ & 51.456388 & 179.167 \\
\hline & \multicolumn{11}{|c|}{ Amchitka Site (Long Shot) (AMC02) } \\
\hline & \multirow{3}{*}{ Chiton } & CHIT-LS-LT1-1 & FAUNA & $7 / 4 / 2011$ & $0.08 \pm 0.04$ & 0.01 & $0.397 \pm 0.198$ & 0.05 & & 51.457555 & 179.195138 \\
\hline & & CHIT-LS-LT2-1 & FAUNA & $7 / 3 / 2011$ & $0.08 \pm 0.04$ & 0.006 & $0.375 \pm 0.187$ & 0.028 & & 51.44925 & 179.205583 \\
\hline & & CHIT-LS-LT3-1 & FAUNA & $7 / 3 / 2011$ & $0.14 \pm 0.02$ & 0.002 & $0.667 \pm 0.095$ & 0.01 & & 51.43 & 179.22667 \\
\hline & \multirow{10}{*}{ Dragon Kelp } & EUAL-LS-DUP & VEG & $7 / 3 / 2011$ & $0.001 \pm 0.002$ & 0.001 & $0.006 \pm 0.012$ & 0.006 & $U$ & 51.44925 & 179.205583 \\
\hline & & EUAL-LS-LT1-1 & VEG & $7 / 4 / 2011$ & $0.001 \pm 0.002$ & 0.002 & $0.009 \pm 0.019$ & 0.019 & $\mathrm{U}$ & 51.457555 & 179.195138 \\
\hline & & EUAL-LS-LT1-2 & VEG & $7 / 4 / 2011$ & $0.004 \pm 0.002$ & 0.0004 & $0.025 \pm 0.012$ & 0.002 & & 51.457555 & 179.195138 \\
\hline है & & EUAL-LS-LT1-3 & VEG & $7 / 4 / 2011$ & $-0.0007 \pm 0.0014$ & 0.004 & $-0.004 \pm 0.008$ & 0.024 & UJ & 51.457555 & 179.195138 \\
\hline $\overrightarrow{\overrightarrow{\hat{N}}}$ & & EUAL-LS-LT2-1 & VEG & $7 / 3 / 2011$ & $0.023 \pm 0.004$ & 0.0009 & $0.139 \pm 0.024$ & 0.005 & & 51.44925 & 179.205583 \\
\hline & & EUAL-LS-LT2-2 & VEG & $7 / 3 / 2011$ & $0.012 \pm 0.002$ & 0.0003 & $0.087 \pm 0.015$ & 0.002 & & 51.44925 & 179.205583 \\
\hline है & & EUAL-LS-LT2-3 & VEG & $7 / 3 / 2011$ & $0.005 \pm 0.004$ & 0.002 & $0.036 \pm 0.029$ & 0.014 & $\mathrm{U}$ & 51.44925 & 179.205583 \\
\hline$\approx$ & & EUAL-LS-LT3-1 & VEG & $7 / 2 / 2011$ & $0.006 \pm 0.002$ & 0.0004 & $0.038 \pm 0.013$ & 0.003 & & 51.43 & 179.22667 \\
\hline & & EUAL-LS-LT3-2 & VEG & $7 / 2 / 2011$ & $0.033 \pm 0.008$ & 0.001 & $0.235 \pm 0.057$ & 0.007 & & 51.43 & 179.22667 \\
\hline 苾. & & EUAL-LS-LT3-3 & VEG & $7 / 2 / 2011$ & $0.007 \pm 0.004$ & 0.0008 & $0.059 \pm 0.034$ & 0.007 & & 51.43 & 179.22667 \\
\hline 10 & \multirow{10}{*}{ Rockweed } & FUCU-LS-DUP & VEG & $6 / 22 / 2011$ & $0.001 \pm 0.002$ & 0.002 & $0.005 \pm 0.011$ & 0.011 & $\mathrm{U}$ & 51.447472 & 179.190888 \\
\hline 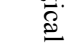 & & FUCU-LS-LT1-1 & VEG & $6 / 22 / 2011$ & $0.003 \pm 0.002$ & 0.0009 & $0.016 \pm 0.011$ & 0.005 & & 51.447527 & 179.189361 \\
\hline$\vec{v}$ & & FUCU-LS-LT1-2 & VEG & $6 / 22 / 2011$ & $0.003 \pm 0.004$ & 0.004 & $0.018 \pm 0.024$ & 0.024 & $U$ & 51.447527 & 179.189361 \\
\hline & & FUCU-LS-LT1-3 & VEG & $6 / 22 / 2011$ & $0.003 \pm 0.002$ & 0.0009 & $0.02 \pm 0.013$ & 0.006 & & 51.448777 & 179.189305 \\
\hline 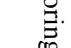 & & FUCU-LS-LT2-1 & VEG & $6 / 22 / 2011$ & $0.0008 \pm 0.0012$ & 0.0007 & $0.006 \pm 0.009$ & 0.006 & $\mathrm{U}$ & 51.447222 & 179.199361 \\
\hline & & FUCU-LS-LT2-2 & VEG & $6 / 22 / 2011$ & $0.002 \pm 0.002$ & 0.001 & $0.014 \pm 0.014$ & 0.007 & $U$ & 51.44625 & 179.196972 \\
\hline & & FUCU-LS-LT2-3 & VEG & $6 / 22 / 2011$ & $0.0003 \pm 0.0006$ & 0.0009 & $0.002 \pm 0.004$ & 0.006 & $\mathrm{U}$ & 51.446722 & 179.191888 \\
\hline & & FUCU-LS-LT3-1 & VEG & $7 / 2 / 2011$ & $0.002 \pm 0.002$ & 0.001 & $0.01 \pm 0.01$ & 0.005 & $\mathrm{U}$ & 51.433611 & 179.219305 \\
\hline & & FUCU-LS-LT3-2 & VEG & $7 / 2 / 2011$ & $0.004 \pm 0.002$ & 0.002 & $0.018 \pm 0.009$ & 0.009 & $\mathrm{~J}$ & 51.430166 & 179.218694 \\
\hline 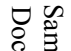 & & FUCU-LS-LT3-3 & VEG & $7 / 2 / 2011$ & $-0.0004 \pm 0.0006$ & 0.002 & $-0.001 \pm 0.002$ & 0.007 & $U$ & 51.430166 & 179.218694 \\
\hline
\end{tabular}




\begin{tabular}{|c|c|c|c|c|c|c|c|c|c|c|}
\hline Matrix Subtype & Location Code & Matrix & $\begin{array}{c}\text { Date } \\
\text { Sampled }\end{array}$ & $\begin{array}{l}\text { Wet Result } \pm \\
\text { Uncertainty }\end{array}$ & $\begin{array}{c}\text { Wet } \\
\text { Detection } \\
\text { Limit }\end{array}$ & $\begin{array}{l}\text { Dry Result } \pm \\
\text { Uncertainty }\end{array}$ & \begin{tabular}{|c|} 
Dry \\
Detection \\
Limit
\end{tabular} & Qualifiers & Latitude & Longitude \\
\hline \multirow{10}{*}{ Greenling - whole } & GREN-LS-DUP & FAUNA & \begin{tabular}{|l|}
$7 / 12 / 2011$ \\
\end{tabular} & $0.01 \pm 0.02$ & 0.01 & $0.049 \pm 0.097$ & 0.049 & $U$ & 51.434527 & 179.234416 \\
\hline & GREN-LS-LT1-1 & FAUNA & $7 / 4 / 2011$ & $0.03 \pm 0.02$ & 0.01 & $0.141 \pm 0.094$ & 0.047 & & 51.457555 & 179.195138 \\
\hline & GREN-LS-LT1-2 & FAUNA & $7 / 4 / 2011$ & $0.02 \pm 0.02$ & 0.02 & $0.095 \pm 0.095$ & 0.095 & $U$ & 51.457555 & 179.195138 \\
\hline & GREN-LS-LT1-3 & FAUNA & $7 / 5 / 2011$ & $0.005 \pm 0.014$ & 0.01 & $0.023 \pm 0.066$ & 0.047 & $U$ & 51.457555 & 179.195138 \\
\hline & GREN-LS-LT2-1 & FAUNA & $7 / 3 / 2011$ & $0.009 \pm 0.018$ & 0.02 & $0.043 \pm 0.087$ & 0.097 & $\bar{U}$ & 51.432194 & 179.230277 \\
\hline & GREN-LS-LT2-2 & FAUNA & $7 / 3 / 2011$ & $0.01 \pm 0.02$ & 0.007 & $0.046 \pm 0.092$ & 0.032 & $\mathrm{U}$ & 51.432194 & 179.230277 \\
\hline & GREN-LS-LT2-3 & FAUNA & $7 / 3 / 2011$ & $0.005 \pm 0.01$ & 0.006 & $0.024 \pm 0.048$ & 0.029 & $U$ & 51.432194 & 179.230277 \\
\hline & GREN-LS-LT3-1 & FAUNA & $7 / 3 / 2011$ & $0.005 \pm 0.012$ & 0.007 & $0.022 \pm 0.053$ & 0.031 & $\bar{U}$ & 51.43 & 179.22667 \\
\hline & GREN-LS-LT3-2 & FAUNA & $7 / 12 / 2011$ & $0.001 \pm 0.016$ & 0.02 & $0.005 \pm 0.078$ & 0.098 & $U$ & 51.434527 & 179.234416 \\
\hline & GREN-LS-LT3-3 & FAUNA & 7/12/2011 & $0.001 \pm 0.014$ & 0.02 & $0.005 \pm 0.068$ & 0.097 & $\bar{U}$ & 51.434527 & 179.234416 \\
\hline Halibut - whole & HALI-LS-L2M-1 & FAUNA & 7/13/2011 & $0.001 \pm 0.002$ & 0.002 & $0.004 \pm 0.008$ & 0.008 & $\bar{U}$ & 51.40025 & 179.283361 \\
\hline \multirow{3}{*}{$\begin{array}{l}\text { Horse Mussel - } \\
\text { soft tissue only }\end{array}$} & HMUS-LS-LT1-1 & FAUNA & $7 / 5 / 2011$ & $0.11 \pm 0.04$ & 0.01 & $0.471 \pm 0.171$ & 0.043 & & 51.457555 & 179.195138 \\
\hline & HMUS-LS-LT2-1 & FAUNA & $7 / 4 / 2011$ & $0.14 \pm 0.02$ & 0.005 & $0.572 \pm 0.082$ & 0.02 & & 51.44925 & 179.205583 \\
\hline & HMUS-LS-LT3-1 & FAUNA & $7 / 3 / 2011$ & $0.4 \pm 0.8$ & 0.8 & $0.839 \pm 1.678$ & 1.678 & $U$ & 51.44925 & 179.205583 \\
\hline \multirow{9}{*}{ Irish Lord - whole } & IRLO-LS-LT1-1 & FAUNA & $7 / 4 / 2011$ & $0.01 \pm 0.02$ & 0.01 & $0.047 \pm 0.094$ & 0.047 & $\bar{U}$ & 51.457555 & 179.195138 \\
\hline & IRLO-LS-LT1-2 & FAUNA & $7 / 4 / 2011$ & $0.02 \pm 0.02$ & 0.009 & $0.095 \pm 0.095$ & 0.043 & $\bar{U}$ & 51.457555 & 179.195138 \\
\hline & IRLO-LS-LT1-3 & FAUNA & $7 / 5 / 2011$ & $0.01 \pm 0.01$ & 0.004 & $0.053 \pm 0.053$ & 0.021 & $\mathrm{U}$ & 51.457555 & 179.195138 \\
\hline & IRLO-LS-LT2-1 & FAUNA & $7 / 3 / 2011$ & $0.01 \pm 0.02$ & 0.01 & $0.043 \pm 0.087$ & 0.043 & $\bar{U}$ & 51.432194 & 179.230277 \\
\hline & IRLO-LS-LT2-2 & FAUNA & $7 / 3 / 2011$ & $0.05 \pm 0.04$ & 0.02 & $0.207 \pm 0.166$ & 0.083 & $U$ & 51.432194 & 179.230277 \\
\hline & IRLO-LS-LT2-3 & FAUNA & $7 / 3 / 2011$ & $0.01 \pm 0.02$ & 0.02 & $0.044 \pm 0.089$ & 0.089 & $U$ & 51.432194 & 179.230277 \\
\hline & IRLO-LS-LT3-1 & FAUNA & $7 / 2 / 2011$ & $0.006 \pm 0.004$ & 0.002 & $0.027 \pm 0.018$ & 0.009 & & 51.43 & 179.22667 \\
\hline & IRLO-LS-LT3-2 & FAUNA & $7 / 12 / 2011$ & $-0.002 \pm 0.014$ & 0.02 & $-0.009 \pm 0.066$ & 0.095 & $U$ & 51.434944 & 179.2315 \\
\hline & IRLO-LS-LT3-3 & FAUNA & $7 / 12 / 2011$ & $0.009 \pm 0.016$ & 0.02 & $0.05 \pm 0.088$ & 0.11 & $\mathrm{U}$ & 51.434944 & 179.2315 \\
\hline Octopus & OCTP-LS-L2M-1 & FAUNA & $7 / 3 / 2011$ & $0.019 \pm 0.002$ & 0.0004 & $0.135 \pm 0.014$ & 0.003 & & 51.44925 & 179.205583 \\
\hline $\begin{array}{l}\text { Pacific Cod - } \\
\text { whole }\end{array}$ & PCOD-LS-L2M-1 & FAUNA & 7/4/2011 & $0.003 \pm 0.004$ & 0.002 & $0.015 \pm 0.02$ & 0.01 & $u$ & 51.451805 & 179.196972 \\
\hline \multirow{10}{*}{ Rockfish - whole } & ROCK-LS-DUP & FAUNA & 7/12/2011 & $0.006 \pm 0.012$ & 0.02 & $0.023 \pm 0.047$ & 0.078 & $\mathrm{U}$ & 51.434944 & 179.2315 \\
\hline & ROCK-LS-DUP2 & FAUNA & \begin{tabular}{|l|}
$7 / 12 / 2011$ \\
\end{tabular} & $0.012 \pm 0.01$ & 0.004 & $0.051 \pm 0.043$ & 0.017 & $U$ & 51.465472 & 179.206722 \\
\hline & ROCK-LS-LT1-1 & FAUNA & $7 / 4 / 2011$ & $0.012 \pm 0.016$ & 0.007 & $0.056 \pm 0.074$ & 0.033 & $U$ & 51.457555 & 179.195138 \\
\hline & ROCK-LS-LT1-2 & FAUNA & 7/12/2011 & $0.013 \pm 0.018$ & 0.008 & $0.054 \pm 0.074$ & 0.033 & $\mathrm{U}$ & 51.465472 & 179.206722 \\
\hline & ROCK-LS-LT1-3 & FAUNA & 7/12/2011 & $0.008 \pm 0.016$ & 0.01 & $0.033 \pm 0.066$ & 0.041 & $\mathrm{U}$ & 51.465472 & 179.206722 \\
\hline & ROCK-LS-LT2-1 & FAUNA & $7 / 4 / 2011$ & $0.001 \pm 0.012$ & 0.02 & $0.004 \pm 0.048$ & 0.08 & UJ & 51.44925 & 179.205583 \\
\hline & ROCK-LS-LT2-2 & FAUNA & \begin{tabular}{|l|}
$7 / 12 / 2011$ \\
\end{tabular} & $0.01 \pm 0.02$ & 0.01 & $0.039 \pm 0.079$ & 0.039 & $\mathrm{U}$ & 51.44625 & 179.223416 \\
\hline & ROCK-LS-LT3-1 & FAUNA & 7/12/2011 & $-0.0004 \pm 0.0008$ & 0.004 & $-0.002 \pm 0.004$ & 0.018 & $U$ & 51.434944 & 179.2315 \\
\hline & ROCK-LS-LT3-2 & FAUNA & \begin{tabular}{|l|}
$7 / 12 / 2011$ \\
\end{tabular} & $-0.004 \pm 0.008$ & 0.02 & $-0.017 \pm 0.034$ & 0.085 & UJ & 51.434944 & 179.2315 \\
\hline & ROCK-LS-LT3-3 & FAUNA & $7 / 3 / 2011$ & $0.008 \pm 0.006$ & 0.003 & $0.028 \pm 0.021$ & 0.01 & $U$ & 51.43 & 179.22667 \\
\hline
\end{tabular}




\begin{tabular}{|c|c|c|c|c|c|c|c|c|c|c|}
\hline Matrix Subtype & Location Code & Matrix & $\begin{array}{c}\text { Date } \\
\text { Sampled }\end{array}$ & $\begin{array}{l}\text { Wet Result } \pm \\
\text { Uncertainty }\end{array}$ & $\begin{array}{c}\text { Wet } \\
\text { Detection } \\
\text { Limit }\end{array}$ & $\begin{array}{l}\text { Dry Result } \pm \\
\text { Uncertainty }\end{array}$ & \begin{tabular}{|c|} 
Dry \\
Detection \\
Limit
\end{tabular} & Qualifiers & Latitude & Longitude \\
\hline \begin{tabular}{|l|} 
Reindeer Lichen \\
\end{tabular} & STRL-AI-LT1-2 & VEG & $6 / 22 / 2011$ & $0.06 \pm 0.06$ & 0.03 & $0.182 \pm 0.182$ & 0.091 & $\bar{U}$ & 51.447361 & 179.189111 \\
\hline \multirow{3}{*}{$\begin{array}{l}\text { Sea Urchin - } \\
\text { whole }\end{array}$} & URCH-LS-LT1-1 & FAUNA & $7 / 4 / 2011$ & $0.11 \pm 0.04$ & 0.005 & $0.43 \pm 0.156$ & 0.02 & & 51.457555 & 179.195138 \\
\hline & URCH-LS-LT2-1 & FAUNA & $7 / 3 / 2011$ & $0.09 \pm 0.02$ & 0.004 & $0.346 \pm 0.077$ & 0.015 & & 51.44925 & 179.205583 \\
\hline & URCH-LS-LT3-1 & FAUNA & $7 / 2 / 2011$ & $0.08 \pm 0.02$ & 0.004 & $0.312 \pm 0.078$ & 0.016 & & 51.43 & 179.22667 \\
\hline \multicolumn{11}{|c|}{ Amchitka Site (Milrow) (AMC03) } \\
\hline \multirow{3}{*}{ Chiton } & CHIT-ML-MT1-1 & FAUNA & $7 / 9 / 2011$ & $0.07 \pm 0.04$ & 0.008 & $0.264 \pm 0.151$ & 0.03 & & 51.408472 & 179.139166 \\
\hline & CHIT-ML-MT2-1 & FAUNA & \begin{tabular}{|l|}
$7 / 10 / 2011$ \\
\end{tabular} & $0.07 \pm 0.02$ & 0.002 & $0.325 \pm 0.093$ & 0.009 & & 51.389416 & 179.173138 \\
\hline & CHIT-ML-MT3-1 & FAUNA & $7 / 10 / 2011$ & $0.09 \pm 0.04$ & 0.006 & $0.408 \pm 0.182$ & 0.027 & & 51.387805 & 179.184 \\
\hline \multirow{10}{*}{ Dragon Kelp } & EUAL-ML-DUP & VEG & 7/9/2011 & $0.003 \pm 0.004$ & 0.002 & $0.023 \pm 0.03$ & 0.015 & $\mathrm{U}$ & 51.408472 & 179.139166 \\
\hline & EUAL-ML-MT1-1 & VEG & $7 / 9 / 2011$ & $0.017 \pm 0.002$ & 0.0003 & $0.136 \pm 0.016$ & 0.002 & & 51.408472 & 179.139166 \\
\hline & EUAL-ML-MT1-2 & VEG & $7 / 9 / 2011$ & $0.009 \pm 0.002$ & 0.0004 & $0.067 \pm 0.015$ & 0.003 & & 51.408472 & 179.139166 \\
\hline & EUAL-ML-MT1-3 & VEG & $7 / 9 / 2011$ & $0.003 \pm 0.002$ & 0.001 & $0.025 \pm 0.017$ & 0.008 & $\mathrm{~J}$ & 51.408472 & 179.139166 \\
\hline & EUAL-ML-MT2-1 & VEG & 7/10/2011 & $0.009 \pm 0.004$ & 0.0008 & $0.067 \pm 0.03$ & 0.006 & & 51.389416 & 179.173138 \\
\hline & EUAL-ML-MT2-2 & VEG & 7/10/2011 & $0.002 \pm 0.002$ & 0.002 & $0.014 \pm 0.014$ & 0.014 & $U$ & 51.389416 & 179.173138 \\
\hline & EUAL-ML-MT2-3 & VEG & 7/10/2011 & $0.0002 \pm 0.0018$ & 0.003 & $0.002 \pm 0.014$ & 0.023 & $\mathrm{U}$ & 51.389416 & 179.173138 \\
\hline & EUAL-ML-MT3-1 & VEG & 7/10/2011 & $0.024 \pm 0.004$ & 0.0004 & $0.169 \pm 0.028$ & 0.003 & & 51.387805 & 179.184 \\
\hline & EUAL-ML-MT3-2 & VEG & $7 / 10 / 2011$ & $0.013 \pm 0.006$ & 0.001 & $0.095 \pm 0.044$ & 0.007 & & 51.387805 & 179.184 \\
\hline & EUAL-ML-MT3-3 & VEG & 7/10/2011 & $0.025 \pm 0.004$ & 0.0006 & $0.172 \pm 0.028$ & 0.004 & & 51.387805 & 179.184 \\
\hline \multirow{10}{*}{ Rockweed } & FUCU-ML-DUP & VEG & \begin{tabular}{|l|}
$6 / 20 / 2011$ \\
\end{tabular} & $-0.0004 \pm 0.0006$ & 0.002 & $-0.002 \pm 0.003$ & 0.009 & $U$ & 51.412449 & 179.160843 \\
\hline & FUCU-ML-MT1-1 & VEG & 6/20/2011 & $-0.0006 \pm 0.0008$ & 0.002 & $-0.002 \pm 0.003$ & 0.008 & $U$ & 51.412449 & 179.160843 \\
\hline & FUCU-ML-MT1-2 & VEG & 6/21/2011 & $0.001 \pm 0.002$ & 0.002 & $0.006 \pm 0.012$ & 0.012 & U & 51.417083 & 179.134083 \\
\hline & FUCU-ML-MT1-3 & VEG & 7/9/2011 & $0.0006 \pm 0.001$ & 0.0006 & $0.003 \pm 0.005$ & 0.003 & $u$ & 51.417083 & 179.134083 \\
\hline & FUCU-ML-MT2-1 & VEG & 6/20/2011 & $-0.0002 \pm 0.0004$ & 0.001 & $-0.001 \pm 0.002$ & 0.005 & U & 51.403305 & 179.167166 \\
\hline & FUCU-ML-MT2-2 & VEG & 6/21/2011 & $-0.002 \pm 0.004$ & 0.006 & $-0.014 \pm 0.028$ & 0.042 & UJ & 51.405694 & 179.161361 \\
\hline & FUCU-ML-MT2-3 & VEG & $6 / 21 / 2011$ & $0.002 \pm 0.004$ & 0.004 & $0.014 \pm 0.028$ & 0.028 & UJ & 51.405694 & 179.161361 \\
\hline & FUCU-ML-MT3-1 & VEG & \begin{tabular}{|l|}
$7 / 10 / 2011$ \\
\end{tabular} & $0.002 \pm 0.002$ & 0.001 & $0.01 \pm 0.01$ & 0.005 & $U$ & 51.389416 & 179.173138 \\
\hline & FUCU-ML-MT3-2 & VEG & \begin{tabular}{|l|}
$7 / 10 / 2011$ \\
\end{tabular} & $0.011 \pm 0.004$ & 0.0006 & $0.053 \pm 0.019$ & 0.003 & & 51.389416 & 179.173138 \\
\hline & FUCU-ML-MT3-3 & VEG & 7/10/2011 & $-0.0008 \pm 0.0016$ & 0.005 & $-0.004 \pm 0.008$ & 0.025 & UJ & 51.389416 & 179.173138 \\
\hline
\end{tabular}




\begin{tabular}{|c|c|c|c|c|c|c|c|c|c|c|}
\hline Matrix Subtype & Location Code & Matrix & $\begin{array}{c}\text { Date } \\
\text { Sampled }\end{array}$ & $\begin{array}{l}\text { Wet Result } \pm \\
\text { Uncertainty }\end{array}$ & $\begin{array}{c}\text { Wet } \\
\text { Detection } \\
\text { Limit }\end{array}$ & $\begin{array}{l}\text { Dry Result } \pm \\
\text { Uncertainty }\end{array}$ & \begin{tabular}{|c|} 
Dry \\
Detection \\
Limit
\end{tabular} & Qualifiers & Latitude & Longitude \\
\hline \multirow{10}{*}{ Greenling - whole } & GREN-ML-DUP & FAUNA & \begin{tabular}{|l|}
$7 / 10 / 2011$ \\
\end{tabular} & $0.02 \pm 0.02$ & 0.007 & $0.097 \pm 0.097$ & 0.034 & $\bar{U}$ & 51.387805 & 179.184 \\
\hline & GREN-ML-MT1-1 & FAUNA & $7 / 9 / 2011$ & $0.005 \pm 0.004$ & 0.002 & $0.022 \pm 0.017$ & 0.009 & $\mathrm{U}$ & 51.408472 & 179.139166 \\
\hline & GREN-ML-MT1-2 & FAUNA & $7 / 9 / 2011$ & $0.01 \pm 0.02$ & 0.01 & $0.046 \pm 0.093$ & 0.046 & $U$ & 51.408472 & 179.139166 \\
\hline & GREN-ML-MT1-3 & FAUNA & $7 / 9 / 2011$ & $-0.001 \pm 0.002$ & 0.01 & $-0.005 \pm 0.01$ & 0.048 & $U$ & 51.408472 & 179.139166 \\
\hline & GREN-ML-MT2-1 & FAUNA & 7/11/2011 & $0.03 \pm 0.02$ & 0.006 & $0.14 \pm 0.093$ & 0.028 & & 51.396944 & 179.1435 \\
\hline & GREN-ML-MT2-2 & FAUNA & 7/111/2011 & $0.02 \pm 0.02$ & 0.005 & $0.094 \pm 0.094$ & 0.023 & $\mathrm{U}$ & 51.396944 & 179.1435 \\
\hline & GREN-ML-MT2-3 & FAUNA & 7/11/2011 & $0.02 \pm 0.02$ & 0.005 & $0.095 \pm 0.095$ & 0.024 & $U$ & 51.396944 & 179.1435 \\
\hline & GREN-ML-MT3-1 & FAUNA & \begin{tabular}{|l|}
$7 / 10 / 2011$ \\
\end{tabular} & $0.01 \pm 0.02$ & 0.005 & $0.048 \pm 0.097$ & 0.024 & $\mathrm{U}$ & 51.387805 & 179.184 \\
\hline & GREN-ML-MT3-2 & FAUNA & 7/10/2011 & $0.05 \pm 0.04$ & 0.02 & $0.241 \pm 0.193$ & 0.096 & $U$ & 51.387805 & 179.184 \\
\hline & GREN-ML-MT3-3 & FAUNA & 7/10/2011 & $0.034 \pm 0.008$ & 0.002 & $0.161 \pm 0.038$ & 0.009 & & 51.387805 & 179.184 \\
\hline Halibut - whole & HALI-ML-M2M-1 & FAUNA & 7/11/2011 & $0.0006 \pm 0.0018$ & 0.001 & $0.002 \pm 0.006$ & 0.003 & $\bar{U}$ & 51.365055 & 179.111472 \\
\hline \multirow{3}{*}{$\begin{array}{l}\text { Horse Mussel - } \\
\text { soft tissue only }\end{array}$} & HMUS-ML-MT1-1 & FAUNA & $7 / 9 / 2011$ & $0.11 \pm 0.04$ & 0.01 & $0.5 \pm 0.182$ & 0.045 & & 51.408472 & 179.139166 \\
\hline & HMUS-ML-MT2-1 & FAUNA & 7/111/2011 & $0.21 \pm 0.08$ & 0.01 & $0.944 \pm 0.36$ & 0.045 & & 51.389416 & 179.173138 \\
\hline & HMUS-ML-MT3-1 & FAUNA & 7/10/2011 & $0.07 \pm 0.08$ & 0.04 & $0.321 \pm 0.367$ & 0.184 & $\mathrm{U}$ & 51.387805 & 179.184 \\
\hline \multirow{9}{*}{ Irish Lord - whole } & IRLO-ML-MT1-1 & FAUNA & $7 / 9 / 2011$ & $0.004 \pm 0.01$ & 0.01 & $0.017 \pm 0.043$ & 0.043 & $\mathrm{U}$ & 51.408472 & 179.139166 \\
\hline & IRLO-ML-MT1-2 & FAUNA & $7 / 9 / 2011$ & $0.02 \pm 0.02$ & 0.01 & $0.095 \pm 0.095$ & 0.047 & $\bar{U}$ & 51.408472 & 179.139166 \\
\hline & IRLO-ML-MT1-3 & FAUNA & $7 / 9 / 2011$ & $0.012 \pm 0.014$ & 0.007 & $0.056 \pm 0.066$ & 0.033 & $U$ & 51.408472 & 179.139166 \\
\hline & IRLO-ML-MT2-1 & FAUNA & \begin{tabular}{|l|}
$7 / 10 / 2011$ \\
\end{tabular} & $0.003 \pm 0.006$ & 0.01 & $0.013 \pm 0.026$ & 0.043 & $\bar{U}$ & 51.389416 & 179.173138 \\
\hline & IRLO-ML-MT2-2 & FAUNA & 7/11/2011 & $0.009 \pm 0.016$ & 0.008 & $0.039 \pm 0.069$ & 0.034 & $U$ & 51.396944 & 179.1435 \\
\hline & IRLO-ML-MT2-3 & FAUNA & 7/11/2011 & $0.005 \pm 0.014$ & 0.01 & $0.024 \pm 0.066$ & 0.047 & $\mathrm{U}$ & 51.396944 & 179.1435 \\
\hline & IRLO-ML-MT3-1 & FAUNA & $7 / 10 / 2011$ & $0.02 \pm 0.02$ & 0.02 & $0.113 \pm 0.113$ & 0.113 & $\mathrm{U}$ & 51.387805 & 179.184 \\
\hline & IRLO-ML-MT3-2 & FAUNA & \begin{tabular}{|l|}
$7 / 10 / 2011$ \\
\end{tabular} & $0.03 \pm 0.02$ & 0.01 & $0.145 \pm 0.097$ & 0.048 & & 51.387805 & 179.184 \\
\hline & IRLO-ML-MT3-3 & FAUNA & $7 / 10 / 2011$ & $0.01 \pm 0.02$ & 0.008 & $0.056 \pm 0.112$ & 0.045 & $\mathrm{U}$ & 51.387805 & 179.184 \\
\hline Octopus & OCTP-ML-M2M-1 & FAUNA & 7/10/2011 & $0.009 \pm 0.012$ & 0.007 & $0.042 \pm 0.057$ & 0.033 & $\mathrm{U}$ & 51.389416 & 179.173138 \\
\hline $\begin{array}{l}\text { Pacific Cod - } \\
\text { whole }\end{array}$ & PCOD-ML-M2M-1 & FAUNA & 7/10/2011 & $0.001 \pm 0.002$ & 0.001 & $0.005 \pm 0.011$ & 0.005 & $U$ & 51.408472 & 179.139166 \\
\hline \multirow{10}{*}{ Rockfish - whole } & ROCK-ML-DUP & FAUNA & $7 / 9 / 2011$ & $0.004 \pm 0.012$ & 0.01 & $0.018 \pm 0.053$ & 0.044 & $\mathrm{U}$ & 51.408472 & 179.139166 \\
\hline & ROCK-ML-MT1-1 & FAUNA & $7 / 9 / 2011$ & $0.004 \pm 0.008$ & 0.004 & $0.018 \pm 0.035$ & 0.018 & $U$ & 51.408472 & 179.139166 \\
\hline & ROCK-ML-MT1-2 & FAUNA & $7 / 9 / 2011$ & $0.011 \pm 0.012$ & 0.009 & $0.048 \pm 0.053$ & 0.04 & $U$ & 51.408472 & 179.139166 \\
\hline & ROCK-ML-MT1-3 & FAUNA & $7 / 9 / 2011$ & $-0.001 \pm 0.002$ & 0.01 & $-0.004 \pm 0.008$ & 0.042 & $\mathrm{U}$ & 51.408472 & 179.139166 \\
\hline & ROCK-ML-MT2-1 & FAUNA & 7/11/2011 & $-0.0008 \pm 0.0012$ & 0.007 & $-0.003 \pm 0.005$ & 0.026 & $\mathrm{U}$ & 51.365055 & 179.111472 \\
\hline & ROCK-ML-MT2-2 & FAUNA & 7/11/2011 & $0.07 \pm 0.04$ & 0.009 & $0.283 \pm 0.162$ & 0.036 & & 51.365055 & 179.111472 \\
\hline & ROCK-ML-MT2-3 & FAUNA & 7/11/2011 & $0.008 \pm 0.012$ & 0.005 & $0.036 \pm 0.054$ & 0.023 & $U$ & 51.365055 & 179.111472 \\
\hline & ROCK-ML-MT3-1 & FAUNA & \begin{tabular}{|l|}
$7 / 10 / 2011$ \\
\end{tabular} & $0.009 \pm 0.006$ & 0.004 & $0.042 \pm 0.028$ & 0.019 & $\mathrm{~J}$ & 51.387805 & 179.184 \\
\hline & ROCK-ML-MT3-2 & FAUNA & 7/10/2011 & $0.002 \pm 0.008$ & 0.01 & $0.007 \pm 0.029$ & 0.036 & $\mathrm{U}$ & 51.387805 & 179.184 \\
\hline & ROCK-ML-MT3-3 & FAUNA & 7/10/2011 & $0.004 \pm 0.006$ & 0.004 & $0.009 \pm 0.014$ & 0.009 & $U$ & 51.387805 & 179.184 \\
\hline
\end{tabular}




\begin{tabular}{|c|c|c|c|c|c|c|c|c|c|c|}
\hline Matrix Subtype & Location Code & Matrix & $\begin{array}{c}\text { Date } \\
\text { Sampled }\end{array}$ & $\begin{array}{l}\text { Wet Result } \pm \\
\text { Uncertainty }\end{array}$ & $\begin{array}{c}\text { Wet } \\
\text { Detection } \\
\text { Limit }\end{array}$ & $\begin{array}{l}\text { Dry Result } \pm \\
\text { Uncertainty }\end{array}$ & \begin{tabular}{|c|} 
Dry \\
Detection \\
Limit
\end{tabular} & Qualifiers & Latitude & Longitude \\
\hline \begin{tabular}{|l|} 
Reindeer Lichen \\
\end{tabular} & STRL-AI-ML-1 & VEG & $6 / 21 / 2011$ & $0.03 \pm 0.02$ & 0.007 & $0.107 \pm 0.072$ & 0.025 & & 51.402583 & 179.183972 \\
\hline \multirow{3}{*}{$\begin{array}{l}\text { Sea Urchin - } \\
\text { whole }\end{array}$} & URCH-ML-MT1-1 & FAUNA & $7 / 9 / 2011$ & $0.15 \pm 0.02$ & 0.002 & $0.556 \pm 0.074$ & 0.007 & & 51.408472 & 179.139166 \\
\hline & URCH-ML-MT2-1 & FAUNA & $7 / 10 / 2011$ & $0.08 \pm 0.04$ & 0.005 & $0.299 \pm 0.149$ & 0.019 & & 51.389416 & 179.173138 \\
\hline & URCH-ML-MT3-1 & FAUNA & $7 / 10 / 2011$ & $0.12 \pm 0.06$ & 0.009 & $0.476 \pm 0.238$ & 0.036 & & 51.387805 & 179.184 \\
\hline \multicolumn{11}{|c|}{ Amchitka Site (Cannikin) (AMC05) } \\
\hline \multirow{3}{*}{ Chiton } & CHIT-CN-CT1-1 & FAUNA & $7 / 8 / 2011$ & $0.05 \pm 0.04$ & 0.01 & $0.234 \pm 0.187$ & 0.047 & $\mathrm{U}$ & 51.482972 & 179.123833 \\
\hline & CHIT-CN-CT2-1 & FAUNA & 7/7/2011 & $0.07 \pm 0.02$ & 0.004 & $0.331 \pm 0.095$ & 0.019 & & 51.472611 & 179.151111 \\
\hline & CHIT-CN-CT3-1 & FAUNA & $7 / 5 / 2011$ & $0.11 \pm 0.04$ & 0.005 & $0.54 \pm 0.196$ & 0.025 & & 51.471416 & 179.172861 \\
\hline \multirow{10}{*}{ Dragon Kelp } & EUAL-CN-CT1-1 & VEG & $7 / 8 / 2011$ & $0.009 \pm 0.004$ & 0.001 & $0.067 \pm 0.03$ & 0.007 & & 51.482972 & 179.123833 \\
\hline & EUAL-CN-CT1-2 & VEG & $7 / 8 / 2011$ & $0.0005 \pm 0.001$ & 0.002 & $0.003 \pm 0.007$ & 0.013 & $\mathrm{U}$ & 51.482972 & 179.123833 \\
\hline & EUAL-CN-CT1-3 & VEG & $7 / 8 / 2011$ & $0.016 \pm 0.004$ & 0.0004 & $0.098 \pm 0.025$ & 0.002 & & 51.482972 & 179.123833 \\
\hline & EUAL-CN-CT2-1 & VEG & $7 / 6 / 2011$ & $0.01 \pm 0.002$ & 0.0005 & $0.064 \pm 0.013$ & 0.003 & & 51.472611 & 179.151111 \\
\hline & EUAL-CN-CT2-2 & VEG & $7 / 6 / 2011$ & $0.02 \pm 0.004$ & 0.0006 & $0.126 \pm 0.025$ & 0.004 & & 51.472611 & 179.151111 \\
\hline & EUAL-CN-CT2-3 & VEG & 7/7/2011 & $0.018 \pm 0.004$ & 0.0005 & $0.13 \pm 0.029$ & 0.004 & & 51.472611 & 179.151111 \\
\hline & EUAL-CN-CT3-1 & VEG & $7 / 5 / 2011$ & $0.023 \pm 0.004$ & 0.0008 & $0.16 \pm 0.028$ & 0.006 & & 51.471416 & 179.172861 \\
\hline & EUAL-CN-CT3-2 & VEG & $7 / 5 / 2011$ & $0.0009 \pm 0.0016$ & 0.002 & $0.006 \pm 0.012$ & 0.014 & U & 51.471416 & 179.172861 \\
\hline & EUAL-CN-CT3-3 & VEG & $7 / 5 / 2011$ & $0.029 \pm 0.004$ & 0.0003 & $0.188 \pm 0.026$ & 0.002 & & 51.471416 & 179.172861 \\
\hline & EUAL-CN-DUP & VEG & $7 / 6 / 2011$ & $0.005 \pm 0.004$ & 0.0014 & $0.033 \pm 0.027$ & 0.009 & U & 51.471416 & 179.172861 \\
\hline \multirow{10}{*}{ Rockweed } & FUCU-CN-CT1-1 & VEG & \begin{tabular}{|c|}
$6 / 23 / 2011$ \\
\end{tabular} & $0.0006 \pm 0.0012$ & 0.002 & $0.003 \pm 0.007$ & 0.012 & $U$ & 51.481888 & 179.118138 \\
\hline & FUCU-CN-CT1-2 & VEG & 6/23/2011 & $0.0007 \pm 0.0024$ & 0.002 & $0.004 \pm 0.015$ & 0.013 & $U$ & 51.481888 & 179.118138 \\
\hline & FUCU-CN-CT1-3 & VEG & 6/23/2011 & $0.002 \pm 0.002$ & 0.0005 & $0.012 \pm 0.012$ & 0.003 & U & 51.481888 & 179.118138 \\
\hline & FUCU-CN-CT2-1 & VEG & $7 / 8 / 2011$ & $0.002 \pm 0.004$ & 0.004 & $0.008 \pm 0.017$ & 0.017 & UJ & 51.476638 & 179.135277 \\
\hline & FUCU-CN-CT2-2 & VEG & $7 / 8 / 2011$ & $0.004 \pm 0.004$ & 0.001 & $0.018 \pm 0.018$ & 0.004 & $\mathrm{U}$ & 51.476638 & 179.135277 \\
\hline & FUCU-CN-CT2-3 & VEG & $7 / 12 / 2011$ & $0.005 \pm 0.002$ & 0.0006 & $0.026 \pm 0.011$ & 0.003 & & 51.467583 & 179.153583 \\
\hline & FUCU-CN-CT3-1 & VEG & $7 / 6 / 2011$ & $0.005 \pm 0.004$ & 0.001 & $0.027 \pm 0.022$ & 0.005 & $\mathrm{U}$ & 51.465027 & 179.173166 \\
\hline & FUCU-CN-CT3-2 & VEG & $7 / 6 / 2011$ & $0.004 \pm 0.002$ & 0.0007 & $0.023 \pm 0.012$ & 0.004 & & 51.465472 & 179.170583 \\
\hline & FUCU-CN-CT3-3 & VEG & $7 / 6 / 2011$ & $-0.0001 \pm 0.0002$ & 0.001 & $-0.001 \pm 0.001$ & 0.006 & $\mathrm{U}$ & 51.464638 & 179.159305 \\
\hline & FUCU-CN-DUP & VEG & 6/23/2011 & $0.001 \pm 0.002$ & 0.001 & $0.006 \pm 0.013$ & 0.006 & $u$ & 51.481888 & 179.118138 \\
\hline
\end{tabular}




\begin{tabular}{|c|c|c|c|c|c|c|c|c|c|c|}
\hline Matrix Subtype & Location Code & Matrix & $\begin{array}{c}\text { Date } \\
\text { Sampled }\end{array}$ & $\begin{array}{l}\text { Wet Result } \pm \\
\text { Uncertainty }\end{array}$ & $\begin{array}{c}\text { Wet } \\
\text { Detection } \\
\text { Limit }\end{array}$ & $\begin{array}{l}\text { Dry Result } \pm \\
\text { Uncertainty }\end{array}$ & \begin{tabular}{|c|} 
Dry \\
Detection \\
Limit
\end{tabular} & Qualifiers & Latitude & Longitude \\
\hline \multirow{10}{*}{ Greenling - whole } & GREN-CN-CT1-1 & FAUNA & $7 / 8 / 2011$ & $0.02 \pm 0.02$ & 0.006 & $0.096 \pm 0.096$ & 0.029 & $\bar{U}$ & 51.482972 & 179.123833 \\
\hline & GREN-CN-CT1-2 & FAUNA & $7 / 8 / 2011$ & $0.01 \pm 0.02$ & 0.007 & $0.047 \pm 0.095$ & 0.033 & $\mathrm{U}$ & 51.482972 & 179.123833 \\
\hline & GREN-CN-CT1-3 & FAUNA & $7 / 8 / 2011$ & $0.005 \pm 0.008$ & 0.004 & $0.023 \pm 0.036$ & 0.018 & $U$ & 51.482972 & 179.123833 \\
\hline & GREN-CN-CT2-1 & FAUNA & 7/7/2011 & $0.003 \pm 0.006$ & 0.005 & $0.015 \pm 0.029$ & 0.024 & $U$ & 51.472611 & 179.151111 \\
\hline & GREN-CN-CT2-2 & FAUNA & 7/7/2011 & $0.01 \pm 0.02$ & 0.004 & $0.046 \pm 0.092$ & 0.018 & $\bar{U}$ & 51.472611 & 179.151111 \\
\hline & GREN-CN-CT2-3 & FAUNA & $7 / 7 / 2011$ & $0.005 \pm 0.006$ & 0.003 & $0.023 \pm 0.028$ & 0.014 & $\mathrm{U}$ & 51.472611 & 179.151111 \\
\hline & GREN-CN-CT3-1 & FAUNA & $7 / 5 / 2011$ & $0.01 \pm 0.02$ & 0.005 & $0.046 \pm 0.091$ & 0.023 & $U$ & 51.471416 & 179.172861 \\
\hline & GREN-CN-CT3-2 & FAUNA & $7 / 5 / 2011$ & $0.02 \pm 0.02$ & 0.01 & $0.087 \pm 0.087$ & 0.044 & $\bar{U}$ & 51.471416 & 179.172861 \\
\hline & GREN-CN-CT3-3 & FAUNA & $7 / 5 / 2011$ & $0.01 \pm 0.02$ & 0.004 & $0.046 \pm 0.092$ & 0.018 & $U$ & 51.471416 & 179.172861 \\
\hline & GREN-CN-DUP & FAUNA & $7 / 8 / 2011$ & $0.03 \pm 0.02$ & 0.007 & $0.145 \pm 0.096$ & 0.034 & & 51.482972 & 179.123833 \\
\hline Halibut - whole & HALI-CN-C2M-1 & FAUNA & 7/13/2011 & $0.001 \pm 0.002$ & 0.001 & $0.004 \pm 0.008$ & 0.004 & $\bar{U}$ & 51.40025 & 179.283361 \\
\hline \multirow{3}{*}{$\begin{array}{l}\text { Horse Mussel - } \\
\text { soft tissue only }\end{array}$} & HMUS-CN-CT1-1 & FAUNA & $7 / 8 / 2011$ & $0.02 \pm 0.04$ & 0.06 & $0.082 \pm 0.164$ & 0.247 & $U$ & 51.482972 & 179.123833 \\
\hline & HMUS-CN-CT2-1 & FAUNA & $7 / 7 / 2011$ & $0.12 \pm 0.06$ & 0.02 & $0.544 \pm 0.272$ & 0.091 & & 51.472611 & 179.151111 \\
\hline & HMUS-CN-CT3-1 & FAUNA & $7 / 5 / 2011$ & $0.11 \pm 0.06$ & 0.01 & $0.451 \pm 0.246$ & 0.041 & & 51.471416 & 179.172861 \\
\hline \multirow{9}{*}{ Irish Lord - whole } & IRLO-CN-CT1-1 & FAUNA & $7 / 8 / 2011$ & $0.013 \pm 0.008$ & 0.005 & $0.059 \pm 0.036$ & 0.023 & $\mathrm{~J}$ & 51.482972 & 179.123833 \\
\hline & IRLO-CN-CT1-2 & FAUNA & $7 / 8 / 2011$ & $0.01 \pm 0.02$ & 0.01 & $0.05 \pm 0.1$ & 0.05 & $\bar{U}$ & 51.482972 & 179.123833 \\
\hline & IRLO-CN-CT1-3 & FAUNA & $7 / 8 / 2011$ & $0.004 \pm 0.012$ & 0.009 & $0.019 \pm 0.056$ & 0.042 & $\mathrm{U}$ & 51.482972 & 179.123833 \\
\hline & IRLO-CN-CT2-1 & FAUNA & 7/7/2011 & $0.011 \pm 0.01$ & 0.009 & $0.052 \pm 0.047$ & 0.042 & $\bar{U}$ & 51.472611 & 179.151111 \\
\hline & IRLO-CN-CT2-2 & FAUNA & $7 / 7 / 2011$ & $0.01 \pm 0.02$ & 0.02 & $0.046 \pm 0.091$ & 0.091 & $U$ & 51.472611 & 179.151111 \\
\hline & IRLO-CN-CT2-3 & FAUNA & $7 / 7 / 2011$ & $0.006 \pm 0.008$ & 0.005 & $0.03 \pm 0.039$ & 0.025 & $\mathrm{U}$ & 51.472611 & 179.151111 \\
\hline & IRLO-CN-CT3-1 & FAUNA & $7 / 5 / 2011$ & $0.014 \pm 0.016$ & 0.01 & $0.072 \pm 0.082$ & 0.052 & $\mathrm{U}$ & 51.471416 & 179.172861 \\
\hline & IRLO-CN-CT3-2 & FAUNA & $7 / 5 / 2011$ & $0.006 \pm 0.008$ & 0.005 & $0.029 \pm 0.039$ & 0.024 & $U$ & 51.471416 & 179.172861 \\
\hline & IRLO-CN-CT3-3 & FAUNA & $7 / 5 / 2011$ & $-0.001 \pm 0.002$ & 0.006 & $-0.005 \pm 0.01$ & 0.029 & $\mathrm{U}$ & 51.471416 & 179.172861 \\
\hline Octopus & OCTP-CN-C2M-1 & FAUNA & $7 / 8 / 2011$ & $0.017 \pm 0.01$ & 0.003 & $0.083 \pm 0.049$ & 0.015 & & 51.482972 & 179.123833 \\
\hline $\begin{array}{l}\text { Pacific Cod - } \\
\text { whole }\end{array}$ & PCOD-CN-C2M-1 & FAUNA & $7 / 6 / 2011$ & $0.0004 \pm 0.0016$ & 0.0019 & $0.002 \pm 0.007$ & 0.009 & U & 51.483527 & 179.187583 \\
\hline \multirow{10}{*}{ Rockfish - whole } & ROCK-CN-CT1-1 & FAUNA & $7 / 8 / 2011$ & $-0.002 \pm 0.004$ & 0.012 & $-0.008 \pm 0.016$ & 0.047 & $\mathrm{U}$ & 51.482972 & 179.123833 \\
\hline & ROCK-CN-CT1-2 & FAUNA & $7 / 8 / 2011$ & $0.007 \pm 0.01$ & 0.01 & $0.027 \pm 0.039$ & 0.039 & $U$ & 51.482972 & 179.123833 \\
\hline & ROCK-CN-CT1-3 & FAUNA & $7 / 8 / 2011$ & $0.03 \pm 0.02$ & 0.03 & $0.121 \pm 0.08$ & 0.121 & $\mathrm{~J}$ & 51.482972 & 179.123833 \\
\hline & ROCK-CN-CT2-1 & FAUNA & $7 / 6 / 2011$ & $0.001 \pm 0.004$ & 0.003 & $0.004 \pm 0.014$ & 0.011 & $\mathrm{U}$ & 51.472611 & 179.151111 \\
\hline & ROCK-CN-CT2-2 & FAUNA & 7/6/2011 & $0.004 \pm 0.008$ & 0.02 & $0.015 \pm 0.029$ & 0.073 & $\mathrm{U}$ & 51.472611 & 179.151111 \\
\hline & ROCK-CN-CT2-3 & FAUNA & $7 / 6 / 2011$ & $0.004 \pm 0.006$ & 0.003 & $0.016 \pm 0.025$ & 0.012 & $U$ & 51.472611 & 179.151111 \\
\hline & ROCK-CN-CT3-1 & FAUNA & $7 / 6 / 2011$ & $0.01 \pm 0.016$ & 0.01 & $0.043 \pm 0.068$ & 0.043 & $\mathrm{U}$ & 51.471416 & 179.172861 \\
\hline & ROCK-CN-CT3-2 & FAUNA & $7 / 6 / 2011$ & $0.006 \pm 0.01$ & 0.004 & $0.023 \pm 0.038$ & 0.015 & $U$ & 51.471416 & 179.172861 \\
\hline & ROCK-CN-CT3-3 & FAUNA & $7 / 6 / 2011$ & $0.01 \pm 0.01$ & 0.005 & $0.042 \pm 0.042$ & 0.021 & $U$ & 51.471416 & 179.172861 \\
\hline & ROCK-CN-DUP & FAUNA & $7 / 6 / 2011$ & $0.003 \pm 0.006$ & 0.004 & $0.012 \pm 0.024$ & 0.016 & $U$ & 51.471416 & 179.172861 \\
\hline
\end{tabular}




\begin{tabular}{|c|c|c|c|c|c|c|c|c|c|c|}
\hline Matrix Subtype & Location Code & Matrix & $\begin{array}{c}\text { Date } \\
\text { Sampled }\end{array}$ & $\begin{array}{l}\text { Wet Result } \pm \\
\text { Uncertainty }\end{array}$ & $\begin{array}{c}\text { Wet } \\
\text { Detection } \\
\text { Limit }\end{array}$ & $\begin{array}{l}\text { Dry Result } \pm \\
\text { Uncertainty }\end{array}$ & \begin{tabular}{|c|} 
Dry \\
Detection \\
Limit
\end{tabular} & Qualifiers & Latitude & Longitude \\
\hline \begin{tabular}{|l|} 
Reindeer Lichen \\
\end{tabular} & STRL-AI-CT1-1 & VEG & 6/23/2011 & $-0.001 \pm 0.002$ & 0.01 & $-0.002 \pm 0.003$ & 0.016 & $\bar{U}$ & 51.480583 & \begin{tabular}{|l}
179.109472 \\
\end{tabular} \\
\hline \multirow{3}{*}{$\begin{array}{l}\text { Sea Urchin - } \\
\text { whole }\end{array}$} & URCH-CN-CT1-1 & FAUNA & $7 / 8 / 2011$ & $0.2 \pm 0.04$ & 0.003 & $0.802 \pm 0.16$ & 0.012 & & 51.482972 & 179.123833 \\
\hline & URCH-CN-CT2-1 & FAUNA & $7 / 7 / 2011$ & $0.1 \pm 0.08$ & 0.01 & $0.343 \pm 0.274$ & 0.034 & $U$ & 51.472611 & $\mid 179.151111$ \\
\hline & URCH-CN-CT3-1 & FAUNA & $7 / 5 / 2011$ & $0.11 \pm 0.04$ & 0.004 & $0.421 \pm 0.153$ & 0.015 & & 51.471416 & \begin{tabular}{|l|}
179.172861 \\
\end{tabular} \\
\hline \multicolumn{11}{|c|}{ Amchitka Site (Adak Island) (AMC13) } \\
\hline $\begin{array}{l}\text { Dolly Varden - } \\
\text { whole }\end{array}$ & DOLL-AD-XXX-1 & FAUNA & $6 / 28 / 2011$ & $0.003 \pm 0.004$ & 0.002 & $0.012 \pm 0.016$ & 0.008 & $u$ & 51.8375 & -176.672555 \\
\hline \multirow{3}{*}{ Reindeer Lichen } & STRL-AD-XXX-1 & VEG & \begin{tabular}{|l|}
$6 / 28 / 2011$ \\
\end{tabular} & $0.12 \pm 0.04$ & 0.004 & $0.235 \pm 0.078$ & 0.008 & & 51.837527 & $\mid-176.670694$ \\
\hline & STRL-AD-XXX-2 & VEG & \begin{tabular}{|l|}
$6 / 29 / 2011$ \\
\end{tabular} & $0.04 \pm 0.02$ & 0.008 & $0.099 \pm 0.049$ & 0.02 & & 51.872833 & \begin{tabular}{|l}
-176.707444 \\
\end{tabular} \\
\hline & STRL-AD-XXX-3 & VEG & \begin{tabular}{|l|}
$6 / 29 / 2011$ \\
\end{tabular} & $0.1 \pm 0.04$ & 0.008 & $0.159 \pm 0.064$ & 0.013 & & 51.900805 & $\mid-176.688527$ \\
\hline \multicolumn{11}{|c|}{ Amchitka Site (Adak Island North) (AMC14) } \\
\hline \multirow{3}{*}{ Chiton } & CHIT-AN-ANT1-1 & FAUNA & $7 / 20 / 2011$ & $0.08 \pm 0.02$ & 0.003 & $0.39 \pm 0.097$ & 0.015 & & 51.838722 & -176.582805 \\
\hline & CHIT-AN-ANT2-1 & FAUNA & $7 / 18 / 2011$ & $0.04 \pm 0.04$ & 0.02 & $0.2 \pm 0.2$ & 0.1 & $\mathrm{U}$ & 51.848361 & -176.506277 \\
\hline & CHIT-AN-ANT3-1 & FAUNA & $7 / 17 / 2011$ & $0.01 \pm 0.02$ & 0.02 & $0.051 \pm 0.102$ & 0.102 & $\mathrm{U}$ & 51.853722 & -176.470694 \\
\hline \multirow{10}{*}{ Dragon Kelp } & EUAL-AN-ANT1-1 & VEG & 7/19/2011 & $0.009 \pm 0.002$ & 0.0006 & $0.075 \pm 0.017$ & 0.005 & & 51.835694 & -176.542138 \\
\hline & EUAL-AN-ANT1-2 & VEG & $7 / 19 / 2011$ & $0.006 \pm 0.002$ & 0.0008 & $0.041 \pm 0.014$ & 0.005 & & 51.835694 & -176.542138 \\
\hline & EUAL-AN-ANT1-3 & VEG & $7 / 19 / 2011$ & $0.01 \pm 0.002$ & 0.0004 & $0.077 \pm 0.015$ & 0.003 & & 51.835694 & -176.542138 \\
\hline & EUAL-AN-ANT2-1 & VEG & 7/18/2011 & $0.011 \pm 0.002$ & 0.0003 & $0.08 \pm 0.015$ & 0.002 & & 51.848361 & -176.506277 \\
\hline & EUAL-AN-ANT2-2 & VEG & $7 / 18 / 2011$ & $0.011 \pm 0.002$ & 0.0005 & $0.07 \pm 0.013$ & 0.003 & & 51.848361 & -176.506277 \\
\hline & EUAL-AN-ANT2-3 & VEG & $7 / 18 / 2011$ & $0.009 \pm 0.002$ & 0.0005 & $0.063 \pm 0.014$ & 0.003 & & 51.848361 & \begin{tabular}{|l|l|}
-176.506277 \\
\end{tabular} \\
\hline & EUAL-AN-ANT3-1 & VEG & \begin{tabular}{|l|}
$7 / 17 / 2011$ \\
\end{tabular} & $0.0007 \pm 0.0016$ & 0.002 & $0.005 \pm 0.012$ & 0.015 & $\bar{U}$ & 51.853722 & \begin{tabular}{|l|}
-176.470694 \\
\end{tabular} \\
\hline & EUAL-AN-ANT3-2 & VEG & $7 / 17 / 2011$ & $0.026 \pm 0.004$ & 0.0004 & $0.177 \pm 0.027$ & 0.003 & & 51.853722 & -176.470694 \\
\hline & EUAL-AN-ANT3-3 & VEG & 7/17/2011 & $0.006 \pm 0.004$ & 0.001 & $0.04 \pm 0.027$ & 0.007 & & 51.853722 & \begin{tabular}{|l|}
-176.470694 \\
\end{tabular} \\
\hline & EUAL-AN-DUP & VEG & 7/17/2011 & $0.002 \pm 0.004$ & 0.007 & $0.013 \pm 0.025$ & 0.044 & UJ & 51.853722 & -176.470694 \\
\hline \multirow{10}{*}{ Rockweed } & FUCU-AN-ANT1-1 & VEG & \begin{tabular}{|l|}
$7 / 19 / 2011$ \\
\end{tabular} & $0.007 \pm 0.004$ & 0.0006 & $0.036 \pm 0.02$ & 0.003 & & 51.841444 & $\mid-176.546083$ \\
\hline & FUCU-AN-ANT1-2 & VEG & $7 / 20 / 2011$ & $0.004 \pm 0.006$ & 0.005 & $0.026 \pm 0.039$ & 0.032 & $\overline{U J}$ & 51.8395 & -176.591305 \\
\hline & FUCU-AN-ANT1-3 & VEG & \begin{tabular}{|l|}
$7 / 20 / 2011$ \\
\end{tabular} & $0.001 \pm 0.002$ & 0.003 & $0.005 \pm 0.011$ & 0.016 & $\mathrm{U}$ & 51.8395 & -176.591305 \\
\hline & FUCU-AN-ANT2-1 & VEG & \begin{tabular}{|l|}
$7 / 18 / 2011$ \\
\end{tabular} & $0.009 \pm 0.004$ & 0.001 & $0.057 \pm 0.025$ & 0.006 & & 51.837666 & \begin{tabular}{|l|}
-176.517527 \\
\end{tabular} \\
\hline & $\begin{array}{l}\text { FUCU-AN-ANT2-2 } \\
\end{array}$ & VEG & \begin{tabular}{|l|}
$7 / 18 / 2011$ \\
\end{tabular} & $0.003 \pm 0.004$ & 0.0014 & $0.016 \pm 0.021$ & 0.007 & $U$ & 51.841444 & -176.546083 \\
\hline & FUCU-AN-ANT2-3 & VEG & \begin{tabular}{|l|}
$7 / 18 / 2011$ \\
\end{tabular} & $0.001 \pm 0.002$ & 0.002 & $0.004 \pm 0.008$ & 0.008 & $U$ & 51.8395 & -176.591305 \\
\hline & FUCU-AN-ANT3-1 & VEG & \begin{tabular}{|l|}
$7 / 18 / 2011$ \\
\end{tabular} & $0.015 \pm 0.008$ & 0.002 & $0.086 \pm 0.046$ & 0.011 & & 51.839944 & -176.515916 \\
\hline & $\begin{array}{l}\text { FUCU-AN-ANT3-2 } \\
\end{array}$ & VEG & \begin{tabular}{|l|}
$7 / 18 / 2011$ \\
\end{tabular} & $0.002 \pm 0.002$ & 0.002 & $0.01 \pm 0.01$ & 0.01 & $\mathrm{U}$ & 51.828972 & $\mid-176.448305$ \\
\hline & FUCU-AN-ANT3-3 & VEG & $7 / 18 / 2011$ & $0.0005 \pm 0.002$ & 0.003 & $0.002 \pm 0.01$ & 0.015 & $U$ & 51.830833 & -176.456361 \\
\hline & FUCU-AN-DUP & VEG & 7/18/2011 & $0.0004 \pm 0.001$ & 0.001 & $0.002 \pm 0.005$ & 0.005 & $\bar{U}$ & 51.828972 & -176.448305 \\
\hline
\end{tabular}




\begin{tabular}{|c|c|c|c|c|c|c|c|c|c|c|}
\hline Matrix Subtype & Location Code & Matrix & $\begin{array}{c}\text { Date } \\
\text { Sampled }\end{array}$ & $\begin{array}{l}\text { Wet Result } \pm \\
\text { Uncertainty }\end{array}$ & $\begin{array}{c}\text { Wet } \\
\text { Detection } \\
\text { Limit }\end{array}$ & $\begin{array}{l}\text { Dry Result } \pm \\
\text { Uncertainty }\end{array}$ & \begin{tabular}{|c|} 
Dry \\
Detection \\
Limit
\end{tabular} & Qualifiers & Latitude & Longitude \\
\hline \multirow{10}{*}{ Greenling - whole } & GREN-AN-ANT1-1 & FAUNA & 7/19/2011 & $0.02 \pm 0.04$ & 0.03 & $0.089 \pm 0.178$ & 0.133 & U & 51.835694 & -176.542138 \\
\hline & GREN-AN-ANT1-2 & FAUNA & 7/19/2011 & $0.01 \pm 0.02$ & 0.02 & $0.042 \pm 0.084$ & 0.084 & U & 51.835694 & -176.542138 \\
\hline & GREN-AN-ANT1-3 & FAUNA & 7/19/2011 & $0.008 \pm 0.016$ & 0.02 & $0.029 \pm 0.058$ & 0.073 & $\mathrm{U}$ & 51.835694 & -176.542138 \\
\hline & GREN-AN-ANT2-1 & FAUNA & $7 / 18 / 2011$ & $0.033 \pm 0.01$ & 0.003 & $0.153 \pm 0.046$ & 0.014 & & 51.848361 & -176.506277 \\
\hline & GREN-AN-ANT2-2 & FAUNA & $7 / 18 / 2011$ & $0.009 \pm 0.004$ & 0.002 & $0.042 \pm 0.019$ & 0.009 & & 51.848361 & -176.506277 \\
\hline & GREN-AN-ANT2-3 & FAUNA & $7 / 18 / 2011$ & $0.004 \pm 0.008$ & 0.02 & $0.019 \pm 0.038$ & 0.095 & $\mathrm{U}$ & 51.848361 & -176.506277 \\
\hline & GREN-AN-ANT3-1 & FAUNA & 7/17/2011 & $0.012 \pm 0.01$ & 0.007 & $0.055 \pm 0.046$ & 0.032 & & 51.853722 & -176.470694 \\
\hline & GREN-AN-ANT3-2 & FAUNA & 7/17/2011 & $0.03 \pm 0.04$ & 0.02 & $0.139 \pm 0.186$ & 0.093 & $\mathrm{U}$ & 51.853722 & -176.470694 \\
\hline & GREN-AN-ANT3-3 & FAUNA & 7/17/2011 & $0.006 \pm 0.016$ & 0.01 & $0.028 \pm 0.074$ & 0.047 & 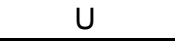 & 51.853722 & -176.470694 \\
\hline & GREN-AN-DUP & FAUNA & $7 / 17 / 2011$ & $0.02 \pm 0.02$ & 0.006 & $0.095 \pm 0.095$ & 0.029 & $U$ & 51.853722 & -176.470694 \\
\hline \multirow{2}{*}{ Halibut - whole } & HALI-AN-AN2M-1 & FAUNA & 7/17/2011 & $0.0005 \pm 0.0014$ & 0.001 & $0.002 \pm 0.005$ & 0.003 & $\mathrm{U}$ & 51.853722 & -176.470694 \\
\hline & HALI-AN-DUP & FAUNA & $7 / 17 / 2011$ & $0.0007 \pm 0.0022$ & 0.002 & $0.002 \pm 0.007$ & 0.007 & $U$ & 51.853722 & -176.470694 \\
\hline \multirow{3}{*}{$\begin{array}{l}\text { Horse Mussel - } \\
\text { soft tissue only }\end{array}$} & HMUS-AN-ANT1-1 & FAUNA & $7 / 20 / 2011$ & $0.09 \pm 0.06$ & 0.01 & $0.492 \pm 0.328$ & 0.055 & & 51.838722 & -176.582805 \\
\hline & HMUS-AN-ANT2-1 & FAUNA & 7/18/2011 & $0.07 \pm 0.08$ & 0.04 & $0.321 \pm 0.367$ & 0.184 & $\mathrm{U}$ & 51.848361 & -176.506277 \\
\hline & HMUS-AN-ANT3-1 & FAUNA & $7 / 18 / 2011$ & $0.03 \pm 0.04$ & 0.01 & $0.134 \pm 0.178$ & 0.045 & $U$ & 51.853722 & -176.470694 \\
\hline \multirow{9}{*}{ Irish Lord - whole } & IRLO-AN-ANT1-1 & FAUNA & $7 / 19 / 2011$ & $0.02 \pm 0.02$ & 0.004 & $0.094 \pm 0.094$ & 0.019 & $\bar{U}$ & 51.835694 & -176.542138 \\
\hline & IRLO-AN-ANT1-2 & FAUNA & 7/19/2011 & $0.007 \pm 0.01$ & 0.006 & $0.031 \pm 0.044$ & 0.027 & $\mathrm{U}$ & 51.835694 & -176.542138 \\
\hline & IRLO-AN-ANT1-3 & FAUNA & 7/19/2011 & $0.02 \pm 0.02$ & 0.008 & $0.088 \pm 0.088$ & 0.035 & $\mathrm{U}$ & 51.835694 & -176.542138 \\
\hline & IRLO-AN-ANT2-1 & FAUNA & $7 / 19 / 2011$ & $0.01 \pm 0.02$ & 0.005 & $0.047 \pm 0.095$ & 0.024 & $U$ & 51.848361 & -176.506277 \\
\hline & IRLO-AN-ANT2-2 & FAUNA & 7/19/2011 & $0.01 \pm 0.02$ & 0.01 & $0.046 \pm 0.093$ & 0.046 & $\mathrm{U}$ & 51.848361 & -176.506277 \\
\hline & IRLO-AN-ANT2-3 & FAUNA & 7/19/2011 & $0.005 \pm 0.01$ & 0.009 & $0.024 \pm 0.048$ & 0.043 & $\mathrm{U}$ & 51.848361 & -176.506277 \\
\hline & IRLO-AN-ANT3-1 & FAUNA & $7 / 17 / 2011$ & $0.005 \pm 0.01$ & 0.01 & $0.024 \pm 0.048$ & 0.048 & $\mathrm{U}$ & 51.853722 & -176.470694 \\
\hline & IRLO-AN-ANT3-2 & FAUNA & $7 / 17 / 2011$ & $0.01 \pm 0.02$ & 0.01 & $0.045 \pm 0.09$ & 0.045 & $\bar{U}$ & 51.853722 & -176.470694 \\
\hline & IRLO-AN-ANT3-3 & FAUNA & 7/17/2011 & $0.01 \pm 0.02$ & 0.01 & $0.045 \pm 0.09$ & 0.045 & $U$ & 51.853722 & -176.470694 \\
\hline Octopus & OCTP-AN-AN2M-1 & FAUNA & 7/18/2011 & $0.015 \pm 0.006$ & 0.002 & $0.076 \pm 0.03$ & 0.01 & & 51.848361 & -176.506277 \\
\hline $\begin{array}{l}\text { Pacific Cod - } \\
\text { whole } \\
\end{array}$ & PCOD-AN-AN2M-1 & FAUNA & 7/17/2011 & $0.0005 \pm 0.001$ & 0.0009 & $0.002 \pm 0.005$ & 0.004 & U & 51.853722 & -176.470694 \\
\hline \multirow{6}{*}{ Rockfish - whole } & ROCK-AN-ANT1-1 & FAUNA & 7/19/2011 & $0.006 \pm 0.01$ & 0.005 & $0.022 \pm 0.037$ & 0.018 & $\mathrm{U}$ & 51.835694 & -176.542138 \\
\hline & ROCK-AN-ANT2-1 & FAUNA & $7 / 18 / 2011$ & $0.001 \pm 0.002$ & 0.003 & $0.004 \pm 0.007$ & 0.011 & $\bar{U}$ & 51.848361 & -176.506277 \\
\hline & ROCK-AN-ANT2-2 & FAUNA & $7 / 18 / 2011$ & $0.003 \pm 0.008$ & 0.007 & $0.012 \pm 0.033$ & 0.029 & $\mathrm{U}$ & 51.848361 & -176.506277 \\
\hline & ROCK-AN-ANT3-1 & FAUNA & 7/17/2011 & $-0.001 \pm 0.002$ & 0.005 & $-0.004 \pm 0.007$ & 0.018 & $\mathrm{U}$ & 51.853722 & -176.470694 \\
\hline & ROCK-AN-ANT3-2 & FAUNA & 7/17/2011 & $0.006 \pm 0.006$ & 0.008 & $0.023 \pm 0.023$ & 0.031 & $\mathrm{U}$ & 51.853722 & -176.470694 \\
\hline & ROCK-AN-ANT3-3 & FAUNA & 7/17/2011 & $0.02 \pm 0.02$ & 0.006 & $0.079 \pm 0.079$ & 0.024 & $\mathrm{U}$ & 51.853722 & -176.470694 \\
\hline \multirow{3}{*}{$\begin{array}{l}\text { Sea Urchin - } \\
\text { whole }\end{array}$} & URCH-AN-ANT1-1 & FAUNA & 7/19/2011 & $0.03 \pm 0.04$ & 0.03 & $0.102 \pm 0.136$ & 0.102 & $\mathrm{UJ}$ & 51.835694 & -176.542138 \\
\hline & URCH-AN-ANT2-1 & FAUNA & $7 / 18 / 2011$ & $0.08 \pm 0.04$ & 0.007 & $0.291 \pm 0.146$ & 0.026 & & 51.848361 & -176.506277 \\
\hline & \begin{tabular}{|l|} 
URCH-AN-ANT3-1 \\
\end{tabular} & FAUNA & $7 / 17 / 2011$ & $0.07 \pm 0.02$ & 0.003 & $0.266 \pm 0.076$ & 0.011 & & 51.853722 & -176.470694 \\
\hline
\end{tabular}




\begin{tabular}{|c|c|c|c|c|c|c|c|c|c|c|}
\hline Matrix Subtype & Location Code & Matrix & $\begin{array}{c}\text { Date } \\
\text { Sampled }\end{array}$ & $\begin{array}{l}\text { Wet Result } \pm \\
\text { Uncertainty }\end{array}$ & $\begin{array}{c}\text { Wet } \\
\text { Detection } \\
\text { Limit }\end{array}$ & $\begin{array}{l}\text { Dry Result } \pm \\
\text { Uncertainty }\end{array}$ & \begin{tabular}{|c|} 
Dry \\
Detection \\
Limit
\end{tabular} & Qualifiers & Latitude & Longitude \\
\hline \multicolumn{11}{|c|}{ Amchitka Site (Adak Island South) (AMC15) } \\
\hline \multirow{3}{*}{ Chiton } & CHIT-AS-AST1-1 & FAUNA & $7 / 15 / 2011$ & $0.06 \pm 0.02$ & 0.003 & $0.247 \pm 0.082$ & 0.012 & & 51.737472 & -176.48025 \\
\hline & CHIT-AS-AST2-1 & FAUNA & $7 / 16 / 2011$ & $0.017 \pm 0.006$ & 0.002 & $0.087 \pm 0.031$ & 0.01 & & 51.745666 & -176.507055 \\
\hline & CHIT-AS-AST3-1 & FAUNA & $7 / 14 / 2011$ & $0.07 \pm 0.02$ & 0.005 & $0.33 \pm 0.094$ & 0.024 & & 51.688333 & -176.63375 \\
\hline \multirow{10}{*}{ Dragon Kelp } & EUAL-AS-AST1-1 & VEG & $7 / 15 / 2011$ & $0.006 \pm 0.004$ & 0.002 & $0.04 \pm 0.026$ & 0.013 & & 51.737472 & -176.48025 \\
\hline & EUAL-AS-AST1-2 & VEG & $7 / 15 / 2011$ & $0.008 \pm 0.002$ & 0.0007 & $0.064 \pm 0.016$ & 0.006 & & 51.737472 & -176.48025 \\
\hline & EUAL-AS-AST1-3 & VEG & 7/15/2011 & $0.008 \pm 0.004$ & 0.0006 & $0.077 \pm 0.038$ & 0.006 & & 51.737472 & -176.48025 \\
\hline & EUAL-AS-AST2-1 & VEG & $7 / 16 / 2011$ & $0.015 \pm 0.002$ & 0.0002 & $0.107 \pm 0.014$ & 0.001 & & 51.745666 & -176.507055 \\
\hline & EUAL-AS-AST2-2 & VEG & 7/16/2011 & $0.027 \pm 0.004$ & 0.0006 & $0.191 \pm 0.028$ & 0.004 & & 51.745666 & -176.507055 \\
\hline & EUAL-AS-AST2-3 & VEG & $7 / 16 / 2011$ & $0.0007 \pm 0.0018$ & 0.002 & $0.005 \pm 0.013$ & 0.015 & $U$ & 51.745666 & -176.507055 \\
\hline & EUAL-AS-AST3-1 & VEG & 7/14/2011 & $0.009 \pm 0.002$ & 0.0006 & $0.066 \pm 0.015$ & 0.004 & & 51.688333 & -176.63375 \\
\hline & EUAL-AS-AST3-2 & VEG & $7 / 14 / 2011$ & $0.006 \pm 0.004$ & 0.002 & $0.057 \pm 0.038$ & 0.019 & & 51.688333 & -176.63375 \\
\hline & $\begin{array}{l}\text { EUAL-AS-AST3-3 } \\
\end{array}$ & VEG & \begin{tabular}{|l|}
$7 / 14 / 2011$ \\
\end{tabular} & $0.004 \pm 0.004$ & 0.002 & $0.035 \pm 0.035$ & 0.018 & $U$ & 51.688333 & -176.63375 \\
\hline & EUAL-AS-DUP & VEG & 7/14/2011 & $0.008 \pm 0.004$ & 0.0006 & $0.059 \pm 0.029$ & 0.004 & & 51.688333 & -176.63375 \\
\hline \multirow{10}{*}{ Rockweed } & FUCU-AS-AST1-1 & VEG & $7 / 16 / 2011$ & $0.007 \pm 0.006$ & 0.003 & $0.043 \pm 0.037$ & 0.019 & $U$ & 51.733666 & -176.470805 \\
\hline & FUCU-AS-AST1-2 & VEG & \begin{tabular}{|l|}
$7 / 16 / 2011$ \\
\end{tabular} & $0.01 \pm 0.006$ & 0.004 & $0.055 \pm 0.033$ & 0.022 & $\mathrm{~J}$ & 51.733666 & $\mid-176.470805$ \\
\hline & FUCU-AS-AST1-3 & VEG & \begin{tabular}{|l|}
$7 / 16 / 2011$ \\
\end{tabular} & $0.008 \pm 0.004$ & 0.0006 & $0.043 \pm 0.022$ & 0.003 & & 51.733666 & \begin{tabular}{|l}
-176.470805 \\
\end{tabular} \\
\hline & FUCU-AS-AST2-1 & VEG & $7 / 16 / 2011$ & $0.006 \pm 0.002$ & 0.0005 & $0.029 \pm 0.01$ & 0.002 & & 51.745666 & -176.507055 \\
\hline & FUCU-AS-AST2-2 & VEG & 7/16/2011 & $0.0007 \pm 0.0012$ & 0.0007 & $0.004 \pm 0.006$ & 0.004 & $\mathrm{U}$ & 51.745666 & -176.507055 \\
\hline & $\begin{array}{l}\text { FUCU-AS-AST2-3 } \\
\end{array}$ & VEG & \begin{tabular}{|l|}
$7 / 16 / 2011$ \\
\end{tabular} & $0.004 \pm 0.004$ & 0.002 & $0.02 \pm 0.02$ & 0.01 & $U$ & 51.745666 & -176.507055 \\
\hline & FUCU-AS-AST3-1 & VEG & $7 / 14 / 2011$ & $0.015 \pm 0.004$ & 0.0007 & $0.079 \pm 0.021$ & 0.004 & & 51.688333 & -176.63375 \\
\hline & FUCU-AS-AST3-2 & VEG & $7 / 14 / 2011$ & $0.002 \pm 0.002$ & 0.001 & $0.01 \pm 0.01$ & 0.005 & $U$ & 51.688333 & -176.63375 \\
\hline & $\begin{array}{l}\text { FUCU-AS-AST3-3 } \\
\end{array}$ & VEG & \begin{tabular}{|l|}
$7 / 14 / 2011$ \\
\end{tabular} & $0.0009 \pm 0.0012$ & 0.001 & $0.005 \pm 0.006$ & 0.005 & $U$ & 51.688333 & -176.63375 \\
\hline & FUCU-AS-DUP & VEG & \begin{tabular}{|l|l|}
$7 / 2011$ \\
\end{tabular} & $0.006 \pm 0.004$ & 0.002 & $0.031 \pm 0.021$ & 0.01 & & 51.733666 & -176.470805 \\
\hline \multirow{10}{*}{ Greenling - whole } & GREN-AS-AST1-1 & FAUNA & $7 / 15 / 2011$ & $0.005 \pm 0.01$ & 0.008 & $0.024 \pm 0.048$ & 0.038 & $U$ & 51.737472 & -176.48025 \\
\hline & GREN-AS-AST1-2 & FAUNA & \begin{tabular}{|l|}
$7 / 15 / 2011$ \\
\end{tabular} & $0.008 \pm 0.016$ & 0.01 & $0.038 \pm 0.075$ & 0.047 & $U$ & 51.737472 & -176.48025 \\
\hline & GREN-AS-AST1-3 & FAUNA & \begin{tabular}{|l|}
$7 / 15 / 2011$ \\
\end{tabular} & $0.02 \pm 0.02$ & 0.02 & $0.096 \pm 0.096$ & 0.096 & $U$ & 51.737472 & -176.48025 \\
\hline & GREN-AS-AST2-1 & FAUNA & 7/16/2011 & $0.01 \pm 0.02$ & 0.006 & $0.04 \pm 0.08$ & 0.024 & $U$ & 51.745666 & -176.507055 \\
\hline & GREN-AS-AST2-2 & FAUNA & $7 / 16 / 2011$ & $0.002 \pm 0.004$ & 0.008 & $0.009 \pm 0.018$ & 0.037 & $U$ & 51.745666 & -176.507055 \\
\hline & GREN-AS-AST2-3 & FAUNA & \begin{tabular}{|l|}
$7 / 16 / 2011$ \\
\end{tabular} & $0.01 \pm 0.02$ & 0.009 & $0.047 \pm 0.093$ & 0.042 & $U$ & 51.745666 & -176.507055 \\
\hline & GREN-AS-AST3-1 & FAUNA & 7/14/2011 & $0.007 \pm 0.012$ & 0.007 & $0.033 \pm 0.057$ & 0.033 & $U$ & 51.688333 & -176.63375 \\
\hline & GREN-AS-AST3-2 & FAUNA & $7 / 14 / 2011$ & $0.01 \pm 0.02$ & 0.02 & $0.047 \pm 0.094$ & 0.094 & $U$ & 51.688333 & -176.63375 \\
\hline & GREN-AS-AST3-3 & FAUNA & $7 / 14 / 2011$ & $0.02 \pm 0.02$ & 0.005 & $0.096 \pm 0.096$ & 0.024 & $U$ & 51.688333 & -176.63375 \\
\hline & GREN-AS-DUP & FAUNA & \begin{tabular}{|l|}
$7 / 14 / 2011$ \\
\end{tabular} & $0.009 \pm 0.012$ & 0.007 & $0.045 \pm 0.06$ & 0.035 & $U$ & 51.688333 & -176.63375 \\
\hline
\end{tabular}




\begin{tabular}{|c|c|c|c|c|c|c|c|c|c|c|}
\hline Matrix Subtype & Location Code & Matrix & $\begin{array}{c}\text { Date } \\
\text { Sampled }\end{array}$ & $\begin{array}{l}\text { Wet Result } \pm \\
\text { Uncertainty }\end{array}$ & $\begin{array}{c}\text { Wet } \\
\text { Detection } \\
\text { Limit }\end{array}$ & $\begin{array}{l}\text { Dry Result } \pm \\
\text { Uncertainty }\end{array}$ & \begin{tabular}{|c|} 
Dry \\
Detection \\
Limit
\end{tabular} & Qualifiers & Latitude & Longitude \\
\hline \multirow{3}{*}{$\begin{array}{l}\text { Horse Mussel - } \\
\text { soft tissue only }\end{array}$} & HMUS-AS-AST1-1 & \begin{tabular}{|l|} 
FAUNA \\
\end{tabular} & $7 / 15 / 2011$ & $0.07 \pm 0.1$ & 0.05 & $0.351 \pm 0.501$ & 0.25 & $U$ & 51.737472 & -176.48025 \\
\hline & HMUS-AS-AST2-1 & FAUNA & $7 / 16 / 2011$ & $0.03 \pm 0.06$ & 0.04 & $0.19 \pm 0.381$ & 0.254 & $\mathrm{U}$ & 51.745666 & -176.507055 \\
\hline & 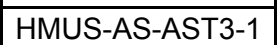 & \begin{tabular}{|l|} 
FAUNA \\
\end{tabular} & $7 / 14 / 2011$ & $0.15 \pm 0.04$ & 0.009 & $0.765 \pm 0.204$ & 0.046 & & 51.688333 & \begin{tabular}{|l|}
-176.63375 \\
\end{tabular} \\
\hline \multirow{9}{*}{ Irish Lord - whole } & IRLO-AS-AST1-1 & \begin{tabular}{|l|} 
FAUNA \\
\end{tabular} & $7 / 15 / 2011$ & $0.02 \pm 0.02$ & 0.006 & $0.088 \pm 0.088$ & 0.026 & $U$ & 51.737472 & \begin{tabular}{|l|}
-176.48025 \\
\end{tabular} \\
\hline & IRLO-AS-AST1-2 & \begin{tabular}{|l|} 
FAUNA \\
\end{tabular} & $7 / 15 / 2011$ & $0.03 \pm 0.04$ & 0.01 & $0.14 \pm 0.187$ & 0.047 & $U$ & 51.737472 & -176.48025 \\
\hline & IRLO-AS-AST1-3 & FAUNA & $7 / 15 / 2011$ & $0.009 \pm 0.014$ & 0.008 & $0.041 \pm 0.064$ & 0.036 & $\mathrm{U}$ & 51.737472 & -176.48025 \\
\hline & IRLO-AS-AST2-1 & \begin{tabular}{|l|} 
FAUNA \\
\end{tabular} & $7 / 16 / 2011$ & $0.01 \pm 0.02$ & 0.01 & $0.047 \pm 0.094$ & 0.047 & $U$ & 51.745666 & -176.507055 \\
\hline & IRLO-AS-AST2-2 & FAUNA & $7 / 16 / 2011$ & $0.02 \pm 0.02$ & 0.01 & $0.085 \pm 0.085$ & 0.043 & $U$ & 51.745666 & \begin{tabular}{|l}
-176.507055 \\
\end{tabular} \\
\hline & IRLO-AS-AST2-3 & FAUNA & $7 / 16 / 2011$ & $0.007 \pm 0.012$ & 0.006 & $0.035 \pm 0.061$ & 0.03 & $U$ & 51.745666 & -176.507055 \\
\hline & IRLO-AS-AST3-1 & \begin{tabular}{|l|} 
FAUNA \\
\end{tabular} & $7 / 14 / 2011$ & $0.01 \pm 0.02$ & 0.007 & $0.045 \pm 0.09$ & 0.031 & $U$ & 51.688333 & \begin{tabular}{|l|}
-176.63375 \\
\end{tabular} \\
\hline & IRLO-AS-AST3-2 & \begin{tabular}{|l|} 
FAUNA \\
\end{tabular} & $7 / 14 / 2011$ & $0.003 \pm 0.014$ & 0.01 & $0.013 \pm 0.059$ & 0.042 & $U$ & 51.688333 & \begin{tabular}{|l|}
-176.63375 \\
\end{tabular} \\
\hline & \begin{tabular}{|l|} 
IRLO-AS-AST3-3 \\
\end{tabular} & FAUNA & $7 / 14 / 2011$ & $0.03 \pm 0.02$ & 0.004 & $0.137 \pm 0.091$ & 0.018 & & 51.688333 & -176.63375 \\
\hline $\begin{array}{l}\text { Pacific Cod - } \\
\text { whole }\end{array}$ & PCOD-AS-AS2M-1 & FAUNA & $7 / 15 / 2011$ & $0.001 \pm 0.002$ & 0.0009 & $0.005 \pm 0.01$ & 0.005 & U & 51.737472 & -176.48025 \\
\hline \multirow{10}{*}{ Rockfish - whole } & ROCK-AS-AST1-1 & FAUNA & $7 / 15 / 2011$ & $0.01 \pm 0.02$ & 0.007 & $0.036 \pm 0.072$ & 0.025 & $U$ & 51.737472 & \begin{tabular}{|l|}
-176.48025 \\
\end{tabular} \\
\hline & ROCK-AS-AST1-2 & FAUNA & $7 / 15 / 2011$ & $-0.002 \pm 0.002$ & 0.01 & $-0.007 \pm 0.007$ & 0.037 & $U$ & 51.737472 & -176.48025 \\
\hline & \begin{tabular}{|l} 
ROCK-AS-AST1-3 \\
\end{tabular} & \begin{tabular}{|l|} 
FAUNA \\
\end{tabular} & $7 / 15 / 2011$ & $0.008 \pm 0.008$ & 0.003 & $0.034 \pm 0.034$ & 0.013 & $U$ & 51.737472 & \begin{tabular}{|l|}
-176.48025 \\
\end{tabular} \\
\hline & \begin{tabular}{|l|} 
ROCK-AS-AST2-1 \\
\end{tabular} & FAUNA & $7 / 16 / 2011$ & $0.005 \pm 0.01$ & 0.009 & $0.019 \pm 0.038$ & 0.035 & $U$ & 51.745666 & -176.507055 \\
\hline & ROCK-AS-AST2-2 & FAUNA & $7 / 16 / 2011$ & $0.003 \pm 0.008$ & 0.008 & $0.011 \pm 0.03$ & 0.03 & $U$ & 51.745666 & -176.507055 \\
\hline & \begin{tabular}{|l} 
ROCK-AS-AST2-3 \\
\end{tabular} & \begin{tabular}{|l|} 
FAUNA \\
\end{tabular} & $7 / 16 / 2011$ & $0.002 \pm 0.004$ & 0.005 & $0.008 \pm 0.015$ & 0.019 & $U$ & 51.745666 & -176.507055 \\
\hline & \begin{tabular}{|l} 
ROCK-AS-AST3-1 \\
\end{tabular} & \begin{tabular}{|l|} 
FAUNA \\
\end{tabular} & $7 / 14 / 2011$ & $0.02 \pm 0.02$ & 0.01 & $0.082 \pm 0.082$ & 0.041 & $U$ & 51.688333 & \begin{tabular}{|l|}
-176.63375 \\
\end{tabular} \\
\hline & \begin{tabular}{|l|} 
ROCK-AS-AST3-2 \\
\end{tabular} & FAUNA & $7 / 14 / 2011$ & $0.02 \pm 0.02$ & 0.008 & $0.085 \pm 0.085$ & 0.034 & $U$ & 51.688333 & -176.63375 \\
\hline & ROCK-AS-AST3-3 & FAUNA & $7 / 14 / 2011$ & $-0.001 \pm 0.002$ & 0.004 & $-0.004 \pm 0.009$ & 0.017 & $U$ & 51.688333 & -176.63375 \\
\hline & ROCK-AS-DUP & \begin{tabular}{|l|} 
FAUNA \\
\end{tabular} & $7 / 14 / 2011$ & $-0.0008 \pm 0.0016$ & 0.005 & $-0.003 \pm 0.006$ & 0.02 & $U$ & 51.688333 & \begin{tabular}{|l|}
-176.63375 \\
\end{tabular} \\
\hline \multirow{3}{*}{$\begin{array}{l}\text { Sea Urchin - } \\
\text { whole }\end{array}$} & URCH-AS-AST1-1 & FAUNA & $7 / 15 / 2011$ & $0.1 \pm 0.04$ & 0.009 & $0.38 \pm 0.152$ & 0.034 & & 51.737472 & \begin{tabular}{|l|}
-176.48025 \\
\end{tabular} \\
\hline & \begin{tabular}{|l|} 
URCH-AS-AST2-1 \\
\end{tabular} & FAUNA & $7 / 16 / 2011$ & $0.12 \pm 0.02$ & 0.004 & $0.484 \pm 0.081$ & 0.016 & & 51.745666 & -176.507055 \\
\hline & URCH-AS-AST3-1 & FAUNA & $7 / 14 / 2011$ & $0.07 \pm 0.02$ & 0.003 & $0.261 \pm 0.075$ & 0.011 & & 51.688333 & -176.63375 \\
\hline
\end{tabular}

Qualifier definitions:

$\mathrm{U}$ - Analytical result below detection limit

$\mathrm{J}$ - Estimated 
Appendix I

Activity Concentration of Plutonium Isotopes 
This page intentionally left blank 


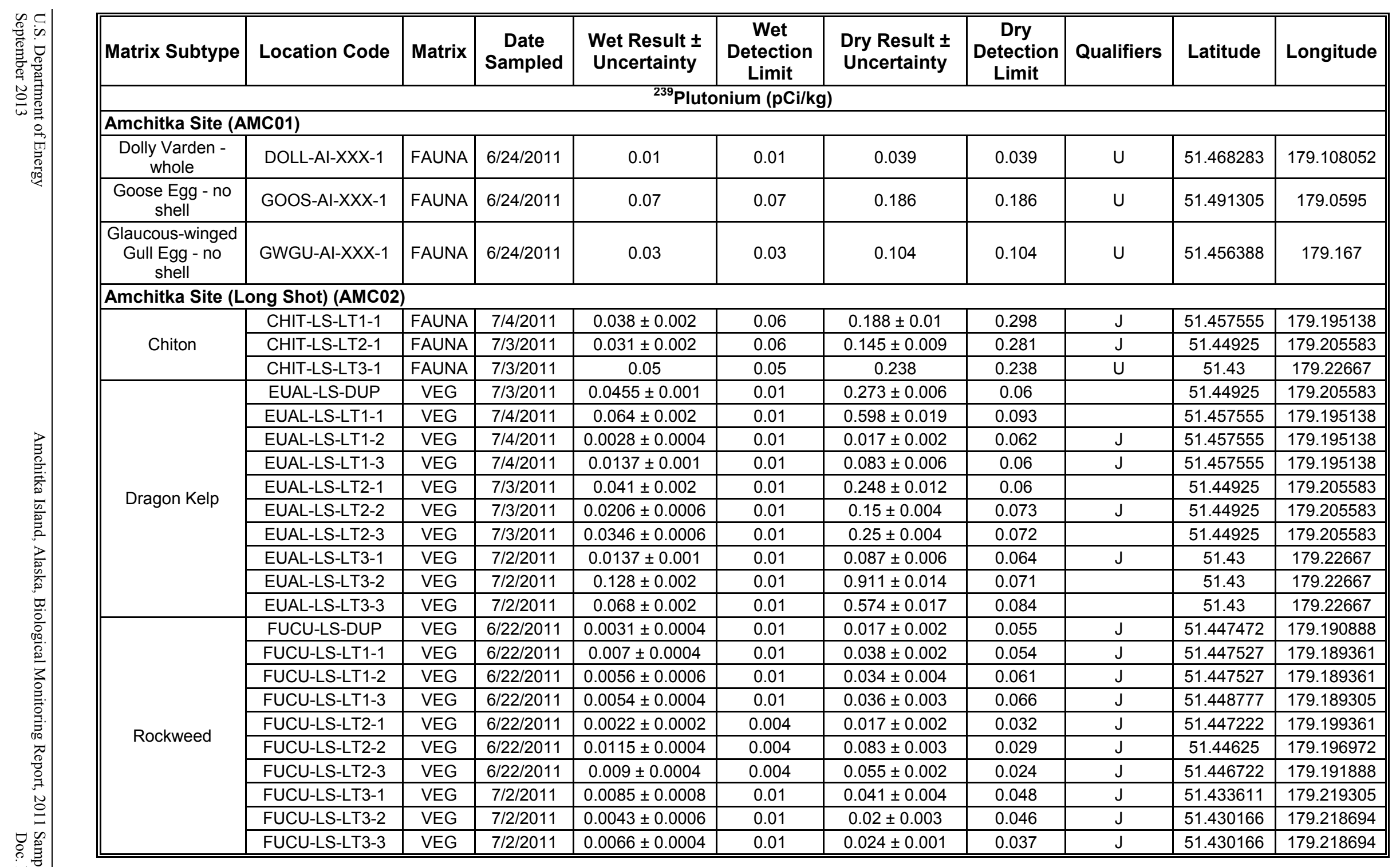




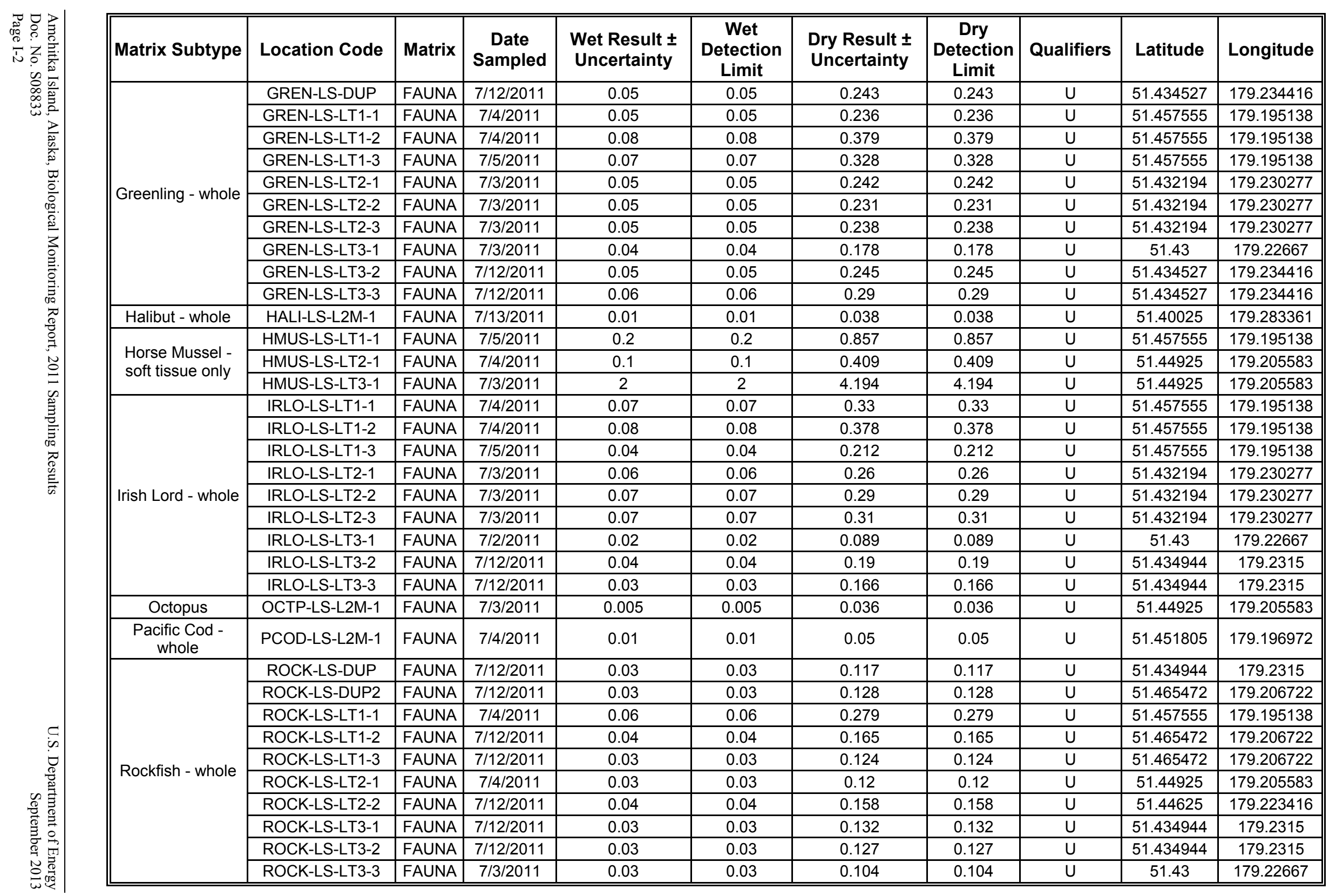




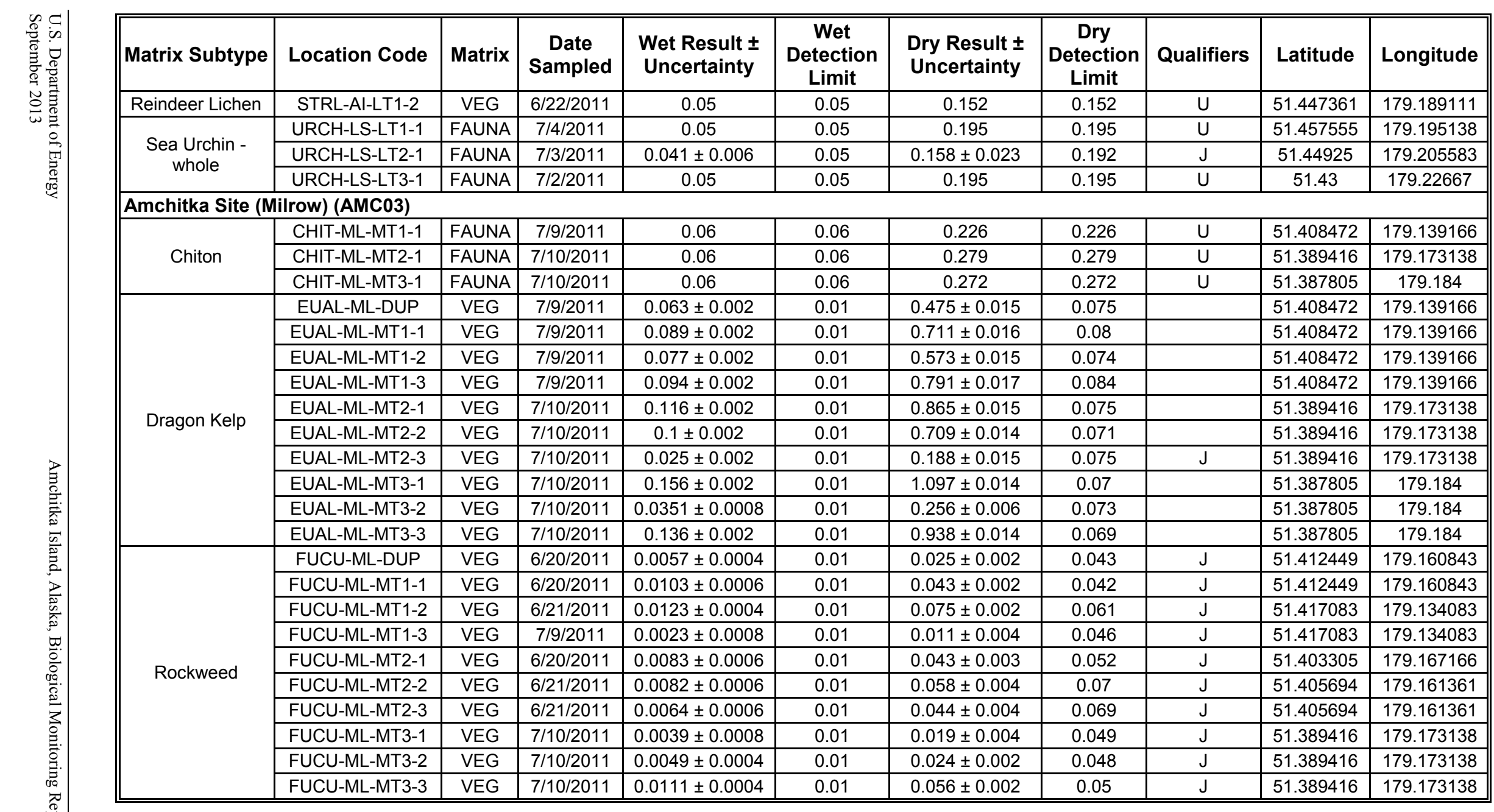




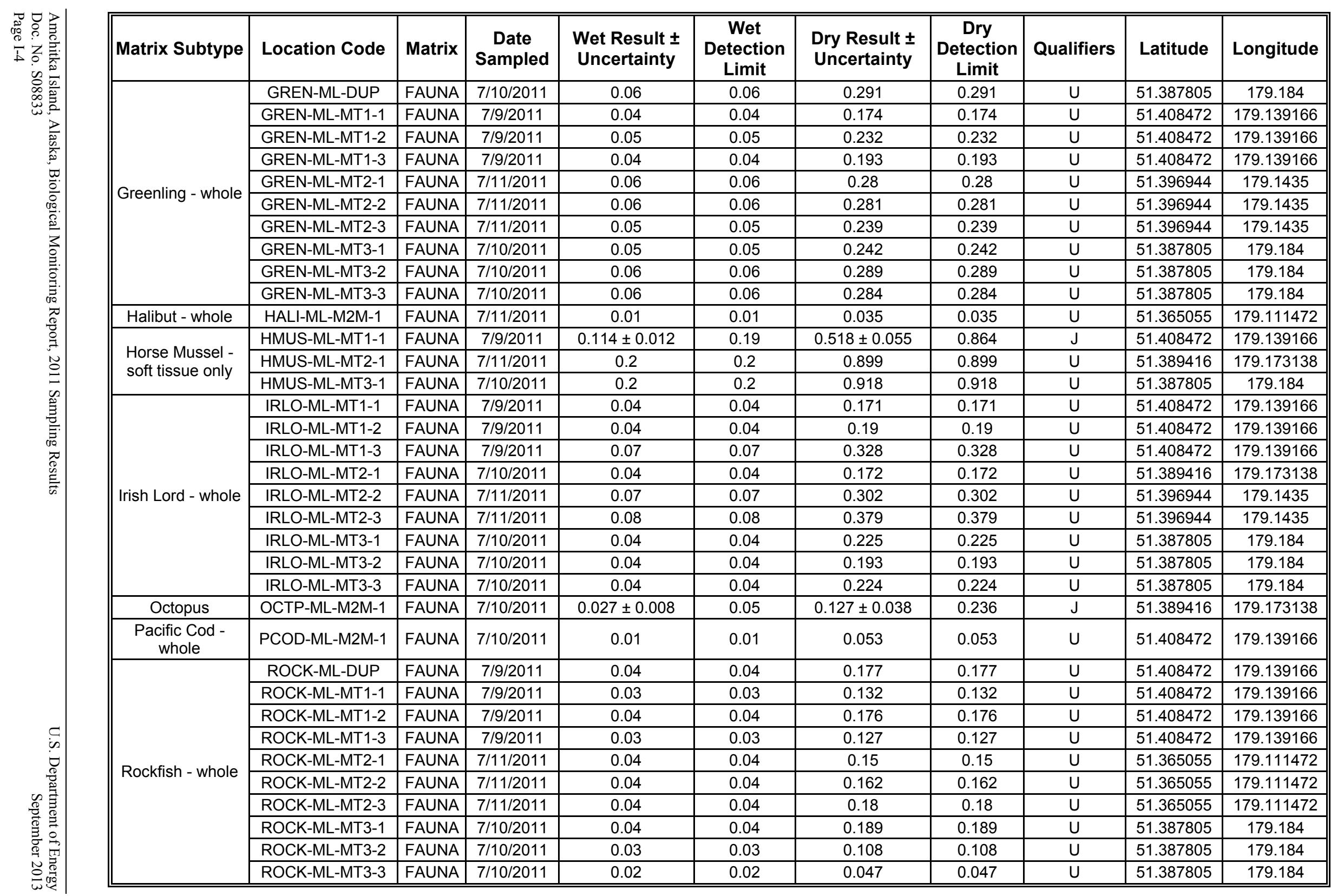




\begin{tabular}{|c|c|c|c|c|c|c|c|c|c|c|}
\hline Matrix Subtype & Location Code & Matrix & $\begin{array}{c}\text { Date } \\
\text { Sampled }\end{array}$ & $\begin{array}{l}\text { Wet Result } \pm \\
\text { Uncertainty }\end{array}$ & $\begin{array}{c}\text { Wet } \\
\text { Detection } \\
\text { Limit }\end{array}$ & $\begin{array}{l}\text { Dry Result } \pm \\
\text { Uncertainty }\end{array}$ & \begin{tabular}{|c|} 
Dry \\
Detection \\
Limit
\end{tabular} & Qualifiers & Latitude & Longitude \\
\hline \begin{tabular}{|l|} 
Reindeer Lichen \\
\end{tabular} & STRL-AI-ML-1 & VEG & $6 / 21 / 2011$ & 0.06 & 0.06 & 0.215 & 0.215 & $\mathrm{U}$ & 51.402583 & 179.183972 \\
\hline \multirow{3}{*}{$\begin{array}{l}\text { Sea Urchin - } \\
\text { whole }\end{array}$} & URCH-ML-MT1-1 & FAUNA & $7 / 9 / 2011$ & 0.04 & 0.04 & 0.148 & 0.148 & $\mathrm{U}$ & 51.408472 & 179.139166 \\
\hline & URCH-ML-MT2-1 & FAUNA & $7 / 10 / 2011$ & 0.05 & 0.05 & 0.187 & 0.187 & $\mathrm{U}$ & 51.389416 & 179.173138 \\
\hline & URCH-ML-MT3-1 & FAUNA & $7 / 10 / 2011$ & 0.06 & 0.06 & 0.238 & 0.238 & $U$ & 51.387805 & 179.184 \\
\hline \multicolumn{11}{|c|}{ Amchitka Site (Cannikin) (AMC05) } \\
\hline \multirow{3}{*}{ Chiton } & CHIT-CN-CT1-1 & FAUNA & $7 / 8 / 2011$ & 0.06 & 0.06 & 0.281 & 0.281 & $\mathrm{U}$ & 51.482972 & 179.123833 \\
\hline & CHIT-CN-CT2-1 & FAUNA & 7/7/2011 & $0.05 \pm 0.02$ & 0.06 & $0.237 \pm 0.095$ & 0.284 & $\mathrm{~J}$ & 51.472611 & 179.151111 \\
\hline & CHIT-CN-CT3-1 & FAUNA & $7 / 5 / 2011$ & 0.07 & 0.07 & 0.344 & 0.344 & $\mathrm{U}$ & 51.471416 & 179.172861 \\
\hline \multirow{10}{*}{ Dragon Kelp } & EUAL-CN-CT1-1 & VEG & $7 / 8 / 2011$ & $0.074 \pm 0.002$ & 0.01 & $0.548 \pm 0.015$ & 0.074 & & 51.482972 & 179.123833 \\
\hline & EUAL-CN-CT1-2 & VEG & $7 / 8 / 2011$ & $0.0113 \pm 0.0006$ & 0.01 & $0.074 \pm 0.004$ & 0.065 & $\mathrm{~J}$ & 51.482972 & 179.123833 \\
\hline & EUAL-CN-CT1-3 & VEG & $7 / 8 / 2011$ & $0.0301 \pm 0.0006$ & 0.01 & $0.184 \pm 0.004$ & 0.061 & & 51.482972 & 179.123833 \\
\hline & EUAL-CN-CT2-1 & VEG & $7 / 6 / 2011$ & $0.0253 \pm 0.001$ & 0.01 & $0.161 \pm 0.006$ & 0.064 & $\mathrm{~J}$ & 51.472611 & 179.151111 \\
\hline & EUAL-CN-CT2-2 & VEG & $7 / 6 / 2011$ & $0.029 \pm 0.002$ & 0.01 & $0.182 \pm 0.013$ & 0.063 & $\mathrm{~J}$ & 51.472611 & 179.151111 \\
\hline & EUAL-CN-CT2-3 & VEG & 7/7/2011 & $0.061 \pm 0.002$ & 0.01 & $0.442 \pm 0.014$ & 0.072 & & 51.472611 & 179.151111 \\
\hline & EUAL-CN-CT3-1 & VEG & $7 / 5 / 2011$ & 0.01 & 0.01 & 0.07 & 0.07 & $\mathrm{U}$ & 51.471416 & 179.172861 \\
\hline & EUAL-CN-CT3-2 & VEG & $7 / 5 / 2011$ & $0.0153 \pm 0.0006$ & 0.01 & $0.11 \pm 0.004$ & 0.072 & $\mathrm{~J}$ & 51.471416 & 179.172861 \\
\hline & EUAL-CN-CT3-3 & VEG & $7 / 5 / 2011$ & $0.0037 \pm 0.0004$ & 0.01 & $0.024 \pm 0.003$ & 0.065 & $\mathrm{~J}$ & 51.471416 & 179.172861 \\
\hline & EUAL-CN-DUP & VEG & $7 / 6 / 2011$ & $0.0326 \pm 0.0008$ & 0.01 & $0.217 \pm 0.005$ & 0.066 & & 51.471416 & 179.172861 \\
\hline \multirow{10}{*}{ Rockweed } & FUCU-CN-CT1-1 & VEG & 6/23/2011 & 0.01 & 0.01 & 0.058 & 0.058 & $U$ & 51.481888 & 179.118138 \\
\hline & FUCU-CN-CT1-2 & VEG & 6/23/2011 & 0.01 & 0.01 & 0.063 & 0.063 & $\mathrm{U}$ & 51.481888 & 179.118138 \\
\hline & FUCU-CN-CT1-3 & VEG & 6/23/2011 & $0.0048 \pm 0.0006$ & 0.01 & $0.028 \pm 0.004$ & 0.059 & $\mathrm{~J}$ & 51.481888 & 179.118138 \\
\hline & FUCU-CN-CT2-1 & VEG & $7 / 8 / 2011$ & $0.0105 \pm 0.0006$ & 0.01 & $0.043 \pm 0.002$ & 0.041 & $\mathrm{~J}$ & 51.476638 & 179.135277 \\
\hline & FUCU-CN-CT2-2 & VEG & $7 / 8 / 2011$ & $0.0083 \pm 0.0004$ & 0.01 & $0.037 \pm 0.002$ & 0.044 & $\mathrm{~J}$ & 51.476638 & 179.135277 \\
\hline & FUCU-CN-CT2-3 & VEG & $7 / 12 / 2011$ & $0.0059 \pm 0.0008$ & 0.01 & $0.031 \pm 0.004$ & 0.053 & $\mathrm{~J}$ & 51.467583 & 179.153583 \\
\hline & FUCU-CN-CT3-1 & VEG & $7 / 6 / 2011$ & $0.0108 \pm 0.0008$ & 0.01 & $0.058 \pm 0.004$ & 0.054 & $\mathrm{~J}$ & 51.465027 & 179.173166 \\
\hline & FUCU-CN-CT3-2 & VEG & $7 / 6 / 2011$ & $0.0041 \pm 0.0004$ & 0.01 & $0.024 \pm 0.002$ & 0.059 & $\mathrm{~J}$ & 51.465472 & 179.170583 \\
\hline & FUCU-CN-CT3-3 & VEG & $7 / 6 / 2011$ & $0.0112 \pm 0.0008$ & 0.01 & $0.071 \pm 0.005$ & 0.063 & $\mathrm{~J}$ & 51.464638 & 179.159305 \\
\hline & FUCU-CN-DUP & VEG & 6/23/2011 & 0.01 & 0.01 & 0.065 & 0.065 & $\mathrm{U}$ & 51.481888 & 179.118138 \\
\hline
\end{tabular}




\begin{tabular}{|c|c|c|c|c|c|c|c|c|c|c|c|}
\hline 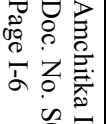 & Matrix Subtype & Location Code & Matrix & $\begin{array}{c}\text { Date } \\
\text { Sampled }\end{array}$ & $\begin{array}{c}\text { Wet Result } \pm \\
\text { Uncertainty }\end{array}$ & $\begin{array}{c}\text { Wet } \\
\text { Detection } \\
\text { Limit }\end{array}$ & $\begin{array}{l}\text { Dry Result } \pm \\
\text { Uncertainty }\end{array}$ & \begin{tabular}{|c|} 
Dry \\
Detection \\
Limit
\end{tabular} & Qualifiers & Latitude & Longitude \\
\hline 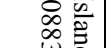 & \multirow{10}{*}{ Greenling - whole } & GREN-CN-CT1-1 & \begin{tabular}{|l|} 
FAUNA \\
\end{tabular} & $7 / 8 / 2011$ & 0.05 & 0.05 & 0.241 & 0.241 & $U$ & 51.482972 & 179.123833 \\
\hline & & GREN-CN-CT1-2 & FAUNA & $7 / 8 / 2011$ & 0.06 & 0.06 & 0.285 & 0.285 & $U$ & 51.482972 & 179.123833 \\
\hline 总 & & GREN-CN-CT1-3 & FAUNA & $7 / 8 / 2011$ & 0.05 & 0.05 & 0.228 & 0.228 & $\mathrm{U}$ & 51.482972 & 179.123833 \\
\hline$\pi$ & & GREN-CN-CT2-1 & FAUNA & $7 / 7 / 2011$ & 0.04 & 0.04 & 0.195 & 0.195 & $\mathrm{U}$ & 51.472611 & 179.151111 \\
\hline$\stackrel{\square}{0}$ & & GREN-CN-CT2-2 & FAUNA & $7 / 7 / 2011$ & 0.05 & 0.05 & 0.229 & 0.229 & $\mathrm{U}$ & 51.472611 & 179.151111 \\
\hline s. & & GREN-CN-CT2-3 & FAUNA & $7 / 7 / 2011$ & 0.04 & 0.04 & 0.185 & 0.185 & $\mathrm{U}$ & 51.472611 & 179.151111 \\
\hline E & & GREN-CN-CT3-1 & FAUNA & $7 / 5 / 2011$ & $0.02 \pm 0.004$ & 0.05 & $0.091 \pm 0.018$ & 0.228 & $\mathrm{~J}$ & 51.471416 & 179.172861 \\
\hline$=$ & & GREN-CN-CT3-2 & FAUNA & $7 / 5 / 2011$ & $0.018 \pm 0.004$ & 0.05 & $0.078 \pm 0.017$ & 0.218 & $\mathrm{~J}$ & 51.471416 & 179.172861 \\
\hline$=$ & & GREN-CN-CT3-3 & FAUNA & $7 / 5 / 2011$ & 0.04 & 0.04 & 0.184 & 0.184 & $\mathrm{U}$ & 51.471416 & 179.172861 \\
\hline 鬲 & & GREN-CN-DUP & \begin{tabular}{|l|} 
FAUNA \\
\end{tabular} & $7 / 8 / 2011$ & 0.06 & 0.06 & 0.289 & 0.289 & $U$ & 51.482972 & 179.123833 \\
\hline$\underset{0}{0}$ & Halibut - whole & HALI-CN-C2M-1 & FAUNA & $7 / 13 / 2011$ & 0.005 & 0.005 & 0.019 & 0.019 & $\mathrm{U}$ & 51.40025 & 179.283361 \\
\hline$\stackrel{8}{9}$ & \multirow{3}{*}{$\begin{array}{l}\text { Horse Mussel - } \\
\text { soft tissue only }\end{array}$} & HMUS-CN-CT1-1 & \begin{tabular}{|l|} 
FAUNA \\
\end{tabular} & $7 / 8 / 2011$ & 0.2 & 0.2 & 0.822 & 0.822 & $U$ & 51.482972 & 179.123833 \\
\hline ĩ & & HMUS-CN-CT2-1 & \begin{tabular}{|l|} 
FAUNA \\
\end{tabular} & $7 / 7 / 2011$ & $0.253 \pm 0.008$ & 0.19 & $1.146 \pm 0.036$ & 0.861 & $\mathrm{~J}$ & 51.472611 & 179.151111 \\
\hline$\infty$ & & HMUS-CN-CT3-1 & FAUNA & $7 / 5 / 2011$ & 0.2 & 0.2 & 0.82 & 0.82 & $U$ & 51.471416 & 179.172861 \\
\hline & \multirow{9}{*}{ || Irish Lord - whole } & IRLO-CN-CT1-1 & FAUNA & $7 / 8 / 2011$ & 0.07 & 0.07 & 0.315 & 0.315 & $\mathrm{U}$ & 51.482972 & 179.123833 \\
\hline & & IRLO-CN-CT1-2 & FAUNA & $7 / 8 / 2011$ & 0.04 & 0.04 & 0.2 & 0.2 & $\mathrm{U}$ & 51.482972 & 179.123833 \\
\hline & & IRLO-CN-CT1-3 & FAUNA & $7 / 8 / 2011$ & 0.07 & 0.07 & 0.329 & 0.329 & $\mathrm{U}$ & 51.482972 & 179.123833 \\
\hline & & IRLO-CN-CT2-1 & FAUNA & $7 / 7 / 2011$ & 0.04 & 0.04 & 0.189 & 0.189 & $\mathrm{U}$ & 51.472611 & 179.151111 \\
\hline & & IRLO-CN-CT2-2 & FAUNA & $7 / 7 / 2011$ & 0.05 & 0.05 & 0.228 & 0.228 & $U$ & 51.472611 & 179.151111 \\
\hline & & IRLO-CN-CT2-3 & FAUNA & $7 / 7 / 2011$ & 0.04 & 0.04 & 0.197 & 0.197 & $\mathrm{U}$ & 51.472611 & 179.151111 \\
\hline & & IRLO-CN-CT3-1 & FAUNA & $7 / 5 / 2011$ & 0.07 & 0.07 & 0.361 & 0.361 & $\mathrm{U}$ & 51.471416 & 179.172861 \\
\hline & & IRLO-CN-CT3-2 & FAUNA & $7 / 5 / 2011$ & 0.04 & 0.04 & 0.194 & 0.194 & $U$ & 51.471416 & 179.172861 \\
\hline & & IRLO-CN-CT3-3 & \begin{tabular}{|l|} 
FAUNA \\
\end{tabular} & $7 / 5 / 2011$ & 0.04 & 0.04 & 0.192 & 0.192 & $\mathrm{U}$ & 51.471416 & 179.172861 \\
\hline & Octopus & OCTP-CN-C2M-1 & \begin{tabular}{|l|} 
FAUNA \\
\end{tabular} & $7 / 8 / 2011$ & $0.031 \pm 0.004$ & 0.02 & $0.152 \pm 0.02$ & 0.098 & $\mathrm{~J}$ & 51.482972 & 179.123833 \\
\hline & $\begin{array}{l}\text { Pacific Cod - } \\
\text { whole }\end{array}$ & PCOD-CN-C2M-1 & FAUNA & $7 / 6 / 2011$ & 0.01 & 0.01 & 0.046 & 0.046 & $U$ & 51.483527 & 179.187583 \\
\hline & \multirow{10}{*}{ Rockfish - whole } & ROCK-CN-CT1-1 & FAUNA & $7 / 8 / 2011$ & 0.04 & 0.04 & 0.158 & 0.158 & $U$ & 51.482972 & 179.123833 \\
\hline & & ROCK-CN-CT1-2 & \begin{tabular}{|l|} 
FAUNA \\
\end{tabular} & $7 / 8 / 2011$ & 0.03 & 0.03 & 0.116 & 0.116 & $\mathrm{U}$ & 51.482972 & 179.123833 \\
\hline & & ROCK-CN-CT1-3 & \begin{tabular}{|l|} 
FAUNA \\
\end{tabular} & $7 / 8 / 2011$ & 0.03 & 0.03 & 0.121 & 0.121 & $U$ & 51.482972 & 179.123833 \\
\hline is & & ROCK-CN-CT2-1 & FAUNA & $7 / 6 / 2011$ & 0.03 & 0.03 & 0.106 & 0.106 & $\mathrm{U}$ & 51.472611 & 179.151111 \\
\hline$\nabla$ & & ROCK-CN-CT2-2 & FAUNA & $7 / 6 / 2011$ & 0.03 & 0.03 & 0.11 & 0.11 & $U$ & 51.472611 & 179.151111 \\
\hline & & ROCK-CN-CT2-3 & FAUNA & $7 / 6 / 2011$ & 0.03 & 0.03 & 0.123 & 0.123 & $\mathrm{U}$ & 51.472611 & 179.151111 \\
\hline $\overrightarrow{\bar{Q}}$ & & ROCK-CN-CT3-1 & \begin{tabular}{|l|} 
FAUNA \\
\end{tabular} & $7 / 6 / 2011$ & 0.03 & 0.03 & 0.128 & 0.128 & $\mathrm{U}$ & 51.471416 & 179.172861 \\
\hline & & ROCK-CN-CT3-2 & \begin{tabular}{|l|} 
FAUNA \\
\end{tabular} & $7 / 6 / 2011$ & 0.03 & 0.03 & 0.115 & 0.115 & $\mathrm{U}$ & 51.471416 & 179.172861 \\
\hline $\begin{array}{c}\sigma \\
\oplus \\
9\end{array}$ & & ROCK-CN-CT3-3 & FAUNA & $7 / 6 / 2011$ & 0.04 & 0.04 & 0.167 & 0.167 & $\mathrm{U}$ & 51.471416 & 179.172861 \\
\hline$e_{0}^{0}$ & & ROCK-CN-DUP & \begin{tabular}{|l|} 
FAUNA \\
\end{tabular} & $7 / 6 / 2011$ & 0.04 & 0.04 & 0.161 & 0.161 & $\mathrm{U}$ & 51.471416 & 179.172861 \\
\hline
\end{tabular}




\begin{tabular}{|c|c|c|c|c|c|c|c|c|c|c|}
\hline Matrix Subtype & Location Code & Matrix & $\begin{array}{c}\text { Date } \\
\text { Sampled }\end{array}$ & $\begin{array}{l}\text { Wet Result } \pm \\
\text { Uncertainty }\end{array}$ & $\begin{array}{c}\text { Wet } \\
\text { Detection } \\
\text { Limit }\end{array}$ & $\begin{array}{l}\text { Dry Result } \pm \\
\text { Uncertainty }\end{array}$ & \begin{tabular}{|c|} 
Dry \\
Detection \\
Limit
\end{tabular} & Qualifiers & Latitude & Longitude \\
\hline Reindeer Lichen & STRL-AI-CT1-1 & VEG & $6 / 23 / 2011$ & $0.064 \pm 0.004$ & 0.07 & $0.101 \pm 0.006$ & 0.111 & $\mathrm{~J}$ & 51.480583 & 179.109472 \\
\hline \multirow{3}{*}{$\begin{array}{l}\text { Sea Urchin - } \\
\text { whole }\end{array}$} & URCH-CN-CT1-1 & FAUNA & $7 / 8 / 2011$ & $0.09 \pm 0.004$ & 0.04 & $0.361 \pm 0.016$ & 0.16 & $\mathrm{~J}$ & 51.482972 & 179.123833 \\
\hline & URCH-CN-CT2-1 & FAUNA & $7 / 7 / 2011$ & 0.06 & 0.06 & 0.206 & 0.206 & $\mathrm{U}$ & 51.472611 & 179.151111 \\
\hline & URCH-CN-CT3-1 & FAUNA & $7 / 5 / 2011$ & $0.033 \pm 0.004$ & 0.04 & $0.126 \pm 0.015$ & 0.153 & $\mathrm{~J}$ & 51.471416 & 179.172861 \\
\hline \multicolumn{11}{|c|}{ Amchitka Site (Adak Island) (AMC13) } \\
\hline $\begin{array}{l}\text { Dolly Varden - } \\
\text { whole }\end{array}$ & DOLL-AD-XXX-1 & FAUNA & $6 / 28 / 2011$ & 0.01 & 0.01 & 0.041 & 0.041 & $u$ & 51.8375 & -176.672555 \\
\hline \multirow{3}{*}{ Reindeer Lichen } & STRL-AD-XXX-1 & VEG & $6 / 28 / 2011$ & $0.308 \pm 0.008$ & 0.05 & $0.603 \pm 0.016$ & 0.098 & & 51.837527 & -176.670694 \\
\hline & STRL-AD-XXX-2 & VEG & $6 / 29 / 2011$ & $0.1 \pm 0.004$ & 0.04 & $0.247 \pm 0.01$ & 0.099 & $\mathrm{~J}$ & 51.872833 & -176.707444 \\
\hline & STRL-AD-XXX-3 & VEG & $6 / 29 / 2011$ & $0.237 \pm 0.008$ & 0.05 & $0.377 \pm 0.013$ & 0.079 & & 51.900805 & -176.688527 \\
\hline \multicolumn{11}{|c|}{ Amchitka Site (Adak Island North) (AMC14) } \\
\hline \multirow{3}{*}{ Chiton } & CHIT-AN-ANT1-1 & FAUNA & $7 / 20 / 2011$ & 0.03 & 0.03 & 0.146 & 0.146 & $\mathrm{U}$ & 51.838722 & -176.582805 \\
\hline & CHIT-AN-ANT2-1 & FAUNA & $7 / 18 / 2011$ & 0.04 & 0.04 & 0.2 & 0.2 & $U$ & 51.848361 & -176.506277 \\
\hline & \begin{tabular}{|l|} 
CHIT-AN-ANT3-1 \\
\end{tabular} & FAUNA & 7/17/2011 & 0.04 & 0.04 & 0.203 & 0.203 & $U$ & 51.853722 & -176.470694 \\
\hline \multirow{10}{*}{ Dragon Kelp } & \begin{tabular}{|l|} 
EUAL-AN-ANT1-1 \\
\end{tabular} & VEG & 7/19/2011 & $0.051 \pm 0.002$ & 0.01 & $0.428 \pm 0.017$ & 0.084 & & 51.835694 & -176.542138 \\
\hline & \begin{tabular}{|l} 
EUAL-AN-ANT1-2 \\
\end{tabular} & VEG & \begin{tabular}{|l|}
$7 / 19 / 2011$ \\
\end{tabular} & $0.0146 \pm 0.0006$ & 0.01 & $0.1 \pm 0.004$ & 0.068 & $\mathrm{~J}$ & 51.835694 & -176.542138 \\
\hline & \begin{tabular}{|l|} 
EUAL-AN-ANT1-3 \\
\end{tabular} & VEG & 7/19/2011 & $0.0362 \pm 0.0008$ & 0.01 & $0.28 \pm 0.006$ & 0.077 & & 51.835694 & -176.542138 \\
\hline & \begin{tabular}{|l} 
EUAL-AN-ANT2-1 \\
\end{tabular} & VEG & $7 / 18 / 2011$ & $0.022 \pm 0.002$ & 0.01 & $0.16 \pm 0.015$ & 0.073 & $\mathrm{~J}$ & 51.848361 & -176.506277 \\
\hline & \begin{tabular}{|l} 
EUAL-AN-ANT2-2 \\
\end{tabular} & VEG & 7/18/2011 & $0.035 \pm 0.002$ & 0.01 & $0.223 \pm 0.013$ & 0.064 & & 51.848361 & \begin{tabular}{|l}
-176.506277 \\
\end{tabular} \\
\hline & \begin{tabular}{|l|l|} 
EUAL-AN-ANT2-3 \\
\end{tabular} & VEG & \begin{tabular}{|l|}
$7 / 18 / 2011$ \\
\end{tabular} & $0.054 \pm 0.002$ & 0.01 & $0.375 \pm 0.014$ & 0.069 & & 51.848361 & -176.506277 \\
\hline & \begin{tabular}{|l|} 
EUAL-AN-ANT3-1 \\
\end{tabular} & VEG & $7 / 17 / 2011$ & $0.049 \pm 0.002$ & 0.01 & $0.366 \pm 0.015$ & 0.075 & & 51.853722 & -176.470694 \\
\hline & \begin{tabular}{|l|} 
EUAL-AN-ANT3-2 \\
\end{tabular} & VEG & 7/17/2011 & $0.0133 \pm 0.0006$ & 0.01 & $0.091 \pm 0.004$ & 0.068 & $\mathrm{~J}$ & 51.853722 & \begin{tabular}{|l}
-176.470694 \\
\end{tabular} \\
\hline & \begin{tabular}{|l} 
EUAL-AN-ANT3-3 \\
\end{tabular} & VEG & \begin{tabular}{|l|}
$7 / 17 / 2011$ \\
\end{tabular} & $0.0136 \pm 0.0008$ & 0.01 & $0.091 \pm 0.005$ & 0.067 & $\mathrm{~J}$ & 51.853722 & -176.470694 \\
\hline & EUAL-AN-DUP & VEG & 7/17/2011 & $0.0208 \pm 0.0008$ & 0.01 & $0.131 \pm 0.005$ & 0.063 & $\mathrm{~J}$ & 51.853722 & -176.470694 \\
\hline \multirow{10}{*}{ Rockweed } & FUCU-AN-ANT1-1 & VEG & 7/19/2011 & $0.019 \pm 0.0006$ & 0.01 & $0.097 \pm 0.003$ & 0.051 & $\mathrm{~J}$ & 51.841444 & -176.546083 \\
\hline & \begin{tabular}{|l} 
FUCU-AN-ANT1-2 \\
\end{tabular} & VEG & \begin{tabular}{|l|}
$7 / 20 / 2011$ \\
\end{tabular} & 0.01 & 0.01 & 0.065 & 0.065 & $\mathrm{U}$ & 51.8395 & -176.591305 \\
\hline & \begin{tabular}{|l|l|l|} 
FUCU-AN-ANT1-3 \\
\end{tabular} & VEG & $7 / 20 / 2011$ & $0.0055 \pm 0.0008$ & 0.01 & $0.03 \pm 0.004$ & 0.055 & $\mathrm{~J}$ & 51.8395 & -176.591305 \\
\hline & FUCU-AN-ANT2-1 & VEG & $7 / 18 / 2011$ & $0.0061 \pm 0.001$ & 0.01 & $0.039 \pm 0.006$ & 0.063 & $\mathrm{~J}$ & 51.837666 & -176.517527 \\
\hline & \begin{tabular}{|l|} 
FUCU-AN-ANT2-2 \\
\end{tabular} & VEG & \begin{tabular}{|l|}
$7 / 18 / 2011$ \\
\end{tabular} & 0.01 & 0.01 & 0.053 & 0.053 & $U$ & 51.841444 & -176.546083 \\
\hline & \begin{tabular}{|l|} 
FUCU-AN-ANT2-3 \\
\end{tabular} & VEG & \begin{tabular}{|l|}
$7 / 18 / 2011$ \\
\end{tabular} & $0.0036 \pm 0.0004$ & 0.01 & $0.015 \pm 0.002$ & 0.042 & $\mathrm{~J}$ & 51.8395 & \begin{tabular}{|l}
-176.591305 \\
\end{tabular} \\
\hline & \begin{tabular}{|l} 
FUCU-AN-ANT3-1 \\
\end{tabular} & VEG & \begin{tabular}{|l|}
$7 / 18 / 2011$ \\
\end{tabular} & $0.004 \pm 0.0008$ & 0.01 & $0.023 \pm 0.005$ & 0.057 & $\mathrm{~J}$ & 51.839944 & -176.515916 \\
\hline & \begin{tabular}{|l} 
FUCU-AN-ANT3-2 \\
\end{tabular} & VEG & 7/18/2011 & $0.0063 \pm 0.0004$ & 0.01 & $0.031 \pm 0.002$ & 0.049 & $\mathrm{~J}$ & 51.828972 & -176.448305 \\
\hline & \begin{tabular}{|l} 
FUCU-AN-ANT3-3 \\
\end{tabular} & VEG & $7 / 18 / 2011$ & $0.0068 \pm 0.0004$ & 0.01 & $0.034 \pm 0.002$ & 0.05 & $\mathrm{~J}$ & 51.830833 & -176.456361 \\
\hline & FUCU-AN-DUP & VEG & 7/18/2011 & $0.0048 \pm 0.0004$ & 0.01 & $0.022 \pm 0.002$ & 0.047 & $\mathrm{~J}$ & 51.828972 & -176.448305 \\
\hline
\end{tabular}




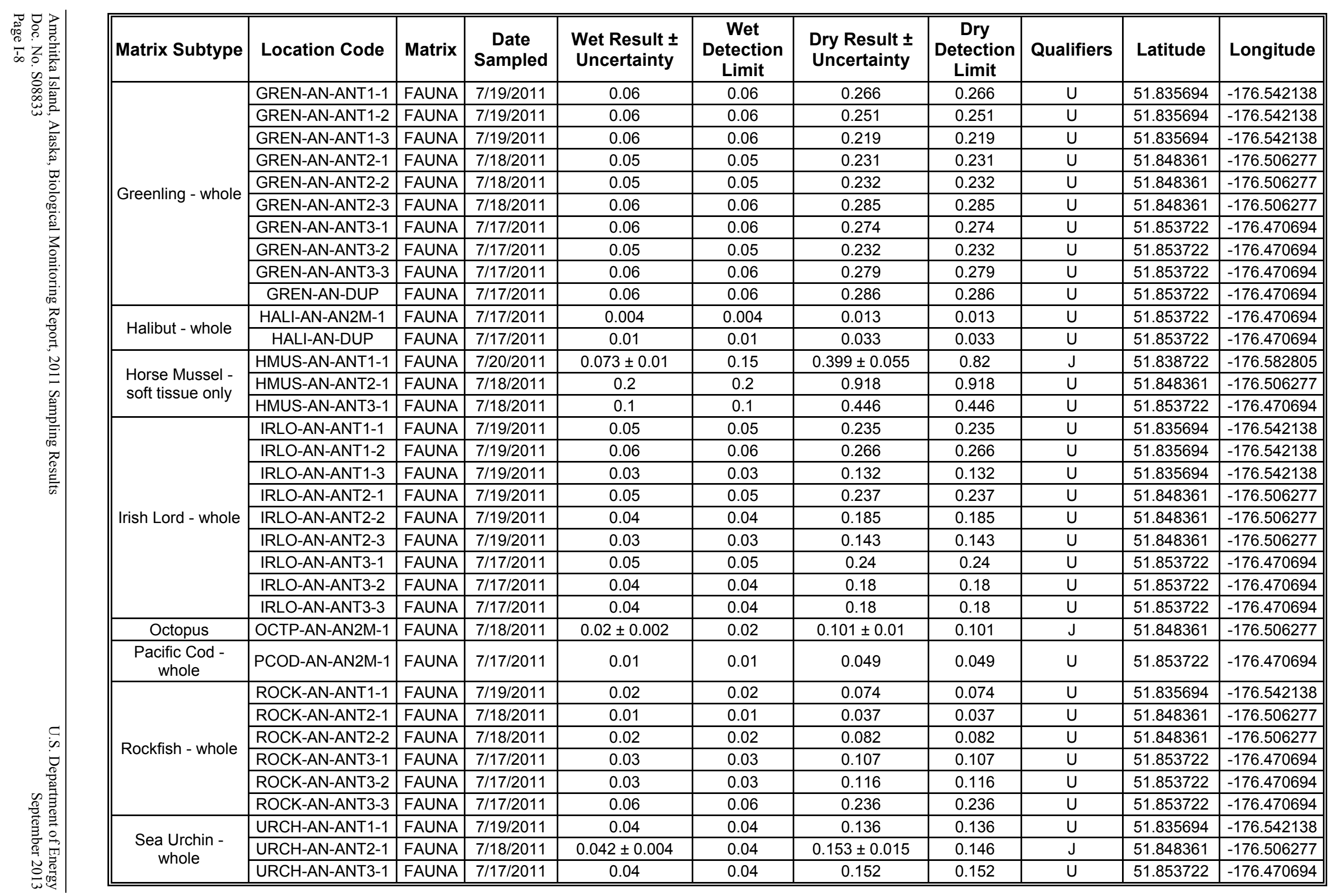




\begin{tabular}{|c|c|c|c|c|c|c|c|c|c|c|}
\hline Matrix Subtype & Location Code & Matrix & $\begin{array}{c}\text { Date } \\
\text { Sampled }\end{array}$ & $\begin{array}{l}\text { Wet Result } \pm \\
\text { Uncertainty }\end{array}$ & $\begin{array}{c}\text { Wet } \\
\text { Detection } \\
\text { Limit }\end{array}$ & $\begin{array}{l}\text { Dry Result } \pm \\
\text { Uncertainty }\end{array}$ & \begin{tabular}{|c|} 
Dry \\
Detection \\
Limit
\end{tabular} & Qualifiers & Latitude & Longitude \\
\hline \multicolumn{11}{|c|}{ Amchitka Site (Adak Island South) (AMC15) } \\
\hline \multirow{3}{*}{ Chiton } & CHIT-AS-AST1-1 & FAUNA & $7 / 15 / 2011$ & 0.06 & 0.06 & 0.247 & 0.247 & $\mathrm{U}$ & 51.737472 & -176.48025 \\
\hline & CHIT-AS-AST2-1 & FAUNA & $7 / 16 / 2011$ & 0.04 & 0.04 & 0.205 & 0.205 & $\mathrm{U}$ & 51.745666 & -176.507055 \\
\hline & CHIT-AS-AST3-1 & FAUNA & $7 / 14 / 2011$ & $0.056 \pm 0.008$ & 0.06 & $0.264 \pm 0.038$ & 0.283 & $\mathrm{~J}$ & 51.688333 & -176.63375 \\
\hline \multirow{10}{*}{ Dragon Kelp } & EUAL-AS-AST1-1 & VEG & $7 / 15 / 2011$ & $0.0193 \pm 0.0008$ & 0.01 & $0.127 \pm 0.005$ & 0.066 & $\mathrm{~J}$ & 51.737472 & -176.48025 \\
\hline & EUAL-AS-AST1-2 & VEG & $7 / 15 / 2011$ & $0.0577 \pm 0.0008$ & 0.01 & $0.46 \pm 0.006$ & 0.08 & & 51.737472 & -176.48025 \\
\hline & EUAL-AS-AST1-3 & VEG & $7 / 15 / 2011$ & $0.1 \pm 0.002$ & 0.01 & $0.96 \pm 0.019$ & 0.096 & & 51.737472 & -176.48025 \\
\hline & EUAL-AS-AST2-1 & VEG & $7 / 16 / 2011$ & $0.0366 \pm 0.0008$ & 0.01 & $0.26 \pm 0.006$ & 0.071 & & 51.745666 & -176.507055 \\
\hline & EUAL-AS-AST2-2 & VEG & \begin{tabular}{|l|}
$7 / 16 / 2011$ \\
\end{tabular} & $0.0206 \pm 0.001$ & 0.01 & $0.146 \pm 0.007$ & 0.071 & $\mathrm{~J}$ & 51.745666 & -176.507055 \\
\hline & EUAL-AS-AST2-3 & VEG & \begin{tabular}{|l|}
$7 / 16 / 2011$ \\
\end{tabular} & $0.0189 \pm 0.0008$ & 0.01 & $0.137 \pm 0.006$ & 0.073 & $\mathrm{~J}$ & 51.745666 & -176.507055 \\
\hline & $\begin{array}{l}\text { EUAL-AS-AST3-1 } \\
\end{array}$ & VEG & \begin{tabular}{|l|}
$7 / 14 / 2011$ \\
\end{tabular} & $0.1 \pm 0.002$ & 0.01 & $0.73 \pm 0.015$ & 0.073 & & 51.688333 & \begin{tabular}{|l|}
-176.63375 \\
\end{tabular} \\
\hline & EUAL-AS-AST3-2 & VEG & \begin{tabular}{|l|}
$7 / 14 / 2011$ \\
\end{tabular} & $0.11 \pm 0.002$ & 0.01 & $1.043 \pm 0.019$ & 0.095 & & 51.688333 & \begin{tabular}{|l|}
-176.63375 \\
\end{tabular} \\
\hline & EUAL-AS-AST3-3 & VEG & $7 / 14 / 2011$ & $0.104 \pm 0.002$ & 0.01 & $0.917 \pm 0.018$ & 0.088 & & 51.688333 & -176.63375 \\
\hline & EUAL-AS-DUP & VEG & 7/14/2011 & $0.039 \pm 0.002$ & 0.01 & $0.287 \pm 0.015$ & 0.074 & & 51.688333 & -176.63375 \\
\hline \multirow{10}{*}{ Rockweed } & FUCU-AS-AST1-1 & VEG & $7 / 16 / 2011$ & $0.0072 \pm 0.0004$ & 0.01 & $0.044 \pm 0.002$ & 0.062 & $\mathrm{~J}$ & 51.733666 & -176.470805 \\
\hline & FUCU-AS-AST1-2 & VEG & \begin{tabular}{|l|l|}
$7 / 2011$ \\
\end{tabular} & $0.0045 \pm 0.0004$ & 0.01 & $0.025 \pm 0.002$ & 0.055 & $\mathrm{~J}$ & 51.733666 & -176.470805 \\
\hline & FUCU-AS-AST1-3 & VEG & $7 / 16 / 2011$ & $0.011 \pm 0.0006$ & 0.01 & $0.06 \pm 0.003$ & 0.054 & $\mathrm{~J}$ & 51.733666 & -176.470805 \\
\hline & $\begin{array}{l}\text { FUCU-AS-AST2-1 } \\
\end{array}$ & VEG & \begin{tabular}{|l|}
$7 / 16 / 2011$ \\
\end{tabular} & $0.0085 \pm 0.0004$ & 0.01 & $0.041 \pm 0.002$ & 0.048 & $\mathrm{~J}$ & 51.745666 & -176.507055 \\
\hline & FUCU-AS-AST2-2 & VEG & \begin{tabular}{|l|}
$7 / 16 / 2011$ \\
\end{tabular} & $0.0086 \pm 0.0004$ & 0.01 & $0.044 \pm 0.002$ & 0.051 & $\mathrm{~J}$ & 51.745666 & -176.507055 \\
\hline & FUCU-AS-AST2-3 & VEG & \begin{tabular}{|l|}
$7 / 16 / 2011$ \\
\end{tabular} & 0.01 & 0.01 & 0.049 & 0.049 & $\mathrm{U}$ & 51.745666 & -176.507055 \\
\hline & FUCU-AS-AST3-1 & VEG & 7/14/2011 & $0.0048 \pm 0.0004$ & 0.01 & $0.025 \pm 0.002$ & 0.053 & $\mathrm{~J}$ & 51.688333 & -176.63375 \\
\hline & FUCU-AS-AST3-2 & VEG & $7 / 14 / 2011$ & $0.0094 \pm 0.0006$ & 0.01 & $0.046 \pm 0.003$ & 0.049 & $\mathrm{~J}$ & 51.688333 & -176.63375 \\
\hline & FUCU-AS-AST3-3 & VEG & $7 / 14 / 2011$ & $0.0022 \pm 0.0002$ & 0.004 & $0.012 \pm 0.001$ & 0.021 & $\mathrm{~J}$ & 51.688333 & -176.63375 \\
\hline & FUCU-AS-DUP & VEG & $7 / 16 / 2011$ & $0.0043 \pm 0.0004$ & 0.01 & $0.022 \pm 0.002$ & 0.052 & $\mathrm{~J}$ & 51.733666 & -176.470805 \\
\hline \multirow{10}{*}{ Greenling - whole } & GREN-AS-AST1-1 & FAUNA & \begin{tabular}{|l|}
$7 / 15 / 2011$ \\
\end{tabular} & 0.06 & 0.06 & 0.286 & 0.286 & $U$ & 51.737472 & \begin{tabular}{|l|}
-176.48025 \\
\end{tabular} \\
\hline & GREN-AS-AST1-2 & FAUNA & \begin{tabular}{|l|}
$7 / 15 / 2011$ \\
\end{tabular} & 0.05 & 0.05 & 0.236 & 0.236 & $U$ & 51.737472 & \begin{tabular}{|l|}
-176.48025 \\
\end{tabular} \\
\hline & GREN-AS-AST1-3 & FAUNA & \begin{tabular}{|l|}
$7 / 15 / 2011$ \\
\end{tabular} & 0.05 & 0.05 & 0.24 & 0.24 & $\mathrm{U}$ & 51.737472 & -176.48025 \\
\hline & GREN-AS-AST2-1 & FAUNA & 7/16/2011 & 0.04 & 0.04 & 0.161 & 0.161 & $\mathrm{U}$ & 51.745666 & -176.507055 \\
\hline & GREN-AS-AST2-2 & FAUNA & $7 / 16 / 2011$ & 0.05 & 0.05 & 0.229 & 0.229 & $U$ & 51.745666 & -176.507055 \\
\hline & GREN-AS-AST2-3 & FAUNA & 7/16/2011 & 0.05 & 0.05 & 0.233 & 0.233 & $U$ & 51.745666 & -176.507055 \\
\hline & GREN-AS-AST3-1 & FAUNA & 7/14/2011 & 0.05 & 0.05 & 0.238 & 0.238 & $U$ & 51.688333 & -176.63375 \\
\hline & GREN-AS-AST3-2 & FAUNA & $7 / 14 / 2011$ & 0.06 & 0.06 & 0.282 & 0.282 & $U$ & 51.688333 & -176.63375 \\
\hline & GREN-AS-AST3-3 & FAUNA & \begin{tabular}{|l|}
$7 / 14 / 2011$ \\
\end{tabular} & 0.07 & 0.07 & 0.334 & 0.334 & $U$ & 51.688333 & -176.63375 \\
\hline & GREN-AS-DUP & FAUNA & $7 / 14 / 2011$ & 0.05 & 0.05 & 0.251 & 0.251 & $U$ & 51.688333 & -176.63375 \\
\hline
\end{tabular}




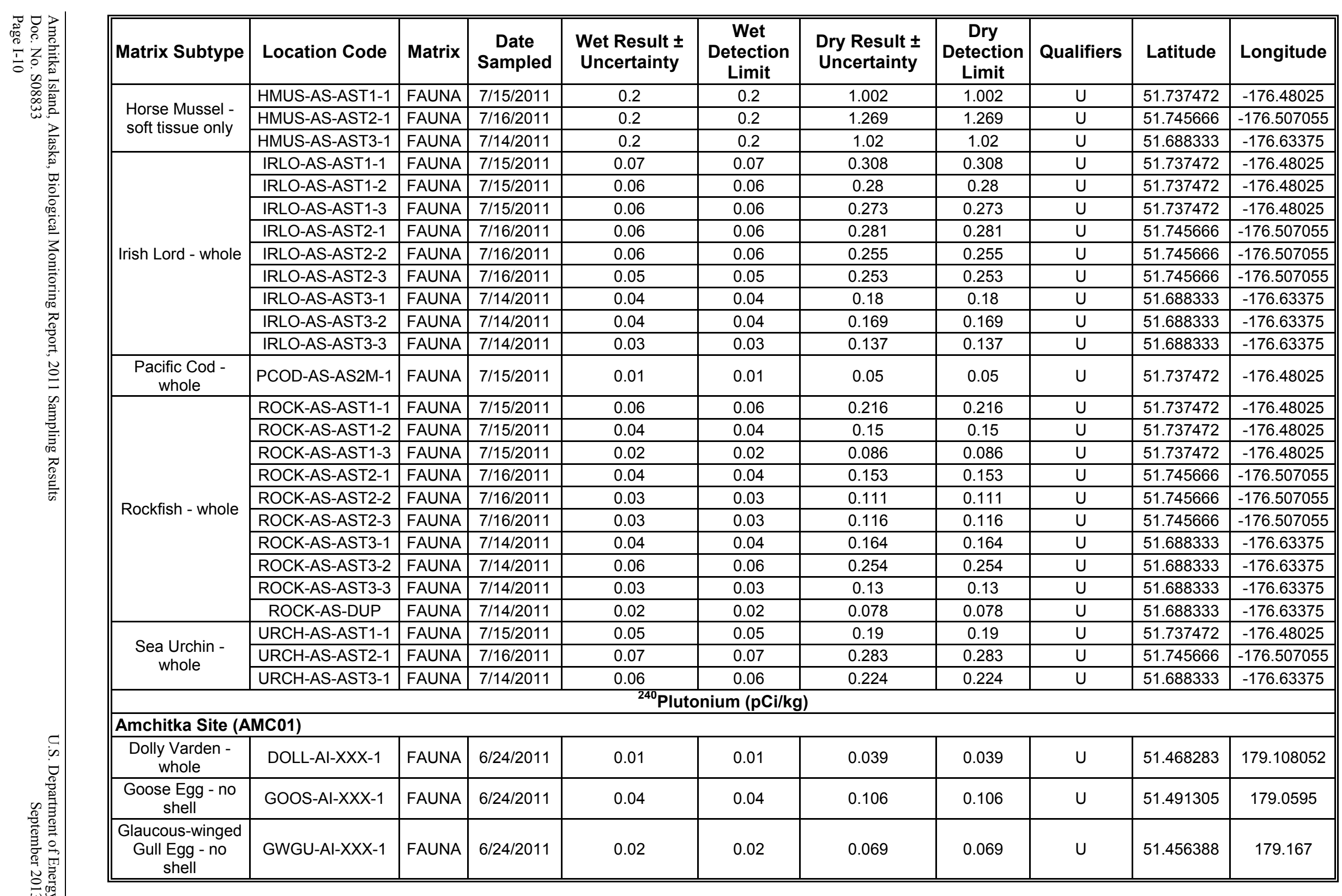




\begin{tabular}{|c|c|c|c|c|c|c|c|c|c|c|}
\hline Matrix Subtype & Location Code & Matrix & $\begin{array}{c}\text { Date } \\
\text { Sampled }\end{array}$ & $\begin{array}{l}\text { Wet Result } \pm \\
\text { Uncertainty }\end{array}$ & $\begin{array}{c}\text { Wet } \\
\text { Detection } \\
\text { Limit }\end{array}$ & $\begin{array}{l}\text { Dry Result } \pm \\
\text { Uncertainty }\end{array}$ & \begin{tabular}{|c|} 
Dry \\
Detection \\
Limit
\end{tabular} & Qualifiers & Latitude & Longitude \\
\hline \multicolumn{11}{|c|}{ Amchitka Site (Long Shot) (AMC02) } \\
\hline \multirow{3}{*}{ Chiton } & CHIT-LS-LT1-1 & FAUNA & $7 / 4 / 2011$ & 0.03 & 0.03 & 0.149 & 0.149 & $U$ & 51.457555 & 179.195138 \\
\hline & CHIT-LS-LT2-1 & FAUNA & $7 / 3 / 2011$ & 0.03 & 0.03 & 0.141 & 0.141 & $U$ & 51.44925 & 179.205583 \\
\hline & CHIT-LS-LT3-1 & FAUNA & $7 / 3 / 2011$ & 0.03 & 0.03 & 0.143 & 0.143 & $\mathrm{U}$ & 51.43 & 179.22667 \\
\hline \multirow{10}{*}{ Dragon Kelp } & EUAL-LS-DUP & VEG & $7 / 3 / 2011$ & $0.034 \pm 0.002$ & 0.003 & $0.204 \pm 0.012$ & 0.018 & & 51.44925 & 179.205583 \\
\hline & EUAL-LS-LT1-1 & VEG & $7 / 4 / 2011$ & $0.051 \pm 0.004$ & 0.004 & $0.477 \pm 0.037$ & 0.037 & & 51.457555 & 179.195138 \\
\hline & EUAL-LS-LT1-2 & VEG & $7 / 4 / 2011$ & 0.003 & 0.003 & 0.019 & 0.019 & $U$ & 51.457555 & 179.195138 \\
\hline & EUAL-LS-LT1-3 & VEG & $7 / 4 / 2011$ & $0.01 \pm 0.002$ & 0.003 & $0.06 \pm 0.012$ & 0.018 & & 51.457555 & 179.195138 \\
\hline & EUAL-LS-LT2-1 & VEG & $7 / 3 / 2011$ & $0.032 \pm 0.002$ & 0.003 & $0.194 \pm 0.012$ & 0.018 & & 51.44925 & 179.205583 \\
\hline & EUAL-LS-LT2-2 & VEG & $7 / 3 / 2011$ & $0.013 \pm 0.002$ & 0.004 & $0.094 \pm 0.015$ & 0.029 & & 51.44925 & 179.205583 \\
\hline & EUAL-LS-LT2-3 & VEG & $7 / 3 / 2011$ & $0.029 \pm 0.002$ & 0.003 & $0.21 \pm 0.014$ & 0.022 & & 51.44925 & 179.205583 \\
\hline & EUAL-LS-LT3-1 & VEG & $7 / 2 / 2011$ & $0.011 \pm 0.002$ & 0.003 & $0.07 \pm 0.013$ & 0.019 & & 51.43 & 179.22667 \\
\hline & EUAL-LS-LT3-2 & VEG & $7 / 2 / 2011$ & $0.103 \pm 0.004$ & 0.004 & $0.733 \pm 0.028$ & 0.028 & & 51.43 & 179.22667 \\
\hline & EUAL-LS-LT3-3 & VEG & $7 / 2 / 2011$ & $0.054 \pm 0.004$ & 0.003 & $0.455 \pm 0.034$ & 0.025 & & 51.43 & 179.22667 \\
\hline \multirow{10}{*}{ Rockweed } & FUCU-LS-DUP & VEG & 6/22/2011 & 0.003 & 0.003 & 0.016 & 0.016 & $\mathrm{U}$ & 51.447472 & 179.190888 \\
\hline & FUCU-LS-LT1-1 & VEG & 6/22/2011 & 0.003 & 0.003 & 0.016 & 0.016 & $\mathrm{U}$ & 51.447527 & 179.189361 \\
\hline & FUCU-LS-LT1-2 & VEG & $6 / 22 / 2011$ & 0.003 & 0.003 & 0.018 & 0.018 & $\mathrm{U}$ & 51.447527 & 179.189361 \\
\hline & FUCU-LS-LT1-3 & VEG & 6/22/2011 & 0.003 & 0.003 & 0.02 & 0.02 & $\mathrm{U}$ & 51.448777 & 179.189305 \\
\hline & FUCU-LS-LT2-1 & VEG & $6 / 22 / 2011$ & 0.002 & 0.002 & 0.016 & 0.016 & $U$ & 51.447222 & 179.199361 \\
\hline & FUCU-LS-LT2-2 & VEG & $6 / 22 / 2011$ & $0.0048 \pm 0.001$ & 0.002 & $0.035 \pm 0.007$ & 0.014 & $\mathrm{~J}$ & 51.44625 & 179.196972 \\
\hline & FUCU-LS-LT2-3 & VEG & 6/22/2011 & 0.002 & 0.002 & 0.012 & 0.012 & $\mathrm{U}$ & 51.446722 & 179.191888 \\
\hline & FUCU-LS-LT3-1 & VEG & $7 / 2 / 2011$ & 0.003 & 0.003 & 0.015 & 0.015 & $\mathrm{U}$ & 51.433611 & 179.219305 \\
\hline & FUCU-LS-LT3-2 & VEG & $7 / 2 / 2011$ & 0.003 & 0.003 & 0.014 & 0.014 & $\mathrm{U}$ & 51.430166 & 179.218694 \\
\hline & FUCU-LS-LT3-3 & VEG & $7 / 2 / 2011$ & 0.003 & 0.003 & 0.011 & 0.011 & $\mathrm{U}$ & 51.430166 & 179.218694 \\
\hline \multirow{10}{*}{ Greenling - whole } & GREN-LS-DUP & FAUNA & \begin{tabular}{|l|l|}
$7 / 12 / 2011$ \\
\end{tabular} & 0.03 & 0.03 & 0.146 & 0.146 & $\mathrm{U}$ & 51.434527 & 179.234416 \\
\hline & GREN-LS-LT1-1 & FAUNA & $7 / 4 / 2011$ & 0.03 & 0.03 & 0.141 & 0.141 & $U$ & 51.457555 & 179.195138 \\
\hline & GREN-LS-LT1-2 & FAUNA & $7 / 4 / 2011$ & 0.04 & 0.04 & 0.189 & 0.189 & $\mathrm{U}$ & 51.457555 & 179.195138 \\
\hline & GREN-LS-LT1-3 & FAUNA & $7 / 5 / 2011$ & 0.04 & 0.04 & 0.187 & 0.187 & $\mathrm{U}$ & 51.457555 & 179.195138 \\
\hline & GREN-LS-LT2-1 & FAUNA & $7 / 3 / 2011$ & 0.03 & 0.03 & 0.145 & 0.145 & $\mathrm{U}$ & 51.432194 & 179.230277 \\
\hline & GREN-LS-LT2-2 & FAUNA & $7 / 3 / 2011$ & 0.02 & 0.02 & 0.092 & 0.092 & $\mathrm{U}$ & 51.432194 & 179.230277 \\
\hline & GREN-LS-LT2-3 & FAUNA & $7 / 3 / 2011$ & 0.02 & 0.02 & 0.095 & 0.095 & $U$ & 51.432194 & 179.230277 \\
\hline & GREN-LS-LT3-1 & FAUNA & $7 / 3 / 2011$ & 0.02 & 0.02 & 0.089 & 0.089 & $\mathrm{U}$ & 51.43 & 179.22667 \\
\hline & GREN-LS-LT3-2 & FAUNA & 7/12/2011 & 0.03 & 0.03 & 0.147 & 0.147 & $\mathrm{U}$ & 51.434527 & 179.234416 \\
\hline & GREN-LS-LT3-3 & FAUNA & $7 / 12 / 2011$ & 0.03 & 0.03 & 0.145 & 0.145 & $U$ & 51.434527 & 179.234416 \\
\hline Halibut - whole & HALI-LS-L2M-1 & FAUNA & 7/13/2011 & 0.003 & 0.003 & 0.012 & 0.012 & $\mathrm{U}$ & 51.40025 & 179.283361 \\
\hline
\end{tabular}




\begin{tabular}{|c|c|c|c|c|c|c|c|c|c|c|}
\hline Matrix Subtype & Location Code & Matrix & $\begin{array}{c}\text { Date } \\
\text { Sampled }\end{array}$ & $\begin{array}{l}\text { Wet Result } \pm \\
\text { Uncertainty }\end{array}$ & $\begin{array}{c}\text { Wet } \\
\text { Detection } \\
\text { Limit }\end{array}$ & $\begin{array}{l}\text { Dry Result } \pm \\
\text { Uncertainty }\end{array}$ & \begin{tabular}{|c|} 
Dry \\
Detection \\
Limit
\end{tabular} & Qualifiers & Latitude & Longitude \\
\hline \multirow{3}{*}{$\begin{array}{l}\text { Horse Mussel - } \\
\text { soft tissue only }\end{array}$} & HMUS-LS-LT1-1 & FAUNA & $7 / 5 / 2011$ & 0.1 & 0.1 & 0.428 & 0.428 & $U$ & 51.457555 & 179.195138 \\
\hline & HMUS-LS-LT2-1 & FAUNA & $7 / 4 / 2011$ & 0.08 & 0.08 & 0.327 & 0.327 & $\mathrm{U}$ & 51.44925 & 179.205583 \\
\hline & HMUS-LS-LT3-1 & FAUNA & $7 / 3 / 2011$ & 1 & 1 & 2.097 & 2.097 & $\bar{U}$ & 51.44925 & 179.205583 \\
\hline \multirow{9}{*}{ || Irish Lord - whole } & IRLO-LS-LT1-1 & FAUNA & $7 / 4 / 2011$ & 0.04 & 0.04 & 0.189 & 0.189 & $U$ & 51.457555 & 179.195138 \\
\hline & IRLO-LS-LT1-2 & FAUNA & $7 / 4 / 2011$ & 0.04 & 0.04 & 0.189 & 0.189 & $\bar{U}$ & 51.457555 & 179.195138 \\
\hline & IRLO-LS-LT1-3 & FAUNA & $7 / 5 / 2011$ & 0.02 & 0.02 & 0.106 & 0.106 & $\mathrm{U}$ & 51.457555 & 179.195138 \\
\hline & IRLO-LS-LT2-1 & FAUNA & $7 / 3 / 2011$ & 0.03 & 0.03 & 0.13 & 0.13 & $\mathrm{U}$ & 51.432194 & 179.230277 \\
\hline & IRLO-LS-LT2-2 & FAUNA & $7 / 3 / 2011$ & 0.04 & 0.04 & 0.166 & 0.166 & $\bar{U}$ & 51.432194 & 179.230277 \\
\hline & IRLO-LS-LT2-3 & FAUNA & $7 / 3 / 2011$ & 0.04 & 0.04 & 0.177 & 0.177 & $U$ & 51.432194 & 179.230277 \\
\hline & IRLO-LS-LT3-1 & FAUNA & $7 / 2 / 2011$ & 0.01 & 0.01 & 0.045 & 0.045 & $\bar{U}$ & 51.43 & 179.22667 \\
\hline & IRLO-LS-LT3-2 & \begin{tabular}{|l|} 
FAUNA \\
\end{tabular} & $7 / 12 / 2011$ & 0.02 & 0.02 & 0.095 & 0.095 & $\bar{U}$ & 51.434944 & 179.2315 \\
\hline & IRLO-LS-LT3-3 & FAUNA & $7 / 12 / 2011$ & 0.02 & 0.02 & 0.11 & 0.11 & $U$ & 51.434944 & 179.2315 \\
\hline Octopus & OCTP-LS-L2M-1 & FAUNA & $7 / 3 / 2011$ & 0.003 & 0.003 & 0.021 & 0.021 & $\mathrm{U}$ & 51.44925 & 179.205583 \\
\hline $\begin{array}{l}\text { Pacific Cod - } \\
\text { whole }\end{array}$ & PCOD-LS-L2M-1 & FAUNA & $7 / 4 / 2011$ & 0.003 & 0.003 & 0.015 & 0.015 & $u$ & 51.451805 & 179.196972 \\
\hline \multirow{10}{*}{ Rockfish - whole } & ROCK-LS-DUP & FAUNA & $7 / 12 / 2011$ & 0.02 & 0.02 & 0.078 & 0.078 & $U$ & 51.434944 & 179.2315 \\
\hline & ROCK-LS-DUP2 & FAUNA & $7 / 12 / 2011$ & 0.02 & 0.02 & 0.086 & 0.086 & $\mathrm{U}$ & 51.465472 & 179.206722 \\
\hline & ROCK-LS-LT1-1 & FAUNA & $7 / 4 / 2011$ & 0.03 & 0.03 & 0.139 & 0.139 & $U$ & 51.457555 & 179.195138 \\
\hline & ROCK-LS-LT1-2 & FAUNA & $7 / 12 / 2011$ & 0.02 & 0.02 & 0.083 & 0.083 & $\bar{U}$ & 51.465472 & 179.206722 \\
\hline & ROCK-LS-LT1-3 & FAUNA & $7 / 12 / 2011$ & 0.02 & 0.02 & 0.083 & 0.083 & $\mathrm{U}$ & 51.465472 & 179.206722 \\
\hline & ROCK-LS-LT2-1 & FAUNA & $7 / 4 / 2011$ & 0.02 & 0.02 & 0.08 & 0.08 & $U$ & 51.44925 & 179.205583 \\
\hline & ROCK-LS-LT2-2 & FAUNA & $7 / 12 / 2011$ & 0.02 & 0.02 & 0.079 & 0.079 & $U$ & 51.44625 & 179.223416 \\
\hline & ROCK-LS-LT3-1 & FAUNA & $7 / 12 / 2011$ & 0.02 & 0.02 & 0.088 & 0.088 & $\mathrm{U}$ & 51.434944 & 179.2315 \\
\hline & ROCK-LS-LT3-2 & FAUNA & $7 / 12 / 2011$ & 0.02 & 0.02 & 0.085 & 0.085 & $\mathrm{U}$ & 51.434944 & 179.2315 \\
\hline & ROCK-LS-LT3-3 & FAUNA & $7 / 3 / 2011$ & 0.02 & 0.02 & 0.069 & 0.069 & $\mathrm{U}$ & 51.43 & 179.22667 \\
\hline Reindeer Lichen & STRL-AI-LT1-2 & VEG & $6 / 22 / 2011$ & 0.03 & 0.03 & 0.091 & 0.091 & $U$ & 51.447361 & 179.189111 \\
\hline \multirow{3}{*}{$\begin{array}{l}\text { Sea Urchin - } \\
\text { whole }\end{array}$} & URCH-LS-LT1-1 & FAUNA & $7 / 4 / 2011$ & 0.03 & 0.03 & 0.117 & 0.117 & $\mathrm{U}$ & 51.457555 & 179.195138 \\
\hline & URCH-LS-LT2-1 & FAUNA & 7/3/2011 & 0.03 & 0.03 & 0.115 & 0.115 & $U$ & 51.44925 & 179.205583 \\
\hline & URCH-LS-LT3-1 & FAUNA & $7 / 2 / 2011$ & 0.03 & 0.03 & 0.117 & 0.117 & $U$ & 51.43 & 179.22667 \\
\hline \multicolumn{11}{|c|}{ Amchitka Site (Milrow) (AMC03) } \\
\hline \multirow{3}{*}{ Chiton } & CHIT-ML-MT1-1 & FAUNA & $7 / 9 / 2011$ & 0.04 & 0.04 & 0.151 & 0.151 & $\mathrm{U}$ & 51.408472 & 179.139166 \\
\hline & CHIT-ML-MT2-1 & FAUNA & $7 / 10 / 2011$ & 0.03 & 0.03 & 0.139 & 0.139 & $\mathrm{U}$ & 51.389416 & 179.173138 \\
\hline & CHIT-ML-MT3-1 & FAUNA & 7/10/2011 & 0.04 & 0.04 & 0.182 & 0.182 & $\mathrm{U}$ & 51.387805 & 179.184 \\
\hline
\end{tabular}




\begin{tabular}{|c|c|c|c|c|c|c|c|c|c|c|}
\hline Matrix Subtype & Location Code & Matrix & $\begin{array}{c}\text { Date } \\
\text { Sampled }\end{array}$ & $\begin{array}{c}\text { Wet Result } \pm \\
\text { Uncertainty }\end{array}$ & $\begin{array}{c}\text { Wet } \\
\text { Detection } \\
\text { Limit }\end{array}$ & $\begin{array}{l}\text { Dry Result } \pm \\
\text { Uncertainty }\end{array}$ & \begin{tabular}{|c|} 
Dry \\
Detection \\
Limit
\end{tabular} & Qualifiers & Latitude & Longitude \\
\hline \multirow{10}{*}{ Dragon Kelp } & EUAL-ML-DUP & VEG & $7 / 9 / 2011$ & $0.053 \pm 0.004$ & 0.003 & $0.399 \pm 0.03$ & 0.023 & & 51.408472 & 179.139166 \\
\hline & EUAL-ML-MT1-1 & VEG & $7 / 9 / 2011$ & $0.074 \pm 0.004$ & 0.003 & $0.592 \pm 0.032$ & 0.024 & & 51.408472 & 179.139166 \\
\hline & EUAL-ML-MT1-2 & VEG & $7 / 9 / 2011$ & $0.06 \pm 0.004$ & 0.003 & $0.446 \pm 0.03$ & 0.022 & & 51.408472 & 179.139166 \\
\hline & EUAL-ML-MT1-3 & VEG & $7 / 9 / 2011$ & $0.078 \pm 0.004$ & 0.003 & $0.656 \pm 0.034$ & 0.025 & & 51.408472 & 179.139166 \\
\hline & EUAL-ML-MT2-1 & VEG & $7 / 10 / 2011$ & $0.096 \pm 0.006$ & 0.004 & $0.715 \pm 0.045$ & 0.03 & & 51.389416 & 179.173138 \\
\hline & EUAL-ML-MT2-2 & VEG & $7 / 10 / 2011$ & $0.085 \pm 0.004$ & 0.003 & $0.602 \pm 0.028$ & 0.021 & & 51.389416 & 179.173138 \\
\hline & EUAL-ML-MT2-3 & VEG & $7 / 10 / 2011$ & $0.022 \pm 0.002$ & 0.004 & $0.166 \pm 0.015$ & 0.03 & & 51.389416 & 179.173138 \\
\hline & EUAL-ML-MT3-1 & VEG & $7 / 10 / 2011$ & $0.129 \pm 0.004$ & 0.003 & $0.907 \pm 0.028$ & 0.021 & & 51.387805 & 179.184 \\
\hline & EUAL-ML-MT3-2 & VEG & $7 / 10 / 2011$ & $0.026 \pm 0.002$ & 0.003 & $0.19 \pm 0.015$ & 0.022 & & 51.387805 & 179.184 \\
\hline & EUAL-ML-MT3-3 & VEG & $7 / 10 / 2011$ & $0.117 \pm 0.004$ & 0.003 & $0.807 \pm 0.028$ & 0.021 & & 51.387805 & 179.184 \\
\hline \multirow{10}{*}{ Rockweed } & FUCU-ML-DUP & VEG & $6 / 20 / 2011$ & 0.003 & 0.003 & 0.013 & 0.013 & $\mathrm{U}$ & 51.412449 & 179.160843 \\
\hline & FUCU-ML-MT1-1 & VEG & $6 / 20 / 2011$ & 0.003 & 0.003 & 0.012 & 0.012 & $\mathrm{U}$ & 51.412449 & 179.160843 \\
\hline & FUCU-ML-MT1-2 & VEG & $6 / 21 / 2011$ & 0.003 & 0.003 & 0.018 & 0.018 & $\mathrm{U}$ & 51.417083 & 179.134083 \\
\hline & FUCU-ML-MT1-3 & VEG & $7 / 9 / 2011$ & 0.003 & 0.003 & 0.014 & 0.014 & $U$ & 51.417083 & 179.134083 \\
\hline & FUCU-ML-MT2-1 & VEG & $6 / 20 / 2011$ & 0.003 & 0.003 & 0.015 & 0.015 & $\mathrm{U}$ & 51.403305 & 179.167166 \\
\hline & FUCU-ML-MT2-2 & VEG & $6 / 21 / 2011$ & 0.003 & 0.003 & 0.021 & 0.021 & $\mathrm{U}$ & 51.405694 & 179.161361 \\
\hline & FUCU-ML-MT2-3 & VEG & $6 / 21 / 2011$ & 0.003 & 0.003 & 0.021 & 0.021 & $\mathrm{U}$ & 51.405694 & 179.161361 \\
\hline & FUCU-ML-MT3-1 & VEG & $7 / 10 / 2011$ & 0.003 & 0.003 & 0.015 & 0.015 & $\mathrm{U}$ & 51.389416 & 179.173138 \\
\hline & FUCU-ML-MT3-2 & VEG & $7 / 10 / 2011$ & 0.003 & 0.003 & 0.014 & 0.014 & $\mathrm{U}$ & 51.389416 & 179.173138 \\
\hline & FUCU-ML-MT3-3 & VEG & $7 / 10 / 2011$ & $0.008 \pm 0.002$ & 0.003 & $0.04 \pm 0.01$ & 0.015 & $\mathrm{~J}$ & 51.389416 & 179.173138 \\
\hline \multirow{10}{*}{ Greenling - whole } & GREN-ML-DUP & FAUNA & $7 / 10 / 2011$ & 0.03 & 0.03 & 0.145 & 0.145 & $\mathrm{U}$ & 51.387805 & 179.184 \\
\hline & GREN-ML-MT1-1 & FAUNA & $7 / 9 / 2011$ & 0.02 & 0.02 & 0.087 & 0.087 & $\mathrm{U}$ & 51.408472 & 179.139166 \\
\hline & GREN-ML-MT1-2 & FAUNA & $7 / 9 / 2011$ & 0.03 & 0.03 & 0.139 & 0.139 & $\mathrm{U}$ & 51.408472 & 179.139166 \\
\hline & GREN-ML-MT1-3 & FAUNA & $7 / 9 / 2011$ & 0.02 & 0.02 & 0.097 & 0.097 & $\mathrm{U}$ & 51.408472 & 179.139166 \\
\hline & GREN-ML-MT2-1 & FAUNA & $7 / 11 / 2011$ & 0.03 & 0.03 & 0.14 & 0.14 & $\mathrm{U}$ & 51.396944 & 179.1435 \\
\hline & GREN-ML-MT2-2 & FAUNA & $7 / 11 / 2011$ & 0.03 & 0.03 & 0.14 & 0.14 & $U$ & 51.396944 & 179.1435 \\
\hline & GREN-ML-MT2-3 & FAUNA & $7 / 11 / 2011$ & 0.03 & 0.03 & 0.143 & 0.143 & $U$ & 51.396944 & 179.1435 \\
\hline & GREN-ML-MT3-1 & FAUNA & $7 / 10 / 2011$ & 0.03 & 0.03 & 0.145 & 0.145 & $\mathrm{U}$ & 51.387805 & 179.184 \\
\hline & GREN-ML-MT3-2 & FAUNA & $7 / 10 / 2011$ & 0.04 & 0.04 & 0.193 & 0.193 & $\mathrm{U}$ & 51.387805 & 179.184 \\
\hline & GREN-ML-MT3-3 & FAUNA & $7 / 10 / 2011$ & 0.03 & 0.03 & 0.142 & 0.142 & $\mathrm{U}$ & 51.387805 & 179.184 \\
\hline Halibut - whole & HALI-ML-M2M-1 & FAUNA & $7 / 11 / 2011$ & 0.003 & 0.003 & 0.01 & 0.01 & $\mathrm{U}$ & 51.365055 & 179.111472 \\
\hline \multirow{3}{*}{$\begin{array}{l}\text { Horse Mussel - } \\
\text { soft tissue only }\end{array}$} & HMUS-ML-MT1-1 & FAUNA & $7 / 9 / 2011$ & 0.1 & 0.1 & 0.455 & 0.455 & $\mathrm{U}$ & 51.408472 & 179.139166 \\
\hline & HMUS-ML-MT2-1 & FAUNA & $7 / 11 / 2011$ & 0.1 & 0.1 & 0.45 & 0.45 & $\mathrm{U}$ & 51.389416 & 179.173138 \\
\hline & HMUS-ML-MT3-1 & FAUNA & $7 / 10 / 2011$ & 0.1 & 0.1 & 0.459 & 0.459 & $\mathrm{U}$ & 51.387805 & 179.184 \\
\hline
\end{tabular}




\begin{tabular}{|c|c|c|c|c|c|c|c|c|c|c|}
\hline Matrix Subtype & Location Code & Matrix & $\begin{array}{c}\text { Date } \\
\text { Sampled }\end{array}$ & $\begin{array}{l}\text { Wet Result } \pm \\
\text { Uncertainty }\end{array}$ & $\begin{array}{c}\text { Wet } \\
\text { Detection } \\
\text { Limit }\end{array}$ & $\begin{array}{l}\text { Dry Result } \pm \\
\text { Uncertainty }\end{array}$ & \begin{tabular}{c|} 
Dry \\
Detection \\
Limit
\end{tabular} & Qualifiers & Latitude & Longitude \\
\hline \multirow{9}{*}{ Irish Lord - whole } & IRLO-ML-MT1-1 & FAUNA & $7 / 9 / 2011$ & 0.02 & 0.02 & 0.085 & 0.085 & $\mathrm{U}$ & 51.408472 & 179.139166 \\
\hline & IRLO-ML-MT1-2 & FAUNA & $7 / 9 / 2011$ & 0.02 & 0.02 & 0.095 & 0.095 & $U$ & 51.408472 & 179.139166 \\
\hline & IRLO-ML-MT1-3 & FAUNA & $7 / 9 / 2011$ & 0.04 & 0.04 & 0.187 & 0.187 & $U$ & 51.408472 & 179.139166 \\
\hline & IRLO-ML-MT2-1 & FAUNA & $7 / 10 / 2011$ & 0.02 & 0.02 & 0.086 & 0.086 & $U$ & 51.389416 & 179.173138 \\
\hline & IRLO-ML-MT2-2 & FAUNA & $7 / 11 / 2011$ & 0.04 & 0.04 & 0.172 & 0.172 & $U$ & 51.396944 & 179.1435 \\
\hline & IRLO-ML-MT2-3 & FAUNA & $7 / 11 / 2011$ & 0.04 & 0.04 & 0.19 & 0.19 & $U$ & 51.396944 & 179.1435 \\
\hline & IRLO-ML-MT3-1 & FAUNA & $7 / 10 / 2011$ & 0.02 & 0.02 & 0.113 & 0.113 & $U$ & 51.387805 & 179.184 \\
\hline & IRLO-ML-MT3-2 & FAUNA & $7 / 10 / 2011$ & 0.02 & 0.02 & 0.097 & 0.097 & $U$ & 51.387805 & 179.184 \\
\hline & IRLO-ML-MT3-3 & FAUNA & $7 / 10 / 2011$ & 0.02 & 0.02 & 0.112 & 0.112 & $U$ & 51.387805 & 179.184 \\
\hline Octopus & OCTP-ML-M2M-1 & FAUNA & $7 / 10 / 2011$ & 0.03 & 0.03 & 0.141 & 0.141 & $U$ & 51.389416 & 179.173138 \\
\hline $\begin{array}{l}\text { Pacific Cod - } \\
\text { whole }\end{array}$ & PCOD-ML-M2M-1 & FAUNA & $7 / 10 / 2011$ & 0.003 & 0.003 & 0.016 & 0.016 & $\mathrm{U}$ & 51.408472 & 179.139166 \\
\hline \multirow{10}{*}{ Rockfish - whole } & ROCK-ML-DUP & FAUNA & $7 / 9 / 2011$ & 0.02 & 0.02 & 0.088 & 0.088 & $U$ & 51.408472 & 179.139166 \\
\hline & ROCK-ML-MT1-1 & FAUNA & $7 / 9 / 2011$ & 0.02 & 0.02 & 0.088 & 0.088 & $U$ & 51.408472 & 179.139166 \\
\hline & ROCK-ML-MT1-2 & FAUNA & $7 / 9 / 2011$ & 0.02 & 0.02 & 0.088 & 0.088 & $U$ & 51.408472 & 179.139166 \\
\hline & ROCK-ML-MT1-3 & FAUNA & $7 / 9 / 2011$ & 0.02 & 0.02 & 0.085 & 0.085 & $\mathrm{U}$ & 51.408472 & 179.139166 \\
\hline & ROCK-ML-MT2-1 & FAUNA & $7 / 11 / 2011$ & 0.02 & 0.02 & 0.075 & 0.075 & $U$ & 51.365055 & 179.111472 \\
\hline & ROCK-ML-MT2-2 & FAUNA & $7 / 11 / 2011$ & 0.02 & 0.02 & 0.081 & 0.081 & $\mathrm{U}$ & 51.365055 & 179.111472 \\
\hline & ROCK-ML-MT2-3 & FAUNA & $7 / 11 / 2011$ & 0.02 & 0.02 & 0.09 & 0.09 & $U$ & 51.365055 & 179.111472 \\
\hline & ROCK-ML-MT3-1 & FAUNA & $7 / 10 / 2011$ & 0.02 & 0.02 & 0.094 & 0.094 & $U$ & 51.387805 & 179.184 \\
\hline & ROCK-ML-MT3-2 & FAUNA & $7 / 10 / 2011$ & 0.01 & 0.01 & 0.036 & 0.036 & $\mathrm{U}$ & 51.387805 & 179.184 \\
\hline & ROCK-ML-MT3-3 & FAUNA & $7 / 10 / 2011$ & 0.01 & 0.01 & 0.023 & 0.023 & $U$ & 51.387805 & 179.184 \\
\hline Reindeer Lichen & STRL-AI-ML-1 & VEG & $6 / 21 / 2011$ & 0.03 & 0.03 & 0.107 & 0.107 & $U$ & 51.402583 & 179.183972 \\
\hline \multirow{3}{*}{$\begin{array}{l}\text { Sea Urchin - } \\
\text { whole }\end{array}$} & URCH-ML-MT1-1 & FAUNA & $7 / 9 / 2011$ & 0.02 & 0.02 & 0.074 & 0.074 & $U$ & 51.408472 & 179.139166 \\
\hline & URCH-ML-MT2-1 & FAUNA & $7 / 10 / 2011$ & 0.03 & 0.03 & 0.112 & 0.112 & $U$ & 51.389416 & 179.173138 \\
\hline & URCH-ML-MT3-1 & FAUNA & $7 / 10 / 2011$ & 0.03 & 0.03 & 0.119 & 0.119 & $U$ & 51.387805 & 179.184 \\
\hline \multicolumn{11}{|c|}{ Amchitka Site (Cannikin) (AMC05) } \\
\hline \multirow{3}{*}{ Chiton } & CHIT-CN-CT1-1 & FAUNA & $7 / 8 / 2011$ & 0.04 & 0.04 & 0.187 & 0.187 & $\mathrm{U}$ & 51.482972 & 179.123833 \\
\hline & CHIT-CN-CT2-1 & FAUNA & $7 / 7 / 2011$ & 0.03 & 0.03 & 0.142 & 0.142 & $\mathrm{U}$ & 51.472611 & 179.151111 \\
\hline & CHIT-CN-CT3-1 & FAUNA & $7 / 5 / 2011$ & 0.04 & 0.04 & 0.196 & 0.196 & $U$ & 51.471416 & 179.172861 \\
\hline
\end{tabular}




\begin{tabular}{|c|c|c|c|c|c|c|c|c|c|c|}
\hline Matrix Subtype & Location Code & Matrix & $\begin{array}{c}\text { Date } \\
\text { Sampled }\end{array}$ & $\begin{array}{l}\text { Wet Result } \pm \\
\text { Uncertainty }\end{array}$ & $\begin{array}{c}\text { Wet } \\
\text { Detection } \\
\text { Limit }\end{array}$ & $\begin{array}{l}\text { Dry Result } \pm \\
\text { Uncertainty }\end{array}$ & \begin{tabular}{|c|} 
Dry \\
Detection \\
Limit
\end{tabular} & Qualifiers & Latitude & Longitude \\
\hline \multirow{10}{*}{ Dragon Kelp } & EUAL-CN-CT1-1 & VEG & $7 / 8 / 2011$ & $0.061 \pm 0.004$ & 0.003 & $0.452 \pm 0.03$ & 0.022 & & 51.482972 & 179.123833 \\
\hline & EUAL-CN-CT1-2 & VEG & $7 / 8 / 2011$ & $0.007 \pm 0.002$ & 0.003 & $0.046 \pm 0.013$ & 0.02 & $\mathrm{~J}$ & 51.482972 & 179.123833 \\
\hline & EUAL-CN-CT1-3 & VEG & $7 / 8 / 2011$ & $0.024 \pm 0.002$ & 0.003 & $0.147 \pm 0.012$ & 0.018 & & 51.482972 & 179.123833 \\
\hline & EUAL-CN-CT2-1 & VEG & $7 / 6 / 2011$ & $0.02 \pm 0.004$ & 0.003 & $0.128 \pm 0.026$ & 0.019 & & \begin{tabular}{|l|}
51.472611 \\
\end{tabular} & 179.151111 \\
\hline & EUAL-CN-CT2-2 & VEG & $7 / 6 / 2011$ & $0.023 \pm 0.004$ & 0.003 & $0.144 \pm 0.025$ & 0.019 & & 51.472611 & 179.151111 \\
\hline & EUAL-CN-CT2-3 & VEG & 7/7/2011 & $0.052 \pm 0.004$ & 0.003 & $0.377 \pm 0.029$ & 0.022 & & \begin{tabular}{|l|}
51.472611 \\
\end{tabular} & 179.151111 \\
\hline & EUAL-CN-CT3-1 & VEG & $7 / 5 / 2011$ & 0.003 & 0.003 & 0.021 & 0.021 & $U$ & \begin{tabular}{|l|}
51.471416 \\
\end{tabular} & 179.172861 \\
\hline & EUAL-CN-CT3-2 & VEG & $7 / 5 / 2011$ & $0.008 \pm 0.002$ & 0.003 & $0.058 \pm 0.014$ & 0.022 & $\bar{J}$ & 51.471416 & 179.172861 \\
\hline & EUAL-CN-CT3-3 & VEG & $7 / 5 / 2011$ & 0.003 & 0.003 & 0.019 & 0.019 & $U$ & \begin{tabular}{|l|}
51.471416 \\
\end{tabular} & 179.172861 \\
\hline & EUAL-CN-DUP & VEG & $7 / 6 / 2011$ & $0.026 \pm 0.002$ & 0.003 & $0.173 \pm 0.013$ & 0.02 & & 51.471416 & 179.172861 \\
\hline \multirow{10}{*}{ Rockweed } & FUCU-CN-CT1-1 & VEG & \begin{tabular}{|l|}
$6 / 23 / 2011$ \\
\end{tabular} & 0.003 & 0.003 & 0.017 & 0.017 & $U$ & \begin{tabular}{|l|}
51.481888 \\
\end{tabular} & 179.118138 \\
\hline & FUCU-CN-CT1-2 & VEG & 6/23/2011 & 0.003 & 0.003 & 0.019 & 0.019 & $U$ & 51.481888 & 179.118138 \\
\hline & FUCU-CN-CT1-3 & VEG & 6/23/2011 & 0.003 & 0.003 & 0.018 & 0.018 & $\bar{U}$ & 51.481888 & 179.118138 \\
\hline & FUCU-CN-CT2-1 & VEG & $7 / 8 / 2011$ & 0.003 & 0.003 & 0.012 & 0.012 & $U$ & 51.476638 & 179.135277 \\
\hline & FUCU-CN-CT2-2 & VEG & $7 / 8 / 2011$ & 0.003 & 0.003 & 0.013 & 0.013 & $U$ & 51.476638 & 179.135277 \\
\hline & FUCU-CN-CT2-3 & VEG & \begin{tabular}{|l|}
$7 / 12 / 2011$ \\
\end{tabular} & 0.003 & 0.003 & 0.016 & 0.016 & $U$ & 51.467583 & 179.153583 \\
\hline & FUCU-CN-CT3-1 & VEG & $7 / 6 / 2011$ & 0.003 & 0.003 & 0.016 & 0.016 & $U$ & \begin{tabular}{|l|}
51.465027 \\
\end{tabular} & 179.173166 \\
\hline & FUCU-CN-CT3-2 & VEG & $7 / 6 / 2011$ & 0.003 & 0.003 & 0.018 & 0.018 & $\bar{U}$ & 51.465472 & 179.170583 \\
\hline & FUCU-CN-CT3-3 & VEG & $7 / 6 / 2011$ & 0.003 & 0.003 & 0.019 & 0.019 & $U$ & 51.464638 & 179.159305 \\
\hline & FUCU-CN-DUP & VEG & \begin{tabular}{|l|}
$6 / 23 / 2011$ \\
\end{tabular} & 0.003 & 0.003 & 0.019 & 0.019 & $U$ & 51.481888 & 179.118138 \\
\hline \multirow{10}{*}{ Greenling - whole } & GREN-CN-CT1-1 & FAUNA & \begin{tabular}{|l|}
$7 / 8 / 2011$ \\
\end{tabular} & 0.03 & 0.03 & 0.145 & 0.145 & $U$ & \begin{tabular}{|l|}
51.482972 \\
\end{tabular} & 179.123833 \\
\hline & GREN-CN-CT1-2 & FAUNA & $7 / 8 / 2011$ & 0.03 & 0.03 & 0.142 & 0.142 & $U$ & 51.482972 & 179.123833 \\
\hline & GREN-CN-CT1-3 & FAUNA & $7 / 8 / 2011$ & 0.03 & 0.03 & 0.137 & 0.137 & $U$ & 51.482972 & 179.123833 \\
\hline & GREN-CN-CT2-1 & FAUNA & $7 / 7 / 2011$ & 0.02 & 0.02 & 0.097 & 0.097 & $U$ & \begin{tabular}{|l|}
51.472611 \\
\end{tabular} & 179.151111 \\
\hline & GREN-CN-CT2-2 & FAUNA & $7 / 7 / 2011$ & 0.03 & 0.03 & 0.138 & 0.138 & $U$ & 51.472611 & 179.151111 \\
\hline & GREN-CN-CT2-3 & FAUNA & 7/7/2011 & 0.02 & 0.02 & 0.092 & 0.092 & $U$ & 51.472611 & 179.151111 \\
\hline & GREN-CN-CT3-1 & FAUNA & $7 / 5 / 2011$ & 0.03 & 0.03 & 0.137 & 0.137 & $U$ & 51.471416 & 179.172861 \\
\hline & GREN-CN-CT3-2 & FAUNA & $7 / 5 / 2011$ & 0.02 & 0.02 & 0.087 & 0.087 & $U$ & \begin{tabular}{|l|}
51.471416 \\
\end{tabular} & 179.172861 \\
\hline & GREN-CN-CT3-3 & FAUNA & $7 / 5 / 2011$ & 0.02 & 0.02 & 0.092 & 0.092 & $U$ & 51.471416 & 179.172861 \\
\hline & GREN-CN-DUP & FAUNA & $7 / 8 / 2011$ & 0.03 & 0.03 & 0.145 & 0.145 & $U$ & \begin{tabular}{|l|}
51.482972 \\
\end{tabular} & 179.123833 \\
\hline Halibut - whole & HALI-CN-C2M-1 & FAUNA & \begin{tabular}{|l|}
$7 / 13 / 2011$ \\
\end{tabular} & 0.003 & 0.003 & 0.012 & 0.012 & $U$ & 51.40025 & 179.283361 \\
\hline \multirow{3}{*}{$\begin{array}{l}\text { Horse Mussel - } \\
\text { soft tissue only }\end{array}$} & HMUS-CN-CT1-1 & FAUNA & $7 / 8 / 2011$ & 0.1 & 0.1 & 0.411 & 0.411 & $U$ & \begin{tabular}{|l|}
51.482972 \\
\end{tabular} & 179.123833 \\
\hline & HMUS-CN-CT2-1 & FAUNA & $7 / 7 / 2011$ & 0.1 & 0.1 & 0.453 & 0.453 & $U$ & 51.472611 & 179.151111 \\
\hline & HMUS-CN-CT3-1 & FAUNA & $7 / 5 / 2011$ & 0.09 & 0.09 & 0.369 & 0.369 & $U$ & \begin{tabular}{|l|}
51.471416 \\
\end{tabular} & 179.172861 \\
\hline
\end{tabular}




\begin{tabular}{|c|c|c|c|c|c|c|c|c|c|c|}
\hline Matrix Subtype & Location Code & Matrix & $\begin{array}{c}\text { Date } \\
\text { Sampled }\end{array}$ & $\begin{array}{l}\text { Wet Result } \pm \\
\text { Uncertainty }\end{array}$ & $\begin{array}{c}\text { Wet } \\
\text { Detection } \\
\text { Limit }\end{array}$ & $\begin{array}{l}\text { Dry Result } \pm \\
\text { Uncertainty }\end{array}$ & \begin{tabular}{|c|} 
Dry \\
Detection \\
Limit
\end{tabular} & Qualifiers & Latitude & Longitude \\
\hline \multirow{9}{*}{ Irish Lord - whole } & IRLO-CN-CT1-1 & \begin{tabular}{|l|} 
FAUNA \\
\end{tabular} & $7 / 8 / 2011$ & 0.04 & 0.04 & 0.18 & 0.18 & $U$ & 51.482972 & 179.123833 \\
\hline & IRLO-CN-CT1-2 & FAUNA & 7/8/2011 & 0.02 & 0.02 & 0.1 & 0.1 & $U$ & 51.482972 & 179.123833 \\
\hline & IRLO-CN-CT1-3 & \begin{tabular}{|l|} 
FAUNA \\
\end{tabular} & $7 / 8 / 2011$ & 0.04 & 0.04 & 0.188 & 0.188 & $U$ & 51.482972 & 179.123833 \\
\hline & IRLO-CN-CT2-1 & FAUNA & 7/7/2011 & 0.02 & 0.02 & 0.094 & 0.094 & $U$ & 51.472611 & 179.151111 \\
\hline & IRLO-CN-CT2-2 & FAUNA & $7 / 7 / 2011$ & 0.03 & 0.03 & 0.137 & 0.137 & 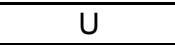 & 51.472611 & 179.151111 \\
\hline & IRLO-CN-CT2-3 & FAUNA & $7 / 7 / 2011$ & 0.02 & 0.02 & 0.098 & 0.098 & $\mathrm{U}$ & 51.472611 & 179.151111 \\
\hline & IRLO-CN-CT3-1 & FAUNA & $7 / 5 / 2011$ & 0.04 & 0.04 & 0.206 & 0.206 & $\bar{U}$ & 51.471416 & 179.172861 \\
\hline & IRLO-CN-CT3-2 & FAUNA & $7 / 5 / 2011$ & 0.02 & 0.02 & 0.097 & 0.097 & $\mathrm{U}$ & 51.471416 & 179.172861 \\
\hline & IRLO-CN-CT3-3 & FAUNA & $7 / 5 / 2011$ & 0.02 & 0.02 & 0.096 & 0.096 & $U$ & 51.471416 & 179.172861 \\
\hline Octopus & OCTP-CN-C2M-1 & FAUNA & $7 / 8 / 2011$ & 0.01 & 0.01 & 0.049 & 0.049 & $\mathrm{U}$ & 51.482972 & 179.123833 \\
\hline $\begin{array}{l}\text { Pacific Cod - } \\
\text { whole }\end{array}$ & PCOD-CN-C2M-1 & FAUNA & 7/6/2011 & 0.003 & 0.003 & 0.014 & 0.014 & u & 51.483527 & 179.187583 \\
\hline \multirow{10}{*}{ Rockfish - whole } & ROCK-CN-CT1-1 & FAUNA & $7 / 8 / 2011$ & 0.02 & 0.02 & 0.079 & 0.079 & $\mathrm{U}$ & 51.482972 & 179.123833 \\
\hline & ROCK-CN-CT1-2 & FAUNA & $7 / 8 / 2011$ & 0.02 & 0.02 & 0.077 & 0.077 & $U$ & 51.482972 & 179.123833 \\
\hline & ROCK-CN-CT1-3 & FAUNA & $7 / 8 / 2011$ & 0.02 & 0.02 & 0.08 & 0.08 & $U$ & 51.482972 & 179.123833 \\
\hline & ROCK-CN-CT2-1 & FAUNA & $7 / 6 / 2011$ & 0.01 & 0.01 & 0.035 & 0.035 & $\mathrm{U}$ & 51.472611 & 179.151111 \\
\hline & ROCK-CN-CT2-2 & FAUNA & $7 / 6 / 2011$ & 0.02 & 0.02 & 0.073 & 0.073 & $\mathrm{U}$ & 51.472611 & 179.151111 \\
\hline & ROCK-CN-CT2-3 & FAUNA & $7 / 6 / 2011$ & 0.02 & 0.02 & 0.082 & 0.082 & $\mathrm{U}$ & 51.472611 & 179.151111 \\
\hline & ROCK-CN-CT3-1 & FAUNA & $7 / 6 / 2011$ & 0.02 & 0.02 & 0.085 & 0.085 & $\bar{U}$ & 51.471416 & 179.172861 \\
\hline & ROCK-CN-CT3-2 & FAUNA & $7 / 6 / 2011$ & 0.01 & 0.01 & 0.038 & 0.038 & $\mathrm{U}$ & 51.471416 & 179.172861 \\
\hline & ROCK-CN-CT3-3 & FAUNA & $7 / 6 / 2011$ & 0.02 & 0.02 & 0.084 & 0.084 & $\mathrm{U}$ & 51.471416 & 179.172861 \\
\hline & ROCK-CN-DUP & FAUNA & $7 / 6 / 2011$ & 0.02 & 0.02 & 0.08 & 0.08 & $\mathrm{U}$ & 51.471416 & 179.172861 \\
\hline \begin{tabular}{|l|} 
Reindeer Lichen \\
\end{tabular} & STRL-AI-CT1-1 & VEG & $6 / 23 / 2011$ & 0.04 & 0.04 & 0.063 & 0.063 & $\mathrm{U}$ & 51.480583 & 179.109472 \\
\hline \multirow{3}{*}{$\begin{array}{l}\text { Sea Urchin - } \\
\text { whole }\end{array}$} & URCH-CN-CT1-1 & FAUNA & $7 / 8 / 2011$ & $0.065 \pm 0.012$ & 0.02 & $0.261 \pm 0.048$ & 0.08 & & 51.482972 & 179.123833 \\
\hline & URCH-CN-CT2-1 & FAUNA & 7/7/2011 & 0.03 & 0.03 & 0.103 & 0.103 & $\mathrm{U}$ & 51.472611 & 179.151111 \\
\hline & URCH-CN-CT3-1 & FAUNA & $7 / 5 / 2011$ & 0.02 & 0.02 & 0.077 & 0.077 & $U$ & 51.471416 & 179.172861 \\
\hline \multicolumn{11}{|c|}{ Amchitka Site (Adak Island) (AMC13) } \\
\hline $\begin{array}{l}\text { Dolly Varden - } \\
\text { whole }\end{array}$ & DOLL-AD-XXX-1 & FAUNA & 6/28/2011 & 0.01 & 0.01 & 0.041 & 0.041 & U & 51.8375 & -176.672555 \\
\hline \multirow{3}{*}{ Reindeer Lichen } & STRL-AD-XXX-1 & VEG & $6 / 28 / 2011$ & $0.19 \pm 0.02$ & 0.03 & $0.372 \pm 0.039$ & 0.059 & & 51.837527 & -176.670694 \\
\hline & STRL-AD-XXX-2 & VEG & $6 / 29 / 2011$ & $0.062 \pm 0.01$ & 0.02 & $0.153 \pm 0.025$ & 0.049 & & 51.872833 & -176.707444 \\
\hline & STRL-AD-XXX-3 & VEG & $6 / 29 / 2011$ & $0.13 \pm 0.02$ & 0.03 & $0.207 \pm 0.032$ & 0.048 & & 51.900805 & -176.688527 \\
\hline \multicolumn{11}{|c|}{ Amchitka Site (Adak Island North) (AMC14) } \\
\hline \multirow{3}{*}{ Chiton } & CHIT-AN-ANT1-1 & FAUNA & $7 / 20 / 2011$ & 0.02 & 0.02 & 0.097 & 0.097 & $U$ & 51.838722 & -176.582805 \\
\hline & CHIT-AN-ANT2-1 & FAUNA & 7/18/2011 & 0.02 & 0.02 & 0.1 & 0.1 & $\mathrm{U}$ & 51.848361 & -176.506277 \\
\hline & CHIT-AN-ANT3-1 & FAUNA & $7 / 17 / 2011$ & 0.02 & 0.02 & 0.102 & 0.102 & $\mathrm{U}$ & 51.853722 & -176.470694 \\
\hline
\end{tabular}




\begin{tabular}{|c|c|c|c|c|c|c|c|c|c|c|}
\hline Matrix Subtype & Location Code & Matrix & $\begin{array}{c}\text { Date } \\
\text { Sampled }\end{array}$ & $\begin{array}{l}\text { Wet Result } \pm \\
\text { Uncertainty }\end{array}$ & $\begin{array}{c}\text { Wet } \\
\text { Detection } \\
\text { Limit }\end{array}$ & $\begin{array}{l}\text { Dry Result } \pm \\
\text { Uncertainty }\end{array}$ & $\begin{array}{c}\text { Dry } \\
\text { Detection } \\
\text { Limit }\end{array}$ & Qualifiers & Latitude & Longitude \\
\hline \multirow{10}{*}{ Dragon Kelp } & EUAL-AN-ANT1-1 & VEG & 7/19/2011 & $0.04 \pm 0.002$ & 0.003 & $0.335 \pm 0.017$ & 0.025 & & 51.835694 & -176.542138 \\
\hline & \begin{tabular}{|l|} 
EUAL-AN-ANT1-2 \\
\end{tabular} & VEG & 7/19/2011 & $0.01 \pm 0.002$ & 0.003 & $0.068 \pm 0.014$ & 0.02 & & 51.835694 & -176.542138 \\
\hline & \begin{tabular}{|l|} 
EUAL-AN-ANT1-3 \\
\end{tabular} & VEG & 7/19/2011 & $0.03 \pm 0.002$ & 0.003 & $0.232 \pm 0.015$ & 0.023 & & 51.835694 & -176.542138 \\
\hline & \begin{tabular}{|l|} 
EUAL-AN-ANT2-1 \\
\end{tabular} & VEG & $7 / 18 / 2011$ & $0.017 \pm 0.002$ & 0.003 & $0.124 \pm 0.015$ & 0.022 & & 51.848361 & -176.506277 \\
\hline & \begin{tabular}{|l|} 
EUAL-AN-ANT2-2 \\
\end{tabular} & VEG & 7/18/2011 & $0.028 \pm 0.004$ & 0.003 & $0.178 \pm 0.025$ & 0.019 & & 51.848361 & -176.506277 \\
\hline & \begin{tabular}{|l|} 
EUAL-AN-ANT2-3 \\
\end{tabular} & VEG & 7/18/2011 & $0.042 \pm 0.004$ & 0.003 & $0.292 \pm 0.028$ & 0.021 & & 51.848361 & -176.506277 \\
\hline & \begin{tabular}{|l|} 
EUAL-AN-ANT3-1 \\
\end{tabular} & VEG & 7/17/2011 & $0.037 \pm 0.004$ & 0.004 & $0.277 \pm 0.03$ & 0.03 & & 51.853722 & -176.470694 \\
\hline & \begin{tabular}{|l|} 
EUAL-AN-ANT3-2 \\
\end{tabular} & VEG & 7/17/2011 & $0.007 \pm 0.002$ & 0.003 & $0.048 \pm 0.014$ & 0.02 & $\mathrm{~J}$ & 51.853722 & -176.470694 \\
\hline & \begin{tabular}{|l|} 
EUAL-AN-ANT3-3 \\
\end{tabular} & VEG & 7/17/2011 & $0.012 \pm 0.002$ & 0.003 & $0.081 \pm 0.013$ & 0.02 & & 51.853722 & -176.470694 \\
\hline & EUAL-AN-DUP & VEG & 7/17/2011 & $0.017 \pm 0.002$ & 0.003 & $0.107 \pm 0.013$ & 0.019 & & 51.853722 & -176.470694 \\
\hline \multirow{10}{*}{ Rockweed } & \begin{tabular}{|l|} 
FUCU-AN-ANT1-1 \\
\end{tabular} & VEG & 7/19/2011 & $0.011 \pm 0.002$ & 0.003 & $0.056 \pm 0.01$ & 0.015 & & 51.841444 & -176.546083 \\
\hline & \begin{tabular}{|l|} 
FUCU-AN-ANT1-2 \\
\end{tabular} & VEG & 7/20/2011 & 0.003 & 0.003 & 0.019 & 0.019 & $\mathrm{U}$ & 51.8395 & -176.591305 \\
\hline & \begin{tabular}{|l|} 
FUCU-AN-ANT1-3 \\
\end{tabular} & VEG & $7 / 20 / 2011$ & 0.003 & 0.003 & 0.016 & 0.016 & U & 51.8395 & -176.591305 \\
\hline & \begin{tabular}{|l|} 
FUCU-AN-ANT2-1 \\
\end{tabular} & VEG & $7 / 18 / 2011$ & 0.003 & 0.003 & 0.019 & 0.019 & $\mathrm{U}$ & 51.837666 & -176.517527 \\
\hline & \begin{tabular}{|l|} 
FUCU-AN-ANT2-2 \\
\end{tabular} & VEG & 7/18/2011 & 0.003 & 0.003 & 0.016 & 0.016 & 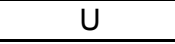 & 51.841444 & -176.546083 \\
\hline & \begin{tabular}{|l|} 
FUCU-AN-ANT2-3 \\
\end{tabular} & VEG & \begin{tabular}{|l|}
$7 / 18 / 2011$ \\
\end{tabular} & 0.003 & 0.003 & 0.013 & 0.013 & $U$ & 51.8395 & -176.591305 \\
\hline & \begin{tabular}{|l|} 
FUCU-AN-ANT3-1 \\
\end{tabular} & VEG & 7/18/2011 & 0.003 & 0.003 & 0.017 & 0.017 & $U$ & 51.839944 & -176.515916 \\
\hline & \begin{tabular}{|l|} 
FUCU-AN-ANT3-2 \\
\end{tabular} & VEG & 7/18/2011 & 0.003 & 0.003 & 0.015 & 0.015 & U & 51.828972 & -176.448305 \\
\hline & \begin{tabular}{|l|} 
FUCU-AN-ANT3-3 \\
\end{tabular} & VEG & 7/18/2011 & 0.003 & 0.003 & 0.015 & 0.015 & $\mathrm{U}$ & 51.830833 & -176.456361 \\
\hline & FUCU-AN-DUP & VEG & 7/18/2011 & 0.003 & 0.003 & 0.014 & 0.014 & $\mathrm{U}$ & 51.828972 & -176.448305 \\
\hline \multirow{10}{*}{ Greenling - whole } & GREN-AN-ANT1-1 & FAUNA & \begin{tabular}{|l|}
$7 / 19 / 2011$ \\
\end{tabular} & 0.03 & 0.03 & 0.133 & 0.133 & $\mathrm{U}$ & 51.835694 & -176.542138 \\
\hline & \begin{tabular}{|l|} 
GREN-AN-ANT1-2 \\
\end{tabular} & FAUNA & 7/19/2011 & 0.03 & 0.03 & 0.125 & 0.125 & $U$ & 51.835694 & -176.542138 \\
\hline & GREN-AN-ANT1-3 & FAUNA & 7/19/2011 & 0.03 & 0.03 & 0.109 & 0.109 & $U$ & 51.835694 & -176.542138 \\
\hline & \begin{tabular}{|l|} 
GREN-AN-ANT2-1 \\
\end{tabular} & FAUNA & 7/18/2011 & 0.03 & 0.03 & 0.139 & 0.139 & $U$ & 51.848361 & -176.506277 \\
\hline & \begin{tabular}{|l} 
GREN-AN-ANT2-2 \\
\end{tabular} & FAUNA & $7 / 18 / 2011$ & 0.03 & 0.03 & 0.139 & 0.139 & $U$ & 51.848361 & -176.506277 \\
\hline & GREN-AN-ANT2-3 & FAUNA & 7/18/2011 & 0.03 & 0.03 & 0.142 & 0.142 & $\mathrm{U}$ & 51.848361 & -176.506277 \\
\hline & \begin{tabular}{|l|} 
GREN-AN-ANT3-1 \\
\end{tabular} & FAUNA & 7/17/2011 & 0.03 & 0.03 & 0.137 & 0.137 & $u$ & 51.853722 & -176.470694 \\
\hline & GREN-AN-ANT3-2 & FAUNA & \begin{tabular}{|l|}
$7 / 17 / 2011$ \\
\end{tabular} & 0.03 & 0.03 & 0.139 & 0.139 & $U$ & 51.853722 & -176.470694 \\
\hline & GREN-AN-ANT3-3 & FAUNA & 7/17/2011 & 0.03 & 0.03 & 0.14 & 0.14 & $U$ & 51.853722 & -176.470694 \\
\hline & GREN-AN-DUP & FAUNA & 7/17/2011 & 0.03 & 0.03 & 0.143 & 0.143 & $U$ & 51.853722 & -176.470694 \\
\hline \multirow{2}{*}{ Halibut - whole } & \begin{tabular}{|l|} 
HALI-AN-AN2M-1 \\
\end{tabular} & FAUNA & 7/17/2011 & 0.002 & 0.002 & 0.007 & 0.007 & $U$ & 51.853722 & -176.470694 \\
\hline & HALI-AN-DUP & FAUNA & $7 / 17 / 2011$ & 0.003 & 0.003 & 0.01 & 0.01 & $U$ & 51.853722 & -176.470694 \\
\hline \multirow{3}{*}{$\begin{array}{l}\text { Horse Mussel - } \\
\text { soft tissue only }\end{array}$} & HMUS-AN-ANT1-1 & FAUNA & $7 / 20 / 2011$ & 0.08 & 0.08 & 0.437 & 0.437 & $U$ & 51.838722 & -176.582805 \\
\hline & HMUS-AN-ANT2-1 & FAUNA & \begin{tabular}{|l|l|}
$7 / 18 / 2011$ \\
\end{tabular} & 0.09 & 0.09 & 0.413 & 0.413 & $\mathrm{U}$ & 51.848361 & -176.506277 \\
\hline & HMUS-AN-ANT3-1 & FAUNA & 7/18/2011 & 0.07 & 0.07 & 0.312 & 0.312 & $\mathrm{U}$ & 51.853722 & -176.470694 \\
\hline
\end{tabular}




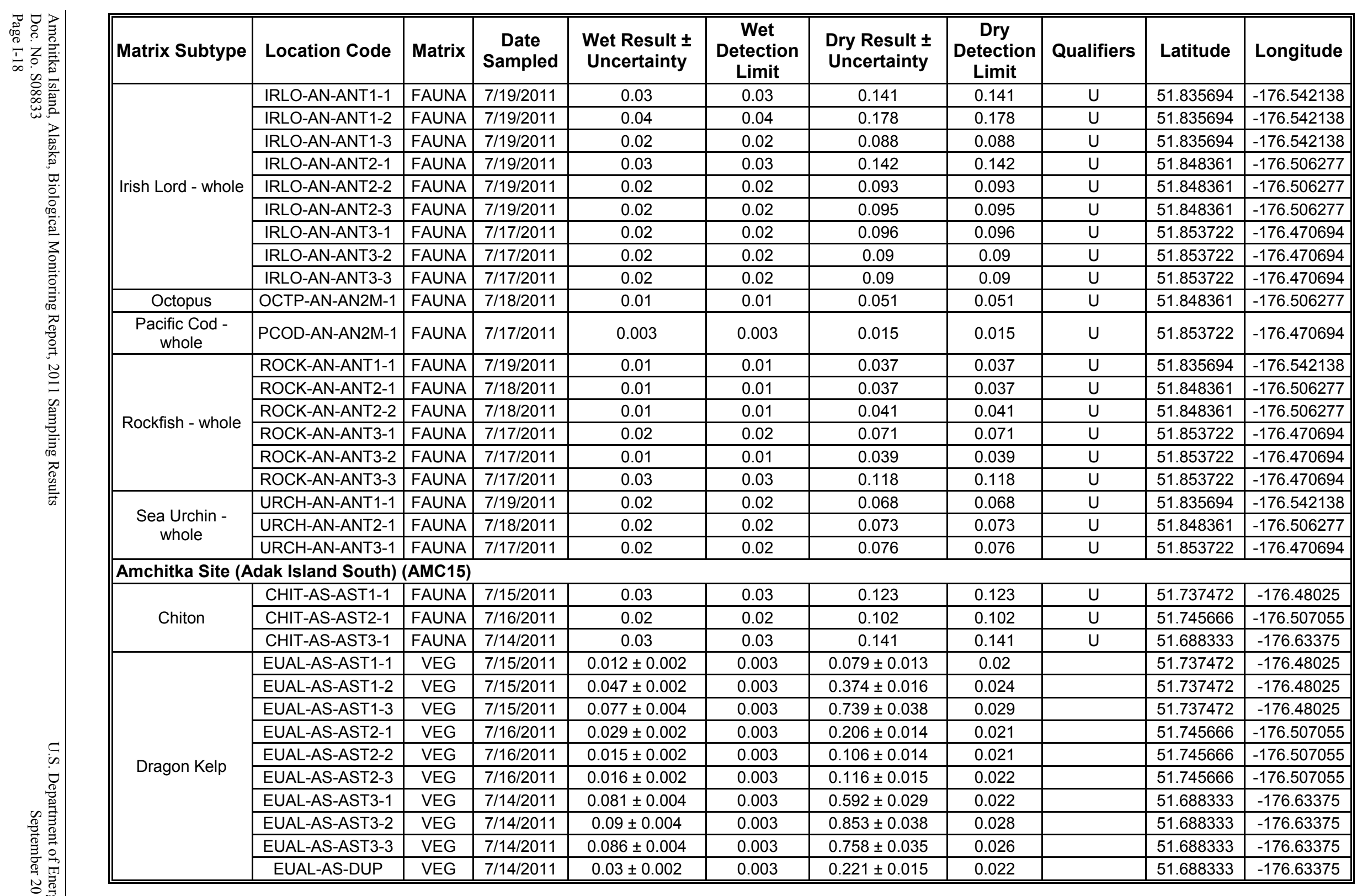




\begin{tabular}{|c|c|c|c|c|c|c|c|c|c|c|}
\hline Matrix Subtype & Location Code & Matrix & $\begin{array}{c}\text { Date } \\
\text { Sampled }\end{array}$ & $\begin{array}{c}\text { Wet Result } \pm \\
\text { Uncertainty }\end{array}$ & $\begin{array}{c}\text { Wet } \\
\text { Detection } \\
\text { Limit }\end{array}$ & $\begin{array}{l}\text { Dry Result } \pm \\
\text { Uncertainty }\end{array}$ & \begin{tabular}{|c|} 
Dry \\
$\begin{array}{c}\text { Detection } \\
\text { Limit }\end{array}$ \\
\end{tabular} & Qualifiers & Latitude & Longitude \\
\hline \multirow{10}{*}{ Rockweed } & FUCU-AS-AST1-1 & VEG & $7 / 16 / 2011$ & 0.003 & 0.003 & 0.019 & 0.019 & $\mathrm{U}$ & 51.733666 & -176.470805 \\
\hline & FUCU-AS-AST1-2 & VEG & $7 / 16 / 2011$ & 0.003 & 0.003 & 0.017 & 0.017 & $\mathrm{U}$ & 51.733666 & -176.470805 \\
\hline & FUCU-AS-AST1-3 & VEG & $7 / 16 / 2011$ & $0.008 \pm 0.002$ & 0.003 & $0.043 \pm 0.011$ & 0.016 & $\mathrm{~J}$ & 51.733666 & -176.470805 \\
\hline & FUCU-AS-AST2-1 & VEG & $7 / 16 / 2011$ & 0.003 & 0.003 & 0.015 & 0.015 & $\mathrm{U}$ & 51.745666 & -176.507055 \\
\hline & FUCU-AS-AST2-2 & VEG & $7 / 16 / 2011$ & 0.003 & 0.003 & 0.015 & 0.015 & $\mathrm{U}$ & 51.745666 & -176.507055 \\
\hline & FUCU-AS-AST2-3 & VEG & $7 / 16 / 2011$ & 0.003 & 0.003 & 0.015 & 0.015 & $\mathrm{U}$ & 51.745666 & -176.507055 \\
\hline & FUCU-AS-AST3-1 & VEG & $7 / 14 / 2011$ & 0.003 & 0.003 & 0.016 & 0.016 & $\mathrm{U}$ & 51.688333 & -176.63375 \\
\hline & FUCU-AS-AST3-2 & VEG & $7 / 14 / 2011$ & $0.006 \pm 0.002$ & 0.003 & $0.03 \pm 0.01$ & 0.015 & $\mathrm{~J}$ & 51.688333 & -176.63375 \\
\hline & FUCU-AS-AST3-3 & VEG & $7 / 14 / 2011$ & 0.002 & 0.002 & 0.011 & 0.011 & $\mathrm{U}$ & 51.688333 & -176.63375 \\
\hline & FUCU-AS-DUP & VEG & $7 / 16 / 2011$ & 0.003 & 0.003 & 0.016 & 0.016 & $\mathrm{U}$ & 51.733666 & -176.470805 \\
\hline \multirow{10}{*}{ Greenling - whole } & GREN-AS-AST1-1 & FAUNA & $7 / 15 / 2011$ & 0.03 & 0.03 & 0.143 & 0.143 & $\mathrm{U}$ & 51.737472 & -176.48025 \\
\hline & GREN-AS-AST1-2 & FAUNA & $7 / 15 / 2011$ & 0.03 & 0.03 & 0.142 & 0.142 & $\mathrm{U}$ & 51.737472 & -176.48025 \\
\hline & GREN-AS-AST1-3 & FAUNA & $7 / 15 / 2011$ & 0.03 & 0.03 & 0.144 & 0.144 & $\mathrm{U}$ & 51.737472 & -176.48025 \\
\hline & GREN-AS-AST2-1 & FAUNA & $7 / 16 / 2011$ & 0.02 & 0.02 & 0.08 & 0.08 & $\mathrm{U}$ & 51.745666 & -176.507055 \\
\hline & GREN-AS-AST2-2 & FAUNA & $7 / 16 / 2011$ & 0.03 & 0.03 & 0.137 & 0.137 & $\mathrm{U}$ & 51.745666 & -176.507055 \\
\hline & GREN-AS-AST2-3 & FAUNA & $7 / 16 / 2011$ & 0.03 & 0.03 & 0.14 & 0.14 & $\mathrm{U}$ & 51.745666 & -176.507055 \\
\hline & GREN-AS-AST3-1 & FAUNA & $7 / 14 / 2011$ & 0.03 & 0.03 & 0.143 & 0.143 & $\mathrm{U}$ & 51.688333 & -176.63375 \\
\hline & GREN-AS-AST3-2 & FAUNA & $7 / 14 / 2011$ & 0.03 & 0.03 & 0.141 & 0.141 & $U$ & 51.688333 & -176.63375 \\
\hline & GREN-AS-AST3-3 & FAUNA & $7 / 14 / 2011$ & 0.04 & 0.04 & 0.191 & 0.191 & $\mathrm{U}$ & 51.688333 & -176.63375 \\
\hline & GREN-AS-DUP & FAUNA & $7 / 14 / 2011$ & 0.03 & 0.03 & 0.15 & 0.15 & $\mathrm{U}$ & 51.688333 & -176.63375 \\
\hline \multirow{3}{*}{$\begin{array}{l}\text { Horse Mussel - } \\
\text { soft tissue only }\end{array}$} & HMUS-AS-AST1-1 & FAUNA & $7 / 15 / 2011$ & 0.1 & 0.1 & 0.501 & 0.501 & $\mathrm{U}$ & 51.737472 & -176.48025 \\
\hline & HMUS-AS-AST2-1 & FAUNA & $7 / 16 / 2011$ & 0.08 & 0.08 & 0.507 & 0.507 & $\mathrm{U}$ & 51.745666 & -176.507055 \\
\hline & HMUS-AS-AST3-1 & FAUNA & $7 / 14 / 2011$ & 0.1 & 0.1 & 0.51 & 0.51 & $\mathrm{U}$ & 51.688333 & -176.63375 \\
\hline \multirow{9}{*}{ Irish Lord - whole } & IRLO-AS-AST1-1 & FAUNA & $7 / 15 / 2011$ & 0.04 & 0.04 & 0.176 & 0.176 & $\mathrm{U}$ & 51.737472 & -176.48025 \\
\hline & IRLO-AS-AST1-2 & FAUNA & $7 / 15 / 2011$ & 0.03 & 0.03 & 0.14 & 0.14 & $\mathrm{U}$ & 51.737472 & -176.48025 \\
\hline & IRLO-AS-AST1-3 & FAUNA & $7 / 15 / 2011$ & 0.03 & 0.03 & 0.137 & 0.137 & $\mathrm{U}$ & 51.737472 & -176.48025 \\
\hline & IRLO-AS-AST2-1 & FAUNA & $7 / 16 / 2011$ & 0.03 & 0.03 & 0.14 & 0.14 & $\mathrm{U}$ & 51.745666 & -176.507055 \\
\hline & IRLO-AS-AST2-2 & FAUNA & $7 / 16 / 2011$ & 0.03 & 0.03 & 0.128 & 0.128 & $\mathrm{U}$ & 51.745666 & -176.507055 \\
\hline & \begin{tabular}{|l|} 
IRLO-AS-AST2-3 \\
\end{tabular} & FAUNA & $7 / 16 / 2011$ & 0.03 & 0.03 & 0.152 & 0.152 & $U$ & 51.745666 & -176.507055 \\
\hline & IRLO-AS-AST3-1 & FAUNA & $7 / 14 / 2011$ & 0.02 & 0.02 & 0.09 & 0.09 & $\mathrm{U}$ & 51.688333 & -176.63375 \\
\hline & IRLO-AS-AST3-2 & FAUNA & $7 / 14 / 2011$ & 0.02 & 0.02 & 0.085 & 0.085 & $U$ & 51.688333 & -176.63375 \\
\hline & IRLO-AS-AST3-3 & FAUNA & $7 / 14 / 2011$ & 0.02 & 0.02 & 0.091 & 0.091 & $U$ & 51.688333 & -176.63375 \\
\hline $\begin{array}{l}\text { Pacific Cod - } \\
\text { whole } \\
\end{array}$ & PCOD-AS-AS2M-1 & FAUNA & $7 / 15 / 2011$ & 0.003 & 0.003 & 0.015 & 0.015 & $U$ & 51.737472 & -176.48025 \\
\hline
\end{tabular}




\begin{tabular}{|c|c|c|c|c|c|c|c|c|c|c|}
\hline Matrix Subtype & Location Code & Matrix & $\begin{array}{c}\text { Date } \\
\text { Sampled }\end{array}$ & $\begin{array}{c}\text { Wet Result } \pm \\
\text { Uncertainty }\end{array}$ & $\begin{array}{c}\text { Wet } \\
\text { Detection } \\
\text { Limit }\end{array}$ & $\begin{array}{l}\text { Dry Result } \pm \\
\text { Uncertainty }\end{array}$ & \begin{tabular}{|c|} 
Dry \\
$\begin{array}{c}\text { Detection } \\
\text { Limit }\end{array}$ \\
\end{tabular} & Qualifiers & Latitude & Longitude \\
\hline \multirow{10}{*}{ Rockfish - whole } & ROCK-AS-AST1-1 & FAUNA & $7 / 15 / 2011$ & 0.03 & 0.03 & 0.108 & 0.108 & $U$ & 51.737472 & -176.48025 \\
\hline & ROCK-AS-AST1-2 & FAUNA & $7 / 15 / 2011$ & 0.02 & 0.02 & 0.075 & 0.075 & $\mathrm{U}$ & 51.737472 & -176.48025 \\
\hline & ROCK-AS-AST1-3 & FAUNA & $7 / 15 / 2011$ & 0.01 & 0.01 & 0.043 & 0.043 & $\mathrm{U}$ & 51.737472 & -176.48025 \\
\hline & ROCK-AS-AST2-1 & FAUNA & $7 / 16 / 2011$ & 0.02 & 0.02 & 0.077 & 0.077 & $U$ & 51.745666 & -176.507055 \\
\hline & ROCK-AS-AST2-2 & FAUNA & $7 / 16 / 2011$ & 0.01 & 0.01 & 0.037 & 0.037 & $\mathrm{U}$ & 51.745666 & -176.507055 \\
\hline & ROCK-AS-AST2-3 & FAUNA & $7 / 16 / 2011$ & 0.02 & 0.02 & 0.077 & 0.077 & $\mathrm{U}$ & 51.745666 & -176.507055 \\
\hline & ROCK-AS-AST3-1 & FAUNA & $7 / 14 / 2011$ & 0.02 & 0.02 & 0.082 & 0.082 & $\mathrm{U}$ & 51.688333 & \begin{tabular}{|l}
-176.63375 \\
\end{tabular} \\
\hline & ROCK-AS-AST3-2 & FAUNA & $7 / 14 / 2011$ & 0.03 & 0.03 & 0.127 & 0.127 & $U$ & 51.688333 & -176.63375 \\
\hline & ROCK-AS-AST3-3 & FAUNA & $7 / 14 / 2011$ & 0.01 & 0.01 & 0.043 & 0.043 & $\mathrm{U}$ & 51.688333 & -176.63375 \\
\hline & ROCK-AS-DUP & FAUNA & $7 / 14 / 2011$ & 0.01 & 0.01 & 0.039 & 0.039 & $\mathrm{U}$ & 51.688333 & -176.63375 \\
\hline \multirow{3}{*}{$\begin{array}{l}\text { Sea Urchin - } \\
\text { whole }\end{array}$} & URCH-AS-AST1-1 & FAUNA & $7 / 15 / 2011$ & 0.03 & 0.03 & 0.114 & 0.114 & $\mathrm{U}$ & 51.737472 & -176.48025 \\
\hline & URCH-AS-AST2-1 & FAUNA & $7 / 16 / 2011$ & 0.04 & 0.04 & 0.161 & 0.161 & $U$ & 51.745666 & -176.507055 \\
\hline & URCH-AS-AST3-1 & FAUNA & $7 / 14 / 2011$ & 0.03 & 0.03 & 0.112 & 0.112 & $\mathrm{U}$ & 51.688333 & -176.63375 \\
\hline
\end{tabular}

Qualifier definitions:

$\mathrm{U}$ - Analytical result below detection limit

$\mathrm{J}$ - Estimated 
Appendix $\mathbf{J}$

Activity Concentration of Uranium Isotopes 
This page intentionally left blank 


\begin{tabular}{|c|c|c|c|c|c|c|c|c|c|c|}
\hline Matrix Subtype & Location Code & Matrix & $\begin{array}{c}\text { Date } \\
\text { Sampled }\end{array}$ & $\begin{array}{l}\text { Wet Result } \pm \\
\text { Uncertainty }\end{array}$ & $\begin{array}{c}\text { Wet } \\
\text { Detection } \\
\text { Limit }\end{array}$ & $\begin{array}{l}\text { Dry Result } \pm \\
\text { Uncertainty }\end{array}$ & $\begin{array}{c}\text { Dry } \\
\text { Detection } \\
\text { Limit }\end{array}$ & Qualifiers & Latitude & Longitude \\
\hline \multicolumn{11}{|c|}{${ }^{234}$ Uranium (pCi/kg) } \\
\hline \multicolumn{11}{|c|}{ Amchitka Site (AMC01) } \\
\hline $\begin{array}{l}\text { Dolly Varden - } \\
\text { whole }\end{array}$ & DOLL-AI-XXX-1 & FAUNA & $6 / 24 / 2011$ & $1.3 \pm 1.2$ & 1 & $5.01 \pm 4.625$ & 3.854 & $U$ & 51.468283 & 179.108052 \\
\hline $\begin{array}{l}\text { Goose Egg - no } \\
\text { shell }\end{array}$ & GOOS-AI-XXX-1 & FAUNA & $6 / 24 / 2011$ & $0.7 \pm 0.8$ & 2 & $1.859 \pm 2.125$ & 5.312 & UJ & 51.491305 & 179.0595 \\
\hline $\begin{array}{c}\text { Glaucous-winged } \\
\text { Gull Egg - no } \\
\text { shell }\end{array}$ & GWGU-AI-XXX-1 & FAUNA & $6 / 24 / 2011$ & $0.6 \pm 0.2$ & 1 & $2.079 \pm 0.693$ & 3.466 & $\mathrm{~J}$ & 51.456388 & 179.167 \\
\hline \multicolumn{11}{|c|}{ Amchitka Site (Long Shot) (AMC02) } \\
\hline \multirow{3}{*}{ Chiton } & CHIT-LS-LT1-1 & FAUNA & $7 / 4 / 2011$ & $5 \pm 1$ & 1 & $24.801 \pm 4.96$ & 4.96 & & 51.457555 & 179.195138 \\
\hline & CHIT-LS-LT2-1 & FAUNA & $7 / 3 / 2011$ & $27 \pm 2.7$ & 1 & $126.477 \pm 12.648$ & 4.684 & & 51.44925 & 179.205583 \\
\hline & CHIT-LS-LT3-1 & FAUNA & $7 / 3 / 2011$ & $19.6 \pm 3.9$ & 1 & $93.324 \pm 18.57$ & 4.761 & & 51.43 & 179.22667 \\
\hline \multirow{10}{*}{ Dragon Kelp } & EUAL-LS-DUP & VEG & $7 / 3 / 2011$ & $25.6 \pm 2.4$ & 1 & $153.746 \pm 14.414$ & 6.006 & & 51.44925 & 179.205583 \\
\hline & EUAL-LS-LT1-1 & VEG & $7 / 4 / 2011$ & $15.7 \pm 2.9$ & 1 & $146.698 \pm 27.097$ & 9.344 & & 51.457555 & 179.195138 \\
\hline & EUAL-LS-LT1-2 & VEG & $7 / 4 / 2011$ & $31.3 \pm 1.2$ & 1 & $194.58 \pm 7.46$ & 6.217 & & 51.457555 & 179.195138 \\
\hline & EUAL-LS-LT1-3 & VEG & $7 / 4 / 2011$ & $30.3 \pm 2.2$ & 1 & $182.931 \pm 13.282$ & 6.037 & & 51.457555 & 179.195138 \\
\hline & EUAL-LS-LT2-1 & VEG & $7 / 3 / 2011$ & $28 \pm 2$ & 1 & $169.37 \pm 12.098$ & 6.049 & & 51.44925 & 179.205583 \\
\hline & EUAL-LS-LT2-2 & VEG & $7 / 3 / 2011$ & $12.4 \pm 1.8$ & 1 & $90.027 \pm 13.068$ & 7.26 & & 51.44925 & 179.205583 \\
\hline & EUAL-LS-LT2-3 & VEG & $7 / 3 / 2011$ & $17.6 \pm 2.7$ & 1 & $127.31 \pm 19.53$ & 7.234 & & 51.44925 & 179.205583 \\
\hline & EUAL-LS-LT3-1 & VEG & $7 / 2 / 2011$ & $34.5 \pm 2.5$ & 1 & $220.189 \pm 15.956$ & 6.382 & & 51.43 & 179.22667 \\
\hline & EUAL-LS-LT3-2 & VEG & $7 / 2 / 2011$ & $44.6 \pm 5.3$ & 1 & $317.361 \pm 37.713$ & 7.116 & & 51.43 & 179.22667 \\
\hline & EUAL-LS-LT3-3 & VEG & $7 / 2 / 2011$ & $12.5 \pm 2.5$ & 1 & $105.437 \pm 21.087$ & 8.435 & & 51.43 & 179.22667 \\
\hline \multirow{10}{*}{ Rockweed } & FUCU-LS-DUP & VEG & $6 / 22 / 2011$ & $67.4 \pm 3.1$ & 1 & $370.394 \pm 17.036$ & 5.495 & & 51.447472 & 179.190888 \\
\hline & FUCU-LS-LT1-1 & VEG & $6 / 22 / 2011$ & $88.5 \pm 2.9$ & 1 & $478.094 \pm 15.666$ & 5.402 & & 51.447527 & 179.189361 \\
\hline & FUCU-LS-LT1-2 & VEG & $6 / 22 / 2011$ & $75.7 \pm 4.5$ & 1 & $463.077 \pm 27.528$ & 6.117 & & 51.447527 & 179.189361 \\
\hline & FUCU-LS-LT1-3 & VEG & $6 / 22 / 2011$ & $61.2 \pm 3.9$ & 1 & $406.922 \pm 25.931$ & 6.649 & & 51.448777 & 179.189305 \\
\hline & FUCU-LS-LT2-1 & VEG & $6 / 22 / 2011$ & $35 \pm 1.2$ & 1 & $275.964 \pm 9.462$ & 7.885 & & 51.447222 & 179.199361 \\
\hline & FUCU-LS-LT2-2 & VEG & $6 / 22 / 2011$ & $56 \pm 2.4$ & 1 & $405.905 \pm 17.396$ & 7.248 & & 51.44625 & 179.196972 \\
\hline & FUCU-LS-LT2-3 & VEG & $6 / 22 / 2011$ & $73.5 \pm 2.9$ & 1 & $449.502 \pm 17.735$ & 6.116 & & 51.446722 & 179.191888 \\
\hline & FUCU-LS-LT3-1 & VEG & $7 / 2 / 2011$ & $89.1 \pm 3.5$ & 1 & $431.578 \pm 16.953$ & 4.844 & & 51.433611 & 179.219305 \\
\hline & FUCU-LS-LT3-2 & VEG & $7 / 2 / 2011$ & $68.1 \pm 3.7$ & 1 & $309.893 \pm 16.837$ & 4.551 & & 51.430166 & 179.218694 \\
\hline & FUCU-LS-LT3-3 & VEG & $7 / 2 / 2011$ & $95.7 \pm 4.9$ & 1 & $350.681 \pm 17.955$ & 3.664 & & 51.430166 & 179.218694 \\
\hline
\end{tabular}




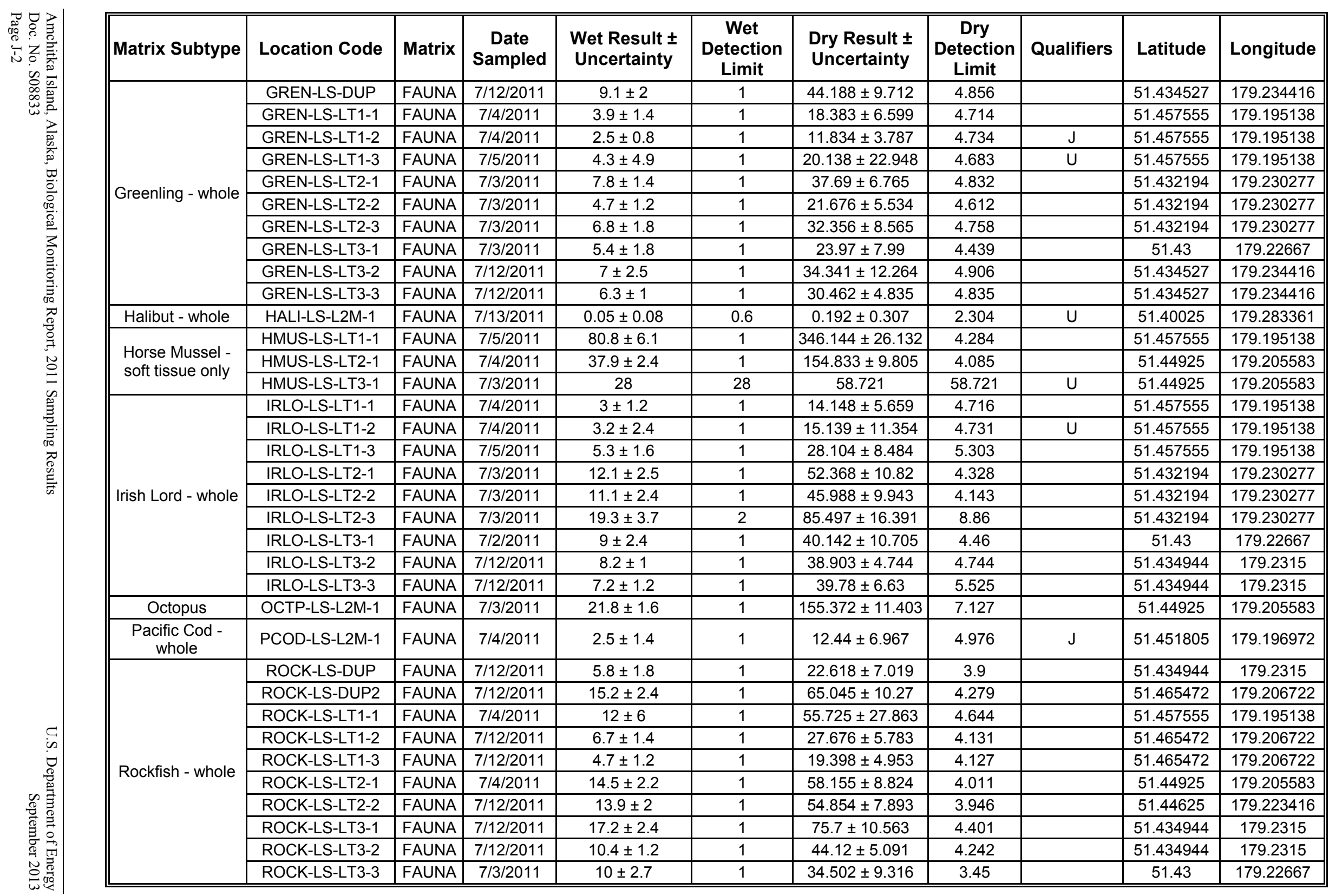




\begin{tabular}{|c|c|c|c|c|c|c|c|c|c|c|}
\hline Matrix Subtype & Location Code & Matrix & $\begin{array}{c}\text { Date } \\
\text { Sampled }\end{array}$ & $\begin{array}{l}\text { Wet Result } \pm \\
\text { Uncertainty }\end{array}$ & $\begin{array}{c}\text { Wet } \\
\text { Detection } \\
\text { Limit }\end{array}$ & $\begin{array}{l}\text { Dry Result } \pm \\
\text { Uncertainty }\end{array}$ & \begin{tabular}{|c|} 
Dry \\
Detection \\
Limit
\end{tabular} & Qualifiers & Latitude & Longitude \\
\hline \begin{tabular}{|l|} 
Reindeer Lichen \\
\end{tabular} & STRL-AI-LT1-2 & VEG & $6 / 22 / 2011$ & $3.3 \pm 0.6$ & 1 & $10.005 \pm 1.819$ & 3.032 & & 51.447361 & 179.189111 \\
\hline \multirow{3}{*}{$\begin{array}{l}\text { Sea Urchin - } \\
\text { whole }\end{array}$} & URCH-LS-LT1-1 & FAUNA & $7 / 4 / 2011$ & $25 \pm 7.3$ & 1 & $97.661 \pm 28.517$ & 3.906 & & 51.457555 & 179.195138 \\
\hline & URCH-LS-LT2-1 & FAUNA & $7 / 3 / 2011$ & $23.8 \pm 7.8$ & 1 & $91.442 \pm 29.968$ & 3.842 & & 51.44925 & 179.205583 \\
\hline & URCH-LS-LT3-1 & FAUNA & $7 / 2 / 2011$ & $26.3 \pm 5.1$ & 1 & $102.444 \pm 19.866$ & 3.895 & & 51.43 & 179.22667 \\
\hline \multicolumn{11}{|c|}{ Amchitka Site (Milrow) (AMC03) } \\
\hline \multirow{3}{*}{ Chiton } & CHIT-ML-MT1-1 & FAUNA & $7 / 9 / 2011$ & $18.7 \pm 7.3$ & 1 & $70.516 \pm 27.528$ & 3.771 & & 51.408472 & 179.139166 \\
\hline & CHIT-ML-MT2-1 & FAUNA & $7 / 10 / 2011$ & $28.9 \pm 6.1$ & 1 & $134.189 \pm 28.324$ & 4.643 & & 51.389416 & 179.173138 \\
\hline & CHIT-ML-MT3-1 & FAUNA & $7 / 10 / 2011$ & $27.9 \pm 4.1$ & 1 & $126.603 \pm 18.605$ & 4.538 & & 51.387805 & 179.184 \\
\hline \multirow{10}{*}{ Dragon Kelp } & EUAL-ML-DUP & VEG & $7 / 9 / 2011$ & $17 \pm 2.4$ & 1 & $128.107 \pm 18.086$ & 7.536 & & 51.408472 & 179.139166 \\
\hline & EUAL-ML-MT1-1 & VEG & $7 / 9 / 2011$ & $18.2 \pm 1.4$ & 1 & $145.492 \pm 11.192$ & 7.994 & & 51.408472 & 179.139166 \\
\hline & EUAL-ML-MT1-2 & VEG & $7 / 9 / 2011$ & $23.2 \pm 2.9$ & 1 & $172.629 \pm 21.579$ & 7.441 & & 51.408472 & 179.139166 \\
\hline & EUAL-ML-MT1-3 & VEG & $7 / 9 / 2011$ & $14.8 \pm 1.8$ & 1 & $124.491 \pm 15.141$ & 8.412 & & 51.408472 & 179.139166 \\
\hline & EUAL-ML-MT2-1 & VEG & $7 / 10 / 2011$ & $41.9 \pm 4.7$ & 2 & $312.28 \pm 35.029$ & 14.906 & & 51.389416 & 179.173138 \\
\hline & EUAL-ML-MT2-2 & VEG & $7 / 10 / 2011$ & $26.8 \pm 3.5$ & 1 & $189.883 \pm 24.798$ & 7.085 & & 51.389416 & 179.173138 \\
\hline & EUAL-ML-MT2-3 & VEG & $7 / 10 / 2011$ & $21.9 \pm 1.2$ & 1 & $165.077 \pm 9.045$ & 7.538 & & 51.389416 & 179.173138 \\
\hline & EUAL-ML-MT3-1 & VEG & $7 / 10 / 2011$ & $21 \pm 3.5$ & 1 & $147.717 \pm 24.62$ & 7.034 & & 51.387805 & 179.184 \\
\hline & EUAL-ML-MT3-2 & VEG & $7 / 10 / 2011$ & $21.5 \pm 2.5$ & 1 & $157.076 \pm 18.265$ & 7.306 & & 51.387805 & 179.184 \\
\hline & EUAL-ML-MT3-3 & VEG & 7/10/2011 & $38.2 \pm 4.1$ & 1 & $263.434 \pm 28.274$ & 6.896 & & 51.387805 & 179.184 \\
\hline \multirow{10}{*}{ Rockweed } & FUCU-ML-DUP & VEG & $6 / 20 / 2011$ & $103 \pm 4$ & 1 & $446.93 \pm 17.356$ & 4.339 & & 51.412449 & 179.160843 \\
\hline & FUCU-ML-MT1-1 & VEG & $6 / 20 / 2011$ & $96.7 \pm 3.5$ & 1 & $402.858 \pm 14.581$ & 4.166 & & 51.412449 & 179.160843 \\
\hline & FUCU-ML-MT1-2 & VEG & $6 / 21 / 2011$ & $89.1 \pm 1.4$ & 1 & $540.504 \pm 8.493$ & 6.066 & & 51.417083 & 179.134083 \\
\hline & FUCU-ML-MT1-3 & VEG & 7/9/2011 & $108 \pm 6$ & 1 & $496.426 \pm 27.579$ & 4.597 & & 51.417083 & 179.134083 \\
\hline & FUCU-ML-MT2-1 & VEG & $6 / 20 / 2011$ & $91.6 \pm 4.3$ & 1 & $472.733 \pm 22.192$ & 5.161 & & 51.403305 & 179.167166 \\
\hline & FUCU-ML-MT2-2 & VEG & $6 / 21 / 2011$ & $55 \pm 2.2$ & 1 & $386.405 \pm 15.456$ & 7.026 & & 51.405694 & 179.161361 \\
\hline & FUCU-ML-MT2-3 & VEG & $6 / 21 / 2011$ & $53 \pm 3.1$ & 1 & $368.012 \pm 21.525$ & 6.944 & & 51.405694 & 179.161361 \\
\hline & FUCU-ML-MT3-1 & VEG & $7 / 10 / 2011$ & $133 \pm 4$ & 1 & $645.882 \pm 19.425$ & 4.856 & & 51.389416 & 179.173138 \\
\hline & FUCU-ML-MT3-2 & VEG & $7 / 10 / 2011$ & $144 \pm 4$ & 1 & $691.579 \pm 19.211$ & 4.803 & & \begin{tabular}{|l|}
51.389416 \\
\end{tabular} & 179.173138 \\
\hline & FUCU-ML-MT3-3 & VEG & 7/10/2011 & $130 \pm 6$ & 1 & $656.317 \pm 30.292$ & 5.049 & & 51.389416 & 179.173138 \\
\hline
\end{tabular}




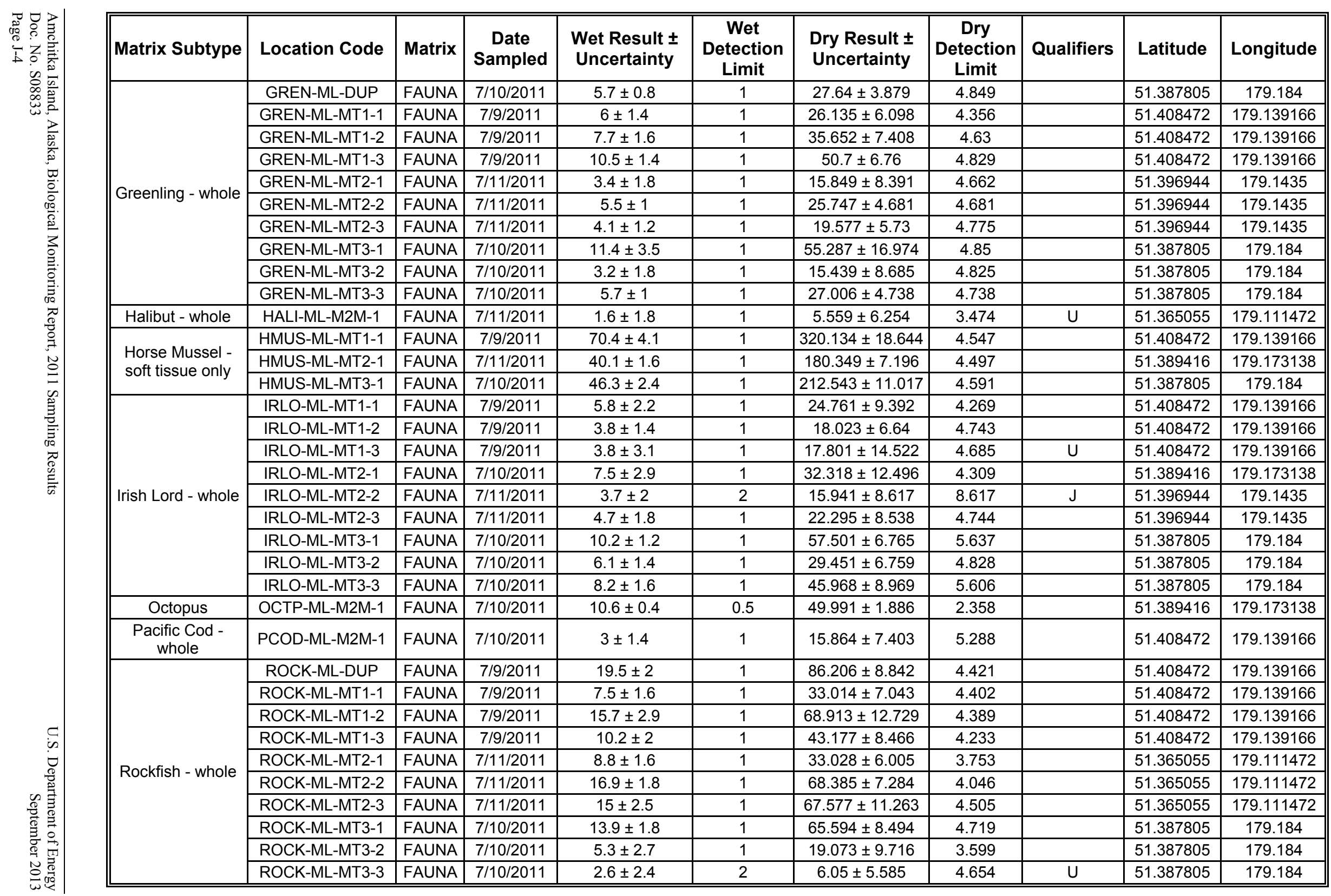




\begin{tabular}{|c|c|c|c|c|c|c|c|c|c|c|}
\hline Matrix Subtype & Location Code & Matrix & $\begin{array}{c}\text { Date } \\
\text { Sampled }\end{array}$ & $\begin{array}{l}\text { Wet Result } \pm \\
\text { Uncertainty }\end{array}$ & $\begin{array}{c}\text { Wet } \\
\text { Detection } \\
\text { Limit }\end{array}$ & $\begin{array}{l}\text { Dry Result } \pm \\
\text { Uncertainty }\end{array}$ & \begin{tabular}{|c|} 
Dry \\
Detection \\
Limit
\end{tabular} & Qualifiers & Latitude & Longitude \\
\hline \begin{tabular}{|l|} 
Reindeer Lichen \\
\end{tabular} & STRL-AI-ML-1 & VEG & 6/21/2011 & $3.2 \pm 0.4$ & 1 & $11.452 \pm 1.432$ & 3.579 & & 51.402583 & 179.183972 \\
\hline \multirow{3}{*}{$\begin{array}{l}\text { Sea Urchin - } \\
\text { whole }\end{array}$} & URCH-ML-MT1-1 & FAUNA & $7 / 9 / 2011$ & $26.4 \pm 7.8$ & 1 & $97.937 \pm 28.936$ & 3.71 & & 51.408472 & 179.139166 \\
\hline & URCH-ML-MT2-1 & FAUNA & $7 / 10 / 2011$ & $24.7 \pm 5.1$ & 1 & $92.247 \pm 19.047$ & 3.735 & & 51.389416 & 179.173138 \\
\hline & URCH-ML-MT3-1 & FAUNA & $7 / 10 / 2011$ & $24.2 \pm 7.1$ & 1 & $96.071 \pm 28.186$ & 3.97 & & 51.387805 & 179.184 \\
\hline \multicolumn{11}{|c|}{ Amchitka Site (Cannikin) (AMC05) } \\
\hline \multirow{3}{*}{ Chiton } & CHIT-CN-CT1-1 & FAUNA & $7 / 8 / 2011$ & $31.6 \pm 3.7$ & 1 & $147.999 \pm 17.329$ & 4.684 & & 51.482972 & 179.123833 \\
\hline & CHIT-CN-CT2-1 & FAUNA & 7/7/2011 & $32.4 \pm 6.1$ & 1 & $153.332 \pm 28.868$ & 4.732 & & 51.472611 & 179.151111 \\
\hline & CHIT-CN-CT3-1 & FAUNA & $7 / 5 / 2011$ & $24.5 \pm 2.9$ & 1 & $120.255 \pm 14.234$ & 4.908 & & 51.471416 & 179.172861 \\
\hline \multirow{10}{*}{ Dragon Kelp } & EUAL-CN-CT1-1 & VEG & $7 / 8 / 2011$ & $23.3 \pm 2.5$ & 1 & $172.471 \pm 18.505$ & 7.402 & & 51.482972 & 179.123833 \\
\hline & EUAL-CN-CT1-2 & VEG & $7 / 8 / 2011$ & $19.1 \pm 1$ & 1 & $124.527 \pm 6.52$ & 6.52 & & 51.482972 & 179.123833 \\
\hline & EUAL-CN-CT1-3 & VEG & $7 / 8 / 2011$ & $28 \pm 2.2$ & 1 & $171.504 \pm 13.475$ & 6.125 & & 51.482972 & 179.123833 \\
\hline & EUAL-CN-CT2-1 & VEG & $7 / 6 / 2011$ & $23.7 \pm 1.6$ & 1 & $151.156 \pm 10.205$ & 6.378 & & 51.472611 & 179.151111 \\
\hline & EUAL-CN-CT2-2 & VEG & $7 / 6 / 2011$ & $39.8 \pm 3.7$ & 1 & $249.804 \pm 23.223$ & 6.276 & & 51.472611 & 179.151111 \\
\hline & EUAL-CN-CT2-3 & VEG & 7/7/2011 & $17.8 \pm 1.2$ & 1 & $129.05 \pm 8.7$ & 7.25 & & 51.472611 & 179.151111 \\
\hline & EUAL-CN-CT3-1 & VEG & $7 / 5 / 2011$ & $20.1 \pm 2$ & 1 & $140.157 \pm 13.946$ & 6.973 & & 51.471416 & 179.172861 \\
\hline & EUAL-CN-CT3-2 & VEG & $7 / 5 / 2011$ & $13.5 \pm 2$ & 1 & $97.061 \pm 14.379$ & 7.19 & & 51.471416 & 179.172861 \\
\hline & EUAL-CN-CT3-3 & VEG & $7 / 5 / 2011$ & $25.1 \pm 2.7$ & 1 & $163.136 \pm 17.548$ & 6.499 & & 51.471416 & 179.172861 \\
\hline & EUAL-CN-DUP & VEG & $7 / 6 / 2011$ & $9.1 \pm 1.8$ & 1 & $60.466 \pm 11.96$ & 6.645 & & 51.471416 & 179.172861 \\
\hline \multirow{10}{*}{ Rockweed } & FUCU-CN-CT1-1 & VEG & \begin{tabular}{|c|}
$6 / 23 / 2011$ \\
\end{tabular} & $53.8 \pm 2.5$ & 1 & $310.26 \pm 14.417$ & 5.767 & & 51.481888 & 179.118138 \\
\hline & FUCU-CN-CT1-2 & VEG & 6/23/2011 & $59.8 \pm 3.7$ & 1 & $376.624 \pm 23.303$ & 6.298 & & 51.481888 & 179.118138 \\
\hline & FUCU-CN-CT1-3 & VEG & 6/23/2011 & $69.5 \pm 2.9$ & 1 & $409.713 \pm 17.096$ & 5.895 & & 51.481888 & 179.118138 \\
\hline & FUCU-CN-CT2-1 & VEG & $7 / 8 / 2011$ & $127 \pm 4$ & 1 & $524.824 \pm 16.53$ & 4.132 & & 51.476638 & 179.135277 \\
\hline & FUCU-CN-CT2-2 & VEG & $7 / 8 / 2011$ & $124 \pm 6$ & 1 & $550.213 \pm 26.623$ & 4.437 & & 51.476638 & 179.135277 \\
\hline & FUCU-CN-CT2-3 & VEG & $7 / 12 / 2011$ & $55.3 \pm 3.5$ & 1 & $292.316 \pm 18.501$ & 5.286 & & 51.467583 & 179.153583 \\
\hline & FUCU-CN-CT3-1 & VEG & $7 / 6 / 2011$ & $51.2 \pm 3.9$ & 1 & $275.897 \pm 21.016$ & 5.389 & & 51.465027 & 179.173166 \\
\hline & FUCU-CN-CT3-2 & VEG & 7/6/2011 & $41.2 \pm 6.3$ & 1 & $241.828 \pm 36.979$ & 5.87 & & 51.465472 & 179.170583 \\
\hline & FUCU-CN-CT3-3 & VEG & $7 / 6 / 2011$ & $77.6 \pm 3.1$ & 1 & $492.451 \pm 19.673$ & 6.346 & & 51.464638 & 179.159305 \\
\hline & FUCU-CN-DUP & VEG & 6/23/2011 & $53.1 \pm 2.2$ & 1 & $342.587 \pm 14.194$ & 6.452 & & 51.481888 & 179.118138 \\
\hline
\end{tabular}




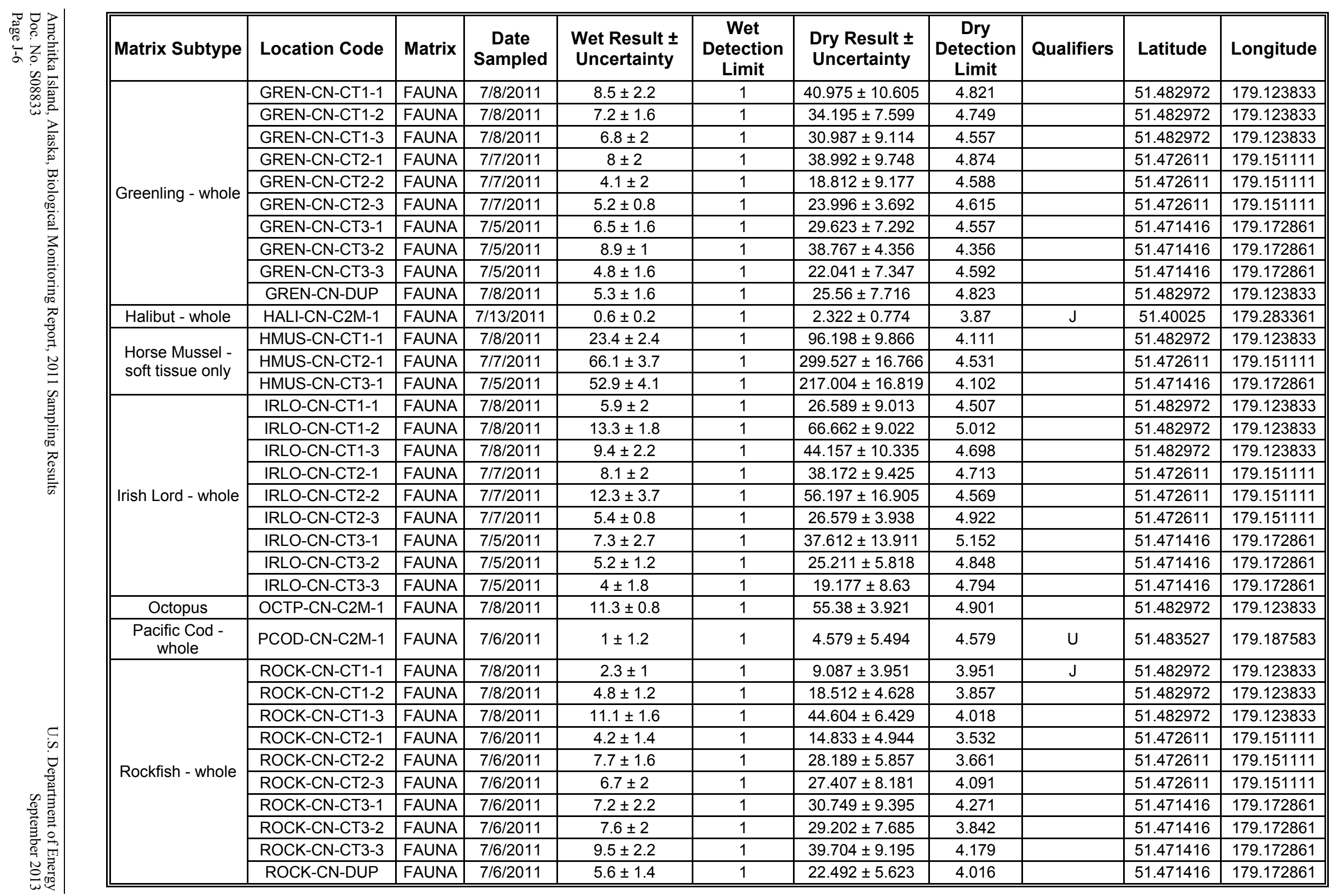




\begin{tabular}{|c|c|c|c|c|c|c|c|c|c|c|}
\hline Matrix Subtype & Location Code & Matrix & $\begin{array}{c}\text { Date } \\
\text { Sampled }\end{array}$ & $\begin{array}{l}\text { Wet Result } \pm \\
\text { Uncertainty }\end{array}$ & $\begin{array}{c}\text { Wet } \\
\text { Detection } \\
\text { Limit }\end{array}$ & $\begin{array}{l}\text { Dry Result } \pm \\
\text { Uncertainty }\end{array}$ & \begin{tabular}{|c|} 
Dry \\
Detection \\
Limit
\end{tabular} & Qualifiers & Latitude & Longitude \\
\hline \begin{tabular}{|l|} 
Reindeer Lichen \\
\end{tabular} & STRL-AI-CT1-1 & VEG & $6 / 23 / 2011$ & $10.8 \pm 3.5$ & 2 & $17.121 \pm 5.548$ & 3.171 & & 51.480583 & 179.109472 \\
\hline \multirow{3}{*}{$\begin{array}{l}\text { Sea Urchin - } \\
\text { whole }\end{array}$} & URCH-CN-CT1-1 & FAUNA & $7 / 8 / 2011$ & $24.6 \pm 6.9$ & 1 & $98.702 \pm 27.685$ & 4.012 & & 51.482972 & 179.123833 \\
\hline & URCH-CN-CT2-1 & FAUNA & $7 / 7 / 2011$ & $17.7 \pm 8.6$ & 2 & $60.652 \pm 29.469$ & 6.853 & & 51.472611 & 179.151111 \\
\hline & URCH-CN-CT3-1 & FAUNA & $7 / 5 / 2011$ & $16.7 \pm 5.1$ & 1 & $\mid 63.969 \pm 19.535$ & 3.83 & & 51.471416 & 179.172861 \\
\hline \multicolumn{11}{|c|}{\begin{tabular}{|l} 
Amchitka Site (Adak Island) (AMC13) \\
\end{tabular}} \\
\hline $\begin{array}{l}\text { Dolly Varden - } \\
\text { whole }\end{array}$ & DOLL-AD-XXX-1 & FAUNA & $6 / 28 / 2011$ & $0.9 \pm 0.4$ & 1 & $3.671 \pm 1.631$ & 4.079 & $\mathrm{~J}$ & 51.8375 & -176.672555 \\
\hline \multirow{3}{*}{ Reindeer Lichen } & STRL-AD-XXX-1 & VEG & $6 / 28 / 2011$ & $4.6 \pm 1$ & 1 & $9.004 \pm 1.957$ & 1.957 & & 51.837527 & -176.670694 \\
\hline & STRL-AD-XXX-2 & VEG & $6 / 29 / 2011$ & $3.7 \pm 0.8$ & 1 & $9.142 \pm 1.977$ & 2.471 & & 51.872833 & -176.707444 \\
\hline & STRL-AD-XXX-3 & VEG & $6 / 29 / 2011$ & $6 \pm 1.4$ & 1 & $9.532 \pm 2.224$ & 1.589 & & 51.900805 & -176.688527 \\
\hline \multicolumn{11}{|c|}{ Amchitka Site (Adak Island North) (AMC14) } \\
\hline \multirow{3}{*}{ Chiton } & \begin{tabular}{|l|} 
CHIT-AN-ANT1-1 \\
\end{tabular} & FAUNA & $7 / 20 / 2011$ & $13.5 \pm 3.7$ & 1 & $65.749 \pm 18.02$ & 4.87 & & 51.838722 & -176.582805 \\
\hline & CHIT-AN-ANT2-1 & FAUNA & \begin{tabular}{|l|}
$7 / 18 / 2011$ \\
\end{tabular} & $9.4 \pm 3.7$ & 1 & $47.116 \pm 18.546$ & 5.012 & & 51.848361 & \begin{tabular}{|l|}
-176.506277 \\
\end{tabular} \\
\hline & \begin{tabular}{|l} 
CHIT-AN-ANT3-1 \\
\end{tabular} & FAUNA & \begin{tabular}{|l|}
$7 / 17 / 2011$ \\
\end{tabular} & $9.9 \pm 3.5$ & 1 & $\mid 50.343 \pm 17.798$ & 5.085 & & 51.853722 & -176.470694 \\
\hline \multirow{10}{*}{ Dragon Kelp } & \begin{tabular}{|l} 
EUAL-AN-ANT1-1 \\
\end{tabular} & VEG & \begin{tabular}{|l|}
$7 / 19 / 2011$ \\
\end{tabular} & $15.7 \pm 1.4$ & 1 & $\mid 131.646 \pm 11.739$ & 8.385 & & 51.835694 & \begin{tabular}{|l|}
-176.542138 \\
\end{tabular} \\
\hline & \begin{tabular}{|l} 
EUAL-AN-ANT1-2 \\
\end{tabular} & VEG & \begin{tabular}{|l|}
$7 / 19 / 2011$ \\
\end{tabular} & $18.2 \pm 2.7$ & 1 & $124.065 \pm 18.405$ & 6.817 & & 51.835694 & \begin{tabular}{|l|}
-176.542138 \\
\end{tabular} \\
\hline & \begin{tabular}{|l|} 
EUAL-AN-ANT1-3 \\
\end{tabular} & VEG & 7/19/2011 & $15.6 \pm 1.2$ & 1 & $120.575 \pm 9.275$ & 7.729 & & 51.835694 & -176.542138 \\
\hline & \begin{tabular}{|l|} 
EUAL-AN-ANT2-1 \\
\end{tabular} & VEG & \begin{tabular}{|l|}
$7 / 18 / 2011$ \\
\end{tabular} & $27.8 \pm 2.7$ & 1 & $202.652 \pm 19.682$ & 7.29 & & 51.848361 & $\mid-176.506277$ \\
\hline & \begin{tabular}{|l} 
EUAL-AN-ANT2-2 \\
\end{tabular} & VEG & \begin{tabular}{|l|}
$7 / 18 / 2011$ \\
\end{tabular} & $33.7 \pm 2$ & 1 & $214.752 \pm 12.745$ & 6.372 & & 51.848361 & -176.506277 \\
\hline & \begin{tabular}{|l|} 
EUAL-AN-ANT2-3 \\
\end{tabular} & VEG & 7/18/2011 & $21.8 \pm 1.8$ & 1 & $151.42 \pm 12.503$ & 6.946 & & 51.848361 & -176.506277 \\
\hline & \begin{tabular}{|l|} 
EUAL-AN-ANT3-1 \\
\end{tabular} & VEG & \begin{tabular}{|l|}
$7 / 17 / 2011$ \\
\end{tabular} & $7.3 \pm 1$ & 0.4 & $54.561 \pm 7.474$ & 2.99 & & 51.853722 & \begin{tabular}{|l|}
-176.470694 \\
\end{tabular} \\
\hline & \begin{tabular}{|l} 
EUAL-AN-ANT3-2 \\
\end{tabular} & VEG & $7 / 17 / 2011$ & $27.9 \pm 1$ & 1 & $190.255 \pm 6.819$ & 6.819 & & 51.853722 & -176.470694 \\
\hline & \begin{tabular}{|l|} 
EUAL-AN-ANT3-3 \\
\end{tabular} & VEG & 7/17/2011 & $17.8 \pm 3.1$ & 1 & $119.448 \pm 20.803$ & 6.711 & & 51.853722 & \begin{tabular}{|l|}
-176.470694 \\
\end{tabular} \\
\hline & EUAL-AN-DUP & VEG & 7/17/2011 & $46.8 \pm 3.9$ & 1 & $294.27 \pm 24.523$ & 6.288 & & 51.853722 & -176.470694 \\
\hline \multirow{10}{*}{ Rockweed } & \begin{tabular}{|l} 
FUCU-AN-ANT1-1 \\
\end{tabular} & VEG & \begin{tabular}{|l|}
$7 / 19 / 2011$ \\
\end{tabular} & $83.9 \pm 3.7$ & 1 & $427.464 \pm 18.851$ & 5.095 & & 51.841444 & $\mid-176.546083$ \\
\hline & \begin{tabular}{|l} 
FUCU-AN-ANT1-2 \\
\end{tabular} & VEG & $7 / 20 / 2011$ & $48.9 \pm 2.7$ & 1 & $315.462 \pm 17.418$ & 6.451 & & 51.8395 & -176.591305 \\
\hline & \begin{tabular}{|l} 
FUCU-AN-ANT1-3 \\
\end{tabular} & VEG & \begin{tabular}{|l|}
$7 / 20 / 2011$ \\
\end{tabular} & $75.7 \pm 3.7$ & 1 & $413.124 \pm 20.192$ & 5.457 & & 51.8395 & -176.591305 \\
\hline & \begin{tabular}{|l|} 
FUCU-AN-ANT2-1 \\
\end{tabular} & VEG & \begin{tabular}{|l|}
$7 / 18 / 2011$ \\
\end{tabular} & $78.6 \pm 4.3$ & 1 & $496.375 \pm 27.155$ & 6.315 & & 51.837666 & \begin{tabular}{|l|}
-176.517527 \\
\end{tabular} \\
\hline & \begin{tabular}{|l|} 
FUCU-AN-ANT2-2 \\
\end{tabular} & VEG & \begin{tabular}{|l|}
$7 / 18 / 2011$ \\
\end{tabular} & $46.1 \pm 2.7$ & 1 & $245.055 \pm 14.352$ & 5.316 & & 51.841444 & -176.546083 \\
\hline & \begin{tabular}{|l|} 
FUCU-AN-ANT2-3 \\
\end{tabular} & VEG & \begin{tabular}{|l|}
$7 / 18 / 2011$ \\
\end{tabular} & $113 \pm 4$ & 1 & $476.681 \pm 16.874$ & 4.218 & & 51.8395 & -176.591305 \\
\hline & \begin{tabular}{|l} 
FUCU-AN-ANT3-1 \\
\end{tabular} & VEG & \begin{tabular}{|l|}
$7 / 18 / 2011$ \\
\end{tabular} & $62 \pm 3.3$ & 1 & $354.728 \pm 18.881$ & 5.721 & & 51.839944 & -176.515916 \\
\hline & \begin{tabular}{|l|} 
FUCU-AN-ANT3-2 \\
\end{tabular} & VEG & \begin{tabular}{|l|}
$7 / 18 / 2011$ \\
\end{tabular} & $87.9 \pm 4.1$ & 1 & $429.686 \pm 20.042$ & 4.888 & & 51.828972 & $\mid-176.448305$ \\
\hline & \begin{tabular}{|l} 
FUCU-AN-ANT3-3 \\
\end{tabular} & VEG & \begin{tabular}{|l|l|}
$7 / 2011$ \\
\end{tabular} & $84.2 \pm 3.5$ & 1 & $418.621 \pm 17.401$ & 4.972 & & 51.830833 & -176.456361 \\
\hline & FUCU-AN-DUP & VEG & 7/18/2011 & $144 \pm 8$ & 1 & $670.078 \pm 37.227$ & 4.653 & & 51.828972 & -176.448305 \\
\hline
\end{tabular}




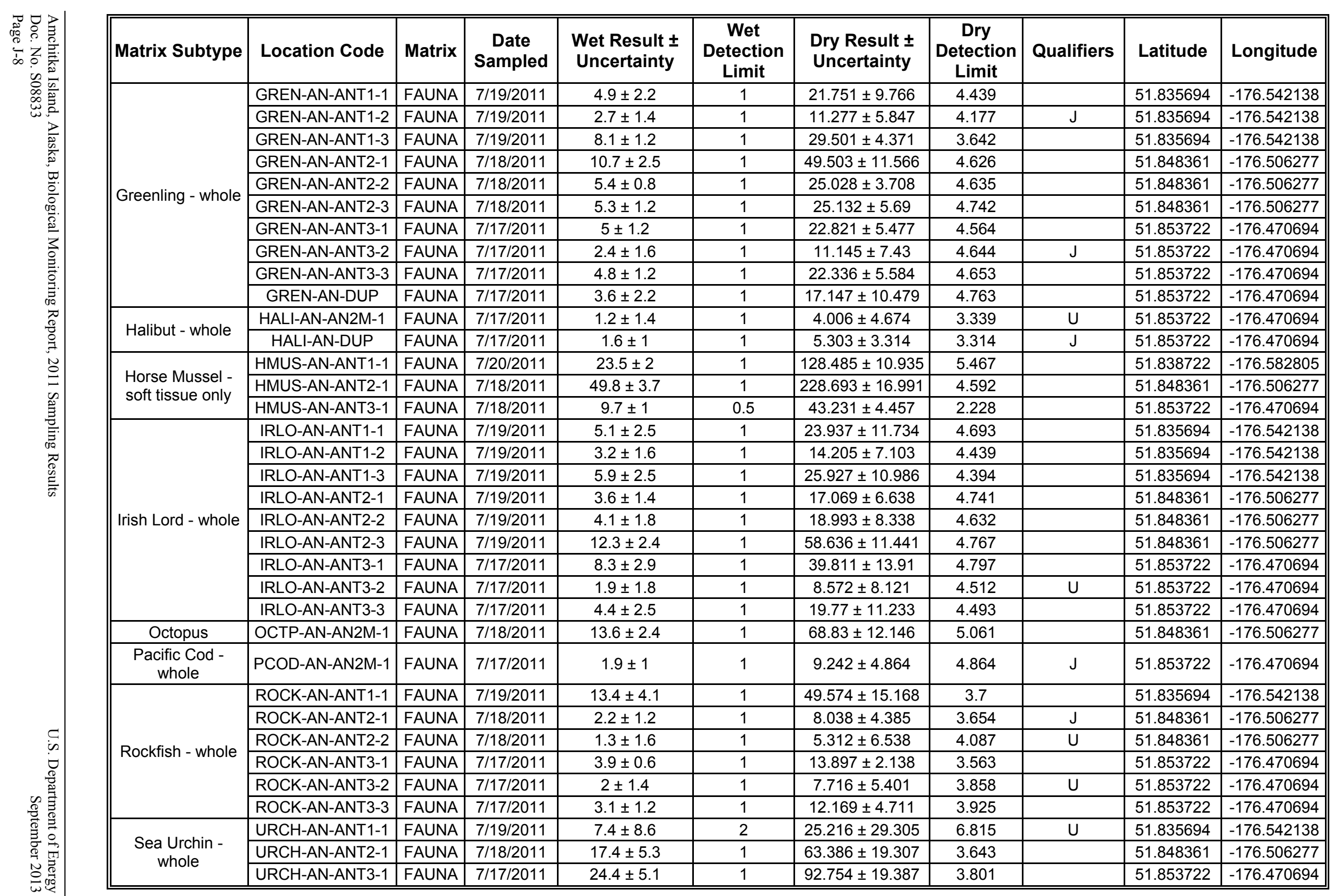




\begin{tabular}{|c|c|c|c|c|c|c|c|c|c|c|}
\hline Matrix Subtype & Location Code & Matrix & $\begin{array}{c}\text { Date } \\
\text { Sampled }\end{array}$ & $\begin{array}{l}\text { Wet Result } \pm \\
\text { Uncertainty }\end{array}$ & $\begin{array}{c}\text { Wet } \\
\text { Detection } \\
\text { Limit }\end{array}$ & $\begin{array}{l}\text { Dry Result } \pm \\
\text { Uncertainty }\end{array}$ & \begin{tabular}{|c|} 
Dry \\
Detection \\
Limit
\end{tabular} & Qualifiers & Latitude & Longitude \\
\hline \multicolumn{11}{|c|}{ Amchitka Site (Adak Island South) (AMC15) } \\
\hline \multirow{3}{*}{ Chiton } & CHIT-AS-AST1-1 & FAUNA & $7 / 15 / 2011$ & $29.4 \pm 4.3$ & 1 & $120.806 \pm 17.669$ & 4.109 & & 51.737472 & -176.48025 \\
\hline & CHIT-AS-AST2-1 & FAUNA & $7 / 16 / 2011$ & $5.1 \pm 3.7$ & 1 & $26.107 \pm 18.94$ & 5.119 & $\mathrm{U}$ & 51.745666 & -176.507055 \\
\hline & CHIT-AS-AST3-1 & FAUNA & $7 / 14 / 2011$ & $32.5 \pm 5.9$ & 1 & $153.096 \pm 27.793$ & 4.711 & & 51.688333 & -176.63375 \\
\hline \multirow{10}{*}{ Dragon Kelp } & EUAL-AS-AST1-1 & VEG & $7 / 15 / 2011$ & $25.7 \pm 2$ & 1 & $169.356 \pm 13.179$ & 6.59 & & 51.737472 & -176.48025 \\
\hline & EUAL-AS-AST1-2 & VEG & $7 / 15 / 2011$ & $17.8 \pm 2.4$ & 1 & $141.769 \pm 19.115$ & 7.965 & & 51.737472 & -176.48025 \\
\hline & EUAL-AS-AST1-3 & VEG & $7 / 15 / 2011$ & $9.4 \pm 2.5$ & 1 & $90.207 \pm 23.991$ & 9.596 & & 51.737472 & -176.48025 \\
\hline & EUAL-AS-AST2-1 & VEG & $7 / 16 / 2011$ & $15.3 \pm 2$ & 1 & $108.779 \pm 14.219$ & 7.11 & & 51.745666 & -176.507055 \\
\hline & EUAL-AS-AST2-2 & VEG & $7 / 16 / 2011$ & $20.5 \pm 2.2$ & 1 & $145.054 \pm 15.567$ & 7.076 & & 51.745666 & -176.507055 \\
\hline & EUAL-AS-AST2-3 & VEG & $7 / 16 / 2011$ & $31.2 \pm 3.1$ & 1 & $226.659 \pm 22.521$ & 7.265 & & 51.745666 & -176.507055 \\
\hline & EUAL-AS-AST3-1 & VEG & $7 / 14 / 2011$ & $27 \pm 2.4$ & 1 & $197.213 \pm 17.53$ & 7.304 & & 51.688333 & -176.63375 \\
\hline & EUAL-AS-AST3-2 & VEG & $7 / 14 / 2011$ & $9.3 \pm 1.6$ & 1 & $88.149 \pm 15.165$ & 9.478 & & 51.688333 & -176.63375 \\
\hline & EUAL-AS-AST3-3 & VEG & $7 / 14 / 2011$ & $13.2 \pm 2.4$ & 1 & $116.388 \pm 21.161$ & 8.817 & & 51.688333 & -176.63375 \\
\hline & EUAL-AS-DUP & VEG & $7 / 14 / 2011$ & $1.4 \pm 0.2$ & 0.1 & $10.301 \pm 1.472$ & 0.736 & & 51.688333 & -176.63375 \\
\hline \multirow{10}{*}{ Rockweed } & FUCU-AS-AST1-1 & VEG & $7 / 16 / 2011$ & $64.3 \pm 3.5$ & 1 & $396.643 \pm 21.59$ & 6.169 & & 51.733666 & -176.470805 \\
\hline & FUCU-AS-AST1-2 & VEG & $7 / 16 / 2011$ & $57.8 \pm 3.9$ & 1 & $320.616 \pm 21.633$ & 5.547 & & 51.733666 & -176.470805 \\
\hline & FUCU-AS-AST1-3 & VEG & $7 / 16 / 2011$ & $86.4 \pm 3.9$ & 1 & $468.95 \pm 21.168$ & 5.428 & & 51.733666 & -176.470805 \\
\hline & FUCU-AS-AST2-1 & VEG & $7 / 16 / 2011$ & $72.4 \pm 3.7$ & 1 & $349.956 \pm 17.884$ & 4.834 & & 51.745666 & -176.507055 \\
\hline & FUCU-AS-AST2-2 & VEG & $7 / 16 / 2011$ & $92.9 \pm 4.5$ & 1 & $470.25 \pm 22.779$ & 5.062 & & 51.745666 & -176.507055 \\
\hline & FUCU-AS-AST2-3 & VEG & $7 / 16 / 2011$ & $84.7 \pm 5.3$ & 1 & $416.093 \pm 26.037$ & 4.913 & & 51.745666 & -176.507055 \\
\hline & FUCU-AS-AST3-1 & VEG & $7 / 14 / 2011$ & $71.4 \pm 3.7$ & 1 & $376.399 \pm 19.505$ & 5.272 & & 51.688333 & -176.63375 \\
\hline & FUCU-AS-AST3-2 & VEG & $7 / 14 / 2011$ & $52.4 \pm 4.5$ & 1 & $258.046 \pm 22.16$ & 4.925 & & 51.688333 & -176.63375 \\
\hline & FUCU-AS-AST3-3 & VEG & $7 / 14 / 2011$ & $45.5 \pm 2$ & 1 & $238.935 \pm 10.503$ & 5.251 & & 51.688333 & -176.63375 \\
\hline & FUCU-AS-DUP & VEG & $7 / 16 / 2011$ & $115 \pm 4$ & 1 & $594.372 \pm 20.674$ & 5.168 & & 51.733666 & -176.470805 \\
\hline \multirow{10}{*}{ Greenling - whole } & GREN-AS-AST1-1 & FAUNA & $7 / 15 / 2011$ & $8.9 \pm 1.6$ & 1 & $42.4 \pm 7.622$ & 4.764 & & 51.737472 & -176.48025 \\
\hline & GREN-AS-AST1-2 & FAUNA & $7 / 15 / 2011$ & $3.9 \pm 1.2$ & 1 & $18.4 \pm 5.662$ & 4.718 & & 51.737472 & -176.48025 \\
\hline & GREN-AS-AST1-3 & FAUNA & $7 / 15 / 2011$ & $5.4 \pm 1.4$ & 1 & $25.937 \pm 6.724$ & 4.803 & & 51.737472 & -176.48025 \\
\hline & GREN-AS-AST2-1 & FAUNA & $7 / 16 / 2011$ & $2 \pm 0.6$ & 1 & $8.033 \pm 2.41$ & 4.017 & $\mathrm{~J}$ & 51.745666 & -176.507055 \\
\hline & GREN-AS-AST2-2 & FAUNA & $7 / 16 / 2011$ & $6.3 \pm 1.4$ & 1 & $28.816 \pm 6.404$ & 4.574 & & 51.745666 & -176.507055 \\
\hline & GREN-AS-AST2-3 & FAUNA & $7 / 16 / 2011$ & $4.2 \pm 2.2$ & 1 & $19.56 \pm 10.246$ & 4.657 & & 51.745666 & -176.507055 \\
\hline & GREN-AS-AST3-1 & FAUNA & $7 / 14 / 2011$ & $2.9 \pm 1.4$ & 1 & $13.812 \pm 6.668$ & 4.763 & $\mathrm{~J}$ & 51.688333 & -176.63375 \\
\hline & GREN-AS-AST3-2 & FAUNA & $7 / 14 / 2011$ & $6.2 \pm 2$ & 1 & $29.184 \pm 9.414$ & 4.707 & & 51.688333 & -176.63375 \\
\hline & GREN-AS-AST3-3 & FAUNA & $7 / 14 / 2011$ & $6.3 \pm 1.6$ & 1 & $30.087 \pm 7.641$ & 4.776 & & 51.688333 & -176.63375 \\
\hline & GREN-AS-DUP & FAUNA & $7 / 14 / 2011$ & $8.3 \pm 6.1$ & 1 & $41.621 \pm 30.589$ & 5.015 & $U$ & 51.688333 & -176.63375 \\
\hline
\end{tabular}




\begin{tabular}{|c|c|c|c|c|c|c|c|c|c|c|}
\hline Matrix Subtype & Location Code & Matrix & $\begin{array}{c}\text { Date } \\
\text { Sampled }\end{array}$ & $\begin{array}{l}\text { Wet Result } \pm \\
\text { Uncertainty }\end{array}$ & $\begin{array}{c}\text { Wet } \\
\text { Detection } \\
\text { Limit }\end{array}$ & $\begin{array}{l}\text { Dry Result } \pm \\
\text { Uncertainty }\end{array}$ & $\begin{array}{c}\text { Dry } \\
\text { Detection } \\
\text { Limit }\end{array}$ & Qualifiers & Latitude & Longitude \\
\hline \multirow{3}{*}{$\begin{array}{l}\text { Horse Mussel - } \\
\text { soft tissue only }\end{array}$} & HMUS-AS-AST1-1 & FAUNA & $7 / 15 / 2011$ & $34.1 \pm 2.4$ & 1 & $170.84 \pm 12.024$ & 5.01 & & 51.737472 & -176.48025 \\
\hline & HMUS-AS-AST2-1 & FAUNA & $7 / 16 / 2011$ & $15.3 \pm 1.2$ & 1 & $97.059 \pm 7.612$ & 6.344 & & 51.745666 & -176.507055 \\
\hline & \begin{tabular}{|l|} 
HMUS-AS-AST3-1 \\
\end{tabular} & FAUNA & $7 / 14 / 2011$ & $45.2 \pm 3.3$ & 1 & $230.447 \pm 16.825$ & 5.098 & & 51.688333 & -176.63375 \\
\hline \multirow{9}{*}{ Irish Lord - whole } & \begin{tabular}{|l|} 
IRLO-AS-AST1-1 \\
\end{tabular} & FAUNA & $7 / 15 / 2011$ & $8.3 \pm 2.5$ & 1 & $36.541 \pm 11.006$ & 4.403 & & 51.737472 & -176.48025 \\
\hline & IRLO-AS-AST1-2 & FAUNA & $7 / 15 / 2011$ & $5.3 \pm 2.7$ & 1 & $24.711 \pm 12.589$ & 4.663 & & 51.737472 & -176.48025 \\
\hline & IRLO-AS-AST1-3 & \begin{tabular}{|l|} 
FAUNA \\
\end{tabular} & $7 / 15 / 2011$ & $13.3 \pm 1.8$ & 1 & $60.623 \pm 8.205$ & 4.558 & & 51.737472 & -176.48025 \\
\hline & IRLO-AS-AST2-1 & FAUNA & $7 / 16 / 2011$ & $16 \pm 3.5$ & 1 & $74.877 \pm 16.379$ & 4.68 & & 51.745666 & -176.507055 \\
\hline & IRLO-AS-AST2-2 & FAUNA & $7 / 16 / 2011$ & $7 \pm 3.1$ & 1 & $29.801 \pm 13.198$ & 4.257 & & 51.745666 & -176.507055 \\
\hline & IRLO-AS-AST2-3 & FAUNA & $7 / 16 / 2011$ & $13.8 \pm 2.7$ & 1 & $69.814 \pm 13.659$ & 5.059 & & 51.745666 & -176.507055 \\
\hline & \begin{tabular}{|l|} 
IRLO-AS-AST3-1 \\
\end{tabular} & \begin{tabular}{|l|} 
FAUNA \\
\end{tabular} & $7 / 14 / 2011$ & $6.4 \pm 1.8$ & 1 & $28.734 \pm 8.081$ & 4.49 & & 51.688333 & -176.63375 \\
\hline & IRLO-AS-AST3-2 & FAUNA & $7 / 14 / 2011$ & $2.9 \pm 1.8$ & 1 & $12.254 \pm 7.606$ & 4.225 & $\mathrm{~J}$ & 51.688333 & -176.63375 \\
\hline & IRLO-AS-AST3-3 & FAUNA & $7 / 14 / 2011$ & $5.2 \pm 0.6$ & 1 & $23.727 \pm 2.738$ & 4.563 & & 51.688333 & -176.63375 \\
\hline $\begin{array}{l}\text { Pacific Cod - } \\
\text { whole }\end{array}$ & PCOD-AS-AS2M-1 & FAUNA & 7/15/2011 & $1.9 \pm 1.2$ & 1 & $9.506 \pm 6.004$ & 5.003 & $J$ & 51.737472 & -176.48025 \\
\hline \multirow{10}{*}{ Rockfish - whole } & ROCK-AS-AST1-1 & FAUNA & $7 / 15 / 2011$ & $5.6 \pm 1.4$ & 1 & $20.133 \pm 5.033$ & 3.595 & & 51.737472 & -176.48025 \\
\hline & \begin{tabular}{|l} 
ROCK-AS-AST1-2 \\
\end{tabular} & \begin{tabular}{|l|} 
FAUNA \\
\end{tabular} & $7 / 15 / 2011$ & $3 \pm 1$ & 1 & $11.24 \pm 3.747$ & 3.747 & & 51.737472 & -176.48025 \\
\hline & \begin{tabular}{|l|} 
ROCK-AS-AST1-3 \\
\end{tabular} & FAUNA & $7 / 15 / 2011$ & $4.8 \pm 1.6$ & 1 & $20.666 \pm 6.889$ & 4.305 & & 51.737472 & -176.48025 \\
\hline & ROCK-AS-AST2-1 & FAUNA & $7 / 16 / 2011$ & $7.2 \pm 3.3$ & 1 & $27.613 \pm 12.656$ & 3.835 & & 51.745666 & -176.507055 \\
\hline & ROCK-AS-AST2-2 & FAUNA & $7 / 16 / 2011$ & $1.7 \pm 2$ & 1 & $6.314 \pm 7.429$ & 3.714 & $\mathrm{U}$ & 51.745666 & -176.507055 \\
\hline & ROCK-AS-AST2-3 & FAUNA & $7 / 16 / 2011$ & $4.9 \pm 1.4$ & 1 & $18.909 \pm 5.403$ & 3.859 & & 51.745666 & -176.507055 \\
\hline & \begin{tabular}{|l|} 
ROCK-AS-AST3-1 \\
\end{tabular} & FAUNA & $7 / 14 / 2011$ & $12.8 \pm 3.9$ & 2 & $52.481 \pm 15.99$ & 8.2 & & 51.688333 & -176.63375 \\
\hline & ROCK-AS-AST3-2 & FAUNA & $7 / 14 / 2011$ & $11.3 \pm 1.8$ & 1 & $47.912 \pm 7.632$ & 4.24 & & 51.688333 & -176.63375 \\
\hline & \begin{tabular}{|l|} 
ROCK-AS-AST3-3 \\
\end{tabular} & FAUNA & $7 / 14 / 2011$ & $3.7 \pm 1.6$ & 1 & $16.056 \pm 6.943$ & 4.34 & & 51.688333 & -176.63375 \\
\hline & ROCK-AS-DUP & FAUNA & $7 / 14 / 2011$ & $2.6 \pm 2.7$ & 1 & $10.179 \pm 10.57$ & 3.915 & $\bar{U}$ & 51.688333 & -176.63375 \\
\hline \multirow{3}{*}{$\begin{array}{l}\text { Sea Urchin - } \\
\text { whole }\end{array}$} & URCH-AS-AST1-1 & FAUNA & $7 / 15 / 2011$ & $25.2 \pm 5.3$ & 1 & $95.718 \pm 20.131$ & 3.798 & & 51.737472 & -176.48025 \\
\hline & \begin{tabular}{|l|} 
URCH-AS-AST2-1 \\
\end{tabular} & FAUNA & $7 / 16 / 2011$ & $26 \pm 3.9$ & 1 & $104.963 \pm 15.744$ & 4.037 & & 51.745666 & -176.507055 \\
\hline & URCH-AS-AST3-1 & FAUNA & $7 / 14 / 2011$ & $28.5 \pm 10.2$ & 1 & $106.423 \pm 38.088$ & 3.734 & & 51.688333 & -176.63375 \\
\hline \multicolumn{11}{|c|}{${ }^{234}$ Uranium (pCi/kg) } \\
\hline \multicolumn{11}{|c|}{ Amchitka Site (AMC01) } \\
\hline $\begin{array}{l}\text { Dolly Varden - } \\
\text { whole }\end{array}$ & DOLL-AI-XXX-1 & FAUNA & $6 / 24 / 2011$ & $0.0104 \pm 0.0008$ & 0.0004 & $0.04 \pm 0.003$ & 0.002 & & 51.468283 & 179.108052 \\
\hline \begin{tabular}{|c|}
$\begin{array}{c}\text { Goose Egg - no } \\
\text { shell }\end{array}$ \\
\end{tabular} & GoOS-AI-XXX-1 & FAUNA & $6 / 24 / 2011$ & $0.0008 \pm 0.0004$ & 0.0008 & $0.002 \pm 0.001$ & 0.002 & J & 51.491305 & 179.0595 \\
\hline $\begin{array}{c}\text { Glaucous-winged } \\
\text { Gull Egg - no } \\
\text { shell }\end{array}$ & GWGU-AI-XXX-1 & FAUNA & $6 / 24 / 2011$ & $0.0007 \pm 0.0002$ & 0.0002 & $0.002 \pm 0.001$ & 0.001 & & 51.456388 & 179.167 \\
\hline
\end{tabular}




\begin{tabular}{|c|c|c|c|c|c|c|c|c|c|c|}
\hline Matrix Subtype & Location Code & Matrix & $\begin{array}{c}\text { Date } \\
\text { Sampled }\end{array}$ & $\begin{array}{l}\text { Wet Result } \pm \\
\text { Uncertainty }\end{array}$ & $\begin{array}{c}\text { Wet } \\
\text { Detection } \\
\text { Limit }\end{array}$ & $\begin{array}{l}\text { Dry Result } \pm \\
\text { Uncertainty }\end{array}$ & \begin{tabular}{|c|} 
Dry \\
Detection \\
Limit
\end{tabular} & Qualifiers & Latitude & Longitude \\
\hline \multicolumn{11}{|c|}{ Amchitka Site (Long Shot) (AMC02) } \\
\hline \multirow{3}{*}{ Chiton } & CHIT-LS-LT1-1 & FAUNA & $7 / 4 / 2011$ & $0.124 \pm 0.004$ & 0.0003 & $0.615 \pm 0.02$ & 0.001 & & 51.457555 & 179.195138 \\
\hline & CHIT-LS-LT2-1 & FAUNA & $7 / 3 / 2011$ & $0.91 \pm 0.02$ & 0.0004 & $4.263 \pm 0.094$ & 0.002 & & 51.44925 & 179.205583 \\
\hline & CHIT-LS-LT3-1 & FAUNA & $7 / 3 / 2011$ & $0.7 \pm 0.02$ & 0.0004 & $3.333 \pm 0.095$ & 0.002 & & 51.43 & 179.22667 \\
\hline \multirow{10}{*}{ Dragon Kelp } & EUAL-LS-DUP & VEG & $7 / 3 / 2011$ & $0.91 \pm 0.04$ & 0.0004 & $5.465 \pm 0.24$ & 0.002 & & 51.44925 & 179.205583 \\
\hline & EUAL-LS-LT1-1 & VEG & $7 / 4 / 2011$ & $0.51 \pm 0.02$ & 0.0003 & $4.765 \pm 0.187$ & 0.003 & & 51.457555 & 179.195138 \\
\hline & EUAL-LS-LT1-2 & VEG & $7 / 4 / 2011$ & $1.13 \pm 0.02$ & 0.0003 & $7.025 \pm 0.124$ & 0.002 & & 51.457555 & 179.195138 \\
\hline & EUAL-LS-LT1-3 & VEG & $7 / 4 / 2011$ & $1.09 \pm 0.04$ & 0.0004 & $6.581 \pm 0.241$ & 0.002 & & 51.457555 & 179.195138 \\
\hline & EUAL-LS-LT2-1 & VEG & $7 / 3 / 2011$ & $0.99 \pm 0.04$ & 0.0004 & $5.988 \pm 0.242$ & 0.002 & & 51.44925 & 179.205583 \\
\hline & EUAL-LS-LT2-2 & VEG & $7 / 3 / 2011$ & $0.406 \pm 0.008$ & 0.0003 & $2.948 \pm 0.058$ & 0.002 & & 51.44925 & 179.205583 \\
\hline & EUAL-LS-LT2-3 & VEG & $7 / 3 / 2011$ & $0.58 \pm 0.02$ & 0.0005 & $4.195 \pm 0.145$ & 0.004 & & 51.44925 & 179.205583 \\
\hline & EUAL-LS-LT3-1 & VEG & $7 / 2 / 2011$ & $1.2 \pm 0.02$ & 0.0004 & $7.659 \pm 0.128$ & 0.003 & & 51.43 & 179.22667 \\
\hline & EUAL-LS-LT3-2 & VEG & $7 / 2 / 2011$ & $1.5 \pm 0.02$ & 0.0005 & $10.674 \pm 0.142$ & 0.004 & & 51.43 & 179.22667 \\
\hline & EUAL-LS-LT3-3 & VEG & $7 / 2 / 2011$ & $0.425 \pm 0.008$ & 0.0004 & $3.585 \pm 0.067$ & 0.003 & & 51.43 & 179.22667 \\
\hline \multirow{10}{*}{ Rockweed } & FUCU-LS-DUP & VEG & $6 / 22 / 2011$ & $2.35 \pm 0.04$ & 0.0003 & $12.914 \pm 0.22$ & 0.002 & & 51.447472 & 179.190888 \\
\hline & FUCU-LS-LT1-1 & VEG & 6/22/2011 & $3.12 \pm 0.04$ & 0.0003 & $16.855 \pm 0.216$ & 0.002 & & 51.447527 & 179.189361 \\
\hline & FUCU-LS-LT1-2 & VEG & \begin{tabular}{|c|}
$6 / 22 / 2011$ \\
\end{tabular} & $2.63 \pm 0.06$ & 0.0004 & $16.088 \pm 0.367$ & 0.002 & & 51.447527 & 179.189361 \\
\hline & FUCU-LS-LT1-3 & VEG & 6/22/2011 & $2.14 \pm 0.04$ & 0.0004 & $14.229 \pm 0.266$ & 0.003 & & 51.448777 & 179.189305 \\
\hline & FUCU-LS-LT2-1 & VEG & 6/22/2011 & $1.22 \pm 0.02$ & 0.0002 & $9.619 \pm 0.158$ & 0.002 & & 51.447222 & 179.199361 \\
\hline & FUCU-LS-LT2-2 & VEG & 6/22/2011 & $1.98 \pm 0.02$ & 0.0002 & $14.352 \pm 0.145$ & 0.001 & & 51.44625 & 179.196972 \\
\hline & FUCU-LS-LT2-3 & VEG & 6/22/2011 & $2.55 \pm 0.04$ & 0.0003 & $15.595 \pm 0.245$ & 0.002 & & 51.446722 & 179.191888 \\
\hline & FUCU-LS-LT3-1 & VEG & $7 / 2 / 2011$ & $3.15 \pm 0.06$ & 0.0004 & $15.258 \pm 0.291$ & 0.002 & & 51.433611 & 179.219305 \\
\hline & FUCU-LS-LT3-2 & VEG & $7 / 2 / 2011$ & $2.37 \pm 0.06$ & 0.0004 & $10.785 \pm 0.273$ & 0.002 & & 51.430166 & 179.218694 \\
\hline & FUCU-LS-LT3-3 & VEG & $7 / 2 / 2011$ & $3.4 \pm 0.08$ & 0.0005 & $12.459 \pm 0.293$ & 0.002 & & 51.430166 & 179.218694 \\
\hline \multirow{10}{*}{ Greenling - whole } & GREN-LS-DUP & FAUNA & $7 / 12 / 2011$ & $0.287 \pm 0.008$ & 0.0004 & $1.394 \pm 0.039$ & 0.002 & & 51.434527 & 179.234416 \\
\hline & GREN-LS-LT1-1 & FAUNA & $7 / 4 / 2011$ & $0.104 \pm 0.004$ & 0.0003 & $0.49 \pm 0.019$ & 0.001 & & 51.457555 & 179.195138 \\
\hline & GREN-LS-LT1-2 & FAUNA & $7 / 4 / 2011$ & $0.075 \pm 0.004$ & 0.0003 & $0.355 \pm 0.019$ & 0.001 & & 51.457555 & 179.195138 \\
\hline & GREN-LS-LT1-3 & FAUNA & $7 / 5 / 2011$ & $0.134 \pm 0.008$ & 0.0005 & $0.628 \pm 0.037$ & 0.002 & & 51.457555 & 179.195138 \\
\hline & GREN-LS-LT2-1 & FAUNA & $7 / 3 / 2011$ & $0.266 \pm 0.006$ & 0.0003 & $1.285 \pm 0.029$ & 0.001 & & 51.432194 & 179.230277 \\
\hline & GREN-LS-LT2-2 & FAUNA & $7 / 3 / 2011$ & $0.142 \pm 0.004$ & 0.0003 & $0.655 \pm 0.018$ & 0.001 & & 51.432194 & 179.230277 \\
\hline & GREN-LS-LT2-3 & FAUNA & $7 / 3 / 2011$ & $0.2 \pm 0.006$ & 0.0003 & $0.952 \pm 0.029$ & 0.001 & & 51.432194 & 179.230277 \\
\hline & GREN-LS-LT3-1 & FAUNA & $7 / 3 / 2011$ & $0.167 \pm 0.004$ & 0.0004 & $0.741 \pm 0.018$ & 0.002 & & 51.43 & 179.22667 \\
\hline & GREN-LS-LT3-2 & FAUNA & 7/12/2011 & $0.25 \pm 0.02$ & 0.0004 & $1.226 \pm 0.098$ & 0.002 & & 51.434527 & 179.234416 \\
\hline & GREN-LS-LT3-3 & FAUNA & $7 / 12 / 2011$ & $0.207 \pm 0.008$ & 0.0004 & $1.001 \pm 0.039$ & 0.002 & & 51.434527 & 179.234416 \\
\hline Halibut - whole & HALI-LS-L2M-1 & FAUNA & 7/13/2011 & $0.011 \pm 0.002$ & 0.0002 & $0.042 \pm 0.008$ & 0.001 & & 51.40025 & 179.283361 \\
\hline
\end{tabular}




\begin{tabular}{|c|c|c|c|c|c|c|c|c|c|c|}
\hline Matrix Subtype & Location Code & Matrix & $\begin{array}{c}\text { Date } \\
\text { Sampled }\end{array}$ & $\begin{array}{l}\text { Wet Result } \pm \\
\text { Uncertainty }\end{array}$ & $\begin{array}{c}\text { Wet } \\
\text { Detection } \\
\text { Limit }\end{array}$ & $\begin{array}{l}\text { Dry Result } \pm \\
\text { Uncertainty }\end{array}$ & $\begin{array}{c}\text { Dry } \\
\text { Detection } \\
\text { Limit }\end{array}$ & Qualifiers & Latitude & Longitude \\
\hline \multirow{3}{*}{$\begin{array}{l}\text { Horse Mussel - } \\
\text { soft tissue only }\end{array}$} & HMUS-LS-LT1-1 & FAUNA & $7 / 5 / 2011$ & $2.86 \pm 0.06$ & 0.0004 & $12.252 \pm 0.257$ & 0.002 & & 51.457555 & 179.195138 \\
\hline & HMUS-LS-LT2-1 & FAUNA & $7 / 4 / 2011$ & $1.41 \pm 0.02$ & 0.0004 & $5.76 \pm 0.082$ & 0.002 & & 51.44925 & 179.205583 \\
\hline & HMUS-LS-LT3-1 & FAUNA & $7 / 3 / 2011$ & $0.53 \pm 0.04$ & 0.0098 & $1.112 \pm 0.084$ & 0.021 & & 51.44925 & 179.205583 \\
\hline \multirow{9}{*}{ || Irish Lord - whole } & IRLO-LS-LT1-1 & FAUNA & $7 / 4 / 2011$ & $0.093 \pm 0.006$ & 0.0004 & $0.439 \pm 0.028$ & 0.002 & & 51.457555 & 179.195138 \\
\hline & IRLO-LS-LT1-2 & FAUNA & $7 / 4 / 2011$ & $0.085 \pm 0.004$ & 0.0004 & $0.402 \pm 0.019$ & 0.002 & & 51.457555 & 179.195138 \\
\hline & IRLO-LS-LT1-3 & FAUNA & $7 / 5 / 2011$ & $0.179 \pm 0.006$ & 0.0004 & $0.949 \pm 0.032$ & 0.002 & & 51.457555 & 179.195138 \\
\hline & IRLO-LS-LT2-1 & FAUNA & $7 / 3 / 2011$ & $0.415 \pm 0.008$ & 0.0004 & $1.796 \pm 0.035$ & 0.002 & & 51.432194 & 179.230277 \\
\hline & IRLO-LS-LT2-2 & FAUNA & $7 / 3 / 2011$ & $0.331 \pm 0.006$ & 0.0004 & $1.371 \pm 0.025$ & 0.002 & & 51.432194 & 179.230277 \\
\hline & IRLO-LS-LT2-3 & FAUNA & $7 / 3 / 2011$ & $0.68 \pm 0.02$ & 0.0007 & $3.012 \pm 0.089$ & 0.003 & & 51.432194 & 179.230277 \\
\hline & IRLO-LS-LT3-1 & FAUNA & $7 / 2 / 2011$ & $0.362 \pm 0.01$ & 0.0004 & $1.615 \pm 0.045$ & 0.002 & & 51.43 & 179.22667 \\
\hline & IRLO-LS-LT3-2 & \begin{tabular}{|l|} 
FAUNA \\
\end{tabular} & $7 / 12 / 2011$ & $0.27 \pm 0.02$ & 0.0003 & $1.281 \pm 0.095$ & 0.001 & & 51.434944 & 179.2315 \\
\hline & IRLO-LS-LT3-3 & FAUNA & $7 / 12 / 2011$ & $0.23 \pm 0.01$ & 0.0003 & $1.271 \pm 0.055$ & 0.002 & & 51.434944 & 179.2315 \\
\hline Octopus & OCTP-LS-L2M-1 & FAUNA & $7 / 3 / 2011$ & $0.77 \pm 0.02$ & 0.0002 & $5.488 \pm 0.143$ & 0.001 & & 51.44925 & 179.205583 \\
\hline $\begin{array}{l}\text { Pacific Cod - } \\
\text { whole }\end{array}$ & PCOD-LS-L2M-1 & FAUNA & $7 / 4 / 2011$ & $0.078 \pm 0.004$ & 0.0003 & $0.388 \pm 0.02$ & 0.001 & & 51.451805 & 179.196972 \\
\hline \multirow{10}{*}{ Rockfish - whole } & ROCK-LS-DUP & FAUNA & $7 / 12 / 2011$ & $0.165 \pm 0.002$ & 0.0004 & $0.643 \pm 0.008$ & 0.002 & & 51.434944 & 179.2315 \\
\hline & ROCK-LS-DUP2 & FAUNA & $7 / 12 / 2011$ & $0.52 \pm 0.004$ & 0.0004 & $2.225 \pm 0.017$ & 0.002 & & 51.465472 & 179.206722 \\
\hline & ROCK-LS-LT1-1 & FAUNA & $7 / 4 / 2011$ & $0.23 \pm 0.02$ & 0.0004 & $1.068 \pm 0.093$ & 0.002 & & 51.457555 & 179.195138 \\
\hline & ROCK-LS-LT1-2 & FAUNA & $7 / 12 / 2011$ & $0.218 \pm 0.006$ & 0.0004 & $0.901 \pm 0.025$ & 0.002 & & 51.465472 & 179.206722 \\
\hline & ROCK-LS-LT1-3 & FAUNA & $7 / 12 / 2011$ & $0.136 \pm 0.004$ & 0.0004 & $0.561 \pm 0.017$ & 0.002 & & 51.465472 & 179.206722 \\
\hline & ROCK-LS-LT2-1 & FAUNA & $7 / 4 / 2011$ & $0.484 \pm 0.008$ & 0.0004 & $1.941 \pm 0.032$ & 0.002 & & 51.44925 & 179.205583 \\
\hline & ROCK-LS-LT2-2 & FAUNA & $7 / 12 / 2011$ & $0.452 \pm 0.01$ & 0.0004 & $1.784 \pm 0.039$ & 0.002 & & 51.44625 & 179.223416 \\
\hline & ROCK-LS-LT3-1 & FAUNA & $7 / 12 / 2011$ & $0.574 \pm 0.004$ & 0.0004 & $2.526 \pm 0.018$ & 0.002 & & 51.434944 & 179.2315 \\
\hline & ROCK-LS-LT3-2 & FAUNA & $7 / 12 / 2011$ & $0.322 \pm 0.006$ & 0.0004 & $1.366 \pm 0.025$ & 0.002 & & 51.434944 & 179.2315 \\
\hline & ROCK-LS-LT3-3 & FAUNA & $7 / 3 / 2011$ & $0.278 \pm 0.008$ & 0.0004 & $0.959 \pm 0.028$ & 0.001 & & 51.43 & 179.22667 \\
\hline Reindeer Lichen & STRL-AI-LT1-2 & VEG & $6 / 22 / 2011$ & $0.115 \pm 0.004$ & 0.0003 & $0.349 \pm 0.012$ & 0.001 & & 51.447361 & 179.189111 \\
\hline \multirow{3}{*}{$\begin{array}{l}\text { Sea Urchin - } \\
\text { whole }\end{array}$} & URCH-LS-LT1-1 & FAUNA & $7 / 4 / 2011$ & $0.77 \pm 0.02$ & 0.0005 & $3.008 \pm 0.078$ & 0.002 & & 51.457555 & 179.195138 \\
\hline & URCH-LS-LT2-1 & FAUNA & 7/3/2011 & $0.67 \pm 0.02$ & 0.0005 & $2.574 \pm 0.077$ & 0.002 & & 51.44925 & 179.205583 \\
\hline & URCH-LS-LT3-1 & FAUNA & $7 / 2 / 2011$ & $0.82 \pm 0.02$ & 0.0005 & $3.194 \pm 0.078$ & 0.002 & & 51.43 & 179.22667 \\
\hline \multicolumn{11}{|c|}{ Amchitka Site (Milrow) (AMC03) } \\
\hline \multirow{3}{*}{ Chiton } & CHIT-ML-MT1-1 & FAUNA & $7 / 9 / 2011$ & $0.63 \pm 0.02$ & 0.0005 & $2.376 \pm 0.075$ & 0.002 & & 51.408472 & 179.139166 \\
\hline & CHIT-ML-MT2-1 & FAUNA & 7/10/2011 & $0.93 \pm 0.04$ & 0.0004 & $4.318 \pm 0.186$ & 0.002 & & 51.389416 & 179.173138 \\
\hline & CHIT-ML-MT3-1 & FAUNA & 7/10/2011 & $0.89 \pm 0.02$ & 0.0004 & $4.039 \pm 0.091$ & 0.002 & & 51.387805 & 179.184 \\
\hline
\end{tabular}




\begin{tabular}{|c|c|c|c|c|c|c|c|c|c|c|}
\hline Matrix Subtype & Location Code & Matrix & $\begin{array}{c}\text { Date } \\
\text { Sampled }\end{array}$ & $\begin{array}{l}\text { Wet Result } \pm \\
\text { Uncertainty }\end{array}$ & $\begin{array}{c}\text { Wet } \\
\text { Detection } \\
\text { Limit }\end{array}$ & $\begin{array}{l}\text { Dry Result } \pm \\
\text { Uncertainty }\end{array}$ & \begin{tabular}{|c|} 
Dry \\
Detection \\
Limit
\end{tabular} & Qualifiers & Latitude & Longitude \\
\hline \multirow{10}{*}{ Dragon Kelp } & EUAL-ML-DUP & VEG & $7 / 9 / 2011$ & $0.64 \pm 0.02$ & 0.0003 & $4.823 \pm 0.151$ & 0.002 & & 51.408472 & 179.139166 \\
\hline & EUAL-ML-MT1-1 & VEG & $7 / 9 / 2011$ & $0.63 \pm 0.02$ & 0.0003 & $5.036 \pm 0.16$ & 0.002 & & 51.408472 & 179.139166 \\
\hline & EUAL-ML-MT1-2 & VEG & $7 / 9 / 2011$ & $0.8 \pm 0.02$ & 0.0004 & $5.953 \pm 0.149$ & 0.003 & & 51.408472 & 179.139166 \\
\hline & EUAL-ML-MT1-3 & VEG & $7 / 9 / 2011$ & $0.494 \pm 0.008$ & 0.0003 & $4.155 \pm 0.067$ & 0.003 & & 51.408472 & 179.139166 \\
\hline & EUAL-ML-MT2-1 & VEG & 7/10/2011 & $1.42 \pm 0.02$ & 0.0007 & $10.583 \pm 0.149$ & 0.005 & & 51.389416 & 179.173138 \\
\hline & EUAL-ML-MT2-2 & VEG & 7/10/2011 & $0.96 \pm 0.04$ & 0.0003 & $6.802 \pm 0.283$ & 0.002 & & 51.389416 & 179.173138 \\
\hline & EUAL-ML-MT2-3 & VEG & 7/10/2011 & $0.77 \pm 0.02$ & 0.0003 & $5.804 \pm 0.151$ & 0.002 & & 51.389416 & 179.173138 \\
\hline & EUAL-ML-MT3-1 & VEG & $7 / 10 / 2011$ & $0.74 \pm 0.02$ & 0.0004 & $5.205 \pm 0.141$ & 0.003 & & 51.387805 & 179.184 \\
\hline & EUAL-ML-MT3-2 & VEG & 7/10/2011 & $0.77 \pm 0.04$ & 0.0004 & $5.626 \pm 0.292$ & 0.003 & & 51.387805 & 179.184 \\
\hline & EUAL-ML-MT3-3 & VEG & \begin{tabular}{|l|}
$7 / 10 / 2011$ \\
\end{tabular} & $1.4 \pm 0.04$ & 0.0004 & $9.655 \pm 0.276$ & 0.003 & & 51.387805 & 179.184 \\
\hline \multirow{10}{*}{ Rockweed } & FUCU-ML-DUP & VEG & \begin{tabular}{|l|}
$6 / 20 / 2011$ \\
\end{tabular} & $3.59 \pm 0.04$ & 0.0004 & $15.577 \pm 0.174$ & 0.002 & & 51.412449 & 179.160843 \\
\hline & FUCU-ML-MT1-1 & VEG & 6/20/2011 & $3.38 \pm 0.02$ & 0.0004 & $14.081 \pm 0.083$ & 0.002 & & 51.412449 & 179.160843 \\
\hline & FUCU-ML-MT1-2 & VEG & 6/21/2011 & $3.14 \pm 0.04$ & 0.0003 & $19.048 \pm 0.243$ & 0.002 & & 51.417083 & 179.134083 \\
\hline & FUCU-ML-MT1-3 & VEG & $7 / 9 / 2011$ & $3.8 \pm 0.2$ & 0.0004 & $17.467 \pm 0.919$ & 0.002 & & 51.417083 & 179.134083 \\
\hline & FUCU-ML-MT2-1 & VEG & \begin{tabular}{|l|}
$6 / 20 / 2011$ \\
\end{tabular} & $3.23 \pm 0.04$ & 0.0004 & $16.67 \pm 0.206$ & 0.002 & & 51.403305 & 179.167166 \\
\hline & FUCU-ML-MT2-2 & VEG & $6 / 21 / 2011$ & $1.94 \pm 0.06$ & 0.0003 & $13.63 \pm 0.422$ & 0.002 & & 51.405694 & 179.161361 \\
\hline & FUCU-ML-MT2-3 & VEG & \begin{tabular}{|l|}
$6 / 21 / 2011$ \\
\end{tabular} & $1.84 \pm 0.02$ & 0.0003 & $12.776 \pm 0.139$ & 0.002 & & 51.405694 & 179.161361 \\
\hline & FUCU-ML-MT3-1 & VEG & \begin{tabular}{|l|}
$7 / 10 / 2011$ \\
\end{tabular} & $4.8 \pm 0.2$ & 0.0004 & $23.31 \pm 0.971$ & 0.002 & & 51.389416 & 179.173138 \\
\hline & FUCU-ML-MT3-2 & VEG & 7/10/2011 & $5.1 \pm 0.2$ & 0.0004 & $24.493 \pm 0.961$ & 0.002 & & 51.389416 & 179.173138 \\
\hline & FUCU-ML-MT3-3 & VEG & 7/10/2011 & $4.58 \pm 0.1$ & 0.0004 & $23.123 \pm 0.505$ & 0.002 & & 51.389416 & 179.173138 \\
\hline \multirow{10}{*}{ Greenling - whole } & GREN-ML-DUP & FAUNA & $7 / 10 / 2011$ & $0.215 \pm 0.006$ & 0.0003 & $1.043 \pm 0.029$ & 0.001 & & 51.387805 & 179.184 \\
\hline & \begin{tabular}{|l} 
GREN-ML-MT1-1 \\
\end{tabular} & FAUNA & \begin{tabular}{|l|}
$7 / 9 / 2011$ \\
\end{tabular} & $0.191 \pm 0.004$ & 0.0004 & $0.832 \pm 0.017$ & 0.002 & & 51.408472 & 179.139166 \\
\hline & GREN-ML-MT1-2 & FAUNA & $7 / 9 / 2011$ & $0.234 \pm 0.006$ & 0.0004 & $1.083 \pm 0.028$ & 0.002 & & 51.408472 & 179.139166 \\
\hline & GREN-ML-MT1-3 & FAUNA & $7 / 9 / 2011$ & $0.385 \pm 0.008$ & 0.0003 & $1.859 \pm 0.039$ & 0.001 & & 51.408472 & 179.139166 \\
\hline & GREN-ML-MT2-1 & FAUNA & 7/11/2011 & $0.118 \pm 0.004$ & 0.0003 & $0.55 \pm 0.019$ & 0.001 & & 51.396944 & 179.1435 \\
\hline & GREN-ML-MT2-2 & FAUNA & $7 / 11 / 2011$ & $0.168 \pm 0.004$ & 0.0003 & $0.786 \pm 0.019$ & 0.001 & & 51.396944 & 179.1435 \\
\hline & GREN-ML-MT2-3 & FAUNA & 7/11/2011 & $0.124 \pm 0.006$ & 0.0003 & $0.592 \pm 0.029$ & 0.001 & & 51.396944 & 179.1435 \\
\hline & GREN-ML-MT3-1 & FAUNA & $7 / 10 / 2011$ & $0.364 \pm 0.012$ & 0.0004 & $1.765 \pm 0.058$ & 0.002 & & 51.387805 & 179.184 \\
\hline & GREN-ML-MT3-2 & FAUNA & 7/10/2011 & $0.111 \pm 0.006$ & 0.0004 & $0.536 \pm 0.029$ & 0.002 & & 51.387805 & 179.184 \\
\hline & GREN-ML-MT3-3 & FAUNA & 7/10/2011 & $0.186 \pm 0.008$ & 0.0003 & $0.881 \pm 0.038$ & 0.001 & & 51.387805 & 179.184 \\
\hline Halibut - whole & HALI-ML-M2M-1 & FAUNA & 7/11/2011 & $0.028 \pm 0.002$ & 0.0004 & $0.097 \pm 0.007$ & 0.001 & & 51.365055 & 179.111472 \\
\hline \multirow{3}{*}{$\begin{array}{l}\text { Horse Mussel - } \\
\text { soft tissue only }\end{array}$} & HMUS-ML-MT1-1 & FAUNA & 7/9/2011 & $2.49 \pm 0.06$ & 0.0004 & $11.323 \pm 0.273$ & 0.002 & & 51.408472 & 179.139166 \\
\hline & HMUS-ML-MT2-1 & FAUNA & $7 / 11 / 2011$ & $1.46 \pm 0.02$ & 0.0004 & $6.566 \pm 0.09$ & 0.002 & & 51.389416 & 179.173138 \\
\hline & HMUS-ML-MT3-1 & FAUNA & 7/10/2011 & $1.63 \pm 0.02$ & 0.0004 & $7.483 \pm 0.092$ & 0.002 & & 51.387805 & 179.184 \\
\hline
\end{tabular}




\begin{tabular}{|c|c|c|c|c|c|c|c|c|c|c|}
\hline Matrix Subtype & Location Code & Matrix & $\begin{array}{c}\text { Date } \\
\text { Sampled }\end{array}$ & $\begin{array}{l}\text { Wet Result } \pm \\
\text { Uncertainty }\end{array}$ & $\begin{array}{c}\text { Wet } \\
\text { Detection } \\
\text { Limit }\end{array}$ & $\begin{array}{l}\text { Dry Result } \pm \\
\text { Uncertainty }\end{array}$ & \begin{tabular}{|c|} 
Dry \\
Detection \\
Limit
\end{tabular} & Qualifiers & Latitude & Longitude \\
\hline \multirow{9}{*}{ Irish Lord - whole } & IRLO-ML-MT1-1 & FAUNA & $7 / 9 / 2011$ & $0.178 \pm 0.008$ & 0.0005 & $0.76 \pm 0.034$ & 0.002 & & 51.408472 & 179.139166 \\
\hline & IRLO-ML-MT1-2 & FAUNA & $7 / 9 / 2011$ & $0.113 \pm 0.002$ & 0.0004 & $0.536 \pm 0.009$ & 0.002 & & 51.408472 & 179.139166 \\
\hline & IRLO-ML-MT1-3 & FAUNA & $7 / 9 / 2011$ & $0.096 \pm 0.004$ & 0.0005 & $0.45 \pm 0.019$ & 0.002 & & 51.408472 & 179.139166 \\
\hline & IRLO-ML-MT2-1 & FAUNA & \begin{tabular}{|l|}
$7 / 10 / 2011$ \\
\end{tabular} & $0.207 \pm 0.008$ & 0.0005 & $0.892 \pm 0.034$ & 0.002 & & 51.389416 & 179.173138 \\
\hline & IRLO-ML-MT2-2 & FAUNA & 7/11/2011 & $0.089 \pm 0.008$ & 0.0006 & $0.383 \pm 0.034$ & 0.003 & & 51.396944 & 179.1435 \\
\hline & IRLO-ML-MT2-3 & FAUNA & \begin{tabular}{|l|}
$7 / 11 / 2011$ \\
\end{tabular} & $0.153 \pm 0.006$ & 0.0004 & $0.726 \pm 0.028$ & 0.002 & & 51.396944 & 179.1435 \\
\hline & IRLO-ML-MT3-1 & FAUNA & \begin{tabular}{|l|}
$7 / 10 / 2011$ \\
\end{tabular} & $0.348 \pm 0.006$ & 0.0003 & $1.962 \pm 0.034$ & 0.002 & & 51.387805 & 179.184 \\
\hline & IRLO-ML-MT3-2 & FAUNA & \begin{tabular}{|l|}
$7 / 10 / 2011$ \\
\end{tabular} & $0.19 \pm 0.004$ & 0.0004 & $0.917 \pm 0.019$ & 0.002 & & 51.387805 & 179.184 \\
\hline & IRLO-ML-MT3-3 & FAUNA & 7/10/2011 & $0.281 \pm 0.006$ & 0.0003 & $1.575 \pm 0.034$ & 0.002 & & 51.387805 & 179.184 \\
\hline Octopus & OCTP-ML-M2M-1 & FAUNA & \begin{tabular}{|l|}
$7 / 10 / 2011$ \\
\end{tabular} & $0.365 \pm 0.004$ & 0.0002 & $1.721 \pm 0.019$ & 0.001 & & 51.389416 & 179.173138 \\
\hline $\begin{array}{l}\text { Pacific Cod - } \\
\text { whole }\end{array}$ & PCOD-ML-M2M-1 & FAUNA & 7/10/2011 & $0.09 \pm 0.004$ & 0.0003 & $0.476 \pm 0.021$ & 0.002 & & 51.408472 & 179.139166 \\
\hline \multirow{10}{*}{ Rockfish - whole } & ROCK-ML-DUP & FAUNA & $7 / 9 / 2011$ & $0.6 \pm 0.02$ & 0.0004 & $2.652 \pm 0.088$ & 0.002 & & 51.408472 & 179.139166 \\
\hline & ROCK-ML-MT1-1 & FAUNA & $7 / 9 / 2011$ & $0.249 \pm 0.016$ & 0.0003 & $1.096 \pm 0.07$ & 0.001 & & 51.408472 & 179.139166 \\
\hline & ROCK-ML-MT1-2 & FAUNA & $7 / 9 / 2011$ & $0.524 \pm 0.016$ & 0.0005 & $2.3 \pm 0.07$ & 0.002 & & 51.408472 & 179.139166 \\
\hline & ROCK-ML-MT1-3 & FAUNA & $7 / 9 / 2011$ & $0.34 \pm 0.006$ & 0.0005 & $1.439 \pm 0.025$ & 0.002 & & 51.408472 & 179.139166 \\
\hline & ROCK-ML-MT2-1 & \begin{tabular}{|l|} 
FAUNA \\
\end{tabular} & 7/11/2011 & $0.269 \pm 0.008$ & 0.0004 & $1.01 \pm 0.03$ & 0.002 & & 51.365055 & 179.111472 \\
\hline & ROCK-ML-MT2-2 & FAUNA & 7/11/2011 & $0.59 \pm 0.02$ & 0.0004 & $2.387 \pm 0.081$ & 0.002 & & 51.365055 & 179.111472 \\
\hline & ROCK-ML-MT2-3 & FAUNA & 7/11/2011 & $0.521 \pm 0.012$ & 0.0003 & $2.347 \pm 0.054$ & 0.001 & & 51.365055 & 179.111472 \\
\hline & ROCK-ML-MT3-1 & FAUNA & \begin{tabular}{|l|}
$7 / 10 / 2011$ \\
\end{tabular} & $0.443 \pm 0.012$ & 0.0004 & $2.091 \pm 0.057$ & 0.002 & & 51.387805 & 179.184 \\
\hline & ROCK-ML-MT3-2 & FAUNA & \begin{tabular}{|l|}
$7 / 10 / 2011$ \\
\end{tabular} & $0.131 \pm 0.008$ & 0.0004 & $0.471 \pm 0.029$ & 0.001 & & 51.387805 & 179.184 \\
\hline & ROCK-ML-MT3-3 & FAUNA & 7/10/2011 & $0.092 \pm 0.006$ & 0.0007 & $0.214 \pm 0.014$ & 0.002 & & 51.387805 & 179.184 \\
\hline Reindeer Lichen & STRL-AI-ML-1 & VEG & $6 / 21 / 2011$ & $0.103 \pm 0.004$ & 0.0005 & $0.369 \pm 0.014$ & 0.002 & & 51.402583 & 179.183972 \\
\hline \multirow{3}{*}{$\begin{array}{l}\text { Sea Urchin - } \\
\text { whole }\end{array}$} & URCH-ML-MT1-1 & FAUNA & $7 / 9 / 2011$ & $0.83 \pm 0.02$ & 0.0005 & $3.079 \pm 0.074$ & 0.002 & & 51.408472 & 179.139166 \\
\hline & URCH-ML-MT2-1 & FAUNA & 7/10/2011 & $0.81 \pm 0.02$ & 0.0005 & $3.025 \pm 0.075$ & 0.002 & & 51.389416 & 179.173138 \\
\hline & URCH-ML-MT3-1 & FAUNA & $7 / 10 / 2011$ & $0.75 \pm 0.02$ & 0.0004 & $2.977 \pm 0.079$ & 0.002 & & 51.387805 & 179.184 \\
\hline \multicolumn{11}{|c|}{ Amchitka Site (Cannikin) (AMC05) } \\
\hline \multirow{3}{*}{ Chiton } & CHIT-CN-CT1-1 & \begin{tabular}{|l|} 
FAUNA \\
\end{tabular} & $7 / 8 / 2011$ & $1.14 \pm 0.02$ & 0.0004 & $5.339 \pm 0.094$ & 0.002 & & 51.482972 & 179.123833 \\
\hline & CHIT-CN-CT2-1 & FAUNA & $7 / 7 / 2011$ & $1.11 \pm 0.04$ & 0.0004 & $5.253 \pm 0.189$ & 0.002 & & 51.472611 & 179.151111 \\
\hline & CHIT-CN-CT3-1 & FAUNA & $7 / 5 / 2011$ & $0.84 \pm 0.02$ & 0.0004 & $4.123 \pm 0.098$ & 0.002 & & 51.471416 & 179.172861 \\
\hline
\end{tabular}




\begin{tabular}{|c|c|c|c|c|c|c|c|c|c|c|}
\hline Matrix Subtype & Location Code & Matrix & $\begin{array}{c}\text { Date } \\
\text { Sampled }\end{array}$ & $\begin{array}{l}\text { Wet Result } \pm \\
\text { Uncertainty }\end{array}$ & $\begin{array}{c}\text { Wet } \\
\text { Detection } \\
\text { Limit }\end{array}$ & $\begin{array}{l}\text { Dry Result } \pm \\
\text { Uncertainty }\end{array}$ & \begin{tabular}{|c|} 
Dry \\
Detection \\
Limit
\end{tabular} & Qualifiers & Latitude & Longitude \\
\hline \multirow{10}{*}{ Dragon Kelp } & EUAL-CN-CT1-1 & VEG & $7 / 8 / 2011$ & $0.82 \pm 0.02$ & 0.0002 & $6.07 \pm 0.148$ & 0.001 & & 51.482972 & 179.123833 \\
\hline & EUAL-CN-CT1-2 & VEG & $7 / 8 / 2011$ & $0.66 \pm 0.02$ & 0.0003 & $4.303 \pm 0.13$ & 0.002 & & 51.482972 & 179.123833 \\
\hline & EUAL-CN-CT1-3 & VEG & $7 / 8 / 2011$ & $0.94 \pm 0.02$ & 0.0003 & $5.758 \pm 0.123$ & 0.002 & & 51.482972 & 179.123833 \\
\hline & EUAL-CN-CT2-1 & VEG & $7 / 6 / 2011$ & $0.85 \pm 0.02$ & 0.0003 & $5.421 \pm 0.128$ & 0.002 & & 51.472611 & 179.151111 \\
\hline & EUAL-CN-CT2-2 & VEG & $7 / 6 / 2011$ & $1.38 \pm 0.06$ & 0.0004 & $8.662 \pm 0.377$ & 0.003 & & 51.472611 & 179.151111 \\
\hline & EUAL-CN-CT2-3 & VEG & 7/7/2011 & $0.6 \pm 0.02$ & 0.0003 & $4.35 \pm 0.145$ & 0.002 & & 51.472611 & 179.151111 \\
\hline & EUAL-CN-CT3-1 & VEG & $7 / 5 / 2011$ & $0.695 \pm 0.008$ & 0.0003 & $4.846 \pm 0.056$ & 0.002 & & 51.471416 & 179.172861 \\
\hline & EUAL-CN-CT3-2 & VEG & $7 / 5 / 2011$ & $0.49 \pm 0.02$ & 0.0005 & $3.523 \pm 0.144$ & 0.004 & & 51.471416 & 179.172861 \\
\hline & EUAL-CN-CT3-3 & VEG & $7 / 5 / 2011$ & $0.84 \pm 0.02$ & 0.0003 & $5.46 \pm 0.13$ & 0.002 & & 51.471416 & 179.172861 \\
\hline & EUAL-CN-DUP & VEG & $7 / 6 / 2011$ & $0.305 \pm 0.008$ & 0.0003 & $2.027 \pm 0.053$ & 0.002 & & 51.471416 & 179.172861 \\
\hline \multirow{10}{*}{ Rockweed } & FUCU-CN-CT1-1 & VEG & \begin{tabular}{|l|}
$6 / 23 / 2011$ \\
\end{tabular} & $1.878 \pm 0.01$ & 0.0003 & $10.83 \pm 0.058$ & 0.002 & & 51.481888 & 179.118138 \\
\hline & FUCU-CN-CT1-2 & VEG & 6/23/2011 & $2.13 \pm 0.04$ & 0.0003 & $13.415 \pm 0.252$ & 0.002 & & 51.481888 & 179.118138 \\
\hline & FUCU-CN-CT1-3 & VEG & 6/23/2011 & $2.44 \pm 0.04$ & 0.0004 & $14.384 \pm 0.236$ & 0.002 & & 51.481888 & 179.118138 \\
\hline & FUCU-CN-CT2-1 & VEG & $7 / 8 / 2011$ & $4.58 \pm 0.08$ & 0.0005 & $18.927 \pm 0.331$ & 0.002 & & 51.476638 & 179.135277 \\
\hline & FUCU-CN-CT2-2 & VEG & $7 / 8 / 2011$ & $4.4 \pm 0.2$ & 0.0004 & $19.524 \pm 0.887$ & 0.002 & & 51.476638 & 179.135277 \\
\hline & FUCU-CN-CT2-3 & VEG & \begin{tabular}{|l|}
$7 / 12 / 2011$ \\
\end{tabular} & $1.87 \pm 0.06$ & 0.0004 & $9.885 \pm 0.317$ & 0.002 & & 51.467583 & 179.153583 \\
\hline & FUCU-CN-CT3-1 & VEG & $7 / 6 / 2011$ & $1.84 \pm 0.04$ & 0.0004 & $9.915 \pm 0.216$ & 0.002 & & 51.465027 & 179.173166 \\
\hline & FUCU-CN-CT3-2 & VEG & $7 / 6 / 2011$ & $1.48 \pm 0.06$ & 0.0004 & $8.687 \pm 0.352$ & 0.002 & & 51.465472 & 179.170583 \\
\hline & FUCU-CN-CT3-3 & VEG & $7 / 6 / 2011$ & $2.73 \pm 0.04$ & 0.0004 & $17.325 \pm 0.254$ & 0.003 & & 51.464638 & 179.159305 \\
\hline & FUCU-CN-DUP & VEG & \begin{tabular}{|l|}
$6 / 23 / 2011$ \\
\end{tabular} & $1.87 \pm 0.02$ & 0.0003 & $12.065 \pm 0.129$ & 0.002 & & 51.481888 & 179.118138 \\
\hline \multirow{10}{*}{ Greenling - whole } & GREN-CN-CT1-1 & FAUNA & \begin{tabular}{|l|}
$7 / 8 / 2011$ \\
\end{tabular} & $0.282 \pm 0.006$ & 0.0003 & $1.359 \pm 0.029$ & 0.001 & & 51.482972 & 179.123833 \\
\hline & GREN-CN-CT1-2 & FAUNA & $7 / 8 / 2011$ & $0.247 \pm 0.008$ & 0.0004 & $1.173 \pm 0.038$ & 0.002 & & 51.482972 & 179.123833 \\
\hline & GREN-CN-CT1-3 & FAUNA & $7 / 8 / 2011$ & $0.23 \pm 0.008$ & 0.0004 & $1.048 \pm 0.036$ & 0.002 & & 51.482972 & 179.123833 \\
\hline & GREN-CN-CT2-1 & FAUNA & $7 / 7 / 2011$ & $0.275 \pm 0.01$ & 0.0003 & $1.34 \pm 0.049$ & 0.001 & & 51.472611 & 179.151111 \\
\hline & GREN-CN-CT2-2 & FAUNA & $7 / 7 / 2011$ & $0.106 \pm 0.006$ & 0.0004 & $0.486 \pm 0.028$ & 0.002 & & 51.472611 & 179.151111 \\
\hline & GREN-CN-CT2-3 & FAUNA & 7/7/2011 & $0.182 \pm 0.006$ & 0.0004 & $0.84 \pm 0.028$ & 0.002 & & 51.472611 & 179.151111 \\
\hline & GREN-CN-CT3-1 & FAUNA & $7 / 5 / 2011$ & $0.206 \pm 0.006$ & 0.0004 & $0.939 \pm 0.027$ & 0.002 & & 51.471416 & 179.172861 \\
\hline & GREN-CN-CT3-2 & FAUNA & $7 / 5 / 2011$ & $0.335 \pm 0.012$ & 0.0004 & $1.459 \pm 0.052$ & 0.002 & & 51.471416 & 179.172861 \\
\hline & GREN-CN-CT3-3 & FAUNA & $7 / 5 / 2011$ & $0.142 \pm 0.004$ & 0.0003 & $0.652 \pm 0.018$ & 0.001 & & 51.471416 & 179.172861 \\
\hline & GREN-CN-DUP & FAUNA & $7 / 8 / 2011$ & $0.188 \pm 0.006$ & 0.0004 & $0.907 \pm 0.029$ & 0.002 & & 51.482972 & 179.123833 \\
\hline Halibut - whole & HALI-CN-C2M-1 & FAUNA & \begin{tabular}{|l|}
$7 / 13 / 2011$ \\
\end{tabular} & $0.0088 \pm 0.0002$ & 0.0002 & $0.034 \pm 0.001$ & 0.001 & & 51.40025 & 179.283361 \\
\hline \multirow{3}{*}{$\begin{array}{l}\text { Horse Mussel - } \\
\text { soft tissue only }\end{array}$} & HMUS-CN-CT1-1 & FAUNA & $7 / 8 / 2011$ & $0.85 \pm 0.02$ & 0.0004 & $3.494 \pm 0.082$ & 0.002 & & 51.482972 & 179.123833 \\
\hline & HMUS-CN-CT2-1 & FAUNA & $7 / 7 / 2011$ & $2.29 \pm 0.02$ & 0.0004 & $10.377 \pm 0.091$ & 0.002 & & 51.472611 & 179.151111 \\
\hline & HMUS-CN-CT3-1 & \begin{tabular}{|l|} 
FAUNA \\
\end{tabular} & $7 / 5 / 2011$ & $1.78 \pm 0.04$ & 0.0004 & $7.302 \pm 0.164$ & 0.002 & & 51.471416 & 179.172861 \\
\hline
\end{tabular}




\begin{tabular}{|c|c|c|c|c|c|c|c|c|c|c|}
\hline Matrix Subtype & Location Code & Matrix & $\begin{array}{c}\text { Date } \\
\text { Sampled }\end{array}$ & $\begin{array}{l}\text { Wet Result } \pm \\
\text { Uncertainty }\end{array}$ & $\begin{array}{c}\text { Wet } \\
\text { Detection } \\
\text { Limit }\end{array}$ & $\begin{array}{l}\text { Dry Result } \pm \\
\text { Uncertainty }\end{array}$ & \begin{tabular}{|c|} 
Dry \\
Detection \\
Limit
\end{tabular} & Qualifiers & Latitude & Longitude \\
\hline \multirow{9}{*}{ Irish Lord - whole } & IRLO-CN-CT1-1 & FAUNA & 7/8/2011 & $0.172 \pm 0.004$ & 0.0004 & $0.775 \pm 0.018$ & 0.002 & & 51.482972 & 179.123833 \\
\hline & IRLO-CN-CT1-2 & FAUNA & 7/8/2011 & $0.43 \pm 0.02$ & 0.0005 & $2.155 \pm 0.1$ & 0.003 & & 51.482972 & 179.123833 \\
\hline & IRLO-CN-CT1-3 & FAUNA & 7/8/2011 & $0.339 \pm 0.006$ & 0.0005 & $1.592 \pm 0.028$ & 0.002 & & 51.482972 & 179.123833 \\
\hline & IRLO-CN-CT2-1 & FAUNA & 7/7/2011 & $0.27 \pm 0.006$ & 0.0004 & $1.272 \pm 0.028$ & 0.002 & & 51.472611 & 179.151111 \\
\hline & IRLO-CN-CT2-2 & FAUNA & 7/7/2011 & $0.377 \pm 0.01$ & 0.0005 & $1.722 \pm 0.046$ & 0.002 & & 51.472611 & 179.151111 \\
\hline & IRLO-CN-CT2-3 & FAUNA & 7/7/2011 & $0.178 \pm 0.006$ & 0.0004 & $0.876 \pm 0.03$ & 0.002 & & 51.472611 & 179.151111 \\
\hline & IRLO-CN-CT3-1 & FAUNA & $7 / 5 / 2011$ & $0.24 \pm 0.01$ & 0.0003 & $1.237 \pm 0.052$ & 0.002 & & 51.471416 & 179.172861 \\
\hline & IRLO-CN-CT3-2 & FAUNA & $7 / 5 / 2011$ & $0.167 \pm 0.006$ & 0.0003 & $0.81 \pm 0.029$ & 0.001 & & 51.471416 & 179.172861 \\
\hline & IRLO-CN-CT3-3 & FAUNA & $7 / 5 / 2011$ & $0.113 \pm 0.004$ & 0.0003 & $0.542 \pm 0.019$ & 0.001 & & 51.471416 & 179.172861 \\
\hline Octopus & OCTP-CN-C2M-1 & FAUNA & $7 / 8 / 2011$ & $0.389 \pm 0.006$ & 0.0003 & $1.906 \pm 0.029$ & 0.001 & & 51.482972 & 179.123833 \\
\hline $\begin{array}{l}\text { Pacific Cod - } \\
\text { whole }\end{array}$ & PCOD-CN-C2M-1 & FAUNA & 7/6/2011 & $0.033 \pm 0.002$ & 0.0003 & $0.151 \pm 0.009$ & 0.001 & & 51.483527 & 179.187583 \\
\hline \multirow{10}{*}{ Rockfish - whole } & ROCK-CN-CT1-1 & FAUNA & $7 / 8 / 2011$ & $0.084 \pm 0.006$ & 0.0004 & $0.332 \pm 0.024$ & 0.002 & & 51.482972 & 179.123833 \\
\hline & ROCK-CN-CT1-2 & FAUNA & 7/8/2011 & $0.143 \pm 0.006$ & 0.0004 & $0.552 \pm 0.023$ & 0.002 & & 51.482972 & 179.123833 \\
\hline & ROCK-CN-CT1-3 & FAUNA & 7/8/2011 & $0.35 \pm 0.004$ & 0.0004 & $1.406 \pm 0.016$ & 0.002 & & 51.482972 & 179.123833 \\
\hline & ROCK-CN-CT2-1 & FAUNA & $7 / 6 / 2011$ & $0.136 \pm 0.008$ & 0.0003 & $0.48 \pm 0.028$ & 0.001 & & 51.472611 & 179.151111 \\
\hline & ROCK-CN-CT2-2 & FAUNA & $7 / 6 / 2011$ & $0.23 \pm 0.01$ & 0.0004 & $0.842 \pm 0.037$ & 0.001 & & 51.472611 & 179.151111 \\
\hline & ROCK-CN-CT2-3 & FAUNA & $7 / 6 / 2011$ & $0.239 \pm 0.008$ & 0.0004 & $0.978 \pm 0.033$ & 0.002 & & 51.472611 & 179.151111 \\
\hline & ROCK-CN-CT3-1 & FAUNA & 7/6/2011 & $0.236 \pm 0.006$ & 0.0004 & $1.008 \pm 0.026$ & 0.002 & & 51.471416 & 179.172861 \\
\hline & ROCK-CN-CT3-2 & FAUNA & 7/6/2011 & $0.232 \pm 0.006$ & 0.0004 & $0.891 \pm 0.023$ & 0.002 & & 51.471416 & 179.172861 \\
\hline & ROCK-CN-CT3-3 & FAUNA & $7 / 6 / 2011$ & $0.337 \pm 0.008$ & 0.0004 & $1.408 \pm 0.033$ & 0.002 & & 51.471416 & 179.172861 \\
\hline & ROCK-CN-DUP & FAUNA & $7 / 6 / 2011$ & $0.193 \pm 0.004$ & 0.0004 & $0.775 \pm 0.016$ & 0.002 & & 51.471416 & 179.172861 \\
\hline Reindeer Lichen & STRL-AI-CT1-1 & VEG & 6/23/2011 & $0.42 \pm 0.02$ & 0.0007 & $0.666 \pm 0.032$ & 0.001 & & 51.480583 & 179.109472 \\
\hline \multirow{3}{*}{$\begin{array}{l}\text { Sea Urchin - } \\
\text { whole }\end{array}$} & URCH-CN-CT1-1 & FAUNA & $7 / 8 / 2011$ & $0.7 \pm 0.02$ & 0.0004 & $2.809 \pm 0.08$ & 0.002 & & 51.482972 & 179.123833 \\
\hline & URCH-CN-CT2-1 & FAUNA & 7/7/2011 & $0.6 \pm 0.02$ & 0.0005 & $2.056 \pm 0.069$ & 0.002 & & 51.472611 & 179.151111 \\
\hline & URCH-CN-CT3-1 & FAUNA & $7 / 5 / 2011$ & $0.573 \pm 0.01$ & 0.0005 & $2.195 \pm 0.038$ & 0.002 & & 51.471416 & 179.172861 \\
\hline \multicolumn{11}{|c|}{ Amchitka Site (Adak Island) (AMC13) } \\
\hline $\begin{array}{l}\text { Dolly Varden - } \\
\text { whole }\end{array}$ & DOLL-AD-XXX-1 & FAUNA & 6/28/2011 & $0.0158 \pm 0.0004$ & 0.0002 & $0.064 \pm 0.002$ & 0.001 & & 51.8375 & -176.672555 \\
\hline \multirow{3}{*}{ Reindeer Lichen } & STRL-AD-XXX-1 & VEG & $6 / 28 / 2011$ & $0.151 \pm 0.008$ & 0.0004 & $0.296 \pm 0.016$ & 0.001 & & 51.837527 & -176.670694 \\
\hline & STRL-AD-XXX-2 & VEG & 6/29/2011 & $0.141 \pm 0.006$ & 0.0004 & $0.348 \pm 0.015$ & 0.001 & & 51.872833 & -176.707444 \\
\hline & STRL-AD-XXX-3 & VEG & 6/29/2011 & $0.232 \pm 0.008$ & 0.0005 & $0.369 \pm 0.013$ & 0.001 & & 51.900805 & -176.688527 \\
\hline \multicolumn{11}{|c|}{ Amchitka Site (Adak Island North) (AMC14) } \\
\hline \multirow{3}{*}{ Chiton } & CHIT-AN-ANT1-1 & FAUNA & \begin{tabular}{|l|}
$7 / 20 / 2011$ \\
\end{tabular} & $0.46 \pm 0.02$ & 0.0004 & $2.24 \pm 0.097$ & 0.002 & & 51.838722 & -176.582805 \\
\hline & CHIT-AN-ANT2-1 & FAUNA & \begin{tabular}{|l|}
$7 / 18 / 2011$ \\
\end{tabular} & $0.32 \pm 0.02$ & 0.0003 & $1.604 \pm 0.1$ & 0.002 & & 51.848361 & -176.506277 \\
\hline & CHIT-AN-ANT3-1 & FAUNA & \begin{tabular}{|l|}
$7 / 17 / 2011$ \\
\end{tabular} & $0.28 \pm 0.02$ & 0.0003 & $1.424 \pm 0.102$ & 0.002 & & 51.853722 & -176.470694 \\
\hline
\end{tabular}




\begin{tabular}{|c|c|c|c|c|c|c|c|c|c|c|}
\hline Matrix Subtype & Location Code & Matrix & $\begin{array}{c}\text { Date } \\
\text { Sampled }\end{array}$ & $\begin{array}{l}\text { Wet Result } \pm \\
\text { Uncertainty }\end{array}$ & $\begin{array}{c}\text { Wet } \\
\text { Detection } \\
\text { Limit }\end{array}$ & $\begin{array}{l}\text { Dry Result } \pm \\
\text { Uncertainty }\end{array}$ & $\begin{array}{c}\text { Dry } \\
\text { Detection } \\
\text { Limit }\end{array}$ & Qualifiers & Latitude & Longitude \\
\hline \multirow{10}{*}{ Dragon Kelp } & EUAL-AN-ANT1-1 & VEG & 7/19/2011 & $0.55 \pm 0.02$ & 0.0004 & $4.612 \pm 0.168$ & 0.003 & & 51.835694 & -176.542138 \\
\hline & EUAL-AN-ANT1-2 & VEG & 7/19/2011 & $0.6 \pm 0.02$ & 0.0004 & $4.09 \pm 0.136$ & 0.003 & & 51.835694 & -176.542138 \\
\hline & EUAL-AN-ANT1-3 & VEG & 7/19/2011 & $0.51 \pm 0.02$ & 0.0004 & $3.942 \pm 0.155$ & 0.003 & & 51.835694 & -176.542138 \\
\hline & EUAL-AN-ANT2-1 & VEG & $7 / 18 / 2011$ & $0.94 \pm 0.02$ & 0.0004 & $6.852 \pm 0.146$ & 0.003 & & 51.848361 & -176.506277 \\
\hline & EUAL-AN-ANT2-2 & VEG & 7/18/2011 & $1.17 \pm 0.04$ & 0.0004 & $7.456 \pm 0.255$ & 0.003 & & 51.848361 & -176.506277 \\
\hline & EUAL-AN-ANT2-3 & VEG & 7/18/2011 & $0.71 \pm 0.02$ & 0.0005 & $4.932 \pm 0.139$ & 0.003 & & 51.848361 & -176.506277 \\
\hline & EUAL-AN-ANT3-1 & VEG & 7/17/2011 & $0.268 \pm 0.008$ & 0.0002 & $2.003 \pm 0.06$ & 0.001 & & 51.853722 & -176.470694 \\
\hline & EUAL-AN-ANT3-2 & VEG & 7/17/2011 & $1 \pm 0.02$ & 0.0003 & $6.819 \pm 0.136$ & 0.002 & & 51.853722 & -176.470694 \\
\hline & EUAL-AN-ANT3-3 & VEG & 7/17/2011 & $0.61 \pm 0.02$ & 0.0003 & $4.093 \pm 0.134$ & 0.002 & & 51.853722 & -176.470694 \\
\hline & EUAL-AN-DUP & VEG & 7/17/2011 & $1.62 \pm 0.04$ & 0.0004 & $10.186 \pm 0.252$ & 0.003 & & 51.853722 & -176.470694 \\
\hline \multirow{10}{*}{ Rockweed } & FUCU-AN-ANT1-1 & VEG & 7/19/2011 & $2.91 \pm 0.04$ & 0.0003 & $14.826 \pm 0.204$ & 0.002 & & 51.841444 & -176.546083 \\
\hline & FUCU-AN-ANT1-2 & VEG & 7/20/2011 & $1.7 \pm 0.02$ & 0.0004 & $10.967 \pm 0.129$ & 0.003 & & 51.8395 & -176.591305 \\
\hline & FUCU-AN-ANT1-3 & VEG & $7 / 20 / 2011$ & $2.7 \pm 0.04$ & 0.0005 & $14.735 \pm 0.218$ & 0.003 & & 51.8395 & -176.591305 \\
\hline & FUCU-AN-ANT2-1 & VEG & $7 / 18 / 2011$ & $2.75 \pm 0.08$ & 0.0004 & $17.367 \pm 0.505$ & 0.003 & & 51.837666 & -176.517527 \\
\hline & FUCU-AN-ANT2-2 & VEG & 7/18/2011 & $1.58 \pm 0.08$ & 0.0004 & $8.399 \pm 0.425$ & 0.002 & & 51.841444 & -176.546083 \\
\hline & FUCU-AN-ANT2-3 & VEG & \begin{tabular}{|l|}
$7 / 18 / 2011$ \\
\end{tabular} & $4.01 \pm 0.08$ & 0.0005 & $16.916 \pm 0.337$ & 0.002 & & 51.8395 & -176.591305 \\
\hline & FUCU-AN-ANT3-1 & VEG & 7/18/2011 & $2.16 \pm 0.04$ & 0.0004 & $12.358 \pm 0.229$ & 0.002 & & 51.839944 & -176.515916 \\
\hline & FUCU-AN-ANT3-2 & VEG & 7/18/2011 & $3.17 \pm 0.06$ & 0.0004 & $15.496 \pm 0.293$ & 0.002 & & 51.828972 & -176.448305 \\
\hline & FUCU-AN-ANT3-3 & VEG & 7/18/2011 & $2.98 \pm 0.02$ & 0.0003 & $14.816 \pm 0.099$ & 0.001 & & 51.830833 & -176.456361 \\
\hline & FUCU-AN-DUP & VEG & 7/18/2011 & $5.1 \pm 0.2$ & 0.0005 & $23.732 \pm 0.931$ & 0.002 & & 51.828972 & -176.448305 \\
\hline \multirow{10}{*}{ Greenling - whole } & GREN-AN-ANT1-1 & FAUNA & \begin{tabular}{|l|}
$7 / 19 / 2011$ \\
\end{tabular} & $0.135 \pm 0.004$ & 0.0004 & $0.599 \pm 0.018$ & 0.002 & & 51.835694 & \begin{tabular}{|l|}
-176.542138 \\
\end{tabular} \\
\hline & GREN-AN-ANT1-2 & FAUNA & 7/19/2011 & $0.066 \pm 0.004$ & 0.0004 & $0.276 \pm 0.017$ & 0.002 & & 51.835694 & -176.542138 \\
\hline & GREN-AN-ANT1-3 & FAUNA & 7/19/2011 & $0.276 \pm 0.01$ & 0.0004 & $1.005 \pm 0.036$ & 0.001 & & 51.835694 & -176.542138 \\
\hline & GREN-AN-ANT2-1 & FAUNA & 7/18/2011 & $0.31 \pm 0.02$ & 0.0005 & $1.434 \pm 0.093$ & 0.002 & & 51.848361 & -176.506277 \\
\hline & GREN-AN-ANT2-2 & FAUNA & $7 / 18 / 2011$ & $0.173 \pm 0.004$ & 0.0003 & $0.802 \pm 0.019$ & 0.001 & & 51.848361 & -176.506277 \\
\hline & GREN-AN-ANT2-3 & FAUNA & 7/18/2011 & $0.148 \pm 0.004$ & 0.0004 & $0.702 \pm 0.019$ & 0.002 & & 51.848361 & -176.506277 \\
\hline & GREN-AN-ANT3-1 & FAUNA & 7/17/2011 & $0.151 \pm 0.006$ & 0.0004 & $0.689 \pm 0.027$ & 0.002 & & 51.853722 & -176.470694 \\
\hline & GREN-AN-ANT3-2 & FAUNA & \begin{tabular}{|l|}
$7 / 17 / 2011$ \\
\end{tabular} & $0.061 \pm 0.002$ & 0.0003 & $0.283 \pm 0.009$ & 0.001 & & 51.853722 & \begin{tabular}{|l|}
-176.470694 \\
\end{tabular} \\
\hline & GREN-AN-ANT3-3 & FAUNA & 7/17/2011 & $0.139 \pm 0.006$ & 0.0003 & $0.647 \pm 0.028$ & 0.001 & & 51.853722 & -176.470694 \\
\hline & GREN-AN-DUP & FAUNA & 7/17/2011 & $0.122 \pm 0.004$ & 0.0004 & $0.581 \pm 0.019$ & 0.002 & & 51.853722 & -176.470694 \\
\hline \multirow{2}{*}{ Halibut - whole } & HALI-AN-AN2M-1 & FAUNA & 7/17/2011 & $0.037 \pm 0.002$ & 0.0004 & $0.124 \pm 0.007$ & 0.001 & & 51.853722 & \begin{tabular}{|l|}
-176.470694 \\
\end{tabular} \\
\hline & HALI-AN-DUP & FAUNA & \begin{tabular}{|l|l|l|l|}
$7 / 2011$ \\
\end{tabular} & $0.035 \pm 0.004$ & 0.0005 & $0.116 \pm 0.013$ & 0.002 & & 51.853722 & \begin{tabular}{|l|}
-176.470694 \\
\end{tabular} \\
\hline \multirow{3}{*}{$\begin{array}{l}\text { Horse Mussel - } \\
\text { soft tissue only }\end{array}$} & HMUS-AN-ANT1-1 & FAUNA & $7 / 20 / 2011$ & $0.813 \pm 0.008$ & 0.0003 & $4.445 \pm 0.044$ & 0.002 & & 51.838722 & -176.582805 \\
\hline & HMUS-AN-ANT2-1 & FAUNA & \begin{tabular}{|l|l|}
$7 / 18 / 2011$ \\
\end{tabular} & $1.75 \pm 0.06$ & 0.0004 & $8.036 \pm 0.276$ & 0.002 & & 51.848361 & -176.506277 \\
\hline & HMUS-AN-ANT3-1 & FAUNA & 7/18/2011 & $0.292 \pm 0.006$ & 0.0002 & $1.301 \pm 0.027$ & 0.001 & & 51.853722 & -176.470694 \\
\hline
\end{tabular}




\begin{tabular}{|c|c|c|c|c|c|c|c|c|c|c|}
\hline Matrix Subtype & Location Code & Matrix & $\begin{array}{c}\text { Date } \\
\text { Sampled }\end{array}$ & $\begin{array}{l}\text { Wet Result } \pm \\
\text { Uncertainty }\end{array}$ & $\begin{array}{c}\text { Wet } \\
\text { Detection } \\
\text { Limit }\end{array}$ & $\begin{array}{l}\text { Dry Result } \pm \\
\text { Uncertainty }\end{array}$ & $\begin{array}{c}\text { Dry } \\
\text { Detection } \\
\text { Limit }\end{array}$ & Qualifiers & Latitude & Longitude \\
\hline & IRLO-AN-ANT1-1 & FAUNA & 7/19/2011 & $0.161 \pm 0.002$ & 0.0004 & $0.756 \pm 0.009$ & 0.002 & & 51.835694 & -176.542138 \\
\hline & IRLO-AN-ANT1-2 & FAUNA & $7 / 19 / 2011$ & $0.097 \pm 0.006$ & 0.0004 & $0.431 \pm 0.027$ & 0.002 & & 51.835694 & -176.542138 \\
\hline & IRLO-AN-ANT1-3 & FAUNA & $7 / 19 / 2011$ & $0.136 \pm 0.006$ & 0.0004 & $0.598 \pm 0.026$ & 0.002 & & 51.835694 & -176.542138 \\
\hline & IRLO-AN-ANT2-1 & FAUNA & $7 / 19 / 2011$ & $0.123 \pm 0.004$ & 0.0004 & $0.583 \pm 0.019$ & 0.002 & & 51.848361 & -176.506277 \\
\hline & IRLO-AN-ANT2-2 & FAUNA & $7 / 19 / 2011$ & $0.144 \pm 0.004$ & 0.0004 & $0.667 \pm 0.019$ & 0.002 & & 51.848361 & -176.506277 \\
\hline & IRLO-AN-ANT2-3 & FAUNA & $7 / 19 / 2011$ & $0.43 \pm 0.02$ & 0.0004 & $2.05 \pm 0.095$ & 0.002 & & 51.848361 & -176.506277 \\
\hline & IRLO-AN-ANT3-1 & FAUNA & $7 / 17 / 2011$ & $0.256 \pm 0.008$ & 0.0005 & $1.228 \pm 0.038$ & 0.002 & & 51.853722 & -176.470694 \\
\hline & IRLO-AN-ANT3-2 & FAUNA & $7 / 17 / 2011$ & $0.062 \pm 0.004$ & 0.0005 & $0.28 \pm 0.018$ & 0.002 & & 51.853722 & -176.470694 \\
\hline & IRLO-AN-ANT3-3 & FAUNA & $7 / 17 / 2011$ & $0.113 \pm 0.004$ & 0.0004 & $0.508 \pm 0.018$ & 0.002 & & 51.853722 & -176.470694 \\
\hline Irish Lord - whole & OCTP-AN-AN2M-1 & FAUNA & $7 / 18 / 2011$ & $0.45 \pm 0.02$ & 0.0004 & $2.277 \pm 0.101$ & 0.002 & & 51.848361 & -176.506277 \\
\hline $\begin{array}{l}\text { Pacific Cod - } \\
\text { whole }\end{array}$ & PCOD-AN-AN2M-1 & FAUNA & 7/17/2011 & $0.05 \pm 0.002$ & 0.0002 & $0.243 \pm 0.01$ & 0.001 & & 51.853722 & -176.470694 \\
\hline \multirow{6}{*}{ Rockfish - whole } & ROCK-AN-ANT1-1 & FAUNA & $7 / 19 / 2011$ & $0.48 \pm 0.02$ & 0.0004 & $1.776 \pm 0.074$ & 0.001 & & 51.835694 & -176.542138 \\
\hline & ROCK-AN-ANT2-1 & FAUNA & $7 / 18 / 2011$ & $0.05 \pm 0.004$ & 0.0002 & $0.183 \pm 0.015$ & 0.001 & & 51.848361 & -176.506277 \\
\hline & ROCK-AN-ANT2-2 & FAUNA & $7 / 18 / 2011$ & $0.046 \pm 0.002$ & 0.0003 & $0.188 \pm 0.008$ & 0.001 & & 51.848361 & -176.506277 \\
\hline & ROCK-AN-ANT3-1 & FAUNA & $7 / 17 / 2011$ & $0.107 \pm 0.004$ & 0.0002 & $0.381 \pm 0.014$ & 0.001 & & 51.853722 & -176.470694 \\
\hline & ROCK-AN-ANT3-2 & FAUNA & $7 / 17 / 2011$ & $0.041 \pm 0.004$ & 0.0004 & $0.158 \pm 0.015$ & 0.002 & & 51.853722 & -176.470694 \\
\hline & ROCK-AN-ANT3-3 & FAUNA & $7 / 17 / 2011$ & $0.083 \pm 0.004$ & 0.0004 & $0.326 \pm 0.016$ & 0.002 & & 51.853722 & -176.470694 \\
\hline \multirow{3}{*}{$\begin{array}{l}\text { Sea Urchin - } \\
\text { whole }\end{array}$} & URCH-AN-ANT1-1 & FAUNA & $7 / 19 / 2011$ & $0.2 \pm 0.02$ & 0.0005 & $0.682 \pm 0.068$ & 0.002 & & 51.835694 & -176.542138 \\
\hline & URCH-AN-ANT2-1 & FAUNA & $7 / 18 / 2011$ & $0.56 \pm 0.02$ & 0.0005 & $2.04 \pm 0.073$ & 0.002 & & 51.848361 & -176.506277 \\
\hline & URCH-AN-ANT3-1 & FAUNA & $7 / 17 / 2011$ & $0.61 \pm 0.02$ & 0.0005 & $2.319 \pm 0.076$ & 0.002 & & 51.853722 & -176.470694 \\
\hline \multicolumn{11}{|c|}{ Amchitka Site (Adak Island South) (AMC15) } \\
\hline \multirow{3}{*}{ Chiton } & CHIT-AS-AST1-1 & FAUNA & $7 / 15 / 2011$ & $0.94 \pm 0.02$ & 0.0004 & $3.863 \pm 0.082$ & 0.002 & & 51.737472 & -176.48025 \\
\hline & CHIT-AS-AST2-1 & FAUNA & $7 / 16 / 2011$ & $0.153 \pm 0.004$ & 0.0004 & $0.783 \pm 0.02$ & 0.002 & & 51.745666 & -176.507055 \\
\hline & CHIT-AS-AST3-1 & FAUNA & $7 / 14 / 2011$ & $0.99 \pm 0.04$ & 0.0004 & $4.664 \pm 0.188$ & 0.002 & & 51.688333 & -176.63375 \\
\hline \multirow{10}{*}{ Dragon Kelp } & EUAL-AS-AST1-1 & VEG & 7/15/2011 & $0.9 \pm 0.02$ & 0.0003 & $5.931 \pm 0.132$ & 0.002 & & 51.737472 & -176.48025 \\
\hline & EUAL-AS-AST1-2 & VEG & $7 / 15 / 2011$ & $0.638 \pm 0.01$ & 0.0004 & $5.081 \pm 0.08$ & 0.003 & & 51.737472 & -176.48025 \\
\hline & EUAL-AS-AST1-3 & VEG & $7 / 15 / 2011$ & $0.32 \pm 0.02$ & 0.0004 & $3.071 \pm 0.192$ & 0.004 & & 51.737472 & -176.48025 \\
\hline & EUAL-AS-AST2-1 & VEG & $7 / 16 / 2011$ & $0.56 \pm 0.02$ & 0.0005 & $3.981 \pm 0.142$ & 0.004 & & 51.745666 & -176.507055 \\
\hline & EUAL-AS-AST2-2 & VEG & $7 / 16 / 2011$ & $0.721 \pm 0.006$ & 0.0003 & $5.102 \pm 0.042$ & 0.002 & & 51.745666 & -176.507055 \\
\hline & EUAL-AS-AST2-3 & VEG & 7/16/2011 & $1.08 \pm 0.04$ & 0.0005 & $7.846 \pm 0.291$ & 0.004 & & 51.745666 & -176.507055 \\
\hline & EUAL-AS-AST3-1 & VEG & 7/14/2011 & $0.88 \pm 0.02$ & 0.0003 & $6.428 \pm 0.146$ & 0.002 & & 51.688333 & -176.63375 \\
\hline & EUAL-AS-AST3-2 & VEG & $7 / 14 / 2011$ & $0.33 \pm 0.02$ & 0.0004 & $3.128 \pm 0.19$ & 0.004 & & 51.688333 & -176.63375 \\
\hline & EUAL-AS-AST3-3 & VEG & $7 / 14 / 2011$ & $0.46 \pm 0.02$ & 0.0003 & $4.056 \pm 0.176$ & 0.003 & & 51.688333 & -176.63375 \\
\hline & EUAL-AS-DUP & VEG & $7 / 14 / 2011$ & $0.05 \pm 0.002$ & 0.00005 & $0.368 \pm 0.015$ & 0 & & 51.688333 & -176.63375 \\
\hline
\end{tabular}




\begin{tabular}{|c|c|c|c|c|c|c|c|c|c|c|}
\hline Matrix Subtype & Location Code & Matrix & $\begin{array}{c}\text { Date } \\
\text { Sampled }\end{array}$ & $\begin{array}{l}\text { Wet Result } \pm \\
\text { Uncertainty }\end{array}$ & $\begin{array}{c}\text { Wet } \\
\text { Detection } \\
\text { Limit }\end{array}$ & $\begin{array}{l}\text { Dry Result } \pm \\
\text { Uncertainty }\end{array}$ & \begin{tabular}{|c|} 
Dry \\
Detection \\
Limit
\end{tabular} & Qualifiers & Latitude & Longitude \\
\hline \multirow{10}{*}{ Rockweed } & FUCU-AS-AST1-1 & VEG & \begin{tabular}{|l|l|}
$7 / 16 / 2011$ \\
\end{tabular} & $2.24 \pm 0.08$ & 0.0004 & $13.818 \pm 0.493$ & 0.002 & & 51.733666 & -176.470805 \\
\hline & FUCU-AS-AST1-2 & VEG & \begin{tabular}{|l|}
$7 / 16 / 2011$ \\
\end{tabular} & $2.03 \pm 0.06$ & 0.0004 & $11.26 \pm 0.333$ & 0.002 & & 51.733666 & -176.470805 \\
\hline & FUCU-AS-AST1-3 & VEG & \begin{tabular}{|l|}
$7 / 16 / 2011$ \\
\end{tabular} & $3.04 \pm 0.08$ & 0.0004 & $16.5 \pm 0.434$ & 0.002 & & 51.733666 & -176.470805 \\
\hline & FUCU-AS-AST2-1 & VEG & $7 / 16 / 2011$ & $2.59 \pm 0.06$ & 0.0004 & $12.519 \pm 0.29$ & 0.002 & & 51.745666 & -176.507055 \\
\hline & FUCU-AS-AST2-2 & VEG & \begin{tabular}{|l|}
$7 / 16 / 2011$ \\
\end{tabular} & $3.25 \pm 0.06$ & 0.0004 & $16.451 \pm 0.304$ & 0.002 & & 51.745666 & -176.507055 \\
\hline & FUCU-AS-AST2-3 & VEG & \begin{tabular}{|l|l|}
$7 / 16 / 2011$ \\
\end{tabular} & $3.01 \pm 0.06$ & 0.0005 & $14.787 \pm 0.295$ & 0.002 & & 51.745666 & -176.507055 \\
\hline & FUCU-AS-AST3-1 & VEG & $7 / 14 / 2011$ & $2.53 \pm 0.06$ & 0.0004 & $13.337 \pm 0.316$ & 0.002 & & 51.688333 & -176.63375 \\
\hline & FUCU-AS-AST3-2 & VEG & \begin{tabular}{|l|}
$7 / 14 / 2011$ \\
\end{tabular} & $1.93 \pm 0.08$ & 0.0004 & $9.504 \pm 0.394$ & 0.002 & & 51.688333 & -176.63375 \\
\hline & FUCU-AS-AST3-3 & VEG & \begin{tabular}{|l|}
$7 / 14 / 2011$ \\
\end{tabular} & $1.65 \pm 0.04$ & 0.0003 & $8.665 \pm 0.21$ & 0.002 & & 51.688333 & -176.63375 \\
\hline & FUCU-AS-DUP & VEG & \begin{tabular}{|l|}
$7 / 16 / 2011$ \\
\end{tabular} & $4.01 \pm 0.04$ & 0.0003 & $20.725 \pm 0.207$ & 0.002 & & 51.733666 & -176.470805 \\
\hline \multirow{10}{*}{ Greenling - whole } & GREN-AS-AST1-1 & FAUNA & 7/15/2011 & $0.291 \pm 0.01$ & 0.0004 & $1.386 \pm 0.048$ & 0.002 & & 51.737472 & -176.48025 \\
\hline & GREN-AS-AST1-2 & FAUNA & $7 / 15 / 2011$ & $0.123 \pm 0.004$ & 0.0003 & $0.58 \pm 0.019$ & 0.001 & & 51.737472 & -176.48025 \\
\hline & GREN-AS-AST1-3 & FAUNA & \begin{tabular}{|l|}
$7 / 15 / 2011$ \\
\end{tabular} & $0.149 \pm 0.004$ & 0.0003 & $0.716 \pm 0.019$ & 0.001 & & 51.737472 & -176.48025 \\
\hline & GREN-AS-AST2-1 & FAUNA & $7 / 16 / 2011$ & $0.048 \pm 0.002$ & 0.0002 & $0.193 \pm 0.008$ & 0.001 & & 51.745666 & -176.507055 \\
\hline & GREN-AS-AST2-2 & FAUNA & \begin{tabular}{|l|}
$7 / 16 / 2011$ \\
\end{tabular} & $0.2 \pm 0.01$ & 0.0004 & $0.915 \pm 0.046$ & 0.002 & & 51.745666 & -176.507055 \\
\hline & GREN-AS-AST2-3 & FAUNA & \begin{tabular}{|l|}
$7 / 16 / 2011$ \\
\end{tabular} & $0.125 \pm 0.006$ & 0.0004 & $0.582 \pm 0.028$ & 0.002 & & 51.745666 & -176.507055 \\
\hline & GREN-AS-AST3-1 & FAUNA & $7 / 14 / 2011$ & $0.093 \pm 0.004$ & 0.0003 & $0.443 \pm 0.019$ & 0.001 & & 51.688333 & -176.63375 \\
\hline & GREN-AS-AST3-2 & FAUNA & 7/14/2011 & $0.201 \pm 0.008$ & 0.0003 & $0.946 \pm 0.038$ & 0.001 & & 51.688333 & -176.63375 \\
\hline & GREN-AS-AST3-3 & FAUNA & \begin{tabular}{|l|l|}
$7 / 14 / 2011$ \\
\end{tabular} & $0.197 \pm 0.006$ & 0.0004 & $0.941 \pm 0.029$ & 0.002 & & 51.688333 & -176.63375 \\
\hline & GREN-AS-DUP & FAUNA & \begin{tabular}{|l|l|}
$7 / 2011$ \\
\end{tabular} & $0.26 \pm 0.02$ & 0.0004 & $1.304 \pm 0.1$ & 0.002 & & 51.688333 & -176.63375 \\
\hline \multirow{3}{*}{$\begin{array}{l}\text { Horse Mussel - } \\
\text { soft tissue only }\end{array}$} & HMUS-AS-AST1-1 & FAUNA & 7/15/2011 & $1.16 \pm 0.02$ & 0.0003 & $5.812 \pm 0.1$ & 0.002 & & 51.737472 & -176.48025 \\
\hline & HMUS-AS-AST2-1 & FAUNA & 7/16/2011 & $0.55 \pm 0.02$ & 0.0003 & $3.489 \pm 0.127$ & 0.002 & & 51.745666 & -176.507055 \\
\hline & HMUS-AS-AST3-1 & FAUNA & \begin{tabular}{|l|}
$7 / 14 / 2011$ \\
\end{tabular} & $1.6 \pm 0.04$ & 0.0003 & $8.157 \pm 0.204$ & 0.002 & & 51.688333 & $\begin{array}{l}-176.63375 \\
\end{array}$ \\
\hline \multirow{9}{*}{ Irish Lord - whole } & IRLO-AS-AST1-1 & FAUNA & \begin{tabular}{|l|l|}
$7 / 15 / 2011$ \\
\end{tabular} & $0.23 \pm 0.02$ & 0.0004 & $1.013 \pm 0.088$ & 0.002 & & 51.737472 & -176.48025 \\
\hline & IRLO-AS-AST1-2 & FAUNA & 7/15/2011 & $0.164 \pm 0.004$ & 0.0004 & $0.765 \pm 0.019$ & 0.002 & & 51.737472 & -176.48025 \\
\hline & IRLO-AS-AST1-3 & FAUNA & $7 / 15 / 2011$ & $0.406 \pm 0.008$ & 0.0004 & $1.851 \pm 0.036$ & 0.002 & & 51.737472 & -176.48025 \\
\hline & IRLO-AS-AST2-1 & FAUNA & \begin{tabular}{|l|l|}
$7 / 16 / 2011$ \\
\end{tabular} & $0.55 \pm 0.01$ & 0.0004 & $2.574 \pm 0.047$ & 0.002 & & 51.745666 & -176.507055 \\
\hline & IRLO-AS-AST2-2 & FAUNA & 7/16/2011 & $0.166 \pm 0.006$ & 0.0005 & $0.707 \pm 0.026$ & 0.002 & & 51.745666 & -176.507055 \\
\hline & IRLO-AS-AST2-3 & FAUNA & $7 / 16 / 2011$ & $0.416 \pm 0.008$ & 0.0004 & $2.105 \pm 0.04$ & 0.002 & & 51.745666 & -176.507055 \\
\hline & IRLO-AS-AST3-1 & FAUNA & \begin{tabular}{|l|l|}
$7 / 2011$ \\
\end{tabular} & $0.202 \pm 0.008$ & 0.0004 & $0.907 \pm 0.036$ & 0.002 & & 51.688333 & -176.63375 \\
\hline & IRLO-AS-AST3-2 & FAUNA & \begin{tabular}{|l|l|}
$7 / 14 / 2011$ \\
\end{tabular} & $0.076 \pm 0.004$ & 0.0005 & $0.321 \pm 0.017$ & 0.002 & & 51.688333 & -176.63375 \\
\hline & IRLO-AS-AST3-3 & FAUNA & 7/14/2011 & $0.142 \pm 0.008$ & 0.0004 & $0.648 \pm 0.037$ & 0.002 & & 51.688333 & -176.63375 \\
\hline $\begin{array}{l}\text { Pacific Cod - } \\
\text { whole } \\
\end{array}$ & PCOD-AS-AS2M-1 & FAUNA & 7/15/2011 & $0.06 \pm 0.004$ & 0.0003 & $0.3 \pm 0.02$ & 0.002 & & 51.737472 & -176.48025 \\
\hline
\end{tabular}




\begin{tabular}{|c|c|c|c|c|c|c|c|c|c|c|}
\hline Matrix Subtype & Location Code & Matrix & $\begin{array}{c}\text { Date } \\
\text { Sampled }\end{array}$ & $\begin{array}{l}\text { Wet Result } \pm \\
\text { Uncertainty }\end{array}$ & $\begin{array}{c}\text { Wet } \\
\text { Detection } \\
\text { Limit }\end{array}$ & $\begin{array}{l}\text { Dry Result } \pm \\
\text { Uncertainty }\end{array}$ & \begin{tabular}{|c|} 
Dry \\
Detection \\
Limit
\end{tabular} & Qualifiers & Latitude & Longitude \\
\hline \multirow{10}{*}{ Rockfish - whole } & ROCK-AS-AST1-1 & FAUNA & 7/15/2011 & $0.152 \pm 0.008$ & 0.0004 & $0.546 \pm 0.029$ & 0.001 & & 51.737472 & -176.48025 \\
\hline & ROCK-AS-AST1-2 & FAUNA & 7/15/2011 & $0.094 \pm 0.004$ & 0.0004 & $0.352 \pm 0.015$ & 0.001 & & 51.737472 & -176.48025 \\
\hline & ROCK-AS-AST1-3 & FAUNA & \begin{tabular}{|l|}
$7 / 15 / 2011$ \\
\end{tabular} & $0.167 \pm 0.004$ & 0.0004 & $0.719 \pm 0.017$ & 0.002 & & 51.737472 & -176.48025 \\
\hline & ROCK-AS-AST2-1 & FAUNA & \begin{tabular}{|l|}
$7 / 16 / 2011$ \\
\end{tabular} & $0.23 \pm 0.02$ & 0.0004 & $0.882 \pm 0.077$ & 0.002 & & 51.745666 & -176.507055 \\
\hline & ROCK-AS-AST2-2 & FAUNA & \begin{tabular}{|l|}
$7 / 16 / 2011$ \\
\end{tabular} & $0.047 \pm 0.002$ & 0.0004 & $0.175 \pm 0.007$ & 0.001 & & 51.745666 & -176.507055 \\
\hline & ROCK-AS-AST2-3 & \begin{tabular}{|l|} 
FAUNA \\
\end{tabular} & 7/16/2011 & $0.156 \pm 0.008$ & 0.0004 & $0.602 \pm 0.031$ & 0.002 & & 51.745666 & -176.507055 \\
\hline & ROCK-AS-AST3-1 & FAUNA & $7 / 14 / 2011$ & $0.384 \pm 0.01$ & 0.0007 & $1.574 \pm 0.041$ & 0.003 & & 51.688333 & -176.63375 \\
\hline & ROCK-AS-AST3-2 & FAUNA & $7 / 14 / 2011$ & $0.38 \pm 0.02$ & 0.0005 & $1.611 \pm 0.085$ & 0.002 & & 51.688333 & -176.63375 \\
\hline & ROCK-AS-AST3-3 & FAUNA & $7 / 14 / 2011$ & $0.113 \pm 0.004$ & 0.0003 & $0.49 \pm 0.017$ & 0.001 & & 51.688333 & -176.63375 \\
\hline & ROCK-AS-DUP & FAUNA & \begin{tabular}{|l|}
$7 / 14 / 2011$ \\
\end{tabular} & $0.068 \pm 0.006$ & 0.0004 & $0.266 \pm 0.023$ & 0.002 & & 51.688333 & -176.63375 \\
\hline \multirow{3}{*}{$\begin{array}{l}\text { Sea Urchin - } \\
\text { whole }\end{array}$} & URCH-AS-AST1-1 & FAUNA & $7 / 15 / 2011$ & $0.72 \pm 0.02$ & 0.0005 & $2.735 \pm 0.076$ & 0.002 & & 51.737472 & -176.48025 \\
\hline & URCH-AS-AST2-1 & FAUNA & \begin{tabular}{|l|}
$7 / 16 / 2011$ \\
\end{tabular} & $0.83 \pm 0.02$ & 0.0005 & $3.351 \pm 0.081$ & 0.002 & & 51.745666 & -176.507055 \\
\hline & URCH-AS-AST3-1 & FAUNA & \begin{tabular}{|l|}
$7 / 14 / 2011$ \\
\end{tabular} & $0.75 \pm 0.04$ & 0.0005 & $2.801 \pm 0.149$ & 0.002 & & 51.688333 & -176.63375 \\
\hline \multicolumn{11}{|c|}{${ }^{238}$ Uranium (pCi/kg) } \\
\hline \multicolumn{11}{|c|}{ Amchitka Site (AMC01) } \\
\hline $\begin{array}{l}\text { Dolly Varden - } \\
\text { whole }\end{array}$ & DOLL-AI-XXX-1 & FAUNA & $6 / 24 / 2011$ & $0.234 \pm 0.004$ & 0.002 & $0.902 \pm 0.015$ & 0.008 & & 51.468283 & 179.108052 \\
\hline $\begin{array}{c}\text { Goose Egg - no } \\
\text { shell }\end{array}$ & GoOS-AI-XXX-1 & FAUNA & 6/24/2011 & $0.015 \pm 0.01$ & 0.003 & $0.04 \pm 0.027$ & 0.008 & & 51.491305 & 179.0595 \\
\hline $\begin{array}{c}\text { Glaucous-winged } \\
\text { Gull Egg - no } \\
\text { shell }\end{array}$ & GWGU-AI-XXX-1 & FAUNA & 6/24/2011 & $0.015 \pm 0.004$ & 0.001 & $0.052 \pm 0.014$ & 0.003 & & 51.456388 & 179.167 \\
\hline \multicolumn{11}{|c|}{ Amchitka Site (Long Shot) (AMC02) } \\
\hline \multirow{3}{*}{ Chiton } & CHIT-LS-LT1-1 & FAUNA & $7 / 4 / 2011$ & $2.88 \pm 0.06$ & 0.001 & $14.286 \pm 0.298$ & 0.005 & & 51.457555 & 179.195138 \\
\hline & CHIT-LS-LT2-1 & FAUNA & $7 / 3 / 2011$ & $21.49 \pm 0.04$ & 0.002 & $100.666 \pm 0.187$ & 0.009 & & 51.44925 & 179.205583 \\
\hline & CHIT-LS-LT3-1 & FAUNA & $7 / 3 / 2011$ & $16.6 \pm 0.2$ & 0.002 & $79.04 \pm 0.952$ & 0.01 & & 51.43 & 179.22667 \\
\hline \multirow{10}{*}{ Dragon Kelp } & EUAL-LS-DUP & VEG & $7 / 3 / 2011$ & $21.4 \pm 0.6$ & 0.002 & $128.522 \pm 3.603$ & 0.012 & & 51.44925 & 179.205583 \\
\hline & EUAL-LS-LT1-1 & VEG & $7 / 4 / 2011$ & $11.9 \pm 0.2$ & 0.001 & $111.191 \pm 1.869$ & 0.009 & & 51.457555 & 179.195138 \\
\hline & EUAL-LS-LT1-2 & VEG & $7 / 4 / 2011$ & $26.6 \pm 0.2$ & 0.001 & $165.362 \pm 1.243$ & 0.006 & & 51.457555 & 179.195138 \\
\hline & EUAL-LS-LT1-3 & VEG & $7 / 4 / 2011$ & $25.8 \pm 0.8$ & 0.002 & $155.763 \pm 4.83$ & 0.012 & & 51.457555 & 179.195138 \\
\hline & EUAL-LS-LT2-1 & VEG & $7 / 3 / 2011$ & $23.3 \pm 0.6$ & 0.002 & $140.94 \pm 3.629$ & 0.012 & & 51.44925 & 179.205583 \\
\hline & EUAL-LS-LT2-2 & VEG & $7 / 3 / 2011$ & $9.58 \pm 0.08$ & 0.001 & $69.553 \pm 0.581$ & 0.007 & & 51.44925 & 179.205583 \\
\hline & EUAL-LS-LT2-3 & VEG & $7 / 3 / 2011$ & $13.7 \pm 0.4$ & 0.002 & $99.099 \pm 2.893$ & 0.014 & & 51.44925 & 179.205583 \\
\hline & EUAL-LS-LT3-1 & VEG & $7 / 2 / 2011$ & $28.2 \pm 0.4$ & 0.002 & $179.98 \pm 2.553$ & 0.013 & & 51.43 & 179.22667 \\
\hline & EUAL-LS-LT3-2 & VEG & $7 / 2 / 2011$ & $35.4 \pm 0.2$ & 0.002 & $251.896 \pm 1.423$ & 0.014 & & 51.43 & 179.22667 \\
\hline & EUAL-LS-LT3-3 & VEG & $7 / 2 / 2011$ & $9.9 \pm 0.2$ & 0.002 & $83.506 \pm 1.687$ & 0.017 & & 51.43 & 179.22667 \\
\hline
\end{tabular}




\begin{tabular}{|c|c|c|c|c|c|c|c|c|c|c|}
\hline Matrix Subtype & Location Code & Matrix & $\begin{array}{c}\text { Date } \\
\text { Sampled }\end{array}$ & $\begin{array}{l}\text { Wet Result } \pm \\
\text { Uncertainty }\end{array}$ & $\begin{array}{c}\text { Wet } \\
\text { Detection } \\
\text { Limit }\end{array}$ & $\begin{array}{l}\text { Dry Result } \pm \\
\text { Uncertainty }\end{array}$ & $\begin{array}{c}\text { Dry } \\
\text { Detection } \\
\text { Limit }\end{array}$ & Qualifiers & Latitude & Longitude \\
\hline \multirow{10}{*}{ Rockweed } & FUCU-LS-DUP & VEG & 6/22/2011 & $55.5 \pm 0.6$ & 0.001 & $304.998 \pm 3.297$ & 0.005 & & 51.447472 & 179.190888 \\
\hline & FUCU-LS-LT1-1 & VEG & $6 / 22 / 2011$ & $73.6 \pm 0.6$ & 0.001 & $397.602 \pm 3.241$ & 0.005 & & 51.447527 & 179.189361 \\
\hline & FUCU-LS-LT1-2 & VEG & \begin{tabular}{|l|}
$6 / 22 / 2011$ \\
\end{tabular} & $62 \pm 2$ & 0.002 & $379.27 \pm 12.235$ & 0.012 & & 51.447527 & 179.189361 \\
\hline & FUCU-LS-LT1-3 & VEG & \begin{tabular}{|c|}
$6 / 22 / 2011$ \\
\end{tabular} & $50.4 \pm 1$ & 0.002 & $335.112 \pm 6.649$ & 0.013 & & 51.448777 & 179.189305 \\
\hline & FUCU-LS-LT2-1 & VEG & 6/22/2011 & $29 \pm 0.2$ & 0.001 & $228.656 \pm 1.577$ & 0.008 & & 51.447222 & 179.199361 \\
\hline & FUCU-LS-LT2-2 & VEG & $6 / 22 / 2011$ & $47 \pm 0.4$ & 0.001 & $340.671 \pm 2.899$ & 0.007 & & 51.44625 & 179.196972 \\
\hline & FUCU-LS-LT2-3 & VEG & 6/22/2011 & $60.5 \pm 0.6$ & 0.001 & $369.998 \pm 3.669$ & 0.006 & & 51.446722 & 179.191888 \\
\hline & FUCU-LS-LT3-1 & VEG & $7 / 2 / 2011$ & $74.1 \pm 0.8$ & 0.002 & $358.922 \pm 3.875$ & 0.01 & & 51.433611 & 179.219305 \\
\hline & FUCU-LS-LT3-2 & VEG & $7 / 2 / 2011$ & $55 \pm 2$ & 0.002 & $250.28 \pm 9.101$ & 0.009 & & 51.430166 & 179.218694 \\
\hline & FUCU-LS-LT3-3 & VEG & $7 / 2 / 2011$ & $80 \pm 2$ & 0.002 & $293.15 \pm 7.329$ & 0.007 & & 51.430166 & 179.218694 \\
\hline \multirow{10}{*}{ | Greenling - whole } & GREN-LS-DUP & FAUNA & \begin{tabular}{|l|}
$7 / 12 / 2011$ \\
\end{tabular} & $6.74 \pm 0.06$ & 0.002 & $32.728 \pm 0.291$ & 0.01 & & 51.434527 & 179.234416 \\
\hline & GREN-LS-LT1-1 & FAUNA & \begin{tabular}{|l|}
$7 / 4 / 2011$ \\
\end{tabular} & $2.45 \pm 0.06$ & 0.001 & $11.548 \pm 0.283$ & 0.005 & & 51.457555 & 179.195138 \\
\hline & GREN-LS-LT1-2 & FAUNA & $7 / 4 / 2011$ & $1.76 \pm 0.04$ & 0.001 & $8.331 \pm 0.189$ & 0.005 & & 51.457555 & 179.195138 \\
\hline & GREN-LS-LT1-3 & FAUNA & $7 / 5 / 2011$ & $3.12 \pm 0.04$ & 0.002 & $14.612 \pm 0.187$ & 0.009 & & 51.457555 & 179.195138 \\
\hline & GREN-LS-LT2-1 & FAUNA & $7 / 3 / 2011$ & $6.2 \pm 0.2$ & 0.002 & $29.959 \pm 0.966$ & 0.01 & & 51.432194 & 179.230277 \\
\hline & GREN-LS-LT2-2 & FAUNA & $7 / 3 / 2011$ & $3.36 \pm 0.06$ & 0.001 & $15.496 \pm 0.277$ & 0.005 & & 51.432194 & 179.230277 \\
\hline & GREN-LS-LT2-3 & FAUNA & $7 / 3 / 2011$ & $4.77 \pm 0.04$ & 0.001 & $22.697 \pm 0.19$ & 0.005 & & 51.432194 & 179.230277 \\
\hline & GREN-LS-LT3-1 & FAUNA & $7 / 3 / 2011$ & $3.84 \pm 0.04$ & 0.002 & $17.045 \pm 0.178$ & 0.009 & & 51.43 & 179.22667 \\
\hline & GREN-LS-LT3-2 & FAUNA & $7 / 12 / 2011$ & $5.93 \pm 0.1$ & 0.002 & $29.091 \pm 0.491$ & 0.01 & & 51.434527 & 179.234416 \\
\hline & GREN-LS-LT3-3 & FAUNA & $7 / 12 / 2011$ & $4.8 \pm 0.2$ & 0.002 & $23.209 \pm 0.967$ & 0.01 & & 51.434527 & 179.234416 \\
\hline Halibut - whole & HALI-LS-L2M-1 & FAUNA & 7/13/2011 & $0.254 \pm 0.006$ & 0.001 & $0.975 \pm 0.023$ & 0.004 & & 51.40025 & 179.283361 \\
\hline \multirow{3}{*}{$\begin{array}{l}\text { Horse Mussel - } \\
\text { soft tissue only }\end{array}$} & HMUS-LS-LT1-1 & FAUNA & $7 / 5 / 2011$ & $67.1 \pm 1.2$ & 0.002 & $287.454 \pm 5.141$ & 0.009 & & 51.457555 & 179.195138 \\
\hline & HMUS-LS-LT2-1 & FAUNA & $7 / 4 / 2011$ & $33.1 \pm 0.2$ & 0.002 & $135.224 \pm 0.817$ & 0.008 & & 51.44925 & 179.205583 \\
\hline & HMUS-LS-LT3-1 & FAUNA & $7 / 3 / 2011$ & $12.2 \pm 0.6$ & 0.043 & $25.586 \pm 1.258$ & 0.09 & & 51.44925 & 179.205583 \\
\hline \multirow{9}{*}{ || Irish Lord - whole } & IRLO-LS-LT1-1 & FAUNA & $7 / 4 / 2011$ & $2.22 \pm 0.02$ & 0.002 & $10.469 \pm 0.094$ & 0.009 & & 51.457555 & 179.195138 \\
\hline & IRLO-LS-LT1-2 & FAUNA & $7 / 4 / 2011$ & $1.99 \pm 0.04$ & 0.002 & $9.415 \pm 0.189$ & 0.009 & & 51.457555 & 179.195138 \\
\hline & IRLO-LS-LT1-3 & FAUNA & $7 / 5 / 2011$ & $4.2 \pm 0.02$ & 0.002 & $22.271 \pm 0.106$ & 0.011 & & 51.457555 & 179.195138 \\
\hline & IRLO-LS-LT2-1 & FAUNA & $7 / 3 / 2011$ & $9.83 \pm 0.08$ & 0.002 & $42.543 \pm 0.346$ & 0.009 & & 51.432194 & 179.230277 \\
\hline & IRLO-LS-LT2-2 & FAUNA & $7 / 3 / 2011$ & $7.74 \pm 0.04$ & 0.002 & $32.067 \pm 0.166$ & 0.008 & & 51.432194 & 179.230277 \\
\hline & IRLO-LS-LT2-3 & FAUNA & $7 / 3 / 2011$ & $16.1 \pm 0.2$ & 0.003 & $71.321 \pm 0.886$ & 0.013 & & 51.432194 & 179.230277 \\
\hline & IRLO-LS-LT3-1 & FAUNA & $7 / 2 / 2011$ & $8.5 \pm 0.2$ & 0.002 & $37.912 \pm 0.892$ & 0.009 & & 51.43 & 179.22667 \\
\hline & IRLO-LS-LT3-2 & FAUNA & \begin{tabular}{|l|}
$7 / 12 / 2011$ \\
\end{tabular} & $6.24 \pm 0.08$ & 0.001 & $29.604 \pm 0.38$ & 0.005 & & 51.434944 & 179.2315 \\
\hline & IRLO-LS-LT3-3 & FAUNA & $7 / 12 / 2011$ & $5.39 \pm 0.04$ & 0.001 & $29.779 \pm 0.221$ & 0.006 & & 51.434944 & 179.2315 \\
\hline Octopus & OCTP-LS-L2M-1 & FAUNA & \begin{tabular}{|l|}
$7 / 3 / 2011$ \\
\end{tabular} & $18.1 \pm 0.2$ & 0.001 & $129.002 \pm 1.425$ & 0.007 & & 51.44925 & 179.205583 \\
\hline $\begin{array}{l}\text { Pacific Cod - } \\
\text { whole }\end{array}$ & PCOD-LS-L2M-1 & FAUNA & 7/4/2011 & $1.84 \pm 0.02$ & 0.001 & $9.156 \pm 0.1$ & 0.005 & & 51.451805 & 179.196972 \\
\hline
\end{tabular}




\begin{tabular}{|c|c|c|c|c|c|c|c|c|c|c|}
\hline Matrix Subtype & Location Code & Matrix & $\begin{array}{c}\text { Date } \\
\text { Sampled }\end{array}$ & $\begin{array}{l}\text { Wet Result } \pm \\
\text { Uncertainty }\end{array}$ & $\begin{array}{c}\text { Wet } \\
\text { Detection } \\
\text { Limit }\end{array}$ & $\begin{array}{l}\text { Dry Result } \pm \\
\text { Uncertainty }\end{array}$ & $\begin{array}{c}\text { Dry } \\
\text { Detection } \\
\text { Limit }\end{array}$ & Qualifiers & Latitude & Longitude \\
\hline \multirow{10}{*}{ Rockfish - whole } & ROCK-LS-DUP & FAUNA & $7 / 12 / 2011$ & $3.95 \pm 0.06$ & 0.002 & $15.404 \pm 0.234$ & 0.008 & & 51.434944 & 179.2315 \\
\hline & ROCK-LS-DUP2 & FAUNA & $7 / 12 / 2011$ & $12.21 \pm 0.06$ & 0.002 & $52.25 \pm 0.257$ & 0.009 & & 51.465472 & 179.206722 \\
\hline & ROCK-LS-LT1-1 & FAUNA & $7 / 4 / 2011$ & $5.5 \pm 0.2$ & 0.002 & $25.541 \pm 0.929$ & 0.009 & & 51.457555 & 179.195138 \\
\hline & ROCK-LS-LT1-2 & FAUNA & $7 / 12 / 2011$ & $5.15 \pm 0.04$ & 0.002 & $21.273 \pm 0.165$ & 0.008 & & 51.465472 & 179.206722 \\
\hline & ROCK-LS-LT1-3 & FAUNA & $7 / 12 / 2011$ & $3.16 \pm 0.06$ & 0.002 & $13.042 \pm 0.248$ & 0.008 & & 51.465472 & 179.206722 \\
\hline & ROCK-LS-LT2-1 & FAUNA & $7 / 4 / 2011$ & $11.3 \pm 0.2$ & 0.002 & $45.321 \pm 0.802$ & 0.008 & & 51.44925 & 179.205583 \\
\hline & ROCK-LS-LT2-2 & FAUNA & $7 / 12 / 2011$ & $10.6 \pm 0.2$ & 0.002 & $41.831 \pm 0.789$ & 0.008 & & 51.44625 & 179.223416 \\
\hline & ROCK-LS-LT3-1 & FAUNA & $7 / 12 / 2011$ & $13.64 \pm 0.06$ & 0.002 & $60.032 \pm 0.264$ & 0.009 & & 51.434944 & 179.2315 \\
\hline & ROCK-LS-LT3-2 & FAUNA & $7 / 12 / 2011$ & $7.58 \pm 0.06$ & 0.002 & $32.157 \pm 0.255$ & 0.008 & & 51.434944 & 179.2315 \\
\hline & ROCK-LS-LT3-3 & FAUNA & $7 / 3 / 2011$ & $6.6 \pm 0.2$ & 0.002 & $22.771 \pm 0.69$ & 0.007 & & 51.43 & 179.22667 \\
\hline Reindeer Lichen & STRL-AI-LT1-2 & VEG & $6 / 22 / 2011$ & $2.72 \pm 0.04$ & 0.001 & $8.246 \pm 0.121$ & 0.003 & & 51.447361 & 179.189111 \\
\hline \multirow{3}{*}{$\begin{array}{l}\text { Sea Urchin - } \\
\text { whole }\end{array}$} & URCH-LS-LT1-1 & FAUNA & $7 / 4 / 2011$ & $18.4 \pm 0.2$ & 0.002 & $71.878 \pm 0.781$ & 0.008 & & 51.457555 & 179.195138 \\
\hline & URCH-LS-LT2-1 & FAUNA & $7 / 3 / 2011$ & $15.8 \pm 0.2$ & 0.002 & $60.705 \pm 0.768$ & 0.008 & & 51.44925 & 179.205583 \\
\hline & URCH-LS-LT3-1 & FAUNA & $7 / 2 / 2011$ & $19.1 \pm 0.2$ & 0.002 & $74.398 \pm 0.779$ & 0.008 & & 51.43 & 179.22667 \\
\hline \multicolumn{11}{|c|}{ Amchitka Site (Milrow) (AMC03) } \\
\hline \multirow{3}{*}{ Chiton } & CHIT-ML-MT1-1 & FAUNA & $7 / 9 / 2011$ & $14.6 \pm 0.2$ & 0.002 & $55.055 \pm 0.754$ & 0.008 & & 51.408472 & 179.139166 \\
\hline & CHIT-ML-MT2-1 & FAUNA & $7 / 10 / 2011$ & $21.7 \pm 0.4$ & 0.002 & $100.758 \pm 1.857$ & 0.009 & & 51.389416 & 179.173138 \\
\hline & CHIT-ML-MT3-1 & FAUNA & $7 / 10 / 2011$ & $20.9 \pm 0.4$ & 0.002 & $94.839 \pm 1.815$ & 0.009 & & 51.387805 & 179.184 \\
\hline \multirow{10}{*}{ Dragon Kelp } & EUAL-ML-DUP & VEG & $7 / 9 / 2011$ & $15 \pm 0.4$ & 0.002 & $113.036 \pm 3.014$ & 0.015 & & 51.408472 & 179.139166 \\
\hline & EUAL-ML-MT1-1 & VEG & $7 / 9 / 2011$ & $14.9 \pm 0.2$ & 0.001 & $119.111 \pm 1.599$ & 0.008 & & 51.408472 & 179.139166 \\
\hline & EUAL-ML-MT1-2 & VEG & 7/9/2011 & $18.7 \pm 0.6$ & 0.002 & $139.145 \pm 4.465$ & 0.015 & & 51.408472 & 179.139166 \\
\hline & EUAL-ML-MT1-3 & VEG & $7 / 9 / 2011$ & $11.7 \pm 0.2$ & 0.001 & $98.416 \pm 1.682$ & 0.008 & & 51.408472 & 179.139166 \\
\hline & EUAL-ML-MT2-1 & VEG & $7 / 10 / 2011$ & $33.3 \pm 0.4$ & 0.003 & $248.184 \pm 2.981$ & 0.022 & & 51.389416 & 179.173138 \\
\hline & EUAL-ML-MT2-2 & VEG & $7 / 10 / 2011$ & $22.9 \pm 0.8$ & 0.001 & $162.251 \pm 5.668$ & 0.007 & & 51.389416 & 179.173138 \\
\hline & EUAL-ML-MT2-3 & VEG & 7/10/2011 & $18.09 \pm 0.08$ & 0.001 & $136.358 \pm 0.603$ & 0.008 & & 51.389416 & 179.173138 \\
\hline & EUAL-ML-MT3-1 & VEG & $7 / 10 / 2011$ & $17.3 \pm 0.4$ & 0.002 & $121.691 \pm 2.814$ & 0.014 & & 51.387805 & 179.184 \\
\hline & EUAL-ML-MT3-2 & VEG & $7 / 10 / 2011$ & $18.1 \pm 0.8$ & 0.002 & $132.236 \pm 5.845$ & 0.015 & & 51.387805 & 179.184 \\
\hline & EUAL-ML-MT3-3 & VEG & 7/10/2011 & $33 \pm 0.8$ & 0.002 & $227.574 \pm 5.517$ & 0.014 & & 51.387805 & 179.184 \\
\hline
\end{tabular}




\begin{tabular}{|c|c|c|c|c|c|c|c|c|c|c|}
\hline Matrix Subtype & Location Code & Matrix & $\begin{array}{c}\text { Date } \\
\text { Sampled }\end{array}$ & $\begin{array}{l}\text { Wet Result } \pm \\
\text { Uncertainty }\end{array}$ & $\begin{array}{c}\text { Wet } \\
\text { Detection } \\
\text { Limit }\end{array}$ & $\begin{array}{l}\text { Dry Result } \pm \\
\text { Uncertainty }\end{array}$ & $\begin{array}{c}\text { Dry } \\
\text { Detection } \\
\text { Limit }\end{array}$ & Qualifiers & Latitude & Longitude \\
\hline \multirow{10}{*}{ Rockweed } & FUCU-ML-DUP & VEG & 6/20/2011 & $85.2 \pm 1$ & 0.002 & $369.693 \pm 4.339$ & 0.009 & & 51.412449 & 179.160843 \\
\hline & FUCU-ML-MT1-1 & VEG & \begin{tabular}{|l|}
$6 / 20 / 2011$ \\
\end{tabular} & $79.9 \pm 0.4$ & 0.002 & $332.868 \pm 1.666$ & 0.008 & & 51.412449 & 179.160843 \\
\hline & FUCU-ML-MT1-2 & VEG & 6/21/2011 & $74.6 \pm 0.8$ & 0.001 & $452.543 \pm 4.853$ & 0.006 & & 51.417083 & 179.134083 \\
\hline & FUCU-ML-MT1-3 & VEG & \begin{tabular}{|l|}
$7 / 9 / 2011$ \\
\end{tabular} & $89 \pm 4$ & 0.002 & $409.092 \pm 18.386$ & 0.009 & & 51.417083 & 179.134083 \\
\hline & FUCU-ML-MT2-1 & VEG & 6/20/2011 & $76.4 \pm 0.6$ & 0.002 & $394.288 \pm 3.097$ & 0.01 & & 51.403305 & 179.167166 \\
\hline & FUCU-ML-MT2-2 & VEG & $6 / 21 / 2011$ & $46 \pm 2$ & 0.001 & $323.175 \pm 14.051$ & 0.007 & & 51.405694 & 179.161361 \\
\hline & FUCU-ML-MT2-3 & VEG & 6/21/2011 & $43.7 \pm 0.2$ & 0.001 & $303.437 \pm 1.389$ & 0.007 & & 51.405694 & 179.161361 \\
\hline & FUCU-ML-MT3-1 & VEG & \begin{tabular}{|l}
$7 / 10 / 2011$ \\
\end{tabular} & $114 \pm 2$ & 0.002 & $553.613 \pm 9.713$ & 0.01 & & 51.389416 & 179.173138 \\
\hline & FUCU-ML-MT3-2 & VEG & 7/10/2011 & $121 \pm 2$ & 0.002 & $581.118 \pm 9.605$ & 0.01 & & 51.389416 & 179.173138 \\
\hline & FUCU-ML-MT3-3 & VEG & $7 / 10 / 2011$ & $108 \pm 2$ & 0.002 & $545.248 \pm 10.097$ & 0.01 & & 51.389416 & 179.173138 \\
\hline \multirow{10}{*}{ Greenling - whole } & GREN-ML-DUP & FAUNA & $7 / 10 / 2011$ & $4.99 \pm 0.06$ & 0.001 & $24.197 \pm 0.291$ & 0.005 & & 51.387805 & 179.184 \\
\hline & GREN-ML-MT1-1 & FAUNA & \begin{tabular}{|l|}
$7 / 9 / 2011$ \\
\end{tabular} & $4.53 \pm 0.06$ & 0.002 & $19.732 \pm 0.261$ & 0.009 & & 51.408472 & 179.139166 \\
\hline & GREN-ML-MT1-2 & FAUNA & $7 / 9 / 2011$ & $5.62 \pm 0.04$ & 0.002 & $26.022 \pm 0.185$ & 0.009 & & 51.408472 & 179.139166 \\
\hline & GREN-ML-MT1-3 & FAUNA & 7/9/2011 & $9.1 \pm 0.2$ & 0.001 & $43.94 \pm 0.966$ & 0.005 & & 51.408472 & 179.139166 \\
\hline & GREN-ML-MT2-1 & FAUNA & 7/11/2011 & $2.74 \pm 0.06$ & 0.002 & $12.773 \pm 0.28$ & 0.009 & & 51.396944 & 179.1435 \\
\hline & GREN-ML-MT2-2 & FAUNA & $7 / 11 / 2011$ & $3.92 \pm 0.04$ & 0.002 & $18.351 \pm 0.187$ & 0.009 & & 51.396944 & 179.1435 \\
\hline & GREN-ML-MT2-3 & FAUNA & 7/11/2011 & $2.8 \pm 0.2$ & 0.001 & $13.37 \pm 0.955$ & 0.005 & & 51.396944 & 179.1435 \\
\hline & GREN-ML-MT3-1 & FAUNA & 7/10/2011 & $8.4 \pm 0.2$ & 0.002 & $40.738 \pm 0.97$ & 0.01 & & 51.387805 & 179.184 \\
\hline & GREN-ML-MT3-2 & FAUNA & $7 / 10 / 2011$ & $2.61 \pm 0.02$ & 0.002 & $12.593 \pm 0.096$ & 0.01 & & 51.387805 & 179.184 \\
\hline & GREN-ML-MT3-3 & FAUNA & \begin{tabular}{|l|}
$7 / 10 / 2011$ \\
\end{tabular} & $4.41 \pm 0.08$ & 0.001 & $20.894 \pm 0.379$ & 0.005 & & 51.387805 & 179.184 \\
\hline Halibut - whole & HALI-ML-M2M-1 & FAUNA & 7/11/2011 & $0.65 \pm 0.02$ & 0.002 & $2.258 \pm 0.069$ & 0.007 & & 51.365055 & 179.111472 \\
\hline \multirow{3}{*}{$\begin{array}{l}\text { Horse Mussel - } \\
\text { soft tissue only }\end{array}$} & HMUS-ML-MT1-1 & FAUNA & 7/9/2011 & $58.4 \pm 0.6$ & 0.002 & $265.566 \pm 2.728$ & 0.009 & & 51.408472 & 179.139166 \\
\hline & HMUS-ML-MT2-1 & FAUNA & 7/11/2011 & $34.5 \pm 0.2$ & 0.002 & $155.163 \pm 0.899$ & 0.009 & & 51.389416 & 179.173138 \\
\hline & HMUS-ML-MT3-1 & FAUNA & 7/10/2011 & $38.2 \pm 0.4$ & 0.002 & $175.359 \pm 1.836$ & 0.009 & & 51.387805 & 179.184 \\
\hline \multirow{9}{*}{ | Irish Lord - whole } & IRLO-ML-MT1-1 & FAUNA & 7/9/2011 & $4.28 \pm 0.04$ & 0.002 & $18.272 \pm 0.171$ & 0.009 & & 51.408472 & 179.139166 \\
\hline & IRLO-ML-MT1-2 & FAUNA & $7 / 9 / 2011$ & $2.69 \pm 0.02$ & 0.002 & $12.758 \pm 0.095$ & 0.009 & & 51.408472 & 179.139166 \\
\hline & IRLO-ML-MT1-3 & FAUNA & $7 / 9 / 2011$ & $2.21 \pm 0.04$ & 0.002 & $10.353 \pm 0.187$ & 0.009 & & 51.408472 & 179.139166 \\
\hline & IRLO-ML-MT2-1 & FAUNA & 7/10/2011 & $4.88 \pm 0.04$ & 0.002 & $21.028 \pm 0.172$ & 0.009 & & 51.389416 & 179.173138 \\
\hline & IRLO-ML-MT2-2 & FAUNA & 7/11/2011 & $2.1 \pm 0.04$ & 0.003 & $9.048 \pm 0.172$ & 0.013 & & 51.396944 & 179.1435 \\
\hline & IRLO-ML-MT2-3 & FAUNA & 7/11/2011 & $3.6 \pm 0.04$ & 0.002 & $17.077 \pm 0.19$ & 0.009 & & 51.396944 & 179.1435 \\
\hline & IRLO-ML-MT3-1 & FAUNA & 7/10/2011 & $8.16 \pm 0.06$ & 0.001 & $46.001 \pm 0.338$ & 0.006 & & 51.387805 & 179.184 \\
\hline & IRLO-ML-MT3-2 & FAUNA & \begin{tabular}{|l|l|} 
\\
\end{tabular} & $4.44 \pm 0.06$ & 0.002 & $21.437 \pm 0.29$ & 0.01 & & 51.387805 & 179.184 \\
\hline & IRLO-ML-MT3-3 & FAUNA & $7 / 10 / 2011$ & $6.46 \pm 0.08$ & 0.001 & $36.214 \pm 0.448$ & 0.006 & & 51.387805 & 179.184 \\
\hline Octopus & OCTP-ML-M2M-1 & FAUNA & $7 / 10 / 2011$ & $8.52 \pm 0.08$ & 0.001 & $40.182 \pm 0.377$ & 0.005 & & 51.389416 & 179.173138 \\
\hline $\begin{array}{l}\text { Pacific Cod - } \\
\text { whole }\end{array}$ & PCOD-ML-M2M-1 & FAUNA & 7/10/2011 & $2.11 \pm 0.04$ & 0.001 & $11.157 \pm 0.212$ & 0.005 & & 51.408472 & 179.139166 \\
\hline
\end{tabular}




\begin{tabular}{|c|c|c|c|c|c|c|c|c|c|c|}
\hline Matrix Subtype & Location Code & Matrix & $\begin{array}{c}\text { Date } \\
\text { Sampled }\end{array}$ & $\begin{array}{l}\text { Wet Result } \pm \\
\text { Uncertainty }\end{array}$ & $\begin{array}{l}\text { Wet } \\
\text { Detection } \\
\text { Limit }\end{array}$ & $\begin{array}{l}\text { Dry Result } \pm \\
\text { Uncertainty }\end{array}$ & \begin{tabular}{|c|} 
Dry \\
Detection \\
Limit
\end{tabular} & Qualifiers & Latitude & Longitude \\
\hline \multirow{10}{*}{ Rockfish - whole } & ROCK-ML-DUP & FAUNA & $7 / 9 / 2011$ & $14.2 \pm 0.2$ & 0.002 & $62.776 \pm 0.884$ & 0.009 & & 51.408472 & 179.139166 \\
\hline & ROCK-ML-MT1-1 & FAUNA & 7/9/2011 & $5.9 \pm 0.2$ & 0.001 & $25.971 \pm 0.88$ & 0.004 & & 51.408472 & 179.139166 \\
\hline & ROCK-ML-MT1-2 & FAUNA & 7/9/2011 & $12.5 \pm 0.2$ & 0.002 & $54.867 \pm 0.878$ & 0.009 & & 51.408472 & 179.139166 \\
\hline & ROCK-ML-MT1-3 & FAUNA & $7 / 9 / 2011$ & $7.96 \pm 0.08$ & 0.002 & $33.695 \pm 0.339$ & 0.008 & & 51.408472 & 179.139166 \\
\hline & ROCK-ML-MT2-1 & FAUNA & $7 / 11 / 2011$ & $6.33 \pm 0.16$ & 0.002 & $23.758 \pm 0.601$ & 0.008 & & 51.365055 & 179.111472 \\
\hline & ROCK-ML-MT2-2 & FAUNA & $7 / 11 / 2011$ & $13.9 \pm 0.2$ & 0.002 & $56.246 \pm 0.809$ & 0.008 & & 51.365055 & 179.111472 \\
\hline & ROCK-ML-MT2-3 & FAUNA & $7 / 11 / 2011$ & $12.2 \pm 0.2$ & 0.001 & $54.962 \pm 0.901$ & 0.005 & & 51.365055 & 179.111472 \\
\hline & ROCK-ML-MT3-1 & FAUNA & 7/10/2011 & $10.3 \pm 0.2$ & 0.002 & $48.606 \pm 0.944$ & 0.009 & & 51.387805 & 179.184 \\
\hline & ROCK-ML-MT3-2 & FAUNA & $7 / 10 / 2011$ & $3.11 \pm 0.06$ & 0.002 & $11.192 \pm 0.216$ & 0.007 & & 51.387805 & 179.184 \\
\hline & ROCK-ML-MT3-3 & FAUNA & $7 / 10 / 2011$ & $2.2 \pm 0.04$ & 0.003 & $5.119 \pm 0.093$ & 0.007 & & 51.387805 & 179.184 \\
\hline Reindeer Lichen & STRL-AI-ML-1 & VEG & $6 / 21 / 2011$ & $2.46 \pm 0.06$ & 0.002 & $8.804 \pm 0.215$ & 0.007 & & 51.402583 & 179.183972 \\
\hline \multirow{3}{*}{$\begin{array}{l}\text { Sea Urchin - } \\
\text { whole }\end{array}$} & URCH-ML-MT1-1 & FAUNA & $7 / 9 / 2011$ & $19.5 \pm 0.4$ & 0.002 & $72.34 \pm 1.484$ & 0.007 & & 51.408472 & 179.139166 \\
\hline & URCH-ML-MT2-1 & FAUNA & $7 / 10 / 2011$ & $18.9 \pm 0.2$ & 0.002 & $70.586 \pm 0.747$ & 0.007 & & 51.389416 & 179.173138 \\
\hline & URCH-ML-MT3-1 & FAUNA & 7/10/2011 & $17.7 \pm 0.2$ & 0.002 & $70.267 \pm 0.794$ & 0.008 & & 51.387805 & 179.184 \\
\hline \multicolumn{11}{|c|}{ Amchitka Site (Cannikin) (AMC05) } \\
\hline \multirow{3}{*}{ Chiton } & CHIT-CN-CT1-1 & FAUNA & $7 / 8 / 2011$ & $26.8 \pm 0.4$ & 0.002 & $125.518 \pm 1.873$ & 0.009 & & 51.482972 & 179.123833 \\
\hline & CHIT-CN-CT2-1 & FAUNA & 7/7/2011 & $25.5 \pm 0.6$ & 0.002 & $120.678 \pm 2.839$ & 0.009 & & 51.472611 & 179.151111 \\
\hline & CHIT-CN-CT3-1 & FAUNA & $7 / 5 / 2011$ & $19.7 \pm 0.2$ & 0.002 & $96.695 \pm 0.982$ & 0.01 & & 51.471416 & 179.172861 \\
\hline \multirow{10}{*}{ Dragon Kelp } & EUAL-CN-CT1-1 & VEG & $7 / 8 / 2011$ & $19.1 \pm 0.4$ & 0.001 & $141.382 \pm 2.961$ & 0.007 & & 51.482972 & 179.123833 \\
\hline & EUAL-CN-CT1-2 & VEG & $7 / 8 / 2011$ & $15.7 \pm 0.4$ & 0.001 & $102.36 \pm 2.608$ & 0.007 & & 51.482972 & 179.123833 \\
\hline & EUAL-CN-CT1-3 & VEG & $7 / 8 / 2011$ & $22.2 \pm 0.2$ & 0.001 & $135.978 \pm 1.225$ & 0.006 & & 51.482972 & 179.123833 \\
\hline & EUAL-CN-CT2-1 & VEG & 7/6/2011 & $20 \pm 0.6$ & 0.001 & $127.558 \pm 3.827$ & 0.006 & & 51.472611 & 179.151111 \\
\hline & EUAL-CN-CT2-2 & VEG & $7 / 6 / 2011$ & $33 \pm 2$ & 0.002 & $207.124 \pm 12.553$ & 0.013 & & 51.472611 & 179.151111 \\
\hline & EUAL-CN-CT2-3 & VEG & $7 / 7 / 2011$ & $14.1 \pm 0.2$ & 0.001 & $102.225 \pm 1.45$ & 0.007 & & 51.472611 & 179.151111 \\
\hline & EUAL-CN-CT3-1 & VEG & 7/5/2011 & $16.3 \pm 0.2$ & 0.001 & $113.66 \pm 1.395$ & 0.007 & & 51.471416 & 179.172861 \\
\hline & EUAL-CN-CT3-2 & VEG & $7 / 5 / 2011$ & $11.6 \pm 0.2$ & 0.002 & $83.4 \pm 1.438$ & 0.014 & & 51.471416 & 179.172861 \\
\hline & EUAL-CN-CT3-3 & VEG & $7 / 5 / 2011$ & $19.8 \pm 0.2$ & 0.001 & $128.689 \pm 1.3$ & 0.006 & & 51.471416 & 179.172861 \\
\hline & EUAL-CN-DUP & VEG & $7 / 6 / 2011$ & $7.15 \pm 0.04$ & 0.001 & $47.509 \pm 0.266$ & 0.007 & & 51.471416 & 179.172861 \\
\hline
\end{tabular}




\begin{tabular}{|c|c|c|c|c|c|c|c|c|c|c|}
\hline Matrix Subtype & Location Code & Matrix & $\begin{array}{c}\text { Date } \\
\text { Sampled }\end{array}$ & $\begin{array}{l}\text { Wet Result } \pm \\
\text { Uncertainty }\end{array}$ & $\begin{array}{c}\text { Wet } \\
\text { Detection } \\
\text { Limit }\end{array}$ & $\begin{array}{l}\text { Dry Result } \pm \\
\text { Uncertainty }\end{array}$ & $\begin{array}{c}\text { Dry } \\
\text { Detection } \\
\text { Limit }\end{array}$ & Qualifiers & Latitude & Longitude \\
\hline \multirow{10}{*}{ Rockweed } & FUCU-CN-CT1-1 & VEG & 6/23/2011 & $44.2 \pm 0.2$ & 0.001 & $254.898 \pm 1.153$ & 0.006 & & 51.481888 & 179.118138 \\
\hline & FUCU-CN-CT1-2 & VEG & 6/23/2011 & $50 \pm 0.8$ & 0.002 & $314.903 \pm 5.038$ & 0.013 & & 51.481888 & 179.118138 \\
\hline & FUCU-CN-CT1-3 & VEG & 6/23/2011 & $57.5 \pm 0.8$ & 0.002 & $338.971 \pm 4.716$ & 0.012 & & 51.481888 & 179.118138 \\
\hline & FUCU-CN-CT2-1 & VEG & \begin{tabular}{|l|}
$7 / 8 / 2011$ \\
\end{tabular} & $108 \pm 2$ & 0.002 & $446.307 \pm 8.265$ & 0.008 & & 51.476638 & 179.135277 \\
\hline & FUCU-CN-CT2-2 & VEG & $7 / 8 / 2011$ & $105 \pm 2$ & 0.002 & $465.906 \pm 8.874$ & 0.009 & & 51.476638 & 179.135277 \\
\hline & FUCU-CN-CT2-3 & VEG & 7/12/2011 & $44 \pm 2$ & 0.002 & $232.584 \pm 10.572$ & 0.011 & & 51.467583 & 179.153583 \\
\hline & FUCU-CN-CT3-1 & VEG & $7 / 6 / 2011$ & $43.4 \pm 1$ & 0.002 & $233.866 \pm 5.389$ & 0.011 & & 51.465027 & 179.173166 \\
\hline & FUCU-CN-CT3-2 & VEG & 7/6/2011 & $34.5 \pm 0.8$ & 0.002 & $202.502 \pm 4.696$ & 0.012 & & 51.465472 & 179.170583 \\
\hline & FUCU-CN-CT3-3 & VEG & $7 / 6 / 2011$ & $64 \pm 2$ & 0.002 & $406.145 \pm 12.692$ & 0.013 & & 51.464638 & 179.159305 \\
\hline & FUCU-CN-DUP & VEG & 6/23/2011 & $44.2 \pm 0.4$ & 0.001 & $285.166 \pm 2.581$ & 0.006 & & 51.481888 & 179.118138 \\
\hline \multirow{10}{*}{ Greenling - whole } & GREN-CN-CT1-1 & FAUNA & $7 / 8 / 2011$ & $6.57 \pm 0.12$ & 0.001 & $31.671 \pm 0.578$ & 0.005 & & 51.482972 & 179.123833 \\
\hline & GREN-CN-CT1-2 & FAUNA & $7 / 8 / 2011$ & $5.73 \pm 0.12$ & 0.002 & $27.214 \pm 0.57$ & 0.009 & & 51.482972 & 179.123833 \\
\hline & GREN-CN-CT1-3 & FAUNA & $7 / 8 / 2011$ & $5.34 \pm 0.1$ & 0.002 & $24.334 \pm 0.456$ & 0.009 & & 51.482972 & 179.123833 \\
\hline & GREN-CN-CT2-1 & FAUNA & 7/7/2011 & $6.49 \pm 0.18$ & 0.001 & $31.632 \pm 0.877$ & 0.005 & & 51.472611 & 179.151111 \\
\hline & GREN-CN-CT2-2 & FAUNA & $7 / 7 / 2011$ & $2.51 \pm 0.02$ & 0.002 & $11.517 \pm 0.092$ & 0.009 & & 51.472611 & 179.151111 \\
\hline & GREN-CN-CT2-3 & FAUNA & $7 / 7 / 2011$ & $4.3 \pm 0.04$ & 0.002 & $19.843 \pm 0.185$ & 0.009 & & 51.472611 & 179.151111 \\
\hline & GREN-CN-CT3-1 & FAUNA & 7/5/2011 & $4.79 \pm 0.06$ & 0.002 & $21.83 \pm 0.273$ & 0.009 & & 51.471416 & 179.172861 \\
\hline & GREN-CN-CT3-2 & FAUNA & $7 / 5 / 2011$ & $7.9 \pm 0.2$ & 0.002 & $34.411 \pm 0.871$ & 0.009 & & 51.471416 & 179.172861 \\
\hline & GREN-CN-CT3-3 & FAUNA & $7 / 5 / 2011$ & $3.32 \pm 0.04$ & 0.002 & $15.245 \pm 0.184$ & 0.009 & & 51.471416 & 179.172861 \\
\hline & GREN-CN-DUP & FAUNA & $7 / 8 / 2011$ & $4.45 \pm 0.06$ & 0.002 & $21.461 \pm 0.289$ & 0.01 & & 51.482972 & 179.123833 \\
\hline Halibut - whole & HALI-CN-C2M-1 & FAUNA & 7/13/2011 & $0.204 \pm 0.004$ & 0.001 & $0.789 \pm 0.015$ & 0.004 & & 51.40025 & 179.283361 \\
\hline \multirow{3}{*}{$\begin{array}{l}\text { Horse Mussel - } \\
\text { soft tissue only }\end{array}$} & HMUS-CN-CT1-1 & FAUNA & $7 / 8 / 2011$ & $20.2 \pm 0.2$ & 0.002 & $83.043 \pm 0.822$ & 0.008 & & 51.482972 & 179.123833 \\
\hline & HMUS-CN-CT2-1 & FAUNA & 7/7/2011 & $54.1 \pm 0.4$ & 0.002 & $245.15 \pm 1.813$ & 0.009 & & 51.472611 & 179.151111 \\
\hline & HMUS-CN-CT3-1 & FAUNA & $7 / 5 / 2011$ & $41.9 \pm 0.6$ & 0.002 & $171.88 \pm 2.461$ & 0.008 & & 51.471416 & 179.172861 \\
\hline \multirow{9}{*}{ | Irish Lord - whole } & IRLO-CN-CT1-1 & FAUNA & $7 / 8 / 2011$ & $4.07 \pm 0.04$ & 0.002 & $18.342 \pm 0.18$ & 0.009 & & 51.482972 & 179.123833 \\
\hline & IRLO-CN-CT1-2 & FAUNA & $7 / 8 / 2011$ & $10.1 \pm 0.2$ & 0.002 & $50.623 \pm 1.002$ & 0.01 & & 51.482972 & 179.123833 \\
\hline & IRLO-CN-CT1-3 & FAUNA & $7 / 8 / 2011$ & $7.94 \pm 0.06$ & 0.002 & $37.299 \pm 0.282$ & 0.009 & & 51.482972 & 179.123833 \\
\hline & IRLO-CN-CT2-1 & FAUNA & 7/7/2011 & $6.33 \pm 0.04$ & 0.002 & $29.831 \pm 0.189$ & 0.009 & & 51.472611 & 179.151111 \\
\hline & IRLO-CN-CT2-2 & FAUNA & 7/7/2011 & $8.8 \pm 0.1$ & 0.002 & $40.206 \pm 0.457$ & 0.009 & & 51.472611 & 179.151111 \\
\hline & IRLO-CN-CT2-3 & FAUNA & 7/7/2011 & $4.16 \pm 0.04$ & 0.002 & $20.475 \pm 0.197$ & 0.01 & & 51.472611 & 179.151111 \\
\hline & IRLO-CN-CT3-1 & FAUNA & $7 / 5 / 2011$ & $5.57 \pm 0.06$ & 0.001 & $28.699 \pm 0.309$ & 0.005 & & 51.471416 & 179.172861 \\
\hline & IRLO-CN-CT3-2 & FAUNA & 7/5/2011 & $3.91 \pm 0.02$ & 0.001 & $18.957 \pm 0.097$ & 0.005 & & 51.471416 & 179.172861 \\
\hline & IRLO-CN-CT3-3 & FAUNA & $7 / 5 / 2011$ & $2.68 \pm 0.02$ & 0.002 & $12.848 \pm 0.096$ & 0.01 & & 51.471416 & 179.172861 \\
\hline Octopus & OCTP-CN-C2M-1 & FAUNA & $7 / 8 / 2011$ & $9.27 \pm 0.06$ & 0.001 & $45.431 \pm 0.294$ & 0.005 & & 51.482972 & 179.123833 \\
\hline $\begin{array}{l}\text { Pacific Cod - } \\
\text { whole }\end{array}$ & PCOD-CN-C2M-1 & FAUNA & 7/6/2011 & $0.79 \pm 0.02$ & 0.001 & $3.617 \pm 0.092$ & 0.005 & & 51.483527 & 179.187583 \\
\hline
\end{tabular}




\begin{tabular}{|c|c|c|c|c|c|c|c|c|c|c|}
\hline Matrix Subtype & Location Code & Matrix & $\begin{array}{c}\text { Date } \\
\text { Sampled }\end{array}$ & $\begin{array}{l}\text { Wet Result } \pm \\
\text { Uncertainty }\end{array}$ & $\begin{array}{c}\text { Wet } \\
\text { Detection } \\
\text { Limit }\end{array}$ & $\begin{array}{l}\text { Dry Result } \pm \\
\text { Uncertainty }\end{array}$ & $\begin{array}{c}\text { Dry } \\
\text { Detection } \\
\text { Limit }\end{array}$ & Qualifiers & Latitude & Longitude \\
\hline \multirow{10}{*}{ Rockfish - whole } & ROCK-CN-CT1-1 & FAUNA & $7 / 8 / 2011$ & $1.93 \pm 0.06$ & 0.002 & $7.625 \pm 0.237$ & 0.008 & & 51.482972 & 179.123833 \\
\hline & ROCK-CN-CT1-2 & FAUNA & 7/8/2011 & $3.33 \pm 0.12$ & 0.002 & $12.843 \pm 0.463$ & 0.008 & & 51.482972 & \begin{tabular}{|l|}
179.123833 \\
\end{tabular} \\
\hline & ROCK-CN-CT1-3 & FAUNA & $7 / 8 / 2011$ & $8.16 \pm 0.04$ & 0.002 & $32.79 \pm 0.161$ & 0.008 & & 51.482972 & 179.123833 \\
\hline & ROCK-CN-CT2-1 & FAUNA & 7/6/2011 & $3.2 \pm 0.04$ & 0.002 & $11.302 \pm 0.141$ & 0.007 & & 51.472611 & 179.151111 \\
\hline & ROCK-CN-CT2-2 & FAUNA & $7 / 6 / 2011$ & $5.36 \pm 0.06$ & 0.002 & $19.622 \pm 0.22$ & 0.007 & & 51.472611 & 179.151111 \\
\hline & ROCK-CN-CT2-3 & \begin{tabular}{|l|} 
FAUNA \\
\end{tabular} & $7 / 6 / 2011$ & $5.61 \pm 0.12$ & 0.002 & $22.948 \pm 0.491$ & 0.008 & & 51.472611 & \begin{tabular}{|l|}
179.151111 \\
\end{tabular} \\
\hline & ROCK-CN-CT3-1 & FAUNA & 7/6/2011 & $5.51 \pm 0.04$ & 0.002 & $23.531 \pm 0.171$ & 0.009 & & 51.471416 & \begin{tabular}{|l|}
179.172861 \\
\end{tabular} \\
\hline & ROCK-CN-CT3-2 & FAUNA & $7 / 6 / 2011$ & $5.4 \pm 0.1$ & 0.002 & $20.749 \pm 0.384$ & 0.008 & & 51.471416 & 179.172861 \\
\hline & ROCK-CN-CT3-3 & FAUNA & $7 / 6 / 2011$ & $7.92 \pm 0.16$ & 0.002 & $33.1 \pm 0.669$ & 0.008 & & 51.471416 & \begin{tabular}{|l|}
179.172861 \\
\end{tabular} \\
\hline & ROCK-CN-DUP & FAUNA & $7 / 6 / 2011$ & $4.53 \pm 0.04$ & 0.002 & $18.194 \pm 0.161$ & 0.008 & & 51.471416 & \begin{tabular}{|l|}
179.172861 \\
\end{tabular} \\
\hline Reindeer Lichen & STRL-AI-CT1-1 & VEG & $6 / 23 / 2011$ & $9.8 \pm 0.2$ & 0.003 & $15.536 \pm 0.317$ & 0.005 & & 51.480583 & 179.109472 \\
\hline \multirow{3}{*}{$\begin{array}{l}\text { Sea Urchin - } \\
\text { whole }\end{array}$} & URCH-CN-CT1-1 & FAUNA & $7 / 8 / 2011$ & $16.3 \pm 0.2$ & 0.002 & $65.4 \pm 0.802$ & 0.008 & & 51.482972 & 179.123833 \\
\hline & URCH-CN-CT2-1 & FAUNA & 7/7/2011 & $13.9 \pm 0.2$ & 0.002 & $47.63 \pm 0.685$ & 0.007 & & 51.472611 & \begin{tabular}{|l|}
179.151111 \\
\end{tabular} \\
\hline & URCH-CN-CT3-1 & FAUNA & 7/5/2011 & $13.5 \pm 0.2$ & 0.002 & $51.711 \pm 0.766$ & 0.008 & & 51.471416 & \begin{tabular}{|l|}
179.172861 \\
\end{tabular} \\
\hline \multicolumn{11}{|c|}{ Amchitka Site (Adak Island) (AMC13) } \\
\hline $\begin{array}{l}\text { Dolly Varden - } \\
\text { whole }\end{array}$ & DOLL-AD-XXX-1 & FAUNA & $6 / 28 / 2011$ & $0.363 \pm 0.002$ & 0.001 & $1.481 \pm 0.008$ & 0.004 & & 51.8375 & -176.672555 \\
\hline \multirow{3}{*}{ Reindeer Lichen } & STRL-AD-XXX-1 & VEG & $6 / 28 / 2011$ & $3.59 \pm 0.1$ & 0.002 & $7.027 \pm 0.196$ & 0.004 & & 51.837527 & \begin{tabular}{|l|}
-176.670694 \\
\end{tabular} \\
\hline & $\begin{array}{l}\text { STRL-AD-XXX-2 } \\
\end{array}$ & VEG & $6 / 29 / 2011$ & $3.33 \pm 0.1$ & 0.002 & $8.228 \pm 0.247$ & 0.005 & & 51.872833 & -176.707444 \\
\hline & STRL-AD-XXX-3 & VEG & $6 / 29 / 2011$ & $5.5 \pm 0.2$ & 0.002 & $8.738 \pm 0.318$ & 0.003 & & 51.900805 & -176.688527 \\
\hline \multicolumn{11}{|c|}{ Amchitka Site (Adak Island North) (AMC14) } \\
\hline \multirow{3}{*}{ Chiton } & CHIT-AN-ANT1-1 & FAUNA & $7 / 20 / 2011$ & $11 \pm 0.2$ & 0.002 & $53.573 \pm 0.974$ & 0.01 & & 51.838722 & -176.582805 \\
\hline & CHIT-AN-ANT2-1 & FAUNA & $7 / 18 / 2011$ & $7.5 \pm 0.2$ & 0.002 & $37.592 \pm 1.002$ & 0.01 & & 51.848361 & \begin{tabular}{|l}
-176.506277 \\
\end{tabular} \\
\hline & CHIT-AN-ANT3-1 & FAUNA & $7 / 17 / 2011$ & $6.6 \pm 0.2$ & 0.001 & $33.562 \pm 1.017$ & 0.005 & & 51.853722 & -176.470694 \\
\hline \multirow{10}{*}{ Dragon Kelp } & EUAL-AN-ANT1-1 & VEG & $7 / 19 / 2011$ & $13.1 \pm 0.2$ & 0.002 & $109.845 \pm 1.677$ & 0.017 & & 51.835694 & \begin{tabular}{|l|}
-176.542138 \\
\end{tabular} \\
\hline & EUAL-AN-ANT1-2 & VEG & $7 / 19 / 2011$ & $14 \pm 0.2$ & 0.002 & $95.434 \pm 1.363$ & 0.014 & & 51.835694 & \begin{tabular}{|l|}
-176.542138 \\
\end{tabular} \\
\hline & EUAL-AN-ANT1-3 & VEG & $7 / 19 / 2011$ & $12.1 \pm 0.4$ & 0.002 & $93.523 \pm 3.092$ & 0.015 & & 51.835694 & -176.542138 \\
\hline & EUAL-AN-ANT2-1 & VEG & $7 / 18 / 2011$ & $21.9 \pm 0.4$ & 0.002 & $159.643 \pm 2.916$ & 0.015 & & 51.848361 & -176.506277 \\
\hline & EUAL-AN-ANT2-2 & VEG & $7 / 18 / 2011$ & $27.7 \pm 0.6$ & 0.002 & $176.517 \pm 3.823$ & 0.013 & & 51.848361 & -176.506277 \\
\hline & EUAL-AN-ANT2-3 & VEG & $7 / 18 / 2011$ & $16.7 \pm 0.2$ & 0.002 & $115.996 \pm 1.389$ & 0.014 & & 51.848361 & -176.506277 \\
\hline & EUAL-AN-ANT3-1 & VEG & $7 / 17 / 2011$ & $6.3 \pm 0.2$ & 0.001 & $47.087 \pm 1.495$ & 0.007 & & 51.853722 & -176.470694 \\
\hline & EUAL-AN-ANT3-2 & VEG & $7 / 17 / 2011$ & $23.7 \pm 0.2$ & 0.001 & $161.615 \pm 1.364$ & 0.007 & & 51.853722 & \begin{tabular}{|l|}
-176.470694 \\
\end{tabular} \\
\hline & EUAL-AN-ANT3-3 & VEG & $7 / 17 / 2011$ & $14.2 \pm 0.2$ & 0.002 & $95.29 \pm 1.342$ & 0.013 & & 51.853722 & \begin{tabular}{|l|}
-176.470694 \\
\end{tabular} \\
\hline & EUAL-AN-DUP & VEG & $7 / 17 / 2011$ & $38.2 \pm 0.8$ & 0.002 & $240.195 \pm 5.03$ & 0.013 & & 51.853722 & \begin{tabular}{|l|}
-176.470694 \\
\end{tabular} \\
\hline
\end{tabular}




\begin{tabular}{|c|c|c|c|c|c|c|c|c|c|c|}
\hline Matrix Subtype & Location Code & Matrix & $\begin{array}{c}\text { Date } \\
\text { Sampled }\end{array}$ & $\begin{array}{l}\text { Wet Result } \pm \\
\text { Uncertainty }\end{array}$ & $\begin{array}{c}\text { Wet } \\
\text { Detection } \\
\text { Limit }\end{array}$ & $\begin{array}{l}\text { Dry Result } \pm \\
\text { Uncertainty }\end{array}$ & \begin{tabular}{|c} 
Dry \\
Detection \\
Limit
\end{tabular} & Qualifiers & Latitude & Longitude \\
\hline \multirow{10}{*}{ Rockweed } & FUCU-AN-ANT1-1 & VEG & \begin{tabular}{|l|}
$7 / 19 / 2011$ \\
\end{tabular} & $68.8 \pm 0.6$ & 0.002 & $350.53 \pm 3.057$ & 0.01 & & 51.841444 & -176.546083 \\
\hline & \begin{tabular}{|l|} 
FUCU-AN-ANT1-2 \\
\end{tabular} & VEG & \begin{tabular}{|l|}
$7 / 20 / 2011$ \\
\end{tabular} & $39.8 \pm 0.6$ & 0.002 & $256.757 \pm 3.871$ & 0.013 & & 51.8395 & -176.591305 \\
\hline & \begin{tabular}{|l|} 
FUCU-AN-ANT1-3 \\
\end{tabular} & VEG & \begin{tabular}{|l|}
$7 / 20 / 2011$ \\
\end{tabular} & $64 \pm 1$ & 0.002 & $349.272 \pm 5.457$ & 0.011 & & 51.8395 & -176.591305 \\
\hline & \begin{tabular}{|l|} 
FUCU-AN-ANT2-1 \\
\end{tabular} & VEG & \begin{tabular}{|l|}
$7 / 18 / 2011$ \\
\end{tabular} & $65 \pm 2$ & 0.002 & $410.488 \pm 12.63$ & 0.013 & & 51.837666 & -176.517527 \\
\hline & \begin{tabular}{|l|} 
FUCU-AN-ANT2-2 \\
\end{tabular} & VEG & 7/18/2011 & $37 \pm 2$ & 0.002 & $196.682 \pm 10.631$ & 0.011 & & 51.841444 & -176.546083 \\
\hline & \begin{tabular}{|l|} 
FUCU-AN-ANT2-3 \\
\end{tabular} & VEG & \begin{tabular}{|l|}
$7 / 18 / 2011$ \\
\end{tabular} & $95 \pm 2$ & 0.002 & $400.75 \pm 8.437$ & 0.008 & & 51.8395 & -176.591305 \\
\hline & \begin{tabular}{|l|} 
FUCU-AN-ANT3-1 \\
\end{tabular} & VEG & \begin{tabular}{|l|}
$7 / 18 / 2011$ \\
\end{tabular} & $50.9 \pm 0.8$ & 0.002 & $291.22 \pm 4.577$ & 0.011 & & 51.839944 & -176.515916 \\
\hline & \begin{tabular}{|l|} 
FUCU-AN-ANT3-2 \\
\end{tabular} & VEG & \begin{tabular}{|l|}
$7 / 18 / 2011$ \\
\end{tabular} & $74 \pm 2$ & 0.002 & \begin{tabular}{|l|}
$361.738 \pm 9.777$ \\
\end{tabular} & 0.01 & & 51.828972 & -176.448305 \\
\hline & \begin{tabular}{|l|} 
FUCU-AN-ANT3-3 \\
\end{tabular} & VEG & \begin{tabular}{|l|}
$7 / 18 / 2011$ \\
\end{tabular} & $70.3 \pm 0.6$ & 0.001 & $349.514 \pm 2.983$ & 0.005 & & 51.830833 & -176.456361 \\
\hline & FUCU-AN-DUP & VEG & \begin{tabular}{|l|}
$7 / 18 / 2011$ \\
\end{tabular} & $120 \pm 4$ & 0.002 & $558.399 \pm 18.613$ & 0.009 & & 51.828972 & -176.448305 \\
\hline \multirow{10}{*}{ Greenling - whole } & GREN-AN-ANT1-1 & FAUNA & \begin{tabular}{|l|}
$7 / 19 / 2011$ \\
\end{tabular} & $3.16 \pm 0.04$ & 0.002 & $14.027 \pm 0.178$ & 0.009 & & 51.835694 & -176.542138 \\
\hline & \begin{tabular}{|l|} 
GREN-AN-ANT1-2 \\
\end{tabular} & FAUNA & 7/19/2011 & $1.59 \pm 0.04$ & 0.002 & $6.641 \pm 0.167$ & 0.008 & & 51.835694 & -176.542138 \\
\hline & \begin{tabular}{|l|} 
GREN-AN-ANT1-3 \\
\end{tabular} & FAUNA & \begin{tabular}{|l|}
$7 / 19 / 2011$ \\
\end{tabular} & $6.4 \pm 0.2$ & 0.002 & $23.31 \pm 0.728$ & 0.007 & & 51.835694 & -176.542138 \\
\hline & \begin{tabular}{|l|} 
GREN-AN-ANT2-1 \\
\end{tabular} & FAUNA & 7/18/2011 & $7.3 \pm 0.2$ & 0.002 & $33.773 \pm 0.925$ & 0.009 & & 51.848361 & -176.506277 \\
\hline & \begin{tabular}{|l|} 
GREN-AN-ANT2-2 \\
\end{tabular} & FAUNA & \begin{tabular}{|l|}
$7 / 18 / 2011$ \\
\end{tabular} & $4.02 \pm 0.1$ & 0.001 & $18.632 \pm 0.463$ & 0.005 & & 51.848361 & -176.506277 \\
\hline & \begin{tabular}{|l} 
GREN-AN-ANT2-3 \\
\end{tabular} & FAUNA & \begin{tabular}{|l|}
$7 / 18 / 2011$ \\
\end{tabular} & $3.52 \pm 0.04$ & 0.002 & $16.691 \pm 0.19$ & 0.009 & & 51.848361 & -176.506277 \\
\hline & \begin{tabular}{|l} 
GREN-AN-ANT3-1 \\
\end{tabular} & FAUNA & \begin{tabular}{|l|}
$7 / 17 / 2011$ \\
\end{tabular} & $3.54 \pm 0.06$ & 0.002 & $16.157 \pm 0.274$ & 0.009 & & 51.853722 & -176.470694 \\
\hline & 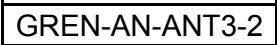 & FAUNA & 7/17/2011 & $1.47 \pm 0.02$ & 0.002 & $6.826 \pm 0.093$ & 0.009 & & 51.853722 & -176.470694 \\
\hline & \begin{tabular}{|l|} 
GREN-AN-ANT3-3 \\
\end{tabular} & FAUNA & 7/17/2011 & $3.28 \pm 0.06$ & 0.002 & $15.263 \pm 0.279$ & 0.009 & & 51.853722 & -176.470694 \\
\hline & GREN-AN-DUP & FAUNA & 7/17/2011 & $2.85 \pm 0.04$ & 0.002 & $13.575 \pm 0.191$ & 0.01 & & 51.853722 & -176.470694 \\
\hline \multirow{2}{*}{ Halibut - whole } & \begin{tabular}{|l} 
HALI-AN-AN2M-1 \\
\end{tabular} & FAUNA & $7 / 17 / 2011$ & $0.86 \pm 0.02$ & 0.002 & $2.871 \pm 0.067$ & 0.007 & & 51.853722 & -176.470694 \\
\hline & HALI-AN-DUP & FAUNA & $7 / 17 / 2011$ & $0.82 \pm 0.02$ & 0.002 & $2.718 \pm 0.066$ & 0.007 & & 51.853722 & -176.470694 \\
\hline \multirow{3}{*}{$\begin{array}{l}\text { Horse Mussel - } \\
\text { soft tissue only }\end{array}$} & HMUS-AN-ANT1-1 & FAUNA & $7 / 20 / 2011$ & $19 \pm 0.2$ & 0.001 & $103.881 \pm 1.093$ & 0.005 & & 51.838722 & -176.582805 \\
\hline & \begin{tabular}{|l|} 
HMUS-AN-ANT2-1 \\
\end{tabular} & FAUNA & \begin{tabular}{|l|}
$7 / 18 / 2011$ \\
\end{tabular} & $41.1 \pm 0.6$ & 0.002 & \begin{tabular}{|l|l}
$188.741 \pm 2.755$ \\
\end{tabular} & 0.009 & & 51.848361 & -176.506277 \\
\hline & \begin{tabular}{|l|} 
HMUS-AN-ANT3-1 \\
\end{tabular} & FAUNA & \begin{tabular}{|l|}
$7 / 18 / 2011$ \\
\end{tabular} & $6.8 \pm 0.2$ & 0.001 & $30.306 \pm 0.891$ & 0.004 & & 51.853722 & -176.470694 \\
\hline \multirow{9}{*}{ |rish Lord - whole } & \begin{tabular}{|l|} 
IRLO-AN-ANT1-1 \\
\end{tabular} & FAUNA & 7/19/2011 & $3.8 \pm 0.02$ & 0.002 & $17.835 \pm 0.094$ & 0.009 & & 51.835694 & -176.542138 \\
\hline & \begin{tabular}{|l|} 
IRLO-AN-ANT1-2 \\
\end{tabular} & FAUNA & \begin{tabular}{|l|}
$7 / 19 / 2011$ \\
\end{tabular} & $2.27 \pm 0.02$ & 0.002 & $10.077 \pm 0.089$ & 0.009 & & 51.835694 & -176.542138 \\
\hline & \begin{tabular}{|l|} 
IRLO-AN-ANT1-3 \\
\end{tabular} & FAUNA & \begin{tabular}{|l|}
$7 / 19 / 2011$ \\
\end{tabular} & $3.18 \pm 0.06$ & 0.002 & $13.974 \pm 0.264$ & 0.009 & & 51.835694 & -176.542138 \\
\hline & \begin{tabular}{|l|} 
IRLO-AN-ANT2-1 \\
\end{tabular} & FAUNA & 7/19/2011 & $2.92 \pm 0.04$ & 0.002 & $13.845 \pm 0.19$ & 0.009 & & 51.848361 & -176.506277 \\
\hline & \begin{tabular}{|l|} 
IRLO-AN-ANT2-2 \\
\end{tabular} & FAUNA & \begin{tabular}{|l|}
$7 / 19 / 2011$ \\
\end{tabular} & $3.39 \pm 0.04$ & 0.002 & $15.704 \pm 0.185$ & 0.009 & & 51.848361 & -176.506277 \\
\hline & \begin{tabular}{|l|} 
IRLO-AN-ANT2-3 \\
\end{tabular} & FAUNA & \begin{tabular}{|l|}
$7 / 19 / 2011$ \\
\end{tabular} & $10.31 \pm 0.08$ & 0.002 & $49.149 \pm 0.381$ & 0.01 & & 51.848361 & -176.506277 \\
\hline & \begin{tabular}{|l|} 
IRLO-AN-ANT3-1 \\
\end{tabular} & FAUNA & \begin{tabular}{|l|}
$7 / 17 / 2011$ \\
\end{tabular} & $6.08 \pm 0.08$ & 0.002 & $29.163 \pm 0.384$ & 0.01 & & 51.853722 & -176.470694 \\
\hline & \begin{tabular}{|l|} 
IRLO-AN-ANT3-2 \\
\end{tabular} & FAUNA & $7 / 17 / 2011$ & $1.5 \pm 0.02$ & 0.002 & $6.767 \pm 0.09$ & 0.009 & & 51.853722 & -176.470694 \\
\hline & \begin{tabular}{|l|} 
IRLO-AN-ANT3-3 \\
\end{tabular} & FAUNA & 7/17/2011 & $2.65 \pm 0.02$ & 0.002 & $11.907 \pm 0.09$ & 0.009 & & 51.853722 & -176.470694 \\
\hline Octopus & OCTP-AN-AN2M-1 & FAUNA & \begin{tabular}{|l|}
$7 / 18 / 2011$ \\
\end{tabular} & $10.7 \pm 0.2$ & 0.002 & $54.153 \pm 1.012$ & 0.01 & & 51.848361 & -176.506277 \\
\hline
\end{tabular}




\begin{tabular}{|c|c|c|c|c|c|c|c|c|c|c|}
\hline Matrix Subtype & Location Code & Matrix & $\begin{array}{c}\text { Date } \\
\text { Sampled }\end{array}$ & $\begin{array}{l}\text { Wet Result } \pm \\
\text { Uncertainty }\end{array}$ & $\begin{array}{c}\text { Wet } \\
\text { Detection } \\
\text { Limit }\end{array}$ & $\begin{array}{l}\text { Dry Result } \pm \\
\text { Uncertainty }\end{array}$ & \begin{tabular}{|c|} 
Dry \\
Detection \\
Limit
\end{tabular} & Qualifiers & Latitude & Longitude \\
\hline $\begin{array}{l}\text { Pacific Cod - } \\
\text { whole }\end{array}$ & PCOD-AN-AN2M-1 & FAUNA & $7 / 17 / 2011$ & $1.18 \pm 0.02$ & 0.001 & $5.74 \pm 0.097$ & 0.005 & & 51.853722 & $\mid-176.470694$ \\
\hline \multirow{6}{*}{ Rockfish - whole } & ROCK-AN-ANT1-1 & FAUNA & $7 / 19 / 2011$ & $11.4 \pm 0.2$ & 0.002 & $42.175 \pm 0.74$ & 0.007 & & 51.835694 & -176.542138 \\
\hline & \begin{tabular}{|l|} 
ROCK-AN-ANT2-1 \\
\end{tabular} & FAUNA & $7 / 18 / 2011$ & $1.16 \pm 0.02$ & 0.001 & $4.238 \pm 0.073$ & 0.004 & & 51.848361 & -176.506277 \\
\hline & ROCK-AN-ANT2-2 & FAUNA & $7 / 18 / 2011$ & $1.4 \pm 0.2$ & 0.002 & $5.721 \pm 0.817$ & 0.008 & & 51.848361 & -176.506277 \\
\hline & \begin{tabular}{|l|} 
ROCK-AN-ANT3-1 \\
\end{tabular} & \begin{tabular}{|l|} 
FAUNA \\
\end{tabular} & $7 / 17 / 2011$ & $2.49 \pm 0.04$ & 0.001 & $8.872 \pm 0.143$ & 0.004 & & 51.853722 & \begin{tabular}{|l|}
-176.470694 \\
\end{tabular} \\
\hline & ROCK-AN-ANT3-2 & FAUNA & $7 / 17 / 2011$ & $0.96 \pm 0.02$ & 0.002 & $3.703 \pm 0.077$ & 0.008 & & 51.853722 & -176.470694 \\
\hline & ROCK-AN-ANT3-3 & FAUNA & $7 / 17 / 2011$ & $1.98 \pm 0.04$ & 0.002 & $7.772 \pm 0.157$ & 0.008 & & 51.853722 & -176.470694 \\
\hline \multirow{3}{*}{$\begin{array}{l}\text { Sea Urchin - } \\
\text { whole }\end{array}$} & URCH-AN-ANT1-1 & FAUNA & $7 / 19 / 2011$ & $4.71 \pm 0.06$ & 0.002 & $16.05 \pm 0.204$ & 0.007 & & 51.835694 & -176.542138 \\
\hline & URCH-AN-ANT2-1 & FAUNA & $7 / 18 / 2011$ & $13.3 \pm 0.2$ & 0.002 & $48.45 \pm 0.729$ & 0.007 & & 51.848361 & -176.506277 \\
\hline & URCH-AN-ANT3-1 & FAUNA & $7 / 17 / 2011$ & $14.2 \pm 0.2$ & 0.002 & $53.98 \pm 0.76$ & 0.008 & & 51.853722 & -176.470694 \\
\hline \multicolumn{11}{|c|}{ Amchitka Site (Adak Island South) (AMC15) } \\
\hline \multirow{3}{*}{ Chiton } & \begin{tabular}{|l|} 
CHIT-AS-AST1-1 \\
\end{tabular} & FAUNA & $7 / 15 / 2011$ & $22 \pm 0.4$ & 0.002 & $90.399 \pm 1.644$ & 0.008 & & 51.737472 & -176.48025 \\
\hline & CHIT-AS-AST2-1 & FAUNA & $7 / 16 / 2011$ & $3.6 \pm 0.04$ & 0.002 & $18.428 \pm 0.205$ & 0.01 & & 51.745666 & -176.507055 \\
\hline & \begin{tabular}{|l|} 
CHIT-AS-AST3-1 \\
\end{tabular} & FAUNA & $7 / 14 / 2011$ & $23.2 \pm 0.2$ & 0.002 & $109.287 \pm 0.942$ & 0.009 & & 51.688333 & \begin{tabular}{|l|}
-176.63375 \\
\end{tabular} \\
\hline \multirow{10}{*}{ Dragon Kelp } & EUAL-AS-AST1-1 & VEG & $7 / 15 / 2011$ & $21.1 \pm 0.6$ & 0.001 & $139.043 \pm 3.954$ & 0.007 & & 51.737472 & \begin{tabular}{|l|}
-176.48025 \\
\end{tabular} \\
\hline & \begin{tabular}{|l|} 
EUAL-AS-AST1-2 \\
\end{tabular} & VEG & $7 / 15 / 2011$ & $15.1 \pm 0.2$ & 0.002 & $120.265 \pm 1.593$ & 0.016 & & 51.737472 & -176.48025 \\
\hline & \begin{tabular}{|l|} 
EUAL-AS-AST1-3 \\
\end{tabular} & VEG & $7 / 15 / 2011$ & $7.5 \pm 0.2$ & 0.002 & $71.973 \pm 1.919$ & 0.019 & & 51.737472 & -176.48025 \\
\hline & \begin{tabular}{|l|} 
EUAL-AS-AST2-1 \\
\end{tabular} & VEG & $7 / 16 / 2011$ & $13.1 \pm 0.4$ & 0.002 & $93.138 \pm 2.844$ & 0.014 & & 51.745666 & \begin{tabular}{|l}
-176.507055 \\
\end{tabular} \\
\hline & \begin{tabular}{|l|} 
EUAL-AS-AST2-2 \\
\end{tabular} & VEG & $7 / 16 / 2011$ & $17.1 \pm 0.2$ & 0.001 & $120.996 \pm 1.415$ & 0.007 & & 51.745666 & -176.507055 \\
\hline & \begin{tabular}{|l|} 
EUAL-AS-AST2-3 \\
\end{tabular} & VEG & $7 / 16 / 2011$ & $25.4 \pm 0.6$ & 0.002 & $184.524 \pm 4.359$ & 0.015 & & 51.745666 & \begin{tabular}{|l|}
-176.507055 \\
\end{tabular} \\
\hline & \begin{tabular}{|l|} 
EUAL-AS-AST3-1 \\
\end{tabular} & VEG & $7 / 14 / 2011$ & $20.89 \pm 0.06$ & 0.002 & $152.584 \pm 0.438$ & 0.015 & & 51.688333 & \begin{tabular}{|l|}
-176.63375 \\
\end{tabular} \\
\hline & \begin{tabular}{|l|} 
EUAL-AS-AST3-2 \\
\end{tabular} & VEG & $7 / 14 / 2011$ & $7.6 \pm 0.2$ & 0.002 & $72.036 \pm 1.896$ & 0.019 & & 51.688333 & -176.63375 \\
\hline & \begin{tabular}{|l|} 
EUAL-AS-AST3-3 \\
\end{tabular} & VEG & $7 / 14 / 2011$ & $10.8 \pm 0.4$ & 0.001 & $95.227 \pm 3.527$ & 0.009 & & 51.688333 & -176.63375 \\
\hline & EUAL-AS-DUP & VEG & $7 / 14 / 2011$ & $1.18 \pm 0.02$ & 0.0002 & $8.683 \pm 0.147$ & 0.001 & & 51.688333 & -176.63375 \\
\hline Rockweed & FUCU-AS-AST1-1 & VEG & $7 / 16 / 2011$ & $53 \pm 2$ & 0.002 & $326.938 \pm 12.337$ & 0.012 & & 51.733666 & -176.470805 \\
\hline \multirow{9}{*}{ Rockweed } & \begin{tabular}{|l|} 
FUCU-AS-AST1-2 \\
\end{tabular} & VEG & $7 / 16 / 2011$ & $48 \pm 2$ & 0.002 & $266.256 \pm 11.094$ & 0.011 & & 51.733666 & -176.470805 \\
\hline & \begin{tabular}{|l|} 
FUCU-AS-AST1-3 \\
\end{tabular} & VEG & $7 / 16 / 2011$ & $71 \pm 2$ & 0.002 & $385.364 \pm 10.855$ & 0.011 & & 51.733666 & -176.470805 \\
\hline & \begin{tabular}{|l|} 
FUCU-AS-AST2-1 \\
\end{tabular} & VEG & $7 / 16 / 2011$ & $60.5 \pm 0.8$ & 0.002 & $292.435 \pm 3.867$ & 0.01 & & 51.745666 & \begin{tabular}{|l}
-176.507055 \\
\end{tabular} \\
\hline & \begin{tabular}{|l|} 
FUCU-AS-AST2-2 \\
\end{tabular} & VEG & $7 / 16 / 2011$ & $77 \pm 2$ & 0.002 & $389.766 \pm 10.124$ & 0.01 & & 51.745666 & -176.507055 \\
\hline & \begin{tabular}{|l|} 
FUCU-AS-AST2-3 \\
\end{tabular} & VEG & $7 / 16 / 2011$ & $71 \pm 2$ & 0.002 & $348.791 \pm 9.825$ & 0.01 & & 51.745666 & -176.507055 \\
\hline & \begin{tabular}{|l|} 
FUCU-AS-AST3-1 \\
\end{tabular} & VEG & 7/14/2011 & $60 \pm 2$ & 0.002 & $316.302 \pm 10.543$ & 0.011 & & 51.688333 & -176.63375 \\
\hline & \begin{tabular}{|l|} 
FUCU-AS-AST3-2 \\
\end{tabular} & VEG & $7 / 14 / 2011$ & $46 \pm 2$ & 0.002 & $226.529 \pm 9.849$ & 0.01 & & 51.688333 & -176.63375 \\
\hline & \begin{tabular}{|l|} 
FUCU-AS-AST3-3 \\
\end{tabular} & VEG & $7 / 14 / 2011$ & $38.8 \pm 0.8$ & 0.001 & $203.752 \pm 4.201$ & 0.005 & & 51.688333 & -176.63375 \\
\hline & FUCU-AS-DUP & VEG & $7 / 16 / 2011$ & $95.2 \pm 0.6$ & 0.001 & $492.037 \pm 3.101$ & 0.005 & & 51.733666 & -176.470805 \\
\hline
\end{tabular}




\begin{tabular}{|c|c|c|c|c|c|c|c|c|c|c|}
\hline Matrix Subtype & Location Code & Matrix & $\begin{array}{c}\text { Date } \\
\text { Sampled }\end{array}$ & $\begin{array}{l}\text { Wet Result } \pm \\
\text { Uncertainty }\end{array}$ & $\begin{array}{c}\text { Wet } \\
\text { Detection } \\
\text { Limit }\end{array}$ & $\begin{array}{l}\text { Dry Result } \pm \\
\text { Uncertainty }\end{array}$ & \begin{tabular}{|c|} 
Dry \\
Detection \\
Limit
\end{tabular} & Qualifiers & Latitude & Longitude \\
\hline \multirow{10}{*}{ Greenling - whole } & GREN-AS-AST1-1 & FAUNA & $7 / 15 / 2011$ & $6.83 \pm 0.08$ & 0.002 & $32.538 \pm 0.381$ & 0.01 & & 51.737472 & -176.48025 \\
\hline & GREN-AS-AST1-2 & FAUNA & \begin{tabular}{|l|}
$7 / 15 / 2011$ \\
\end{tabular} & $2.91 \pm 0.04$ & 0.001 & $13.729 \pm 0.189$ & 0.005 & & 51.737472 & -176.48025 \\
\hline & GREN-AS-AST1-3 & FAUNA & \begin{tabular}{|l|}
$7 / 15 / 2011$ \\
\end{tabular} & $3.51 \pm 0.04$ & 0.001 & $16.859 \pm 0.192$ & 0.005 & & 51.737472 & -176.48025 \\
\hline & GREN-AS-AST2-1 & FAUNA & $7 / 16 / 2011$ & $1.13 \pm 0.02$ & 0.001 & $4.539 \pm 0.08$ & 0.004 & & 51.745666 & -176.507055 \\
\hline & GREN-AS-AST2-2 & FAUNA & $7 / 16 / 2011$ & $4.71 \pm 0.08$ & 0.002 & $21.543 \pm 0.366$ & 0.009 & & 51.745666 & -176.507055 \\
\hline & GREN-AS-AST2-3 & FAUNA & \begin{tabular}{|l|}
$7 / 16 / 2011$ \\
\end{tabular} & $2.95 \pm 0.04$ & 0.002 & $13.739 \pm 0.186$ & 0.009 & & 51.745666 & -176.507055 \\
\hline & GREN-AS-AST3-1 & FAUNA & \begin{tabular}{|l|}
$7 / 14 / 2011$ \\
\end{tabular} & $2.15 \pm 0.08$ & 0.001 & $10.24 \pm 0.381$ & 0.005 & & 51.688333 & -176.63375 \\
\hline & GREN-AS-AST3-2 & FAUNA & $7 / 14 / 2011$ & $4.7 \pm 0.2$ & 0.001 & $22.123 \pm 0.941$ & 0.005 & & 51.688333 & -176.63375 \\
\hline & GREN-AS-AST3-3 & FAUNA & 7/14/2011 & $4.65 \pm 0.08$ & 0.002 & $22.207 \pm 0.382$ & 0.01 & & 51.688333 & -176.63375 \\
\hline & GREN-AS-DUP & FAUNA & \begin{tabular}{|l|}
$7 / 14 / 2011$ \\
\end{tabular} & $6.06 \pm 0.08$ & 0.002 & $30.388 \pm 0.401$ & 0.01 & & 51.688333 & $\begin{array}{l}-176.63375 \\
\end{array}$ \\
\hline \multirow{3}{*}{$\begin{array}{l}\text { Horse Mussel - } \\
\text { soft tissue only }\end{array}$} & HMUS-AS-AST1-1 & FAUNA & $7 / 15 / 2011$ & $27.3 \pm 0.2$ & 0.001 & $136.772 \pm 1.002$ & 0.005 & & 51.737472 & -176.48025 \\
\hline & HMUS-AS-AST2-1 & FAUNA & 7/16/2011 & $13 \pm 0.2$ & 0.001 & $82.469 \pm 1.269$ & 0.006 & & 51.745666 & -176.507055 \\
\hline & HMUS-AS-AST3-1 & FAUNA & $7 / 14 / 2011$ & $37.3 \pm 0.4$ & 0.001 & $190.169 \pm 2.039$ & 0.005 & & 51.688333 & -176.63375 \\
\hline \multirow{9}{*}{ Irish Lord - whole } & IRLO-AS-AST1-1 & FAUNA & 7/15/2011 & $5.4 \pm 0.2$ & 0.002 & $23.774 \pm 0.881$ & 0.009 & & 51.737472 & -176.48025 \\
\hline & IRLO-AS-AST1-2 & FAUNA & \begin{tabular}{|l|}
$7 / 15 / 2011$ \\
\end{tabular} & $3.85 \pm 0.04$ & 0.002 & $17.951 \pm 0.187$ & 0.009 & & 51.737472 & -176.48025 \\
\hline & IRLO-AS-AST1-3 & \begin{tabular}{|l|} 
FAUNA \\
\end{tabular} & \begin{tabular}{|l|}
$7 / 15 / 2011$ \\
\end{tabular} & $9.61 \pm 0.12$ & 0.002 & $43.803 \pm 0.547$ & 0.009 & & 51.737472 & -176.48025 \\
\hline & IRLO-AS-AST2-1 & FAUNA & \begin{tabular}{|l|}
$7 / 16 / 2011$ \\
\end{tabular} & $13 \pm 0.2$ & 0.002 & $60.838 \pm 0.936$ & 0.009 & & 51.745666 & -176.507055 \\
\hline & IRLO-AS-AST2-2 & FAUNA & $7 / 16 / 2011$ & $3.99 \pm 0.04$ & 0.002 & $16.987 \pm 0.17$ & 0.009 & & 51.745666 & -176.507055 \\
\hline & IRLO-AS-AST2-3 & FAUNA & 7/16/2011 & $11 \pm 0.2$ & 0.002 & $55.649 \pm 1.012$ & 0.01 & & 51.745666 & -176.507055 \\
\hline & IRLO-AS-AST3-1 & FAUNA & 7/14/2011 & $4.71 \pm 0.02$ & 0.002 & $21.146 \pm 0.09$ & 0.009 & & 51.688333 & -176.63375 \\
\hline & IRLO-AS-AST3-2 & FAUNA & 7/14/2011 & $1.78 \pm 0.02$ & 0.002 & $7.521 \pm 0.085$ & 0.008 & & 51.688333 & -176.63375 \\
\hline & IRLO-AS-AST3-3 & FAUNA & 7/14/2011 & $3.36 \pm 0.02$ & 0.002 & $15.331 \pm 0.091$ & 0.009 & & 51.688333 & -176.63375 \\
\hline $\begin{array}{l}\text { Pacific Cod - } \\
\text { whole }\end{array}$ & PCOD-AS-AS2M-1 & FAUNA & 7/15/2011 & $1.38 \pm 0.02$ & 0.001 & $6.904 \pm 0.1$ & 0.005 & & 51.737472 & -176.48025 \\
\hline \multirow{10}{*}{ Rockfish - whole } & ROCK-AS-AST1-1 & FAUNA & $7 / 15 / 2011$ & $3.55 \pm 0.08$ & 0.002 & $12.763 \pm 0.288$ & 0.007 & & 51.737472 & -176.48025 \\
\hline & ROCK-AS-AST1-2 & FAUNA & \begin{tabular}{|l|}
$7 / 15 / 2011$ \\
\end{tabular} & $2.25 \pm 0.04$ & 0.002 & $8.43 \pm 0.15$ & 0.007 & & 51.737472 & -176.48025 \\
\hline & ROCK-AS-AST1-3 & FAUNA & \begin{tabular}{|l|}
$7 / 15 / 2011$ \\
\end{tabular} & $3.92 \pm 0.06$ & 0.002 & $16.878 \pm 0.258$ & 0.009 & & 51.737472 & -176.48025 \\
\hline & ROCK-AS-AST2-1 & FAUNA & $7 / 16 / 2011$ & $5.36 \pm 0.06$ & 0.002 & $20.556 \pm 0.23$ & 0.008 & & 51.745666 & -176.507055 \\
\hline & ROCK-AS-AST2-2 & FAUNA & 7/16/2011 & $1.1 \pm 0.02$ & 0.002 & $4.086 \pm 0.074$ & 0.007 & & 51.745666 & -176.507055 \\
\hline & ROCK-AS-AST2-3 & FAUNA & 7/16/2011 & $3.71 \pm 0.08$ & 0.002 & $14.317 \pm 0.309$ & 0.008 & & 51.745666 & -176.507055 \\
\hline & ROCK-AS-AST3-1 & FAUNA & \begin{tabular}{|l|}
$7 / 14 / 2011$ \\
\end{tabular} & $9.01 \pm 0.1$ & 0.003 & $36.942 \pm 0.41$ & 0.012 & & 51.688333 & -176.63375 \\
\hline & ROCK-AS-AST3-2 & FAUNA & 7/14/2011 & $9 \pm 0.2$ & 0.002 & $38.16 \pm 0.848$ & 0.008 & & 51.688333 & -176.63375 \\
\hline & ROCK-AS-AST3-3 & FAUNA & $7 / 14 / 2011$ & $2.67 \pm 0.06$ & 0.001 & $11.587 \pm 0.26$ & 0.004 & & 51.688333 & -176.63375 \\
\hline & ROCK-AS-DUP & FAUNA & \begin{tabular}{|l|}
$7 / 14 / 2011$ \\
\end{tabular} & $1.61 \pm 0.02$ & 0.002 & $6.303 \pm 0.078$ & 0.008 & & 51.688333 & -176.63375 \\
\hline
\end{tabular}




\begin{tabular}{|c|c|c|c|c|c|c|c|c|c|c|}
\hline Matrix Subtype & Location Code & Matrix & $\begin{array}{c}\text { Date } \\
\text { Sampled }\end{array}$ & $\begin{array}{l}\text { Wet Result } \pm \\
\text { Uncertainty }\end{array}$ & $\begin{array}{c}\text { Wet } \\
\text { Detection } \\
\text { Limit }\end{array}$ & $\begin{array}{l}\text { Dry Result } \pm \\
\text { Uncertainty }\end{array}$ & \begin{tabular}{|c|} 
Dry \\
Detection \\
Limit
\end{tabular} & Qualifiers & Latitude & Longitude \\
\hline \multirow{3}{*}{$\begin{array}{l}\text { Sea Urchin - } \\
\text { whole }\end{array}$} & URCH-AS-AST1-1 & FAUNA & \begin{tabular}{|l|}
$7 / 15 / 2011$ \\
\end{tabular} & $16.8 \pm 0.2$ & 0.002 & $63.812 \pm 0.76$ & \begin{tabular}{|l|}
0.008 \\
\end{tabular} & & 51.737472 & -176.48025 \\
\hline & URCH-AS-AST2-1 & FAUNA & \begin{tabular}{|l|}
$7 / 16 / 2011$ \\
\end{tabular} & $19.3 \pm 0.4$ & 0.002 & $77.915 \pm 1.615$ & 0.008 & & 51.745666 & -176.507055 \\
\hline & URCH-AS-AST3-1 & FAUNA & \begin{tabular}{|l|}
$7 / 14 / 2011$ \\
\end{tabular} & $17.5 \pm 0.2$ & 0.002 & $65.347 \pm 0.747$ & 0.007 & & 51.688333 & -176.63375 \\
\hline
\end{tabular}

Qualifier definitions:

$U$ - Analytical result below detection limit

$\mathrm{J}$ - Estimated 
Appendix K

\section{Tritium Results}


This page intentionally left blank 


\begin{tabular}{|c|c|c|c|c|c|c|c|c|c|c|c|}
\hline $\begin{array}{c}\text { Matrix } \\
\text { Subtype }\end{array}$ & Location Code & $\begin{array}{c}\text { Date } \\
\text { Sampled }\end{array}$ & Result & Uncertainty & $\begin{array}{c}\text { Detection } \\
\text { Limit }\end{array}$ & $\begin{array}{c}\text { Lab } \\
\text { Qualifiers }\end{array}$ & \begin{tabular}{|c|} 
Data \\
Validation \\
Qualifiers
\end{tabular} & Matrix & Latitude & Longitude & $\begin{array}{c}\text { Salinity } \\
(\% \circ)\end{array}$ \\
\hline \multicolumn{12}{|c|}{ Tritium (pCi/kg) } \\
\hline \multicolumn{12}{|c|}{ Amchitka Site (Long Shot) (AMC02) } \\
\hline & LAKE-AI-LT1-1 & $6 / 22 / 2011$ & 8.8 & 0.3 & 0.3 & & & WATER & 51.432611 & 179.183444 & \\
\hline & LAKE-AI-LT1-1 & $6 / 22 / 2011$ & 10 & 0.3 & 0.3 & & & WATER & 51.432611 & 179.183444 & \\
\hline \multirow{10}{*}{ Sea-Water } & SEAW-LS-DUP & $7 / 12 / 2011$ & 1.7 & 0.3 & 0.3 & & & WATER & 51.442694 & 179.22925 & 35 \\
\hline & SEAW-LS-LT1-1 & $7 / 4 / 2011$ & 2 & 0.3 & 0.3 & & & WATER & 51.457555 & 179.195138 & 36 \\
\hline & SEAW-LS-LT1-2 & $7 / 4 / 2011$ & 1.6 & 0.3 & 0.3 & & & WATER & 51.457555 & 179.195138 & 36 \\
\hline & SEAW-LS-LT1-3 & $7 / 5 / 2011$ & 1.7 & 0.3 & 0.3 & & & WATER & 51.457555 & 179.195138 & 36 \\
\hline & SEAW-LS-LT2-1 & $7 / 3 / 2011$ & 1.8 & 0.3 & 0.3 & & & WATER & 51.44925 & 179.205583 & 33 \\
\hline & SEAW-LS-LT2-2 & $7 / 3 / 2011$ & 1.7 & 0.3 & 0.3 & & & WATER & 51.44925 & 179.205583 & 34 \\
\hline & SEAW-LS-LT2-3 & $7 / 3 / 2011$ & 1.5 & 0.3 & 0.3 & & & WATER & 51.44925 & 179.205583 & 35 \\
\hline & SEAW-LS-LT3-1 & $7 / 3 / 2011$ & 1.9 & 0.3 & 0.3 & & & WATER & 51.43 & 179.22667 & 35 \\
\hline & SEAW-LS-LT3-2 & $7 / 3 / 2011$ & 1.9 & 0.3 & 0.3 & & & WATER & 51.43 & 179.22667 & 35 \\
\hline & SEAW-LS-LT3-3 & $7 / 3 / 2011$ & 1.5 & 0.3 & 0.3 & & & WATER & 51.43 & 179.22667 & 35 \\
\hline \multicolumn{12}{|c|}{ Amchitka Site (Milrow) (AMC03) } \\
\hline \multirow{10}{*}{ Sea-Water } & SEAW-ML-MT1-1 & 7/9/2011 & 2.2 & 0.3 & 0.3 & & & WATER & 51.408472 & 179.139166 & 36 \\
\hline & SEAW-ML-MT1-2 & $7 / 9 / 2011$ & 1.8 & 0.3 & 0.3 & & & WATER & 51.408472 & 179.139166 & 34 \\
\hline & SEAW-ML-MT1-3 & $7 / 9 / 2011$ & 1.6 & 0.3 & 0.3 & & & WATER & 51.408472 & 179.139166 & 36 \\
\hline & SEAW-ML-MT1-3 & $7 / 9 / 2011$ & 1.9 & 0.3 & 0.3 & & & WATER & 51.408472 & 179.139166 & 36 \\
\hline & SEAW-ML-MT2-1 & $7 / 10 / 2011$ & 2 & 0.3 & 0.3 & & & WATER & 51.389416 & 179.173138 & 34 \\
\hline & SEAW-ML-MT2-2 & $7 / 10 / 2011$ & 1.8 & 0.3 & 0.3 & & & WATER & 51.389416 & 179.173138 & 34 \\
\hline & SEAW-ML-MT2-3 & $7 / 10 / 2011$ & 1.9 & 0.3 & 0.3 & & & WATER & 51.389416 & 179.173138 & 34 \\
\hline & SEAW-ML-MT3-1 & $7 / 10 / 2011$ & 1.8 & 0.3 & 0.3 & & & WATER & 51.387805 & 179.184 & 35 \\
\hline & SEAW-ML-MT3-2 & $7 / 10 / 2011$ & 2 & 0.3 & 0.3 & & & WATER & 51.387805 & 179.184 & 35 \\
\hline & SEAW-ML-MT3-3 & $7 / 10 / 2011$ & 1.6 & 0.3 & 0.3 & & & WATER & 51.387805 & 179.184 & 34 \\
\hline \multicolumn{12}{|c|}{ Amchitka Site (Cannikin) (AMC05) } \\
\hline & LAKE-AI-CN-1 & $6 / 24 / 2011$ & 7.9 & 0.3 & 0.3 & & & WATER & 51.468277 & 179.108055 & \\
\hline & LAKE-AI-CN-1 & 6/24/2011 & 7.6 & 0.3 & 0.3 & & & WATER & 51.468277 & 179.108055 & \\
\hline \multirow{6}{*}{ Sea-Water } & SEAW-CN-C2M-1A-D & $7 / 8 / 2011$ & 1.8 & 0.3 & 0.3 & & & WATER & 51.485027 & 179.12075 & 35 \\
\hline & SEAW-CN-C2M-1A-S & $7 / 8 / 2011$ & 1.5 & 0.3 & 0.3 & & & WATER & 51.485027 & 179.12075 & 35 \\
\hline & SEAW-CN-C2M-1B-D & $7 / 8 / 2011$ & 1.8 & 0.3 & 0.3 & & & WATER & 51.482555 & 179.132861 & 35 \\
\hline & SEAW-CN-C2M-1B-S & $7 / 8 / 2011$ & 1.6 & 0.3 & 0.3 & & & WATER & 51.482555 & 179.132861 & 35 \\
\hline & $\begin{array}{l}\text { SEAW-CN-C2M- } \\
\text { 1CAN-D }\end{array}$ & $6 / 24 / 2011$ & 1.6 & 0.3 & 0.3 & & & WATER & 51.48715 & 179.19055 & 34 \\
\hline & $\begin{array}{c}\text { SEAW-CN-C2M- } \\
\text { 1CAN-D } \\
\end{array}$ & 6/24/2011 & 1.7 & 0.3 & 0.3 & & & WATER & 51.48715 & 179.19055 & 34 \\
\hline
\end{tabular}




\begin{tabular}{|c|c|c|c|c|c|c|c|c|c|c|c|c|}
\hline 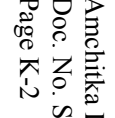 & $\begin{array}{l}\text { Matrix } \\
\text { Subtype }\end{array}$ & Location Code & $\begin{array}{l}\text { Date } \\
\text { Sampled }\end{array}$ & Result & Uncertainty & $\begin{array}{l}\text { Detection } \\
\text { Limit }\end{array}$ & $\begin{array}{c}\text { Lab } \\
\text { Qualifiers }\end{array}$ & $\begin{array}{l}\text { Data } \\
\text { Validation } \\
\text { Qualifiers }\end{array}$ & Matrix & Latitude & Longitude & $\underset{(\%)}{\text { Salinity }}$ \\
\hline $\begin{array}{l}\infty \\
\infty \\
\infty \\
w \\
\omega \\
\omega\end{array}$ & & $\begin{array}{l}\text { SEAW-CN-C2M- } \\
\text { 1CAN-S }\end{array}$ & $6 / 24 / 2011$ & 1.7 & 0.3 & 0.3 & & & WATER & 51.48715 & 179.19055 & 33 \\
\hline $\begin{array}{l}\vec{B} \\
\frac{\hat{W}}{\hat{N}} \\
\frac{1}{2}\end{array}$ & & $\begin{array}{c}\text { SEAW-CN-C2M- } \\
\text { 1CAN-S } \\
\end{array}$ & $6 / 24 / 2011$ & 1.3 & 0.3 & 0.3 & & & WATER & 51.48715 & 179.19055 & 34 \\
\hline 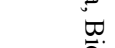 & & SEAW-CN-C2M-1C-D & $7 / 6 / 2011$ & 2.1 & 0.3 & 0.3 & & & WATER & 51.478366 & 179.153183 & 35 \\
\hline$\frac{0}{\circ}$ & & SEAW-CN-C2M-1C-S & $7 / 6 / 2011$ & 1.6 & 0.3 & 0.3 & & & WATER & 51.478366 & 179.153183 & 35 \\
\hline 承. & & SEAW-CN-C2M-1D-D & $7 / 3 / 2011$ & 1.4 & 0.3 & 0.3 & & & WATER & 51.455333 & 179.197483 & 35 \\
\hline 3 & & SEAW-CN-C2M-1D-S & $7 / 3 / 2011$ & 1.7 & 0.3 & 0.3 & & & WATER & 51.455333 & 179.197483 & 35 \\
\hline 兽. & & SEAW-CN-C2M-1E-D & $6 / 24 / 2011$ & 1.6 & 0.3 & 0.3 & & & WATER & 51.465983 & 179.223666 & 35 \\
\hline 官. & & SEAW-CN-C2M-1E-S & $6 / 24 / 2011$ & 1.6 & 0.3 & 0.3 & & & WATER & 51.465983 & 179.223666 & 35 \\
\hline 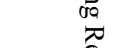 & & SEAW-CN-C2M-1F-D & $7 / 3 / 2011$ & 1.5 & 0.3 & 0.3 & & & WATER & 51.450716 & 179.20635 & 35 \\
\hline 8 & & SEAW-CN-C2M-1F-S & $7 / 3 / 2011$ & 1.8 & 0.3 & 0.3 & & & WATER & 51.450716 & 179.20635 & 35 \\
\hline 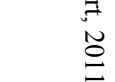 & & \begin{tabular}{|c|} 
SEAW-CN-C2M-1SB- \\
D
\end{tabular} & $7 / 3 / 2011$ & 1.6 & 0.3 & 0.3 & & & WATER & 51.450916 & 179.195316 & 35 \\
\hline $\overrightarrow{\tilde{N}}$ & & \begin{tabular}{|c|} 
SEAW-CN-C2M-1SB- \\
D
\end{tabular} & $7 / 3 / 2011$ & 1.8 & 0.3 & 0.3 & & & WATER & 51.450916 & 179.195316 & 35 \\
\hline 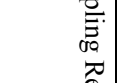 & & \begin{tabular}{|c|} 
SEAW-CN-C2M-1SB- \\
S
\end{tabular} & $7 / 3 / 2011$ & 1.7 & 0.3 & 0.3 & & & WATER & 51.450916 & 179.195316 & 35 \\
\hline$\stackrel{\mathscr{Q}}{=}$ & Sea-Water & \begin{tabular}{|c|} 
SEAW-CN-C2M-1SB- \\
S
\end{tabular} & $7 / 3 / 2011$ & 1.7 & 0.3 & 0.3 & & & WATER & 51.450916 & 179.195316 & 35 \\
\hline & & SEAW-CN-C2M-2A-D & $7 / 6 / 2011$ & 1.6 & 0.3 & 0.3 & & & WATER & 51.489316 & 179.1338 & 35 \\
\hline & & SEAW-CN-C2M-2A-S & $7 / 6 / 2011$ & 1.6 & 0.3 & 0.3 & & & WATER & 51.489316 & 179.1338 & 35 \\
\hline & & SEAW-CN-C2M-2B-D & $7 / 6 / 2011$ & 1.5 & 0.3 & 0.3 & & & WATER & 51.486233 & 179.139033 & 34 \\
\hline & & SEAW-CN-C2M-2B-D & $7 / 6 / 2011$ & 1.7 & 0.3 & 0.3 & & & WATER & 51.486233 & 179.139033 & 34 \\
\hline & & SEAW-CN-C2M-2B-S & $7 / 6 / 2011$ & 1.5 & 0.3 & 0.3 & & & WATER & 51.486233 & 179.139033 & 35 \\
\hline & & SEAW-CN-C2M-2B-S & $7 / 6 / 2011$ & 1.5 & 0.3 & 0.3 & & & WATER & 51.486233 & 179.139033 & 35 \\
\hline & & $\begin{array}{l}\text { SEAW-CN-C2M- } \\
\text { 2CAN-D } \\
\end{array}$ & $7 / 6 / 2011$ & 1.8 & 0.3 & 0.3 & & & WATER & 51.474533 & 179.169866 & 35 \\
\hline & & $\begin{array}{l}\text { SEAW-CN-C2M- } \\
\text { 2CAN-S } \\
\end{array}$ & $7 / 6 / 2011$ & 1.9 & 0.3 & 0.3 & & & WATER & 51.474533 & 179.169866 & 35 \\
\hline$c$ & & SEAW-CN-C2M-2C-D & $7 / 6 / 2011$ & 1.3 & 0.3 & 0.3 & & & WATER & 51.489316 & 179.168783 & 35 \\
\hline & & SEAW-CN-C2M-2C-S & $7 / 6 / 2011$ & 1.7 & 0.3 & 0.3 & & & WATER & 51.489316 & 179.168783 & 35 \\
\hline 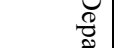 & & SEAW-CN-C2M-2D-D & $6 / 24 / 2011$ & 1.6 & 0.3 & 0.3 & & & WATER & 51.463616 & 179.23645 & 35 \\
\hline $9 E$ & & SEAW-CN-C2M-2D-S & $6 / 24 / 2011$ & 1.3 & 0.3 & 0.3 & & & WATER & 51.463616 & 179.23645 & 35 \\
\hline $\overrightarrow{\vec{c}}$ & & SEAW-CN-C2M-2E-D & $6 / 24 / 2011$ & 1.6 & 0.3 & 0.3 & & & WATER & 51.48365 & 179.268433 & 35 \\
\hline $\begin{array}{cc}\sigma & \vec{T}\end{array}$ & & SEAW-CN-C2M-2E-S & $6 / 24 / 2011$ & 1.9 & 0.3 & 0.3 & & & WATER & 51.48365 & 179.268433 & 35 \\
\hline $\overrightarrow{0}$ & & SEAW-CN-C2M-2F-D & $6 / 24 / 2011$ & 1.5 & 0.3 & 0.3 & & & WATER & 51.479066 & 179.272866 & 34 \\
\hline
\end{tabular}




\begin{tabular}{|c|c|c|c|c|c|c|c|c|c|c|c|}
\hline $\begin{array}{c}\text { Matrix } \\
\text { Subtype }\end{array}$ & Location Code & $\begin{array}{c}\text { Date } \\
\text { Sampled }\end{array}$ & Result & Uncertainty & $\begin{array}{l}\text { Detection } \\
\text { Limit }\end{array}$ & $\begin{array}{c}\text { Lab } \\
\text { Qualifiers }\end{array}$ & \begin{tabular}{|c|} 
Data \\
Validation \\
Qualifiers
\end{tabular} & Matrix & Latitude & Longitude & $\begin{array}{c}\text { Salinity } \\
(\%)\end{array}$ \\
\hline Sea-Water & SEAW-CN-C2M-2F-S & $6 / 24 / 2011$ & 1.2 & 0.3 & 0.3 & & & WATER & 51.479066 & 179.272866 & 35 \\
\hline & \begin{tabular}{|c|} 
SEAW-CN-C2M-2SB- \\
D
\end{tabular} & 7/3/2011 & 1.7 & 0.3 & 0.3 & & & WATER & 51.448283 & 179.217383 & 35 \\
\hline & $\begin{array}{c}\text { SEAW-CN-C2M-2SB- } \\
\text { S }\end{array}$ & $7 / 3 / 2011$ & 1.4 & 0.3 & 0.3 & & & WATER & 51.448283 & 179.217383 & 35 \\
\hline & SEAW-CN-C2M-3A-D & $7 / 6 / 2011$ & 1.5 & 0.3 & 0.3 & & & WATER & 51.4936 & 179.149683 & 35 \\
\hline & SEAW-CN-C2M-3A-S & $7 / 6 / 2011$ & 1.7 & 0.3 & 0.3 & & & WATER & 51.4936 & 179.149683 & 35 \\
\hline & SEAW-CN-C2M-3B-D & $6 / 24 / 2011$ & 1.3 & 0.3 & 0.3 & & & WATER & 51.511816 & 179.18085 & 33 \\
\hline & SEAW-CN-C2M-3B-S & $6 / 24 / 2011$ & 1.8 & 0.3 & 0.3 & & & WATER & 51.511816 & 179.18085 & 33 \\
\hline & $\begin{array}{l}\text { SEAW-CN-C2M- } \\
\text { 3CAN-D }\end{array}$ & $6 / 24 / 2011$ & 1.8 & 0.3 & 0.3 & & & WATER & 51.511416 & 179.240416 & 34 \\
\hline & $\begin{array}{l}\text { SEAW-CN-C2M- } \\
\text { 3CAN-S }\end{array}$ & $6 / 24 / 2011$ & 1.6 & 0.3 & 0.3 & & & WATER & 51.511416 & 179.240416 & 34 \\
\hline & SEAW-CN-C2M-3C-D & $6 / 24 / 2011$ & 1.6 & 0.3 & 0.3 & & & WATER & 51.523666 & 179.218083 & 34 \\
\hline & SEAW-CN-C2M-3C-S & $6 / 24 / 2011$ & 1.6 & 0.3 & 0.3 & & & WATER & 51.523666 & 179.218083 & 34 \\
\hline & SEAW-CN-C2M-3D-D & $6 / 24 / 2011$ & 1.3 & 0.3 & 0.3 & & & WATER & 51.4735 & 179.282333 & 35 \\
\hline & SEAW-CN-C2M-3D-S & $6 / 24 / 2011$ & 2 & 0.3 & 0.3 & & & WATER & 51.4735 & 179.282333 & 35 \\
\hline & SEAW-CN-C2M-4A-D & $6 / 24 / 2011$ & 1.5 & 0.3 & 0.3 & & & WATER & 51.504033 & 179.18895 & 34 \\
\hline & SEAW-CN-C2M-4A-S & $6 / 24 / 2011$ & 1.7 & 0.3 & 0.3 & & & WATER & 51.504033 & 179.18895 & 34 \\
\hline & SEAW-CN-C2M-4B-D & $6 / 24 / 2011$ & 1.4 & 0.3 & 0.3 & & & WATER & 51.529133 & 179.209066 & 35 \\
\hline & SEAW-CN-C2M-4B-S & $6 / 24 / 2011$ & 1.7 & 0.3 & 0.3 & & & WATER & 51.529133 & 179.209066 & 34 \\
\hline & SEAW-CN-C2M-5A-D & $6 / 24 / 2011$ & 1.3 & 0.3 & 0.3 & & & WATER & 51.5156 & 179.232333 & 34 \\
\hline & SEAW-CN-C2M-5A-S & $6 / 24 / 2011$ & 1.7 & 0.3 & 0.3 & & & WATER & 51.5156 & 179.232333 & 34 \\
\hline & $\begin{array}{c}\text { SEAW-CN-C2M-C1- } \\
\text { 2-D }\end{array}$ & $6 / 24 / 2011$ & 1.6 & 0.3 & 0.3 & & & WATER & 51.47845 & 179.219516 & 35 \\
\hline & $\begin{array}{c}\text { SEAW-CN-C2M-C1- } \\
2-S\end{array}$ & $6 / 24 / 2011$ & 1.8 & 0.3 & 0.3 & & & WATER & 51.47845 & 179.219516 & 35 \\
\hline & $\begin{array}{l}\text { SEAW-CN-C2M-C3- } \\
\text { 4-D }\end{array}$ & $6 / 24 / 2011$ & 1.4 & 0.3 & 0.3 & & & WATER & 51.497233 & 179.2102 & 34 \\
\hline & $\begin{array}{c}\text { SEAW-CN-C2M-C3- } \\
4-\mathrm{S}\end{array}$ & $6 / 24 / 2011$ & 1.4 & 0.3 & 0.3 & & & WATER & 51.497233 & 179.2102 & 34 \\
\hline & \begin{tabular}{|c|} 
SEAW-CN-C2M-C4- \\
$1-D$ \\
\end{tabular} & $7 / 6 / 2011$ & 1.6 & 0.3 & 0.3 & & & WATER & 51.482933 & 179.1861 & 35 \\
\hline & $\begin{array}{c}\text { SEAW-CN-C2M-C4- } \\
1-\mathrm{S}\end{array}$ & $7 / 6 / 2011$ & 1.7 & 0.3 & 0.3 & & & WATER & 51.482933 & 179.1861 & 35 \\
\hline & $\begin{array}{c}\text { SEAW-CN-C2M-C4- } \\
4-\mathrm{D} \\
\end{array}$ & $6 / 24 / 2011$ & 1.6 & 0.3 & 0.3 & & & WATER & 51.496283 & 179.18915 & 34 \\
\hline
\end{tabular}




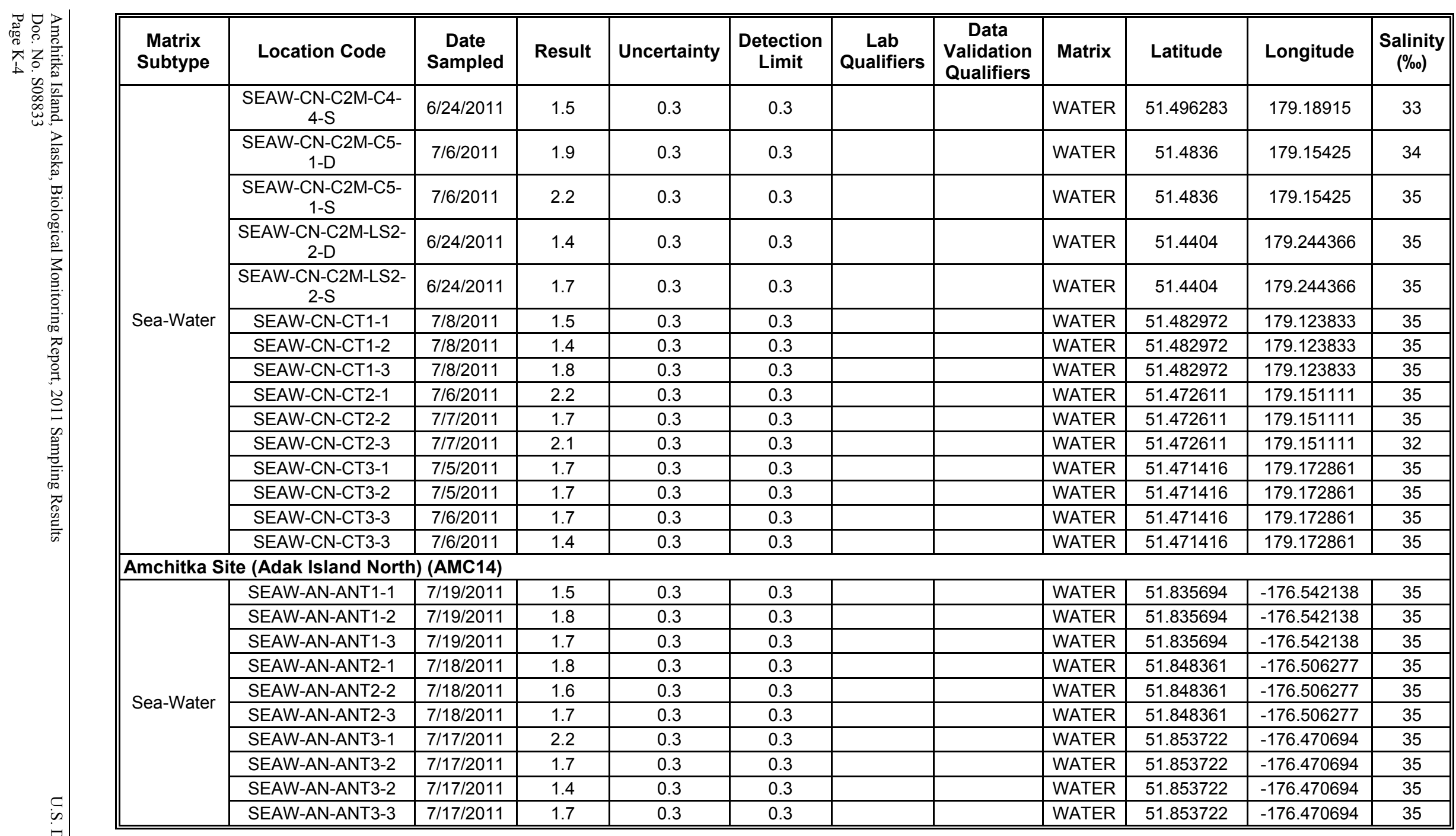




\begin{tabular}{|c|c|c|c|c|c|c|c|c|c|c|c|}
\hline $\begin{array}{c}\text { Matrix } \\
\text { Subtype }\end{array}$ & Location Code & $\begin{array}{c}\text { Date } \\
\text { Sampled }\end{array}$ & Result & Uncertainty & $\begin{array}{c}\text { Detection } \\
\text { Limit }\end{array}$ & $\begin{array}{c}\text { Lab } \\
\text { Qualifiers }\end{array}$ & $\begin{array}{c}\text { Data } \\
\text { Validation } \\
\text { Qualifiers }\end{array}$ & Matrix & Latitude & Longitude & $\begin{array}{c}\text { Salinity } \\
(\%)\end{array}$ \\
\hline \multicolumn{12}{|c|}{\begin{tabular}{|l} 
Amchitka Site (Adak Island South) (AMC15) \\
\end{tabular}} \\
\hline \multirow{10}{*}{ Sea-Water } & SEAW-AS-AST1-1 & $7 / 15 / 2011$ & 2 & 0.3 & 0.3 & & & WATER & 51.737472 & -176.48025 & 34 \\
\hline & SEAW-AS-AST1-2 & $7 / 15 / 2011$ & 2.5 & 0.3 & 0.3 & & & WATER & 51.737472 & -176.48025 & 35 \\
\hline & SEAW-AS-AST1-3 & $7 / 15 / 2011$ & 1.6 & 0.3 & 0.3 & & & WATER & 51.737472 & -176.48025 & 35 \\
\hline & SEAW-AS-AST2-1 & $7 / 16 / 2011$ & 1.8 & 0.3 & 0.3 & & & WATER & 51.745666 & -176.507055 & 35 \\
\hline & SEAW-AS-AST2-2 & 7/16/2011 & 1.8 & 0.3 & 0.3 & & & WATER & 51.745666 & -176.507055 & 35 \\
\hline & SEAW-AS-AST2-3 & $7 / 16 / 2011$ & 2.1 & 0.3 & 0.3 & & & WATER & 51.745666 & -176.507055 & 35 \\
\hline & SEAW-AS-AST3-1 & $7 / 14 / 2011$ & 2.8 & 0.3 & 0.3 & & & WATER & 51.688333 & -176.63375 & 36 \\
\hline & SEAW-AS-AST3-2 & $7 / 14 / 2011$ & 1.4 & 0.3 & 0.3 & & & WATER & 51.688333 & -176.63375 & 35 \\
\hline & SEAW-AS-AST3-3 & $7 / 14 / 2011$ & 1.4 & 0.3 & 0.3 & & & WATER & 51.688333 & -176.63375 & 37 \\
\hline & SEAW-AS-AST3-3 & $7 / 14 / 2011$ & 1.9 & 0.3 & 0.3 & & & WATER & 51.688333 & -176.63375 & 36 \\
\hline
\end{tabular}

Qualifier definitions:

$\mathrm{U}$ - Analytical result below detection limit

$\mathrm{J}$ - Estimated 
This page intentionally left blank 


\section{Appendix L}

2004 (CRESP) and 2011 Data Comparison Summary 
This page intentionally left blank 
Table L-1. Summary of Marine Species Sampled in Both 2004 (CRESP) and 2011

\begin{tabular}{|c|c|c|c|c|c|c|}
\hline Species & ${ }^{137} \mathrm{Cs}$ & ${ }^{241} \mathrm{Am}$ & ${ }^{239,240} \mathrm{Pu}$ & ${ }^{234} \mathrm{U}$ & ${ }^{235} \mathrm{U}$ & ${ }^{238} \mathrm{U}$ \\
\hline Dragon kelp & $\mathrm{X}$ & $\mathrm{X}$ & $\mathrm{X}$ & $\mathrm{X}$ & $\mathrm{X}$ & $\mathrm{X}$ \\
\hline Rockweed & $\mathrm{X}$ & $x$ & $\mathrm{X}$ & $\mathrm{X}$ & $\mathrm{X}$ & $\mathrm{X}$ \\
\hline Reindeer lichen & See Note & & & & & \\
\hline Gumboot or Pacific chiton & $\mathrm{X}$ & $\mathrm{X}$ & & & & \\
\hline Horse mussel & See Note & $\mathrm{X}$ & $\mathrm{X}$ & $\mathrm{X}$ & $\mathrm{X}$ & $\mathrm{X}$ \\
\hline Sea urchin & $\mathrm{X}$ & $\mathrm{X}$ & & & & \\
\hline Rockfish & $x$ & $x$ & $x$ & $x$ & $x$ & $x$ \\
\hline Red or yellow Irish lord & $x$ & $x$ & $x$ & $\mathrm{X}$ & $x$ & $x$ \\
\hline Rock greenling & $\mathrm{X}$ & $\mathrm{X}$ & & & & \\
\hline Pacific halibut & $\mathrm{X}$ & $\mathrm{x}$ & $x$ & $\mathrm{X}$ & $\mathrm{X}$ & $\mathrm{X}$ \\
\hline Pacific cod & $x$ & $x$ & $x$ & $x$ & $x$ & $x$ \\
\hline Octopus & $\mathrm{X}$ & $\mathrm{X}$ & & & & \\
\hline Dolly Varden & $\mathrm{X}$ & $\mathrm{X}$ & & & & \\
\hline Glaucous-winged gull eggs & $\mathrm{X}$ & $x$ & & & & \\
\hline
\end{tabular}

\section{Notes:}

Reindeer lichen samples were collected only in 2011 (for ${ }^{137} \mathrm{Cs}$ analysis only).

Because horse mussel samples were not analyzed for ${ }^{137} \mathrm{Cs}$ in 2004,2011 results for ${ }^{137} \mathrm{Cs}$ in horse mussel were compared with 2004 blue mussel results given the same genus.

In 2004, samples of glaucous-winged gull soft tissue and bone were also collected.

Species sampled in 2004 but not in 2011 are listed below; most marine fauna samples were soft tissue samples unless otherwise noted.

Marine flora and fauna species sampled in 2004 (CRESP) but not in 2011

- $\quad$ Alaria nana (ribbon kelp)

- $\quad$ Atka mackerel (bone and soft tissue)

- Bald eagle

- Blue mussel

- $\quad$ Common eider (egg and soft tissue)

- Limpets

- Northern rock sole

- $\quad$ Pacific Ocean perch (bone and soft tissue)

- $\quad$ Pigeon guillemot (bone and soft tissue)

- Rock jingle

- Sea lion

- $\quad$ Tufted puffin (bone and soft tissue)

- $\quad$ Tufted puffin (soft tissue)

- Ulva latuca (see lettuce)

- Walleye pollock 
Table L-2. Summary of ${ }^{137}$ Cs Results for Marine Flora and Fauna: 2004 (CRESP) vs. 2011 Sampling

\begin{tabular}{|c|c|c|c|c|c|c|c|c|c|c|c|}
\hline \multirow{3}{*}{$\begin{array}{l}\text { Species } \\
\text { Area }^{b} \\
\text { Dragon Kelp }\end{array}$} & \multicolumn{5}{|c|}{2004 (CRESP) $^{\mathrm{a}}$} & \multicolumn{6}{|c|}{$2011^{a}$} \\
\hline & \multirow[t]{2}{*}{$n$} & \multirow[t]{2}{*}{$\begin{array}{l}\%> \\
M D C\end{array}$} & \multirow[t]{2}{*}{$\begin{array}{l}\text { Range of } \\
\text { Detects }\end{array}$} & \multicolumn{2}{|r|}{$\begin{array}{c}\text { Range of } \\
\text { MDCs }\end{array}$} & \multirow[t]{2}{*}{$n$} & \multirow[t]{2}{*}{$\begin{array}{l}\%> \\
M D C\end{array}$} & $\begin{array}{l}\text { Range of } \\
\text { Detects }^{c}\end{array}$ & \multicolumn{3}{|c|}{$\begin{array}{c}\text { Range of } \\
\text { MDCs }\end{array}$} \\
\hline & & & & & & & & & & & \\
\hline Amchitka & 5 & $0 \%$ & & 2.8 & -146 & 27 & $100 \%$ & $0.24-3.2$ & 0.1 & - & 0.7 \\
\hline Kiska/Adak* & 3 & $0 \%$ & & 2.8 & -182 & 18 & $100 \%$ & $0.2-2.0$ & 0.2 & - & 0.5 \\
\hline \multicolumn{12}{|c|}{ *Kiska (2004 only)/Adak (2011 only); formatting applies to all subsequent entries. } \\
\hline \multicolumn{12}{|l|}{ Sea Urchin } \\
\hline Amchitka & 5 & $0 \%$ & & 2.4 & -234 & 9 & $56 \%$ & $1.8-9.9$ & 1.1 & - & 4.3 \\
\hline Kiska/Adak & 2 & $0 \%$ & & 8.5 & -206 & 6 & $33 \%$ & $2.7-9.4$ & 1.2 & - & 3.7 \\
\hline \multicolumn{12}{|c|}{ Horse Mussel (Blue Mussel used for 2004) } \\
\hline Amchitka & 1 & $0 \%$ & & & 19.9 & 9 & $89 \%$ & $1.4-14$ & 2.6 & - & 60 \\
\hline Kiska/Adak & 1 & $0 \%$ & & & 11.7 & 6 & $100 \%$ & $3.4-15$ & 2.7 & - & 5.0 \\
\hline \multicolumn{12}{|l|}{ Chiton } \\
\hline Amchitka & 1 & $0 \%$ & & & 9.4 & 9 & $33 \%$ & $1.6-4.1$ & 1.2 & - & 2.8 \\
\hline Kiska/Adak & 0 & & & & & 6 & $67 \%$ & $0.8-1.3$ & 0.7 & - & 4.0 \\
\hline \multicolumn{12}{|l|}{ Rockfish } \\
\hline Amchitka & 14 & $14 \%$ & $3.0-5.1$ & 2.5 & -207 & 26 & $88 \%$ & $1.1-6.4$ & 0.7 & - & 2.2 \\
\hline Kiska/Adak & 4 & $25 \%$ & 3.5 & 2.6 & -106 & 15 & $93 \%$ & $1.2-9.4$ & 0.5 & - & 2.4 \\
\hline \multicolumn{12}{|l|}{ Greenling } \\
\hline Amchitka & 23 & $0 \%$ & & 4.6 & -119 & 27 & $96 \%$ & $1.9-7.8$ & 1.5 & - & 2.8 \\
\hline Kiska/Adak & 7 & $0 \%$ & & 2.7 & -107 & 18 & $100 \%$ & $0.9-7.4$ & 1.3 & - & 3.6 \\
\hline \multicolumn{12}{|l|}{ Irish Lord } \\
\hline Amchitka & 19 & $11 \%$ & $3.5-30.0$ & 2.6 & -300 & 27 & $67 \%$ & $1.2-9.5$ & 0.8 & - & 3.9 \\
\hline Kiska/Adak & 9 & $0 \%$ & & 2.8 & -620 & 18 & $56 \%$ & $1.8-13$ & 0.9 & - & 2.5 \\
\hline \multicolumn{12}{|l|}{ Octopus } \\
\hline Amchitka & 4 & $100 \%$ & $6.40-8.2$ & 2.4 & -2.6 & 3 & $100 \%$ & $0.23-2.1$ & 0.2 & - & 1.1 \\
\hline Kiska/Adak & 0 & & & & & 1 & $100 \%$ & 0.7 & & & 0.5 \\
\hline \multicolumn{12}{|l|}{ Pacific Cod } \\
\hline Amchitka & 24 & $21 \%$ & $4.8-16.3$ & 2.6 & -210 & 3 & $100 \%$ & $2.1-3.8$ & 0.2 & - & 0.5 \\
\hline Kiska/Adak & 9 & $22 \%$ & $5.1-8.5$ & 2.8 & -250 & 2 & $100 \%$ & $2.0-3.0$ & 0.2 & - & 0.3 \\
\hline \multicolumn{12}{|l|}{ Halibut } \\
\hline Amchitka & 2 & $50 \%$ & 12.0 & 2.5 & -8.8 & 3 & $100 \%$ & $0.82-1.5$ & 0.1 & - & 0.2 \\
\hline Kiska/Adak & 2 & $100 \%$ & $5.1-8.5^{d}$ & 2.7 & 2.8 & 1 & $100 \%$ & 1.0 & & & 0.2 \\
\hline Rockweed & & & & & & & & & & & \\
\hline Amchitka & 4 & $0 \%$ & & 9.1 & -200 & 27 & $100 \%$ & $1.3-5.8$ & 0.2 & - & 0.5 \\
\hline Kiska/Adak & 2 & $0 \%$ & & 8.6 & -160 & 18 & $100 \%$ & $1.0-7.3$ & 0.3 & - & 0.5 \\
\hline Star Reindeer Lic & & & & & & & & & & & \\
\hline Amchitka & 0 & & & & & 3 & $100 \%$ & $404-1523$ & 8.0 & - & 15.0 \\
\hline Kiska/Adak & 0 & & & & & 3 & $100 \%$ & $2855-6236$ & 16.0 & - & 33.0 \\
\hline Gull Eggs (Adak $n$ & pled & 2011) & & & & & & & & & \\
\hline Amchitka & 1 & $0 \%$ & & 5.8 & -5.8 & 1 & $100 \%$ & 4.2 & & & 1.5 \\
\hline Kiska/Adak & 1 & $0 \%$ & & 7.2 & -7.2 & 0 & & & & & \\
\hline Dolly Varden & & & & & & & & & & & \\
\hline Amchitka & 10 & $20 \%$ & $18.8-21.1$ & 3.0 & -109 & 1 & $100 \%$ & 98 & & & 1.0 \\
\hline Kiska/Adak & 0 & & & & & 1 & $100 \%$ & 447 & & & 1.0 \\
\hline
\end{tabular}

$n=$ number of samples; MDC = minimum detectable concentration

a A more detailed summary of ${ }^{137} \mathrm{Cs}$ data for the 2011 sampling effort is provided in Table 6 of the main report.

b Reference (background) area Kiska was sampled in 2004 only, whereas Adak Island was sampled in 2011 only.

${ }^{c}$ Duplicate samples were not easily identified in the 2004 database, which may be reflected in the sample numbers and ranges reported for the 2004 sampling period. Due to the large proportion of results <MDC in 2004, maximum detections for both years are highlighted (yellow) to facilitate comparison.

d Maximum detected ${ }^{137} \mathrm{Cs}$ concentration in halibut in $2004,8.5 \mathrm{pCi} / \mathrm{kg}$, was from Rat (now referred to as Hawadax) Island. 
Table L-3. Summary of ${ }^{241}$ Am Results for Marine Flora and Fauna: 2004 (CRESP) vs. 2011 Sampling

\begin{tabular}{|c|c|c|c|c|c|c|c|c|}
\hline \multirow[b]{2}{*}{$\begin{array}{l}\text { Species } \\
\text { Area }^{b}\end{array}$} & \multicolumn{4}{|c|}{2004 (CRESP) $^{\mathrm{a}}$} & \multicolumn{4}{|c|}{$2011^{a}$} \\
\hline & $n$ & $\begin{array}{l}\%> \\
M D C\end{array}$ & $\begin{array}{l}\text { Range of } \\
\text { Detects }^{c}\end{array}$ & $\begin{array}{c}\text { Range of } \\
\text { MDCs }\end{array}$ & $n$ & $\begin{array}{l}\%> \\
M D C\end{array}$ & $\begin{array}{l}\text { Range of } \\
\text { Detects }^{c}\end{array}$ & $\begin{array}{c}\text { Range of } \\
\text { MDCs }\end{array}$ \\
\hline \multicolumn{9}{|l|}{ Dragon Kelp } \\
\hline Amchitka & 17 & $0 \%$ & & $0.3-5.5$ & 27 & $74 \%$ & $0.003-0.03$ & $0.0003-0.004$ \\
\hline Kiska/Adak* & 6 & $0 \%$ & & $0.6-15$ & 18 & $83 \%$ & $0.006-0.03$ & $0.0002-0.002$ \\
\hline \multicolumn{9}{|c|}{ *Kiska (2004 only)/Adak (2011 only); formatting applies to all subsequent entries. } \\
\hline \multicolumn{9}{|l|}{ Sea Urchin } \\
\hline Amchitka & 2 & $0 \%$ & & $4.1-9.9$ & 9 & $89 \%$ & $0.08-0.20$ & $0.002-0.01$ \\
\hline Kiska/Adak & 1 & $0 \%$ & & $9.7-9.7$ & 6 & $83 \%$ & $0.07-0.12$ & $0.003-0.03$ \\
\hline \multicolumn{9}{|l|}{ Horse Mussel } \\
\hline Amchitka & 6 & $0 \%$ & & $0.63-1.3$ & 9 & $67 \%$ & $0.11-0.21$ & $0.005-0.80$ \\
\hline Kiska/Adak & 2 & $50 \%$ & 0.57 & $0.33-0.69$ & 6 & $33 \%$ & $0.09-0.15$ & $0.009-0.05$ \\
\hline \multicolumn{9}{|l|}{ Chiton } \\
\hline Amchitka & 1 & $0 \%$ & & 9.4 & 9 & $89 \%$ & $0.07-0.14$ & $0.002-0.01$ \\
\hline Kiska/Adak & 0 & & & & 6 & $67 \%$ & $0.02-0.08$ & $0.002-0.02$ \\
\hline \multicolumn{9}{|l|}{ Rockfish } \\
\hline Amchitka & 14 & $0 \%$ & & $6.6-814$ & 26 & $12 \%$ & $0.01-0.07$ & $0.003-0.03$ \\
\hline Kiska/Adak & 5 & $20 \%$ & 0.78 & $0.8-473$ & 15 & $0 \%$ & & $0.003-0.01$ \\
\hline \multicolumn{9}{|l|}{ Greenling } \\
\hline Amchitka & 25 & $0 \%$ & & $10.9-678$ & 27 & $11 \%$ & $0.03-0.03$ & $0.002-0.02$ \\
\hline Kiska/Adak & 7 & $0 \%$ & & $7.2-470$ & 18 & $17 \%$ & $0.01-0.03$ & $0.002-0.03$ \\
\hline \multicolumn{9}{|l|}{ Irish Lord } \\
\hline Amchitka & 21 & $0 \%$ & & $0.7-495$ & 27 & $11 \%$ & 0.01 & $0.002-0.02$ \\
\hline Kiska/Adak & 9 & $0 \%$ & & $0.3-590$ & 18 & $6 \%$ & 0.03 & $0.004-0.01$ \\
\hline \multicolumn{9}{|l|}{ Octopus } \\
\hline Amchitka & 4 & $100 \%$ & & $7.2-7.3$ & 3 & $67 \%$ & $0.02-0.02$ & $0.0004-0.01$ \\
\hline Kiska/Adak & 0 & & & & 1 & $100 \%$ & 0.02 & 0.002 \\
\hline \multicolumn{9}{|l|}{ Pacific Cod } \\
\hline Amchitka & 33 & $0 \%$ & & $0.30-473$ & 3 & $0 \%$ & & $0.001-0.002$ \\
\hline Kiska/Adak & 15 & $7 \%$ & 0.39 & $0.33-481$ & 2 & $0 \%$ & & 0.001 \\
\hline \multicolumn{9}{|l|}{ Halibut } \\
\hline Amchitka & 6 & $0 \%$ & & $0.59-11.6$ & 3 & $0 \%$ & & $0.001-0.002$ \\
\hline Kiska/Adak & $5^{d}$ & $0 \%$ & & $0.30-7.5$ & 1 & $0 \%$ & & 0.001 \\
\hline \multicolumn{9}{|l|}{ Rockweed } \\
\hline Amchitka & 11 & $18 \%$ & $0.75-0.94$ & $0.3-14$ & 27 & $22 \%$ & $0.003-0.01$ & $0.001-0.01$ \\
\hline Kiska/Adak & 5 & $0 \%$ & & $0.7-11$ & 18 & $39 \%$ & $0.01-0.02$ & $0.001-0.01$ \\
\hline \multicolumn{9}{|c|}{ Gull Eggs (Adak not sampled in 2011) } \\
\hline Amchitka & 1 & $0 \%$ & & 16.3 & 1 & $0 \%$ & & 0.01 \\
\hline Kiska/Adak & 1 & $0 \%$ & & 20.1 & 0 & & & \\
\hline \multicolumn{9}{|l|}{ Dolly Varden } \\
\hline Amchitka & 10 & $0 \%$ & & $3.0-109$ & 1 & $0 \%$ & & 0.002 \\
\hline Kiska/Adak & 0 & & & & 1 & $0 \%$ & & 0.002 \\
\hline
\end{tabular}

$n=$ number of samples; MDC = minimum detectable concentration

a A more detailed summary of ${ }^{241} \mathrm{Am}$ data for the 2011 sampling effort is provided in Table 7 of the main report.

${ }^{b}$ Reference (background) area Kiska was sampled in 2004 only, whereas Adak Island was sampled in 2011 only.

${ }^{c}$ Duplicate samples were not easily identified in the 2004 database, which may be reflected in the sample numbers and ranges reported for the 2004 sampling period. Due to the large proportion of results <MDC in 2004, maximum detections for both years are highlighted (yellow) to facilitate comparison.

' In 2004, 3 halibut samples were collected from Kiska island, and 2 from Rat Island. 
Table L-4. Summary of ${ }^{239,240}$ Pu Results for Marine Flora and Fauna: 2004 (CRESP) vs. 2011 Sampling

\begin{tabular}{|c|c|c|c|c|c|c|c|c|}
\hline & \multicolumn{4}{|c|}{2004 (CRESP) } & \multicolumn{4}{|c|}{$2011^{a}$} \\
\hline $\begin{array}{r}\text { Species } \\
\text { Area }^{b}\end{array}$ & $n$ & $\begin{array}{l}\%> \\
M D C\end{array}$ & $\begin{array}{l}\text { Range of } \\
\text { Detects }\end{array}$ & $\begin{array}{c}\text { Range of } \\
\text { MDCs }\end{array}$ & $n$ & $\begin{array}{l}\%> \\
M D C\end{array}$ & $\begin{array}{l}\text { Range of } \\
\text { Detects }^{c}\end{array}$ & $\begin{array}{c}\text { Range of } \\
\text { MDCs }\end{array}$ \\
\hline \multicolumn{9}{|l|}{ Dragon Kelp } \\
\hline Amchitka & 15 & $33 \%$ & $1.1-5.6$ & $0.6-4.1$ & 27 & $96 \%$ & $0.003-0.29$ & $0.003-0.01$ \\
\hline Kiska/Adak* & 4 & $25 \%$ & 2.4 & $1.1-2.1$ & 18 & $100 \%$ & $0.020-0.20$ & $0.003-0.01$ \\
\hline \multicolumn{9}{|c|}{ *Kiska (2004 only)/Adak (2011 only); formatting applies to all subsequent entries. } \\
\hline \multicolumn{9}{|l|}{ Horse Mussel } \\
\hline Amchitka & 6 & $17 \%$ & 1.3 & $0.71-1.2$ & 9 & $22 \%$ & $0.11-0.25$ & $0.08-2.0$ \\
\hline Kiska/Adak & 2 & $0 \%$ & & $0.98-1.06$ & 6 & $17 \%$ & 0.073 & $0.07-0.2$ \\
\hline \multicolumn{9}{|l|}{ Rockfish } \\
\hline Amchitka & 0 & & & & 26 & $0 \%$ & & $0.01-0.06$ \\
\hline Kiska/Adak & 1 & $0 \%$ & & 0.84 & 15 & $0 \%$ & & $0.01-0.06$ \\
\hline \multicolumn{9}{|l|}{ Irish Lord } \\
\hline Amchitka & 2 & $0 \%$ & & $0.78-1.2$ & 27 & $0 \%$ & & $0.01-0.08$ \\
\hline Kiska/Adak & 1 & $0 \%$ & & $0.76-0.76$ & 18 & $0 \%$ & & $0.02-0.07$ \\
\hline \multicolumn{9}{|l|}{ Pacific Cod } \\
\hline Amchitka & 14 & $0 \%$ & & $0.38-2.6$ & 3 & $0 \%$ & & $0.003-0.01$ \\
\hline Kiska/Adak & 7 & $0 \%$ & & $0.81-3.8$ & 2 & $0 \%$ & & $0.003-0.01$ \\
\hline \multicolumn{9}{|l|}{ Halibut } \\
\hline Amchitka & 4 & $25 \%$ & 0.47 & $0.38-2.5$ & 3 & $0 \%$ & & $0.003-0.01$ \\
\hline Kiska/Adak & $3^{d}$ & $0 \%$ & & $0.92-2.1$ & 1 & $0 \%$ & & $0.002-0.004$ \\
\hline \multicolumn{9}{|l|}{ Rockweed } \\
\hline Amchitka & 10 & $50 \%$ & $1.2-1.6$ & $0.90-1.8$ & 27 & $93 \%$ & $0.002-0.03$ & $0.002-0.01$ \\
\hline Kiska/Adak & 4 & $0 \%$ & & $0.78-1.0$ & 18 & $83 \%$ & $0.002-0.03$ & $0.002-0.01$ \\
\hline
\end{tabular}

$n=$ number of samples; MDC = minimum detectable concentration

a A more detailed summary of ${ }^{239,}{ }^{240} \mathrm{Pu}$ data for the 2011 sampling effort is provided in Table 10 . Results for individual Pu isotopes are provided in Tables 8 and 9 . In 2011, ${ }^{240} \mathrm{Pu}$ was detected in only 3 species: dragon kelp, rockweed, and sea urchin (latter not sampled in 2004).

b Reference (background) area Kiska was sampled in 2004 only, whereas Adak Island was sampled in 2011 only.

c Due to the large proportion of results <MDC in 2004, maximum detections for both years are highlighted (yellow) to facilitate comparison.

d In 2004, 2 halibut samples were collected from Kiska island, and 1 from Rat Island. 
Table L-5. Summary of ${ }^{234}$ U Results for Marine Flora and Fauna: 2004 (CRESP) vs. 2011 Sampling

\begin{tabular}{|c|c|c|c|c|c|c|c|c|}
\hline & \multicolumn{4}{|c|}{2004 (CRESP) } & \multicolumn{4}{|c|}{$2011^{a}$} \\
\hline $\begin{array}{l}\text { Species } \\
\text { Area }^{b} \\
\end{array}$ & $n$ & $\begin{array}{l}\%> \\
M D C\end{array}$ & $\begin{array}{l}\text { Range of } \\
\text { Detects }{ }^{c}\end{array}$ & $\begin{array}{c}\text { Range of } \\
\text { MDCs }\end{array}$ & $n$ & $\begin{array}{l}\%> \\
M D C\end{array}$ & $\begin{array}{l}\text { Range of } \\
\text { Detects }{ }^{c}\end{array}$ & $\begin{array}{c}\text { Range of } \\
\text { MDCs }\end{array}$ \\
\hline \multicolumn{9}{|l|}{ Dragon Kelp } \\
\hline Amchitka & 15 & $100 \%$ & $5.9-55.7$ & $0.5-2.7$ & 27 & $100 \%$ & $12.4-44.6$ & $1.0-2.0$ \\
\hline Kiska/Adak* & 4 & $100 \%$ & $7.9-31.6$ & $1.2-1.5$ & 18 & $100 \%$ & $7.3-33.7$ & $0.4-1.0$ \\
\hline \multicolumn{9}{|c|}{ *Kiska (2004 only)/Adak (2011 only). } \\
\hline \multicolumn{9}{|l|}{ Horse Mussel } \\
\hline Amchitka & 6 & $100 \%$ & $12-75.1$ & $0.8-1.4$ & 9 & $89 \%$ & $23.4-80.8$ & $1.0-28$ \\
\hline Kiska/Adak & 2 & $100 \%$ & $8.4-12.6$ & $1.0-1.0$ & 6 & $100 \%$ & $9.7-49.8$ & $0.5-1.0$ \\
\hline \multicolumn{9}{|l|}{ Rockfish } \\
\hline Amchitka & 0 & & & & 26 & $96 \%$ & $2.3-17.2$ & $1.0-2.0$ \\
\hline Kiska/Adak & 1 & $100 \%$ & 58.9 & 1.1 & 15 & $80 \%$ & $2.2-13.4$ & $1.0-2.0$ \\
\hline \multicolumn{9}{|l|}{ Irish Lord } \\
\hline Amchitka & 2 & $100 \%$ & $9.9-13.1$ & $0.73-1.0$ & 27 & $93 \%$ & $3.0-19.3$ & $1.0-2.0$ \\
\hline Kiska/Adak & 1 & $100 \%$ & 15.3 & 1.0 & 18 & $94 \%$ & $2.9-16.0$ & 1.0 \\
\hline \multicolumn{9}{|l|}{ Pacific Cod } \\
\hline Amchitka & 14 & $93 \%$ & $1.1-6.6$ & $0.8-1.4$ & 3 & $67 \%$ & $2.5-3.0$ & 1.0 \\
\hline Kiska/Adak & 7 & $57 \%$ & $-0.5-7.8$ & $0.8-1.3$ & 2 & $100 \%$ & $1.9-1.9$ & 1.0 \\
\hline \multicolumn{9}{|l|}{ Halibut } \\
\hline Amchitka & 4 & $100 \%$ & $1.9-32$ & $0.8-1.9$ & 3 & $33 \%$ & 0.6 & $0.6-1.0$ \\
\hline Kiska/Adak & $3^{a}$ & $67 \%$ & $0.7-4.3$ & $1.1-1.3$ & 1 & $0 \%$ & & 1.0 \\
\hline \multicolumn{9}{|l|}{ Rockweed } \\
\hline Amchitka & 10 & $100 \%$ & $50-130$ & $0.8-1.5$ & 27 & $100 \%$ & $35.0-144$ & 1.0 \\
\hline Kiska/Adak & 4 & $100 \%$ & $74-140$ & $0.4-1.3$ & 18 & $100 \%$ & $45.5-113$ & 1.0 \\
\hline
\end{tabular}

$n=$ number of samples; $\mathrm{MDC}=$ minimum detectable concentration

a A more detailed summary of ${ }^{234} \mathrm{U}$ data for the 2011 sampling effort is provided in Table 11.

${ }^{b}$ Reference (background) area Kiska was sampled in 2004 only, whereas Adak Island was sampled in 2011 only.

c Maximum detections for both years are highlighted (yellow) to facilitate comparison.

d In 2004, 2 halibut samples were collected from Kiska island, and 1 from Rat Island. 
Table L-6. Summary of ${ }^{235}$ U Results for Marine Flora and Fauna: 2004 (CRESP) vs. 2011 Sampling

\begin{tabular}{|c|c|c|c|c|c|c|c|c|}
\hline & \multicolumn{4}{|c|}{2004 (CRESP) } & \multicolumn{4}{|c|}{$2011^{a}$} \\
\hline $\begin{array}{l}\text { Species } \\
\text { Area }^{b}\end{array}$ & $n$ & $\begin{array}{l}\%> \\
M D C\end{array}$ & $\begin{array}{l}\text { Range of } \\
\text { Detects }^{c}\end{array}$ & $\begin{array}{l}\text { Range of } \\
\text { MDCs }\end{array}$ & $n$ & $\begin{array}{l}\%> \\
M D C\end{array}$ & $\begin{array}{l}\text { Range of } \\
\text { Detects }^{c}\end{array}$ & $\begin{array}{l}\text { Range of } \\
\text { MDCs }\end{array}$ \\
\hline \multicolumn{9}{|l|}{ Dragon Kelp } \\
\hline Amchitka & 15 & $47 \%$ & $1.3-4.3$ & $1.1-3.3$ & 27 & $100 \%$ & $0.4-1.5$ & $0.0002-0.001$ \\
\hline Kiska/Adak* & 4 & $25 \%$ & 1.6 & $1.0-1.6$ & 18 & $100 \%$ & $0.3-1.2$ & $0.0002-0.001$ \\
\hline \multicolumn{9}{|c|}{ *Kiska (2004 only)/Adak (2011 only). } \\
\hline \multicolumn{9}{|l|}{ Horse Mussel } \\
\hline Amchitka & 6 & $33 \%$ & $1.3-3.8$ & $0.4-1.5$ & 9 & $100 \%$ & $0.5-2.9$ & $0.0004-0.010$ \\
\hline Kiska/Adak & 2 & $0 \%$ & & $0.9-1.3$ & 6 & $100 \%$ & $0.3-1.8$ & $0.0002-0.0004$ \\
\hline \multicolumn{9}{|l|}{ Rockfish } \\
\hline Amchitka & 0 & & & & 26 & $100 \%$ & $0.1-0.59$ & $0.0003-0.0007$ \\
\hline Kiska/Adak & 1 & $100 \%$ & 3.1 & 1.6 & 15 & $100 \%$ & $0.0-0.48$ & $0.0002-0.0007$ \\
\hline \multicolumn{9}{|l|}{ Irish Lord } \\
\hline Amchitka & 2 & $100 \%$ & $9.9-13.1$ & $1.20-1.5$ & 27 & $100 \%$ & $0.1-0.68$ & $0.0003-0.0007$ \\
\hline Kiska/Adak & 1 & $100 \%$ & 15.3 & $1.20-1.2$ & 18 & $100 \%$ & $0.1-0.55$ & $0.0004-0.0005$ \\
\hline \multicolumn{9}{|l|}{ Pacific Cod } \\
\hline Amchitka & 14 & $0 \%$ & & $0.9-2.0$ & 3 & $100 \%$ & $0.0-0.09$ & 0.0003 \\
\hline Kiska/Adak & 7 & $0 \%$ & & $0.9-1.4$ & 2 & $100 \%$ & $0.1-0.06$ & $0.0002-0.0003$ \\
\hline \multicolumn{9}{|l|}{ Halibut } \\
\hline Amchitka & 4 & $25 \%$ & 1.3 & $0.9-2.1$ & 3 & $100 \%$ & $0.0-0.03$ & $0.0002-0.0004$ \\
\hline Kiska/Adak & $3^{a}$ & $0 \%$ & & $1.1-1.7$ & 1 & $100 \%$ & 0.04 & 0.0004 \\
\hline \multicolumn{9}{|l|}{ Rockweed } \\
\hline Amchitka & 10 & $100 \%$ & $2.5-5.4$ & $0.6-1.7$ & 27 & $100 \%$ & $1.2-5.10$ & $0.0002-0.0005$ \\
\hline Kiska/Adak & 4 & $75 \%$ & $3.2-6.9$ & $1.0-1.6$ & 18 & $100 \%$ & $1.6-4.01$ & $0.0003-0.0005$ \\
\hline
\end{tabular}

$n=$ number of samples; MDC = minimum detectable concentration

a A more detailed summary of ${ }^{235} \mathrm{U}$ data for the 2011 sampling effort is provided in Table 12.

${ }^{b}$ Reference (background) area Kiska was sampled in 2004 only, whereas Adak Island was sampled in 2011 only.

c Maximum detections for both years are highlighted (yellow) to facilitate comparison.

d In 2004, 2 halibut samples were collected from Kiska island, and 1 from Rat Island. 
Table L-7. Summary of ${ }^{238}$ U Results for Marine Flora and Fauna: 2004 (CRESP) vs. 2011 Sampling

\begin{tabular}{|c|c|c|c|c|c|c|c|c|}
\hline & \multicolumn{4}{|c|}{2004 (CRESP) } & \multicolumn{4}{|c|}{$2011^{\mathrm{a}}$} \\
\hline $\begin{array}{l}\text { Species } \\
\text { Area }^{b}\end{array}$ & $n$ & $\begin{array}{l}\%> \\
M D C\end{array}$ & $\begin{array}{l}\text { Range of } \\
\text { Detects }\end{array}$ & $\begin{array}{c}\text { Range of } \\
\text { MDCs }\end{array}$ & $n$ & $\begin{array}{l}\%> \\
M D C\end{array}$ & $\begin{array}{l}\text { Range of } \\
\text { Detects }\end{array}$ & $\begin{array}{c}\text { Range of } \\
\text { MDCs }\end{array}$ \\
\hline \multicolumn{9}{|l|}{ Dragon Kelp } \\
\hline Amchitka & 15 & $100 \%$ & $5.2-57.0$ & $0.9-2.0$ & 27 & $100 \%$ & $9.6-35.4$ & $0.001-0.003$ \\
\hline Kiska/Adak* & 4 & $100 \%$ & $10.7-33.8$ & $0.9-1.3$ & 18 & $100 \%$ & $6.3-27.7$ & $0.001-0.002$ \\
\hline \multicolumn{9}{|c|}{ *Kiska (2004 only)/Adak (2011 only). } \\
\hline \multicolumn{9}{|l|}{ Horse Mussel } \\
\hline Amchitka & 6 & $100 \%$ & $11-61.6$ & $0.7-1.4$ & 9 & $100 \%$ & $12.2-67.1$ & $0.002-0.04$ \\
\hline Kiska/Adak & 2 & $100 \%$ & $7.5-12.6$ & 1.0 & 6 & $100 \%$ & $6.8-41.1$ & $0.001-0.002$ \\
\hline \multicolumn{9}{|l|}{ Rockfish } \\
\hline Amchitka & 0 & & & & 26 & $100 \%$ & $1.9-13.9$ & $0.001-0.003$ \\
\hline Kiska/Adak & 1 & $100 \%$ & 49.5 & 1.0 & 15 & $100 \%$ & $1.0-11.4$ & $0.001-0.003$ \\
\hline \multicolumn{9}{|l|}{ Irish Lord } \\
\hline Amchitka & 2 & $100 \%$ & $8.9-16.4$ & $1.0-1.4$ & 27 & $100 \%$ & $2.0-16.1$ & $0.001-0.003$ \\
\hline Kiska/Adak & 1 & $100 \%$ & $12.7-12.7$ & 1.0 & 18 & $100 \%$ & $1.5-13.0$ & 0.002 \\
\hline \multicolumn{9}{|l|}{ Pacific Cod } \\
\hline Amchitka & 14 & $93 \%$ & $1.0-7.0$ & $0.7-1.5$ & 3 & $100 \%$ & $0.8-2.1$ & 0.001 \\
\hline Kiska/Adak & 7 & $57 \%$ & $-0.004-6.7$ & $0.7-1.3$ & 2 & $100 \%$ & $1.2-1.4$ & 0.001 \\
\hline \multicolumn{9}{|l|}{ Halibut } \\
\hline Amchitka & 4 & $100 \%$ & $1.6-20$ & $1.0-2.2$ & 3 & $100 \%$ & $0.2-0.65$ & $0.001-0.002$ \\
\hline Kiska/Adak & $3^{a}$ & $100 \%$ & $3.0-3.4$ & $0.8-0.9$ & 1 & $100 \%$ & 0.86 & 0.002 \\
\hline \multicolumn{9}{|l|}{ Rockweed } \\
\hline Amchitka & 10 & $100 \%$ & $46-118$ & $0.8-1.5$ & 27 & $100 \%$ & $29.0-121$ & $0.001-0.002$ \\
\hline Kiska/Adak & 4 & $100 \%$ & $60-121$ & $0.9-1.2$ & 18 & $100 \%$ & $37.0-95$ & $0.001-0.002$ \\
\hline
\end{tabular}

$n=$ number of samples; MDC = minimum detectable concentration

${ }^{\text {a }}$ A more detailed summary of ${ }^{238} \mathrm{U}$ data for the 2011 sampling effort is provided in Table 13.

b Reference (background) area Kiska was sampled in 2004 only, whereas Adak Island was sampled in 2011 only.

${ }^{c}$ Maximum detections for both years are highlighted (yellow) to facilitate comparison.

${ }^{d}$ In 2004, 2 halibut samples were collected from Kiska island, and 1 from Rat Island. 
This page intentionally left blank 